\title{
LOW-FLOW CHARACTERISTICS OF OHIO STREAMS
}

U.S. Geological Survey

Open-File Report 81-1195

Prepared in cooperation with the Ohio Environmental Protection Agency

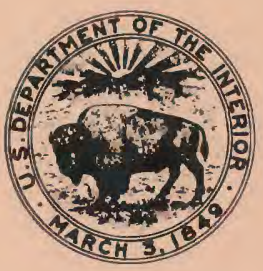





\section{UNI'TED S'TATES \\ DEPARTMENT OF THE INTERIOR \\ Geological Survey}

LOW-FLOW CHARACTERISTICS OF OHIO STREAMS

by Dorothy Pompey Johnson and Kevin D. Metzker

U.S. GEOLOGICAL SURVEY

Open-File Report 81-1195

Prepared in cooperation with the

OHIO ENVIRONMENTAL PROTECTION AGENCY

Columbus, Ohio

1981 


\author{
UNITED STATES DEPARTMENT OF THE INTERIOR \\ JAMES G. WATT, Secretary \\ GEOLOGICAL SURVEY \\ Dallas L. Peck, Director
}

For additional information write to:

U.S. Geological Survey

975 West Third Avenue

Columbus, Ohio 43212 
Abstract -

Introduction

Climatic and geologic Eactors of surface water

$$
\text { in Ohio }
$$

Low-flow characteristics

Streamflow characteristics

Presentation of data

Accuracy of data

Regionalization techniques for estimating low flows - -

Analysis of base flow measurements _-

Multiple regression analysis

Conclusion -

Selected references -

Appendixes -

1. List of low-flow sites in numerical order ---

2. List of low-flow sites in alphabetical order--

3. Low-flow data

\section{ILLUSTRATIONS}

Page

Figure 1 Map of Ohio showing glaciated and

unglaciated areas -

2 Graph showing frequency curve of the annual 7-day low flows of Little Beaver Creek near East Liverpool, Ohio, 1917-78 - - -

3 Graph showing flow-duration curve for Little Beaver Creek near East Liverpool, Ohio, 1917-78 _-

4 Graph showing relation of daily mean base flows of Yellow Creek near Hammondsville, Ohio to concurrent flows of Little Beaver Creek near East Liverpool, Ohio -

5 Graph showing frequency curves of the annual 7-day low flows of Little Beaver Creek near East Liverpool, Ohio $1917-78,1929-38,1969-78$ 
Figure 6 Graph showing relation of daily mean base flows of Yellow Creek near Hammondsville, Ohio and Short Creek near Dillonvale, Ohio to concurrent flows of Little Creek near East Liverpool, Ohio -

7 Map of Stillwater River basin measuring sites used in the gain and

loss study, October 31, $1979 \ldots$

8 Map of Great Miami River basin showing sites used in the multiple regression analysis - - - - -

9 Map showing precipitation of the Upper Great Miami River basin, 1931-1960 -

10 Hydrologic soil-grouping map of Upper Great Miami River basin _...-

11 Graph of flow-duration curves showing effect of basin geology on low flow -

TABLES

Table 1 Results of gain and loss study, stillwater River basin, uctober 31, 1979 -

2 Comparison of $7 \mathrm{Q}_{2}$ and $7 \mathrm{Q}_{10}$ low filo:s calculated Erom station data to estimates calculated from gain and loss study (Stillwater River at Pleasant Hill 03265000 is index station)

3 Summary of regression equations for annual low flows for Upper Great Miami River basin 
Factors for converting inch-pound units to the International system of metric units (SI) are given below:

To convert from

inch (in)

foot ( $f t$ )

mile $(\mathrm{mi})$

mile $\mathrm{e}^{2}\left(\mathrm{mi}^{2}\right)$

million gallons (Mga1/d)
To

millimeter (mm)

meter ( $\mathrm{m}$ )

$\mathrm{kilometer}(\mathrm{km})$
$\mathrm{kilometer}\left(\mathrm{km}^{2}\right)$

meter ${ }^{3}$ per second $\left(\mathrm{m}^{3} / \mathrm{s}\right)$

meters ${ }^{3}$ per second

$\left(\mathrm{m}^{3} / \mathrm{s}\right)$
Multiply by

$$
\begin{aligned}
& 25.4 \\
& 0.3048 \\
& 1.609 \\
& 2.590 \\
& 0.02832 \\
& 0.0438
\end{aligned}
$$





\title{
LOW-FLOW CHAFACTFRISTICS OF CHIC STREAMS \\ by Dorothy Pompey Johnson and Kevin D. Metzker
}

\section{ABSTFACT}

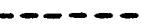

Adequacy of streamflcw for water supplies or waste water assimilation is commenly evaluated in terms of low-flow characteristics. This report presents annual and seasonal lowflow characteristics fcr 237 sites on regulated and unregulated streams in Ohio.

In adition to compilation of site data, two regionalization methcds--an analysis of base flow measurements and multiple regression analysis--were applied to test basins to determine the feasibility and accuracy of each method. An analysis of base flow measurements (gain and loss studies) was made on the stillwater River basin. with error ranging from -39 to 59 percent. Multiple regression analyses were made on the hydrologic data from the Great Miami River kasin north of Dayton, which resulted in a standard error of estimate rariging from 110 to 139 percent. Through the use of an index of relative infiltration, the standard error of estimate was reduced to a range cf 33 to 40 percent.
\end{abstract}

INTRODUCIION

A stream's adequacy as a water supply, its capacity to assirilate liquid wastes, and its suitability as a habitat for aquatic life are all commonly evaluated in terms of low-flow characteristics (Riggs, 1972). In Ohio, the legal basis for wat $\in$ r pollution control is tied to water guality standards that inccrporate the 7-day 1C-year low-flow characteristics of the receiving stream. 
In the most recent comprehensive work on low-flow characteristics of Ohio streams, Anttila, (1970) utilized streamflow records thrcugh the 1967 water year for 180 sites in Ohio having 10 or more years of essentially unregulated flow. Low-flow characteristics reported are the 7-day annual minimum mean discharges for the 2-, 10-, and 20-year recurrence intervals. Attempts tc use regression analysis to regionalize low-flcw frequency characteristics in that study were unsuccessful.

This report is an update and extension cf Antilla's work. It presents annual and seasonal low-flow characteristics for 237 sites. Also included are the results of two regionalization techniques: a multiple regression analysis, and an analysis of base flow measurements in test basins.

\section{CLIMATIC AND GEOLOGICAL FACTCRS OF SURFACE WATER HYDROLOGY IN OHIO}

The climate of 0 hio is temperate. Mean annual temperature is $51.6^{\circ} \mathrm{F},\left(10.9^{\circ} \mathrm{C}\right)$ ranging from $49.6^{\circ} \mathrm{F}\left(9.8^{\circ} \mathrm{C}\right)$ in the northeast to $56.9^{\circ} \mathrm{F}\left(13.8^{\circ} \mathrm{C}\right)$ in the south. Mear annual precipitation is 38 inches and ranges from 31 inches in the northwest to 44 inches in the scuth. Runoff averages 13 inches per year, with a range of 10 inches per year in the northwest to 17 inches per year in the northeast and southeast farts.

Chio is divided diagonally from southwest to northeast by the roundary of glaciation (fig. 1). The northern and western two-thirds of Ohio is covered by glacial arift, consisting mainly of clayey till, with outwash sand and gravel in major valleys. Sand and gravel also occurs as lenses within the till. The southeastern one-third of the state is underlain mainly by shale and sandstone of Pennsylvanian age. Glacial till has low permeability; outwash has moderate to high permeability; and bedrock has low to moderate permeability.

\section{LOW-FIOW CHARACTERISTICS}

Annual and seasonal low-flow data are presented for 156 longterm continuous-record gaging stations and 81 low-flow partialrocord sites for unregulated and regulated streams.

Iong-term continuous-record gaging stations are sites where daily flow data have been systematically okserved for 10 or more years. The long-term stations in this study include some regulated streams. 


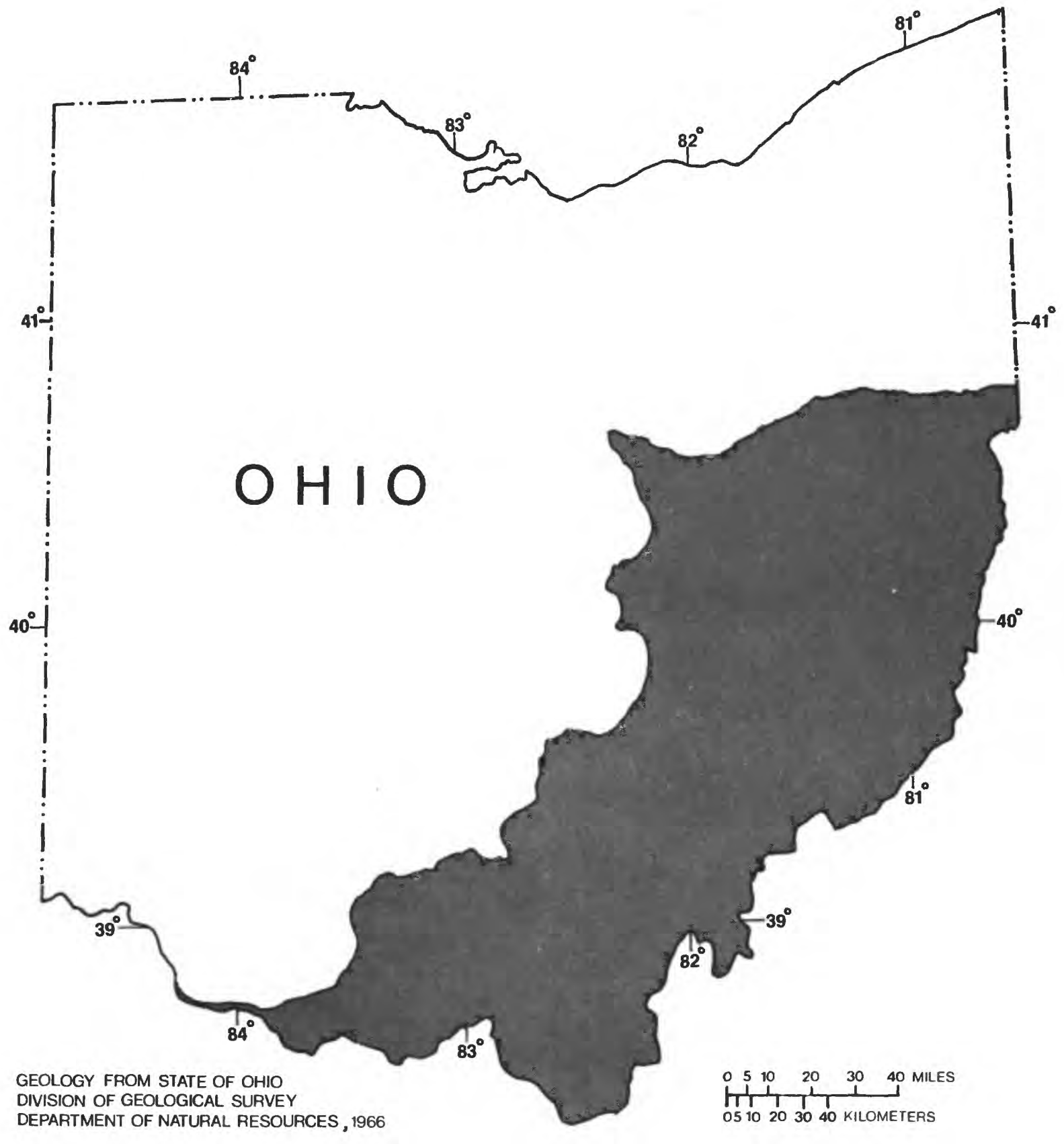

EXPLANATION

Unglaciated

Glaciated

Figure I. --Glaciated and unglaciated areas. 
Iow-fiow partial-reccrd sites are sites where sparse low-flow data have been collected over a several years for use in hydrologic analyses. Sites where systematic observations of daily flow data have been obtained for 2 or more but less than 10 years (short-term continucus-record gaging station) are also considered here as low-flow partial-record sites.

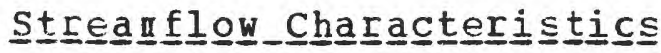

Streamflow characteristics presented in this report are average discharge, minimum discharge recorded, low-flow Erequency, and flow duration. These characteristics represent current flow conditions at the site without adjustments for withdrawals, diversions, $\mathrm{cr}$ inflows.

Average discharge is the arithmetic average of the daily mean flows Ecr all complete water years.

Minimum discharge is the lowest discharge measured at a site or the lowest daily discharge recorded.

A low-flow frequency curve is a grach showing magnitude and frequency of minimum flows for a given $1 \in$ ngth of time (figure 2). Frequency is usually expressed as the average interval, in years, between occurrences of ar annual or seasonal minimum flow equal to or less than that shown by the magnitude scale (Langbein and Iseri, 1960). Time pericds in frequency analysis are: Annual (April 1-March 31), warm weather (May 1-November 30), recreation (Jun€ 1-August 31), autumn (September 1-November 30), winter (December 1-February 28/29), and fish spawn (March 1-llay 31).

Iow-flow frequency curves are computed by: (a) Listing the lowest daily flow value for each time period; (b) arranging the values in order of magnitude and assigning order numbers, beginning with the smallest as number 1; (c) computing the recurrence interval (average interval of time between hydrologic events of a given or lesser magnitude) for $\in a c h$ value through the use of the formula

$$
R I=(n+1) / m
$$

where

FI is the recurrence interval in years.

$n$ is the total number of years.

$m$ is the order number. 


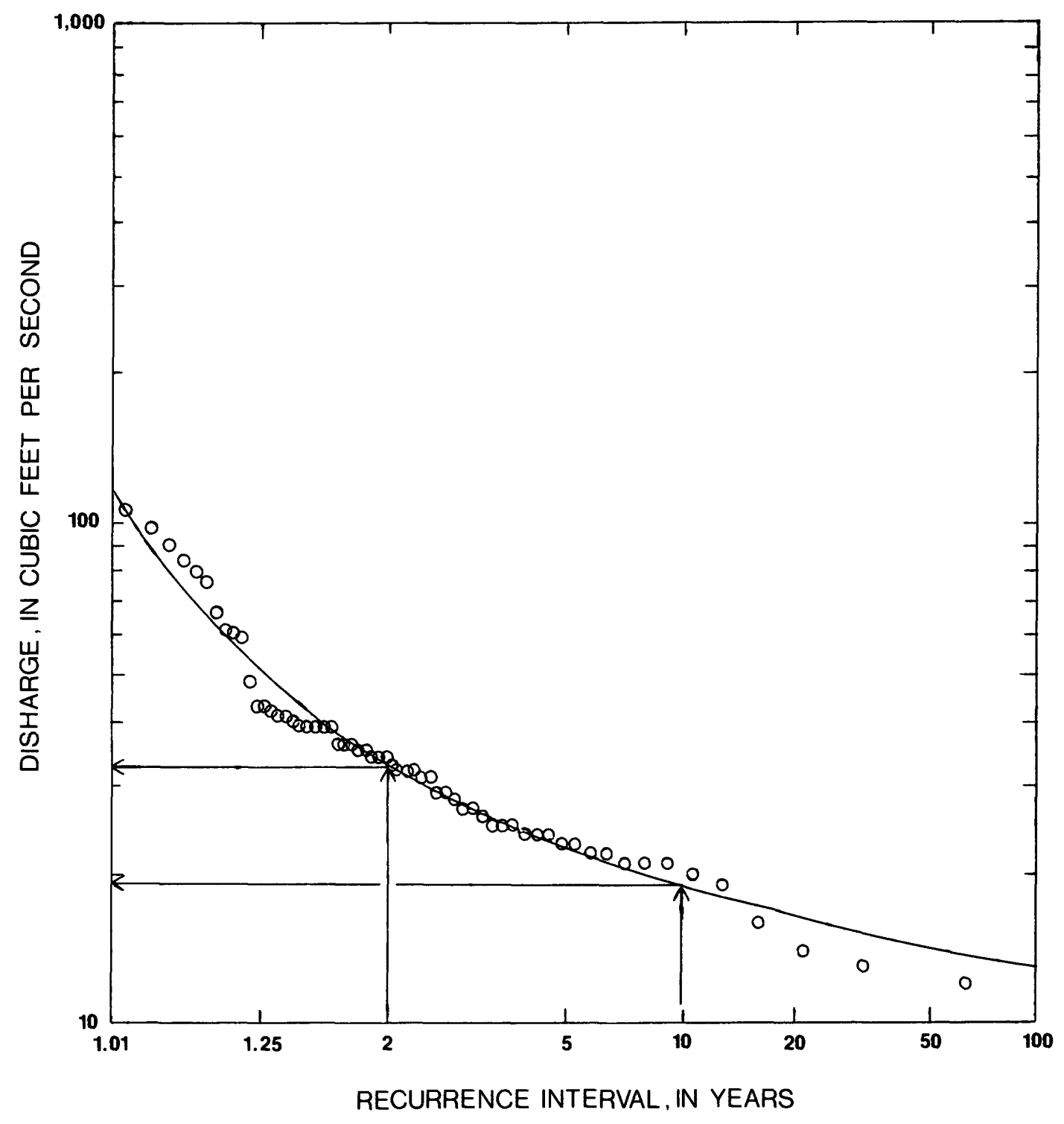

Figure 2. --Frequency curve of the annual 7-day low flows of Little Beaver Creek near East Liverpool, Ohio, 1917-78. 
(d) Flotting each flow value against its computed recurrence interval on log-probability paper; and (e) drawing a smooth curve through the plotted points, either graphically or mathematically, by assuming a log pearscn Type III frequency distribution of the data. A Log Fearson Type III frequency distribution is the preferred method. A Iog-Pearson Type III frequency curve is comfuted using the formula

$$
Q_{i}=M=K_{i} S
$$

where

$i$ is a sfecified recurrence interval, $i$

$Q$ is the discharge $c n$ the frequency curve corresponding to i.

$M$ is the mean of $t h \in$ logarithms (base 10) of the data,

$S$ is the standard deviation of the logarithms (base 10) of the data.

$K$ is a frequency factor that is a function of recurrence interval and the coefficient of $s k \in w$.

The curve in figure 2 is a mathematical fit of the annual 7day low flows (values of the lowest mean discharge for 7 consecutive days) of Iittle Beaver Creek near East Liverpool for climatic years (April 1 to March 31) 1917-78. The 7-day 10-year $(7010)$ is $19 \mathrm{ft} 3 / \mathrm{s}$; that is, the probability is 0.1 that the lowest average 7 days of flow in any given year will be less than or equal to $19 \mathrm{ft} 3 / \mathrm{s}$. Frobability is equal to the reciprocal of the recurrence interval. In other words, the average 7 days of flow will be less than or equal to $19 \mathrm{ft} 3 / \mathrm{s}$ at intervals zveraging 10 years in length. The $7 \mathrm{Q}_{2}$ is $33 \mathrm{ft} / \mathrm{s}$; there is a 50-percent chance each year that the lowest average 7 days of flow will be less than $33 \mathrm{ft} 3 / \mathrm{s}$; or the 1 owest average 7 days of flow will be less than or equal to $33 \mathrm{ft} 3 / \mathrm{s}$ every 2 years.

Historically, values for the $7 Q_{2}$ and $7 Q_{10}$ have been calculated for only the annual period. More recently, these values have been calculated for periods shorter than 1 year which correspond to various seasonal activities or occurrences (e.g. reareation and fish spawing).

A flow-duration curve is a cumulative frequency curve that shows the percentage cf time that specified discharges are equale or exceeded during a given period (figure 3 ). 


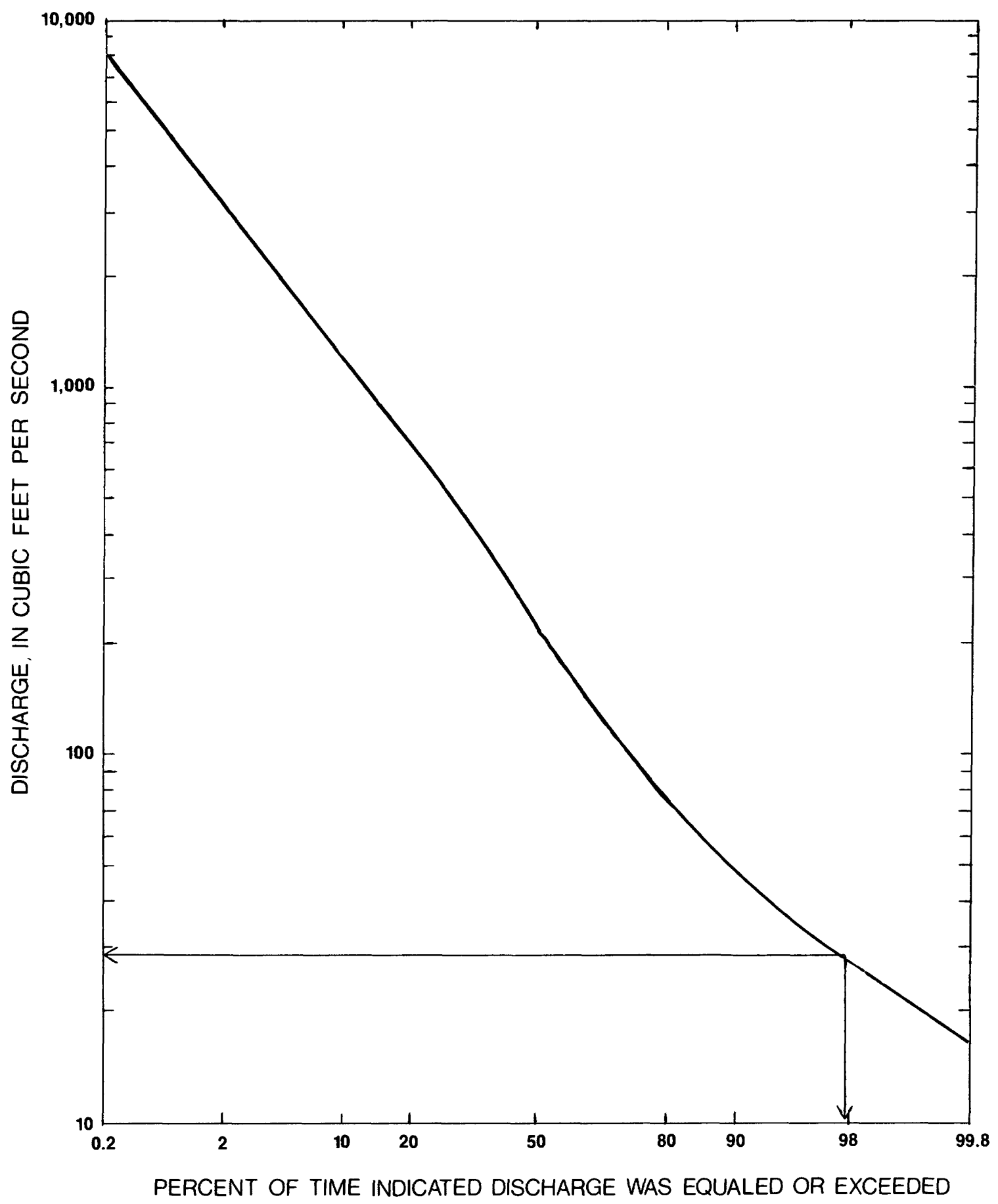

Figure 3. --Flow duration curve for Little Beaver Creek near East Liverpool, Ohio, 1917-78. 
Flow-duration curves are computed using the "total-period method" by: (a) Placing daily discharges in classes according to their magnitude; (b) summing the occurrences of each class beginning with the lowest class; (c) computing the cumulative percent of time each class is exceeded; and (d) plotting the data with the discharge as the ordinate and the time in percentage of total time as the abscissa (Searcy, 1959).

The flow-duration curve is a cumulative frequency curve of the flows for the period of data used. It does not represent the distribution cf the yearly flows. The curve in figure 3 shows the duration of daily flows of Little Eeaver Creek near East Liverfool for climatic years 1917-78. Flows less than $27 \mathrm{ft} 3 / \mathrm{s}$, which were equaled or exceeded 98 fercent of the time, have occurred 2 percent of the 62-year period, nct 2 percent of the time each year. The duration curve is a summary of past hydrologic events, not a predictor of the future.

Icw-flow partial-reccrd sites do not have enough data for either the frequency cr duration analyses as described above. Instead, available data are correlated with those from a hydrologically similar lcng-term continucus-record gaging station (index station) at which the low-flow characteristics are well defined. The correlations are made by plotting on log-log paper either daily mean rase flows or discharge measurements of base Elow aqainst concurrent flows at the index station. A straight line through the plotted points defines the relationship of the partial-record site and the index statior. The relationship defined is valid only withir the range of general discharges used to $d \in f$ ine it.

An example of the correlation method is illustrated in figure 4. To demonstrate the method, concurrent flows were plotted for two long-term-continuous record gaging stations: Yellow creek near Hammondsville and Little Beaver Creek near East Iiverpool. The 7-day 10-year flow is $1.9 \mathrm{ft} / \mathrm{s}$ for Yellow creek near Hammondsville. (See Appendix 3.)

Yellow Creek near Hammondsville was used as the index station and Little $B \in a v \in I$ Creek near East Iiverpool as a low-flow partial-record site. using the method described the 7-day 10year low flow for little Beaver creek is $18 \mathrm{ft} / \mathrm{s}$, which compares quite favorably with the long term 7-day 10-year low flow of 19 $f \pm 3 / s$. 


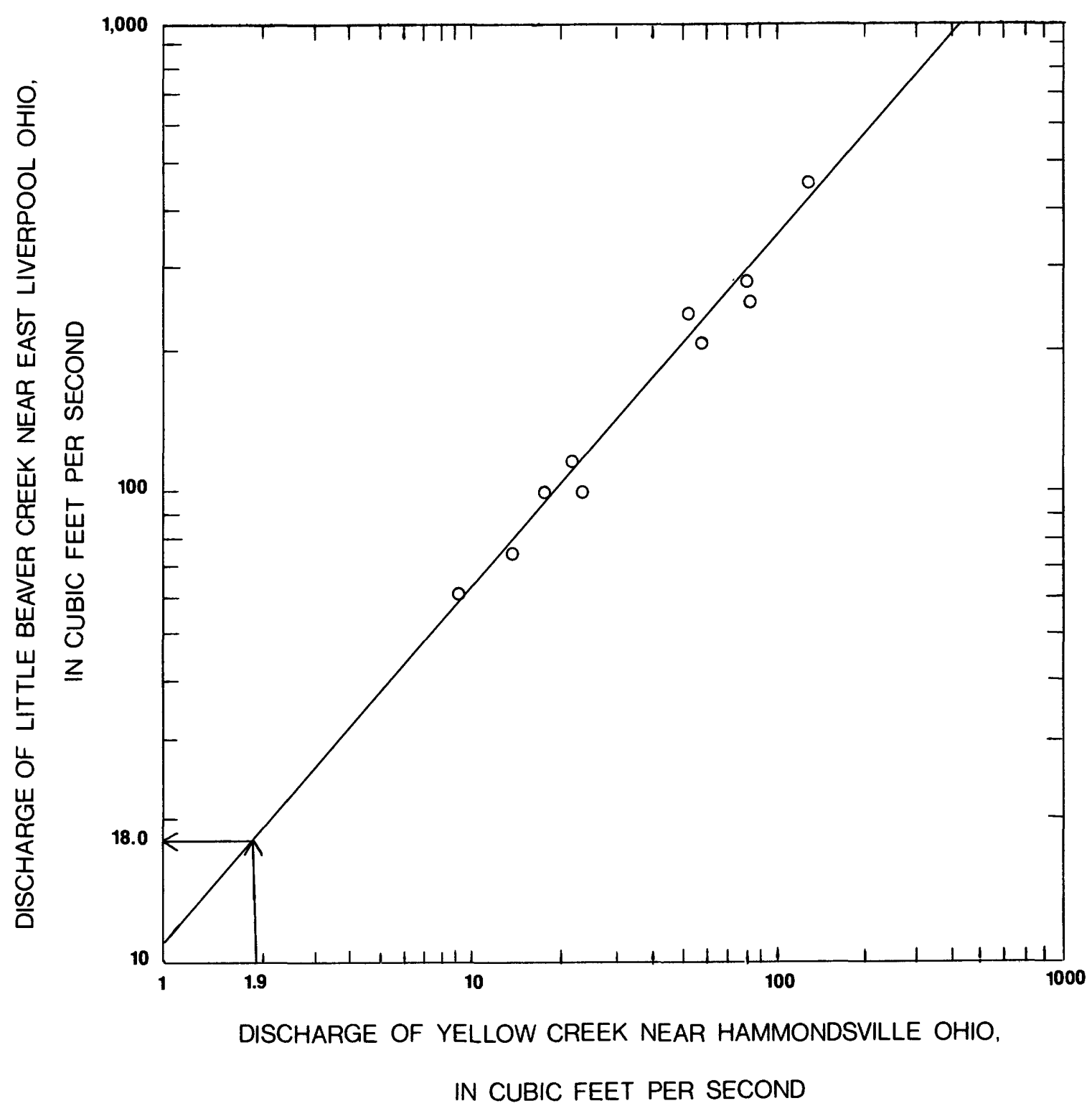

Figure 4. --Relation of daily mean base flows of Yellow Creek near Hammondsville, Ohio to concurrent flows of Little Beaver Creek near East Liverpool, Ohio. 


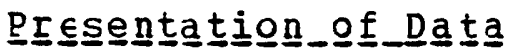

station descriptions and low-flow data for 237 sites (156 long-term sites, 81 partial-record sites) are given in Appendix 3. The stations within the Ohio Piver basin are listed first, in downstream order, follcwed by stations within the St. Lawrence Piver (Lake Erie) basin, also in downstream order. Appendix 1 is an index to each site by U.S. Geological survey station number. Appendix 2 is an alpharetical index by stream name. Information presented for each of the long-term continuous record gaging stations inclides: the location, drainage area, tributary to, discharge data used, remarks, and selected discharge characteristics.

The latitude, longitude, county name, hydrologic unit number, and the location of the gage with respect to the municipality mentioned in the station name are given in the "locaticn" paragraph. Hydrologic unit number is an identification number of a gecgraphic area representing part or all of a surface drainage basin.

"rrainage area" is the area, measured in a horizontal plane, frow which direct surface water runoff drairs into a stream.

"Tributary to" indicates the larger stream into which the repcrted stream flows.

The "discharge data used" section provides a period of continucus record for the long-term stations. For most of these stations, the 1978 water year was the last year of discharge data included in the low-flow analyses. For stations having only 9 years of discharge data thrcugh 1978 water year, data for the 1979 water year was included. Discharge data for discontinued staticns were used if the record refresented current flow conditions of the stream.

Under "remarks," significant factors affecting low-flow are descrited, such as effluent point discharges, municipal or industrial withorawals, dams, or diversions.

Tho "selected discharge characteristics" are average aischarge, minimum daily discharge, low-tlow frequency, and flow-duration information. Low-flow frequency and flow-duration characteristics are presented for six periods: Annual (April 1March 31); warm weather (May 1-November 30); recreation (June 1August 31); autumn (September 1-November 30); winter (December 1February 28/29); and fish spawn (March 1 - May 31). Trhe low-flow Erequency characteristics are 2-, 5-, 10-, 20-, and 50-year recurrence intervals fcr the 1-, 7-, and 30-day durations (pericds of consecutive days). Flow-duration characteristics are flows which were equaled or exceeded $98,95,90,85,80,75$, $70,60,50,40,30,20$, and 10 percent of the time. 
Information presented for $e$ ach of the partial-record sites includes location, drainage area, tributary to, discharge data used, index station, remarks, and selected discharge characteristics.

"Iischarge data used" for the partial-record stations were discharge measurements made during base flow periods (i.e. when streamflow is primarily derived from grcund water storage). These data were used to develop plots similar to the one shown in figure 4 .

The "index station" is a long-term continuous-record gaging staticn used to define the base flow at the low-flow partialreccrd site.

"Selected discharge characteristics" presented for the partial-record stations are minimum discharge observed, low-flow frequency, and flow-duration. Low-flow frequency and flowduration characteristics are for the climatic year, warm weather, recreation, and autumn periods. Iow - flow frequency characteristics are the 2 -year and $10-y \in a r$ recurrence intervals for the 7-day and 30-day duraticns. Flow-duration characteristics given are flows that were equaled or exceeded 98 . 95. $90,85,80$ percent cf the time. The $1 \mathrm{cw} \in$ nd of the duration curve describes the low-flow characteristics.

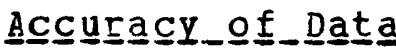

The reliability of gagirg-station reccrds for predicting the behavior of a stream depends upon the accuracy and consistency of the record and how well the period of reccrd represents the longterm flow of the stream (Searcy 1959). The variability of the frequency curve for these different periods of record is shown in figure 5 for Little Beaver Creek near East Iiverpool. The annual 7-day 10-year low flow is, for example, $19 \mathrm{ft} / \mathrm{s}$ for the entire period, but $14 \mathrm{ft} 3 / \mathrm{s}$ for $1929-38$, and $37 \mathrm{ft} 3 / \mathrm{s}$ for $1969-78$.

The reliability cf low-flow partial-record data for predicting the flow of a stream defends on how well the base-flow relationsipip is defined, how far that relationship has to be extrapolated, and the reliability of the estimate at the index station. In figure 6, daily mean base flows of Yellow creek near Hammondsville and Short Creek near Dillonvale for 1977 and 1978 climatic years are plotted with concurrent flcws of Little Beaver Cre $\epsilon$ near East Liverpoo1. The 7-day 10-year low flow for Little Eeaver Creek is $19 \mathrm{ft} 3 / \mathrm{s}$ when the gaging-station record is used. However, it is $18 \mathrm{ft} 3 / \mathrm{s}$ when Yellcw Creek is the index station, and $20 \mathrm{ft} 3 / \mathrm{s}$ when short creok is the index station. 


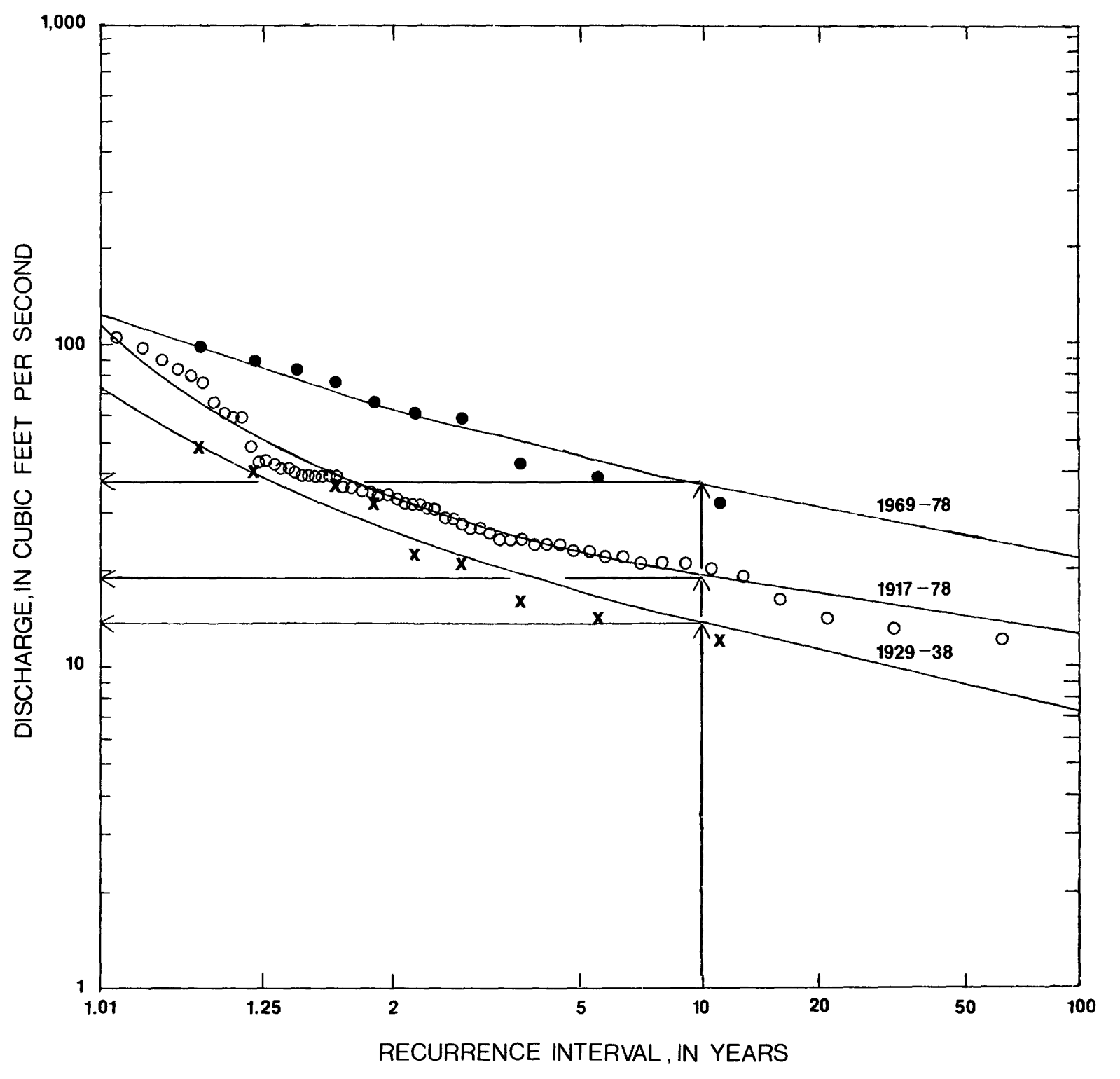

Figure 5. --Frequency curves of the annual 7-day low flows of Little Beaver Creek near East Liverpool, Ohio, 1917-78, 1929-38, 1969-78. 


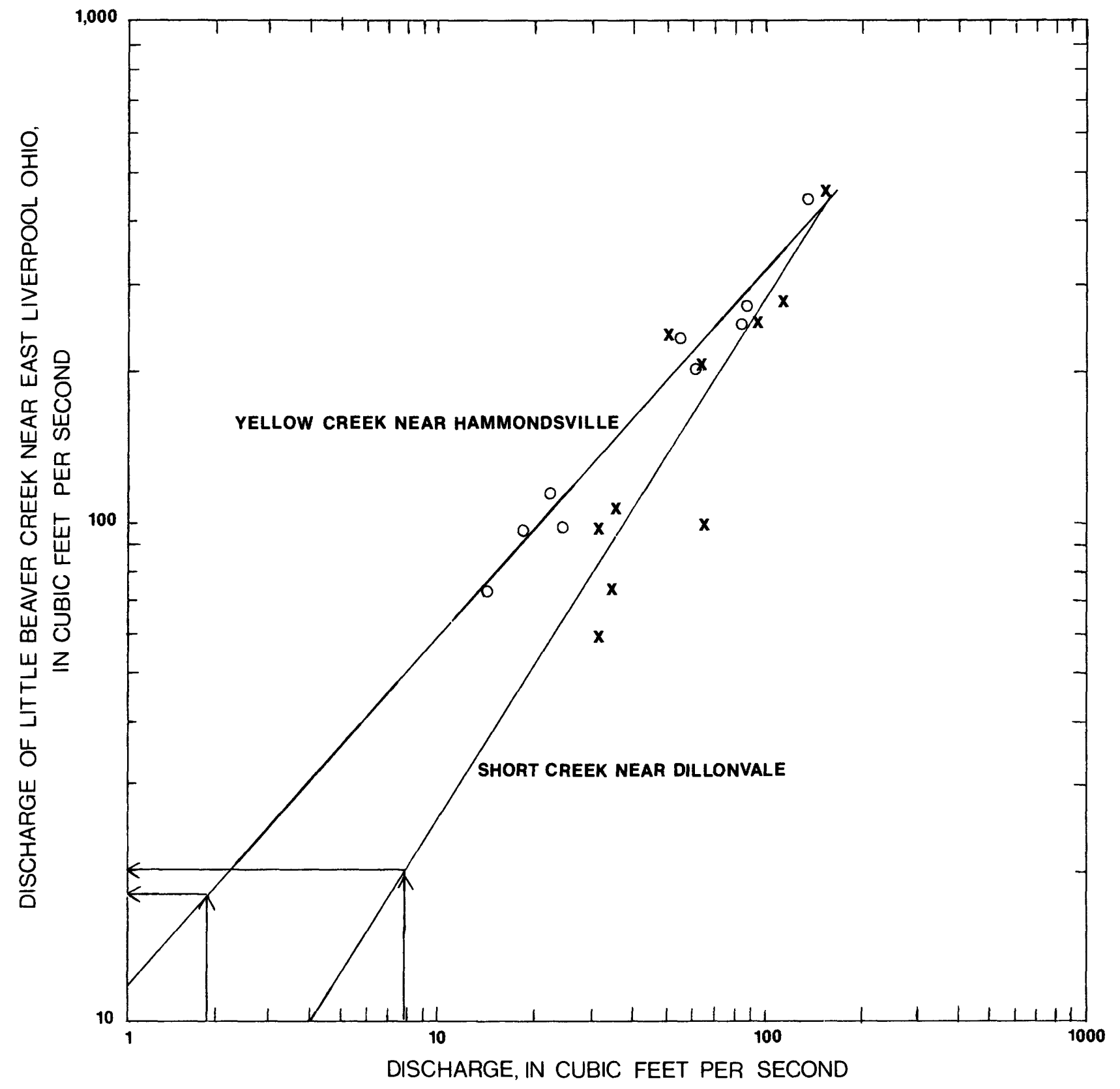

\section{EXPLANATION}

Y Yellow Creek near Hammondsville, Ohio

$x$ Short Creek near Dillonvale, Ohio

Figure 6. --Relation of daily mean base flows of Yellow Creek near Hammondsville, Ohio and Short Creek near Dillonvale, Ohio to concurrent flows of Little Beaver Creek near East Liverpool, Ohio. 
The preceding examples were cited to show the variability of the lcw flow characteristics due to the nature of statistics. Low-flow frequency characteristics are statistical estimates of future events based on past record. Extended periods of record make these estimates more reliable.

\section{PEGIONALIZATION TECHNIQUES FOR ESIIYATING FLOWS}

A method for estimating low-flow characteristics at ungaged sites is needed because streamflow records are collected at only a $f \in w$ of the many pcssible sites where data may be needed. segionalization is the extension of streamflow records in space.

Two regionalization methods examined in this refort are analysis of base-flow measurements and multiple regression analysis. To determine the accuracy and feasibility of each, the methcds are applied in test basins rather tran statewide.

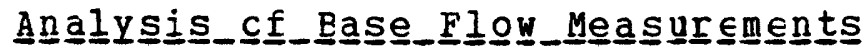

Analysis of base flcw measurements consists of making a gain and lcss study (seepage run) within a basin and correlating the jischarge measurements with the low-flow characteristics of a long-term continuous-reccrd gaging station within the basin.

A gain and loss study (discharge measurements made at intervals along a stream during a period cf base flow) was made on stillwater River and its tributaries. stillwater River is in west central ohio and drains into the Great Miami River upstream Erom Layton. The 33 sites. listed in table 1 and shown in figure 7. refresent drainage areas ranging from 11.3 to 650 square miles. Cf the 33 sites, three are of long-term continuousreccrd, two are of short-term continuous-record, and one is a low Elow fartial-record site. Discharge measurements were made at all the sites on Dctober 31, 1979, during base flow. Base flow varies in magnitude throughout the year and consists solely of ground-water discharge, no surface runoff.

A large field crew is needed to gather the gain and loss field data, as the measurements must all be made in one day.

Icw-flow characteristics presented for 6 ach site are 7-day 2year, and 7-day 10-year low flows. Fcr the three long-term continucus-record gaging stations and the three low-flow partialreccrd sites. low-flow characteristics were computed as described in the section "Streamflow Characteristics Presented" of this repcrt. 
Iow-flow data for the remaining 27 sites were computed using the following formula:

$$
\frac{7 Q_{R I} \text { site }}{Q \text { site }}=\frac{7 Q_{R I} \text { index }}{Q \text { index }}
$$

wher $€$

$$
\begin{aligned}
& 7 Q_{R I} \text { is the } 7 \text {-day } 2 \text {-year low flow or the } 7 \text {-day } 10 \text {-year } \\
& \mathbb{Q} \text { is the flow measurement made }
\end{aligned}
$$

Greenville creek at Bradford was used as an index station for the six ungaged sites on Greenville creek and its tributaries. stillwater River at Pleasant Hill was used as an index station for the remaining 21 ungaged sites.

There is no universally accepted method for computing the accuracy of an analysis of base flow measurements. In table 2 , 7-day 10-year and 7-day 2-year low flcws for the six sites computed from station data are compared with the estimates computed by using stillwater River at Pleasant Hill as the index station. Percentage errcr of the estimates were calculated using the fcllowing formula:

Percent error $=\frac{7 Q_{R I} \text { Seepage run }-7 Q_{R I} \text { Station data }}{7 Q_{R I} \text { Station data }} \times 100$

where

7QRI is the 7-day 2-year or the 7-day 10-year low flow. The error ranges from -39 to 59 percent. 


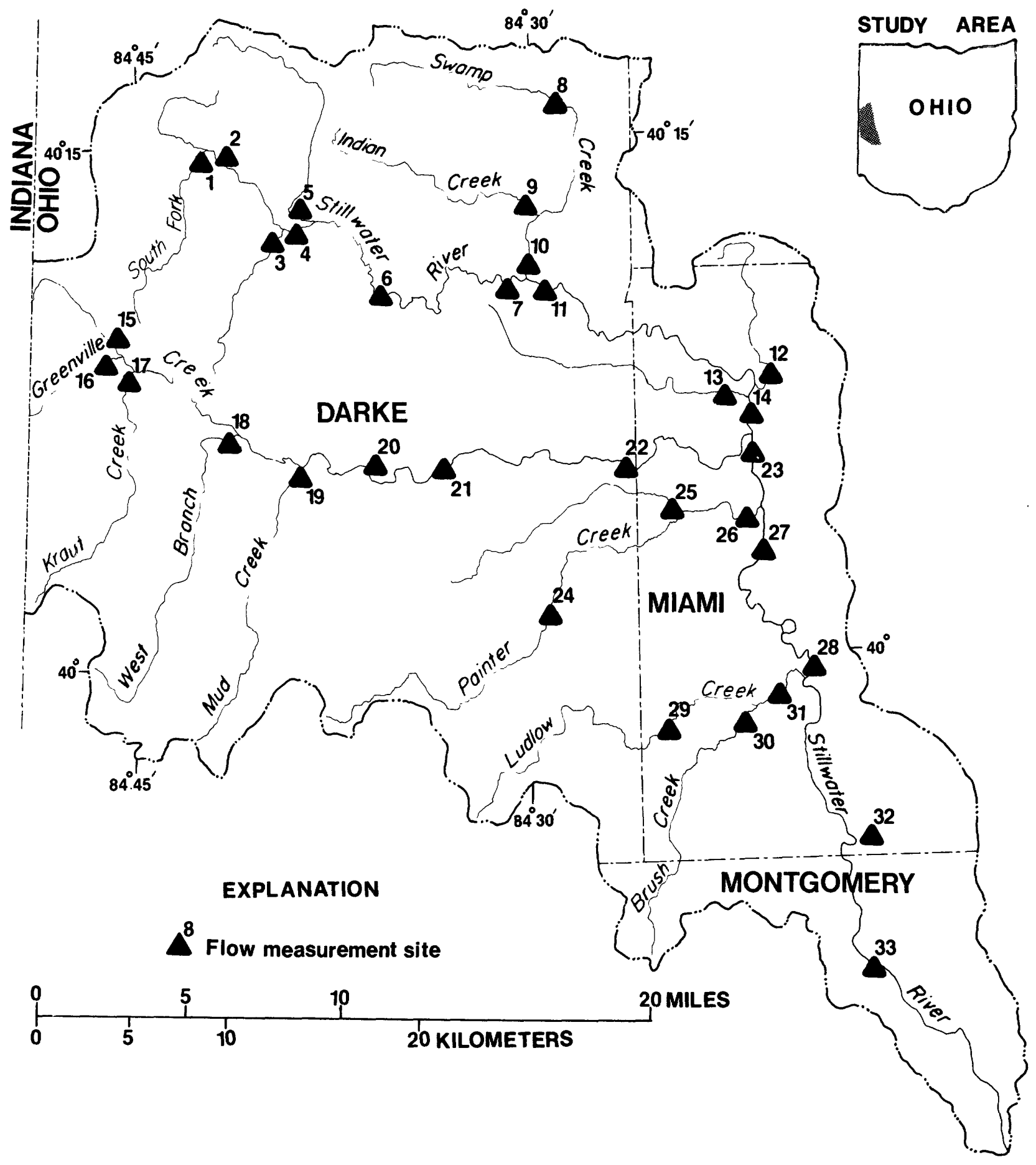

Figure 7. --Stillwater River basin measuring sites used in the gain and loss study, October 31, 1979. 


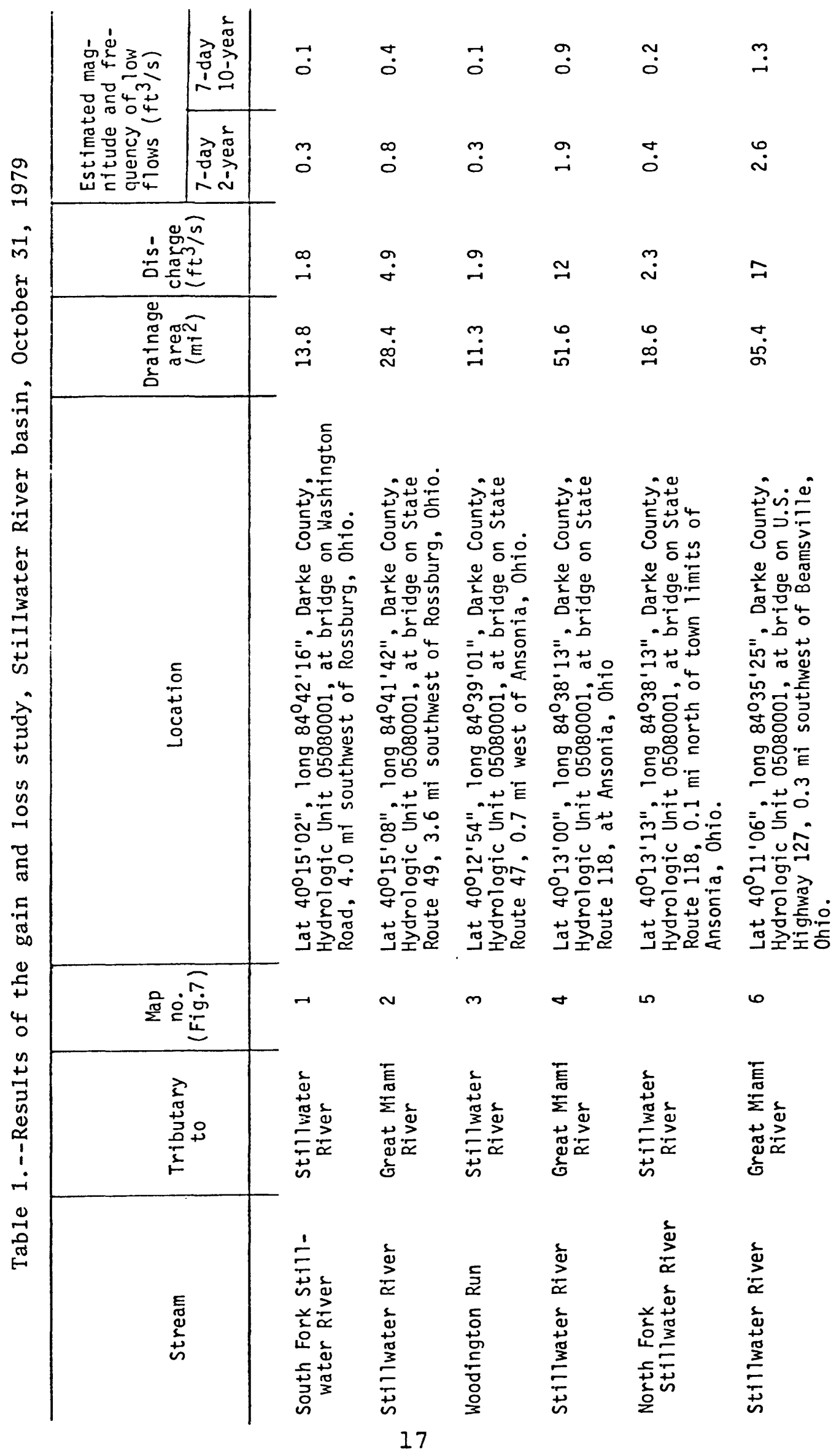




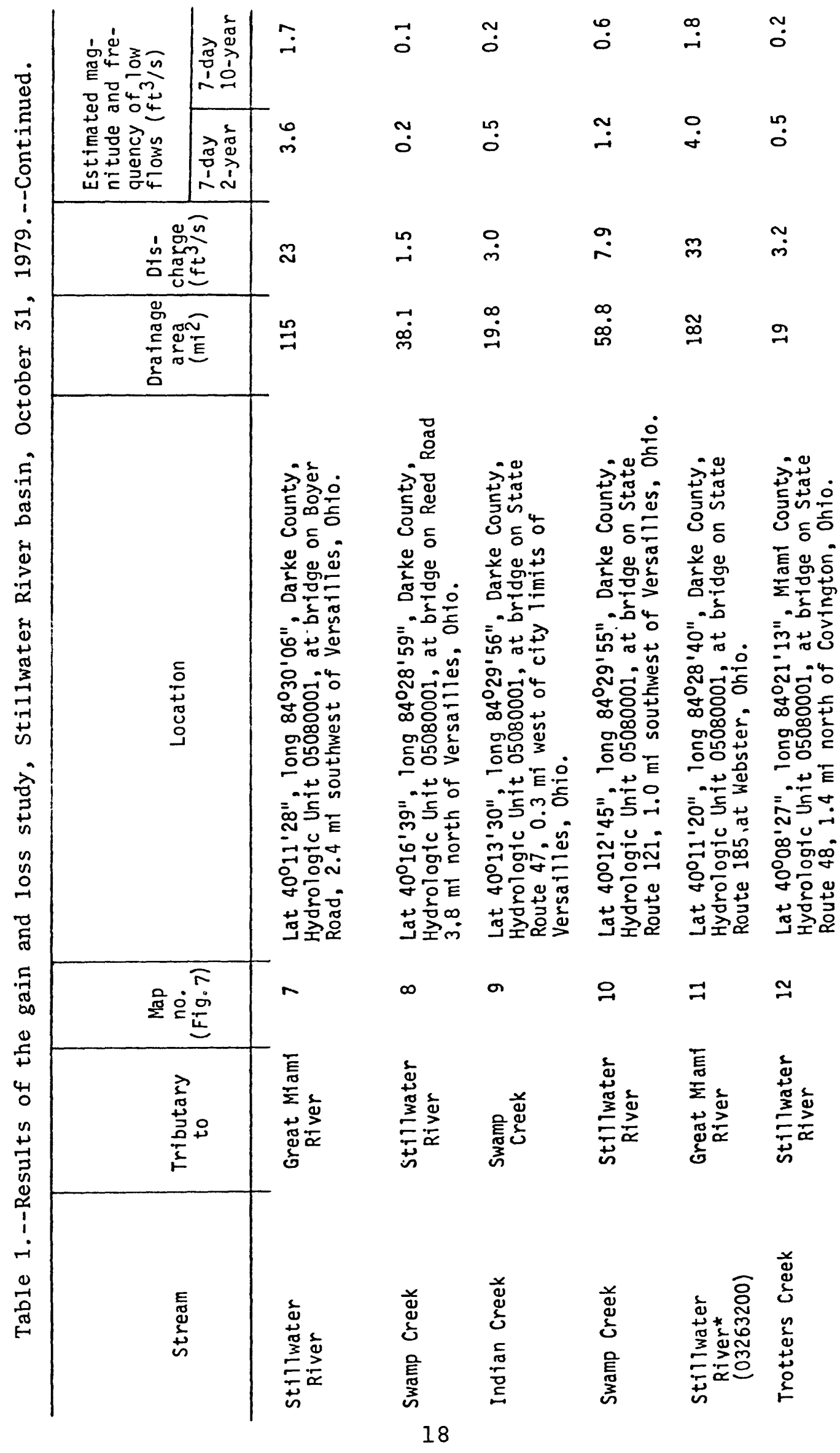




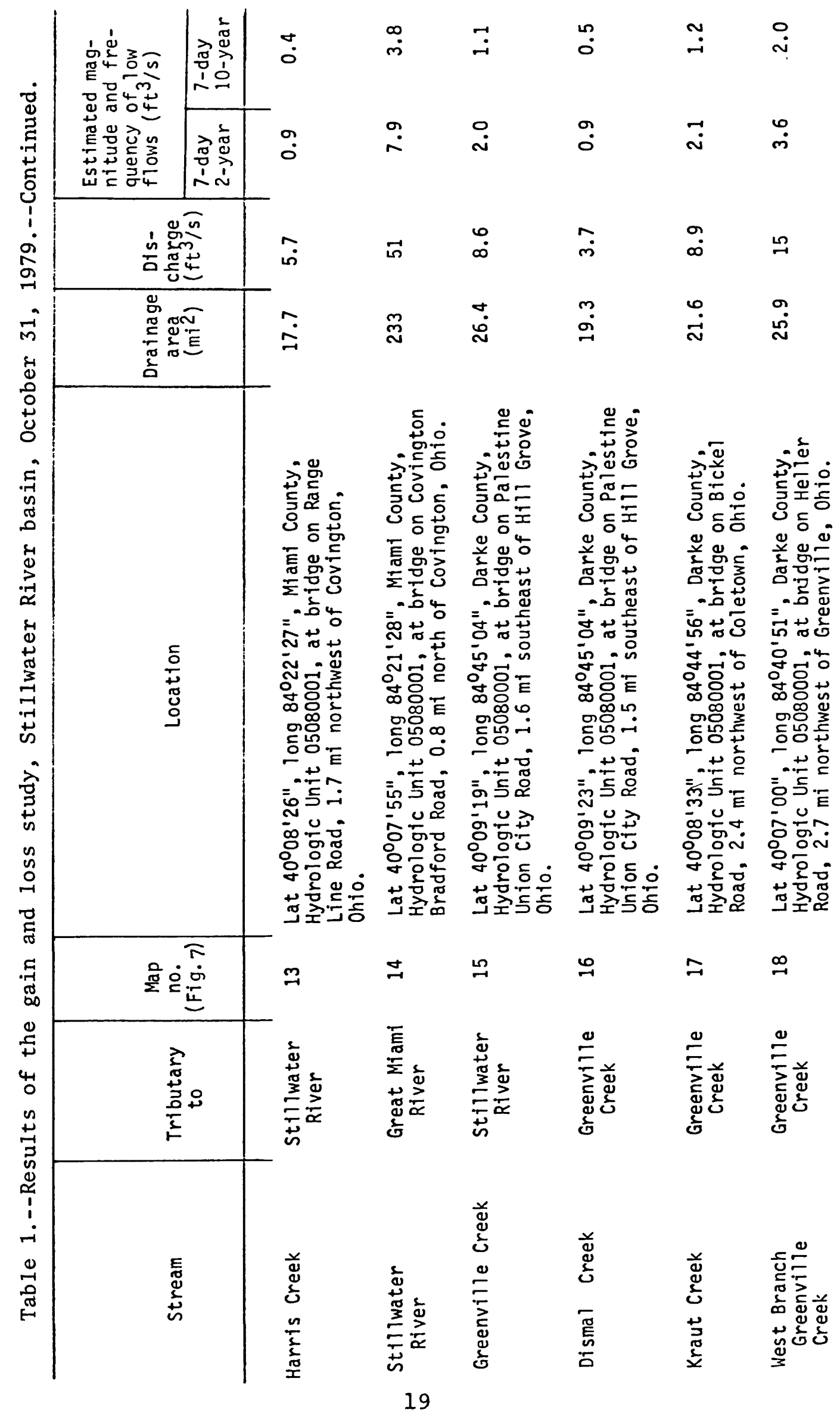




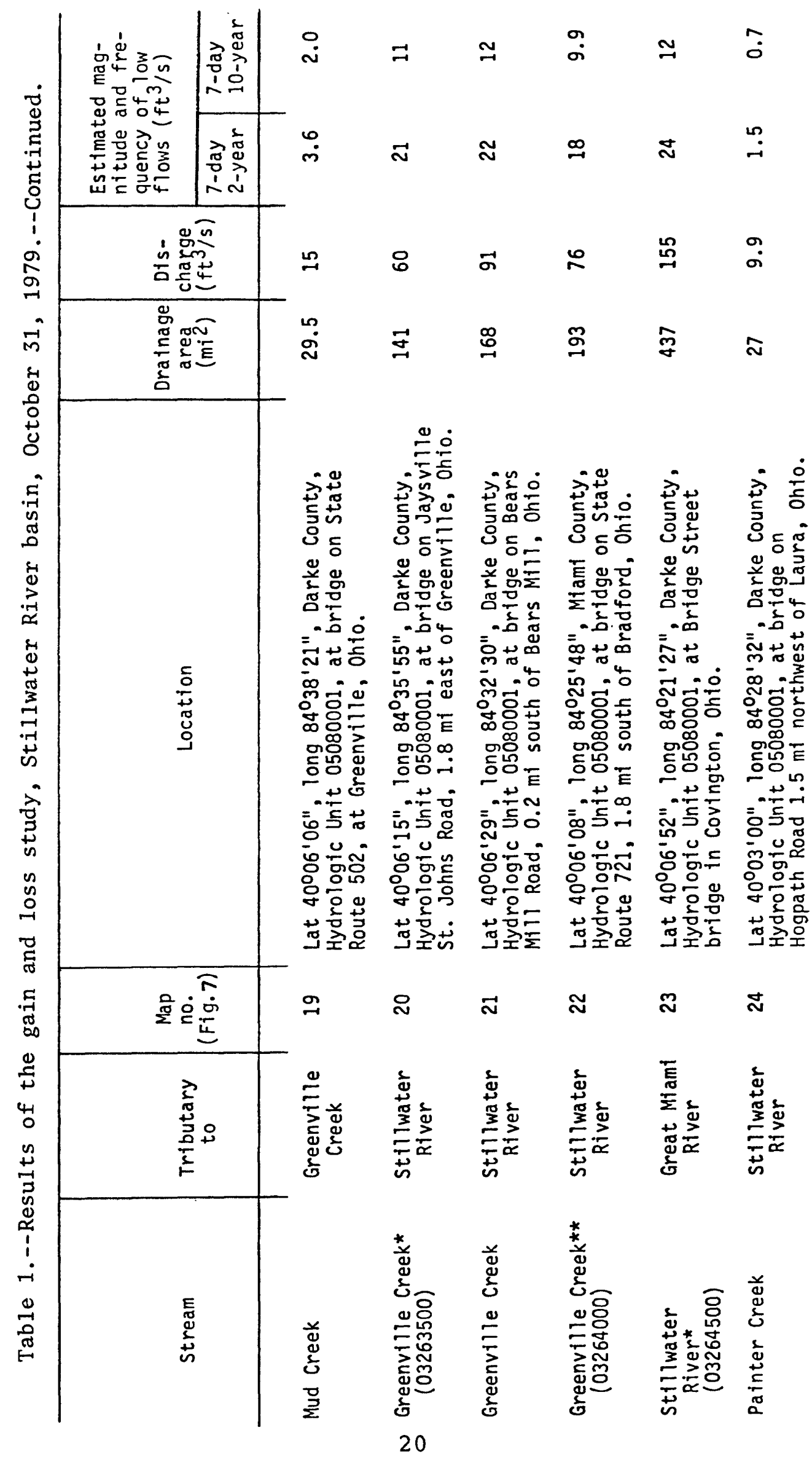




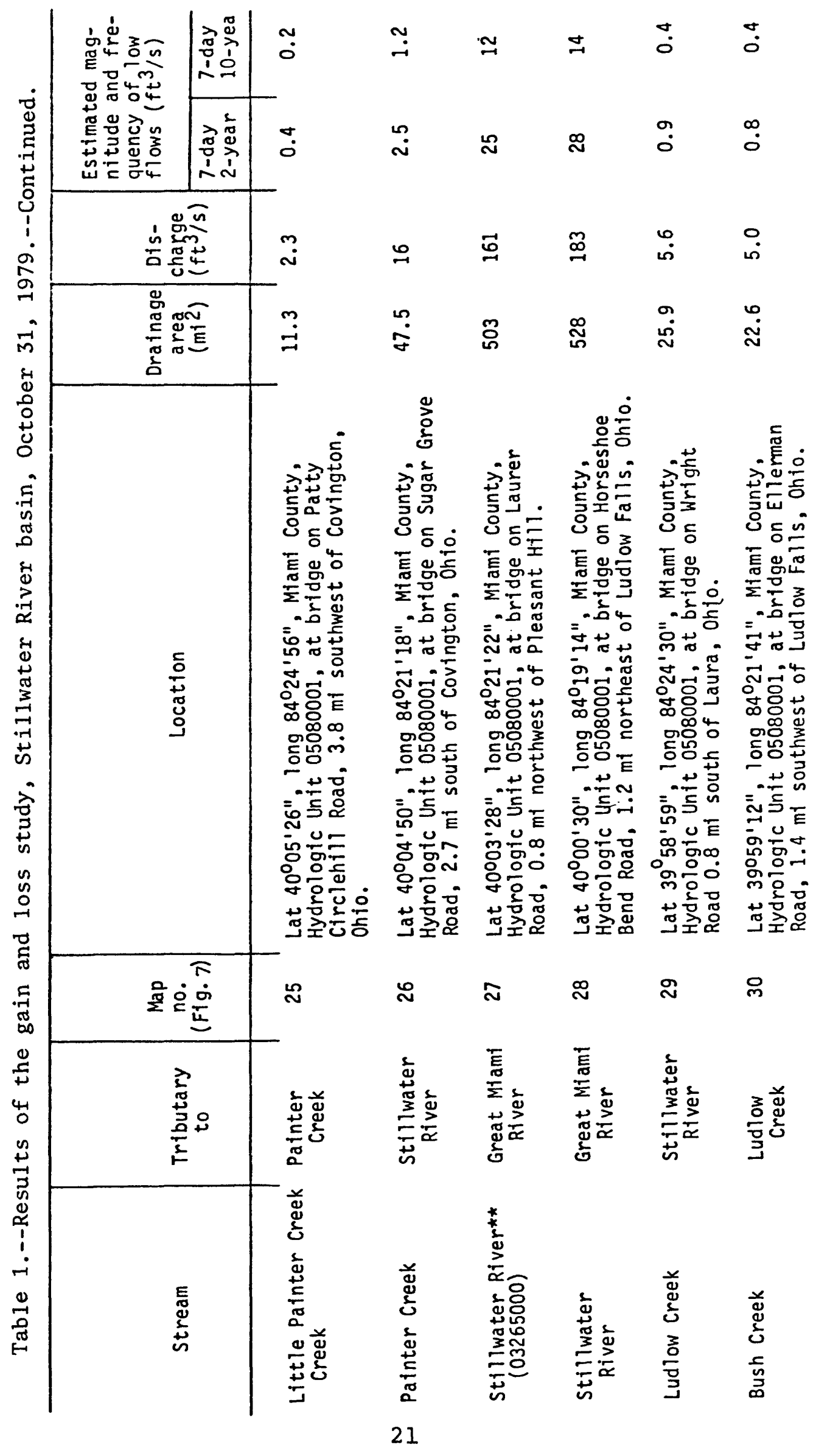




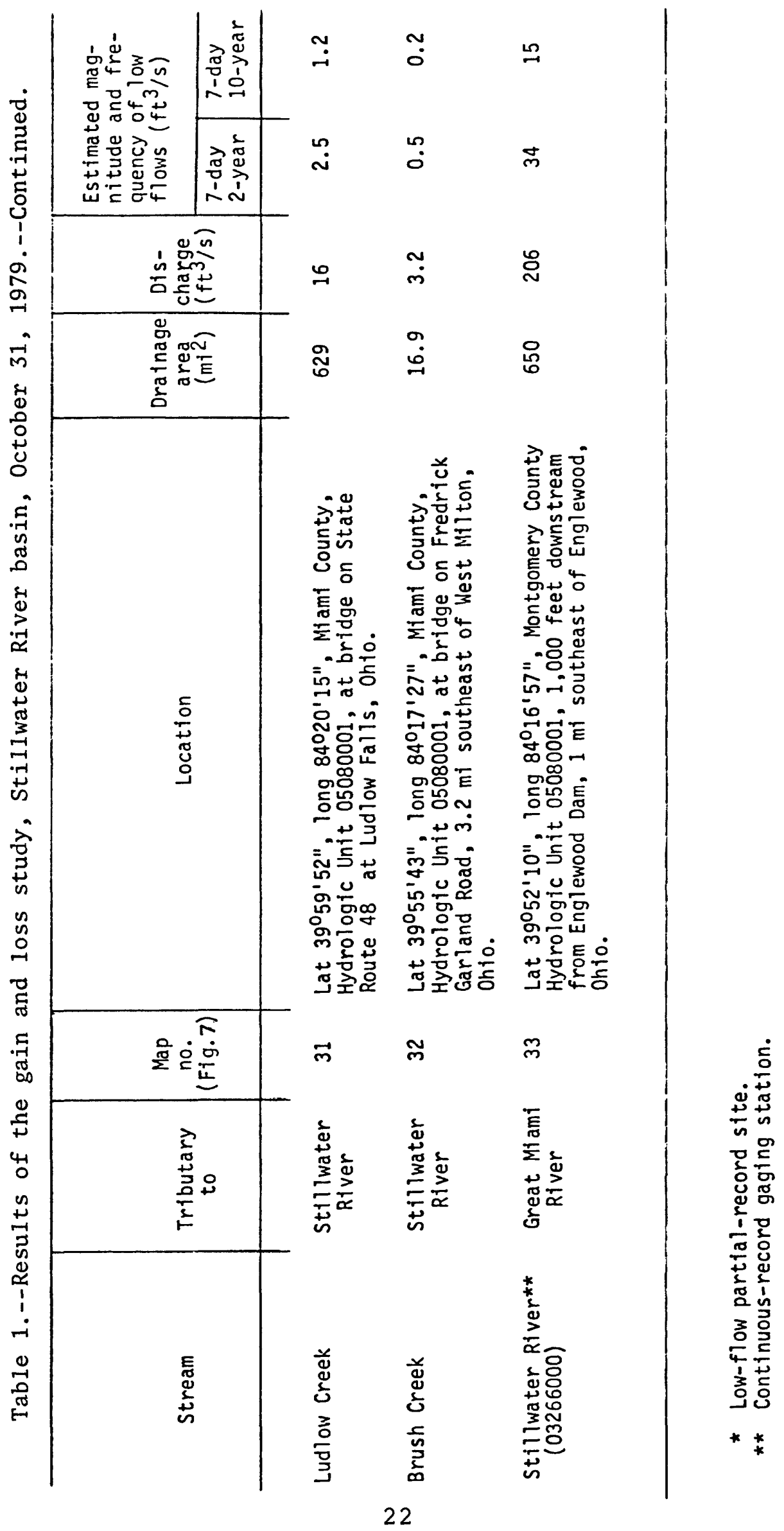




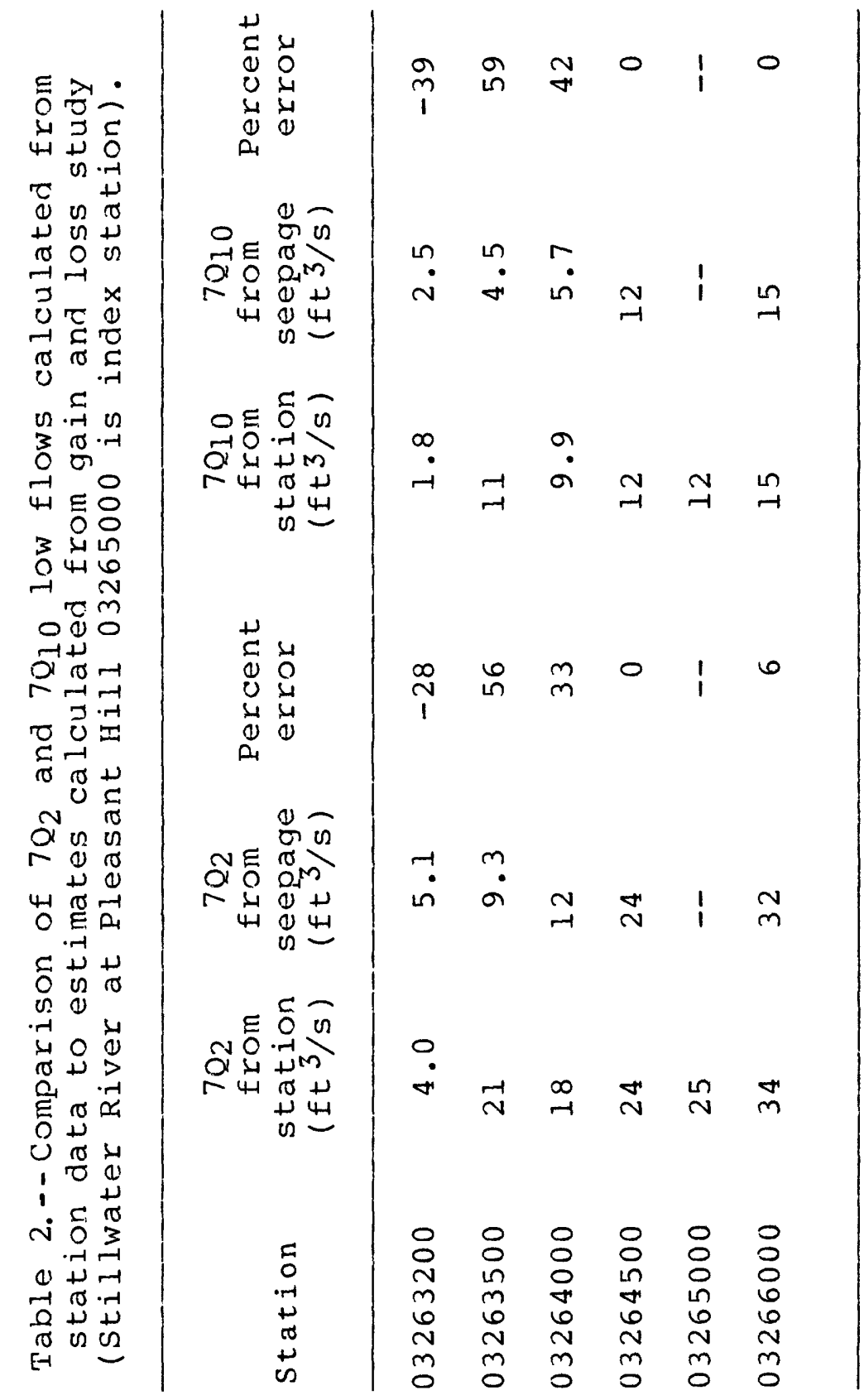




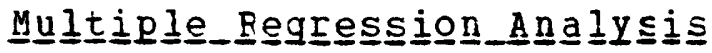

Multiple regression analysis provides a mathematical model for estimating or predicting flow characteristics when basin characteristics are known.

Multiple regression analyses were made on hydrologic data fror the Great Miami River and its trikutaries upstream from Dayton at 28 essentially unregulated sites. Of these 28 sites, 15 are continuous-record gaging stations, 13 are $10 \mathrm{w}-\mathrm{fl}$ low partial-record sites. The sites, shown in figure 8 , range in drainage area from 2.08 to 1,149 square Iiles. The low-flow characteristics used in the analyses are the 7-day 2-year, 7-day 10-year, 30-day 2-year, and 30-day 10-year low flows.

Easin characteristics used in the analyses are drainage area, main-channel slope, main-channel length, average basin elevation. percentage of surface stcrage, percentage of forest cover, index of relative infiltraticn, and average annual precipitation. Of the eight basin characteristics used, the four found to be significant at the 5-percent level are listed below.

rrainage area (A).--Area of watershed, in square miles, was determined from U.S. Geological survey topographic maps and listed by Cross (1967).

Main channel slope (S).--The slope, feet per mile, computed as the difference betweer the elevation, in feet, at 10 and 85 percent of the main-channel length (distance from the station to the basin divide), divided by the main-chanrel length, in miles, between the two points, determined from o.s. Geological survey topcgrachic maps.

Average annual precipitation (P).--Average amount of precipitation on a watershed in a year minus 27 (to approximate average runoff), in inches, determined frcm the precipitation map shown in figure 9 .

Index of relative infiltration (I).--Felative capacity of soils to accept and $r \in l \in a s \in$ water, dimensicnless, was determined as described by Armbruster (1976) by using the hydrologic soilgrouping map (figure 10) and the formula

where

$$
I=\frac{\sum(\mathrm{pa} \times \mathrm{wf})}{100}
$$

I is the index of relative infiltration,

pa is the percentage of drainage area covered by a soil grouping.

wf is the weighting factor of that soil group. 


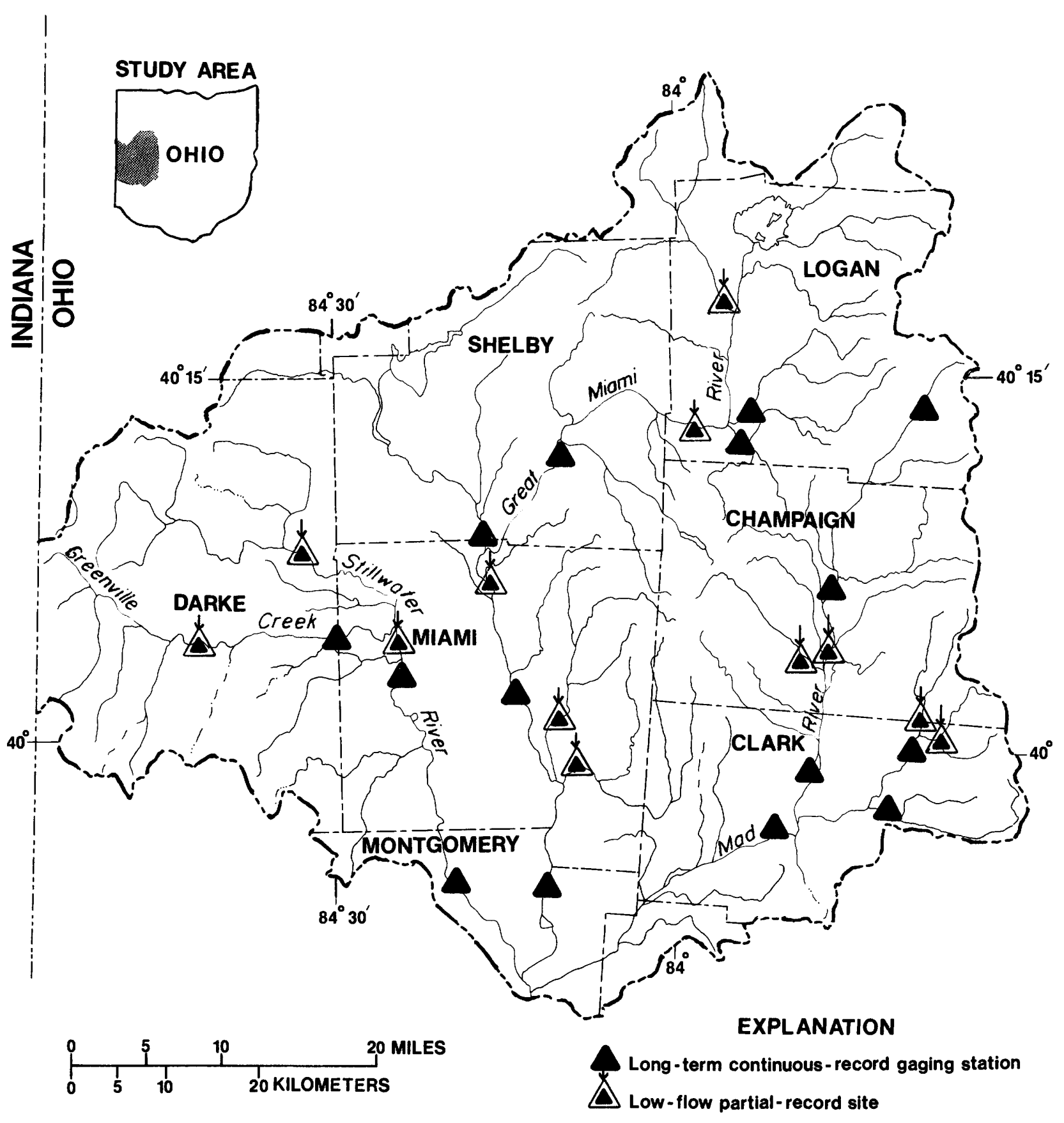

Figure 8. --Great Miami River basin sites used in the multiple regression analysis. 


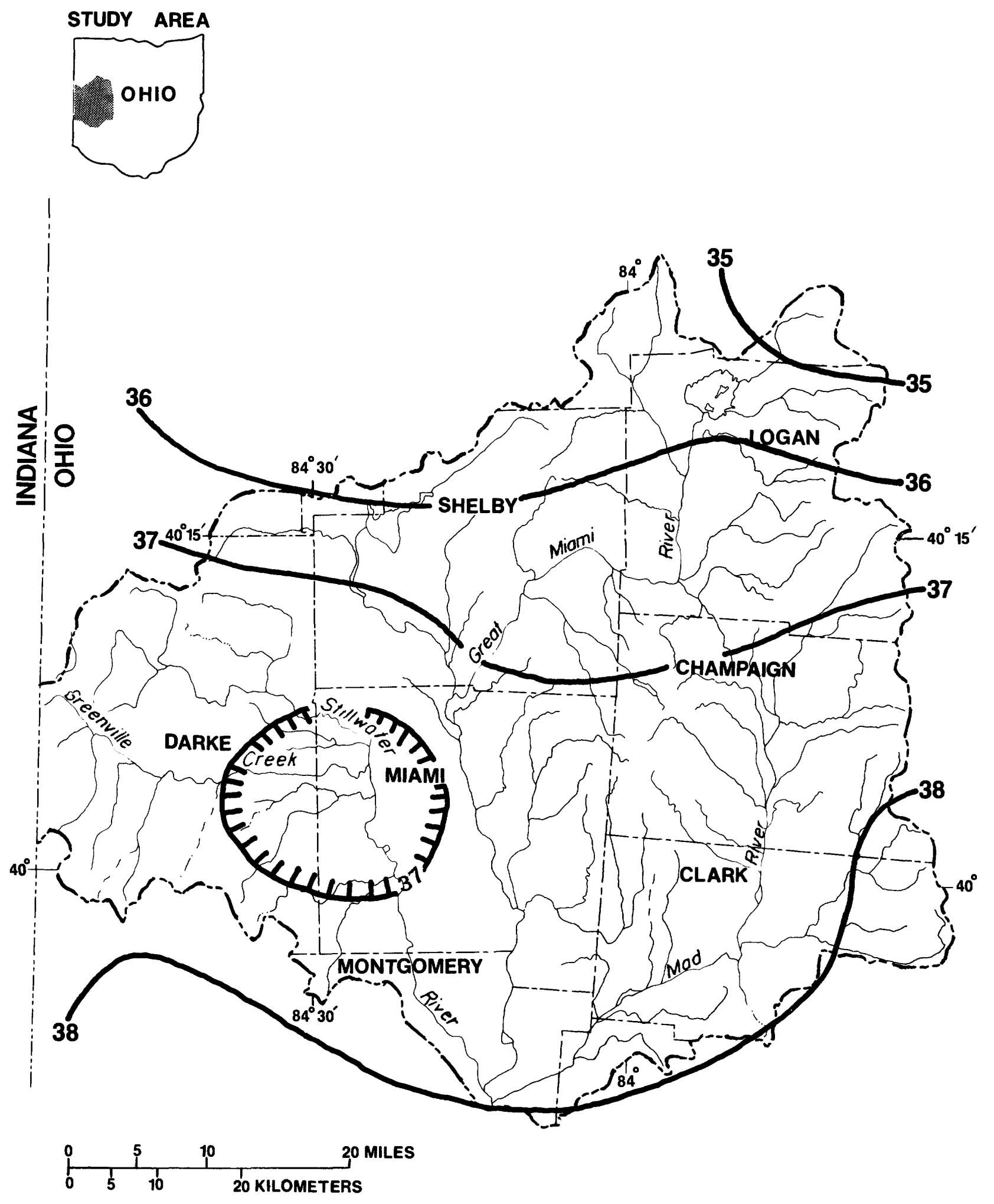

Figure 9. --Precipitation of the Upper Great Miami River basin, in inches, $1931-60$ (modified from Ohio Division of Water, 1962). 


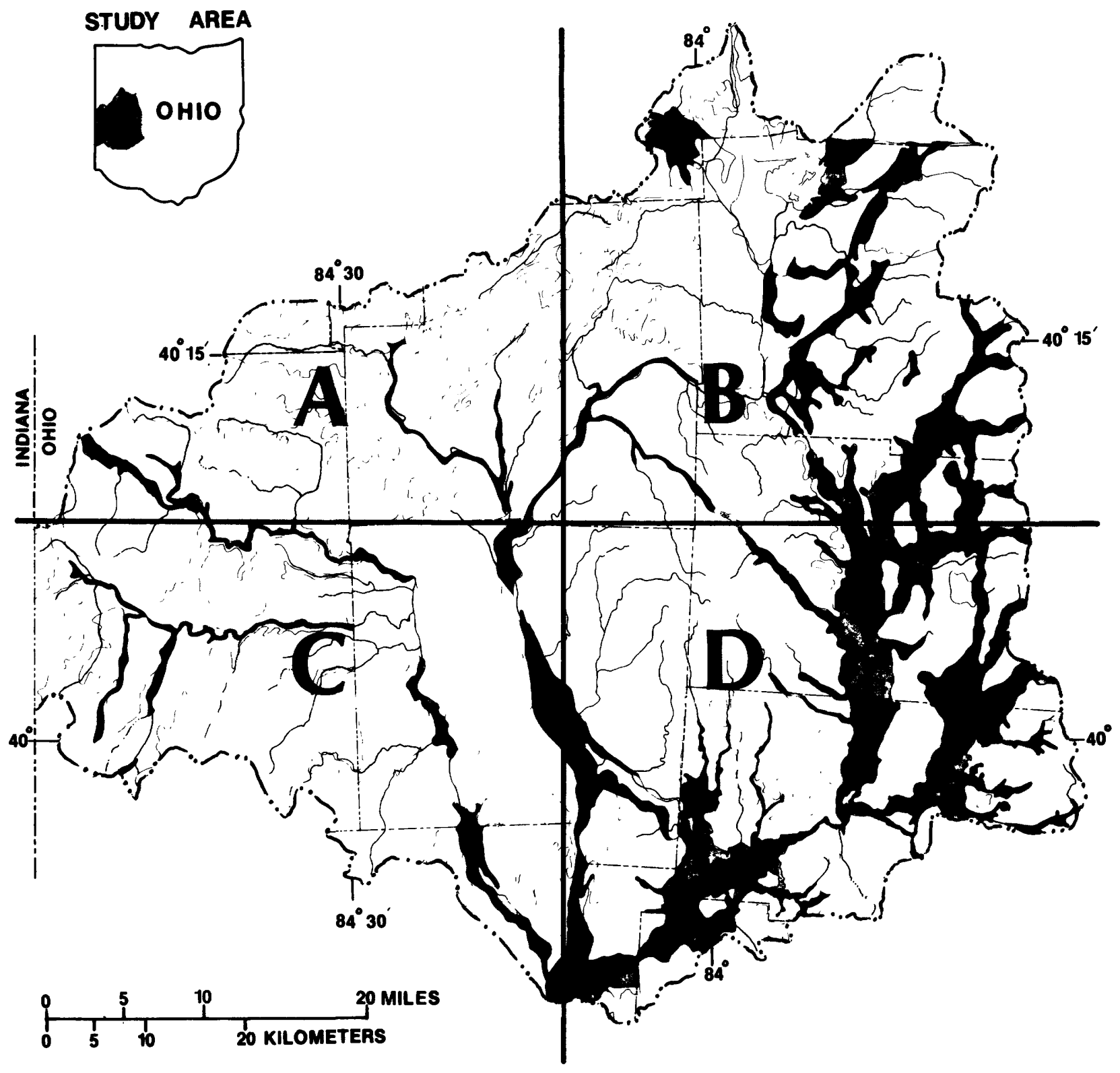

Figure 10. --Hydrologic soli-grouping map of Upper Great Miami River basin. 


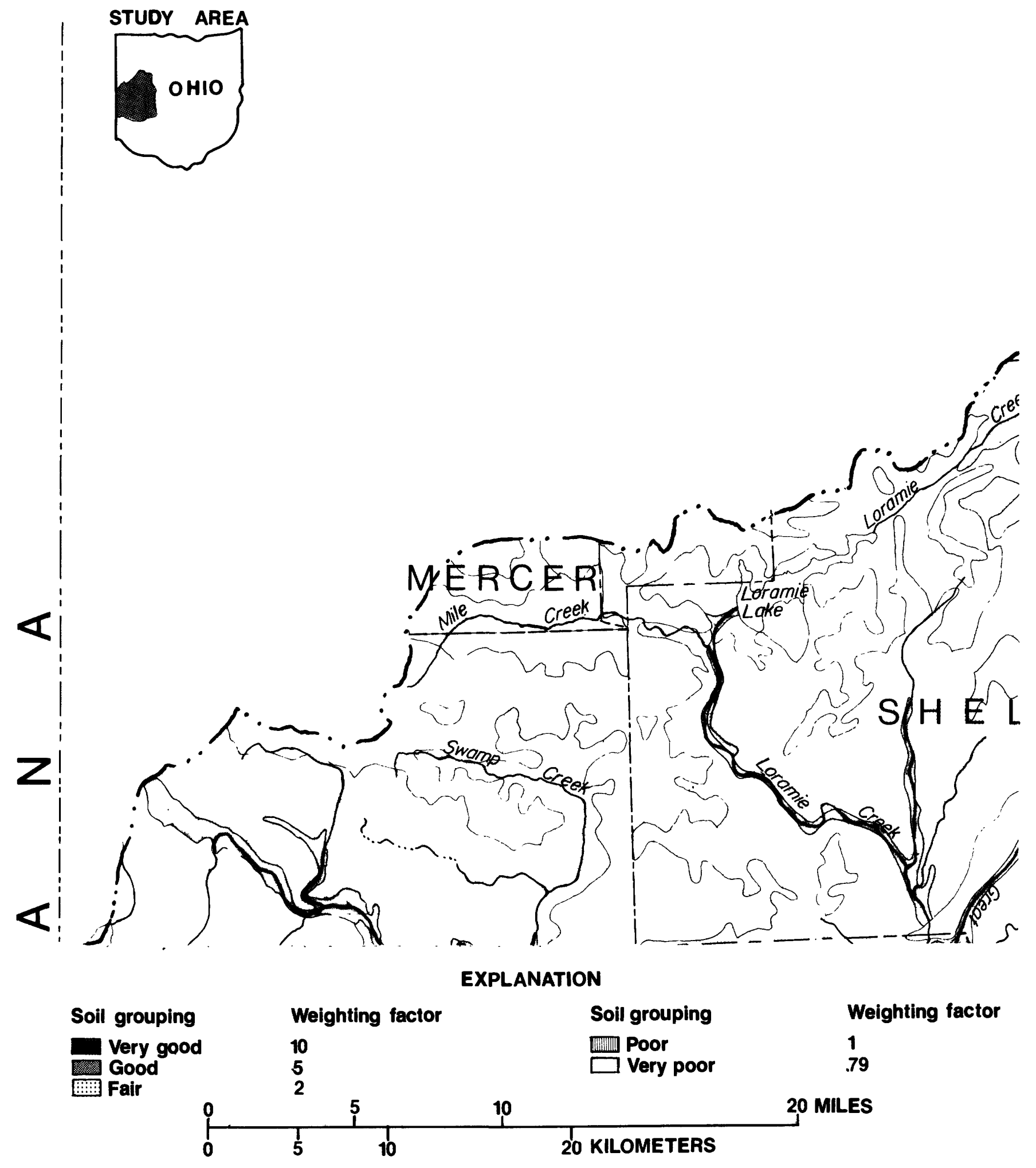

Figure 10A. --Hydrologic soil-grouping map of Upper Great Miami River basin, area A. 


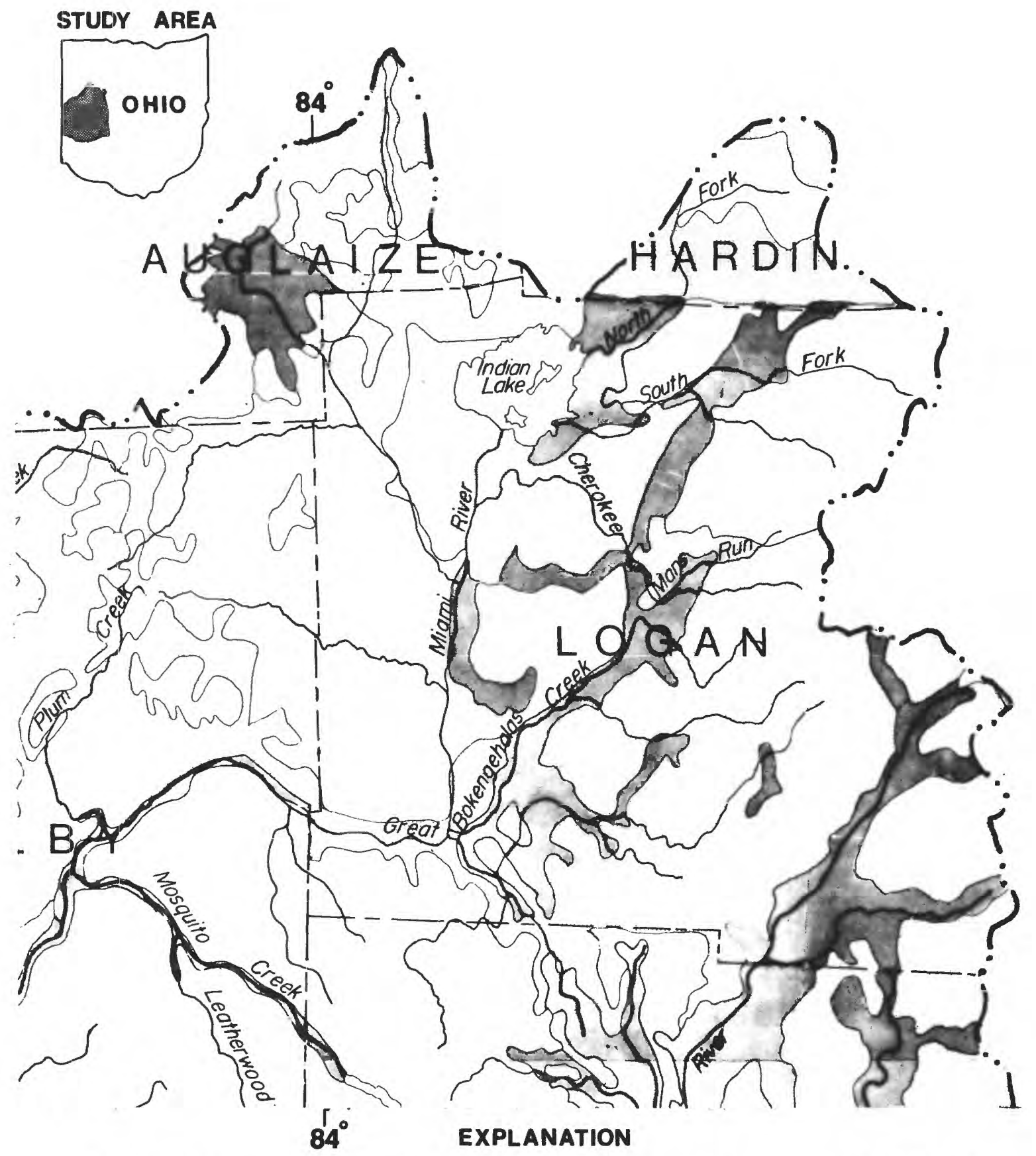

Soil grouping
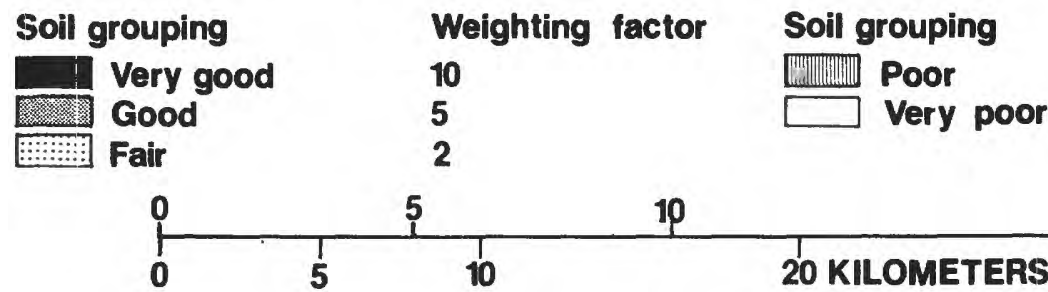

10

20 KILOMETERS

Figure 10B. --Hydrologic soil-grouping map of Upper Great Miami River basin, area B. 

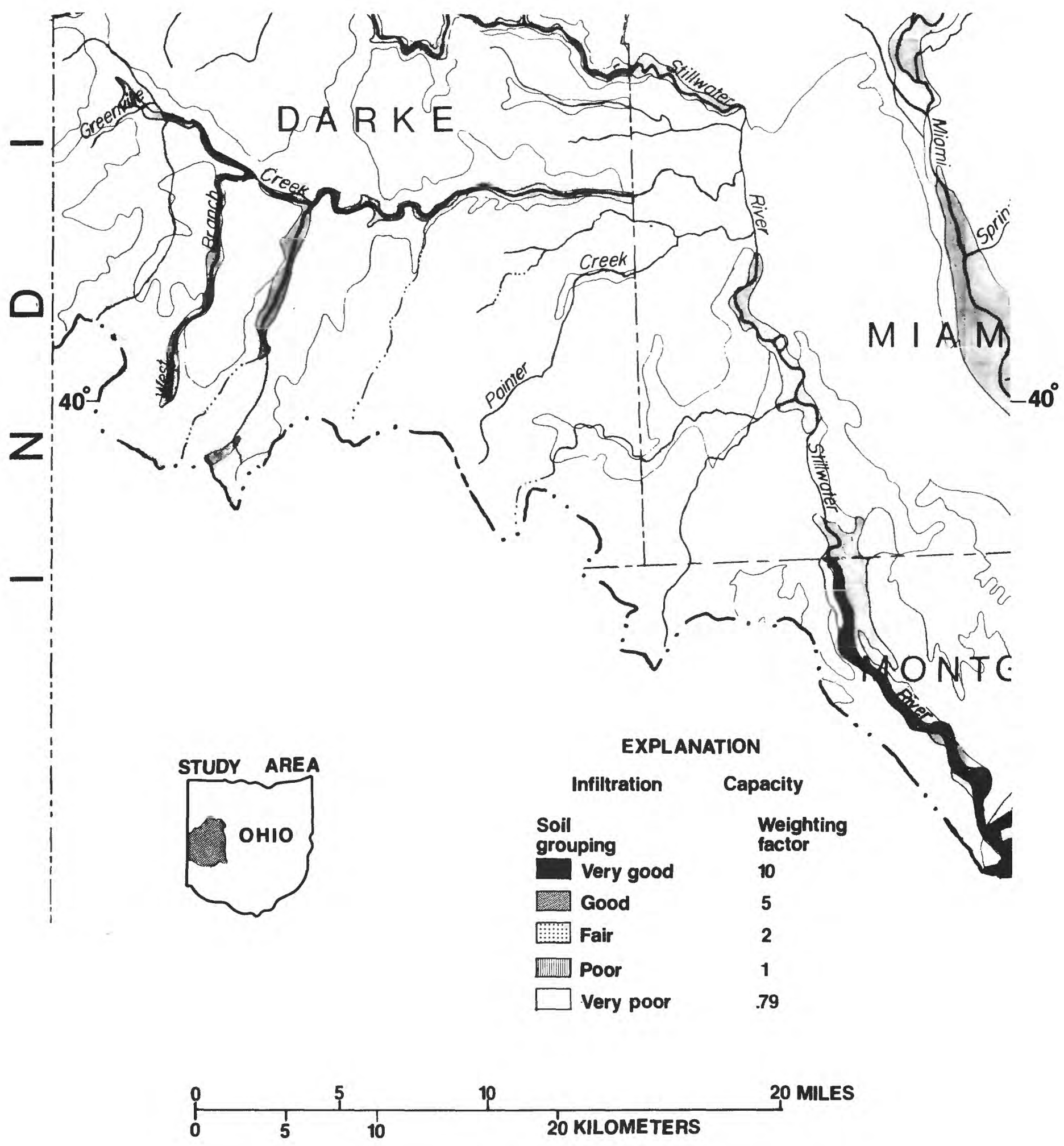

Figure 10C. --Hydrologic soll-grouping map of Upper Great Mlami River basin, area C. 


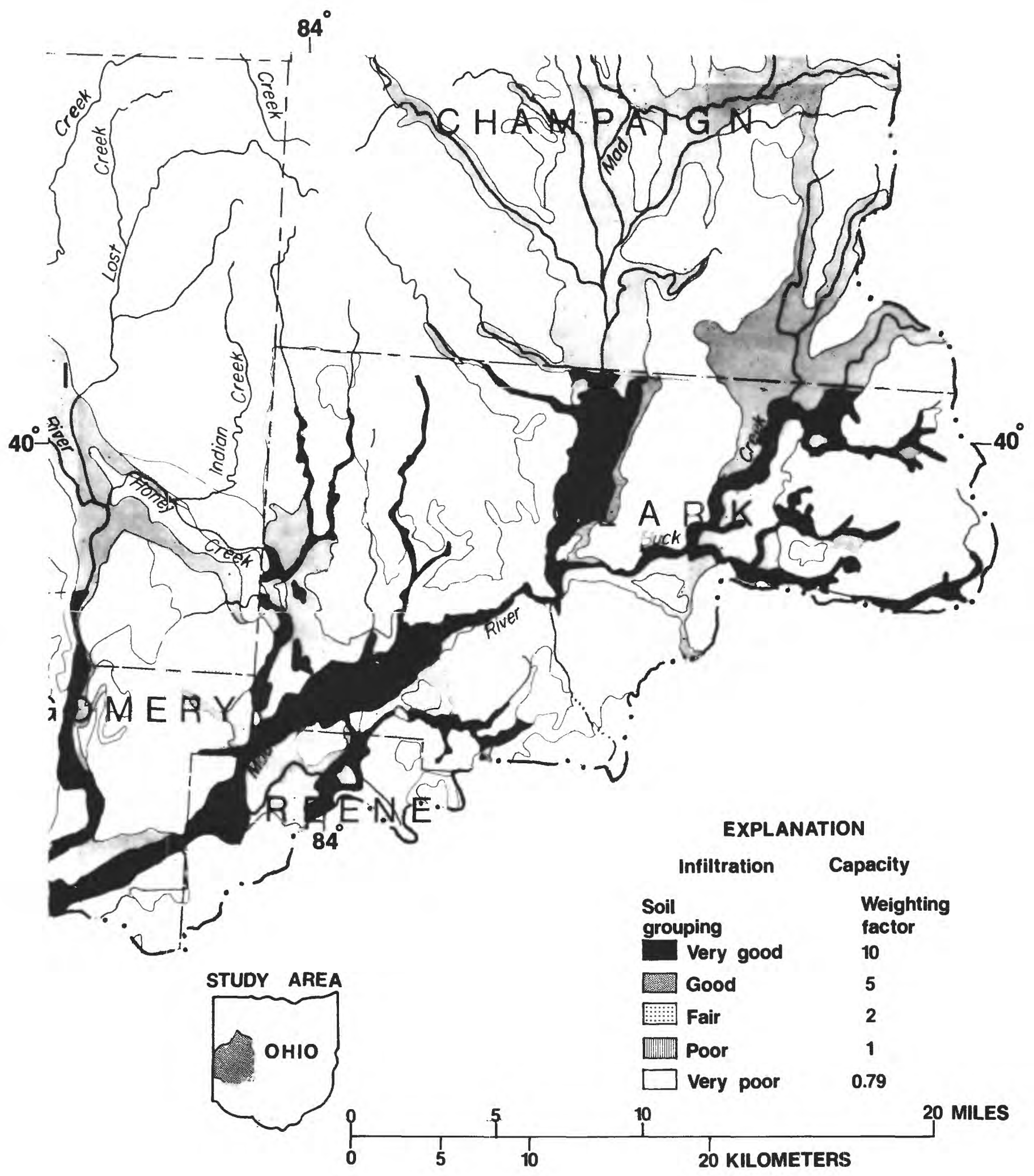

Figure 10D. --Hydrologic soil-grouping map of Upper Great Miami River basin, area D. 
The hydrologic soil-grcuping map was compiled using the county soil maps of the U.S. Soil conservation Service.

Basin characteristics are among the hydrologic factors affecting low flows. The distribution cf low flow is controlled chiefly by the geology of the basin. The flow-duration curves shown in figure 11 are expressed in cubic feet per second per square mile tc compare the effect of basin geology on low flows. The Mad River in the southwestern glacial outwash area has a high flat curve and high sustained flow. Whiteoak creek in the southwestern glacial till area has a steep curve and low sustained flow. Hocking River in the southeastern unglaciated area has a high flat curve but lower sustained flow than Mad Piver. The index of relative infiltraticn (a measure of water passing through the porous ground media) is a guantitative index of the geology of a basin.

The regression model shown below was $d \in v \in l o p e d$ by Armbruster $(1976)$ :

$$
Q=C A^{b_{1}} \quad S^{b_{2}} \quad P^{b_{3}} \quad 10^{b_{4} I}
$$

where

$C$ is a low-flow characteristic,

$c$ is the regression constant,

$k_{1}, b_{2}, b_{3}$, and $b_{4}$ are the regressicn coefficients,

A, S, P, and I are drainage area, main-channel slope, average annual precipitation, and index of relative infiltration.

The results of the multifle regression analyses are listed in $t a b l \in 3$.

The standard error of estimate is a measure of accuracy of multiple regression analysis and was computed by the method lescribed by Riggs (1968b). In the initial multiple regression analysis made on the Upper Great Miami Fiver basin (excluding infiltraticn index), only drainage area was significant at the 5percent level. The standard errors ranged from 110 to 138 percent. Adding the relative infiltration index to other basin characteristics, slope and precipitation became significant at the 5-percent level. The standard error of estimate was reduced to a range of 33 to 39 percent with the addition of the new variables. 


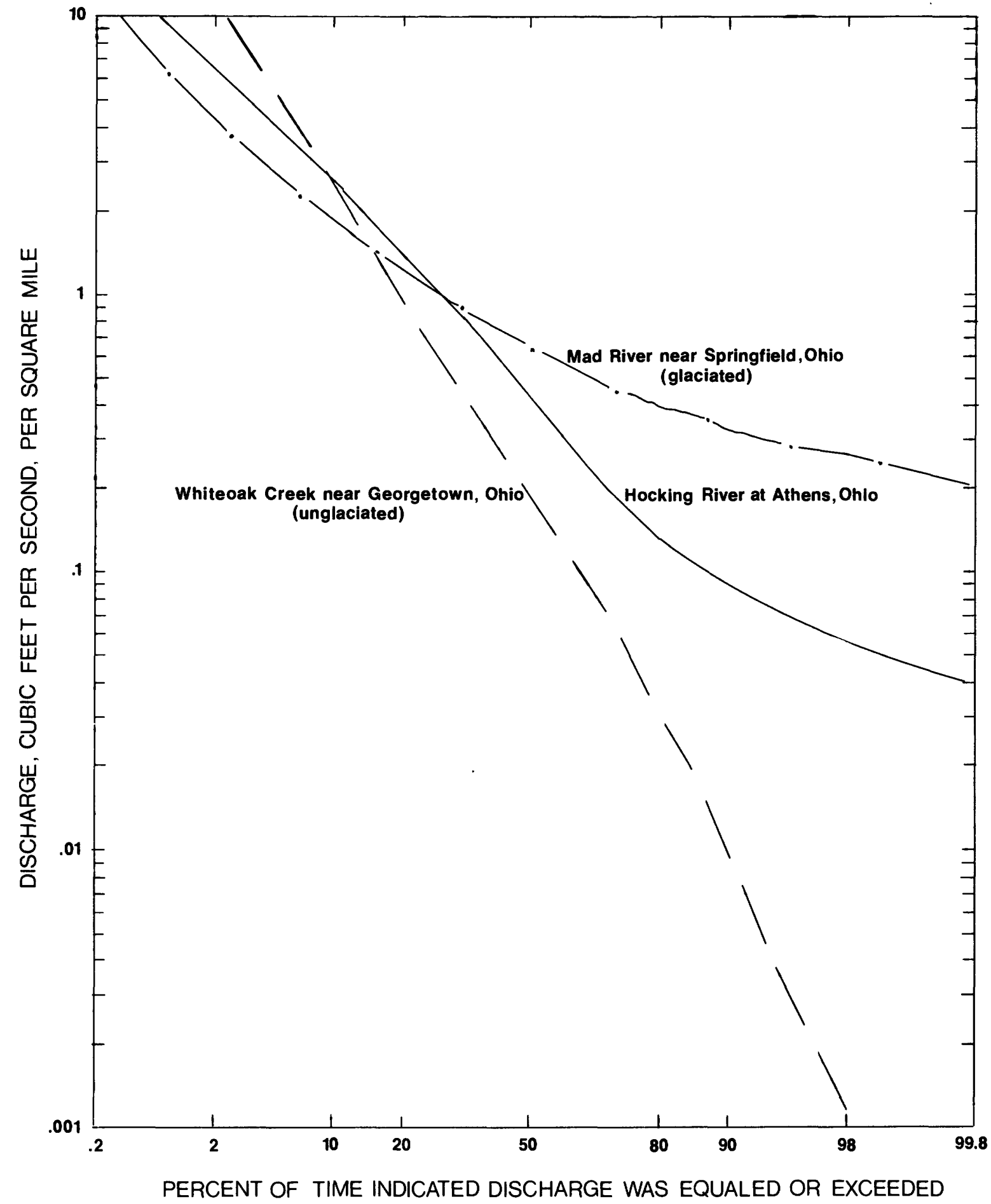

Figure 11. --Flow duration curves showing effect of basin geology on low flow. 

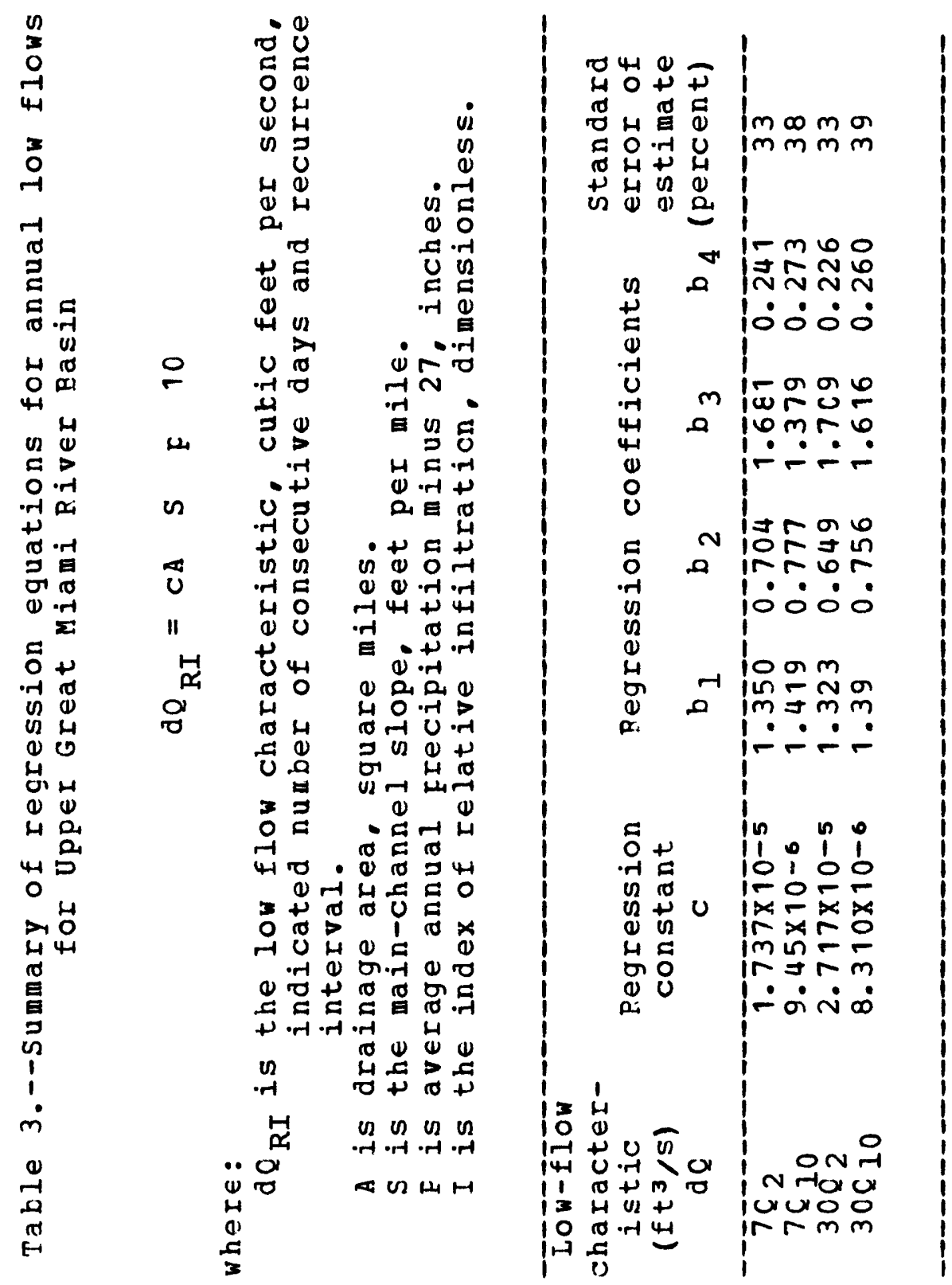
The regression model should only be used to estimate low-flow characteristics at unregulated and ungaged sites or where streamflow records are insufficient for log pearson III computations. At sites near continuous-reccrd gaging stations or low-flow partial-record sites, low-flow characteristics of those sites should be used. The regression mcdel (table 3 ) is only valid for sites with drainage area of 2.08 to 1,149 square miles. a main channel slope of 2.92 to 49.8 feet per mile, average annual precipitation of 35.5 to 38 inches, and index of relative infiltration ranging frow 1 to 10 .

To estimate annual $7 \varepsilon_{2}, 7 Q_{10}, 30 Q_{2}$, and $30 Q_{10}$ low-flow characteristics at ungaged sites in the ofper Great Miami River basin use the regression model described previously. For example, the 7-day 2-year low-flow is calculated for Beaver creek near springfield using the regression model. It is then compared to the 7-day 2-year low-flow computed frcm gaging station records. The basin characteristics are:

Irainage area $(A)=\$ 9.2$ square miles.

Main-channel length $(L)=17.1$ miles.

Elevation at $0.1 \mathrm{I}=\$ 82$ feet.

Elevation at $0.85 I=1.180$ feet.

Slope $(S)=\frac{1180-982}{0.75 \times 17.1}=15.4$ feet per mile.

Average annual precifitation $(P)=38$ irches $-27=11$ inches.

Index of relative infiltration (I).

Soil group Percent Weighting factor

(Fa)

(wf)

$\begin{array}{rrrrrr}\text { A } & \varepsilon & X & 10 & = & 80 \\ \text { E } & 17 & X & 5 & = & 85 \\ \text { C } & 72 & X & 2 & = & 144 \\ \text { D } & \Xi & X & 1 & = & 3 \\ \text { E } & C & X & 0.75 & = & 0\end{array}$

$I=312 / 100=3.12$ 
The regression equation is:

$$
7 \varepsilon_{2}=\left(1.737 \times 10^{-5}\right)\left(A^{1 \cdot 35}\right)\left(5^{0.704)}\left(\mathrm{P}^{1.681}\right)(100 \cdot 241 \times I) .\right.
$$

Substituting

$$
\begin{aligned}
& 7 \mathrm{c}_{2}=(1.737 \times 10-5)(39.21 \cdot 35)(15.40 \cdot 704)(111 \cdot 681) \\
& (100 \cdot 2413 \cdot 12) . \\
& 7 \varepsilon_{2}=5.4 \mathrm{ft} / \mathrm{s} .
\end{aligned}
$$

From the gaging station records, the 70 low flow of Beaver Creek is $6.5 \mathrm{ft} 3 / \mathrm{s}$. The regressicn model was used to estimate the $7 Q$ low flow for Beaver Creek for illustrative furposes only, because it is a continuous-recora gaging station.

\section{CONCLUSION}

Annual and seasonal low-flow characteristics are presented for 237 sites on regulated and unregulated streams. Low-flow characteristics for each site are afflicable only as long as present patterns of flow regulations (withdrawals, diversions, or inflow) are maintained.

Two regionalization methods, an analysis of base flow measurements and multiple regression analysis, were made in test basins to determine the cost and accuracy of each. Errors in the analysis of base-flow measurements ranged from 39 to 59 percent. while the standard error of estimate in the regression analyses ranged from 33 to 40 percent. Accuracy of rcth methods is within accertable limits. However, a manpower analysis indicates that the base-flow-measurements method of analysis requires four times more manpower than $t h \in$ multiple regressicn method of analysis. Statewide multiple regression analyses could provide Ohio planners and regulatory agencies with a good regionalization technigue for estimating low-flow characteristics statewide. 
Anttila, P. W., 1970, A proposed streamflcw data program for Chio: D.S. Geological survey Open-File Refort, 62 p.

Armbruster, J. T., 1964, An infiltration index useful in estimating low-flow characteristics of drainage basins: U.S. Geological Survey, Journal of Research, v. 4, no. 5, p. 533538 .

1976. Technical manual for estimating low-flow frequency characteristics of streams in the susquehanna River basin: U.S. Geological Survey Water Resources Investigations 76-51, $51 \mathrm{p}$.

Cross, W. P., 1949, The relation of geology to dry-weather stream flow in Ohio: Transactions of the American Geophysical Union, v. 30, no. 4, p. 563-566.

1967. Drainage areas of Ohio streams, supplement to Gazetteer of Ohio streams: Ohio Department of Natural Resources, Division of Water. Chio Water Plan Inventory Fieport $12 \mathrm{a}, 61 \mathrm{p}$.

Haan, C. T., 1977, Statistical methods in hydrology: Iowa, Iowa Stzte University Press, $378 \mathrm{p}$.

Hardison, C. H.. 1969, Accuracy of streamflow characteristics: U.S. Geological Survey Professional Eaper 650-D, p. 210-214.

Langtein, W. B., and Iseri, K. T., 1960, Manual of hydrology; pt. 1. General surface-water techniques; General introduction and hydrologic definitions: U.S. Geological Survey Water-supply Eaper 1541-A, p. 13 .

Matalas, N. C., 1963, Probability distribution of low flows: U.S. Geological survey Prcfessional Paper 434-A, 27 p.

Thic Division of Water, 1962, Hydrologic atlas of average arnual frecipitation, temperature, stream-flow, and water loss in Chio: Chio Department Natural Resources, Division of Water, Chio Water Plan Inventory Report 13, 4 maps.

Siggs, H. C., 1967, Regional analyses of streamflow characteristics: J.S. Geological Survey Techniques of WaterFescurces Investigations, book 4, chap. B3, 14 p. 1968a. Frequency curves: U.S. Geological Survey Techniques cf Water-Fesources Investigations, book 4, chap. A2, 15 p.

1968b, Some statistical tools in hydrology: U.S. Geological Survey Techniques of Water-Resources Irvestigations, book 4 , chap. A1, 37 p. 


\section{SELECTEL REFERENCES--Continued}

1972, Low-flow investigations: U.S. Geological Survey $\bar{T} \in \bar{c} h i q u e s$ of water-Resources Investigations, book 4 , chap. Bl, $18 \mathrm{p}$.

Searcy, J. K., 1959, Flow-duration curves: U.S. Geological Survey Water-Supply Paper 1542-A, 33 p. 


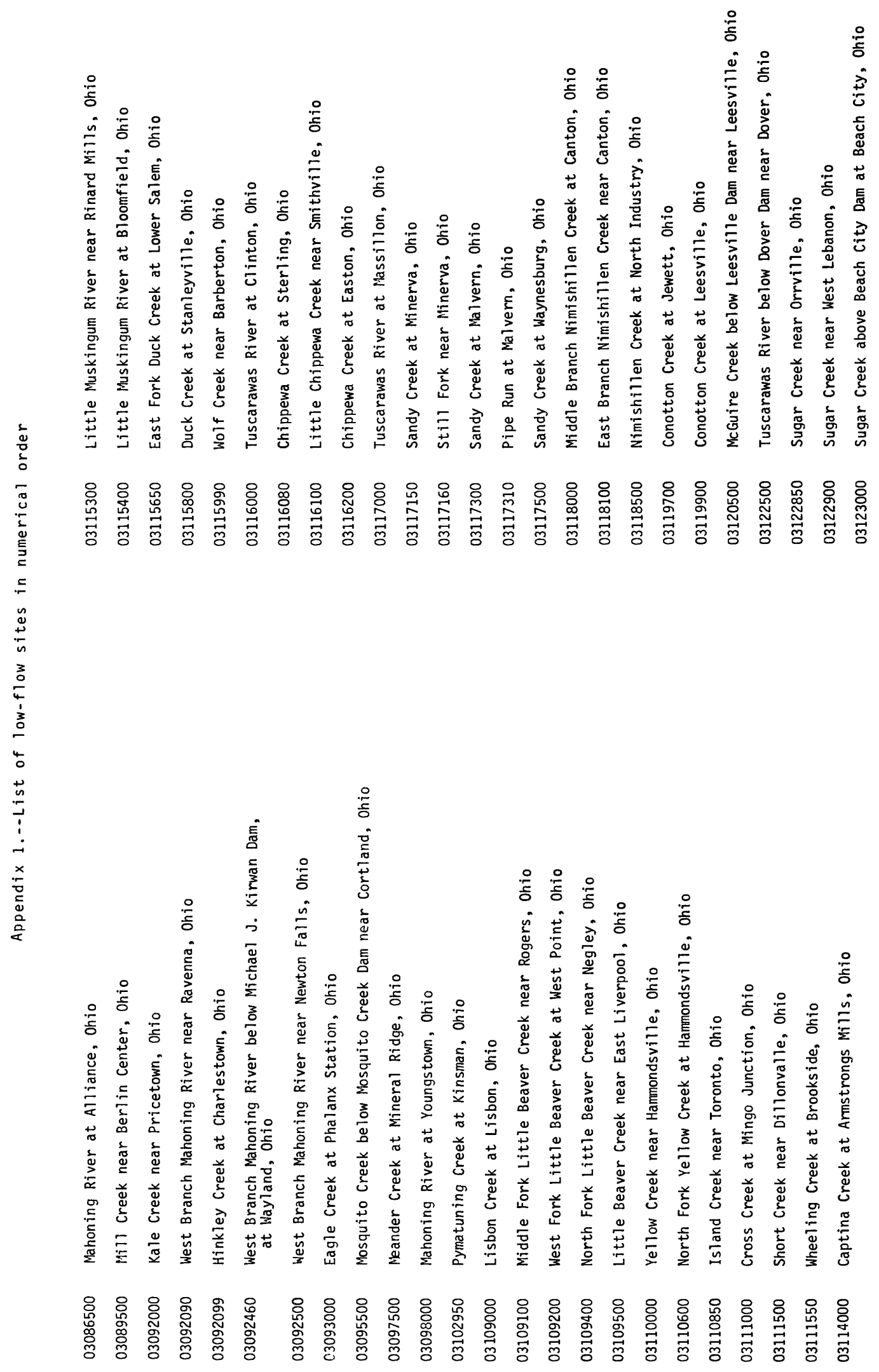




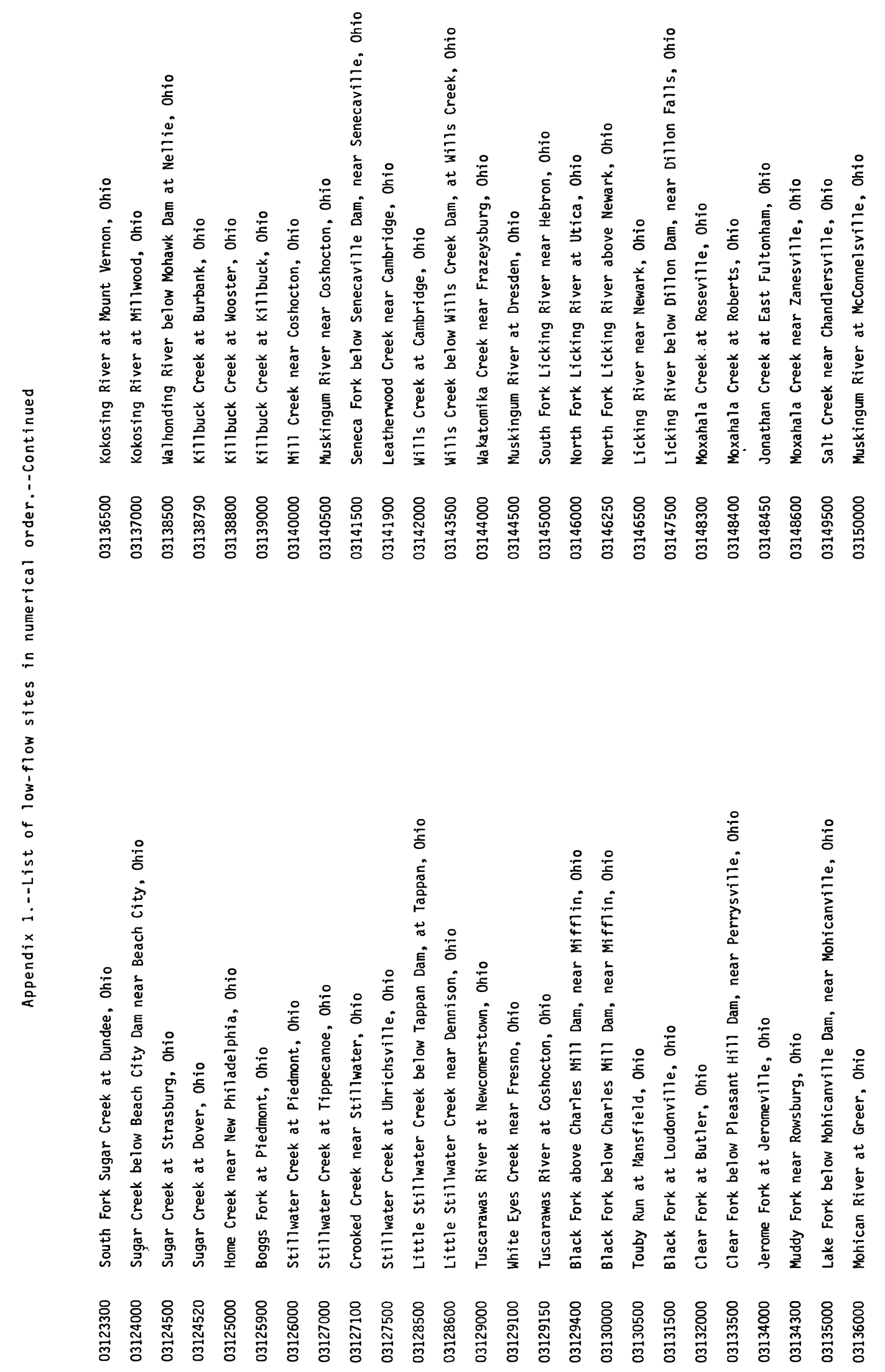




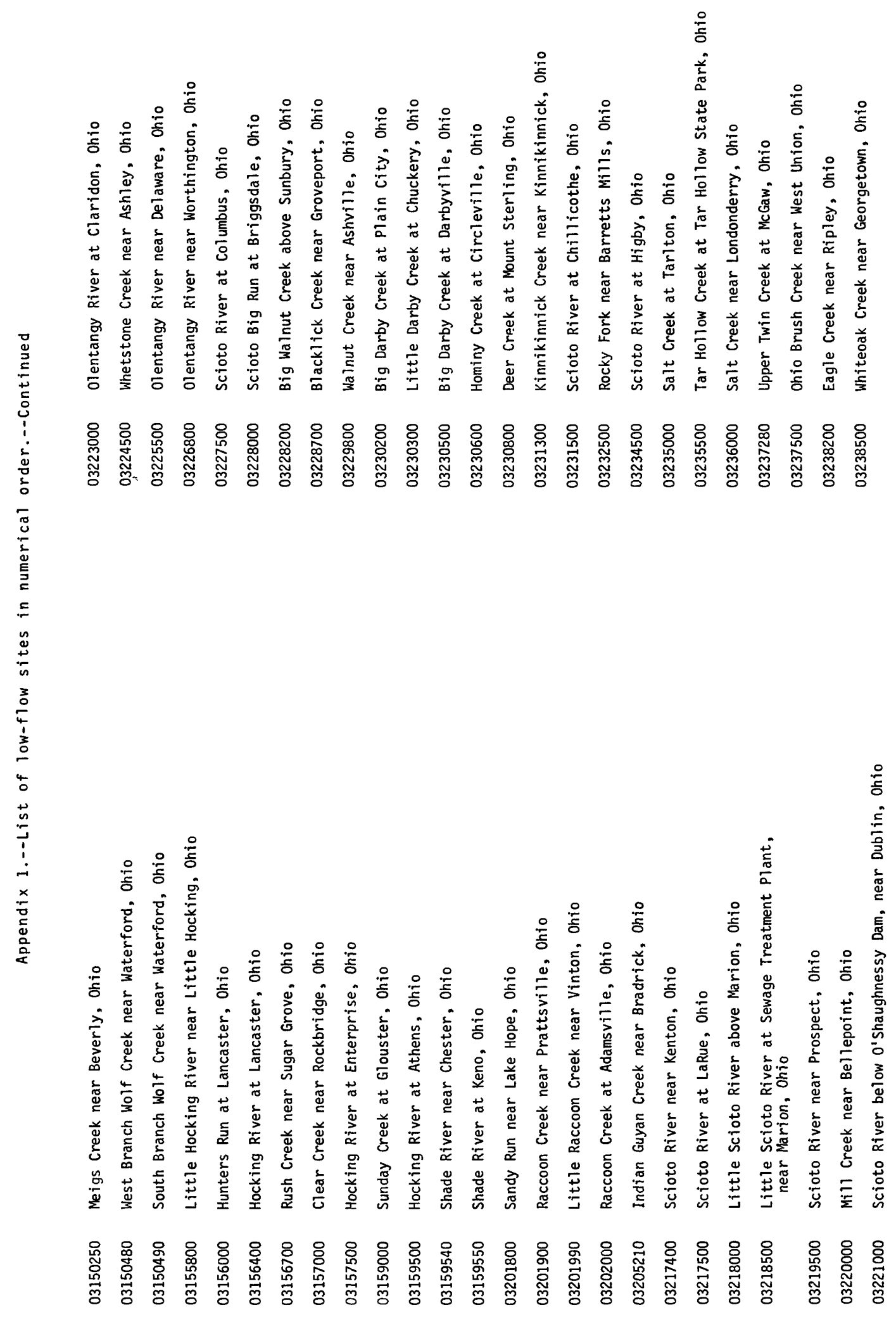




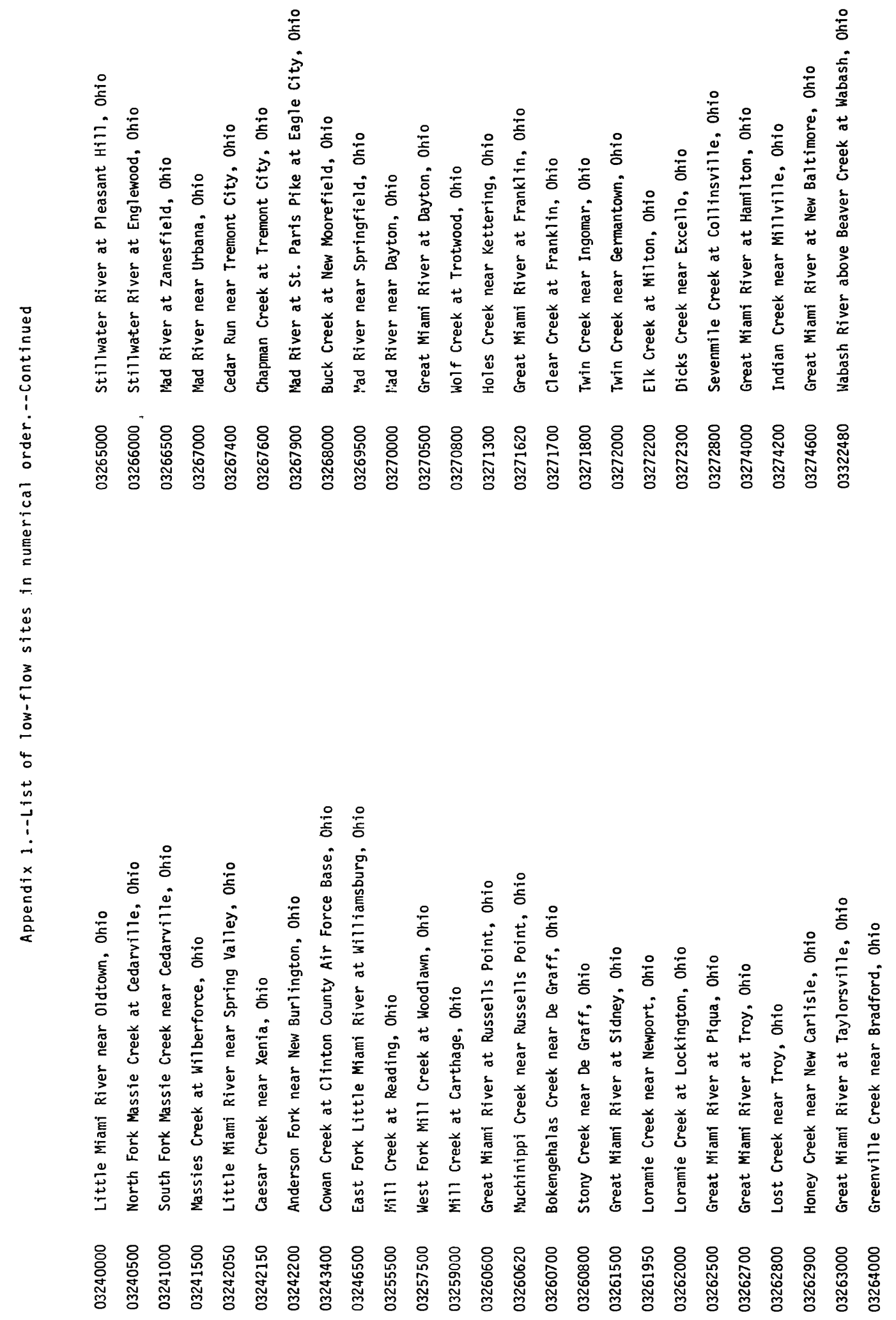




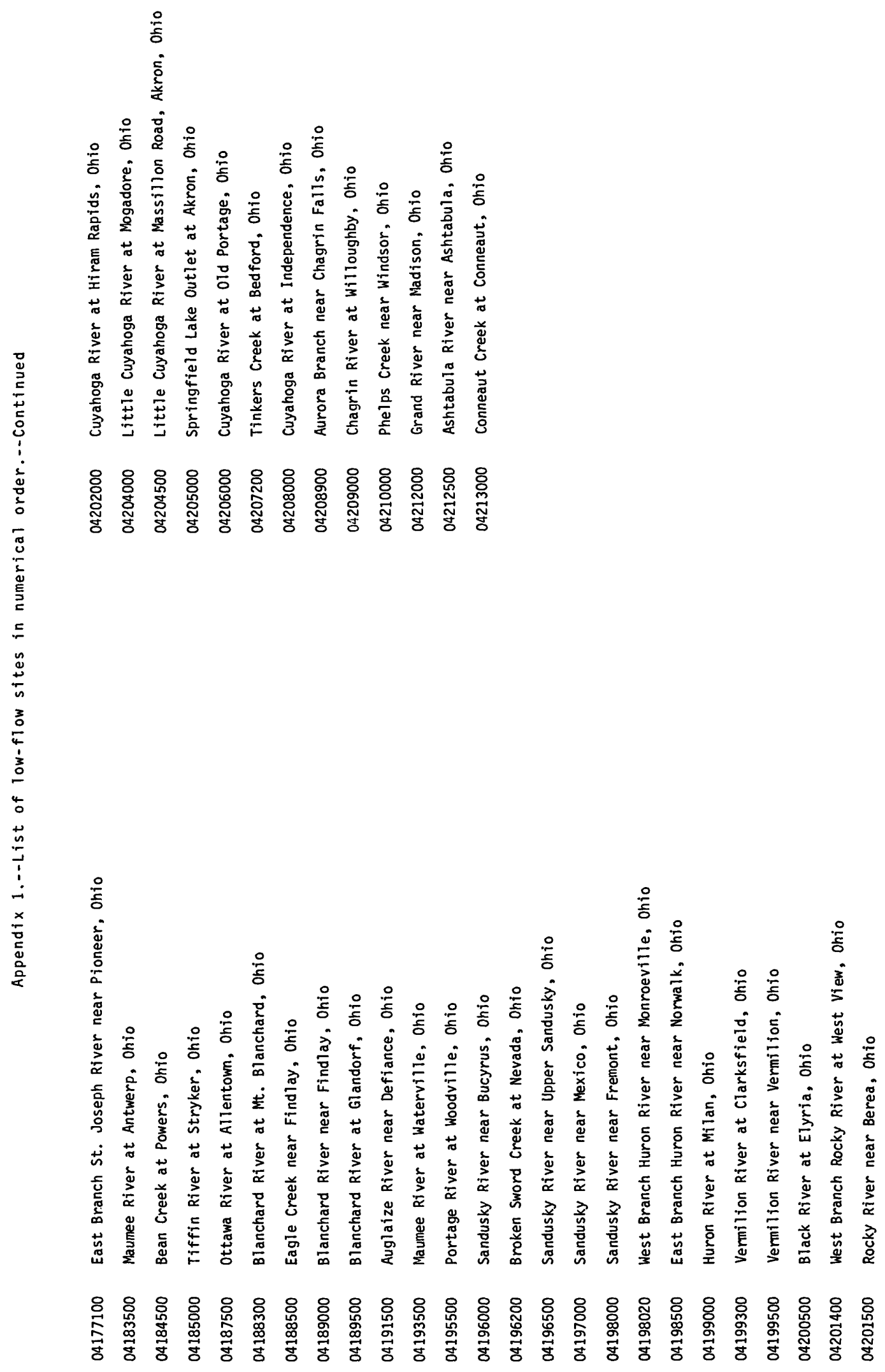




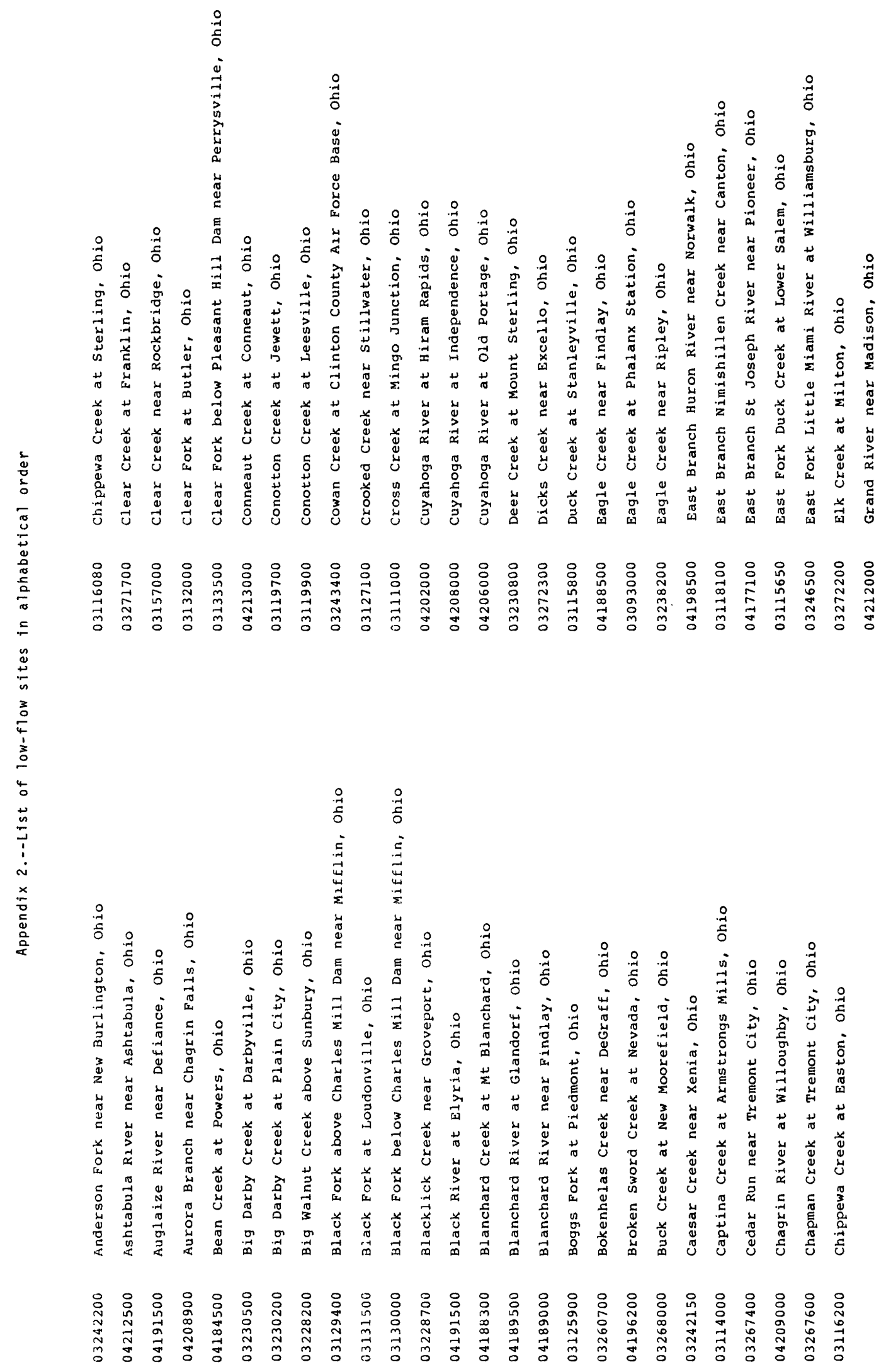




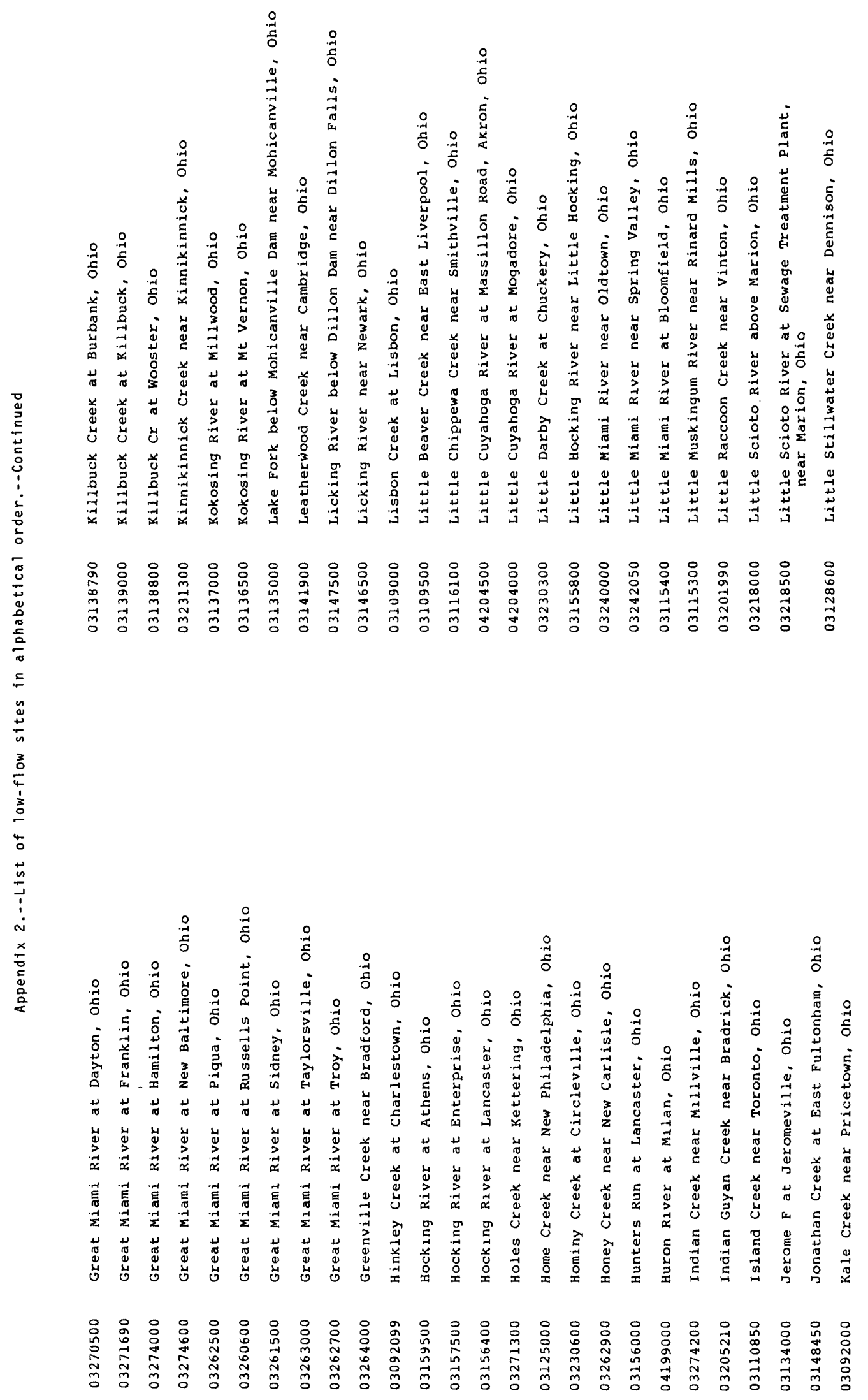




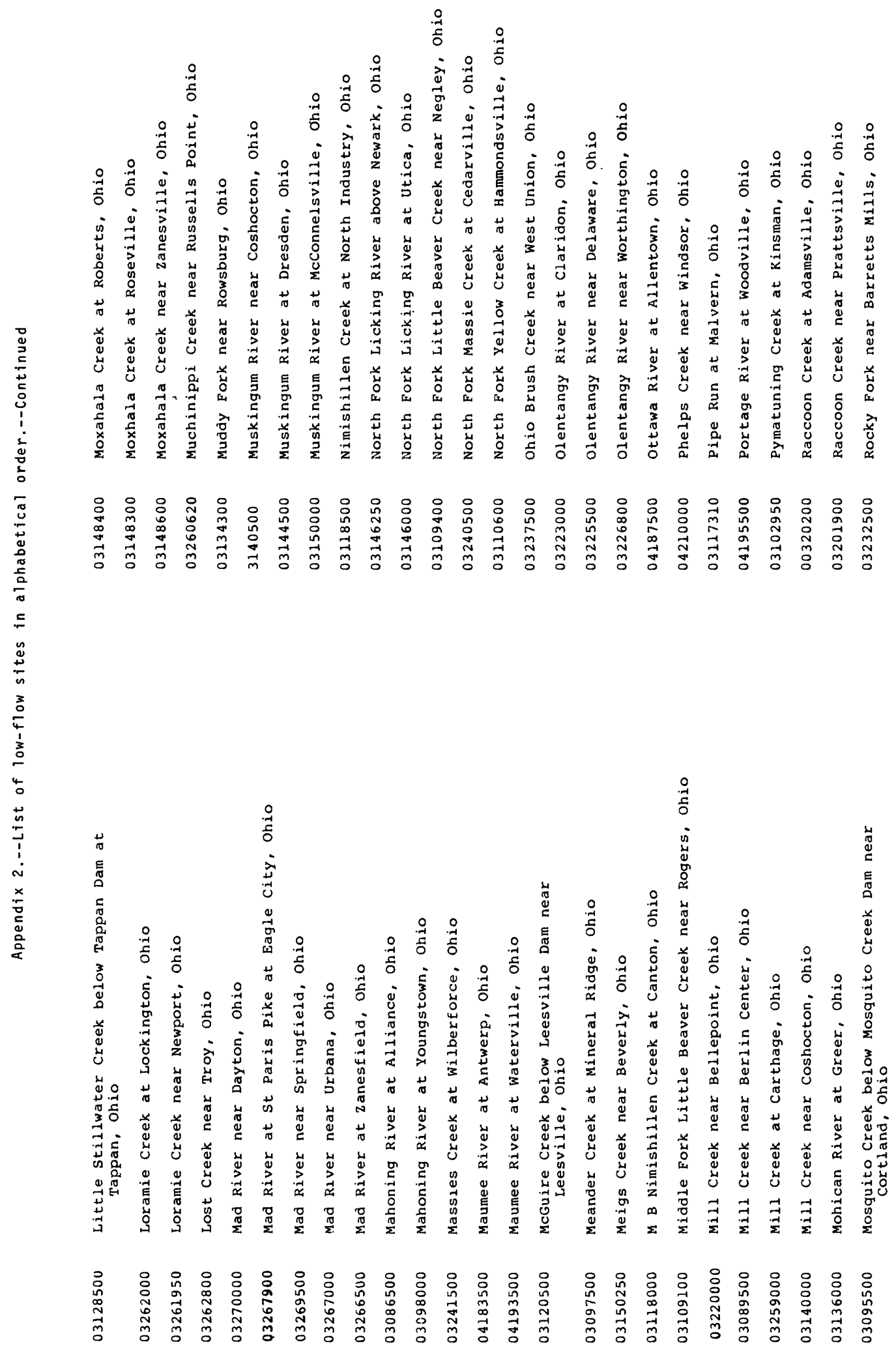




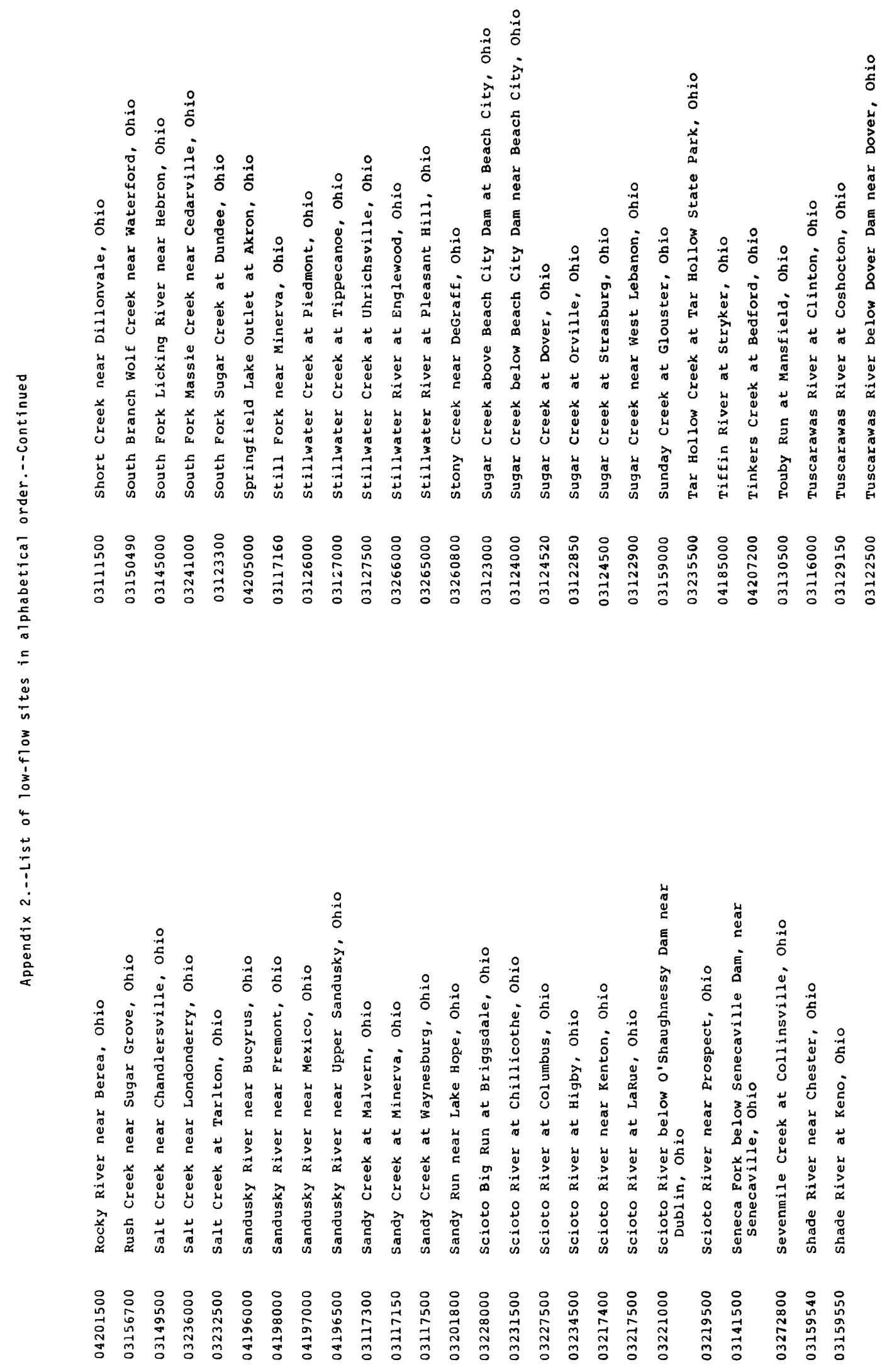


With 
MAHONING RIVER BASIN

03086500 Mahoning River at Alliance, Ohio

LOCATION:

Lat $40^{\circ} 55^{\prime} 58^{\prime \prime}$, long $81^{\circ} 05^{\prime} 41^{\prime \prime}$ in SEl/4 sec. 24, T.19 N., R.6 W., Stark

County, Hydrologic Unit 05030103, on right bank $15 \mathrm{ft}$ upstream from

Webb Avenue Bridge in Alliance, $0.2 \mathrm{mi}$ upstream from water works dam,

and $4 \mathrm{mi}$ upstream from Beech Creek.

DRAINAGE AREA: $\quad 89.2 \mathrm{mi}^{2}$.

TRIBUTARY TO: Head of Beaver River.

DISCHARGE DATA USED: October 1954 to September 1978.

REMARKS :

Flow slightly regulated by Westville Reservoir 9.3 miles upstream from station.

SELECTED DISCHARGE CHARACTERISTICS: Average discharge: $91.2 \mathrm{ft}^{3} / \mathrm{s}$ (24 years).

Minimum daily discharge: No flow August i955, 1958.

Magnitude and frequency of low flow for indicated periods

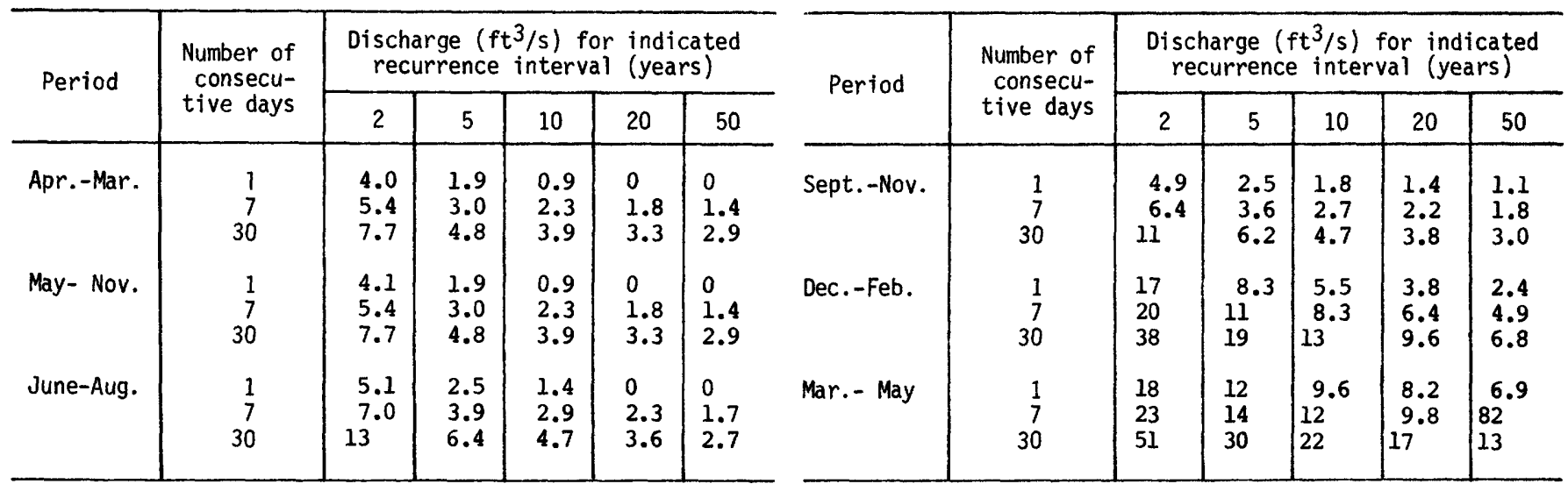

Duration of daily flow for indicated periods

\begin{tabular}{|c|c|c|c|c|c|c|c|c|c|c|c|c|c|}
\hline \multirow{2}{*}{ Period } & \multicolumn{13}{|c|}{ Discharge $\left(\mathrm{ft}^{3} / \mathrm{s}\right)$ which was equaled or exceeded for indicated percent of time } \\
\hline & 98 & 95 & 90 & 85 & 80 & 75 & 70 & 60 & 50 & 40 & 30 & 20 & 10 \\
\hline Apr. - Mar. & 3.7 & 5.2 & 7.2 & 9.4 & 13 & 15 & 18 & 24 & 33 & 48 & 69 & 110 & 210 \\
\hline $\begin{array}{l}\text { May - Nov. } \\
\text { June - Aug. } \\
\text { Sept. - Nov. } \\
\text { Dec. - Feb. } \\
\text { Mar. - May }\end{array}$ & $\begin{array}{l}3.2 \\
3.0 \\
3.2 \\
7.7 \\
13\end{array}$ & $\begin{array}{l}4.4 \\
4.0 \\
4.3 \\
11 \\
18\end{array}$ & $\begin{array}{l}5.6 \\
5.2 \\
5.5 \\
15 \\
25\end{array}$ & $\begin{array}{l}6.8 \\
6.2 \\
6.5 \\
18 \\
30\end{array}$ & $\begin{array}{l}8.2 \\
7.3 \\
7.6 \\
21 \\
36\end{array}$ & $\begin{array}{r}9.7 \\
8.8 \\
8.7 \\
25 \\
41\end{array}$ & $\begin{array}{l}12 \\
11 \\
10 \\
29 \\
48\end{array}$ & $\begin{array}{l}16 \\
14 \\
14 \\
38 \\
62\end{array}$ & $\begin{array}{l}20 \\
18 \\
19 \\
51 \\
81\end{array}$ & $\begin{array}{r}26 \\
23 \\
24 \\
70 \\
100\end{array}$ & $\begin{array}{r}36 \\
30 \\
32 \\
100 \\
140\end{array}$ & $\begin{array}{r}54 \\
44 \\
48 \\
160 \\
200\end{array}$ & $\begin{array}{r}110 \\
87 \\
89 \\
300 \\
350\end{array}$ \\
\hline
\end{tabular}


LOCATION:

DRAINAGE AREA:

TRIBUTARY TO:

DISCHARGE DATA USED:

REMARKS:
Lat $41^{\circ} 00^{\prime} 01^{\prime \prime}$, long $80^{\circ} 58^{\prime} 07^{\prime \prime}$, in T.1 N., R.5 W., Mahoning County, Hydrologic Unit 05030103, on left bank at downstream side of county road bridge, $1.0 \mathrm{mi}$ upstream from flow line of Berlin Reservoir, $1.2 \mathrm{mi}$ upstream from Turkeybroth Creek and $2.0 \mathrm{mi}$ southwest of Berlin Center:

$19.1 \mathrm{mi}^{2}$.

Mahoning River.

October 1941 to September 1971.

None.

SELECTED DISCHARGE CHARACTERISTICS: Average discharge: $16.7 \mathrm{ft}^{3} / \mathrm{s}$ (30 years).

Minimum daily discharge: No flow at times in 1953-1955, 1962, 1965-1967.

Magnitude and frequency of low flow for indicated periods

\begin{tabular}{|c|c|c|c|c|c|c|c|c|c|c|c|c|c|}
\hline \multirow{2}{*}{ Period } & \multirow{2}{*}{$\begin{array}{l}\text { Number of } \\
\text { consecu- } \\
\text { tive days }\end{array}$} & \multicolumn{5}{|c|}{$\begin{array}{l}\text { Discharge }\left(\mathrm{ft}^{3} / \mathrm{s}\right) \text { for indicated } \\
\text { recurrence interval (years) }\end{array}$} & \multirow{2}{*}{ Period } & \multirow{2}{*}{$\begin{array}{l}\text { Number of } \\
\text { consecu- } \\
\text { tive days }\end{array}$} & \multicolumn{5}{|c|}{$\begin{array}{l}\text { Discharge }\left(\mathrm{ft}^{3} / \mathrm{s}\right) \text { for indicated } \\
\text { recurrence interval (years) }\end{array}$} \\
\hline & & 2 & 5 & 10 & 20 & 50 & & & 2 & 5 & 10 & 20 & 50 \\
\hline Apr.-Mar. & $\begin{array}{r}1 \\
7 \\
30\end{array}$ & $\begin{array}{l}0.2 \\
0.2 \\
0.4\end{array}$ & $\begin{array}{l}0 \\
0 \\
0.2\end{array}$ & $\begin{array}{l}0 \\
0 \\
0.1\end{array}$ & $\begin{array}{l}0 \\
0 \\
0.1\end{array}$ & $\begin{array}{l}0 \\
0 \\
0.1\end{array}$ & Sept.-Nov. & $\begin{array}{r}1 \\
7 \\
30\end{array}$ & $\begin{array}{l}0.2 \\
0.3 \\
0.6\end{array}$ & $\begin{array}{l}0.1 \\
0.1 \\
0.3\end{array}$ & $\begin{array}{l}0 \\
0.1 \\
0.2\end{array}$ & $\begin{array}{l}0 \\
0.1 \\
0.2\end{array}$ & $\begin{array}{l}0 \\
0 \\
0.1\end{array}$ \\
\hline June-Aug: & $\begin{array}{r}1 \\
7 \\
30\end{array}$ & $\begin{array}{l}0.2 \\
0.3 \\
0.6\end{array}$ & $\begin{array}{l}0 \\
0.1 \\
0.2\end{array}$ & $\begin{array}{l}0 \\
0 \\
0.2\end{array}$ & $\begin{array}{l}0 \\
0 \\
0.1\end{array}$ & $\begin{array}{l}0 \\
0 \\
0.1\end{array}$ & Mar. - May & $\begin{array}{r}1 \\
7 \\
30\end{array}$ & $\begin{array}{l}1.5 \\
2.2 \\
8.3\end{array}$ & $\begin{array}{l}1.0 \\
1.4 \\
4.4\end{array}$ & $\begin{array}{l}0.8 \\
1.1 \\
3.1\end{array}$ & $\begin{array}{l}0.7 \\
1.0 \\
2.3\end{array}$ & $\begin{array}{l}0.6 \\
0.8 \\
1.7\end{array}$ \\
\hline
\end{tabular}

Duration of daily flow for indicated periods

\begin{tabular}{l|c|c|c|c|c|c|c|c|c|c|c|c|c}
\hline \multirow{2}{*}{ Period } & \multicolumn{8}{|c}{ Discharge $\left(\mathrm{ft}^{3} / \mathrm{s}\right)$ which was equaled or exceeded for indicated percent of time } \\
\cline { 2 - 12 } & 98 & 95 & 90 & 85 & 80 & 75 & 70 & 60 & 50 & 40 & 30 & 20 & 10 \\
\hline Apr. - Mar. & 0.2 & 0.3 & 0.4 & 0.6 & 0.8 & 1.0 & 1.2 & 1.8 & 2.9 & 5.0 & 8.6 & 17 & 37 \\
May - Nov. & 0.1 & 0.2 & 0.3 & 0.4 & 0.5 & 0.6 & 0.7 & 1.0 & 1.3 & 1.8 & 2.8 & 5.1 & 13 \\
June - Aug. & 0.1 & 0.2 & 0.3 & 0.3 & 0.4 & 0.5 & 0.6 & 0.8 & 1.0 & 1.3 & 1.9 & 3.2 & 7.8 \\
Sept. - Nov. & 0.1 & 0.2 & 0.3 & 0.4 & 0.5 & 0.5 & 0.6 & 0.8 & 1.1 & 1.4 & 1.9 & 3.0 & 6.7 \\
Dec. - Feb. & 0.8 & 1.0 & 1.3 & 1.6 & 1.9 & 2.3 & 2.7 & 3.9 & 5.5 & 8.5 & 14 & 26 & 58 \\
Mar. - May & 1.4 & 1.9 & 2.8 & 3.8 & 4.7 & 5.7 & 6.7 & 9.0 & 12 & 17 & 25 & 37 & 73 \\
\hline
\end{tabular}


MAHONING RIVER BASIN

03092000 Kale Creek near Pricetown, Ohio

LOCATION:

Lat $41^{\circ} 08^{\prime} 23^{\prime \prime}$, long $80^{\circ} 59^{\prime} 43^{\prime \prime}$, in T.3 N., R.5 W., Trumbull County, Hydrologic Unit 05030103, on right bank at downstream side of county line road bridge, $0.4 \mathrm{mi}$ north of Mahoning-Trumbull County line, $1.5 \mathrm{mi}$ northwest of pricetown, $2.2 \mathrm{mi}$ upstream from mouth, and $3.5 \mathrm{mi}$ south of Newton Falls.

DRAINAGE AREA:

$21.9 \mathrm{mi}^{2}$.

TRIBUTARY TO:

Mahoning River.

DISCHARGE DATA USED:

REMARKS:

May 1941 to September 1978.

None.

SELECTED DISCHARGE CHARACTERISTICS: Average discharge: $23.1 \mathrm{ft}^{3} / \mathrm{s}$ (37 years).

Minimum daily discharge: No flow at times in 1952-1955, 1962-1966.

Magnitude and frequency of low flow for indicated periods

\begin{tabular}{c|c|c|l|l|l|l}
\hline \multirow{2}{*}{ Period } & \multirow{2}{*}{$\begin{array}{c}\text { Number of } \\
\text { consecu- } \\
\text { tive days }\end{array}$} & \multicolumn{4}{|c|}{$\begin{array}{c}\text { Discharge }\left(\mathrm{ft}^{3} / \mathrm{s} \text { ) for indicated }\right. \\
\text { recurrence interval (years) }\end{array}$} \\
\cline { 3 - 7 } Apr.-Mar. & 1 & 0.1 & 0 & 0 & 0 & 0 \\
& 7 & 0.1 & 0 & 0 & 0 & 0 \\
& 30 & 0.2 & 0.1 & 0.1 & 0 & 0 \\
May- Nov. & 1 & 0.1 & 0 & 0 & 0 & 0 \\
& 7 & 0.1 & 0 & 0 & 0 & 0 \\
& 30 & 0.2 & 0.1 & 0.1 & 0 & 0 \\
& 1 & 0.1 & 0 & 0 & 0 & 0 \\
& 7 & 0.2 & 0.1 & 0 & 0 & 0 \\
& 30 & 0.4 & 0.1 & 0.1 & 0.1 & 0 \\
\hline
\end{tabular}

\begin{tabular}{|c|c|c|c|c|c|c|}
\hline \multirow{2}{*}{ Period } & \multirow{2}{*}{$\begin{array}{l}\text { Number of } \\
\text { consecu- } \\
\text { tive days }\end{array}$} & \multicolumn{5}{|c|}{$\begin{array}{l}\text { Discharge }\left(\mathrm{ft}^{3} / \mathrm{s}\right) \text { for indicated } \\
\text { recurrence interval (years) }\end{array}$} \\
\hline & & 2 & 5 & 10 & 20 & 50 \\
\hline Sept.-Nov. & $\begin{array}{r}1 \\
7 \\
30\end{array}$ & $\begin{array}{l}0.1 \\
0.2 \\
0.4\end{array}$ & $\begin{array}{l}0 \\
0.1 \\
0.2\end{array}$ & $\begin{array}{l}0 \\
0 \\
0.1\end{array}$ & $\begin{array}{l}0 \\
0 \\
0.1\end{array}$ & $\begin{array}{l}0 \\
0 \\
0\end{array}$ \\
\hline Dec.-Feb. & $\begin{array}{r}1 \\
7 \\
30\end{array}$ & $\begin{array}{l}1.0 \\
1.3 \\
5.0\end{array}$ & $\begin{array}{l}0.5 \\
0.6 \\
1.7\end{array}$ & $\begin{array}{l}0.3 \\
0.4 \\
0.9\end{array}$ & $\begin{array}{l}0.3 \\
0.3 \\
0.6\end{array}$ & $\begin{array}{l}0.2 \\
0.2 \\
0.3\end{array}$ \\
\hline Mar. - May & $\begin{array}{r}1 \\
7 \\
30\end{array}$ & $\begin{array}{l}1.2 \\
1.7 \\
8.7\end{array}$ & $\begin{array}{l}0.7 \\
1.0 \\
4.4\end{array}$ & $\begin{array}{l}0.5 \\
0.8 \\
3.0\end{array}$ & $\begin{array}{l}0.4 \\
0.6 \\
2.2\end{array}$ & $\begin{array}{l}0.3 \\
0.5 \\
1.5\end{array}$ \\
\hline
\end{tabular}

Duration of daily flow for indicated periods

\begin{tabular}{|c|c|c|c|c|c|c|c|c|c|c|c|c|c|c|}
\hline \multirow{2}{*}{\multicolumn{2}{|c|}{ Period }} & \multicolumn{13}{|c|}{ Discharge $\left(\mathrm{ft}^{3} / \mathrm{s}\right)$ which was equaled or exceeded for indicated percent of time } \\
\hline & & 98 & 95 & 90 & 85 & 80 & 75 & 70 & 60 & 50 & 40 & 30 & 20 & 10 \\
\hline $\begin{array}{l}\text { Apr. - } \\
\text { May - } \\
\text { June - } \\
\text { Sept. - } \\
\text { Dec. - } \\
\text { Mar. - }\end{array}$ & $\begin{array}{l}\text { Mar. } \\
\text { Nov. } \\
\text { Aug. } \\
\text { Nov. } \\
\text { Feb. } \\
\text { May }\end{array}$ & $\begin{array}{l}0.1 \\
0 \\
0 \\
0 \\
0.5 \\
1.0\end{array}$ & $\begin{array}{l}0.2 \\
0.1 \\
0.1 \\
0.1 \\
0.7 \\
1.5\end{array}$ & $\begin{array}{l}0.3 \\
0.2 \\
0.2 \\
0.2 \\
1.0 \\
2.3\end{array}$ & $\begin{array}{l}0.4 \\
0.3 \\
0.2 \\
0.2 \\
1.4 \\
3.2\end{array}$ & $\begin{array}{l}0.5 \\
0.3 \\
0.3 \\
0.3 \\
1.8 \\
4.2\end{array}$ & $\begin{array}{l}0.7 \\
0.4 \\
0.3 \\
0.3 \\
2.2 \\
5.2\end{array}$ & $\begin{array}{l}1.0 \\
0.5 \\
0.4 \\
0.4 \\
2.7 \\
6.2\end{array}$ & $\begin{array}{l}1.7 \\
0.7 \\
0.6 \\
0.6 \\
4.2 \\
8.7\end{array}$ & $\begin{array}{l}3.0 \\
1.1 \\
0.8 \\
0.9 \\
6.5 \\
13\end{array}$ & $\begin{array}{l}5.4 \\
1.7 \\
1.1 \\
1.4 \\
11 \\
18\end{array}$ & $\begin{array}{l}9.5 \\
2.9 \\
1.6 \\
2.5 \\
18 \\
28\end{array}$ & $\begin{array}{c}20 \\
6.0 \\
2.9 \\
5.0 \\
34 \\
47\end{array}$ & $\begin{array}{c}50 \\
17 \\
8.1 \\
12 \\
86 \\
94\end{array}$ \\
\hline
\end{tabular}


MAHONING RIVER BASIN

LOCATION:

\author{
03092090 West Branch Mahoning River near Ravenna, Ohio
}

Lat 41.09'41", long $81^{\circ} 11^{\prime} 50^{\prime \prime}$, in T.3 N., R.8 W., Portage County, Hydrologic Unit 05030103 , on left bank at downstream side of bridge on Newton Falls Road, $2.5 \mathrm{mi}$ east of Ravenna.

DRAINAGE AREA: $\quad 21.8 \mathrm{mi}^{2}$.

TRIBUTARY TO: Mahoning River.

OISCHARGE DATA USED: October 1965 to September 1978.

REMARKS:

None.

SELECTED DISCHARGE CHARACTERISTICS: Average discharge: $27.4 \mathrm{ft}^{3} / \mathrm{s}$ ( 13 years).

Minimum daily discharge: $0.7 \mathrm{ft} / \mathrm{s}$ September 1972.

Magnitude and frequency of low flow for indicated periods

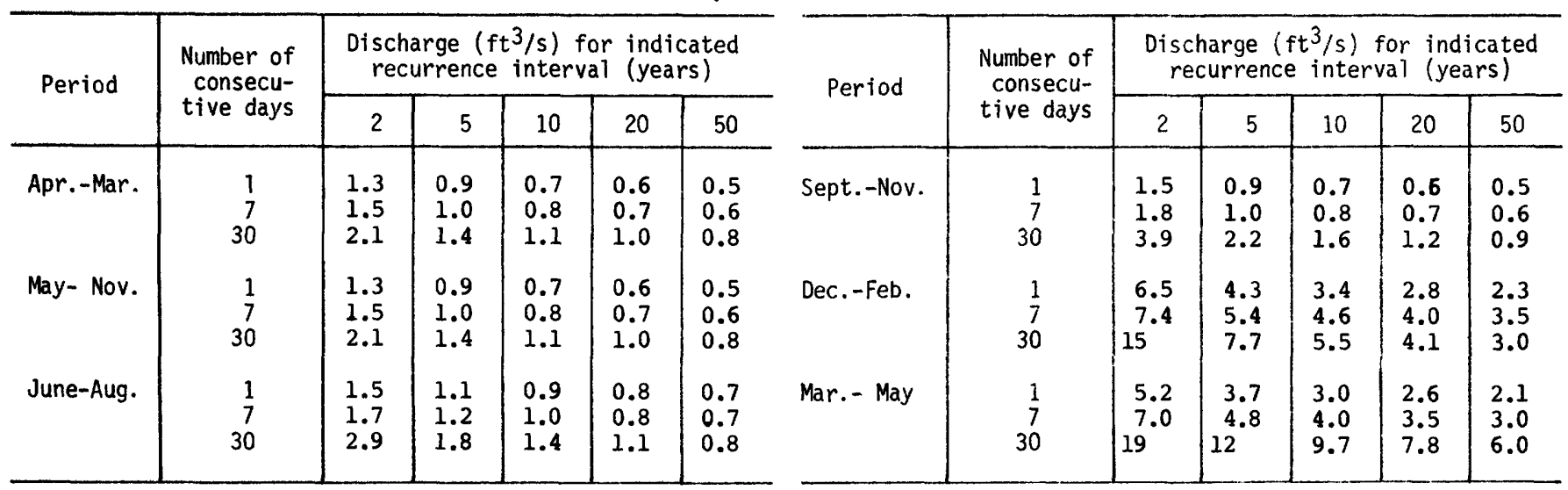

Duration of daily flow for indicated periods

\begin{tabular}{|c|c|c|c|c|c|c|c|c|c|c|c|c|c|}
\hline \multirow{2}{*}{ Period } & \multicolumn{13}{|c|}{ Discharge $\left(\mathrm{ft}^{3} / \mathrm{s}\right)$ which was equaled or exceeded for indicated percent of time } \\
\hline & 98 & 95 & 90 & 85 & 80 & 75 & 70 & 60 & 50 & 40 & 30 & 20 & 10 \\
\hline $\begin{array}{l}\text { Apr. - Mar. } \\
\text { May - Nov. } \\
\text { June - Aug. } \\
\text { Sept. - Nov. } \\
\text { Dec. - Feb. } \\
\text { Mar. - May }\end{array}$ & $\begin{array}{l}1.2 \\
1.0 \\
1.1 \\
1.0 \\
4.4 \\
4.6\end{array}$ & $\begin{array}{l}1.5 \\
1.3 \\
1.3 \\
1.2 \\
4.9 \\
6.3\end{array}$ & $\begin{array}{l}2.3 \\
1.6 \\
1.6 \\
1.5 \\
6.2 \\
8.2\end{array}$ & $\begin{array}{l}3.0 \\
2.1 \\
1.8 \\
1.9 \\
7.8 \\
9.5\end{array}$ & $\begin{array}{r}3.7 \\
2.5 \\
2.2 \\
2.4 \\
8.8 \\
11\end{array}$ & $\begin{array}{l}4.4 \\
2.9 \\
2.5 \\
2.9 \\
9.8 \\
13\end{array}$ & $\begin{array}{l}5.2 \\
3.3 \\
2.8 \\
3.3 \\
12 \\
15\end{array}$ & $\begin{array}{l}7.9 \\
4.2 \\
3.3 \\
4.6 \\
15 \\
19\end{array}$ & $\begin{array}{l}11 \\
5.4 \\
3.9 \\
6.0 \\
18 \\
23\end{array}$ & $\begin{array}{l}16 \\
7.4 \\
4.6 \\
8.2 \\
24 \\
30\end{array}$ & $\begin{array}{l}22 \\
10 \\
5.8 \\
11 \\
32 \\
38\end{array}$ & $\begin{array}{l}33 \\
17 \\
8.1 \\
17 \\
50 \\
55\end{array}$ & $\begin{array}{r}65 \\
33 \\
17 \\
31 \\
100 \\
99\end{array}$ \\
\hline
\end{tabular}


Appendix 3.--Low-flow data--Continued

MAHONING RIVER BASIN

03092099 Hinkley Creek at Charlestown, Ohio

LOCATION:

Lat $41^{\circ} 09^{\prime} 16^{\prime \prime}$, long $81^{\circ} 08^{\prime} 51^{\prime \prime}$, Portage County, Hydrologic

Unit 05030103 , at bridge on Rock Spring Road $0.6 \mathrm{mi}$ south of

Charlestown, 2.2 mi upstream from mouth.

DRAINAGE AREA:

$7.85 \mathrm{mi}^{2}$.

TRIBUTARY TO:

West Branch Mahoning River.

DISCHARGE DATA USED:

Low-flow measurements 1969-1977 water years.

INDEX STATION:

03093000 Eagle Creek at Phalanx Station, Ohio.

REMARKS:

None.

SELECTED DISCHARGE CHARACTERISTICS: Minimum discharge observed: $0.1 \mathrm{ft}^{3} / \mathrm{s}$ August 1971.

\begin{tabular}{c|c|c|c}
\multicolumn{4}{c}{$\begin{array}{c}\text { Magnitude and frequency of low flow for } \\
\text { indicated periods }\end{array}$} \\
\hline \multirow{3}{*}{ Period } & $\begin{array}{c}\text { Number of } \\
\text { consecu- } \\
\text { tive days }\end{array}$ & $\begin{array}{c}\text { Discharge (ft } 3 / \mathrm{s} \text { ) for } \\
\text { indicated recurrence } \\
\text { interval (years) }\end{array}$ \\
\cline { 3 - 4 } & & 2 & 10 \\
\hline Apr. - Mar. & 7 & 0.3 & 0.2 \\
& 30 & 0.4 & 0.2 \\
May - Nov. & 7 & 0.3 & 0.2 \\
& 30 & 0.4 & 0.2 \\
June - Aug. & 7 & 0.3 & 0.2 \\
& 30 & 0.5 & 0.3 \\
Sept. - Nov. & 7 & 0.3 & 0.2 \\
& 30 & 0.4 & 0.2 \\
\hline
\end{tabular}

Duration of daily flow for indicated periods

\begin{tabular}{|c|c|c|c|c|c|}
\hline \multirow{2}{*}{ Period } & \multicolumn{5}{|c|}{$\begin{array}{l}\text { Discharge }\left(\mathrm{ft}^{3} / \mathrm{s}\right) \text { which was equaled or } \\
\text { exceeded for indicated percent of time }\end{array}$} \\
\hline & 98 & 95 & 90 & 85 & 80 \\
\hline $\begin{array}{l}\text { Apr. - Mar. } \\
\text { May - Nov. } \\
\text { June - Aug. } \\
\text { Sept. - Nov. }\end{array}$ & $\begin{array}{l}0.2 \\
0.2 \\
0.2 \\
0.2\end{array}$ & $\begin{array}{l}0.3 \\
0.2 \\
0.2 \\
0.2\end{array}$ & $\begin{array}{l}0.4 \\
0.3 \\
0.3 \\
0.3\end{array}$ & $\begin{array}{l}0.5 \\
0.4 \\
0.4 \\
0.3\end{array}$ & $\begin{array}{l}0.6 \\
0.4 \\
0.4 \\
0.4\end{array}$ \\
\hline
\end{tabular}


MAHONING RIVER BASIN

03092460 West Branch Mahoning River below Michael J. Kirwan Dam, at Wayland, Ohio

LOCATION:

Lat $4^{\circ} 09^{\prime} 2^{\prime \prime}$, long $81^{\circ} 04^{\prime} 1^{\prime \prime}$, in T.3 N., R.6 W., Portage County, Hydrologic Unit 05030103, on right bank $200 \mathrm{ft}$ upstream from bridge on Wayland Road, $0.4 \mathrm{mi}$ downstream from Michael J. Kirwan Dam, and $0.2 \mathrm{mi}$ south of Wayland.

DRAINAGE AREA: $\quad 81.7 \mathrm{mi}^{2}$.

TRIBUTARY TO: Mahoning River.

DISCHARGE DATA USED: October 1968 to September 1979.

REMARKS: Flow completely regulated by Michael J. Kirwan Reservoir since December 1966.

SELECTED DISCHARGE CHARACTERISTICS: Average discharge: $106 \mathrm{ft}^{3} / \mathrm{s}$ (1) years). Minimum daily discharge: 2.5 It $^{3} / \mathrm{s}$ Aprii 1969.

Magnitude and frequency of low flow for indicated periods

\begin{tabular}{|c|c|c|c|c|c|c|c|c|c|c|c|c|c|}
\hline \multirow{2}{*}{ Period } & \multirow{2}{*}{$\begin{array}{l}\text { Number of } \\
\text { consecu- } \\
\text { tive days }\end{array}$} & \multicolumn{5}{|c|}{$\begin{array}{l}\text { Discharge }\left(\mathrm{ft}^{3} / \mathrm{s}\right) \text { for indicated } \\
\text { recurrence interval (years) }\end{array}$} & \multirow{2}{*}{ Period } & \multirow{2}{*}{$\begin{array}{l}\text { Number of } \\
\text { consecu- } \\
\text { tive days }\end{array}$} & \multicolumn{5}{|c|}{$\begin{array}{l}\text { Discharge }\left(\mathrm{ft}^{3} / \mathrm{s}\right) \text { for indicated } \\
\text { recurrence interval (years) }\end{array}$} \\
\hline & & 2 & 5 & 10 & 20 & 50 & & & 2 & 5 & 10 & 20 & 50 \\
\hline Apr.-Mar. & $\begin{array}{r}1 \\
7 \\
30\end{array}$ & $\begin{array}{l}16 \\
19 \\
20\end{array}$ & $\begin{array}{l}8.6 \\
11 \\
16\end{array}$ & $\begin{array}{l}5.4 \\
6.7 \\
15\end{array}$ & $\begin{array}{r}3.5 \\
4.2 \\
14\end{array}$ & $\begin{array}{c}1.9 \\
2.2 \\
13\end{array}$ & Sept.-Nov. & $\begin{array}{r}1 \\
7 \\
30\end{array}$ & $\begin{array}{l}27 \\
31 \\
44\end{array}$ & $\begin{array}{l}18 \\
19 \\
26\end{array}$ & $\begin{array}{l}14 \\
15 \\
20\end{array}$ & $\begin{array}{l}12 \\
12 \\
16\end{array}$ & $\begin{array}{c}9.4 \\
9.2 \\
12\end{array}$ \\
\hline May- Nov. & $\begin{array}{r}1 \\
7 \\
30\end{array}$ & $\begin{array}{l}17 \\
20 \\
35\end{array}$ & $\begin{array}{l}12 \\
15 \\
22\end{array}$ & $\begin{array}{l}9.5 \\
12 \\
17\end{array}$ & $\begin{array}{l}8.1 \\
11 \\
14\end{array}$ & $\begin{array}{r}6.7 \\
9.6 \\
11\end{array}$ & Dec.-Feb. & $\begin{array}{r}1 \\
7 \\
30\end{array}$ & $\begin{array}{l}19 \\
19 \\
34\end{array}$ & $\begin{array}{l}14 \\
14 \\
20\end{array}$ & $\begin{array}{l}12 \\
13 \\
15\end{array}$ & $\begin{array}{l}11 \\
12 \\
12\end{array}$ & $\begin{array}{l}9.6 \\
11 \\
10\end{array}$ \\
\hline June-Aug. & $\begin{array}{r}1 \\
7 \\
30\end{array}$ & $\begin{array}{l}29 \\
35 \\
61\end{array}$ & $\begin{array}{l}19 \\
21 \\
40\end{array}$ & $\begin{array}{l}14 \\
16 \\
30\end{array}$ & $\begin{array}{l}10 \\
12 \\
22\end{array}$ & $\begin{array}{l}7.4 \\
8.8 \\
15\end{array}$ & Mar. - May & $\begin{array}{r}1 \\
7 \\
30\end{array}$ & $\begin{array}{l}18 \\
23 \\
34\end{array}$ & $\begin{array}{l}9.4 \\
12 \\
16\end{array}$ & $\begin{array}{l}5.9 \\
7.3 \\
9.8\end{array}$ & $\begin{array}{l}3.8 \\
4.5 \\
6.2\end{array}$ & $\begin{array}{l}2.1 \\
2.3 \\
3.6\end{array}$ \\
\hline
\end{tabular}

Duration of daily flow for indicated periods

\begin{tabular}{|c|c|c|c|c|c|c|c|c|c|c|c|c|c|}
\hline \multirow{2}{*}{ Period } & \multicolumn{13}{|c|}{ Discharge $\left(\mathrm{ft}^{3} / \mathrm{s}\right)$ which was equaled or exceeded for indicated percent of time } \\
\hline & 98 & 95 & 90 & 85 & 80 & 75 & 70 & 60 & 50 & 40 & 30 & 20 & 10 \\
\hline $\begin{array}{l}\text { Apr. - Mar. } \\
\text { May - Nov. } \\
\text { June - Aug. } \\
\text { Sept. - Nov. } \\
\text { Dec. - Feb. } \\
\text { Mar. - May }\end{array}$ & $\begin{array}{l}13 \\
17 \\
17 \\
17 \\
12 \\
4.5\end{array}$ & $\begin{array}{l}17 \\
21 \\
24 \\
19 \\
13 \\
13\end{array}$ & $\begin{array}{l}20 \\
25 \\
30 \\
23 \\
17 \\
18\end{array}$ & $\begin{array}{l}23 \\
29 \\
41 \\
26 \\
19 \\
21\end{array}$ & $\begin{array}{l}26 \\
41 \\
49 \\
41 \\
21 \\
23\end{array}$ & $\begin{array}{l}35 \\
46 \\
56 \\
46 \\
24 \\
24\end{array}$ & $\begin{array}{l}43 \\
56 \\
66 \\
59 \\
31 \\
26\end{array}$ & $\begin{array}{l}58 \\
72 \\
77 \\
74 \\
47 \\
39\end{array}$ & $\begin{array}{l}77 \\
83 \\
87 \\
90 \\
69 \\
44\end{array}$ & $\begin{array}{r}96 \\
97 \\
96 \\
110 \\
130 \\
60\end{array}$ & $\begin{array}{r}120 \\
110 \\
110 \\
120 \\
160 \\
90\end{array}$ & $\begin{array}{l}150 \\
130 \\
120 \\
160 \\
190 \\
140\end{array}$ & $\begin{array}{l}210 \\
180 \\
140 \\
220 \\
300 \\
240\end{array}$ \\
\hline
\end{tabular}


LOCATION: Lat 41\%10'18", long 81.01'16", in T.3 N., R.6 W., Portage County, Hydrologic Unit 05030103 , on right bank $250 \mathrm{ft}$ downstream from bridge on Newton Falls Road, $2.5 \mathrm{mi}$ southwest of Newton Falls, $6 \mathrm{mi}$ upstream from mouth, and $5 \mathrm{mi}$ downstream from Michael J. Kirwan Dam.

DRAINAGE AREA:

$96.3 \mathrm{mi}^{2}$

TRIBUTARY TO:

Mahoning River.

DISCHARGE DATA USED:

REMARKS :

April 1967 to September 1978.

Flow regulated by Michael J. Kirwan Reservoir since December 1966 .

SELECTED DISCHARGE CHARACTERISTICS: Average discharge: $114 \mathrm{ft}^{3} / \mathrm{s}$ (11 years).

Minimum daily discharge: $7.0 \mathrm{ft}^{3} / \mathrm{s}$ January 1968.

Magnitude and frequency of low flow for indicated periods

\begin{tabular}{|c|c|c|c|c|c|c|c|c|c|c|c|c|c|}
\hline \multirow{2}{*}{ Period } & \multirow{2}{*}{$\begin{array}{l}\text { Number of } \\
\text { consecu- } \\
\text { tive days }\end{array}$} & \multicolumn{5}{|c|}{$\begin{array}{l}\text { Discharge }\left(\mathrm{ft}^{3} / \mathrm{s}\right) \text { for indicated } \\
\text { recurrence interval (years) }\end{array}$} & \multirow{2}{*}{ Period } & \multirow{2}{*}{$\begin{array}{l}\text { Number of } \\
\text { consecu- } \\
\text { tive days }\end{array}$} & \multicolumn{5}{|c|}{$\begin{array}{l}\text { Discharge }\left(\mathrm{ft}^{3} / \mathrm{s}\right) \text { for indicated } \\
\text { recurrence interval (years) }\end{array}$} \\
\hline & & 2 & 5 & 10 & 20 & 50 & & & 2 & 5 & 10 & 20 & 50 \\
\hline Apr.-Mar. & $\begin{array}{r}1 \\
7 \\
30\end{array}$ & $\begin{array}{l}17 \\
22 \\
29\end{array}$ & $\begin{array}{l}11 \\
13 \\
18\end{array}$ & $\begin{array}{l}8.7 \\
9.8 \\
13\end{array}$ & $\begin{array}{r}6.9 \\
7.5 \\
10^{7}\end{array}$ & $\begin{array}{l}5.2 \\
5.3 \\
7.6\end{array}$ & Sept.-Nov. & $\begin{array}{r}1 \\
7 \\
30\end{array}$ & $\begin{array}{l}25 \\
27 \\
40\end{array}$ & $\begin{array}{l}14 \\
17 \\
23\end{array}$ & $\begin{array}{l}10 \\
13 \\
17\end{array}$ & $\begin{array}{l}8.0 \\
11 \\
13\end{array}$ & $\begin{array}{l}5.9 \\
8.7 \\
9.8\end{array}$ \\
\hline June-Aug. & $\begin{array}{r}1 \\
7 \\
30\end{array}$ & $\begin{array}{l}26 \\
35 \\
56\end{array}$ & $\begin{array}{l}17 \\
23 \\
38\end{array}$ & $\begin{array}{l}14 \\
19 \\
30\end{array}$ & $\begin{array}{l}12 \\
16 \\
24\end{array}$ & $\begin{array}{l}10 \\
13 \\
18\end{array}$ & Mar. - May & $\begin{array}{r}1 \\
7 \\
30\end{array}$ & $\begin{array}{l}24 \\
29 \\
43\end{array}$ & $\begin{array}{l}15 \\
19 \\
27\end{array}$ & $\begin{array}{l}12 \\
14 \\
22\end{array}$ & $\begin{array}{l}9.5 \\
11 \\
18\end{array}$ & $\begin{array}{l}7.2 \\
7.6 \\
14\end{array}$ \\
\hline
\end{tabular}

Duration of daily flow for indicated periods

\begin{tabular}{l|c|c|c|c|c|c|c|c|c|c|c|c|c}
\hline \multirow{2}{*}{ Period } & \multicolumn{8}{|c|}{ Discharge $\left(\mathrm{ft}^{3} / \mathrm{s}\right)$ which was equaled or exceeded for indicated percent of time } \\
\cline { 2 - 12 } & 98 & 95 & 90 & 85 & 80 & 75 & 70 & 60 & 50 & 40 & 30 & 20 & 10 \\
\hline Apr. - Mar. & 14 & 19 & 23 & 27 & 32 & 41 & 48 & 66 & 84 & 100 & 130 & 160 & 230 \\
May - Nov. & 15 & 19 & 23 & 29 & 40 & 49 & 57 & 75 & 89 & 100 & 120 & 140 & 190 \\
June - Aug. & 20 & 23 & 30 & 43 & 49 & 55 & 61 & 77 & 87 & 95 & 100 & 120 & 140 \\
Sept.- Nov. & 14 & 16 & 20 & 22 & 26 & 32 & 49 & 76 & 95 & 110 & 140 & 160 & 220 \\
Dec. - Feb. & 11 & 16 & 20 & 23 & 26 & 30 & 35 & 51 & 76 & 120 & 160 & 220 & 330 \\
Mar. - May & 15 & 21 & 26 & 29 & 32 & 35 & 40 & 49 & 57 & 82 & 110 & 150 & 220 \\
\hline
\end{tabular}


LOCATION:

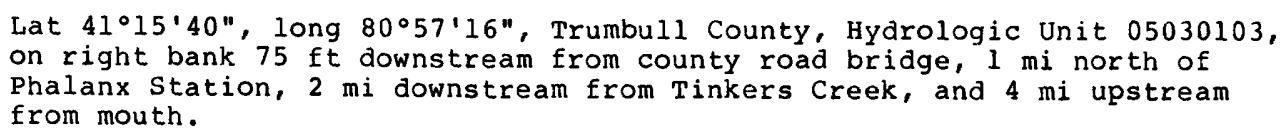

DRAINAGE AREA:

$97.6 \mathrm{mi}^{2}$

TRIBUTARY TO:

Mahoning River.

DISCHARGE DATA USED:

June 1926 to September 1934, October 1937 to September 1978 .

REMARKS :

Low flow slightly regulated by mill several miles upstream from station.

SELECTED DISCHARGE CHARACTERISTICS: Average discharge: $108 \mathrm{ft}^{3} / \mathrm{s}$ (49 years).

Minimum daily discharge: $0.9 \mathrm{ft}^{3} / \mathrm{s}$ August 1939.

Magnitude and frequency of low flow for indicated periods

\begin{tabular}{|c|c|c|c|c|c|c|c|c|c|c|c|c|c|}
\hline \multirow{2}{*}{ Period } & \multirow{2}{*}{$\begin{array}{l}\text { Number of } \\
\text { consecu- } \\
\text { tive days }\end{array}$} & \multicolumn{5}{|c|}{$\begin{array}{l}\text { Discharge }\left(\mathrm{ft}^{3} / \mathrm{s}\right) \text { for indicated } \\
\text { recurrence interval (years) }\end{array}$} & \multirow{2}{*}{ Period } & \multirow{2}{*}{$\begin{array}{l}\text { Number of } \\
\text { consecu- } \\
\text { tive days }\end{array}$} & \multicolumn{5}{|c|}{$\begin{array}{l}\text { Discharge }\left(\mathrm{ft}^{3} / \mathrm{s}\right) \text { for indicated } \\
\text { recurrence interval (years) }\end{array}$} \\
\hline & & 2 & 5 & 10 & 20 & 50 & & & 2 & 5 & 10 & 20 & 50 \\
\hline Apr. -Mar. & $\begin{array}{r}1 \\
7 \\
30\end{array}$ & $\begin{array}{l}7.2 \\
9.6 \\
12\end{array}$ & $\begin{array}{l}4.0 \\
7.2 \\
9.2\end{array}$ & $\begin{array}{l}2.8 \\
6.2 \\
7.9\end{array}$ & $\begin{array}{l}2.0 \\
5.5 \\
7.0\end{array}$ & $\begin{array}{l}1.3 \\
4.9 \\
6.2\end{array}$ & Sept.-Nov. & $\begin{array}{r}1 \\
7 \\
30\end{array}$ & $\begin{array}{l}7.9 \\
9.8 \\
14\end{array}$ & $\begin{array}{l}4.8 \\
7.5 \\
9.8\end{array}$ & $\begin{array}{l}3.7 \\
6.9 \\
8.7\end{array}$ & $\begin{array}{l}3.0 \\
6.5 \\
8.1\end{array}$ & $\begin{array}{l}2.3 \\
6.3 \\
7.7\end{array}$ \\
\hline June-Aug. & $\begin{array}{r}1 \\
7 \\
30\end{array}$ & $\begin{array}{l}8.6 \\
11 \\
15\end{array}$ & $\begin{array}{l}4.5 \\
8.1 \\
11\end{array}$ & $\begin{array}{l}2.9 \\
6.8 \\
9.4\end{array}$ & $\begin{array}{l}2.0 \\
6.0 \\
\text { B.5 }\end{array}$ & $\begin{array}{l}1.2 \\
5.2 \\
7.8\end{array}$ & Mar.- May & $\begin{array}{r}1 \\
7 \\
30\end{array}$ & $\begin{array}{l}30 \\
34 \\
71\end{array}$ & $\begin{array}{l}20 \\
23 \\
44\end{array}$ & $\begin{array}{l}14 \\
19 \\
33\end{array}$ & $\begin{array}{l}11 \\
16 \\
26\end{array}$ & $\begin{array}{l}7.4 \\
14 \\
19\end{array}$ \\
\hline
\end{tabular}

Duration of daily flow for indicated periods

\begin{tabular}{l|c|c|c|c|c|c|c|c|c|r|r|r|r}
\hline \multirow{2}{*}{ Period } & \multicolumn{8}{|c|}{ Discharge $\left(\mathrm{ft}^{3} / \mathrm{s}\right)$ which was equaled or exceeded for indicated percent of time } \\
\cline { 2 - 13 } & 98 & 95 & 90 & 85 & 80 & 75 & 70 & 60 & 50 & 40 & 30 & 20 & 10 \\
\hline Apr. - Mar. & 7.4 & 9.9 & 13 & 15 & 17 & 20 & 23 & 30 & 40 & 56 & 80 & 130 & 260 \\
May - Nov. & 6.7 & 8.4 & 11 & 12 & 14 & 15 & 17 & 20 & 25 & 30 & 39 & 58 & 110 \\
June - Aug. & 6.5 & 8.3 & 10 & 12 & 13 & 15 & 16 & 19 & 22 & 27 & 33 & 44 & 76 \\
Sept. - Nov. & 6.0 & 7.6 & 9.6 & 11 & 12 & 14 & 15 & 18 & 21 & 26 & 34 & 48 & 96 \\
Dec. - Feb. & 15 & 18 & 22 & 26 & 30 & 34 & 39 & 50 & 66 & 87 & 120 & 200 & 380 \\
Mar. - May & 22 & 28 & 35 & 42 & 48 & 55 & 61 & 77 & 97 & 130 & 170 & 250 & 420 \\
\hline
\end{tabular}


MAHONING RIVER BASIN

03095500 Mosquito Creek below Mosquito Creek Dam near Cortland, Ohio

LOCATION:

Lat $41^{\circ} 17^{\prime} 59^{\prime \prime}$, long $80^{\circ} 45^{\prime} 31^{\prime \prime}$, in T.5 N., R. 3 W., Trumbull county Hydrologic Unit 05030103 , on right bank $100 \mathrm{ft}$ downstream from Mosquito Creek Dam, $0.8 \mathrm{mi}$ upstream from Confusion Run, and $2.5 \mathrm{mi}$ southwest of Cortland.

DRAINAGE AREA:

$97.5 \mathrm{mi}^{2}$

TRIBUTTARY TO:

Mahoning River.

DISCHARGE DATA USED:

October 1954 to September 1978.

REMARKS:

Flow completely regulated by Mosquito Creek Lake beginning 1943. Diversion at lake outlet for municipal supply of city of Warren since May 1954; diversion not included in figures of daily discharge.

SELECTED DISCHARGE CHARACTERISTICS: Average discharge: $76.7 \mathrm{ft}^{3} / \mathrm{s}$ (24 years).

Minimum daily discharge: $2.0 \mathrm{ft} / \mathrm{s}$ April 1968.

Magnitude and frequency of low flow for indicated perrods

\begin{tabular}{|c|c|c|c|c|c|c|c|c|c|c|c|c|c|}
\hline \multirow{2}{*}{ Period } & \multirow{2}{*}{$\begin{array}{l}\text { Number of } \\
\text { consecu- } \\
\text { tive days }\end{array}$} & \multicolumn{5}{|c|}{$\begin{array}{l}\text { Discharge }\left(\mathrm{ft}^{3} / \mathrm{s}\right) \text { for indicated } \\
\text { recurrence interval (years) }\end{array}$} & \multirow{2}{*}{ Period } & \multirow{2}{*}{$\begin{array}{l}\text { Number of } \\
\text { consecu- } \\
\text { tive days }\end{array}$} & \multicolumn{5}{|c|}{$\begin{array}{l}\text { Discharge }\left(\mathrm{ft}^{3} / \mathrm{s}\right) \text { for indicated } \\
\text { recurrence interva? (years) }\end{array}$} \\
\hline & & 2 & 5 & 10 & 20 & 50 & & & 2 & 5 & 10 & 20 & 50 \\
\hline Apr.-Mar. & $\begin{array}{r}1 \\
7 \\
30\end{array}$ & $\begin{array}{l}4.7 \\
5.3 \\
6.2\end{array}$ & $\begin{array}{l}3.3 \\
3.7 \\
4.2\end{array}$ & $\begin{array}{l}2.8 \\
3.3 \\
3.5\end{array}$ & $\begin{array}{l}2.5 \\
2.9 \\
3.1\end{array}$ & $\begin{array}{l}2.3 \\
2.7 \\
2.7\end{array}$ & Sept.-Nov. & $\begin{array}{r}1 \\
7 \\
30\end{array}$ & $\begin{array}{l}8.7 \\
10 \\
21\end{array}$ & $\begin{array}{l}4.6 \\
5.5 \\
11\end{array}$ & $\begin{array}{l}3.5 \\
4.1 \\
7.0\end{array}$ & $\begin{array}{l}2.8 \\
3.3 \\
4.8\end{array}$ & $\begin{array}{l}2.3 \\
2.6 \\
3.0\end{array}$ \\
\hline June-Aug. & $\begin{array}{r}1 \\
7 \\
30\end{array}$ & $\begin{array}{l}6.6 \\
9.8 \\
48\end{array}$ & $\begin{array}{r}4.5 \\
5.7 \\
22\end{array}$ & $\begin{array}{c}3.8 \\
4.4 \\
14\end{array}$ & $\begin{array}{l}3.3 \\
3.5 \\
9.1\end{array}$ & $\begin{array}{l}2.9 \\
2.8 \\
5.5\end{array}$ & Mar. - May & $\begin{array}{r}1 \\
7 \\
30\end{array}$ & $\begin{array}{l}5.8 \\
6.2 \\
9.8\end{array}$ & $\begin{array}{l}3.7 \\
4.2 \\
5.0\end{array}$ & $\begin{array}{l}3.0 \\
3.6 \\
3.7\end{array}$ & $\begin{array}{l}2.6 \\
3.2 \\
2.9\end{array}$ & $\begin{array}{l}2.2 \\
2.9 \\
2.2\end{array}$ \\
\hline
\end{tabular}

Duration of daily flow for indicated periods

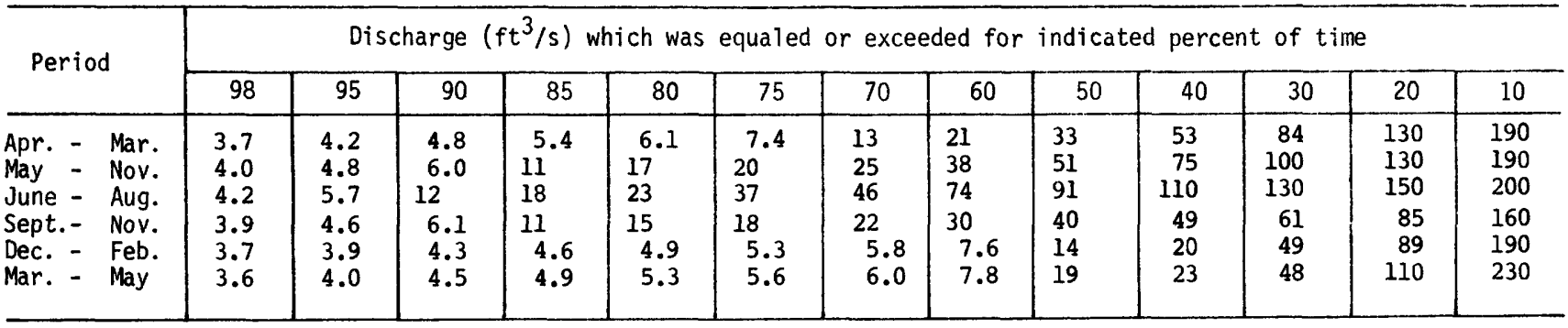


MAHONING RIVER BASIN

03097500 Meander Creek at Mineral Ridge, Ohio

LOCATION:

Lat 41.09'26", long $80^{\circ} 46^{\prime} 31^{\prime \prime}$, in T.3 N., R.3 W., Trumbull county, Hydrologic Unit 05030103 , on right bank $0.4 \mathrm{mi}$ upstream from highway bridge, $0.8 \mathrm{mi}$ downstream from Mineral Ridge Dam, and $1.0 \mathrm{mi}$ northwest of Mineral Ridge.

DRAINAGE AREA: $\quad 84.3 \mathrm{mi}^{2}$.

TRIBUTARY TO: Mahoning River.

DISCHARGE DATA USED: August 1929 to September 1951.

REMARKS :

Some diversion upstream from station for municipal supply for McDonald, Niles, and Youngstown. Flow regulated by Meander Creek Reservoir.

SELECTED DISCHARGE CHARACTERISTICS: Average discharge: $44.1 \mathrm{ft}^{3} / \mathrm{s}(22$ years).

Minimum daily discharge: No flow August 1934 (affected by ponding of water above new concrete control).

Magnitude and frequency of low flow for indicated periods

\begin{tabular}{c|c|c|c|c|l|l}
\hline \multirow{2}{*}{ Period } & \multirow{2}{*}{$\begin{array}{c}\text { Number of } \\
\text { consecu- } \\
\text { tive days }\end{array}$} & \multicolumn{4}{|c}{$\begin{array}{c}\text { Discharge } \\
\text { recurrence } 3 / \mathrm{f} \text { ) for interval indicated } \\
\text { (years) }\end{array}$} \\
\cline { 3 - 7 } Apr.-Mar. & 1 & 2 & 5 & 10 & 20 & 50 \\
\hline \multirow{3}{*}{ May- Nov. } & 7 & 0.4 & 0.2 & 0.1 & 0 & 0 \\
& 30 & 0.7 & 0.3 & 0.2 & 0.1 & 0.1 \\
& 1 & 0.4 & 0.2 & 0.1 & 0 & 0 \\
& 7 & 0.6 & 0.3 & 0.2 & 0.1 & 0.1 \\
& 30 & 0.8 & 0.5 & 0.4 & 0.3 & 0.3 \\
& 1 & 0.5 & 0.3 & 0.2 & 0.2 & 0 \\
& 7 & 0.8 & 0.4 & 0.3 & 0.2 & 0.1 \\
& 30 & 1.0 & 0.7 & 0.6 & 0.5 & 0.4 \\
\hline
\end{tabular}

\begin{tabular}{|c|c|c|c|c|c|c|}
\hline \multirow{2}{*}{ Period } & \multirow{2}{*}{$\begin{array}{l}\text { Number of } \\
\text { consecu- } \\
\text { tive days }\end{array}$} & \multicolumn{5}{|c|}{$\begin{array}{l}\text { Discharge }\left(\mathrm{ft}^{3} / \mathrm{s}\right) \text { for indicated } \\
\text { recurrence interval (years) }\end{array}$} \\
\hline & & 2 & 5 & 10 & 20 & 50 \\
\hline Sept.-Nov. & $\begin{array}{r}1 \\
7 \\
30\end{array}$ & $\begin{array}{l}0.5 \\
0.7 \\
0.9\end{array}$ & $\begin{array}{l}0.2 \\
0.4 \\
0.5\end{array}$ & $\begin{array}{l}0.2 \\
0.3 \\
0.4\end{array}$ & $\begin{array}{l}0.1 \\
0.2 \\
0.4\end{array}$ & $\begin{array}{l}0.1 \\
0.2 \\
0.3\end{array}$ \\
\hline Dec. -Feb. & $\begin{array}{r}1 \\
7 \\
30\end{array}$ & $\begin{array}{l}0.8 \\
1.0 \\
2.8\end{array}$ & $\begin{array}{l}0.4 \\
0.5 \\
0.8\end{array}$ & $\begin{array}{l}0.3 \\
0.4 \\
0.5\end{array}$ & $\begin{array}{l}0.3 \\
0.3 \\
0.3\end{array}$ & $\begin{array}{l}0.2 \\
0.3 \\
0.2\end{array}$ \\
\hline Mar. - May & $\begin{array}{r}1 \\
7 \\
30\end{array}$ & $\begin{array}{c}1.0 \\
1.5 \\
10\end{array}$ & $\begin{array}{l}0.6 \\
0.9 \\
3.1\end{array}$ & $\begin{array}{l}0.5 \\
0.5 \\
1.6\end{array}$ & $\begin{array}{l}0.4 \\
0.5 \\
0.9\end{array}$ & $\begin{array}{l}0.3 \\
0.4 \\
0.5\end{array}$ \\
\hline
\end{tabular}

Duration of daily flow for indicated periods

\begin{tabular}{l|r|c|c|c|c|c|c|c|c|r|r|r|r}
\hline \multirow{2}{*}{ Period } & \multicolumn{8}{c|}{ Discharge $\left(\mathrm{ft}^{3} / \mathrm{s}\right)$ which was equaled or exceeded for indicated percent of time } \\
\cline { 2 - 12 } & 98 & 95 & 90 & 85 & 80 & 75 & 70 & 60 & 50 & 40 & 30 & 20 & 10 \\
\hline Apr. - Mar. & 0.4 & 0.5 & 0.6 & 0.7 & 0.8 & 0.9 & 1.1 & 1.4 & 1.8 & 2.8 & 6.3 & 28 & 100 \\
May - Nov. & 0.4 & 0.5 & 0.6 & 0.7 & 0.7 & 0.8 & 0.9 & 1.1 & 1.4 & 1.7 & 2.3 & 14.3 & 19.1 \\
June - Aug. & 0.4 & 0.5 & 0.6 & 0.7 & 0.8 & 0.9 & 1.0 & 1.3 & 1.5 & 1.7 & 2.3 & 3.9 & 9.7 \\
Sept. - Nov. & 0.3 & 0.4 & 0.5 & 0.6 & 0.7 & 0.7 & 0.8 & 0.9 & 1.1 & 1.4 & 1.7 & 2.2 & 3.9 \\
Dec. - Feb. & 0.4 & 0.5 & 0.7 & 0.8 & 0.8 & 0.9 & 1.2 & 1.8 & 2.5 & 5.5 & 16 & 55 & 140 \\
Mar. - May & 0.7 & 0.8 & 1.2 & 1.7 & 2.7 & 3.9 & 5.1 & 13 & 27 & 49 & 81 & 140 & 280 \\
\hline
\end{tabular}


DRAINAGE AREA: $\quad 898 \mathrm{mi}^{2}$.

TRIBUTARY TO: Head of Beaver River.

DISCHARGE DATA USED: October 1966 to September 1978.

REMARKS :

Water diverted upstream from station for municipal supply for city of Youngstown. Some sewage returned to river upstream from station. Water also diverted upstream and downstream from station by a private company for industrial use, some of which is returned to river upstream from station. Flow regulated by Berlin Lake, $48 \mathrm{mi}$ upstream, beginning in 1942, by Milton Reservoir, $40 \mathrm{mi}$ upstream, by Michael J. Kirwan Reservoir, $43 \mathrm{mi}$ upstream on West Branch, beginning in 1966, by Mosquito Creek Lake, $22 \mathrm{mi}$ upstream, beginning in 1943, by Meander Creek Reservoir, $11 \mathrm{mi}$ upstream, beginning in 1929, and by reşervoir on Squaw Creek, $5 \mathrm{mi}$ upstream.

SELECTED DISCHARGE CHARACTERISTICS: Average discharge: $988 \mathrm{ft}^{3} / \mathrm{s}$ (12 years).

Minimum daily discharge: $177 \mathrm{ft}^{3} / \mathrm{s}$ October 1966.

Magnitude and frequency of low flow for indicated periods

\begin{tabular}{|c|c|c|c|c|c|c|}
\hline \multirow{2}{*}{ Period } & \multirow{2}{*}{$\begin{array}{l}\text { Number of } \\
\text { consecu- } \\
\text { tive days }\end{array}$} & \multicolumn{5}{|c|}{$\begin{array}{l}\text { Discharge }\left(\mathrm{ft}^{3} / \mathrm{s}\right) \text { for indicated } \\
\text { recurrence interval (years) }\end{array}$} \\
\hline & & 2 & 5 & 10 & 20 & 50 \\
\hline Apr.-Mar. & $\begin{array}{r}1 \\
7 \\
30\end{array}$ & $\begin{array}{l}250 \\
269 \\
336\end{array}$ & $\begin{array}{l}212 \\
225 \\
275\end{array}$ & $\begin{array}{l}194 \\
206 \\
246\end{array}$ & $\begin{array}{l}181 \\
191 \\
223\end{array}$ & $\begin{array}{l}167 \\
176 \\
200\end{array}$ \\
\hline May- Nov. & $\begin{array}{r}1 \\
7 \\
30\end{array}$ & $\begin{array}{l}275 \\
297 \\
358\end{array}$ & $\begin{array}{l}234 \\
251 \\
292\end{array}$ & $\begin{array}{l}213 \\
227 \\
258\end{array}$ & $\begin{array}{l}196 \\
208 \\
231\end{array}$ & $\begin{array}{l}177 \\
187 \\
202\end{array}$ \\
\hline June-Aug. & $\begin{array}{r}1 \\
7 \\
30\end{array}$ & $\begin{array}{l}333 \\
371 \\
454\end{array}$ & $\begin{array}{l}297 \\
345 \\
416\end{array}$ & $\begin{array}{l}278 \\
332 \\
401\end{array}$ & $\begin{array}{l}262 \\
321 \\
391\end{array}$ & $\begin{array}{l}243 \\
310 \\
381\end{array}$ \\
\hline
\end{tabular}

\begin{tabular}{|c|c|c|c|c|c|c|}
\hline \multirow{2}{*}{ Period } & \multirow{2}{*}{$\begin{array}{l}\text { Number of } \\
\text { consecu- } \\
\text { tive days }\end{array}$} & \multicolumn{5}{|c|}{$\begin{array}{l}\text { Discharge }\left(\mathrm{ft}^{3} / \mathrm{s}\right) \text { for indicated } \\
\text { recurrence interval (years) }\end{array}$} \\
\hline & & 2 & 5 & 10 & 20 & 50 \\
\hline Sept.-Nov. & $\begin{array}{r}1 \\
7 \\
30\end{array}$ & $\begin{array}{l}291 \\
327 \\
405\end{array}$ & $\begin{array}{l}232 \\
255 \\
298\end{array}$ & $\begin{array}{l}207 \\
222 \\
254\end{array}$ & $\begin{array}{l}189 \\
198 \\
223\end{array}$ & $\begin{array}{l}170 \\
174 \\
193\end{array}$ \\
\hline Dec. -Feb. & $\begin{array}{r}1 \\
7 \\
30\end{array}$ & $\begin{array}{l}258 \\
305 \\
552\end{array}$ & $\begin{array}{l}208 \\
241 \\
324\end{array}$ & $\begin{array}{l}197 \\
224 \\
249\end{array}$ & $\begin{array}{l}192 \\
215 \\
201\end{array}$ & $\begin{array}{l}189 \\
209 \\
160\end{array}$ \\
\hline Mar. - May & $\begin{array}{r}1 \\
7 \\
30\end{array}$ & $\begin{array}{l}299 \\
312 \\
524\end{array}$ & $\begin{array}{l}246 \\
255 \\
375\end{array}$ & $\begin{array}{l}224 \\
240 \\
324\end{array}$ & $\begin{array}{l}210 \\
232 \\
292\end{array}$ & $\begin{array}{l}196 \\
227 \\
263\end{array}$ \\
\hline
\end{tabular}

Duration of daily flow for indicated periods

\begin{tabular}{|c|c|c|c|c|c|c|c|c|c|c|c|c|c|}
\hline \multirow{2}{*}{ Period } & \multicolumn{13}{|c|}{ Discharge $\left(\mathrm{ft}^{3} / \mathrm{s}\right)$ which was equaled or exceeded for indicated percent of time } \\
\hline & 98 & 95 & 90 & 85 & 80 & 75 & 70 & 60 & 50 & 40 & 30 & 20 & 10 \\
\hline $\begin{array}{l}\text { Apr. - Mar. } \\
\text { May - Nov. } \\
\text { June - Aug. } \\
\text { Sept. - Nov. } \\
\text { Dec. - Feb. } \\
\text { Mar. - May }\end{array}$ & $\begin{array}{l}240 \\
240 \\
320 \\
220 \\
220 \\
250\end{array}$ & $\begin{array}{l}270 \\
270 \\
340 \\
240 \\
240 \\
300\end{array}$ & $\begin{array}{l}320 \\
320 \\
370 \\
270 \\
280 \\
340\end{array}$ & $\begin{array}{l}350 \\
340 \\
390 \\
290 \\
320 \\
360\end{array}$ & $\begin{array}{l}380 \\
370 \\
410 \\
320 \\
360 \\
400\end{array}$ & $\begin{array}{l}400 \\
390 \\
420 \\
350 \\
400 \\
440\end{array}$ & $\begin{array}{l}430 \\
410 \\
430 \\
370 \\
460 \\
500\end{array}$ & $\begin{array}{l}480 \\
440 \\
450 \\
410 \\
600 \\
670\end{array}$ & $\begin{array}{l}560 \\
480 \\
470 \\
460 \\
790 \\
960\end{array}$ & $\begin{array}{r}720 \\
540 \\
510 \\
540 \\
1100 \\
1300\end{array}$ & $\begin{array}{r}1000 \\
670 \\
570 \\
730 \\
1600 \\
1800\end{array}$ & $\begin{array}{r}1600 \\
910 \\
680 \\
1100 \\
2100 \\
2300\end{array}$ & $\begin{array}{r}2300 \\
1500 \\
890 \\
1700 \\
2800 \\
3000\end{array}$ \\
\hline
\end{tabular}


SHENANGO RIVER BASIN

03102950 Pymatuning Creek at Kinsman, Ohio

LOCATION:

Lat $41^{\circ} 26^{\prime} 34^{\prime \prime}$, long $80^{\circ} 35^{\prime} 18^{\prime \prime}$, in T.7 N., R.I W., Trumbull County, Hydrologic Unit 05030102 , on left bank at downstream side of bridge on State Route 7 at Kinsman, $0.8 \mathrm{mi}$ downstream from Sugar Creek, and $1.2 \mathrm{mi}$ upstream from stratton creek.

DRAINAGE AREA: $\quad 96.7 \mathrm{mi}^{2}$.

TRIBUTARY TO: Shenango River.

DISCHARGE DATA USED: October 1965 to September 1978.

REMARKS :

None.

SELECTED DISCHARGE CHARACTERISTICS: Average discharge: $120 \mathrm{ft}^{3} / \mathrm{s}$ ( 13 years).

Minimum daily discharge: $0.1 \mathrm{ft}^{3} / \mathrm{s}$ August 1972.

Magnitude and frequency of low flow for indicated periods

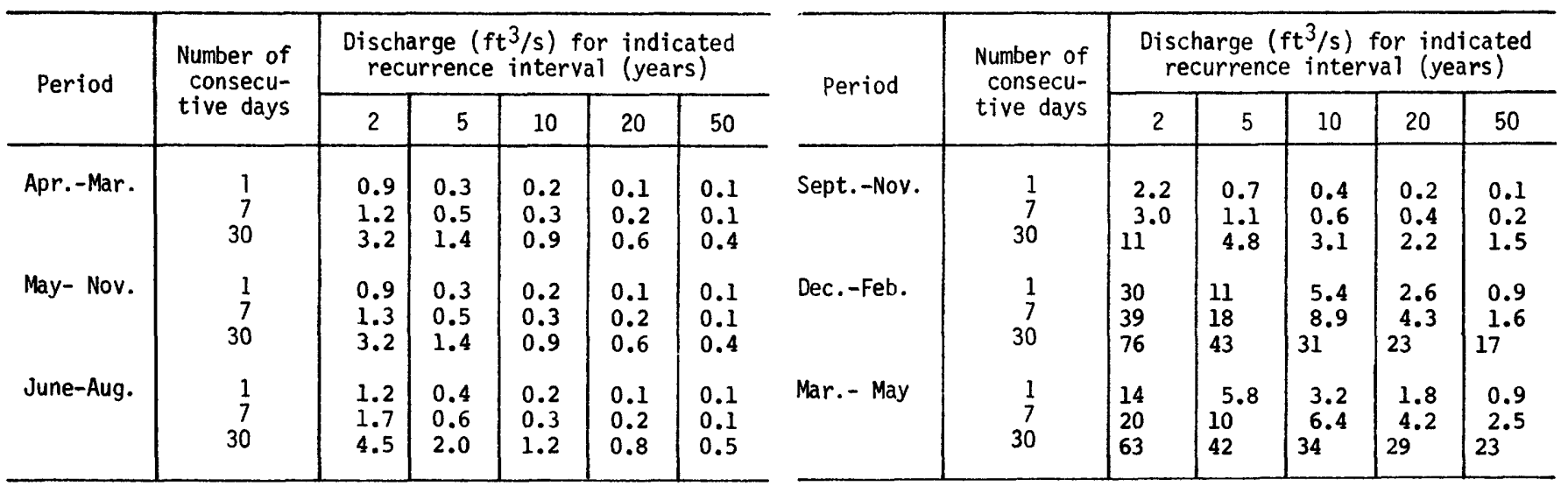

Duration of daily flow for indicated periods

\begin{tabular}{|c|c|c|c|c|c|c|c|c|c|c|c|c|c|}
\hline \multirow{2}{*}{ Period } & \multicolumn{13}{|c|}{ Discharge $\left(\mathrm{ft}^{3} / \mathrm{s}\right)$ which was equaled or exceeded for indicated percent of time } \\
\hline & 98 & 95 & 90 & 85 & 80 & 75 & 70 & 60 & 50 & 40 & 30 & 20 & 10 \\
\hline $\begin{array}{l}\text { Apr. - Mar. } \\
\text { May - Nov. } \\
\text { June - Aug. } \\
\text { Sept. - Nov. } \\
\text { Dec. - Feb. } \\
\text { Mar. - May }\end{array}$ & $\begin{array}{c}1.0 \\
0.6 \\
0.3 \\
1.4 \\
18 \\
8.6\end{array}$ & $\begin{array}{l}2.1 \\
1.4 \\
0.8 \\
2.2 \\
22 \\
19\end{array}$ & $\begin{array}{l}4.0 \\
2.4 \\
1.7 \\
3.1 \\
30 \\
28\end{array}$ & $\begin{array}{l}6.7 \\
3.5 \\
2.3 \\
4.5 \\
38 \\
35\end{array}$ & $\begin{array}{l}10 \\
5.0 \\
3.0 \\
6.2 \\
45 \\
43\end{array}$ & $\begin{array}{l}17 \\
6.7 \\
3.9 \\
8.2 \\
52 \\
51\end{array}$ & $\begin{array}{l}23 \\
8.7 \\
5.1 \\
11 \\
61 \\
60\end{array}$ & $\begin{array}{l}37 \\
15 \\
7.4 \\
19 \\
83 \\
84\end{array}$ & $\begin{array}{r}53 \\
25 \\
11 \\
30 \\
110 \\
110\end{array}$ & $\begin{array}{r}81 \\
36 \\
18 \\
42 \\
150 \\
150\end{array}$ & $\mid \begin{array}{r}120 \\
52 \\
28 \\
64 \\
200 \\
210\end{array}$ & $\begin{array}{r}190 \\
88 \\
41 \\
110 \\
280 \\
290\end{array}$ & $\begin{array}{r}320 \\
170 \\
89 \\
180 \\
460 \\
430\end{array}$ \\
\hline
\end{tabular}




\section{LITTLE GEAVER CREEK BASIN}

03109000 Lisbon Creek at Lisbon, Ohio

LOCATION:

Lat $40^{\circ} 46^{\prime} 55^{\prime \prime}$, long $80^{\circ} 45^{\prime} 50^{\prime \prime}$, in NWl/4 sec. 13, T.14 N., R. 3 W., Columbiana County, Hydrologic Unit 05030101 , on left bank at Citý Water Works of Lisbon, $800 \mathrm{ft}$ upstream from bridge on State

Route 164 and $1.0 \mathrm{mi}$ upstream from mouth.

DRAINAGE AREA: $\quad 6.19 \mathrm{mi}^{2}$.

TRIBUTARY T0: Middle Fork Little Beaver Creek.

DISCHARGE DATA USED: October 1946 to September 1962.

REMARKS :

Water supply for city of Lisbon is pumped from wells adjacent to Lisbon Creek just upstream from station and is returned as sewage downstream from the station.

SELECTED DISCHARGE CHARACTERISTICS: Average discharge: $5.84 \mathrm{ft}^{3} / \mathrm{s}$ ( 36 years).

Minimum daily discharge: $0.1 \mathrm{ft} / \mathrm{s}$ at times during 1946 , 1951-1955, 1957, 1959-1962.

Magnitude and frequency of low flow for indicated periods

\begin{tabular}{|c|c|c|c|c|c|c|}
\hline \multirow{2}{*}{ Period } & \multirow{2}{*}{$\begin{array}{l}\text { Number of } \\
\text { consecu- } \\
\text { tive days }\end{array}$} & \multicolumn{5}{|c|}{$\begin{array}{l}\text { Discharge }\left(\mathrm{ft}^{3} / \mathrm{s}\right) \text { for indicated } \\
\text { recurrence interval (years) }\end{array}$} \\
\hline & & 2 & 5 & 10 & 20 & 50 \\
\hline Apr.-Mar. & $\begin{array}{r}1 \\
7 \\
30\end{array}$ & $\begin{array}{l}0.1 \\
0.2 \\
0.2\end{array}$ & $\begin{array}{l}0.1 \\
0.1 \\
0.2\end{array}$ & $\begin{array}{l}0.1 \\
0.1 \\
0.1\end{array}$ & $\begin{array}{l}0.1 \\
0.1 \\
0.1\end{array}$ & $\begin{array}{l}0.1 \\
0.1 \\
0.1\end{array}$ \\
\hline May- Nov. & $\begin{array}{r}1 \\
7 \\
30\end{array}$ & $\begin{array}{l}0.1 \\
0.2 \\
0.2\end{array}$ & $\begin{array}{l}0.1 \\
0.1 \\
0.2\end{array}$ & $\begin{array}{l}0.1 \\
0.1 \\
0.1\end{array}$ & $\begin{array}{l}0.1 \\
0.1 \\
0.1\end{array}$ & $\begin{array}{l}0.1 \\
0.1 \\
0.1\end{array}$ \\
\hline June-Aug. & $\begin{array}{r}1 \\
7 \\
30\end{array}$ & $\begin{array}{l}0.2 \\
0.2 \\
0.4\end{array}$ & $\begin{array}{l}0.1 \\
0.1 \\
0.2\end{array}$ & $\begin{array}{l}0.1 \\
0.1 \\
0.1\end{array}$ & $\begin{array}{l}0.1 \\
0.1 \\
0.1\end{array}$ & $\mid \begin{array}{l}0.1 \\
0.1 \\
0.1\end{array}$ \\
\hline
\end{tabular}

\begin{tabular}{|c|c|c|c|c|c|c|}
\hline \multirow{2}{*}{ Period } & \multirow{2}{*}{$\begin{array}{l}\text { Number of } \\
\text { consecu- } \\
\text { tive days }\end{array}$} & \multicolumn{5}{|c|}{$\begin{array}{l}\text { Discharge }\left(\mathrm{ft}^{3} / \mathrm{s}\right) \text { for indicated } \\
\text { recurrence interval (years) }\end{array}$} \\
\hline & & 2 & 5 & 10 & 20 & 50 \\
\hline Sept.-Nov. & $\begin{array}{r}1 \\
7 \\
30\end{array}$ & $\begin{array}{l}0.2 \\
0.2 \\
0.3\end{array}$ & $\begin{array}{l}0.1 \\
0.1 \\
0.2\end{array}$ & $\begin{array}{l}0.1 \\
0.1 \\
0.1\end{array}$ & $\begin{array}{l}0.1 \\
0.1 \\
0.1\end{array}$ & $\begin{array}{l}0.1 \\
0.1 \\
0.1\end{array}$ \\
\hline Dec.-Feb. & $\begin{array}{r}1 \\
7 \\
30\end{array}$ & $\begin{array}{l}0.7 \\
0.9 \\
1.9\end{array}$ & $\begin{array}{l}0.3 \\
0.4 \\
0.7\end{array}$ & $\begin{array}{l}0.2 \\
0.3 \\
0.4\end{array}$ & $\begin{array}{l}0.2 \\
0.2 \\
0.2\end{array}$ & $\begin{array}{l}0.1 \\
0.1 \\
0.1\end{array}$ \\
\hline Mar. - May & $\begin{array}{r}1 \\
7 \\
30\end{array}$ & $\begin{array}{l}1.5 \\
2.0 \\
4.6\end{array}$ & $\begin{array}{l}1.0 \\
1.2 \\
3.1\end{array}$ & $\begin{array}{l}0.8 \\
1.0 \\
2.5\end{array}$ & $\begin{array}{l}0.7 \\
0.8 \\
2.0\end{array}$ & $\begin{array}{l}0.6 \\
0.7 \\
1.6\end{array}$ \\
\hline
\end{tabular}

Duration of daily flow for indicated periods

\begin{tabular}{l|c|c|c|c|c|c|c|c|c|c|c|c|c}
\hline \multirow{2}{*}{ Period } & \multicolumn{8}{|c|}{ Discharge $\left(\mathrm{ft}^{3} / \mathrm{s}\right.$ ) which was equaled or exceeded for indicated percent of time } \\
\cline { 2 - 13 } & 98 & 95 & 90 & 85 & 80 & 75 & 70 & 60 & 50 & 40 & 30 & 20 & 10 \\
\hline Apr. - Mar. & 0.2 & 0.2 & 0.3 & 0.4 & 0.5 & 0.6 & 0.8 & 1.3 & 2.2 & 3.7 & 5.3 & 7.8 & 14 \\
May - Nov. & 0.1 & 0.2 & 0.2 & 0.3 & 0.3 & 0.4 & 0.5 & 0.7 & 1.0 & 1.4 & 2.2 & 3.6 & 6.5 \\
June - Aug. & 0.1 & 0.2 & 0.2 & 0.3 & 0.3 & 0.4 & 0.4 & 0.6 & 0.9 & 1.2 & 1.7 & 2.8 & 5.4 \\
Sept.- Nov. & 0.1 & 0.2 & 0.2 & 0.3 & 0.3 & 0.3 & 0.4 & 0.4 & 0.6 & 0.8 & 1.2 & 1.9 & 3.0 \\
Dec. - Feb. & 0.3 & 0.4 & 0.5 & 0.6 & 0.9 & 1.2 & 1.5 & 2.7 & 4.2 & 5.4 & 7.3 & 11 & 19 \\
Mar. - May & 1.2 & 1.7 & 2.5 & 3.1 & 3.7 & 4.2 & 4.6 & 5.4 & 6.5 & 7.9 & 10 & 13 & 20 \\
\hline
\end{tabular}


Appendix 3.--Low-flow data--Continued

LITTLE BEAVER CREEK BASIN

03109100 Middle Fork Little Beaver Creek near Rogers, Ohio

LOCATION: Lat $40^{\circ} 43^{\prime} 22^{\prime \prime}$, long $80^{\circ} 38^{\prime} 03^{\prime \prime}$, Columbiana County, Hydrologic Unit $05030101,0.4 \mathrm{mi}$ upstream from West Fork Little Beaver Creek, at state Route 7 bridge, $5.0 \mathrm{mi}$ south of Rogers.

DRAINAGE AREA: $\quad 149 \mathrm{mi}^{2}$.

TRIBUTARY TO: Head of Little Beaver Creek.

DISCHARGE DATA USED: Low-flow measurements 1959, 1960, 1972-77 water yeàrs.

INDEX STATION: $\quad 03109500$ Little Beaver Creek near East Liverpool, Ohio.

REMARKS:

Salem, Leetonia, and Lisbon (total population 20,000) discharge sewage several miles upstream. Effect is minimal.

SELECTED DISCHARGE CHARACTERISTICS: Minimum discharge observed: $11 \mathrm{ft}^{3} / \mathrm{s}$ August 1973.

\begin{tabular}{l|c|c|c}
\multicolumn{4}{c}{$\begin{array}{c}\text { Magnitude and frequency of low flow for } \\
\text { indicated periods }\end{array}$} \\
\hline Period & $\begin{array}{c}\text { Number of } \\
\text { consecu- } \\
\text { tive days }\end{array}$ & $\begin{array}{c}\text { Discharge (ft3/s) for } \\
\text { indicated recurrence } \\
\text { interval (years) }\end{array}$ \\
\cline { 3 - 4 } & 7 & 15 & 10 \\
\hline Apr. - Mar. & 30 & 15 & 10 \\
& 7 & 15 & 13 \\
May - Nov. & 30 & 19 & 13 \\
June - Aug. & 7 & 18 & 11 \\
& 30 & 25 & 15 \\
Sept. - Nov. & 7 & 16 & 12 \\
& 30 & 22 & 14 \\
\hline
\end{tabular}

Duration of daily flow for indicated periods

\begin{tabular}{l|l|l|l|l|l}
\hline \multirow{2}{*}{ Period } & \multicolumn{5}{|c}{$\begin{array}{c}\text { Discharge }\left(\mathrm{ft}^{3} / \mathrm{s}\right) \text { which was equaled or } \\
\text { exceeded for indicated percent of time }\end{array}$} \\
\cline { 2 - 6 } & 98 & 95 & 90 & 85 & 80 \\
\hline Apr. - Mar. & 13 & 16 & 19 & 22 & 26 \\
May - Nov. & 12 & 14 & 16 & 18 & 20 \\
June - Aug. & 12 & 14 & 17 & 19 & 22 \\
Sept. - Nov. & 12 & 14 & 16 & 16 & 18 \\
\hline
\end{tabular}


Appendix 3.--Low-flow data--Continued

LITTLE BEAVER CREEK BASIN

03109200 West Fork Little Beaver Creek at West Point, Ohio

LOCATION: $\quad$ Lat $40^{\circ} 42^{\prime} 38^{\prime \prime}$, long $80^{\circ} 41^{\prime} 49^{\prime \prime}$, Columbiana County, Hydrologic Unit 05030101 , at bridge on U'.s. Highway $30,0.3$ mi downstream from Patterson Creek, at West Point.

DRAINAGE AREA: $\quad 99.9 \mathrm{mi}^{2}$.

TRIBUTARY TO:

Little Beaver Creek.

DISCHARGE DATA USED:

Low-flow measurements 1959, 1972-1977 water years.

INDEX STATION:

03109500 Little Beaver Creek near East Liverpool, Ohio.

REMARKS:

No diversion, but possible slight regulation at Guilford Lake in headwater upstream.

SELECTED DISCHARGE CHARACTERISTICS: Minimum discharge observed: $5.3 \mathrm{ft}^{3} / \mathrm{s}$ September 1959.

\begin{tabular}{l|r|c|c}
\multicolumn{4}{c}{$\begin{array}{l}\text { Magnitude and frequency of low flow for } \\
\text { indicated periods }\end{array}$} \\
\hline \multirow{3}{*}{ Period } & $\begin{array}{c}\text { Number of } \\
\text { consecu- } \\
\text { tive days }\end{array}$ & $\begin{array}{c}\text { Discharge ( } \mathrm{ft}^{3} / \mathrm{s} \text { ) for } \\
\text { indicated recurrence } \\
\text { interval (years) }\end{array}$ \\
\cline { 3 - 4 } & 7 & 2 & 10 \\
\hline Apr. - Mar. & 30 & 4.1 & 2.2 \\
May - Nov. & 7 & 5.9 & 3.2 \\
& 30 & 4.1 & 2.2 \\
June - Aug. & 7 & 5.9 & 3.2 \\
& 30 & 5.5 & 2.4 \\
Sept.- Nov. & 7 & 9.8 & 4.2 \\
& 30 & 4.3 & 2.7 \\
\hline
\end{tabular}

Duration of daily flow for indicated periods

\begin{tabular}{|c|c|c|c|c|c|}
\hline \multirow{2}{*}{ Period } & \multicolumn{5}{|c|}{$\begin{array}{l}\text { Discharge }\left(\mathrm{ft}^{3} / \mathrm{s}\right) \text { which was equaled or } \\
\text { exceeded for indicated percent of time }\end{array}$} \\
\hline & 98 & 95 & 90 . & 85 & 80 \\
\hline $\begin{array}{l}\text { Apr. - Mar. } \\
\text { May - Nov. } \\
\text { June - Aug. } \\
\text { Sept. - Nov. }\end{array}$ & $\begin{array}{l}3.2 \\
2.8 \\
2.6 \\
2.8\end{array}$ & $\begin{array}{l}4.3 \\
3.8 \\
3.7 \\
3.5\end{array}$ & $\begin{array}{l}5.9 \\
4.8 \\
4.9 \\
4.2\end{array}$ & $\begin{array}{l}7.7 \\
5.6 \\
6.1 \\
4.8\end{array}$ & $\begin{array}{l}10 \\
6.6 \\
7.4 \\
5.5\end{array}$ \\
\hline
\end{tabular}


Appendix 3.--Low-flow data--Continued

LITTLE BEAVER CREEK BASIN

03109400 North Fork Little Beaver Creek near Negley, Ohio

LOCATION:

Lat $40^{\circ} 46^{\prime} 30^{\prime \prime}$, long $80^{\circ} 32^{\prime} 36^{\prime \prime}$, Columbiana County, Hydrologic

Unit $05030101,0.5 \mathrm{mi}$ downstream from Bull Creek at unnamed

road bridge at Achor, $1.1 \mathrm{mi}$ south of Negley.

DRAINAGE AREA: $\quad 166 \mathrm{mi}^{2}$.

TRIBUTARY TO:

Little Beaver Creek.

DISCHARGE DATA USED:

Low-flow measurements 1959, 1972-1976 water years.

INDEX STATION:

03109500 Little Beaver Creek near East Liverpool, Ohio.

REMARKS:

No major regulation or diversion known. East Palestine (population 5,600) sewage is discharged into Leslie Run $4.0 \mathrm{mi}$ upstream. Although quantity of sewage is unknown, the effect is considered to be minor.

SELECTED DISCHARGE CHARACTERISTICS: Minimum discharge observed: $16 \mathrm{ft}^{3} / \mathrm{s}$ september 1959.

Magnitude and frequency of low flow for indicated periods

\begin{tabular}{c|c|c|c}
\hline \multirow{2}{*}{ Period } & $\begin{array}{c}\text { Number of } \\
\text { consecu- } \\
\text { tive days }\end{array}$ & \multicolumn{2}{|c}{$\begin{array}{c}\text { Discharge (ft } 3 / \mathrm{s} \text { ) for } \\
\text { indicated recurrence } \\
\text { interval (years) }\end{array}$} \\
\cline { 2 - 4 } & & 2 & 10 \\
\hline Apr. - Mar. & 7 & 16 & 10 \\
May - Nov. & 30 & 21 & 13 \\
& 7 & 16 & 10 \\
June - Aug. & 30 & 21 & 13 \\
& 7 & 20 & 11 \\
Sept.- Nov. & 30 & 30 & 16 \\
& 30 & 17 & 12 \\
& & 23 & 15 \\
\hline
\end{tabular}

Duration of daily flow for indicated periods

\begin{tabular}{c|c|c|c|c|c}
\hline \multirow{2}{*}{ Period } & \multicolumn{5}{|c}{$\begin{array}{c}\text { Discharge ( } \mathrm{ft}^{3 / \mathrm{s}} \text { ) which was equaled or } \\
\text { exceeded for indicated percent of time }\end{array}$} \\
\cline { 2 - 6 } & 98 & 95 & 90 & 85 & 80 \\
\hline Apr. - Mar. & 14 & 17 & 21 & 25 & 30 \\
May - Nov. & 12 & 15 & 18 & 20 & 22 \\
June - Aug. & 11 & 15 & 18 & 21 & 24 \\
Sept. - Nov. & 12 & 14 & 16 & 18 & 20 \\
\hline
\end{tabular}


03109500 Little Beaver Creek near East Liverpool, Ohio

LOCATION:

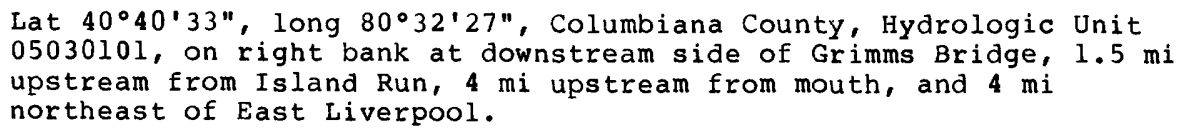

DRAINAGE AREA:

$496 \mathrm{mi}^{2}$.

TRIBUTARY TO:

Ohio River.

DISCHARGE DATA USED:

REMARKS :

October 1915 to September 1978.

None.

SELECTED DISCHARGE CHARACTERISTICS: Average discharge: $517 \mathrm{ft}^{3} / \mathrm{s}$ ( $\xi_{3}$ years).

Minimum daily discharge: $12 \mathrm{ft} / \mathrm{s}$ several days in 1918 and 1932 .

Magnitude and frequency of low flow for indicated periods

\begin{tabular}{|c|c|c|c|c|c|c|}
\hline \multirow{2}{*}{ Period } & \multirow{2}{*}{$\begin{array}{l}\text { Number of } \\
\text { consecu- } \\
\text { tive days }\end{array}$} & \multicolumn{5}{|c|}{$\begin{array}{l}\text { Discharge }\left(\mathrm{ft}^{3} / \mathrm{s}\right) \text { for indicated } \\
\text { recurrence interval (years) }\end{array}$} \\
\hline & & 2 & 5 & 10 & 20 & 50 \\
\hline Apr.-Mar. & $\begin{array}{r}1 \\
7 \\
30\end{array}$ & $\begin{array}{l}29 \\
33 \\
46\end{array}$ & $\begin{array}{l}20 \\
22 \\
32\end{array}$ & $\begin{array}{l}17 \\
19 \\
27\end{array}$ & $\begin{array}{l}15 \\
16 \\
23\end{array}$ & $\begin{array}{l}12 \\
14 \\
20\end{array}$ \\
\hline May- Nov. & $\begin{array}{r}1 \\
7 \\
30\end{array}$ & $\begin{array}{l}29 \\
33 \\
46\end{array}$ & $\begin{array}{l}20 \\
22 \\
32\end{array}$ & $\begin{array}{l}17 \\
19 \\
27\end{array}$ & $\begin{array}{l}15 \\
16 \\
23\end{array}$ & $\begin{array}{l}12 \\
14 \\
20\end{array}$ \\
\hline June-Aug. & $\begin{array}{r}1 \\
7 \\
30\end{array}$ & $\begin{array}{l}36 \\
43 \\
72\end{array}$ & $\begin{array}{l}23 \\
26 \\
43\end{array}$ & $\begin{array}{l}18 \\
21 \\
34\end{array}$ & $\begin{array}{l}15 \\
17 \\
28\end{array}$ & $\begin{array}{l}12 \\
14 \\
23\end{array}$ \\
\hline
\end{tabular}

\begin{tabular}{c|c|c|c|c|c|c}
\hline \multirow{2}{*}{ Period } & \multirow{2}{*}{$\begin{array}{c}\text { Number of } \\
\text { consecu- } \\
\text { tive days }\end{array}$} & \multicolumn{4}{|c|}{\begin{tabular}{c} 
Discharge (ft $3 / \mathrm{s}$ ) \\
recurrence interval indicated \\
\cline { 4 - 7 } (years)
\end{tabular}} \\
\cline { 3 - 7 } Sept.-Nov. & 1 & 31 & 23 & 20 & 19 & 18 \\
& 7 & 35 & 25 & 23 & 21 & 20 \\
Dec.-Feb. & 30 & 53 & 35 & 30 & 28 & 26 \\
& 1 & 117 & 67 & 50 & 39 & 29 \\
& 7 & 137 & 77 & 56 & 42 & 31 \\
& 30 & 251 & 128 & 89 & 65 & 45 \\
& 1 & 164 & 106 & 84 & 68 & 54 \\
& 7 & 196 & 128 & 105 & 89 & 76 \\
& 30 & 386 & 242 & 187 & 151 & 117 \\
\hline
\end{tabular}

Duration of daily flow for indicated periods

\begin{tabular}{l|r|r|r|r|r|r|r|r|r|r|r|r|r}
\hline \multirow{2}{*}{ Period } & \multicolumn{8}{|c|}{ Discharge $\left(\mathrm{ft}^{3} / \mathrm{s}\right)$ which was equaled or exceeded for indicated percent of time } \\
\cline { 2 - 12 } & 98 & 95 & 90 & 85 & 80 & 75 & 70 & 60 & 50 & 40 & 30 & 20 & 10 \\
\hline Apr. - Mar. & 27 & 35 & 46 & 58 & 73 & 90 & 110 & 160 & 230 & 330 & 480 & 720 & 1200 \\
May - Nov. & 24 & 31 & 38 & 44 & 51 & 59 & 69 & 93 & 120 & 170 & 230 & 350 & 640 \\
June - Aug. & 22 & 30 & 39 & 47 & 56 & 65 & 74 & 94 & 120 & 150 & 200 & 290 & 520 \\
Sept. - Nov. & 24 & 29 & 34 & 38 & 43 & 47 & 52 & 65 & 85 & 110 & 160 & 240 & 420 \\
Dec. - Feb. & 53 & 74 & 99 & 120 & 150 & 180 & 210 & 280 & 370 & 480 & 640 & 950 & 1600 \\
Mar. - May & 120 & 160 & 210 & 250 & 290 & 340 & 390 & 490 & 610 & 750 & 940 & 1300 & 1900 \\
\hline
\end{tabular}


LOCATION: Lat $40^{\circ} 32^{\prime} 16^{\prime \prime}$, long $80^{\circ} 43^{\prime} 31^{\prime \prime}$, in sec. $29, \mathrm{~T} .8 \mathrm{~N}, \mathrm{R.2}$ W. Jefferson
County, Hydrologic Unit 05030101 , on right bani $1,000 \mathrm{ft}$ upstream from
Lowery Run, $0.9 \mathrm{mi}$ upstream from Brush Creek, and $1.6 \mathrm{mi}$ southwest of
Hammondsville.

DRAINAGE AREA:

$147 \mathrm{mi}^{2}$

TRIBUTARY TO:

Ohio River.

DISCHARGE DATA USED: October 1940 to September 1978.

REMARKS:

None.

SELECTED DISCHARGE CHARACTERISTICS: Average discharge: $158 \mathrm{ft}^{3} / \mathrm{s}$ ( 38 years).

Minimum daily discharge: $0.8 \mathrm{ft}^{3} / \mathrm{s}$ September, October 1963.

Magnitude and frequency of low flow for indicated periods

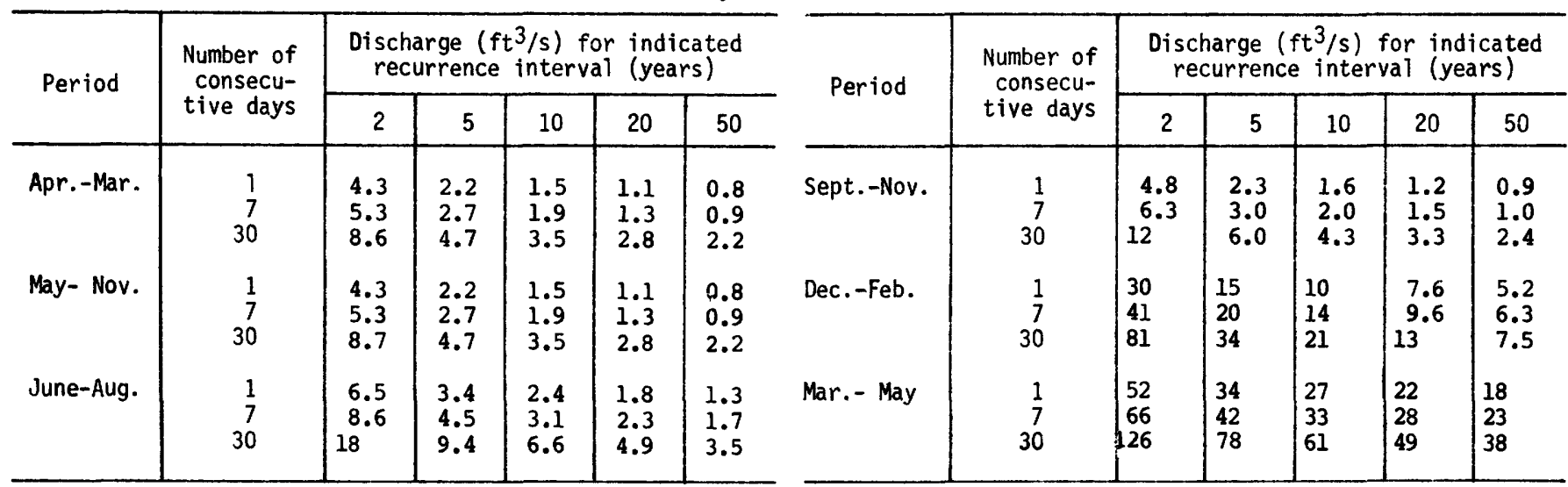

Duration of daily flow for indicated periods

\begin{tabular}{l|c|c|c|c|c|c|c|c|c|c|c|c|c}
\hline \multirow{2}{*}{ Period } & \multicolumn{8}{|c}{ Discharge $\left(\mathrm{ft}^{3} / \mathrm{s}\right)$ which was equaled or exceeded for indicated percent of time } \\
\cline { 2 - 13 } & 98 & 95 & 90 & 85 & 80 & 75 & 70 & 60 & 50 & 40 & 30 & 20 & 10 \\
\hline Apr. - Mar. & 3.7 & 6.0 & 9.8 & 14 & 18 & 23 & 29 & 44 & 69 & 100 & 150 & 230 & 390 \\
May - Nov. & 3.0 & 4.4 & 6.8 & 9.0 & 12 & 15 & 18 & 24 & 33 & 46 & 67 & 100 & 190 \\
June - Aug. & 3.5 & 5.5 & 8.1 & 11 & 13 & 16 & 19 & 24 & 31 & 41 & 54 & 78 & 140 \\
Sept. - Nov. & 2.4 & 3.5 & 4.9 & 6.4 & 8.0 & 9.9 & 12 & 17 & 23 & 31 & 44 & 67 & 120 \\
Dec. - Feb. & 11 & 17 & 23 & 31 & 41 & 52 & 65 & 90 & 120 & 160 & 210 & 310 & 510 \\
Mar. - May & 37 & 49 & 69 & 87 & 100 & 120 & 130 & 160 & 200 & 250 & 310 & 400 & 610 \\
\hline
\end{tabular}


Appendix 3.--Low-flow data--Continued

YELLOW CREEK BASIN

03110600 North Fork Yellow Creek at Hammondsville, Ohio

LOCATION:

Lat $40^{\circ} 33^{\prime} 27^{\prime \prime}$, long $80^{\circ} 42^{\prime} 20^{\prime \prime}$, Jefferson County, Hydrologic Unit 05030101 , at bridge on state Route 213, at north edge of Hammondsville.

DRAINAGE AREA:

$59.4 \mathrm{mi}^{2}$.

TRIBUTARY TO:

Yellow Creek.

DISCHARGE DATA USED:

Low-flow measurements 1959, 1962-1974 water years.

INDEX STATION:

03109500 Little Beaver Creek near East Liverpool, Ohio.

REMARKS:

None.

SELECTED DISCHARGE CHARACTERISTICS: Minimum discharge observed: $1.0 \mathrm{ft} / \mathrm{s}^{3}$ September 1959.

\begin{tabular}{c|r|r|r}
\multicolumn{4}{c}{ Magnitude and frequency of low flow for } \\
indicated periods \\
\hline & $\begin{array}{c}\text { Number of } \\
\text { consecu- } \\
\text { tive days }\end{array}$ & $\begin{array}{c}\text { Discharge (ft } 3 / \mathrm{s} \text { ) for } \\
\text { indicated recurrence } \\
\text { interval (years) }\end{array}$ \\
\cline { 3 - 4 } & & 2 & 10 \\
\hline Apr. - Mar. & 7 & 1.5 & 0.8 \\
& 30 & 2.4 & 1.2 \\
May - Nov. & 7 & 1.5 & 0.8 \\
& 30 & 2.4 & 1.2 \\
June - Aug. & 7 & 2.2 & 0.9 \\
& 30 & 4.1 & 1.6 \\
Sept. - Nov. & 7 & 1.7 & 1.0 \\
& 30 & 2.8 & 1.4 \\
\hline
\end{tabular}

Duration of daily flow for indicated periods

\begin{tabular}{|c|c|c|c|c|c|}
\hline \multirow{2}{*}{ Period } & \multicolumn{5}{|c|}{$\begin{array}{l}\text { Discharge }\left(\mathrm{ft}^{3} / \mathrm{s}\right) \text { which was equaled or } \\
\text { exceeded for indicated percent of time }\end{array}$} \\
\hline & 98 & 95 & 90 & 85 & 80 \\
\hline $\begin{array}{l}\text { Apr. - Mar. } \\
\text { May - Nov. } \\
\text { June - Aug. } \\
\text { Sept. - Nov. }\end{array}$ & $\begin{array}{l}1.2 \\
1.0 \\
0.9 \\
1.0\end{array}$ & $\begin{array}{l}1.7 \\
1.4 \\
1.4 \\
1.3\end{array}$ & $\begin{array}{l}2.4 \\
1.8 \\
1.9 \\
1.6\end{array}$ & $\begin{array}{l}3.2 \\
2.2 \\
2.4 \\
1.8\end{array}$ & $\begin{array}{l}4.2 \\
2.7 \\
3.0 \\
2.2\end{array}$ \\
\hline
\end{tabular}


Appendix 3.--Low-flow data--Continued

ISLAND CREEK BASIN

03110850 Island Creek near Toronto, Ohio

LOCATION:

Lat $40^{\circ} 25^{\prime} 44^{\prime \prime}$, long $80^{\circ} 37^{\prime} 00^{\prime \prime}$, Jefferson County, Hydrologic Unit 05030101 , at boat ramp on state Route 7, downstream

from Little Island Creek, and $2 \mathrm{mi}$ south of Toronto.

DRAINAGE AREA: $\quad 26.4 \mathrm{mi}^{2}$.

TRIBUTARY TO:

Ohio River.

DISCHARGE DATA USED: Low-flow measurements 1976-1979 water years.

INDEX STATION: $\quad 03110000$ Yellow Creek near Hammondsville, Ohio.

REMARKS: $\quad$ None.

SELECTED DISCHARGE CHARACTERISTICS: Minimum discharge observed: $2.3 \mathrm{ft}^{3} / \mathrm{s}$ September 1976.

\begin{tabular}{|c|c|c|c|}
\hline \multirow[t]{2}{*}{ Period } & \multirow{2}{*}{$\begin{array}{l}\text { Number of } \\
\text { consecu- } \\
\text { tive days }\end{array}$} & \multicolumn{2}{|c|}{$\begin{array}{l}\left.\text { Discharge ( } \mathrm{ft}^{3} / \mathrm{s}\right) \text { for } \\
\text { indicated recurrence } \\
\text { interval (years) }\end{array}$} \\
\hline & & 2 & 10 \\
\hline Apr. - Mar. & $\begin{array}{r}7 \\
30\end{array}$ & $\begin{array}{l}1.4 \\
2.1\end{array}$ & $\begin{array}{l}0.6 \\
1.0\end{array}$ \\
\hline May - Nov. & $\begin{array}{r}7 \\
30\end{array}$ & $\begin{array}{l}1.4 \\
2.2\end{array}$ & $\begin{array}{l}0.6 \\
1.0\end{array}$ \\
\hline June - Aug. & $\begin{array}{r}7 \\
30\end{array}$ & $\begin{array}{l}2.1 \\
3.8\end{array}$ & $\begin{array}{l}0.9 \\
1.7\end{array}$ \\
\hline Sept.- Nov. & $\begin{array}{r}7 \\
30\end{array}$ & $\begin{array}{l}1.7 \\
2.8\end{array}$ & $\begin{array}{l}0.7 \\
1.2\end{array}$ \\
\hline
\end{tabular}

Duration of daily flow for indicated periods

\begin{tabular}{l|c|c|c|c|c}
\hline \multirow{2}{*}{ Period } & \multicolumn{5}{|c}{$\begin{array}{l}\text { Discharge }\left(\mathrm{ft}^{3} / \mathrm{s}\right) \text { which was equaled or } \\
\text { exceeded for indicated percent of time }\end{array}$} \\
\cline { 2 - 6 } & 98 & 95 & 90 & 85 & 80 \\
\hline Apr. - Mar. & 1.1 & 1.6 & 2.4 & 3.1 & 3.8 \\
May - Nov. & 0.9 & 1.3 & 1.8 & 2.2 & 2.8 \\
June - Aug. & 1.0 & 1.5 & 2.0 & 2.6 & 3.0 \\
Sept. - Nov. & 0.8 & 1.0 & 1.4 & 1.7 & 2.0 \\
\hline
\end{tabular}


Appendix 3.--Low-flow data--Continued

CROSS CREEK BASIN

03111000 Cross Creek at Mingo Junction, Ohio

LOCATION:

Lat $40^{\circ} 18^{\prime} 55^{\prime \prime}$, long $80^{\circ} 36^{\prime} 45^{\prime \prime}$, Jefferson County, Hydrologic

Unit 05030101 , at bridge on State Route 7 at Mingo Junction, and $0.8 \mathrm{mi}$ upstream from mouth.

DRAINAGE AREA: $\quad 127 \mathrm{mi}^{2}$.

TRIBUTARY TO: Ohio River.

DISCHARGE DATA USED: LOW-flow measurements 1950, 1951, 1953, 1959, 1962-1971 water years.

INDEX STATION:

03111500 Short Creek near Dillonvale, Ohio

REMARKS:

None.

SELECTED DISCHARGE CHARACTERISTICS: Minimum discharge observed: $2.1 \mathrm{ft}^{3} / \mathrm{s}$ September 1963.

Magnitude and frequency of low flow for indicated periods

\begin{tabular}{c|c|c|c}
\hline \multirow{2}{*}{ Period } & $\begin{array}{c}\text { Number of } \\
\text { consecu- } \\
\text { tive days }\end{array}$ & \multicolumn{2}{|c}{$\begin{array}{c}\text { Discharge (ft } 3 / \mathrm{s} \text { ) for } \\
\text { indicated recurrence } \\
\text { interval (years) }\end{array}$} \\
\cline { 3 - 4 } & & 2 & 10 \\
\hline Apr. - Mar. & 7 & 3.7 & 0.5 \\
& 30 & 6.9 & 1.3 \\
May - Nov. & 7 & 3.7 & 0.7 \\
& 30 & 6.9 & 1.7 \\
June - Aug. & 7 & 8.9 & 1.3 \\
& 30 & 27 & 3.7 \\
Sept. - Nov. & 7 & 5.1 & 0.7 \\
& 30 & 13 & 2.1 \\
\hline
\end{tabular}

Duration of daily flow for indicated periods

\begin{tabular}{|c|c|c|c|c|c|}
\hline \multirow{2}{*}{ Period } & \multicolumn{5}{|c|}{$\begin{array}{l}\text { Discharge }\left(\mathrm{ft}^{3} / \mathrm{s}\right) \text { which was equaled or } \\
\text { exceeded for indicated percent of time }\end{array}$} \\
\hline & 98 & 95 & 90 & 85 & 80 \\
\hline $\begin{array}{l}\text { Apr. - Mar. } \\
\text { May - Nov. } \\
\text { June - Aug. } \\
\text { Sept. - Nov. }\end{array}$ & $\begin{array}{l}1.7 \\
1.3 \\
1.7 \\
1.0\end{array}$ & $\begin{array}{l}3.7 \\
2.6 \\
3.1 \\
2.1\end{array}$ & $\begin{array}{l}6.9 \\
4.4 \\
6.0 \\
3.1\end{array}$ & $\begin{array}{l}13 \\
6.9 \\
11 \\
4.4\end{array}$ & $\begin{array}{l}19 \\
11 \\
16 \\
6.0\end{array}$ \\
\hline
\end{tabular}


SHORT CREEK BASIN

03111500 Short Creek near Dillonvale, Ohio

LOCATION:

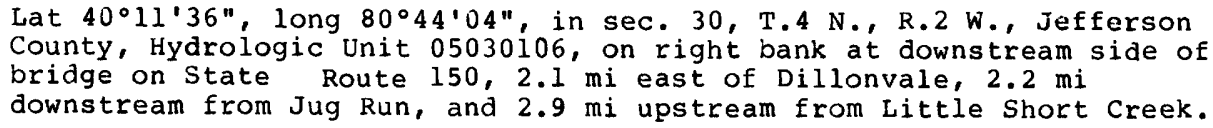

DRAINAGE AREA:

$123 \mathrm{mi}^{2}$.

TRIBUTARY TO:

Ohio River.

DISCHARGE DATA USED: October 1941 to September 1978.

REMARKS :

None.

SELECTED DISCHARGE CHARACTERISTICS:

Average discharge: $125 \mathrm{ft}^{3} / \mathrm{s}$ ( 37 years).

Minimum daily discharge: $2.8 \mathrm{ft}^{3} / \mathrm{s}$ September 1947.

Magnitude and frequency of low flow for indicated periods

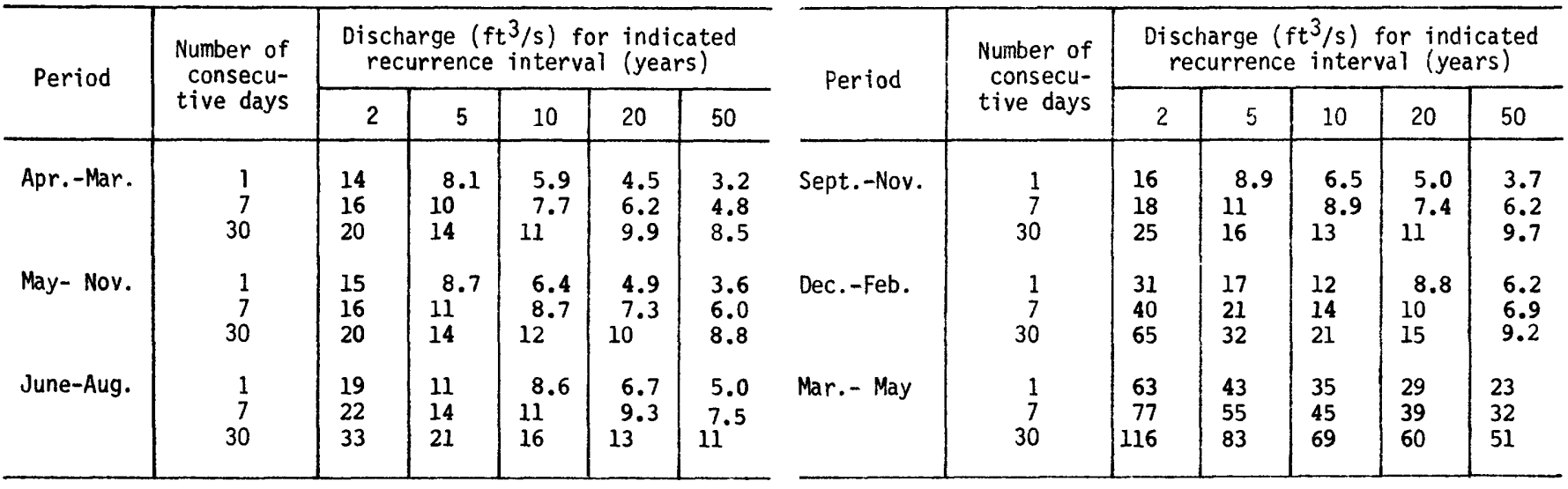

Duration of daily flow for indicated periods

\begin{tabular}{|c|c|c|c|c|c|c|c|c|c|c|c|c|c|}
\hline \multirow{2}{*}{ Period } & \multicolumn{13}{|c|}{ Discharge $\left(\mathrm{ft}^{3} / \mathrm{s}\right)$ which was equaled or exceeded for indicated percent of time } \\
\hline & 98 & 95 & 90 & 85 & 80 & 75 & 70 & 60 & 50 & 40 & 30 & 20 & 10 \\
\hline $\begin{array}{l}\text { Apr. - Mar. } \\
\text { May - Nov. } \\
\text { June - Aug. } \\
\text { Sept. - Nov. } \\
\text { Dec. - Feb. } \\
\text { Mar. - May }\end{array}$ & $\begin{array}{l}12 \\
11 \\
12 \\
10 \\
13 \\
48\end{array}$ & $\begin{array}{l}16 \\
14 \\
15 \\
13 \\
18 \\
60\end{array}$ & $\begin{array}{l}20 \\
17 \\
19 \\
15 \\
26 \\
75\end{array}$ & $\begin{array}{l}25 \\
20 \\
24 \\
17 \\
32 \\
88\end{array}$ & $\begin{array}{l}29 \\
24 \\
27 \\
19 \\
37 \\
98\end{array}$ & $\begin{array}{r}34 \\
27 \\
31 \\
21 \\
45 \\
110\end{array}$ & $\begin{array}{r}39 \\
30 \\
35 \\
23 \\
54 \\
120\end{array}$ & $\begin{array}{r}53 \\
37 \\
42 \\
27 \\
71 \\
140\end{array}$ & $\begin{array}{r}70 \\
47 \\
50 \\
32 \\
98 \\
170\end{array}$ & $\begin{array}{r}97 \\
59 \\
59 \\
38 \\
130 \\
200\end{array}$ & $\begin{array}{r}130 \\
75 \\
71 \\
48 \\
160 \\
230\end{array}$ & $\begin{array}{r}180 \\
100 \\
92 \\
66 \\
210 \\
290\end{array}$ & $\begin{array}{l}270 \\
150 \\
130 \\
100 \\
330 \\
400\end{array}$ \\
\hline
\end{tabular}


Appendix 3.--Low-flow data--Continued

WHEELING CREEK BASIN

03111550 wheeling Creek at Brookside, Ohio

LOCATION:

Lat $40^{\circ} 04^{\prime} 05^{\prime \prime}$, long $80^{\circ} 46^{\prime} 50^{\prime \prime}$, Belmont County, Hydrologic Unit 05030106, at bridge on County Road 28, in Brookside, $0.1 \mathrm{mi}$ downstream from Mutton Hollow.

DRAINAGE AREA: $\quad 103 \mathrm{mi}^{2}$.

TRIBUTARY TO:

Ohio River.

DISCHARGE DATA USED:

Low-flow measurements 1959, 1962-1972 water years.

INDEX STATION:

03111500 Short Creek near Dillonvale, Ohio.

REMARKS:

None.

SELECTED DISCHARGE CHARACTERISTICS: Minimum discharge observed: $8.2 \mathrm{ft}^{3} / \mathrm{s}$ August 1962.

Magnitude and frequency of low flow for indicated periods

\begin{tabular}{c|c|c|c}
\hline \multirow{2}{*}{ Period } & $\begin{array}{c}\text { Number of } \\
\text { consecu- } \\
\text { tive days }\end{array}$ & \multicolumn{2}{|c}{$\begin{array}{c}\text { Discharge (ft3/s) for } \\
\text { indicated recurrence } \\
\text { interval (years) }\end{array}$} \\
\cline { 3 - 4 } & & 2 & 10 \\
\hline Apr. - Mar. & 7 & 11 & 4.5 \\
& 30 & 15 & 7.0 \\
May - Nov. & 7 & 11 & 5.3 \\
& 30 & 15 & 7.8 \\
June - Aug. & 7 & 16 & 7.0 \\
& 30 & 27 & 11 \\
Sept. - Nov. & 7 & 13 & 5.5 \\
& 30 & 19 & 8.6 \\
\hline
\end{tabular}

Duration of dafly flow for indicated periods

\begin{tabular}{c|c|c|c|c|c}
\hline \multirow{2}{*}{ Period } & \multicolumn{4}{|c|}{$\begin{array}{c}\text { Discharge }\left(\mathrm{ft}^{3} / \mathrm{s} \text { ) which was equaled or }\right. \\
\text { exceeded for indicated percent of time }\end{array}$} \\
\cline { 2 - 6 } & 98 & 95 & 90 & 85 & 80 \\
\hline Apr. - Mar. & 7.8 & 11 & 15 & 19 & 23 \\
May - Nov. & 7.0 & 9.4 & 12 & 15 & 18 \\
June - Aug. & 7.8 & 10 & 14 & 18 & 21 \\
Sept. - Nov. & 6.3 & 8.6 & 10 & 12 & 14 \\
\hline
\end{tabular}


Appendix 3.--Low-flow data--Continued

\section{CAPTINA CREEK BASIN}

03114000 Captina Creek at Armstrongs Mills, Ohio

LOCATION:

Lat $39^{\circ} 54^{\prime} 31^{\prime \prime}$, long $80^{\circ} 55^{\prime} 27^{\prime \prime}$, in NEl/4 sec. 10, T.5 N., R.4 W.,

Belmont County, Hydrologic Unit 05030106, on left bank at downstream side of bridge on State Route $148,0.5 \mathrm{mi}$ east of Armstrongs Mills, and $0.7 \mathrm{mi}$ downstream from Anderson Run.

DRAINAGE AREA: $\quad 134 \mathrm{mi}^{2}$.

TRIBUTARY TO: Ohio River.

DISCHARGE DATA USED: August 1926 to September 1935, October 1958 to September 1978.

REMARKS: None.

SELECTED DISCHARGE CHARACTERISTICS: Average discharge: $160 \mathrm{ft}^{3} / \mathrm{s}$ (29 years).

Minimum daily discharge: No flow many times.

Magnitude and frequency of low flow for indicated periods

\begin{tabular}{|c|c|c|c|c|c|c|c|c|c|c|c|c|c|}
\hline \multirow{2}{*}{ Period } & \multirow{2}{*}{$\begin{array}{l}\text { Number of } \\
\text { consecu- } \\
\text { tive days }\end{array}$} & \multicolumn{5}{|c|}{$\begin{array}{l}\text { Discharge }\left(\mathrm{ft}^{3} / \mathrm{s}\right) \text { for indicated } \\
\text { recurrence interval (years) }\end{array}$} & \multirow{2}{*}{ Period } & \multirow{2}{*}{$\begin{array}{l}\text { Number of } \\
\text { consecu- } \\
\text { tive days }\end{array}$} & \multicolumn{5}{|c|}{$\begin{array}{l}\text { Discharge }\left(\mathrm{ft}^{3} / \mathrm{s}\right) \text { for indicated } \\
\text { recurrence interval (years) }\end{array}$} \\
\hline & & 2 & 5 & 10 & 20 & 50 & & & 2 & 5 & 10 & 20 & 50 \\
\hline Apr.-Mar. & $\begin{array}{r}1 \\
7 \\
30\end{array}$ & $\begin{array}{l}0.6 \\
1.0 \\
4.7\end{array}$ & $\begin{array}{l}0 \\
0.1 \\
0.9\end{array}$ & $\begin{array}{l}0 \\
0 \\
0.2\end{array}$ & $\begin{array}{l}0 \\
0 \\
0\end{array}$ & $\begin{array}{l}0 \\
0 \\
0\end{array}$ & Sept.-Nov. & $\begin{array}{r}1 \\
7 \\
30\end{array}$ & $\begin{array}{l}1.2 \\
2.3 \\
7.6\end{array}$ & $\begin{array}{l}0 \\
0.2 \\
1.3\end{array}$ & $\begin{array}{l}0 \\
0 \\
0.4\end{array}$ & $\begin{array}{l}0 \\
0 \\
0.1\end{array}$ & $\begin{array}{l}0 \\
0 \\
0\end{array}$ \\
\hline June-Aug. & $\begin{array}{r}1 \\
7 \\
30\end{array}$ & $\begin{array}{l}1.5 \\
2.5 \\
13\end{array}$ & $\begin{array}{l}0.2 \\
0.7 \\
4.4\end{array}$ & $\begin{array}{l}0 \\
0.3 \\
2.1\end{array}$ & $\begin{array}{l}0 \\
0 \\
0.7\end{array}$ & $\begin{array}{l}0 \\
0 \\
0\end{array}$ & Mar. - May & $\begin{array}{r}1 \\
7 \\
30\end{array}$ & $\begin{array}{r}33 \\
43 \\
103\end{array}$ & $\begin{array}{l}19 \\
23 \\
54\end{array}$ & $\begin{array}{l}13 \\
17 \\
37\end{array}$ & $\begin{array}{l}9.9 \\
12 \\
26\end{array}$ & $\begin{array}{r}6.9 \\
8.7 \\
17\end{array}$ \\
\hline
\end{tabular}

Duration of daily flow for indicated periods

\begin{tabular}{l|c|c|c|c|c|c|c|c|c|c|c|c|c|c}
\hline \multirow{2}{*}{ Period } & \multicolumn{10}{|c|}{ Discharge $\left(\mathrm{ft}^{3} / \mathrm{s}\right)$ which was equaled or exceeded for indicated percent of time } \\
\cline { 2 - 13 } & 98 & 95 & 90 & 85 & 80 & 75 & 70 & 60 & 50 & 40 & 30 & 20 & 10 \\
\hline Apr. - Mar. & 0.2 & 1.2 & 3.5 & 6.4 & 10 & 15 & 20 & 35 & 58 & 93 & 140 & 220 & 370 \\
May - Nov. & 0.1 & 0.5 & 1.6 & 2.9 & 4.6 & 6.4 & 8.7 & 15 & 22 & 34 & 53 & 90 & 180 \\
June - Aug. & 0.1 & 0.7 & 1.8 & 3.1 & 4.5 & 6.0 & 7.8 & 13 & 19 & 27 & 43 & 70 & 160 \\
Sept. - Nov. & 0.1 & 0.2 & 0.9 & 1.7 & 3.0 & 4.4 & 6.1 & 10 & 16 & 22 & 35 & 60 & 130 \\
Dec. - Feb. & 8.6 & 17 & 25 & 35 & 45 & 57 & 70 & 100 & 130 & 170 & 230 & 310 & 510 \\
Mar. - May & 21 & 31 & 44 & 57 & 70 & 83 & 97 & 130 & 160 & 200 & 260 & 360 & 580 \\
\hline
\end{tabular}


Appendix 3.--Low-flow data--Continued

LITTLE MUSKINGUM RIVER BASIN

03115300 Little Muskingum River near Rinard Mills, Ohio

LOCATION:

Lat $39^{\circ} 36^{\prime} 25^{\prime}$, long $81^{\circ} 07^{\prime} 21^{\prime \prime}$, Monroe County, Hydrologic Unit

05030201 , at County Road 68 bridge, $1.5 \mathrm{mi}$ upstream from

straight Fork, and $2.3 \mathrm{mi}$ northeast of Rinard Mills.

DRAINAGE AREA: $\quad 130 \mathrm{mi}^{2}$.

TRIBUTARY TO: Ohio River.

DISCHARGE DATA USED: LOw-flow measurements 1972-1977 water years.

INDEX STATION: $\quad 03115400$ Little Muskingum River at Bloomfield, Ohio.

REMARKS: None.

SELECTED DISCHARGE CHARACTERISTICS: Minimum discharge observed: $1.3 \mathrm{ft}^{3} / \mathrm{s}$ August 1973.

Magnitude and frequency of low flow for indicated periods

\begin{tabular}{c|c|c|c}
\hline \multirow{2}{*}{ Period } & \multirow{2}{*}{$\begin{array}{c}\text { Number of } \\
\text { consecu- } \\
\text { tive days }\end{array}$} & $\begin{array}{c}\text { Discharge (ft } 3 / \mathrm{s} \text { ) for } \\
\text { indicated recurrence } \\
\text { interval (years) }\end{array}$ \\
\cline { 3 - 4 } & & 2 & 10 \\
\hline Apr. - Mar. & 7 & 0.7 & 0.1 \\
& 30 & 1.9 & 0.3 \\
May - Nov. & 7 & 0.7 & 0.1 \\
& 30 & 1.9 & 0.3 \\
June - Aug. & 7 & 2.5 & 0.4 \\
& 30 & 9.4 & 1.3 \\
Sept. - Nov. & 7 & 1.0 & 0.1 \\
& 30 & 3.3 & 0.4 \\
\hline
\end{tabular}

Duration of daily flow for indicated periods

\begin{tabular}{l|c|c|c|c|c}
\hline \multirow{2}{*}{ Period } & \multicolumn{6}{|c}{$\begin{array}{c}\text { Discharge }\left(\mathrm{ft}^{3} / \mathrm{s} \text { ) which was equaled or }\right. \\
\text { exceeded for indicated percent of time }\end{array}$} \\
\cline { 2 - 6 } & 98 & 95 & 90 & 85 & 80 \\
\hline Apr. - Mar. & 0.3 & 0.8 & 2.1 & 4.0 & 7.0 \\
May - Nov. & 0.2 & 0.4 & 0.9 & 1.7 & 2.7 \\
June - Aug. & 0.4 & 0.8 & 1.5 & 2.3 & 3.4 \\
Sept. - Nov. & 0.2 & 0.3 & 0.5 & 0.8 & 1.3 \\
\hline
\end{tabular}




\section{LITTLE MUSKINGUM RIVER BASIN}

03115400 Little Muskingum River at Bloomfield, Ohio

LOCATION:

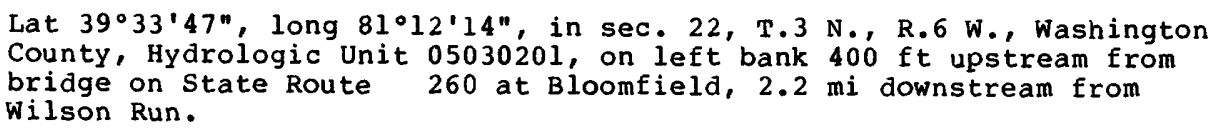

DRAINAGE AREA: $\quad 210 \mathrm{mi}^{2}$.

TRIBUTARY TO: Ohio River.

DISCHARGE DATA USED: October 1958 to September 1978.

REMARKS:

None.

SELECTED DISCHARGE CHARACTERISTICS: Average discharge: $251 \mathrm{ft}^{3} / \mathrm{s}$ (20 years).

Minimum daily discharge : No flow September 1967.

Magnitude and frequency of low flow for indicated periods

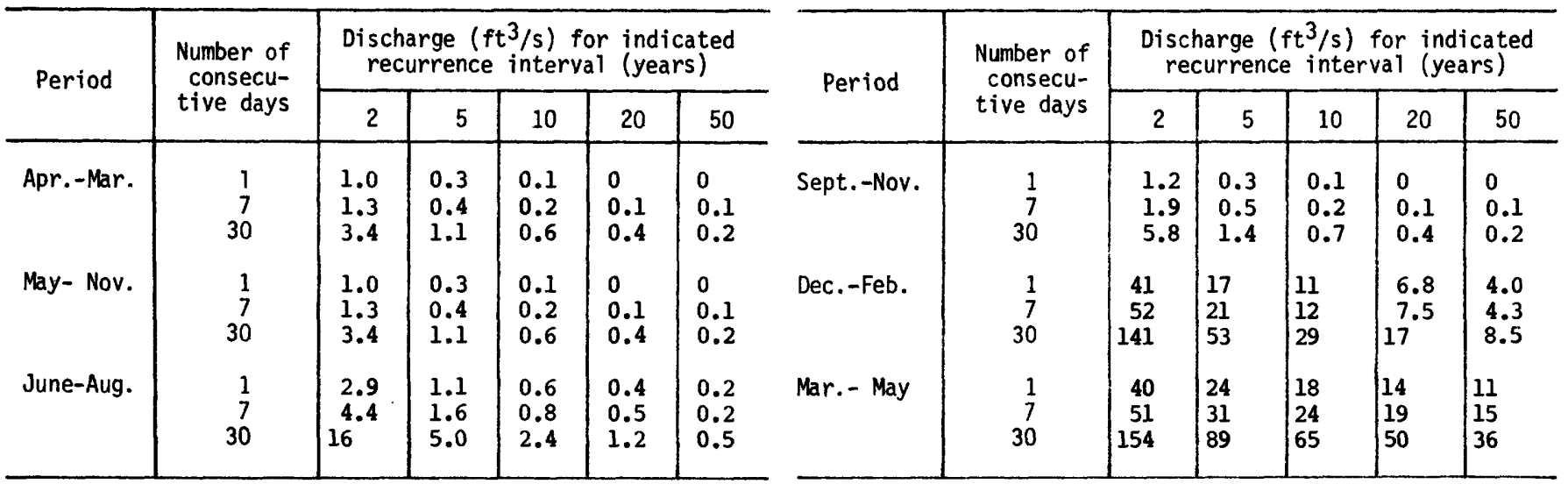

Duration of daily flow for indicated periods

\begin{tabular}{|c|c|c|c|c|c|c|c|c|c|c|c|c|c|}
\hline \multirow{2}{*}{ Period } & \multicolumn{13}{|c|}{ Discharge $\left(\mathrm{ft}^{3} / \mathrm{s}\right)$ which was equaled or exceeded for indicated percent of time } \\
\hline & 98 & 95 & 90 & 85 & 80 & 75 & 70 & 60 & 50 & 40 & 30 & 20 & 10 \\
\hline $\begin{array}{l}\text { Apr. - Mar. } \\
\text { May - Nov. } \\
\text { June - Aug. } \\
\text { Sept. - Nov. } \\
\text { Dec. - Feb. } \\
\text { Mar. - May }\end{array}$ & $\begin{array}{l}0.6 \\
0.4 \\
0.8 \\
0.3 \\
14 \\
29\end{array}$ & $\begin{array}{l}1.4 \\
0.8 \\
1.4 \\
0.5 \\
22 \\
44\end{array}$ & $\begin{array}{l}3.7 \\
1.7 \\
2.7 \\
0.9 \\
31 \\
63\end{array}$ & $\begin{array}{l}7.1 \\
3.0 \\
4.2 \\
1.4 \\
41 \\
77\end{array}$ & $\begin{array}{l}12 \\
4.8 \\
6.0 \\
2.3 \\
60 \\
95\end{array}$ & $\begin{array}{c}18 \\
7.0 \\
7.8 \\
3.4 \\
80 \\
110\end{array}$ & $\begin{array}{c}25 \\
9.5 \\
9.7 \\
5.0 \\
100 \\
140\end{array}$ & $\begin{array}{c}45 \\
17 \\
16 \\
9.7 \\
140 \\
190\end{array}$ & $\begin{array}{r}81 \\
27 \\
23 \\
17 \\
190 \\
240\end{array}$ & $\begin{array}{r}140 \\
43 \\
34 \\
27 \\
250 \\
320\end{array}$ & $\begin{array}{r}210 \\
73 \\
52 \\
48 \\
340 \\
420\end{array}$ & $\begin{array}{r}340 \\
120 \\
84 \\
99 \\
490 \\
590\end{array}$ & $\begin{array}{l}620 \\
270 \\
190 \\
230 \\
890 \\
960\end{array}$ \\
\hline
\end{tabular}


Appendix 3.--Low-flow data--Continued

DUCK CREEK BASIN

03115650 East Fork Duck Creek at Lower Salem, Ohio

LOCATION:

Lat $39^{\circ} 34^{\prime} 26^{\prime \prime}$, long $81^{\circ} 23^{\prime} 25^{\prime \prime}$, Washington County, Hydrologic Unit 05030201 , at bridge on Township Road $319,0.9 \mathrm{mi}$ northeast of Lower Salem, $1.0 \mathrm{mi}$ upstream from Pawpaw Creek.

DRAINAGE AREA: $\quad 111 \mathrm{mi}^{2}$

TRIBUTARY TO: Head of Duck Creek.

DISCHARGE DATA USED: LOW-flow measurements 1959, 1972-1976 water years.

INDEX STATION: $\quad 03115400$ Little Muskingum River at Bloomfield, Ohio.

REMARKS:

None.

SELECTED DISCHARGE CHARACTERISTICS: Minimum discharge observed: $0.6 \mathrm{ft}^{3} / \mathrm{s}$ September 1959.

\begin{tabular}{|c|c|c|c|}
\hline \multirow[t]{2}{*}{ Period } & \multirow{2}{*}{$\begin{array}{l}\text { Number of } \\
\text { consecu- } \\
\text { tive days }\end{array}$} & \multicolumn{2}{|c|}{$\begin{array}{l}\left.\text { Discharge ( } \mathrm{ft}^{3} / \mathrm{s}\right) \text { for } \\
\text { indicated recurrence } \\
\text { interval (years) }\end{array}$} \\
\hline & & 2 & 10 \\
\hline Apr. - Mar. & $\begin{array}{r}7 \\
30\end{array}$ & $\begin{array}{l}2.0 \\
4.3\end{array}$ & $\begin{array}{l}0.4 \\
1.1\end{array}$ \\
\hline May - Nov. & $\begin{array}{r}7 \\
30\end{array}$ & $\begin{array}{l}2.0 \\
4.3\end{array}$ & $\begin{array}{l}0.4 \\
1.1\end{array}$ \\
\hline June - Aug. & $\begin{array}{r}7 \\
30\end{array}$ & $\begin{array}{l}5.4 \\
15\end{array}$ & $\begin{array}{l}1.3 \\
3.3\end{array}$ \\
\hline Sept.- Nov. & $\begin{array}{r}7 \\
30\end{array}$ & $\begin{array}{l}2.7 \\
6.8\end{array}$ & $\begin{array}{l}0.4 \\
1.2\end{array}$ \\
\hline
\end{tabular}

Duration of daily flow for indicated periods

\begin{tabular}{l|c|c|c|c|c}
\hline \multirow{2}{*}{ Period } & \multicolumn{5}{|c}{$\begin{array}{l}\text { Discharge }\left(\mathrm{ft}^{3} / \mathrm{s}\right) \\
\text { exceeded for } \text { which was equaled or }\end{array}$} \\
\cline { 2 - 6 } & 98 & 95 & 90 & 85 & 80 \\
\hline Apr. - Mar. & 1.1 & 2.1 & 4.7 & 7.8 & 12 \\
May - Nov. & 0.8 & 1.3 & 2.5 & 4.0 & 5.8 \\
June - Aug. & 1.3 & 2.1 & 3.6 & 5.2 & 6.9 \\
Sept. - Nov. & 0.6 & 0.9 & 1.5 & 2.1 & 3.2 \\
\hline
\end{tabular}


Appendix 3.--Low-flow data--Continued

DUCK CREEK BASIN

03115800 Duck Creek at Stanleyville, Ohio

LOCATION:

Lat $39^{\circ} 28^{\prime}, 1^{\prime \prime}$, long $81^{\circ} 24^{\prime} 40^{\prime \prime}$, Washington County, Hydrologic Unit 05030201 , at highway bridge in Stanleyville and $1.0 \mathrm{mi}$ upstream from Sugar Creek.

DRAINAGE AREA: $\quad 267 \mathrm{mi}^{2}$.

TRIBUTARY TO: Ohio River

DISCHARGE DATA USED: LOW-flow measurements 1959, 1962-1973 water years.

INDEX STATION: $\quad 03115400$ Little Muskingum River near Bloomfield, Ohio.

REMARKS: $\quad$ None.

SELECTED DISCHARGE CHARACTERISTICS: Minimum discharge observed: $0.6 \mathrm{ft} / \mathrm{s}$ October 1963 .

\begin{tabular}{|c|c|c|c|}
\hline \multirow[t]{2}{*}{ Period } & \multirow{2}{*}{$\begin{array}{l}\text { Number of } \\
\text { consecu- } \\
\text { tive days }\end{array}$} & \multicolumn{2}{|c|}{$\begin{array}{l}\text { Discharge }\left(\mathrm{ft}^{3} / \mathrm{s}\right) \text { for } \\
\text { indicated recurrence } \\
\text { interval (years) }\end{array}$} \\
\hline & & 2 & 10 \\
\hline Apr. - Mar. & $\begin{array}{r}7 \\
30\end{array}$ & $\begin{array}{l}2.7 \\
6.8\end{array}$ & $\begin{array}{l}0.5 \\
1.3\end{array}$ \\
\hline May - Nov. & $\begin{array}{r}7 \\
30\end{array}$ & $\begin{array}{l}2.7 \\
6.8\end{array}$ & $\begin{array}{l}0.5 \\
1.3\end{array}$ \\
\hline June - Aug. & $\begin{array}{r}7 \\
30\end{array}$ & $\begin{array}{l}8.6 \\
29\end{array}$ & $\begin{array}{l}1.7 \\
4.8\end{array}$ \\
\hline Sept.- Nov. & $\begin{array}{r}7 \\
30\end{array}$ & $11^{3.9}$ & $\begin{array}{l}0.5 \\
1.5\end{array}$ \\
\hline
\end{tabular}

Duration of daily flow for indicated periods

\begin{tabular}{l|c|c|c|c|c}
\hline \multirow{2}{*}{ Period } & \multicolumn{4}{|c|}{$\begin{array}{l}\text { Discharge }\left(\mathrm{ft}^{3} / \mathrm{s}\right) \\
\text { exceeded } \text { which was equaled or } \\
\text { exdicated percent of time }\end{array}$} \\
\cline { 2 - 6 } & 98 & 95 & 90 & 85 & 80 \\
\hline Apr. - Mar. & 1.3 & 2.9 & 7.2 & 13 & 22 \\
May - Nov. & 0.9 & 1.7 & 3.5 & 6.0 & 9.3 \\
June - Aug. & 1.7 & 2.9 & 5.4 & 8.2 & 11 \\
Sept. - Nov. & 0.7 & 1.1 & 1.9 & 2.9 & 4.6 \\
\hline
\end{tabular}




\section{MUSKINGUM RIVER BASIN}

03115990 Wolf Creek near Barberton, Ohio

LOCATION:

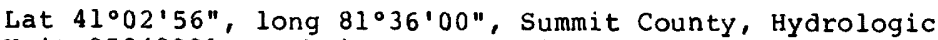

Unit 05040001 , at bridge on Summit Road, $200 \mathrm{ft}$ downstream

from mouth of Pigeon Creek, $2.5 \mathrm{mi}$ north of Barberton.

DRAINAGE AREA: $\quad 53.9 \mathrm{mi}^{2}$.

TRIBUTARY TO: TUscarawas River.

DISCHARGE DATA USED: LOW-flow measurements 1950, 1960, 1974-1978, 1980 water years.

INDEX STATION:

03116000 Tuscarawas River at Clinton, Ohio.

REMARKS:

A pumping station, just upstream from bridge, pumps creek water into Barberton Reservoir on Wolf Creek $1.2 \mathrm{mi}$ upstream. Water supply for Barberton is diverted around site.

SELECTED DISCHARGE CHARACTERISTICS: Minimum discharge observed: $4.2 \mathrm{ft}^{3} / \mathrm{s}$ october 1960.

Magnitude and frequency of low flow for indicated periods

\begin{tabular}{c|c|c|c}
\hline \multirow{2}{*}{ Period } & $\begin{array}{c}\text { Number of } \\
\text { consecu- } \\
\text { tive days }\end{array}$ & \multicolumn{2}{|c}{$\begin{array}{c}\text { Discharge }\left(\mathrm{ft}^{3 / \mathrm{s})} \text { for }\right. \\
\text { indicated recurrence } \\
\text { interval (years) }\end{array}$} \\
\cline { 3 - 4 } & & 2 & 10 \\
\hline Apr. - Mar. & 7 & 3.5 & 1.5 \\
& 30 & 4.1 & 1.8 \\
May - Nov. & 7 & 3.5 & 1.5 \\
& 30 & 4.3 & 2.0 \\
June - Aug. & 7 & 4.7 & 2.2 \\
& 30 & 6.7 & 3.4 \\
Sept. - Nov. & 7 & 3.6 & 1.6 \\
& 30 & 4.7 & 2.1 \\
\hline
\end{tabular}

Duration of daily flow for indicated periods

\begin{tabular}{l|c|c|c|c|c}
\hline \multirow{2}{*}{ Period } & \multicolumn{5}{|c|}{$\begin{array}{c}\text { Discharge }\left(\mathrm{ft}^{3} / \mathrm{s} \text { ) which was equaled or }\right. \\
\text { exceeded for indicated percent of time }\end{array}$} \\
\cline { 2 - 6 } & 98 & 95 & 90 & 85 & 80 \\
\hline Apr. - Mar. & 1.8 & 2.5 & 3.4 & 4.1 & 5.0 \\
May - Nov. & 1.7 & 2.3 & 3.1 & 3.6 & 4.3 \\
June - Aug. & 2.1 & 2.7 & 3.5 & 4.1 & 4.9 \\
Sept. - Nov. & 1.6 & 2.0 & 2.5 & 3.1 & 3.5 \\
\hline
\end{tabular}




\section{MUSKINGUM RIVER BASIN \\ 03116000 Tuscarawas River at Clinton, Ohio}

LOCATION:

Lat $40^{\circ} 55^{\prime} 40^{\prime \prime}$, long $81^{\circ} 37^{\prime} 58^{\prime \prime}$, in NWl/4 sec. 32, T. $^{2}$ N., R.10 W., Summit County, Hydrologic Unit 05040001 , on right bank $100 \mathrm{ft}$ downstream from highway bridge at $\mathrm{Clinton,}$ and $1.0 \mathrm{mi}$ upstream from Chippewa Creek.

DRAINAGE AREA:

$174 \mathrm{mi}^{2}$

TRIBUTARY TO:

Head of Muskingum River.

DISCHARGE DATA USED: October 1939 to September 1978.

REMARKS :

Some water diverted through the Portage Lakes into the Ohio Canal at Long Lake $12 \mathrm{mi}$ upstream and $3 \mathrm{mi}$ south of Akron. Flow affected by industrial plants upstream from station and supplemented at times by diversion from Nimisila Reservoir, capacity, 6,500 acre-ft since. 1939.

SELECTED DISCHARGE CHARACTERISTICS: Average discharge: $157 \mathrm{ft}^{3} / \mathrm{s}$ (39 years).

Minimum daily discharge; $20 \mathrm{ft} / \mathrm{s}$ October 1944.

Magnitude and frequency of low flow for indicated periods

\begin{tabular}{c|c|c|c|c|c|c}
\hline \multirow{2}{*}{ Period } & \multirow{2}{*}{$\begin{array}{c}\text { Number of } \\
\text { consecu- } \\
\text { tive days }\end{array}$} & \multicolumn{4}{|c|}{$\begin{array}{c}\text { Discharge (ft } 3 / \mathrm{s} \text { ) for indicated } \\
\text { recurrence interval (years) }\end{array}$} \\
\cline { 4 - 7 } & & 2 & 5 & 10 & 20 & 50 \\
\hline \multirow{3}{*}{ Apr.-Mar. } & 1 & 46 & 35 & 30 & 26 & 22 \\
& 7 & 50 & 39 & 35 & 31 & 27 \\
& 30 & 54 & 43 & 38 & 35 & 31 \\
& 1 & 47 & 36 & 31 & 27 & 23 \\
Jun- Nov. & 7 & 50 & 40 & 35 & 32 & 28 \\
& 30 & 55 & 44 & 39 & 36 & 32 \\
& 1 & 53 & 42 & 37 & 33 & 29 \\
& 7 & 57 & 46 & 41 & 38 & 34 \\
& 30 & 66 & 54 & 49 & 45 & 41 \\
\hline
\end{tabular}

\begin{tabular}{|c|c|c|c|c|c|c|}
\hline \multirow{2}{*}{ Period } & \multirow{2}{*}{$\begin{array}{l}\text { Number of } \\
\text { consecu- } \\
\text { tive days }\end{array}$} & \multicolumn{5}{|c|}{$\begin{array}{l}\text { Discharge }\left(\mathrm{ft}^{3} / \mathrm{s}\right) \text { for indicated } \\
\text { recurrence interval (years) }\end{array}$} \\
\hline & & 2 & 5 & 10 & 20 & 50 \\
\hline Sept.-Nov. & $\begin{array}{r}1 \\
7 \\
30\end{array}$ & $\begin{array}{l}47 \\
51 \\
57\end{array}$ & $\begin{array}{l}36 \\
40 \\
45\end{array}$ & $\begin{array}{l}31 \\
36 \\
40\end{array}$ & $\begin{array}{l}27 \\
32 \\
37\end{array}$ & $\begin{array}{l}23 \\
28 \\
34\end{array}$ \\
\hline Dec.-Feb. & $\begin{array}{r}1 \\
7 \\
30\end{array}$ & $\begin{array}{l}55 \\
61 \\
83\end{array}$ & $\begin{array}{l}40 \\
45 \\
53\end{array}$ & $\begin{array}{l}34 \\
39 \\
43\end{array}$ & $\begin{array}{l}30 \\
35 \\
37\end{array}$ & $\begin{array}{c}26 \\
31 \\
31\end{array}$ \\
\hline Mar. - May & $\begin{array}{r}1 \\
7 \\
30\end{array}$ & $\begin{array}{r}71 \\
81 \\
118\end{array}$ & $\begin{array}{l}55 \\
64 \\
88\end{array}$ & $\begin{array}{l}49 \\
57 \\
75\end{array}$ & $\begin{array}{l}44 \\
52 \\
66\end{array}$ & $\begin{array}{l}38 \\
46 \\
57\end{array}$ \\
\hline
\end{tabular}

Duration of daily flow for indicated periods

\begin{tabular}{|c|c|c|c|c|c|c|c|c|c|c|c|c|c|}
\hline \multirow{2}{*}{ Period } & \multicolumn{13}{|c|}{ Discharge $\left(\mathrm{ft}^{3} / \mathrm{s}\right)$ which was equaled or exceeded for indicated percent of time } \\
\hline & 98 & 95 & 90 & 85 & 80 & 75 & 70 & 60 & 50 & 40 & 30 & 20 & 10 \\
\hline $\begin{array}{l}\text { Apr. - Mar. } \\
\text { May - Nov. } \\
\text { June - Aug. } \\
\text { Sept. - Nov. } \\
\text { Dec. - Feb. } \\
\text { Mar. - May }\end{array}$ & $\begin{array}{l}38 \\
37 \\
40 \\
36 \\
36 \\
58\end{array}$ & $\begin{array}{l}43 \\
42 \\
45 \\
39 \\
41 \\
71\end{array}$ & $\begin{array}{l}49 \\
47 \\
50 \\
43 \\
48 \\
80\end{array}$ & $\begin{array}{l}54 \\
51 \\
54 \\
47 \\
54 \\
88\end{array}$ & $\begin{array}{l}59 \\
55 \\
58 \\
50 \\
59 \\
99\end{array}$ & $\begin{array}{r}63 \\
58 \\
61 \\
53 \\
64 \\
100\end{array}$ & $\begin{array}{r}67 \\
61 \\
64 \\
55 \\
70 \\
110\end{array}$ & $\begin{array}{r}76 \\
67 \\
69 \\
60 \\
80 \\
130\end{array}$ & $\begin{array}{r}87 \\
74 \\
76 \\
66 \\
98 \\
160\end{array}$ & $\begin{array}{r}110 \\
82 \\
83 \\
72 \\
120 \\
190\end{array}$ & $\begin{array}{r}130 \\
95 \\
95 \\
78 \\
160 \\
250\end{array}$ & $\begin{array}{r}190 \\
120 \\
110 \\
92 \\
240 \\
350\end{array}$ & $\begin{array}{l}340 \\
180 \\
170 \\
130 \\
470 \\
550\end{array}$ \\
\hline
\end{tabular}


Appendix 3.--Low-flow data--Continued

MUSKINGUM RIVER BASIN

03116080 Chippewa Creek at Sterling, Ohio

LOCATION:

Lat $40^{\circ} 57^{\prime} 24^{\prime \prime}$, long $81^{\circ} 50^{\prime} 31^{\prime \prime}$, Wayne County, Hydrologic Unit

05040001 , at bridge on County Road $60,0.8 \mathrm{mi}$ south of

sterling.

DRAINAGE AREA: $\quad 64.4 \mathrm{mi}^{2}$.

TRIBUTARY TO: Tuscarawas River.

DISCHARGE DATA USED: Low-flow measurements 1974-1978, 1980 water years.

INDEX STATION: $\quad 03116200$ Chippewa Creek at Easton, Ohio.

REMARKS: Possible regulation at Chippewa Lake $8.0 \mathrm{mi}$ upstream.

SELECTED DISCHARGE CHARACTERISTICS: Minimum discharge observed: $1.7 \mathrm{ft}^{3} / \mathrm{s}$ July 1975.

Magnitude and frequency of low flow for indicated periods

\begin{tabular}{|c|c|c|c|}
\hline \multirow[t]{2}{*}{ Period } & \multirow{2}{*}{$\begin{array}{l}\text { Number of } \\
\text { consecu- } \\
\text { tive days }\end{array}$} & \multicolumn{2}{|c|}{$\begin{array}{l}\text { Discharge }\left(\mathrm{ft}^{3} / \mathrm{s}\right) \text { for } \\
\text { indicated recurrence } \\
\text { interval (years) }\end{array}$} \\
\hline & & 2 & 10 \\
\hline Apr. - Mar. & $\begin{array}{r}7 \\
30\end{array}$ & $\begin{array}{l}1.9 \\
2.8\end{array}$ & $\begin{array}{l}0.8 \\
1.1\end{array}$ \\
\hline May - Nov. & $\begin{array}{r}7 \\
30\end{array}$ & $\begin{array}{l}1.9 \\
2.8\end{array}$ & $\begin{array}{l}0.8 \\
1.1\end{array}$ \\
\hline June - Aug. & $\begin{array}{r}7 \\
30\end{array}$ & $\begin{array}{l}2.5 \\
4.6\end{array}$ & $\begin{array}{l}0.9 \\
1.3\end{array}$ \\
\hline Sept. - Nov. & $\begin{array}{r}7 \\
30\end{array}$ & $\begin{array}{l}2.5 \\
4.1\end{array}$ & $\begin{array}{l}1.1 \\
1.6\end{array}$ \\
\hline
\end{tabular}

Duration of daily flow for indicated periods

\begin{tabular}{|c|c|c|c|c|c|}
\hline \multirow{2}{*}{ Period } & \multicolumn{5}{|c|}{$\begin{array}{l}\text { Discharge }\left(\mathrm{ft}^{3} / \mathrm{s}\right) \text { which was equaled or } \\
\text { exceeded for indicated percent of time }\end{array}$} \\
\hline & 98 & 95 & 90 & 85 & 80 \\
\hline $\begin{array}{l}\text { Apr. - Mar. } \\
\text { May - Nov. } \\
\text { June - Aug. } \\
\text { Sept. - Nov. }\end{array}$ & $\begin{array}{l}1.2 \\
1.0 \\
1.0 \\
1.1\end{array}$ & $\begin{array}{l}1.6 \\
1.4 \\
1.3 \\
1.4\end{array}$ & $\begin{array}{l}2.5 \\
1.8 \\
1.7 \\
1.7\end{array}$ & $\begin{array}{l}3.3 \\
2.5 \\
2.1 \\
2.1\end{array}$ & $\begin{array}{l}4.6 \\
2.8 \\
2.5 \\
2.5\end{array}$ \\
\hline
\end{tabular}


Appendix 3.--Low-flow data--Continued

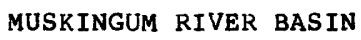

03116100 Little Chippewa Creek near Smithville, Ohio

LOCATION: $\quad$ Lat $40^{\circ} 53^{\prime} 40^{\prime \prime}$, long $81^{\circ} 48^{\prime} 50^{\prime \prime}$, Wayne County, Hydrologic Unit 05040001 , in sec. $3, T .17 \mathrm{~N} ., \mathrm{R} .12 \mathrm{~W}$. , on left downstream pier of bridge on state Route $5,3.3 \mathrm{mi}$ northeast from center of Smithville.

DRAINAGE AREA:

$16.4 \mathrm{mi}^{2}$.

TRIBUTARY TO:

Chippewa Creek.

DISCHARGE DATA USED: LOW-flow measurements 1965-1967, 1969, 1971-1972 water years.

INDEX STATION: $\quad 03116200$ Chippewa Creek at Easton, Ohio.

REMARKS:

Dam upstream. Construction of dam has resulted in 95 percent controlled drainage area.

SELECTED DISCHARGE CHARACTERISTICS: Minimum discharge observed: $1.2 \mathrm{ft}^{3} / \mathrm{s}$ September 1967 .

\begin{tabular}{l|c|c|c}
\multicolumn{4}{c}{$\begin{array}{c}\text { Magnitude and frequency of low flow for } \\
\text { indicated periods }\end{array}$} \\
\hline Period & $\begin{array}{r}\text { Number of } \\
\text { consecu- } \\
\text { tive days }\end{array}$ & $\begin{array}{c}\text { Discharge (ft3/s) for } \\
\text { indicated recurrence } \\
\text { interval }\end{array}$ \\
\cline { 3 - 4 } (years)
\end{tabular}

Duration of daily flow for indicated periods

\begin{tabular}{l|c|c|c|c|c}
\hline \multirow{2}{*}{ Period } & \multicolumn{5}{|c|}{$\begin{array}{l}\text { Discharge } \\
\text { exceeded for } \mathrm{ft} / \mathrm{s})\end{array}$ which was equaled or } \\
& \multicolumn{4}{|c|}{ indicated percent of time } \\
\cline { 2 - 6 } & 98 & 95 & 90 & 85 & 80 \\
\hline Apr. - Mar. & 1.4 & 1.8 & 2.5 & 3.0 & 4.0 \\
May - Nov. & 1.2 & 1.6 & 1.9 & 2.5 & 2.7 \\
June - Aug. & 1.2 & 1.5 & 1.8 & 2.2 & 2.5 \\
Sept. - Nov. & 1.3 & 1.6 & 1.9 & 2.2 & 2.5 \\
\hline
\end{tabular}




\section{MUSKINGUM RIVER BASIN \\ 03116200 Chippewa Creek at Easton, Ohio}

LOCATION:

Lat $40^{\circ} 56^{\prime} 4^{\prime \prime}$, long $81^{\circ} 44^{\prime} 35^{\prime \prime}$, in SWl/4 sec. 17, T.18 N., R.11 W., Wayne County, Hydrologic Unit 05040001 , on left bank at downstream side of bridge on State Route $585,0.5 \mathrm{mi}$ southwest of Easton, and $1.5 \mathrm{mi}$ upstream from Red Run.

DRAINAGE AREA: $\quad 146 \mathrm{mi}^{2}$.

TRIBUTARY TO: Tuscarawas River.

DISCHARGE DATA USED: October 1960 to September 1978.

REMARKS :

Low flow slightly regulated by industry at Rittman $2.5 \mathrm{mi}$ upstream.

SELECTED DISCHARGE CHARACTERISTICS: Average discharge: $131 \mathrm{ft}^{3} / \mathrm{s}$ (1\& years). Minimum daily discharge: $3.2 \mathrm{ft}^{3} / \mathrm{s}$ July 1963.

Magnitude and frequency of low flow for indicated periods

\begin{tabular}{|c|c|c|c|c|c|c|c|c|c|c|c|c|c|}
\hline \multirow{2}{*}{ Period } & \multirow{2}{*}{$\begin{array}{l}\text { Number of } \\
\text { consecu- } \\
\text { tive days }\end{array}$} & \multicolumn{5}{|c|}{$\begin{array}{l}\text { Discharge }\left(\mathrm{ft}^{3} / \mathrm{s}\right) \text { for indicated } \\
\text { recurrence interval (years) }\end{array}$} & \multirow{2}{*}{ Period } & \multirow{2}{*}{$\begin{array}{l}\text { Number of } \\
\text { consecu- } \\
\text { tive days }\end{array}$} & \multicolumn{5}{|c|}{$\begin{array}{l}\text { Discharge }\left(\mathrm{ft}^{3} / \mathrm{s}\right) \text { for indicated } \\
\text { recurrence interval (years) }\end{array}$} \\
\hline & & 2 & 5 & 10 & 20 & 50 & & & 2 & 5 & 10 & 20 & 50 \\
\hline Apr.-Mar. & $\begin{array}{r}1 \\
7 \\
30\end{array}$ & $\begin{array}{l}8.2 \\
9.3 \\
12\end{array}$ & $\begin{array}{l}5.5 \\
6.6 \\
8.0\end{array}$ & $\begin{array}{l}4.5 \\
5.6 \\
6.8\end{array}$ & $\begin{array}{l}3.8 \\
5.0 \\
6.0\end{array}$ & $\begin{array}{l}3.2 \\
4.4 \\
5.3\end{array}$ & Sept.-Nov. & $\begin{array}{r}1 \\
7 \\
30\end{array}$ & $\begin{array}{l}9.5 \\
11 \\
15\end{array}$ & $\begin{array}{l}6.3 \\
7.8 \\
9.7\end{array}$ & $\begin{array}{l}5.1 \\
6.7 \\
8.6\end{array}$ & $\begin{array}{l}4.3 \\
5.9 \\
8.0\end{array}$ & $\begin{array}{l}3.5 \\
5.2 \\
7.6\end{array}$ \\
\hline May- Nov. & $\begin{array}{r}1 \\
7 \\
30\end{array}$ & $\begin{array}{l}8.2 \\
9.3 \\
12\end{array}$ & $\begin{array}{l}5.5 \\
6.6 \\
8.0\end{array}$ & $\begin{array}{l}4.5 \\
5.6 \\
6.8\end{array}$ & $\begin{array}{l}3.8 \\
5.0 \\
6.0\end{array}$ & $\begin{array}{l}3.2 \\
4.4 \\
5.3\end{array}$ & Dec.-Feb. & $\begin{array}{r}1 \\
7 \\
30\end{array}$ & $\begin{array}{l}24 \\
31 \\
49\end{array}$ & $\begin{array}{l}13 \\
17 \\
23\end{array}$ & $\begin{array}{l}9.6 \\
13 \\
16\end{array}$ & $\begin{array}{l}7.6 \\
10 \\
12\end{array}$ & $\begin{array}{l}5.9 \\
7.6 \\
8.6\end{array}$ \\
\hline June-Aug. & $\begin{array}{r}1 \\
7 \\
30\end{array}$ & $\begin{array}{l}9.2 \\
11 \\
16\end{array}$ & $\begin{array}{l}6.0 \\
7.2 \\
9.7\end{array}$ & $\begin{array}{l}4.7 \\
5.9 \\
7.5\end{array}$ & $\begin{array}{l}3.9 \\
5.0 \\
6.1\end{array}$ & $\begin{array}{l}3.1 \\
4.2 \\
4.8\end{array}$ & Mar. - May & $\begin{array}{r}1 \\
7 \\
30\end{array}$ & $\begin{array}{l}27 \\
35 \\
75\end{array}$ & $\begin{array}{l}18 \\
24 \\
44\end{array}$ & $\begin{array}{l}15 \\
19 \\
34\end{array}$ & $\begin{array}{l}12 \\
16 \\
27\end{array}$ & $\begin{array}{l}10 \\
14 \\
21\end{array}$ \\
\hline
\end{tabular}

Duration of daily flow for indicated periods

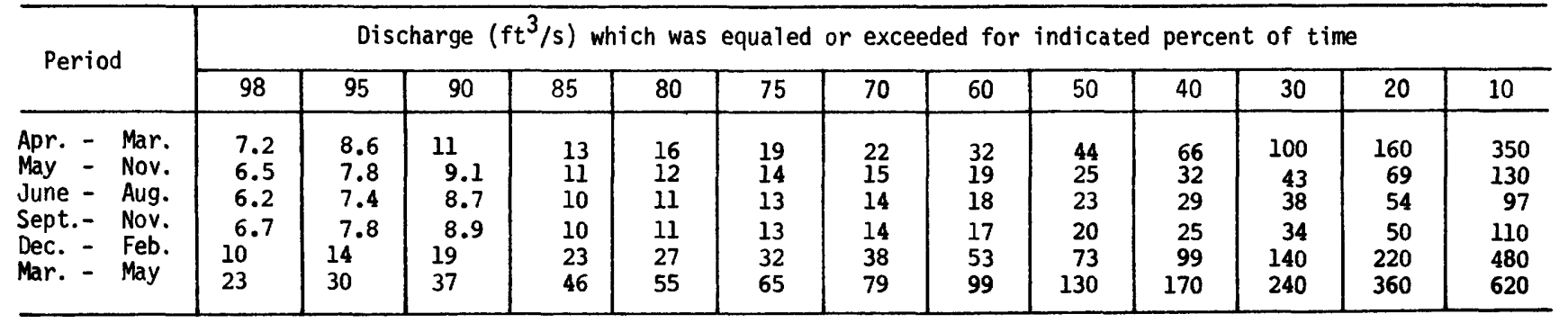


Appendix 3.--Low-flow data--Continued

\author{
MUSKINGUM RIVER BASIN \\ 03117000 Tuscarawas River at Massillon, Ohio
}

LOCATION:

Lat $40^{\circ} 46^{\prime} 13^{\prime \prime}$, long $81^{\circ} 31^{\prime} 27^{\prime \prime}$, in sec. 20 , T.10 N., R.9 W., Stark County, Hydrologic Unit 05040001 , on left bank at sewage-treatment works, $0.7 \mathrm{mi}$ south of Massillon, and $3 \mathrm{mi}$ downstream from Newman Creek.

DRAINAGE AREA: $\quad 518 \mathrm{mi}^{2}$.

TRIBUTARY TO: Head of Muskingum River.

DISCHARGE DATA USED: October 1939 to September 1978.

REMARKS: Diversion from basin and regulation at Portage Lakes (including Nimisila Reservoir since 1939).

SELECTED DISCHARGE CHARACTERISTICS: Average discharge: $433 \mathrm{ft}^{3} / \mathrm{s}$ (39 years).

Minimum daily discharge: $57 \mathrm{ft} / \mathrm{s}$ October 1963.

Magnitude and frequency of low flow for indicated periods

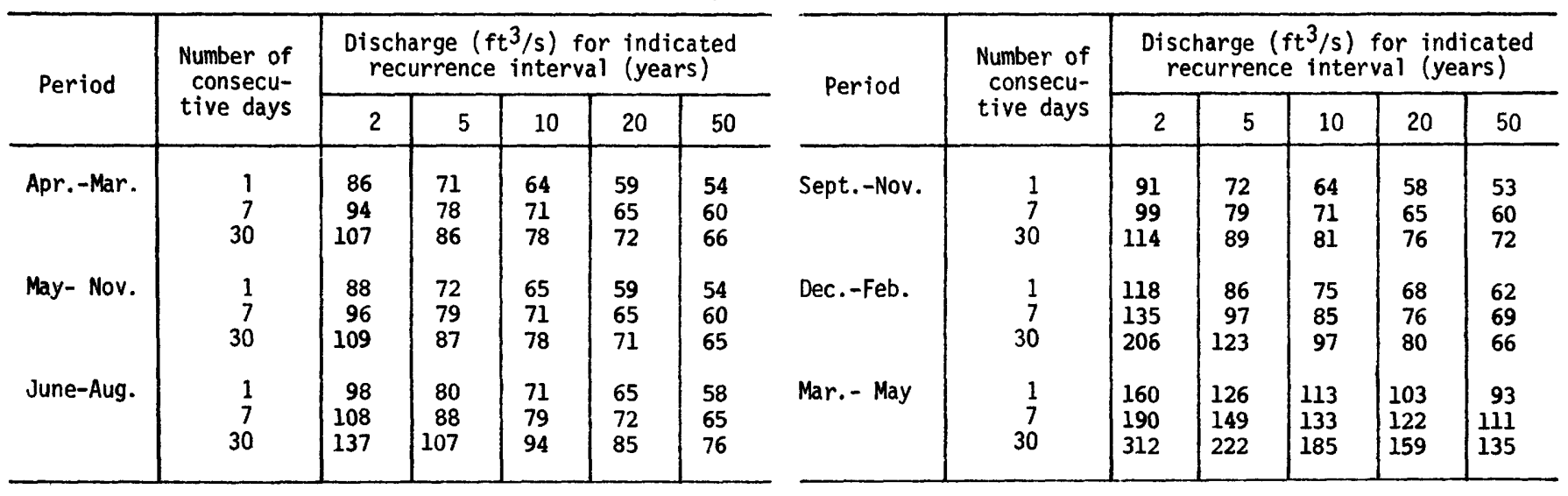

Duration of daily flow for indicated periods

\begin{tabular}{l|r|r|r|r|r|r|r|r|r|r|r|r|r}
\hline \multirow{2}{*}{ Period } & \multicolumn{8}{|c|}{ Discharge $\left(\mathrm{ft}^{3} / \mathrm{s}\right)$ which was equaled or exceeded for indicated percent of time } \\
\cline { 2 - 12 } & 98 & 95 & 90 & 85 & 80 & 75 & 70 & 60 & 50 & 40 & 30 & 20 & 10 \\
\hline Apr. - Mar. & 78 & 88 & 99 & 110 & 120 & 130 & 140 & 170 & 210 & 280 & 370 & 550 & 1000 \\
May - Nov. & 73 & 82 & 93 & 100 & 110 & 120 & 120 & 140 & 160 & 190 & 230 & 310 & 520 \\
June - Aug. & 78 & 87 & 96 & 100 & 110 & 120 & 130 & 140 & 160 & 190 & 220 & 290 & 490 \\
Sept.- Nov. & 69 & 77 & 85 & 92 & 98 & 100 & 110 & 120 & 130 & 150 & 170 & 220 & 350 \\
Dec. - Feb. & 83 & 93 & 110 & 120 & 130 & 140 & 160 & 210 & 270 & 350 & 460 & 710 & 1400 \\
Mar. - May & 140 & 160 & 190 & 220 & 250 & 270 & 300 & 360 & 450 & 560 & 730 & 1000 & 1600 \\
\hline
\end{tabular}


Appendix 3.--Low-flow data--Continued

MUSKINGUM RIVER BASIN

03117150 Sandy Creek at Minerva, Ohio

LOCATION:

Lat $40^{\circ} 43^{\prime} 53^{\prime \prime}$, long $81^{\circ} 05^{\prime} 7^{\prime \prime}$, Stark County, Hydrologic Unit 05040001 , at bridge on U.S. Highway 30 in Minerva.

DRAINAGE AREA:

$61.9 \mathrm{mi}^{2}$

TRIBUTARY TO:

Tuscarawas River.

DISCHARGE DATA USED:

Low-flow measurements 1974-1978, 1980 water years.

INDEX STATION: $\quad 03117500$ Sandy Creek at Waynesburg, Ohio.

REMARKS:

None.

SELECTED DISCHARGE CHARACTERISTICS: Minimum discharge observed: $8.7 \mathrm{ft}^{3} / \mathrm{s}$ September 1978.

\begin{tabular}{|c|c|c|c|}
\hline \multirow[t]{2}{*}{ Period } & \multirow{2}{*}{$\begin{array}{l}\text { Number of } \\
\text { consecu- } \\
\text { tive days }\end{array}$} & \multicolumn{2}{|c|}{$\begin{array}{l}\text { Discharge }\left(\mathrm{ft}^{3} / \mathrm{s}\right) \text { for } \\
\text { indicated recurrence } \\
\text { interval (years) }\end{array}$} \\
\hline & & 2 & 10 \\
\hline Apr. - Mar. & $\begin{array}{r}7 \\
30\end{array}$ & $\begin{array}{l}4.4 \\
5.6\end{array}$ & $\begin{array}{l}2.7 \\
3.2\end{array}$ \\
\hline May - Nov. & $\begin{array}{r}7 \\
30\end{array}$ & $\begin{array}{l}4.6 \\
5.6\end{array}$ & $\begin{array}{l}2.7 \\
3.2\end{array}$ \\
\hline June - Aug. & $\begin{array}{r}7 \\
30\end{array}$ & $\begin{array}{l}6.1 \\
8.5\end{array}$ & $\begin{array}{l}3.5 \\
4.6\end{array}$ \\
\hline Sept.- Nov. & $\begin{array}{r}7 \\
30\end{array}$ & $\begin{array}{l}4.6 \\
6.3\end{array}$ & $\begin{array}{l}2.7 \\
3.4\end{array}$ \\
\hline
\end{tabular}

Duration of daily flow for indicated periods

\begin{tabular}{l|c|c|c|c|c}
\hline \multirow{2}{*}{ Period } & \multicolumn{5}{|c|}{$\begin{array}{c}\text { Discharge }\left(\mathrm{ft}^{3} / \mathrm{s} \text { ) which was equaled or }\right. \\
\text { exceeded for indicated percent of time }\end{array}$} \\
\cline { 2 - 6 } & 98 & 95 & 90 & 85 & 80 \\
\hline Apr. - Mar. & 3.4 & 4.4 & 5.1 & 6.1 & 7.3 \\
May - Nov. & 3.2 & 3.9 & 4.6 & 5.2 & 5.8 \\
June - Aug. & 3.7 & 4.6 & 5.4 & 6.1 & 6.7 \\
Sept. - Nov. & 2.7 & 3.4 & 3.9 & 4.4 & 4.7 \\
\hline
\end{tabular}


Appendix 3.--Low-flow data--Continued

MUSKINGUM RIVER BASIN

03117160 Still Fork near Minerva, Ohio

LOCATION:

Lat $40^{\circ} 39^{\prime} 49^{\prime \prime}$, long $81^{\circ} 02^{\prime} 24^{\prime \prime}$, Carroll County, Hydrologic Unit 05040001 , at bridge on State Route $9,1.4 \mathrm{mi}$ downstream from Pipes Fork, $5.5 \mathrm{mi}$ southeast of Minerva.

DRAINAGE AREA:

$36.2 \mathrm{mi}^{2}$

TRIBUTARY TO:

Sandy Creek.

DISCHARGE DATA USED:

Low-flow measurements 1974-1978, 1980 water years.

INDEX STATION: $\quad 03117500$ Sandy Creek at Waynesburg, Ohio.

REMARKS:

None.

SELECTED DISCHARGE CHARACTERISTICS: Minimum discharge observed: $2.4 \mathrm{ft}^{3} / \mathrm{s}$ September 1978 .

\begin{tabular}{|c|c|c|c|}
\hline \multirow[t]{2}{*}{ Period } & \multirow{2}{*}{$\begin{array}{l}\text { Number of } \\
\text { consecu- } \\
\text { tive days }\end{array}$} & \multicolumn{2}{|c|}{$\begin{array}{l}\text { Discharge }\left(\mathrm{ft}^{3} / \mathrm{s}\right) \text { for } \\
\text { indicated recurrence } \\
\text { interval (years) }\end{array}$} \\
\hline & & 2 & 10 \\
\hline Apr. - Mar. & $\begin{array}{r}7 \\
30\end{array}$ & $\begin{array}{l}0.6 \\
1.0\end{array}$ & $\begin{array}{l}0.3 \\
0.4\end{array}$ \\
\hline May - Nov. & $\begin{array}{r}7 \\
30\end{array}$ & $\begin{array}{l}0.7 \\
1.0\end{array}$ & $\begin{array}{l}0.3 \\
0.4\end{array}$ \\
\hline June - Aug. & $\begin{array}{r}7 \\
30\end{array}$ & $\begin{array}{l}1.1 \\
1.9\end{array}$ & $\begin{array}{l}C .4 \\
0.7\end{array}$ \\
\hline Sept. - Nov. & $\begin{array}{r}7 \\
30\end{array}$ & $\begin{array}{l}0.7 \\
1.1\end{array}$ & $\begin{array}{l}0.3 \\
0.4\end{array}$ \\
\hline
\end{tabular}

Duration of daily flow for indicated periods

\begin{tabular}{|c|c|c|c|c|c|}
\hline \multirow{2}{*}{ Period } & \multicolumn{5}{|c|}{$\begin{array}{l}\text { Discharge }\left(\mathrm{ft}^{3} / \mathrm{s}\right) \text { which was equaled or } \\
\text { exceeded for indicated percent of time }\end{array}$} \\
\hline & 98 & 95 & 90 & 85 & 80 \\
\hline $\begin{array}{l}\text { Apr. - Mar. } \\
\text { May - Nov. } \\
\text { June - Aug. } \\
\text { Sept. - Nov. }\end{array}$ & $\begin{array}{l}0.4 \\
0.4 \\
0.5 \\
0.3\end{array}$ & $\begin{array}{l}0.6 \\
0.5 \\
0.7 \\
0.4\end{array}$ & $\begin{array}{l}0.9 \\
0.7 \\
0.9 \\
0.5\end{array}$ & $\begin{array}{l}1.1 \\
0.9 \\
1.1 \\
0.6\end{array}$ & $\begin{array}{l}1.5 \\
1.0 \\
1.3 \\
0.7\end{array}$ \\
\hline
\end{tabular}


Appendix 3.--Low-flow data--Continued

MUSKINGUM RIVER BASIN

03117300 Sandy Creek at Malvern, Ohio

LOCATION:

Lat $40^{\circ} 41^{\prime} 27^{\prime \prime}$, long $81^{\circ} 10^{\prime} 50^{\prime \prime}$, Carroll County, Hydrologic Unit

05040001 , at bridge on State Route 43 and State Route 183,

in Malvern.

DRAINAGE AREA: $\quad 163 \mathrm{mi}^{2}$.

TRIBUTARY TO: Tuscarawas River.

DISCHARGE DATA USED: Low-flow measurements 1976-1978, 1980 water years.

INDEX STATION: $\quad 03117500$ Sandy Creek at Waynesburg, Ohio.

REMARKS: None.

SELECTED DISCHARGE CHARACTERISTICS: Minimum discharge observed: $33 \mathrm{ft}^{3} / \mathrm{s}$ September 1978.

\begin{tabular}{c|c|c|c}
\multicolumn{4}{c}{$\begin{array}{c}\text { Magnitude and frequency of low flow for } \\
\text { indicated periods }\end{array}$} \\
\hline Period & $\begin{array}{c}\text { Number of } \\
\text { consecu- } \\
\text { tive days }\end{array}$ & $\begin{array}{c}\text { Discharge (ft } 3 / \mathrm{s}) \text { for } \\
\text { indicated recurrence } \\
\text { interval }\end{array}$ \\
\cline { 3 - 4 } & & 2 & 10 \\
\hline Apr. - Mar. & 7 & 20 & 14 \\
\hline May - Nov. & 30 & 24 & 16 \\
& 7 & 21 & 14 \\
June - Aug. & 30 & 24 & 16 \\
& 30 & 26 & 17 \\
Sept. - Nov. & 7 & 34 & 21 \\
& 30 & 21 & 14 \\
\hline
\end{tabular}

Duration of daily flow for indicated periods

\begin{tabular}{|c|c|c|c|c|c|}
\hline \multirow{2}{*}{ Period } & \multicolumn{5}{|c|}{$\begin{array}{l}\text { Discharge }\left(\mathrm{ft}^{3} / \mathrm{s}\right) \text { which was equaled or } \\
\text { exceeded for indicated percent of time }\end{array}$} \\
\hline & 98 & 95 & 90 & 85 & 80 \\
\hline $\begin{array}{l}\text { Apr. - Mar. } \\
\text { May - Nov. } \\
\text { June - Aug. } \\
\text { Sept. - Nov. }\end{array}$ & $\begin{array}{l}17 \\
16 \\
18 \\
14\end{array}$ & $\begin{array}{l}20 \\
19 \\
21 \\
17\end{array}$ & $\begin{array}{l}23 \\
21 \\
24 \\
19\end{array}$ & $\begin{array}{l}26 \\
23 \\
26 \\
20\end{array}$ & $\begin{array}{l}30 \\
25 \\
28 \\
21\end{array}$ \\
\hline
\end{tabular}


Appendix 3.--Low-flow data--Continued

MUSKINGUM RIVER BASIN

03117310 Pipe Run at Malvern, Ohio

LOCATION: Lat $40^{\circ} 41^{\prime} 16^{\prime \prime}$, long $81^{\circ} 11^{\prime} 02^{\prime \prime}$, Carroll County, Hydrologic Unit 05040001 , at bridge in Malvern, $200 \mathrm{ft}$ upstream from mouth.

DRAINAGE AREA: $\quad 27.7 \mathrm{mi}^{2}$.

TRIBUTARY TO: Sandy Creek.

DISCHARGE DATA USED: Low-flow measurements 1976-1978, 1980 water years.

INDEX STATION: $\quad 03117500$ Sandy Creek at Waynesburg, Ohio.

REMARKS: $\quad$ None.

SELECTED DISCHARGE CHARACTERISTICS: Minimum discharge observed: $0.8 \mathrm{ft} / / \mathrm{s}$ September 1978 .

Magnitude and frequency of low flow for indicated periods

\begin{tabular}{c|c|c|c}
\hline \multirow{2}{*}{ Period } & \multirow{2}{*}{$\begin{array}{c}\text { Number of } \\
\text { consecu- } \\
\text { tive days }\end{array}$} & \multicolumn{2}{|c}{$\begin{array}{c}\text { Discharge }\left(\mathrm{ft}^{3} / \mathrm{s}\right) \text { for } \\
\text { indicated recurrence } \\
\text { interval (years) }\end{array}$} \\
\cline { 3 - 4 } Apr. - Mar. & 7 & 2 & 10 \\
\hline May - Nov. & 30 & 0.2 & 0.1 \\
& 7 & 0.3 & 0.1 \\
June - Aug. & 30 & 0.3 & 0.1 \\
& 7 & 0.1 & 0.1 \\
Sept. - Nov. & 30 & 0.7 & 0.1 \\
& 30 & 0.2 & 0.1 \\
& & 0.4 & 0.1 \\
\hline
\end{tabular}

Duration of daily flow for indicated periods

\begin{tabular}{c|c|c|c|c|c}
\hline \multirow{2}{*}{ Period } & \multicolumn{5}{|c}{ Discharge $\left(\mathrm{ft}^{3} / \mathrm{s}\right.$ ) which was equaled or } \\
exceeded for indicated percent of time \\
\cline { 2 - 6 } & 98 & 95 & 90 & 85 & 80 \\
\hline Apr. - Mar. & 0.1 & 0.2 & 0.3 & 0.4 & 0.5 \\
May - Nov. & 0.1 & 0.1 & 0.2 & 0.3 & 0.3 \\
June - Aug. & 0.1 & 0.2 & 0.3 & 0.4 & 0.4 \\
Sept. - Nov. & 0.1 & 0.1 & 0.1 & 0.2 & 0.2 \\
\hline
\end{tabular}


MUSKINGUM RIVER BASIN

03117500 Sandy Creek at Waynesburg, Ohio

LOCATION:

Lat $40^{\circ} 40^{\prime} 21^{\prime \prime}$, long $81^{\circ} 15^{\prime} 36^{\prime \prime}$, in sec. 21 , T. 17 N., R./ W., stark county Hydrologic Unit 05040001 , on upstream side of left pier of bridge on State Route 183 in Waynesburg, $300 \mathrm{ft}$ downstream from Little Sandy Creek, and $0.6 \mathrm{mi}$ upstream from Indian Run.

DRAINAGE AREA:

$253 \mathrm{mi}^{2}$.

TRIBUTARY TO:

Tuscarawas River.

DISCHARGE DATA USED: December 1938 to September 1978.

REMARKS:

None.

SELECTED DISCHARGE CHARACTERISTICS: Average discharge: $265 \mathrm{ft}^{3} / \mathrm{s}$ (39 years).

Minimum daily discharge: $12 \mathrm{ft} 3 / \mathrm{s}$ September, October, November 1963 and October 1966.

Magnitude and frequency of low flow for indicated periods

\begin{tabular}{c|c|c|c|c|c|c}
\hline \multirow{2}{*}{ Period } & \multirow{2}{*}{$\begin{array}{c}\text { Number of } \\
\text { consecu- } \\
\text { tive days }\end{array}$} & \multicolumn{4}{|c}{$\begin{array}{c}\text { Discharge }(\mathrm{ft} / \mathrm{s} / \mathrm{s} \text { ) for indicated } \\
\text { recurrence interva1 }\end{array}$} \\
\cline { 3 - 7 } (years)
\end{tabular}

\begin{tabular}{c|c|c|c|c|c|c}
\hline \multirow{2}{*}{ Period } & \multirow{2}{*}{$\begin{array}{c}\text { Number of } \\
\text { consecu- } \\
\text { tive days }\end{array}$} & \multicolumn{4}{|c|}{$\begin{array}{c}\text { Discharge }\left(\mathrm{ft}^{3} / \mathrm{s}\right) \\
\text { recurrence interval indicated }\end{array}$} \\
\cline { 3 - 7 } (years)
\end{tabular}

Duration of daily flow for indicated periods

\begin{tabular}{|c|c|c|c|c|c|c|c|c|c|c|c|c|c|c|}
\hline \multirow{2}{*}{\multicolumn{2}{|c|}{ Period }} & \multicolumn{13}{|c|}{ Discharge $\left(\mathrm{ft}^{3} / \mathrm{s}\right)$ which was equaled or exceeded for indicated percent of time } \\
\hline & & 98 & 95 & 90 & 85 & 80 & 75 & 70 & 60 & 50 & 40 & 30 & 20 & 10 \\
\hline $\begin{array}{l}\text { Apr. - } \\
\text { May - } \\
\text { June - } \\
\text { Sept - } \\
\text { Dec. - } \\
\text { Mar. - }\end{array}$ & $\begin{array}{l}\text { Mar. } \\
\text { Nov. } \\
\text { Aug. } \\
\text { Nov. } \\
\text { Feb. } \\
\text { May }\end{array}$ & $\begin{array}{l}21 \\
20 \\
23 \\
17 \\
27 \\
74\end{array}$ & $\begin{array}{l}27 \\
24 \\
28 \\
21 \\
34 \\
92\end{array}$ & $\begin{array}{r}32 \\
28 \\
33 \\
24 \\
45 \\
120\end{array}$ & $\begin{array}{r}37 \\
32 \\
37 \\
27 \\
57 \\
150\end{array}$ & $\begin{array}{r}44 \\
35 \\
41 \\
29 \\
67 \\
170\end{array}$ & $\begin{array}{r}53 \\
39 \\
46 \\
31 \\
80 \\
200\end{array}$ & $\begin{array}{r}63 \\
44 \\
51 \\
34 \\
97 \\
220\end{array}$ & $\begin{array}{r}87 \\
57 \\
62 \\
39 \\
140 \\
270\end{array}$ & $\begin{array}{r}120 \\
74 \\
75 \\
48 \\
200 \\
330\end{array}$ & $\begin{array}{r}180 \\
96 \\
92 \\
63 \\
270 \\
410\end{array}$ & $\begin{array}{r}260 \\
130 \\
120 \\
87 \\
360 \\
500\end{array}$ & $\begin{array}{l}380 \\
190 \\
150 \\
130 \\
520 \\
640\end{array}$ & $\begin{array}{l}630 \\
320 \\
240 \\
240 \\
870 \\
950\end{array}$ \\
\hline
\end{tabular}


LOCATION:

03118000 Middle Branch Nimishillen Creek at Canton, Ohio Lat $40^{\circ} 50^{\prime} 29^{\prime \prime}$, long $81^{\circ} 21^{\prime} 14^{\prime \prime}$ in NEl/4 sec. 27, T.11 N., R.8 W., Stark County, Hydrologic Unit 05040001, on right bank at downstream side of bridge on Martindale Road, $2.4 \mathrm{mi}$ upstream from mouth, and $0.5 \mathrm{mi}$ northeast of Canton.

DRAINAGE AREA: $\quad 43.1 \mathrm{mi}^{2}$.

TRIBUTARY TO: $\quad$ Sandy Creek.

DISCHARGE DATA USED: October 1941 to September 1978.

REMARKS:

Part of municipal water supply for city of Canton is pumped from its northeast well field; a portion of pumpage is believed to be derived from creek as recharge to aquifer supplying well field. Niealı purnuage for water year 1977 is $12.7 \mathrm{ft} 3 / \mathrm{s}$, At times low flow regulated by small pools above station.

SELECTED DISCHARGE CHARACTERISTICS: Average discharge: $34.6 \mathrm{ft}^{3} / \mathrm{s}$ (37 years).

Minimum daily discharge: $0.3 \mathrm{ft} 3 / \mathrm{s}$ September 1962 .

Magnitude and frequency of low flow for indicated periods

\begin{tabular}{|c|c|c|c|c|c|c|c|c|c|c|c|c|c|}
\hline \multirow{2}{*}{ Period } & \multirow{2}{*}{$\begin{array}{l}\text { Number of } \\
\text { consecu- } \\
\text { tive days }\end{array}$} & \multicolumn{5}{|c|}{$\begin{array}{l}\text { Discharge }\left(\mathrm{ft}^{3} / \mathrm{s}\right) \text { for indicated } \\
\text { recurrence interval (years) }\end{array}$} & \multirow{2}{*}{ Period } & \multirow{2}{*}{$\begin{array}{l}\text { Number of } \\
\text { consecu- } \\
\text { tive days }\end{array}$} & \multicolumn{5}{|c|}{$\begin{array}{l}\text { Discharge }\left(\mathrm{ft}^{3} / \mathrm{s}\right) \text { for indicated } \\
\text { recurrence interval (years) }\end{array}$} \\
\hline & & 2 & 5 & 10 & 20 & 50 & & & 2 & 5 & 10 & 20 & 50 \\
\hline Apr.-Mar. & $\begin{array}{r}1 \\
7 \\
30\end{array}$ & $\begin{array}{l}2.8 \\
3.6 \\
4.4\end{array}$ & $\begin{array}{l}1.2 \\
1.7 \\
2.4\end{array}$ & $\begin{array}{l}0.8 \\
1.1 \\
1.8\end{array}$ & $\begin{array}{l}0.5 \\
0.8 \\
1.4\end{array}$ & $\begin{array}{l}0.3 \\
0.5 \\
1.1\end{array}$ & Sept.-Nov. & $\begin{array}{r}1 \\
7 \\
30\end{array}$ & $\begin{array}{l}3.1 \\
3.7 \\
5.3\end{array}$ & $\begin{array}{l}1.4 \\
1.9 \\
2.8\end{array}$ & $\begin{array}{l}0.9 \\
1.4 \\
2.0\end{array}$ & $\begin{array}{l}0.6 \\
1.1 \\
1.5\end{array}$ & $\begin{array}{l}0.4 \\
0.8 \\
1.1\end{array}$ \\
\hline June-Aug. & $\begin{array}{r}1 \\
7 \\
30\end{array}$ & $\begin{array}{l}4.2 \\
5.1 \\
7.6\end{array}$ & $\begin{array}{l}1.9 \\
2.5 \\
4.0\end{array}$ & $\begin{array}{l}1.3 \\
1.7 \\
2.8\end{array}$ & $\begin{array}{l}0.9 \\
1.2 \\
2.1\end{array}$ & $\begin{array}{l}0.6 \\
0.8 \\
1.5\end{array}$ & Mar. - May & $\begin{array}{r}1 \\
7 \\
30\end{array}$ & $\begin{array}{l}14 \\
14 \\
26\end{array}$ & $\begin{array}{l}7.5 \\
10^{\circ} \\
17\end{array}$ & $\begin{array}{c}4.6 \\
9.1 \\
14\end{array}$ & $\begin{array}{c}2.7 \\
8.3 \\
12\end{array}$ & $\begin{array}{l}1.3 \\
7.6 \\
10\end{array}$ \\
\hline
\end{tabular}

Duration of daily flow for indicated periods

\begin{tabular}{l|c|c|c|c|c|c|c|c|c|c|c|c|c}
\hline \multirow{2}{*}{ Period } & \multicolumn{8}{|c|}{ Discharge $\left(\mathrm{ft}^{3} / \mathrm{s}\right)$ which was equaled or exceeded for indicated percent of time } \\
\cline { 2 - 12 } & 98 & 95 & 90 & 85 & 80 & 75 & 70 & 60 & 50 & 40 & 30 & 20 & 10 \\
\hline Apr. - Mar. & 1.8 & 2.5 & 3.5 & 4.6 & 5.8 & 7.2 & 8.7 & 12 & 17 & 22 & 31 & 46 & 78 \\
May - Nov. & 1.6 & 2.2 & 3.0 & 3.7 & 4.5 & 5.3 & 6.3 & 8.4 & 11 & 15 & 19 & 26 & 43 \\
June - Aug. & 2.0 & 2.7 & 3.5 & 4.2 & 5.0 & 6.0 & 7.0 & 9.3 & 12 & 15 & 19 & 24 & 39 \\
Sept.- Nov. & 1.5 & 1.8 & 2.3 & 2.8 & 3.3 & 3.9 & 4.5 & 5.8 & 7.5 & 9.9 & 13 & 19 & 31 \\
Dec. - Feb. & 2.0 & 2.9 & 4.3 & 5.5 & 7.3 & 8.9 & 11 & 15 & 21 & 29 & 40 & 59 & 110 \\
Mar. - May & 9.8 & 12 & 15 & 17 & 20 & 22 & 25 & 31 & 37 & 46 & 58 & 77 & 120 \\
\hline
\end{tabular}


Appendix 3.--Low-flow data--Continued

\section{MUSKINGUM RIVER BASIN}

03118100 East Branch Nimishillen Creek near Canton, Ohio

LOCATION:

Lat $40^{\circ} 49^{\prime} 24^{\prime \prime}$, long $81^{\circ} 17^{\prime} 55^{\prime \prime}$, Stark County, Hydrologic Unit 05040001 , at bridge on Broadway Avenue, $1 \mathrm{mi}$ east of Canton city limits, $3.5 \mathrm{mi}$ upstream from Middle Branch.

DRAINAGE AREA: $\quad 33.4 \mathrm{mi}^{2}$.

TRIBUTARY TO: Nimishillen Creek.

DISCHARGE DATA USED: LOW-flow measurements 1974-1978, 1980 water years.

INDEX STATION: $\quad 03117500$ Sandy Creek at Waynesburg, Ohio.

REMARKS:

Canton northeast well field affect flow in Middle Branch

Nimishillen Creek, but is not known to affect flow in East Branch.

SELECTED DISCHARGE CHARACTERISTICS: Minimum discharge observed: $5.7 \mathrm{ft}^{3} / \mathrm{s}$ June 1977.

\begin{tabular}{|c|c|c|c|}
\hline \multirow[t]{2}{*}{ Period } & \multirow{2}{*}{$\begin{array}{l}\text { Number of } \\
\text { consecu- } \\
\text { tive days }\end{array}$} & \multicolumn{2}{|c|}{$\begin{array}{l}\text { Discharge }\left(\mathrm{ft}^{3} / \mathrm{s}\right) \text { for } \\
\text { indicated recurrence } \\
\text { interval (years) }\end{array}$} \\
\hline & & 2 & 10 \\
\hline Apr. - Mar. & $\begin{array}{r}7 \\
30\end{array}$ & $\begin{array}{l}3.7 \\
4.4\end{array}$ & $\begin{array}{l}2.7 \\
3.0\end{array}$ \\
\hline May - Nov. & $\begin{array}{r}7 \\
30\end{array}$ & $\begin{array}{l}3.8 \\
4.4\end{array}$ & $\begin{array}{l}2.7 \\
3.0\end{array}$ \\
\hline June - Aug. & $\begin{array}{r}7 \\
30\end{array}$ & $\begin{array}{l}4.7 \\
6.0\end{array}$ & $\begin{array}{l}3.2 \\
3.8\end{array}$ \\
\hline Sept. - Nov. & $\begin{array}{r}7 \\
30\end{array}$ & $\begin{array}{l}3.8 \\
4.8\end{array}$ & $\begin{array}{l}2.7 \\
3.1\end{array}$ \\
\hline
\end{tabular}

Duration of daily flow for indicated periods

\begin{tabular}{l|c|c|c|c|c}
\hline \multirow{2}{*}{ Period } & \multicolumn{5}{|c}{$\begin{array}{c}\text { Discharge ( } \mathrm{ft} 3 / \mathrm{s} \text { ) which was equaled or } \\
\text { exceeded for indicated percent of time }\end{array}$} \\
\cline { 2 - 6 } & 98 & 95 & 90 & 85 & 80 \\
\hline Apr. - Mar. & 3.1 & 3.7 & 4.2 & 4.7 & 5.4 \\
May - Nov. & 3.0 & 3.4 & 3.8 & 4.2 & 4.5 \\
June - Aug. & 3.3 & 3.8 & 4.3 & 4.7 & 5.1 \\
Sept. - Nov. & 2.7 & 3.1 & 3.4 & 3.7 & 3.9 \\
\hline
\end{tabular}




\section{MUSKINGUM RIVER BASIN}

03118500 Nimishillen Creek at North Industry, Ohio

LOCATION:

Lat $40^{\circ} 44^{\prime} 03^{\prime \prime}$, long $81^{\circ} 21^{\prime} 08^{\prime \prime}$, in sec.35, T.10 N., R.8 W., Stark County, Hydrologic Unit 05040001 , on left bank just downstream from railroad bridge, $1 \mathrm{mi}$ southeast of North Industry, and $3 \mathrm{mi}$ downstream from Sherrick Run.

DRAINAGE AREA:

$175 \mathrm{mi}^{2}$.

TRIBUTARY TO:

Sandy Creek.

DISCHARGE DATA USED:

October 1921 to September 1978.

REMARKS:

Low flow slightly regulated by plants at Canton. Records include diversion from Sugar Creek well field. Mean pumpage for the 1976 water year, $16.0 \mathrm{ft}^{3} / \mathrm{s}$.

SELECTED DISCHARGE CHARACTERISTICS: Average discharge: $178 \mathrm{ft} 3 / \mathrm{s}$ ( 57 years).

Minimum daily discharge: $14 \mathrm{ft} 3 / \mathrm{s}$ August 1923.

Magnitude and frequency of low flow for indicated periods

\begin{tabular}{|c|c|c|c|c|c|c|}
\hline \multirow{2}{*}{ Period } & \multirow{2}{*}{$\begin{array}{l}\text { Number of } \\
\text { consecu- } \\
\text { tive days }\end{array}$} & \multicolumn{5}{|c|}{$\begin{array}{l}\text { Discharge }\left(\mathrm{ft}^{3} / \mathrm{s}\right) \text { for indicated } \\
\text { recurrence interval (years) }\end{array}$} \\
\hline & & 2 & 5 & 10 & 20 & 50 \\
\hline Apr.-Mar. & $\begin{array}{r}1 \\
7 \\
30\end{array}$ & $\begin{array}{l}44 \\
50 \\
57\end{array}$ & $\begin{array}{l}29 \\
36 \\
41\end{array}$ & $\begin{array}{l}23 \\
30 \\
35\end{array}$ & $\begin{array}{l}19 \\
25 \\
30\end{array}$ & $\begin{array}{l}15 \\
21 \\
26\end{array}$ \\
\hline May- Nov. & $\begin{array}{r}1 \\
7 \\
30\end{array}$ & $\begin{array}{l}44 \\
51 \\
58\end{array}$ & $\begin{array}{l}30 \\
36 \\
42\end{array}$ & $\begin{array}{l}23 \\
30 \\
35\end{array}$ & $\begin{array}{l}19 \\
25 \\
30\end{array}$ & $\begin{array}{l}15 \\
21 \\
26\end{array}$ \\
\hline June-Aug. & $\begin{array}{r}1 \\
7 \\
30\end{array}$ & $\begin{array}{l}51 \\
59 \\
77\end{array}$ & $\begin{array}{l}34 \\
41 \\
54\end{array}$ & $\begin{array}{l}26 \\
33 \\
44\end{array}$ & $\begin{array}{l}21 \\
28 \\
37\end{array}$ & $\begin{array}{l}16 \\
22 \\
30\end{array}$ \\
\hline
\end{tabular}

\begin{tabular}{c|c|c|c|c|c|c}
\hline \multirow{2}{*}{ Period } & \multirow{2}{*}{$\begin{array}{c}\text { Number of } \\
\text { consecu- } \\
\text { tive days }\end{array}$} & \multicolumn{4}{|c}{$\begin{array}{c}\text { Discharge } \\
\text { recurrence } \mathrm{ft}^{3} / \mathrm{s} \text { ) }\end{array}$ for interval indicated } \\
\cline { 3 - 7 } & 2 & 5 & 10 & 20 & 50 \\
\hline Sept.-Nov. & 1 & 46 & 32 & 25 & 21 & 17 \\
& 7 & 53 & 37 & 31 & 27 & 22 \\
& 30 & 62 & 44 & 38 & 33 & 29 \\
Dec.-Feb. & 1 & 60 & 43 & 36 & 32 & 27 \\
& 7 & 70 & 49 & 41 & 36 & 31 \\
& 30 & 102 & 65 & 51 & 42 & 34 \\
& 1 & 85 & 61 & 49 & 41 & 33 \\
& 7 & 100 & 71 & 58 & 48 & 39 \\
& 30 & 147 & 100 & 80 & 65 & 51 \\
\hline
\end{tabular}

Duration of daily flow for indicated periods

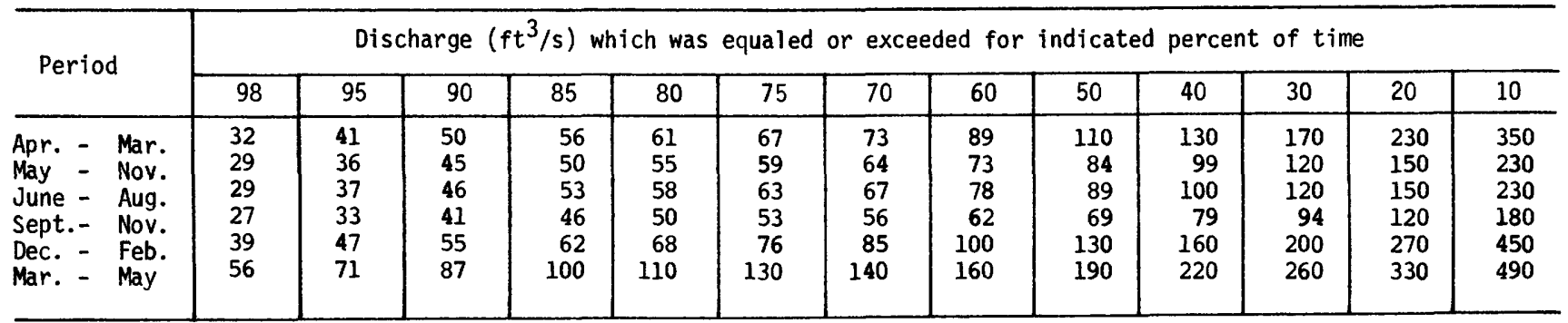


Appendix 3.--Low-flow data--Continued

MUSKINGUM RIVER BASIN

03119700 Conotton Creek at Jewett, Ohio

LOCATION:

Lat $40^{\circ} 21^{\prime} 55^{\prime \prime}$, long $81^{\circ} 00^{\prime} 15^{\prime \prime}$, Harrison County, Hydrologic Unit 05040001 , in NWl $/ 4$ sec. $5, T .11$ N., R. 5 W., on left downstream wingwall of bridge on State Route 9 in Jewett.

DRAINAGE AREA: $\quad 14.3 \mathrm{mi}^{2}$.

TRIBUTARY TO: Tuscarawas River.

DISCHARGE DATA USED: Low-flow measurements 1965-1970, 1972-1974 water years.

INDEX STATION: $\quad 03111500$ Short Creek near Dillonville, Ohio.

REMARKS: None.

SELECTED DISCHARGE CHARACTERISTICS: Minimum discharge observed: $0.2 \mathrm{ft}^{3} / \mathrm{s}$ July 1965.

Magnitude and frequency of low flow for indicated periods

\begin{tabular}{c|c|c|c}
\hline \multirow{2}{*}{ Period } & \multirow{2}{*}{$\begin{array}{c}\text { Number of } \\
\text { consecu- } \\
\text { tive days }\end{array}$} & \multicolumn{2}{|c}{$\begin{array}{c}\text { Discharge (ft } 3 / \mathrm{s}) \text { for } \\
\text { indicated recurrence } \\
\text { interval (years) }\end{array}$} \\
\cline { 3 - 4 } Apr. - Mar. & 7 & 2 & 10 \\
\hline May - Nov. & 30 & 0.3 & 0.1 \\
& 7 & 0.5 & 0.2 \\
June - Aug. & 30 & 0.3 & 0.1 \\
& 7 & 0.5 & 0.2 \\
Sept. - Nov. & 7 & 0.6 & 0.2 \\
& 30 & 0.4 & 0.3 \\
& & 0.8 & 0.1 \\
\hline
\end{tabular}

Duration of daily flow for indicated periods

\begin{tabular}{l|c|c|c|c|c}
\hline \multirow{2}{*}{ Period } & \multicolumn{5}{|c}{$\begin{array}{c}\text { Discharge ( } \mathrm{ft} 3 / \mathrm{s} \text { ) which was equaled or } \\
\text { exceeded for indicated percent of time }\end{array}$} \\
\cline { 2 - 6 } & 98 & 95 & 90 & 85 & 80 \\
\hline Apr. - Mar. & 0.2 & 0.3 & 0.5 & 0.8 & 1.0 \\
May - Nov. & 0.2 & 0.3 & 0.4 & 0.5 & 0.7 \\
June - Aug. & 0.2 & 0.3 & 0.5 & 0.7 & 0.9 \\
Sept. - Nov. & 0.1 & 0.2 & 0.3 & 0.4 & 0.5 \\
\hline
\end{tabular}


MUSKINGUM RIVER BASIN

03119900 Conotton Creek at Leesville, Ohio

LOCATION:

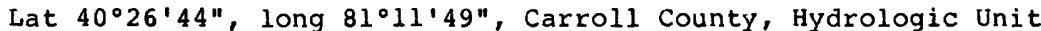

$05040001,2.5 \mathrm{mi}$ upstream from McGuire Creek, at State Route 164 bridge, $0.9 \mathrm{mi}$ southeast of Leesville.

DRAINAGE AREA: $\quad 87.1 \mathrm{mi}^{2}$.

TRIBUTARY TO: Tuscarawas River.

DISCHARGE DATA USED: Low-flow measurements 1959, 1972-1977 water years.

INDEX STATION: $\quad 03111500$ Short Creek near Dillonvale, Ohio.

REMARKS:

Scio sewage (untreated) discharges into Conotton Creek $8.0 \mathrm{mi}$ upstream of station. $80,000 \mathrm{gal} / \mathrm{d}$ is pumped from ground water for water supply. No record of flow.

SELECTED DISCHARGE CHARACTERISTICS: Minimum discharge observed: $3.6 \mathrm{ft}^{3} / \mathrm{s}$ September 1972 .

\begin{tabular}{c|c|c|c}
\multicolumn{4}{c}{ Magnitude and frequency of low flow for } \\
indicated periods \\
\hline Period & $\begin{array}{c}\text { Number of } \\
\text { consecu- } \\
\text { tive days }\end{array}$ & $\begin{array}{c}\text { Discharge }\left(\mathrm{ft}^{3} / \mathrm{s}\right) \text { for } \\
\text { indicated recurrence } \\
\text { interval }\end{array}$ \\
\cline { 3 - 4 } (years)
\end{tabular}

Duration of daily flow for indicated periods

\begin{tabular}{c|c|c|c|c|c}
\hline \multirow{2}{*}{ Period } & \multicolumn{6}{|c|}{$\begin{array}{c}\text { Discharge }(\mathrm{ft} 3 / \mathrm{s} \text { ) which was equaled or } \\
\text { exceeded for indicated percent of timie }\end{array}$} \\
\cline { 2 - 6 } & 98 & 95 & 90 & 85 & 80 \\
\hline Apr. - Mar. & 2.6 & 3.7 & 4.9 & 6.5 & 7.8 \\
May - Nov. & 2.3 & 3.1 & 4.0 & 4.9 & 6.1 \\
June - Aug. & 2.6 & 3.4 & 4.6 & 6.1 & 7.1 \\
Sept. - Nov. & 2.1 & 2.8 & 3.4 & 4.0 & 4.6 \\
\hline
\end{tabular}


Appendix 3.--Low-flow data--Continued

MUSKINGUM RIVER BASIN

03120500 McGuire Creek below Leesville Dam, near Leesville, Ohio

LOCATION:

Lat $40^{\circ} 28^{\prime} 13^{\prime \prime}$, long $81^{\circ} 11^{\prime} 48^{\prime \prime}$, in El/2 sec.36, T.13 N., R.6 W.,

Carroll County, Hydrologic Unit 050400001 , on left bank at outlet of Leesville Dam, $1.3 \mathrm{mi}$ upstream from mouth, and $1.4 \mathrm{mi}$ nortineast of Leesville.

DRAINAGE AREA: $\quad 48.3 \mathrm{mi}^{2}$.

TRIBUTARY TO: Conotton Creek.

DISCHARGE DATA USED: October 1938 to September 1978.

REMARKS: $\quad$ Flow regulated by Leesville Lake.

SELECTED DISCHARGE CHARACTERISTICS: Average discharge: $52.3 \mathrm{ft}^{3} / \mathrm{s}$ (40 years).

Minimum daily discharge: No flow several days during 1939-41.

Magnitude and frequency of low flow for indicated periods

\begin{tabular}{|c|c|c|c|c|c|c|c|c|c|c|c|c|c|}
\hline \multirow{2}{*}{ Period } & \multirow{2}{*}{$\begin{array}{l}\text { Number of } \\
\text { consecu- } \\
\text { tive days }\end{array}$} & \multicolumn{5}{|c|}{$\begin{array}{l}\text { Discharge }\left(\mathrm{ft}^{3} / \mathrm{s}\right) \text { for indicated } \\
\text { recurrence interval (years) }\end{array}$} & \multirow{2}{*}{ Period } & \multirow{2}{*}{$\begin{array}{l}\text { Number of } \\
\text { consecu- } \\
\text { tive days }\end{array}$} & \multicolumn{5}{|c|}{$\begin{array}{l}\text { Discharge }\left(\mathrm{ft}^{3} / \mathrm{s}\right) \text { for indicated } \\
\text { recurrence interval (years) }\end{array}$} \\
\hline & & 2 & 5 & 10 & 20 & 50 & & & 2 & 5 & 10 & 20 & 50 \\
\hline Apr.-Mar. & $\begin{array}{r}1 \\
7 \\
30\end{array}$ & $\begin{array}{l}1.3 \\
1.5 \\
2.1\end{array}$ & $\begin{array}{l}1.0 \\
0.9 \\
1.2\end{array}$ & $\begin{array}{l}0.8 \\
0.7 \\
0.9\end{array}$ & $\begin{array}{l}0 \\
0 \\
0.7\end{array}$ & $\begin{array}{l}0 \\
0 \\
0\end{array}$ & Sept.-Nov. & $\begin{array}{r}1 \\
7 \\
30\end{array}$ & $\begin{array}{l}1.6 \\
2.3 \\
3.2\end{array}$ & $\begin{array}{l}1.0 \\
0.9 \\
1.4\end{array}$ & $\begin{array}{l}0.8 \\
0.4 \\
1.0\end{array}$ & $\begin{array}{l}0 \\
0 \\
0.6\end{array}$ & $\begin{array}{l}0 \\
0 \\
0\end{array}$ \\
\hline June-Aug. & $\begin{array}{r}1 \\
7 \\
30\end{array}$ & $\begin{array}{l}2.3 \\
2.8 \\
5.3\end{array}$ & $\begin{array}{l}1.4 \\
1.6 \\
2.3\end{array}$ & $\begin{array}{l}1.1 \\
1.2 \\
1.5\end{array}$ & $\begin{array}{l}0.9 \\
1.0 \\
1.0\end{array}$ & $\begin{array}{l}0 \\
0 \\
0.7\end{array}$ & Mar. - May & $\begin{array}{r}1 \\
7 \\
30\end{array}$ & $\begin{array}{l}3.6 \\
7.1 \\
32\end{array}$ & $\begin{array}{l}1.2 \\
2.3 \\
13\end{array}$ & $\begin{array}{l}0.7 \\
1.2 \\
6.9\end{array}$ & $\begin{array}{l}0.4 \\
0.6 \\
4.0\end{array}$ & $\begin{array}{l}0 \\
0 \\
2.0\end{array}$ \\
\hline
\end{tabular}

Duration of daily flow for indicated periods

\begin{tabular}{|c|c|c|c|c|c|c|c|c|c|c|c|c|c|}
\hline \multirow{2}{*}{ Period } & \multicolumn{13}{|c|}{ Discharge $\left(\mathrm{ft}^{3} / \mathrm{s}\right)$ which was equaled or exceeded for indicated percent of time } \\
\hline & 98 & 95 & 90 & 85 & 80 & 75 & 70 & 60 & 50 & 40 & 30 & 20 & 10 \\
\hline $\begin{array}{l}\text { Apr. - Mar. } \\
\text { May - Nov. } \\
\text { June - Aug. } \\
\text { Sept. - Nov. } \\
\text { Dec. - Feb. } \\
\text { Mar. - May }\end{array}$ & $\begin{array}{l}1.0 \\
0.3 \\
1.3 \\
0.7 \\
1.5 \\
1.2\end{array}$ & $\begin{array}{l}1.4 \\
1.3 \\
1.6 \\
1.1 \\
1.9 \\
1.9\end{array}$ & $\begin{array}{l}1.8 \\
1.6 \\
2.0 \\
1.3 \\
2.2 \\
2.9\end{array}$ & $\begin{array}{l}2.2 \\
1.9 \\
2.4 \\
1.5 \\
2.8 \\
10\end{array}$ & $\begin{array}{l}2.7 \\
2.3 \\
3.1 \\
1.7 \\
4.4 \\
17\end{array}$ & $\begin{array}{l}4.3 \\
2.8 \\
4.6 \\
2.0 \\
6.0 \\
25\end{array}$ & $\begin{array}{l}6.0 \\
4.3 \\
5.7 \\
2.2 \\
8.6 \\
33\end{array}$ & $\begin{array}{l}9.8 \\
7.2 \\
7.7 \\
3.0 \\
19 \\
50\end{array}$ & $\begin{array}{l}17 \\
9.9 \\
9.9 \\
6.2 \\
30 \\
65\end{array}$ & $\begin{array}{c}33 \\
15 \\
13 \\
9.0 \\
49 \\
82\end{array}$ & $\begin{array}{r}60 \\
27 \\
20 \\
15 \\
78 \\
100\end{array}$ & $\begin{array}{r}93 \\
58 \\
36 \\
50 \\
120 \\
140\end{array}$ & $\begin{array}{r}150 \\
110 \\
75 \\
120 \\
190 \\
200\end{array}$ \\
\hline
\end{tabular}


MUSKINGUM RIVER BASIN

03122500 Tuscarawas River below Dover Dam, near Dover, Ohio

LOCATION:

Lat $40^{\circ} 31^{\prime} 47^{\prime \prime}$, long $81^{\circ} 25^{\prime} 48^{\prime \prime}$, in T.9 N., R.2 W., Tuscarawas county, Hydrologic Unit 05040001 , on left bank at downstream side of bridge on State Route $416,2.2 \mathrm{mi}$ downstream from Dover Dam, $1.5 \mathrm{mi}$ east of Dover, and $3.4 \mathrm{mi}$ upstream from Sugar creek.

DRAINAGE AREA: $\quad 1,405 \mathrm{mi}^{2}$.

TRIBUTARY TO: Head of Muskingum River.

DISCHARGE DATA USED: October 1937 to september 1978.

REMARKS:

Diversion from basin at Portage Lakes. Records include diversion from Sugar Creek well field. Mean pumpage for the 1978 water year, $16.0 \mathrm{ft} 3 / \mathrm{s}$. Flow regulated by four flood-control reservoirs since 1936 at points $2.2 \mathrm{mi}$ to $25 \mathrm{mi}$ upstream.

SELECTED DISCHARGE CHARACTERISTICS: Average discharge: $1,410 \mathrm{ft}^{3} / \mathrm{s}$ (41 years). Minimum daily discharge: $6.5 \mathrm{ft} 3 / \mathrm{s}$ October 1948 .

Magnitude and frequency of low flow for indicated periods

\begin{tabular}{|c|c|c|c|c|c|c|c|c|c|c|c|c|c|}
\hline \multirow{2}{*}{ Period } & \multirow{2}{*}{$\begin{array}{l}\text { Number of } \\
\text { consecu- } \\
\text { tive days }\end{array}$} & \multicolumn{5}{|c|}{$\begin{array}{l}\text { Discharge }\left(\mathrm{ft}^{3} / \mathrm{s}\right) \text { for indicated } \\
\text { recurrence interval (years) }\end{array}$} & \multirow{2}{*}{ Period } & \multirow{2}{*}{$\begin{array}{l}\text { Number of } \\
\text { consecu- } \\
\text { tive days }\end{array}$} & \multicolumn{5}{|c|}{$\begin{array}{l}\text { Discharge }\left(\mathrm{ft}^{3} / \mathrm{s}\right) \text { for indicated } \\
\text { recurrence interval (years) }\end{array}$} \\
\hline & & 2 & 5 & 10 & 20 & 50 & & & 2 & 5 & 10 & 20 & 50 \\
\hline Apr.-Mar. & $\begin{array}{r}1 \\
7 \\
30\end{array}$ & $\begin{array}{l}217 \\
266 \\
320\end{array}$ & $\begin{array}{r}97 \\
211 \\
250\end{array}$ & $\begin{array}{r}46 \\
190 \\
222\end{array}$ & $\begin{array}{r}19 \\
174 \\
202\end{array}$ & $\begin{array}{r}40 \\
159 \\
183\end{array}$ & Sept.-Nov. & $\begin{array}{r}1 \\
7 \\
30\end{array}$ & $\begin{array}{l}266 \\
282 \\
348\end{array}$ & $\begin{array}{l}180 \\
217 \\
257\end{array}$ & $\begin{array}{l}130 \\
192 \\
224\end{array}$ & $\begin{array}{r}92 \\
175 \\
203\end{array}$ & $\begin{array}{r}54 \\
159 \\
183\end{array}$ \\
\hline June-Aug. & $\begin{array}{r}1 \\
7 \\
30\end{array}$ & $\begin{array}{l}290 \\
328 \\
440\end{array}$ & $\begin{array}{l}224 \\
256 \\
324\end{array}$ & $\begin{array}{l}196 \\
226 \\
278\end{array}$ & $\begin{array}{l}176 \\
205 \\
245\end{array}$ & $\begin{array}{l}156 \\
184 \\
213\end{array}$ & Mar. - May & $\begin{array}{r}1 \\
7 \\
30\end{array}$ & $\begin{array}{r}588 \\
682 \\
1140\end{array}$ & $\begin{array}{l}281 \\
506 \\
791\end{array}$ & $\begin{array}{l}154 \\
441 \\
650\end{array}$ & $\begin{array}{r}82 \\
398 \\
553\end{array}$ & $\begin{array}{r}35 \\
357 \\
460\end{array}$ \\
\hline
\end{tabular}

Duration of daily flow for indicated periods

\begin{tabular}{l|r|r|r|r|r|r|r|r|r|r|r|r|r}
\hline \multirow{2}{*}{ Period } & \multicolumn{8}{|c|}{ Discharge $\left(\mathrm{ft}^{3} / \mathrm{s}\right)$ which was equaled or exceeded for indicated percent of time } \\
\cline { 2 - 11 } & 98 & 95 & 90 & 85 & 80 & 75 & 70 & 60 & 50 & 40 & 30 & 20 & 10 \\
\hline Apr. - Mar. & 220 & 250 & 300 & 340 & 380 & 420 & 480 & 600 & 780 & 1000 & 1400 & 2100 & 3700 \\
May - Nov. & 210 & 230 & 270 & 300 & 330 & 360 & 390 & 460 & 550 & 670 & 840 & 1200 & 1900 \\
June - Aug. & 220 & 250 & 290 & 320 & 350 & 390 & 420 & 480 & 560 & 660 & 810 & 1100 & 1800 \\
Sept.- Nov. & 190 & 220 & 240 & 260 & 280 & 300 & 330 & 380 & 430 & 520 & 640 & 840 & 1400 \\
Dec. - Feb. & 240 & 290 & 340 & 390 & 450 & 520 & 600 & 800 & 1100 & 1400 & 1900 & 2800 & 4500 \\
Mar. - May & 440 & 550 & 680 & 800 & 920 & 1100 & 1200 & 1400 & 1800 & 2200 & 2800 & 3900 & 5200 \\
\hline
\end{tabular}


MUSKINGUM RIVER BASIN

03122850 Sugar Creek near Orrville, Ohio

LOCATION: $\quad$ Lat $40^{\circ} 48^{\prime} 4^{\prime \prime}$, long $81^{\circ} 45^{\prime} 56^{\prime \prime}$, wayne County, Hydrologic Unit 05040001 , at bridge on state Route $57,2.0 \mathrm{mi}$ south of orrville.

DRAINAGE AREA: $\quad 47.2 \mathrm{mi}^{2}$.

TRIBUTARY TO: Tuscarawas River.

DISCHARGE DATA USED: Low-flow measurements 1959, 1976-1978, 1980 water years.

INDEX STATION: $\quad 03123000$ Sugar Creek above Beach City Dam at Beach City, ohio. REMARKS: None.

SELECTED DISCHARGE CHARACTERISTICS: Minimum discharge observed: $2.5 \mathrm{ft}^{3} / \mathrm{s}$ September 1978 .

Magnitude and frequency of low flow for indicated periods

\begin{tabular}{c|c|c|c}
\hline \multirow{2}{*}{ Period } & \multirow{2}{*}{$\begin{array}{c}\text { Number of } \\
\text { consecu- } \\
\text { tive days }\end{array}$} & \multicolumn{2}{|c}{$\begin{array}{c}\text { ischarge }\left(\mathrm{ft}^{3} / \mathrm{s} \text { ) for }\right. \\
\text { indicated recurrence } \\
\text { interval (years) }\end{array}$} \\
\cline { 3 - 4 } & & 2 & 10 \\
\hline Apr. - Mar. & 7 & 1.5 & 0.7 \\
& 30 & 2.2 & 1.0 \\
May - Nov. & 7 & 1.5 & 0.7 \\
& 30 & 2.2 & 1.0 \\
June - Aug. & 7 & 2.0 & 0.9 \\
& 30 & 3.6 & 1.7 \\
Sept. - Nov. & 7 & 1.8 & 0.8 \\
& 30 & 2.7 & 1.1 \\
\hline
\end{tabular}

Duration of daily flow for indicated periods

\begin{tabular}{l|c|c|c|c|c}
\hline \multirow{2}{*}{ Period } & \multicolumn{5}{|c}{$\begin{array}{c}\text { Discharge ( } \mathrm{ft}^{3 / \mathrm{s}} \text { ) which was equaled or } \\
\text { exceeded for indicated percent of time }\end{array}$} \\
\cline { 2 - 6 } & 98 & 95 & 90 & 85 & 80 \\
\hline Apr. - Mar. & 1.0 & 1.5 & 2.0 & 2.5 & 2.9 \\
May - Nov. & 0.9 & 1.2 & 1.6 & 2.0 & 2.4 \\
June - Aug. & 1.0 & 1.3 & 1.6 & 2.0 & 2.4 \\
Sept. - Nov. & 0.8 & 1.0 & 1.4 & 1.7 & 1.9 \\
\hline
\end{tabular}


Appendix 3.--Low-flow data--Continued

MUSKINGUM RIVER BASIN

03122900 sugar Creek near West Lebanon, Ohio

LOCATION: $\quad$ Lat $40^{\circ} 44^{\prime} 1^{\prime \prime}$, long $81^{\circ} 39^{\prime} 12^{\prime \prime}$, Wayne County, Hydrologic Unit

05040001 , at bridge on county road, $1.1 \mathrm{mi}$ northeast of West Lebanon.

DRAINAGE AREA: $\quad 69.8 \mathrm{mi}^{2}$.

TRIBUTARY TO: Tuscarawas River.

DISCHARGE DATA USED: LOW-flow measurements 1973, 1976-1978, 1980 water years.

INDEX STATION: $\quad 03123000$ Sugar Creek above Beach City Dam at Beach City, ohio.

REMARKS: None.

SELECTED DISCHARGE CHARACTERISTICS: Minimum discharge observed: $4.7 \mathrm{ft}^{3} / \mathrm{s}$ September 1978 .

Magnitude and frequency of low flow for indicated periods

\begin{tabular}{c|c|c|c}
\hline \multirow{2}{*}{ Period } & \multirow{2}{*}{$\begin{array}{c}\text { Number of } \\
\text { consecu- } \\
\text { tive days }\end{array}$} & \multicolumn{2}{|c}{$\begin{array}{c}\text { Discharge }\left(\mathrm{ft}^{3} / \mathrm{s} \text { ) for }\right. \\
\text { indicated recurrence } \\
\text { interval (years) }\end{array}$} \\
\cline { 3 - 4 } (ypr. - Mar. & 7 & 2 & 10 \\
\hline May - Nov. & 30 & 2.3 & 0.9 \\
& 7 & 3.4 & 1.4 \\
June - Aug. & 30 & 2.3 & 0.9 \\
& 7 & 3.4 & 1.4 \\
Sept. - Nov. & 30 & 3.0 & 1.3 \\
& 7 & 6.0 & 2.5 \\
& 30 & 2.8 & 1.0 \\
& & 4.4 & 1.6 \\
\hline
\end{tabular}

Duration of daily flow for indicated periods

\begin{tabular}{c|c|c|c|c|c}
\hline \multirow{2}{*}{ Period } & \multicolumn{5}{|c}{$\begin{array}{c}\text { oischarge }\left(\mathrm{ft}^{3} / \mathrm{s} \text { ) which was equaled or }\right. \\
\text { exceeded for indicated percent of time }\end{array}$} \\
\cline { 2 - 6 } & 98 & 95 & 90 & 85 & 80 \\
\hline Apr. - Mar. & 1.4 & 2.2 & 3.1 & 4.1 & 4.7 \\
May - Nov. & 1.2 & 1.7 & 2.3 & 3.0 & 3.7 \\
June - Aug. & 1.4 & 1.8 & 2.4 & 3.1 & 3.7 \\
Sept. - Nov. & 1.0 & 1.3 & 2.0 & 2.5 & 2.9 \\
\hline
\end{tabular}


MUSKINGUM RIVER BASIN

03123000 Sugar Creek above Beach City Dam, at Beach City, Ohio

LOCATION:

Lat $40^{\circ} 39^{\prime} 24^{\prime \prime}$, long $81^{\circ} 34^{\prime} 37^{\prime \prime}$, in NEl/4 sec.35, T.11 N., R.10 W., Stark County, Hydrologic Unit 05040001 , on right bank at downstream side of Third Avenue Bridge at Beach City, $2.3 \mathrm{mi}$ upstream from Beach City Dam.

DRAINAGE AREA: $\quad 160 \mathrm{mi}^{2}$.

TRIBUTARY TO:

Tuscarawas River.

DISCHARGE DATA USED: April 1945 to September 1975.

REMARKS: None.

SELECTED DISCHARGE CHARACTERISTICS: Average discharge: $141 \mathrm{ft} 3 / \mathrm{s}$ (30 years).

Minimum daily discharge: $1.5 \mathrm{ft}^{3} / \mathrm{s}$ August 1954.

Magnitude and frequency of low flow for indicated periods

\begin{tabular}{|c|c|c|c|c|c|c|c|c|c|c|c|c|c|}
\hline \multirow{2}{*}{ Period } & \multirow{2}{*}{$\begin{array}{l}\text { Number of } \\
\text { consecu- } \\
\text { tive days }\end{array}$} & \multicolumn{5}{|c|}{$\begin{array}{l}\text { Discharge }\left(\mathrm{ft}^{3} / \mathrm{s}\right) \text { for indicated } \\
\text { recurrence interval (years) }\end{array}$} & \multirow{2}{*}{ Period } & \multirow{2}{*}{$\begin{array}{l}\text { Number of } \\
\text { consecu- } \\
\text { tive days }\end{array}$} & \multicolumn{5}{|c|}{$\begin{array}{l}\text { Discharge }\left(\mathrm{ft}^{3} / \mathrm{s}\right) \text { for indicated } \\
\text { recurrence interval (years) }\end{array}$} \\
\hline & & 2 & 5 & 10 & 20 & 50 & & & 2 & 5 & 10 & 20 & 50 \\
\hline Apr.-Mar. & $\begin{array}{r}1 \\
7 \\
30\end{array}$ & $\begin{array}{l}6.0 \\
7.4\end{array}$ & $\begin{array}{l}3.3 \\
4.1 \\
6.3\end{array}$ & $\begin{array}{l}2.5 \\
3.0 \\
4.8\end{array}$ & $\begin{array}{l}1.9 \\
2.3 \\
3.9\end{array}$ & $\begin{array}{l}1.5 \\
1.7 \\
3.0\end{array}$ & Sept.-Nov. & $\begin{array}{r}1 \\
7 \\
30\end{array}$ & $\begin{array}{l}7.2 \\
9.1 \\
14\end{array}$ & $\begin{array}{l}3.9 \\
4.9 \\
7.2\end{array}$ & $\begin{array}{l}2.9 \\
3.5 \\
5.2\end{array}$ & $\begin{array}{l}2.2 \\
2.6 \\
4.0\end{array}$ & $\begin{array}{l}1.6 \\
1.8 \\
3.0\end{array}$ \\
\hline June-Aug. & $\begin{array}{r}1 \\
7 \\
30\end{array}$ & $\begin{array}{r}7.7 \\
9.9 \\
19\end{array}$ & $\begin{array}{r}4.3 \\
5.7 \\
11\end{array}$ & $\begin{array}{l}3.2 \\
4.3 \\
8.3\end{array}$ & $\begin{array}{l}2.5 \\
3.4 \\
6.8\end{array}$ & $\begin{array}{l}1.9 \\
2.6 \\
5.4\end{array}$ & Mar.- May & $\begin{array}{r}1 \\
7 \\
30\end{array}$ & $\begin{array}{l}36 \\
46 \\
91\end{array}$ & $\begin{array}{l}25 \\
31 \\
57\end{array}$ & $\begin{array}{l}20 \\
25 \\
44\end{array}$ & $\begin{array}{l}16 \\
21 \\
34\end{array}$ & $\begin{array}{l}13 \\
17 \\
26\end{array}$ \\
\hline
\end{tabular}

Duration of daily flow for indicated periods

\begin{tabular}{|c|c|c|c|c|c|c|c|c|c|c|c|c|c|}
\hline \multirow{2}{*}{ Period } & \multicolumn{13}{|c|}{ Discharge $\left(\mathrm{ft}^{3} / \mathrm{s}\right)$ which was equaled or exceeded for indicated percent of time } \\
\hline & 98 & 95 & 90 & 85 & 80 & 75 & 70 & 60 & 50 & 40 & 30 & 20 & 10 \\
\hline $\begin{array}{l}\text { Apr. - Mar. } \\
\text { May - Nov. } \\
\text { June - Aug. } \\
\text { Sept. - Nov. } \\
\text { Dec. - Feb. } \\
\text { Mar. - May }\end{array}$ & $\begin{array}{r}4.7 \\
4.0 \\
4.6 \\
3.5 \\
9.9 \\
26\end{array}$ & $\begin{array}{l}7.1 \\
5.6 \\
5.9 \\
4.5 \\
13 \\
36\end{array}$ & $\begin{array}{l}10 \\
7.7 \\
7.9 \\
6.7 \\
18 \\
48\end{array}$ & $\begin{array}{c}13 \\
9.8 \\
10 \\
8.2 \\
23 \\
58\end{array}$ & $\begin{array}{l}15 \\
12 \\
12 \\
9.6 \\
28 \\
68\end{array}$ & $\begin{array}{l}19 \\
13 \\
14 \\
11 \\
33 \\
79\end{array}$ & $\begin{array}{l}23 \\
15 \\
16 \\
12 \\
39 \\
91\end{array}$ & $\begin{array}{r}35 \\
20 \\
22 \\
14 \\
56 \\
110\end{array}$ & $\begin{array}{r}51 \\
28 \\
28 \\
18 \\
84 \\
140\end{array}$ & $\begin{array}{r}77 \\
38 \\
37 \\
24 \\
120 \\
180\end{array}$ & $\begin{array}{r}120 \\
55 \\
50 \\
32 \\
180 \\
230\end{array}$ & $\begin{array}{r}190 \\
84 \\
76 \\
49 \\
270 \\
320\end{array}$ & $\begin{array}{r}350 \\
170 \\
150 \\
88 \\
520 \\
510\end{array}$ \\
\hline
\end{tabular}


Appendix 3.--Low-flow data--Continued

MUSKINGUM RIVER BASIN

03123300 South Fork Sugar Creek at Dundee, Ohio

LOCATION: $\quad$ Lat $40^{\circ} 35^{\prime} 3^{\prime \prime}$, long $81^{\circ} 36^{\prime} 55^{\prime \prime}$, Tuscarawas County, Hydrologic Unit $05040001,200 \mathrm{ft}$ upstream from county road bridge and $0.5 \mathrm{mi}$ northwest of Dundee.

DRAINAGE AREA: $\quad 124 \mathrm{mi}^{2}$.

TRIBUTARY TO: Sugar Creek.

DISCHARGE DATA USED: LOW-flow measurements 1945-1957, 1960, 1962 water years.

INDEX STATION: $\quad 03123000$ Sugar Creek above Beach City Dam at Beach City, Ohio. REMARKS: $\quad$ None.

SELECTED DISCHARGE CHARACTERISTICS: Minimum discharge observed: $1.0 \mathrm{ft}^{3} / \mathrm{s}$ September 1953.

\begin{tabular}{|c|c|c|c|}
\hline \multirow[t]{2}{*}{ Period } & \multirow{2}{*}{$\begin{array}{l}\text { Number of } \\
\text { consecu- } \\
\text { tive days }\end{array}$} & \multicolumn{2}{|c|}{$\begin{array}{l}\text { Discharge }\left(\mathrm{ft}^{3} / \mathrm{s}\right) \text { for } \\
\text { indicated recurrence } \\
\text { interval (years) }\end{array}$} \\
\hline & & 2 & 10 \\
\hline Apr. - Mar. & $\begin{array}{r}7 \\
30\end{array}$ & $\begin{array}{l}3.8 \\
6.4\end{array}$ & $\begin{array}{l}1.1 \\
2.1\end{array}$ \\
\hline May - Nov. & $\begin{array}{r}7 \\
30\end{array}$ & $\begin{array}{l}3.8 \\
6.4\end{array}$ & $\begin{array}{l}1.1 \\
2.1\end{array}$ \\
\hline June - Aug. & $\begin{array}{r}7 \\
30\end{array}$ & $14^{5.6}$ & $\begin{array}{l}1.8 \\
4.4\end{array}$ \\
\hline Sept. - Nov. & $\begin{array}{r}7 \\
30\end{array}$ & $\begin{array}{l}5.0 \\
8.9\end{array}$ & $\begin{array}{l}1.4 \\
2.4\end{array}$ \\
\hline
\end{tabular}

Duration of daily flow for indicated periods

\begin{tabular}{c|c|c|c|c|c}
\hline \multirow{2}{*}{ Period } & \multicolumn{5}{|c}{ Discharge $\left(\mathrm{ft}^{3} / \mathrm{s}\right)$ which was equaled or } \\
exceeded for indicated percent of time
\end{tabular}




\section{MUSKINGUM RIVER BASIN}

LOCATION:

03124000 Sugar Creek below Beach City Dam near Beach City, Ohio

Lat $40^{\circ} 38^{\prime} 08^{\prime \prime}$, long $81^{\circ} 33^{\prime} 11^{\prime \prime}$, in T.10 N., R.3 W., Tuscarawas

County, Hydrologic Unit 05040001 , on right bank $i, 000 \mathrm{ft}$ down-

stream from Beach City Dam, $0.4 \mathrm{mi}$ downstream from South Fork, and $1.8 \mathrm{mi}$ southeast of Beach City.

DRAINAGE AREA: $\quad 300 \mathrm{mi}^{2}$.

TRIBUTARY TO:

Tuscarawas River.

DISCHARGE DATA USED: October 1938 to September 1978.

REMARKS :

Flow regulated by Beach City Lake since 1937.

SELECTED DISCHARGE CHARACTERISTICS: Average discharge: $268 \mathrm{ft} 3 / \mathrm{s}$ (40 years)

Minimum daily discharge: No flow October 1963.

Magnitude and frequency of low flow for indicated periods

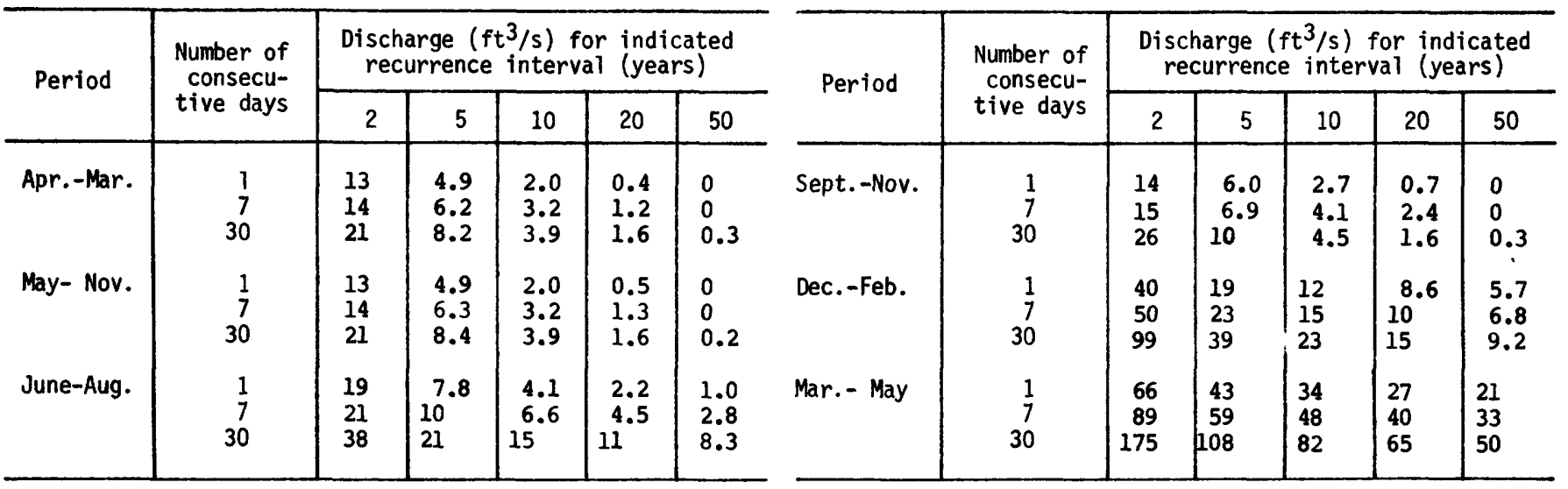

Duration of daily flow for indicated periods

\begin{tabular}{|c|c|c|c|c|c|c|c|c|c|c|c|c|c|}
\hline \multirow{2}{*}{ Period } & \multicolumn{13}{|c|}{ Discharge $\left(\mathrm{ft}^{3} / \mathrm{s}\right)$ which was equaled or exceeded for indicated percent of time } \\
\hline & 98 & 95 & 90 & 85 & 80 & 75 & 70 & 60 & 50 & 40 & 30 & 20 & 10 \\
\hline $\begin{array}{l}\text { Apr. - Mar. } \\
\text { May - Nov. } \\
\text { June - Aug. } \\
\text { Sept. - Nov. } \\
\text { Dec. - Feb. } \\
\text { Mar. - May }\end{array}$ & $\begin{array}{l}7.4 \\
5.9 \\
6.9 \\
3.8 \\
15 \\
50\end{array}$ & $\begin{array}{l}12 \\
9.4 \\
11 \\
7.2 \\
20 \\
67\end{array}$ & $\begin{array}{l}18 \\
14 \\
16 \\
11 \\
29 \\
92\end{array}$ & $\begin{array}{r}24 \\
18 \\
21 \\
13 \\
37 \\
110\end{array}$ & $\begin{array}{r}30 \\
21 \\
26 \\
16 \\
47 \\
140\end{array}$ & $\begin{array}{r}37 \\
26 \\
31 \\
18 \\
60 \\
160\end{array}$ & $\begin{array}{r}46 \\
30 \\
36 \\
21 \\
75 \\
180\end{array}$ & $\begin{array}{r}69 \\
42 \\
47 \\
27 \\
110 \\
230\end{array}$ & $\begin{array}{r}100 \\
57 \\
59 \\
34 \\
170 \\
300\end{array}$ & $\begin{array}{r}160 \\
78 \\
78 \\
46 \\
240 \\
380\end{array}$ & $\begin{array}{r}240 \\
110 \\
110 \\
67 \\
340 \\
490\end{array}$ & $\begin{array}{l}380 \\
170 \\
160 \\
100 \\
530 \\
700\end{array}$ & $\begin{array}{r}730 \\
330 \\
320 \\
190 \\
1300 \\
1300\end{array}$ \\
\hline
\end{tabular}


MUSKINGUM RIVER BASIN

03124500 Sugar Creek at Strasburg, Ohio

LOCATION:

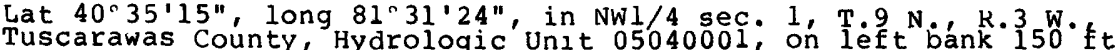
upstream from bridge on State Route $21,0.8 \mathrm{mi}$ upstream rrom

Broad Run, and $0.1 \mathrm{mi}$ southeast of strasburg.

DRAINAGE AREA: $\quad 311 \mathrm{mi}^{2}$.

TRIBUTARY TO: Tuscarawas River.

DISCHARGE DATA USED: October 1962 to September 1573.

REMARKS: $\quad$ Flow regulated by Beach $\mathrm{City}$ Lake $5.0 \mathrm{mi}$ upstream, since August 1937. Part of municipal water supply for city of canton, starting May 1962, is pumped from well field $4.3 \mathrm{mi}$ upstream; pumpage is returned to Nimishillen Creek. Mean pumpage for water year 1978 , $16.0 \mathrm{ft}^{3} / \mathrm{s}$.

SELECTED DISCHARGE CHARACTERISTICS: Average discharge; $293 \mathrm{ft}^{3} / \mathrm{s}$ (16 years).

Minimum daily discharge: No flow September, October, November 1963 .

Magnitude and frequency of low flow for indicated periods

\begin{tabular}{|c|c|c|c|c|c|c|c|c|c|c|c|c|c|}
\hline \multirow{2}{*}{ Period } & \multirow{2}{*}{$\begin{array}{l}\text { Number of } \\
\text { consecu- } \\
\text { tive days }\end{array}$} & \multicolumn{5}{|c|}{$\begin{array}{l}\text { Discharge }\left(\mathrm{ft}^{3} / \mathrm{s}\right) \text { for indicated } \\
\text { recurrence interval (years) }\end{array}$} & \multirow{2}{*}{ Period } & \multirow{2}{*}{$\begin{array}{l}\text { Number of } \\
\text { consecu- } \\
\text { tive days }\end{array}$} & \multicolumn{5}{|c|}{$\begin{array}{l}\text { Discharge }\left(\mathrm{ft}^{3} / \mathrm{s}\right) \text { for indicated } \\
\text { recurrence interval (years) }\end{array}$} \\
\hline & & 2 & 5 & 10 & 20 & 50 & & & 2 & 5 & 10 & 20 & 50 \\
\hline Apr.-Mar. & $\begin{array}{r}1 \\
7 \\
30\end{array}$ & $\begin{array}{l}16 \\
21 \\
30\end{array}$ & $\begin{array}{l}3.3 \\
4.4 \\
8.7\end{array}$ & $\begin{array}{l}0.6 \\
0.8 \\
2.7\end{array}$ & $\begin{array}{l}0 \\
0 \\
0\end{array}$ & $\begin{array}{l}0 \\
0 \\
0\end{array}$ & Sept.-Nov. & $\begin{array}{r}1 \\
7 \\
30\end{array}$ & $\begin{array}{l}23 \\
28 \\
41\end{array}$ & $\begin{array}{r}4.6 \\
5.4 \\
11\end{array}$ & $\begin{array}{l}0.8 \\
0.9 \\
2.2\end{array}$ & $\begin{array}{l}0 \\
0 \\
0\end{array}$ & $\begin{array}{l}0 \\
0 \\
0\end{array}$ \\
\hline June-Aug. & $\begin{array}{r}1 \\
7 \\
30\end{array}$ & $\begin{array}{l}21 \\
24 \\
46\end{array}$ & $\begin{array}{l}6.9 \\
11 \\
24\end{array}$ & $\begin{array}{l}2.6 \\
6.5 \\
16\end{array}$ & $\begin{array}{r}0.9 \\
4.1 \\
11\end{array}$ & $\begin{array}{l}0.2 \\
2.3 \\
7.4\end{array}$ & Mar. - May & $\begin{array}{r}1 \\
7 \\
30\end{array}$ & $\begin{array}{r}80 \\
98 \\
181\end{array}$ & $\begin{array}{r}49 \\
68 \\
116\end{array}$ & $\begin{array}{l}37 \\
57 \\
92\end{array}$ & $\begin{array}{l}29 \\
49 \\
77\end{array}$ & $\begin{array}{l}22 \\
42 \\
63\end{array}$ \\
\hline
\end{tabular}

Duration of daily flow for indicated periods

\begin{tabular}{|c|c|c|c|c|c|c|c|c|c|c|c|c|c|}
\hline \multirow{2}{*}{ Period } & \multicolumn{13}{|c|}{ Discharge $\left(\mathrm{ft}^{3} / \mathrm{s}\right)$ which was equaled or exceeded for indicated percent of time } \\
\hline & 98 & 95 & 90 & 85 & 80 & 75 & 70 & 60 & 50 & 40 & 30 & 20 & 10 \\
\hline $\begin{array}{l}\text { Apr. - Mar. } \\
\text { May - Nov. } \\
\text { June - Aug. } \\
\text { Sept. - Nov. } \\
\text { Dec. - Feb. } \\
\text { Mar. - May }\end{array}$ & $\begin{array}{l}3.9 \\
0.9 \\
5.6 \\
0.1 \\
9.0 \\
60\end{array}$ & $\begin{array}{l}9.4 \\
5.6 \\
11 \\
1.4 \\
31 \\
80\end{array}$ & $\begin{array}{c}21 \\
14 \\
19 \\
5.8 \\
40 \\
100\end{array}$ & $\begin{array}{r}32 \\
21 \\
25 \\
13 \\
51 \\
120\end{array}$ & $\begin{array}{r}41 \\
27 \\
31 \\
19 \\
65 \\
150\end{array}$ & $\begin{array}{r}52 \\
34 \\
37 \\
23 \\
80 \\
170\end{array}$ & $\begin{array}{r}63 \\
41 \\
44 \\
29 \\
97 \\
200\end{array}$ & $\begin{array}{r}90 \\
57 \\
57 \\
41 \\
140 \\
260\end{array}$ & $\begin{array}{r}130 \\
75 \\
72 \\
56 \\
190 \\
320\end{array}$ & $\begin{array}{r}180 \\
99 \\
89 \\
76 \\
260 \\
420\end{array}$ & $\begin{array}{l}270 \\
130 \\
120 \\
100 \\
360 \\
560\end{array}$ & $\begin{array}{l}420 \\
200 \\
170 \\
150 \\
570 \\
790\end{array}$ & $\begin{array}{r}790 \\
380 \\
320 \\
300 \\
1000 \\
1400\end{array}$ \\
\hline
\end{tabular}


Appendix 3.--Low-flow data--Continued

MUSKINGUM RIVER BASIN

03124520 Sugar Creek at Dover, Ohio

LOCATION:

Lat $40^{\circ} 31^{\prime} 40^{\prime \prime}$, long $81^{\circ} 29^{\prime} 43^{\prime \prime}$, Tuscarawas County, Hydrologic Unit 05040001 , at bridge on State Route $39,0.2 \mathrm{mi}$ west of Dover city limits, $1.8 \mathrm{mi}$ upstream from mouth.

DRAINAGE AREA: $\quad 348 \mathrm{mi}^{2}$.

TRIBUTARY TO: TUscarawas River.

DISCHARGE DATA USED: Low-flow measurements 1940, 1974-1978, 1980 water years.

INDEX STATION: $\quad 03123000$ Sugar Creek at Strasburg, ohio.

REMARKS: $\quad$ None.

SELECTED DISCHARGE CHARACTERISTICS: Minimum discharge observed: $52 \mathrm{ft}^{3} / \mathrm{s}$ September 1978 .

Magnitude and frequency of low flow for indicated periods

\begin{tabular}{c|c|c|c}
\hline \multirow{2}{*}{ Period } & $\begin{array}{c}\text { Number of } \\
\text { consecu- } \\
\text { tive days }\end{array}$ & \multicolumn{2}{|c}{$\begin{array}{c}\text { Discharge (ft3/s) for } \\
\text { indicated recurrence } \\
\text { interval (years) }\end{array}$} \\
\cline { 3 - 4 } (ypr. - Mar. & 7 & 2 & 10 \\
\hline May - Nov. & 30 & 20 & 8.1 \\
& 7 & 30 & 13 \\
June - Aug. & 30 & 20 & 8.1 \\
& 7 & 30 & 13 \\
Sept.- Nov. & 30 & 27 & 12 \\
& 7 & 50 & 22 \\
& 30 & 25 & 9.4 \\
\hline
\end{tabular}

Duration of daily flow for indicated periods

\begin{tabular}{l|c|c|c|c|c}
\hline \multirow{2}{*}{ Period } & \multicolumn{5}{|c}{$\begin{array}{c}\text { Discharge (ft3/s) which was equaled or } \\
\text { exceeded for indicated percent of time }\end{array}$} \\
\cline { 2 - 6 } & 98 & 95 & 90 & 85 & 80 \\
\hline Apr. - Mar. & 13 & 19 & 27 & 35 & 40 \\
May - Nov. & 11 & 15 & 21 & 26 & 32 \\
June - Aug. & 12 & 16 & 21 & 27 & 32 \\
Sept. - Nov. & 9.4 & 12 & 18 & 22 & 26 \\
\hline
\end{tabular}


Appendix 3.--Low-flow data--Continued

MUSKINGUM RIVER BASIN

03125000 Home Creek near New Philadelphia, Ohio

LOCATION:

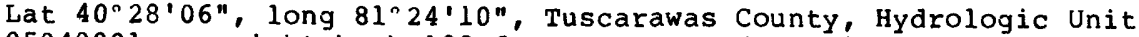
05040001 , on right bank $100 \mathrm{ft}$ downstream from highway bridge, 0.5 mi upstream from the mouth, and $1.5 \mathrm{mi}$ southeast of New Philadelphia.

DRAINAGE AREA: $\quad 1.64 \mathrm{mi}^{2}$.

TRIBUTARY TO: Beaverdam Creek.

DISCHARGE DATA USED: December 1936 to September 1978.

REMARKS: None.

SELECTED DISCHARGE CHARACTERISTICS: Average discharge: $1.27 \mathrm{ft}^{3} / \mathrm{s}$ (41 years).

Minimum daily discharge: No flow many times.

Magnitude and frequency of low flow for indicated periods

\begin{tabular}{c|c|c|c|c|c|c}
\hline \multirow{2}{*}{ Period } & \multirow{2}{*}{$\begin{array}{c}\text { Number of } \\
\text { consecu- } \\
\text { tive days }\end{array}$} & \multicolumn{4}{|c|}{$\begin{array}{c}\text { Discharge (ft } 3 / \mathrm{s} \text { ) for indicated } \\
\text { recurrence interval }\end{array}$} \\
\cline { 3 - 7 } (years)
\end{tabular}

\begin{tabular}{|c|c|c|c|c|c|c|}
\hline \multirow{2}{*}{ Period } & \multirow{2}{*}{$\begin{array}{l}\text { Number of } \\
\text { consecu- } \\
\text { tive days }\end{array}$} & \multicolumn{5}{|c|}{$\begin{array}{l}\text { Discharge }\left(\mathrm{ft}^{3} / \mathrm{s}\right) \text { for indicated } \\
\text { recurrence interval (years) }\end{array}$} \\
\hline & & 2 & 5 & 10 & 20 & 50 \\
\hline Sept.-Nov. & $\begin{array}{r}1 \\
7 \\
30\end{array}$ & $\begin{array}{l}0 \\
0 \\
0\end{array}$ & $\begin{array}{l}0 \\
0 \\
0\end{array}$ & $\begin{array}{l}0 \\
0 \\
0\end{array}$ & $\begin{array}{l}0 \\
0 \\
0\end{array}$ & $\begin{array}{l}0 \\
0 \\
0\end{array}$ \\
\hline Dec. -Feb. & $\begin{array}{r}1 \\
7 \\
30\end{array}$ & $\begin{array}{l}0.1 \\
0.1 \\
0.4\end{array}$ & $\begin{array}{l}0 \\
0 \\
0.1\end{array}$ & $\begin{array}{l}0 \\
0 \\
0.1\end{array}$ & $\begin{array}{l}0 \\
0 \\
0\end{array}$ & $\begin{array}{l}0 \\
0 \\
0\end{array}$ \\
\hline Mar. - May & $\begin{array}{r}1 \\
7 \\
30\end{array}$ & $\begin{array}{l}0.2 \\
0.3 \\
0.7\end{array}$ & $\begin{array}{l}0.1 \\
0.1 \\
0.4\end{array}$ & $\begin{array}{l}0.1 \\
0.1 \\
0.3\end{array}$ & $\begin{array}{l}0.1 \\
0.1 \\
0.2\end{array}$ & $\begin{array}{l}0 \\
0.1 \\
0.2\end{array}$ \\
\hline
\end{tabular}

Duration of daily flow for indicated periods

\begin{tabular}{l|c|c|c|c|c|c|c|c|c|c|c|c|c}
\hline \multirow{2}{*}{ Period } & \multicolumn{8}{|c|}{ Discharge $\left(\mathrm{ft}^{3} / \mathrm{s}\right)$ which was equaled or exceeded for indicated percent of time } \\
\cline { 2 - 11 } & 98 & 95 & 90 & 85 & 80 & 75 & 70 & 60 & 50 & 40 & 30 & 20 & 10 \\
\hline Apr. - Mar. & 0.0 & 0.0 & 0.0 & 0.0 & 0.1 & 0.1 & 0.1 & 0.2 & 0.4 & 0.6 & 1.0 & 1.6 & 2.9 \\
May - Nov. & 0.0 & 0.0 & 0.0 & 0.0 & 0.0 & 0.0 & 0.0 & 0.1 & 0.2 & 0.2 & 0.4 & 0.7 & 1.4 \\
June - Aug. & 0.0 & 0.0 & 0.0 & 0.0 & 0.0 & 0.0 & 0.0 & 0.1 & 0.1 & 0.2 & 0.3 & 0.5 & 1.1 \\
Sept.- Nov. & 0.0 & 0.0 & 0.0 & 0.0 & 0.0 & 0.0 & 0.0 & 0.1 & 0.1 & 0.2 & 0.3 & 0.5 & 0.9 \\
Dec. - Fei & 0.0 & 0.0 & 0.1 & 0.1 & 0.2 & 0.2 & 0.3 & 0.5 & 0.8 & 1.1 & 1.5 & 2.2 & 4.2 \\
Mar. - Mai & 0.1 & 0.2 & 0.3 & 0.4 & 0.6 & 0.7 & 0.8 & 1.0 & 1.2 & 1.6 & 2.0 & 2.8 & 4.6 \\
\hline
\end{tabular}


Appendix 3.--Low-flow data--Continued

MUSKINGUM RIVER BASIN

03125900 Boggs Fork at Piedmont, Ohio

LOCATION :

Lat $40^{\circ} 11^{\prime} 40^{\prime \prime}$, long $81^{\circ} 12^{\prime} 35^{\prime \prime}$, Belmont County, Hydrologic Unit 05040001 , in sec. 35, T.10 N., R. 6 W., at bridge on U.S. Highway $22,0.3 \mathrm{mi}$ upstream from mouth and outlet of piedmont Reservoir, $0.5 \mathrm{mi}$ upstream from gage on Stillwater Creek $0.5 \mathrm{mi}$ downstream from Plum Run and $0.5 \mathrm{mi}$ northwest of Piedmont.

DRAINAGE AREA:

$36.5 \mathrm{mi}^{2}$.

TRIBUTARY TO:

Stillwater Creek.

DISCHARGE DATA USED: Low-flow measurements 1935-1952, 1959, 1962 water years.

INDEX STATION: $\quad 03144000$ Wakatomika Creek near Frazeyburg, ohio.

REMARKS:

None.

SELECTED DISCHARGE CHARACTERISTICS: Minimum discharge observed: $0.2 \mathrm{ft}^{3} / \mathrm{s}$. August 1945 .

\begin{tabular}{|c|c|c|c|}
\hline \multirow[t]{2}{*}{ Period } & \multirow{2}{*}{$\begin{array}{l}\text { Number of } \\
\text { consecu- } \\
\text { tive days }\end{array}$} & \multicolumn{2}{|c|}{$\begin{array}{l}\text { Discharge }\left(\mathrm{ft}^{3 / \mathrm{s}}\right) \text { for } \\
\text { indicated recurrence } \\
\text { interval (years) }\end{array}$} \\
\hline & & 2 & 10 \\
\hline Apr. - Mar. & $\begin{array}{r}7 \\
30\end{array}$ & $\begin{array}{l}0.5 \\
1.1\end{array}$ & $\begin{array}{l}0.1 \\
0.2\end{array}$ \\
\hline May - Nov. & $\begin{array}{r}7 \\
30\end{array}$ & $\begin{array}{l}0.6 \\
1.1\end{array}$ & $\begin{array}{l}0.1 \\
0.2\end{array}$ \\
\hline June - Aug. & $\begin{array}{r}7 \\
30\end{array}$ & $\begin{array}{l}1.1 \\
3.1\end{array}$ & $\begin{array}{l}0.2 \\
0.6\end{array}$ \\
\hline Sept. - Nov. & $\begin{array}{r}7 \\
30\end{array}$ & $\begin{array}{l}0.7 \\
1.7\end{array}$ & $\begin{array}{l}0.1 \\
0.3\end{array}$ \\
\hline
\end{tabular}

Duration of daily flow for indicated periods

\begin{tabular}{l|c|c|c|c|c}
\hline \multirow{2}{*}{ Period } & \multicolumn{3}{|c}{$\begin{array}{l}\text { Discharge } \\
\text { exceeded for } \mathrm{ft} \text { (s) which was equaled or }\end{array}$} \\
\cline { 2 - 6 } & 98 & 95 & 90 & 85 & 80 \\
\hline Apr. - Mar. & 0.3 & 0.5 & 1.1 & 1.7 & 2.5 \\
May - Nov. & 0.2 & 0.3 & 0.6 & 0.9 & 1.3 \\
June - Aug. & 0.2 & 0.5 & 0.8 & 1.3 & 1.7 \\
Sept. - Nov. & 0.1 & 0.2 & 0.4 & 0.6 & 0.8 \\
\hline
\end{tabular}


DRAINAGE AREA: $\quad 122 \mathrm{mi}^{2}$.

TRIBUTARY TO: Tuscarawas River.

DISCHARGE DATA USED: February 1939 to September 1978.

REMARKS: $\quad$ Flow regulated by piedmont Lake.

SELECTED DISCHARGE CHARACTERISTICS: Average discharge: $133 \mathrm{ft}^{3} / \mathrm{s}$ (39 years).

Minimum daily discharge: $0.2 \mathrm{ft}^{3} / \mathrm{s}$ September 1953.

Magnitude and frequency of low flow for indicated periods

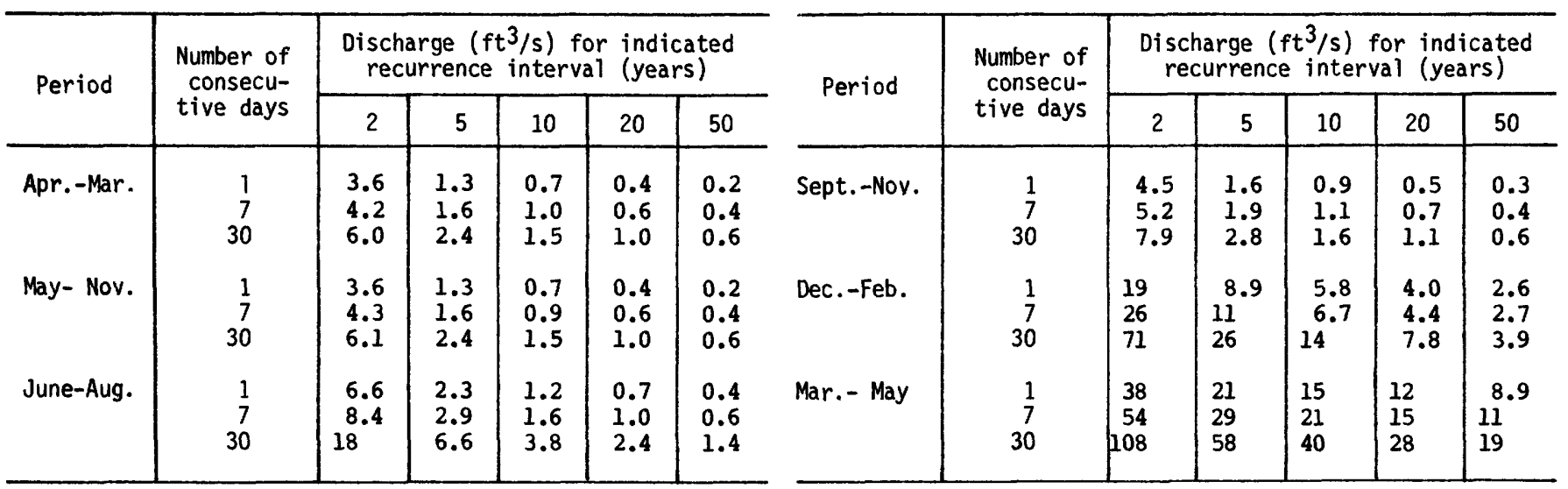

Duration of daily flow ior indicated periods

\begin{tabular}{|c|c|c|c|c|c|c|c|c|c|c|c|c|c|}
\hline \multirow{2}{*}{ Period } & \multicolumn{13}{|c|}{ Discharge $\left(\mathrm{ft}^{3} / \mathrm{s}\right)$ which was equaled or exceeded for indicated percent of time } \\
\hline & 98 & 95 & 90 & 85 & 80 & 75 & 70 & 60 & 50 & 40 & 30 & 20 & 10 \\
\hline $\begin{array}{l}\text { Apr. - Mar. } \\
\text { May - Nov. } \\
\text { June - Aug. } \\
\text { Sept. - Nov. } \\
\text { Dec. - Feb. } \\
\text { Mar. - May }\end{array}$ & $\begin{array}{l}1.9 \\
1.5 \\
1.9 \\
0.9 \\
4.8 \\
20\end{array}$ & $\begin{array}{l}3.3 \\
2.4 \\
2.9 \\
1.8 \\
8.4 \\
33\end{array}$ & $\begin{array}{l}6.1 \\
3.7 \\
5.1 \\
2.7 \\
14 \\
50\end{array}$ & $\begin{array}{l}9.6 \\
5.8 \\
7.8 \\
3.6 \\
20 \\
64\end{array}$ & $\begin{array}{l}14 \\
8.0 \\
11 \\
4.7 \\
28 \\
79\end{array}$ & $\begin{array}{c}19 \\
11 \\
14 \\
6.3 \\
38 \\
93\end{array}$ & $\begin{array}{c}25 \\
14 \\
18 \\
7.7 \\
49 \\
100\end{array}$ & $\begin{array}{r}43 \\
23 \\
28 \\
12 \\
79 \\
130\end{array}$ & $\begin{array}{r}67 \\
36 \\
41 \\
17 \\
110 \\
170\end{array}$ & $\begin{array}{r}100 \\
53 \\
53 \\
25 \\
170 \\
220\end{array}$ & $\begin{array}{r}150 \\
80 \\
71 \\
40 \\
250 \\
280\end{array}$ & $\begin{array}{r}240 \\
130 \\
110 \\
85 \\
320 \\
370\end{array}$ & $\begin{array}{l}360 \\
220 \\
190 \\
190 \\
440 \\
480\end{array}$ \\
\hline
\end{tabular}


LOCATION:

Lat $40^{\circ} 16^{\prime} 13^{\prime \prime}$, long $81^{\circ} 17^{\prime} 26^{\prime \prime}$, in NWl/4 sec. 22, T.12 N., R.7 W., Harrison County, Hydrologic Unit 05040001 , on left bank at downstream side of highway bridge at Tippecanoe, $0.4 \mathrm{mi}$ downstream from Brushy Fork, $3.6 \mathrm{mi}$ upstream from Weaver Run, $6 \mathrm{mi}$ upstream from Laurel Creek, and $9 \mathrm{mi}$ south of Dennison.

DRAINAGE AREA: $282 \mathrm{mi}^{2}$.

TRIBUTARY TO:

Tuscarawas River.

DISCHARGE DATA USED:

REMARKS:

January 1939 to September 1978.

Flow regulated by Clendening Lake on Brushy Fork, $1.9 \mathrm{mi}$ upstream and Piedmont Lake, $16 \mathrm{mi}$ upstream.

SELECTED DISCHARGE CHARACTERISTICS: Average discharge: $309 \mathrm{ft}^{3} / \mathrm{s}$ ( 39 years).

Minimum daily discharge: $1.1 \mathrm{ft}^{3} / \mathrm{s}$ October 1939.

Magnitude and frequency of low flow for indicated periods

\begin{tabular}{c|c|c|c|c|c|c}
\hline \multirow{2}{*}{ Period } & \multirow{2}{*}{$\begin{array}{c}\text { Number of } \\
\text { consecu- } \\
\text { tive days }\end{array}$} & \multicolumn{4}{|c|}{$\begin{array}{c}\text { Discharge }\left(\mathrm{ft}^{3} / \mathrm{s}\right) \text { for indicated } \\
\text { recurrence interval (years) }\end{array}$} \\
\cline { 3 - 7 } & 2 & 5 & 10 & 20 & 50 \\
\hline \multirow{3}{*}{ Apr.-Mar. } & 1 & 9.5 & 4.9 & 3.3 & 2.3 & 1.5 \\
& 7 & 11 & 5.4 & 3.7 & 2.6 & 1.8 \\
& 30 & 14 & 7.1 & 4.9 & 3.6 & 2.6 \\
& 1 & 9.5 & 4.9 & 3.3 & 2.3 & 1.5 \\
Juy- Nov. & 7 & 11 & 5.4 & 3.7 & 2.6 & 1.8 \\
& 30 & 14 & 7.1 & 4.9 & 3.6 & 2.6 \\
& 1 & 14 & 6.8 & 4.6 & 3.3 & 2.3 \\
& 7 & 17 & 8.0 & 5.4 & 4.0 & 2.8 \\
& 30 & 35 & 16 & 10 & 7.1 & 4.8 \\
\hline
\end{tabular}

\begin{tabular}{|c|c|c|c|c|c|c|}
\hline \multirow{2}{*}{ Period } & \multirow{2}{*}{$\begin{array}{l}\text { Number of } \\
\text { consecu- } \\
\text { tive days }\end{array}$} & \multicolumn{5}{|c|}{$\begin{array}{l}\text { Discharge }\left(\mathrm{ft}^{3} / \mathrm{s}\right) \text { for indicated } \\
\text { recurrence interval (years) }\end{array}$} \\
\hline & & 2 & 5 & 10 & 20 & 50 \\
\hline Sept.-Nov. & $\begin{array}{r}1 \\
7 \\
30\end{array}$ & $\begin{array}{l}10 \\
11 \\
17\end{array}$ & $\begin{array}{l}4.9 \\
5.5 \\
7.8\end{array}$ & $\begin{array}{l}3.3 \\
3.9 \\
5.5\end{array}$ & $\begin{array}{l}2.4 \\
3.0 \\
4.2\end{array}$ & $\begin{array}{l}1.6 \\
2.2 \\
3.2\end{array}$ \\
\hline Dec.-Feb. & $\begin{array}{r}1 \\
7 \\
30\end{array}$ & $\begin{array}{r}49 \\
69 \\
170\end{array}$ & $\begin{array}{l}22 \\
29 \\
61\end{array}$ & $\begin{array}{l}14 \\
18 \\
33\end{array}$ & $\begin{array}{l}9.2 \\
11 \\
19\end{array}$ & $\begin{array}{l}5.7 \\
6.7 \\
9.2\end{array}$ \\
\hline Mar. - May & $\begin{array}{r}1 \\
7 \\
30\end{array}$ & $\begin{array}{r}90 \\
122 \\
238\end{array}$ & $\begin{array}{r}54 \\
72 \\
135\end{array}$ & $\begin{array}{l}40 \\
53 \\
97\end{array}$ & $\begin{array}{l}32 \\
41 \\
73\end{array}$ & $\begin{array}{l}24 \\
30 \\
52\end{array}$ \\
\hline
\end{tabular}

Duration of daily flow for indicated periods

\begin{tabular}{|c|c|c|c|c|c|c|c|c|c|c|c|c|c|c|}
\hline \multirow{2}{*}{\multicolumn{2}{|c|}{ Period }} & \multicolumn{13}{|c|}{ Discharge $\left(\mathrm{ft}^{3} / \mathrm{s}\right)$ which was equaled or exceeded for indicated percent of time } \\
\hline & & 98 & 95 & 90 & 85 & 80 & 75 & 70 & 60 & 50 & 40 & 30 & 20 & 10 \\
\hline $\begin{array}{l}\text { Apr. - } \\
\text { May - } \\
\text { June - } \\
\text { Sept. - } \\
\text { Dec. - } \\
\text { Mar. - }\end{array}$ & $\begin{array}{l}\text { Mar. } \\
\text { Nov. } \\
\text { Aug. } \\
\text { Nov. } \\
\text { Feb. } \\
\text { May }\end{array}$ & $\begin{array}{r}6.1 \\
5.1 \\
6.4 \\
3.9 \\
12 \\
55\end{array}$ & $\begin{array}{r}8.9 \\
7.2 \\
8.6 \\
5.7 \\
36 \\
120\end{array}$ & $\begin{array}{c}15 \\
9.9 \\
13 \\
7.7 \\
36 \\
120\end{array}$ & $\begin{array}{c}21 \\
14 \\
18 \\
9.4 \\
55 \\
150\end{array}$ & $\begin{array}{r}29 \\
18 \\
22 \\
12 \\
74 \\
180\end{array}$ & $\begin{array}{r}40 \\
22 \\
27 \\
14 \\
94 \\
210\end{array}$ & $\begin{array}{r}54 \\
28 \\
34 \\
17 \\
120 \\
230\end{array}$ & $\begin{array}{r}91 \\
44 \\
50 \\
24 \\
180 \\
290\end{array}$ & $\begin{array}{r}150 \\
68 \\
69 \\
35 \\
270 \\
380\end{array}$ & $\begin{array}{r}220 \\
100 \\
93 \\
54 \\
400 \\
510\end{array}$ & $\begin{array}{r}340 \\
160 \\
130 \\
93 \\
540 \\
690\end{array}$ & $\begin{array}{l}530 \\
260 \\
200 \\
190 \\
740 \\
950\end{array}$ & $\begin{array}{r}890 \\
480 \\
360 \\
380 \\
1100 \\
1200\end{array}$ \\
\hline
\end{tabular}


Appendix 3.--Low-flow data--Continued

MUSKINGUM RIVER BASIN

03127100 Crooked Creek near stillwater, Ohio

LOCATION:

Lat $40^{\circ} 18^{\prime} 29^{\prime \prime}$, long $81^{\circ} 19^{\prime} 26^{\prime \prime}$, Tuscarawas County, Hydrologic Unit 05040001 , at bridge on State Route $258,0.7 \mathrm{mi}$ upstream from mouth, $1.2 \mathrm{mi}$ southwest of Stillwater.

DRAINAGE AREA: $\quad 47.5 \mathrm{mi}^{2}$.

TRIBUTARY TO: Stillwater Creek.

DISCHARGE DATA USED: LOW-flow measurements 1974-1978, 1980 water years.

INDEX STATION: $\quad 03140000$ Mill Creek near Coshocton, Ohio.

REMARKS: None.

SELECTED DISCHARGE CHARACTERISTICS: Minimum discharge observed: $2.3 \mathrm{ft}^{3} / \mathrm{s}$ September 1976.

Magnitude and frequency of low flow for indicated periods

\begin{tabular}{c|c|c|c}
\hline \multirow{2}{*}{ Period } & \multirow{2}{*}{$\begin{array}{c}\text { Number of } \\
\text { consecu- } \\
\text { tive days }\end{array}$} & \multicolumn{2}{|c}{$\begin{array}{c}\text { Discharge }\left(\mathrm{ft}^{3} / \mathrm{s} \text { ) for }\right. \\
\text { indicated recurrence } \\
\text { interval (years) }\end{array}$} \\
\cline { 3 - 4 } & & 2 & 10 \\
\hline Apr. - Mar. & 7 & 0.4 & 0.1 \\
& 30 & 1.0 & 0.2 \\
May - Nov. & 7 & 0.4 & 0.1 \\
& 30 & 1.0 & 0.2 \\
June - Aug. & 7 & 1.0 & 0.2 \\
& 30 & 3.0 & 0.7 \\
Sept. - Nov. & 7 & 0.6 & 0.1 \\
& 30 & 1.5 & 0.3 \\
\hline
\end{tabular}

Duration of daily flow for indicated periods

\begin{tabular}{l|c|c|c|c|c}
\hline \multirow{2}{*}{ Period } & \multicolumn{5}{|c}{$\begin{array}{c}\text { Discharge ( } \mathrm{ft}^{3} / \mathrm{s} \text { ) which was equaled or } \\
\text { exceeded for indicated percent of time }\end{array}$} \\
\cline { 2 - 6 } & 98 & 95 & 90 & 85 & 80 \\
\hline Apr. - Mar. & 0.2 & 0.4 & 1.1 & 1.7 & 2.4 \\
May - Nov. & 0.2 & 0.3 & 0.6 & 1.0 & 1.4 \\
June - Aug. & 0.2 & 0.4 & 0.8 & 1.2 & 1.7 \\
Sept. - Nov. & 0.1 & 0.2 & 0.3 & 0.6 & 0.8 \\
\hline
\end{tabular}


MUSKINGUM RIVER BASIN

03127500 Stillwater Creek at Uhrichsville, Ohio

LOCATION:

Lat $40^{\circ} 23^{\prime} 10^{\prime \prime}$, long $81^{\circ} 20^{\prime} 50^{\prime \prime}$, Tuscarawas County, Hydrologic Unit 05040001 , on left bank at concrete dam of Dennison Water Supply Co. at Uhrichsville, $2.2 \mathrm{mi}$ upstream from Little Stillwater Creek.

DRAINAGE AREA: $\quad 367 \mathrm{mi}^{2}$.

TRIBLTTARY TO: Tuscarawas River.

DISCHARGE DATA USED: October 1937 to September 1978.

REMARKS:

Flow regulated by Piedmont Lake, $35 \mathrm{mi}$ upstream, and clendening Lake

on Brushy Fork, $22 \mathrm{mi}$ upstream, beginning in 1938. Water is diverted

from Uhrichsville; diversion not included in figures on daily discharge.

SELECTED DISCHARGE CHARACTERISTICS: Average discharge: $414 \mathrm{ft}^{3} / \mathrm{s}$ (41 years).

Minimum ddily discharge: No flow September 1973.

Magnitude and frequency of low flow for indicated periods

\begin{tabular}{|c|c|c|c|c|c|c|c|c|c|c|c|c|c|}
\hline \multirow{2}{*}{ Period } & \multirow{2}{*}{$\begin{array}{l}\text { Number of } \\
\text { consecu- } \\
\text { tive days }\end{array}$} & \multicolumn{5}{|c|}{$\begin{array}{l}\text { Discharge }\left(\mathrm{ft}^{3} / \mathrm{s}\right) \text { for indicated } \\
\text { recurrence interval (years) }\end{array}$} & \multirow{2}{*}{ Period } & \multirow{2}{*}{$\begin{array}{l}\text { Number of } \\
\text { consecu- } \\
\text { tive days }\end{array}$} & \multicolumn{5}{|c|}{$\begin{array}{l}\text { Discharge }\left(\mathrm{ft}^{3} / \mathrm{s}\right) \text { for indicated } \\
\text { recurrence interval (years) }\end{array}$} \\
\hline & & 2 & 5 & 10 & 20 & 50 & & & 2 & 5 & 10 & 20 & 50 \\
\hline Apr.-Mar. & $\begin{array}{r}1 \\
7 \\
30\end{array}$ & $\begin{array}{l}9.1 \\
12 \\
19\end{array}$ & $\begin{array}{l}2.7 \\
4.3 \\
7.6\end{array}$ & $\begin{array}{l}1.1 \\
2.1 \\
4.4\end{array}$ & $\begin{array}{l}0.4 \\
1.1 \\
2.6\end{array}$ & $\begin{array}{l}0 \\
0.5 \\
1.4\end{array}$ & Sept.-Nov. & $\begin{array}{r}1 \\
7 \\
30\end{array}$ & $\begin{array}{l}11 \\
15 \\
25\end{array}$ & $\begin{array}{l}2.9 \\
4.6 \\
9.0\end{array}$ & $\begin{array}{l}1.1 \\
2.3 \\
5.0\end{array}$ & $\begin{array}{l}0.4 \\
1.2 \\
3.0\end{array}$ & $\begin{array}{l}0 \\
0.5 \\
1.7\end{array}$ \\
\hline May - Nov. & $\begin{array}{r}1 \\
7 \\
30\end{array}$ & $\begin{array}{l}9.1 \\
12 \\
19\end{array}$ & $\begin{array}{l}2.7 \\
4.3 \\
7.6\end{array}$ & $\begin{array}{l}1.1 \\
2.1 \\
4.4\end{array}$ & $\begin{array}{l}0.4 \\
1.1 \\
2.6\end{array}$ & $\begin{array}{l}0 \\
0.5 \\
1.4\end{array}$ & Dec.-Feb. & $\begin{array}{r}1 \\
7 \\
30\end{array}$ & $\begin{array}{r}61 \\
86 \\
221\end{array}$ & $\begin{array}{l}28 \\
36 \\
82\end{array}$ & $\begin{array}{l}18 \\
23 \\
45\end{array}$ & $\begin{array}{l}13 \\
15 \\
26\end{array}$ & $\begin{array}{r}8.3 \\
9.4 \\
13\end{array}$ \\
\hline June-Aug. & $\begin{array}{r}1 \\
7 \\
30\end{array}$ & $\begin{array}{l}16 \\
21 \\
50\end{array}$ & $\begin{array}{r}6.9 \\
9.3 \\
21\end{array}$ & $\begin{array}{l}4.3 \\
5.8 \\
13\end{array}$ & $\begin{array}{l}2.9 \\
3.8 \\
9.0\end{array}$ & $\begin{array}{l}1.8 \\
2.4 \\
5.7\end{array}$ & Mar. - May & $\begin{array}{r}1 \\
7 \\
30\end{array}$ & $\begin{array}{l}117 \\
159 \\
325\end{array}$ & $\begin{array}{r}72 \\
93 \\
186\end{array}$ & $\begin{array}{r}55 \\
69 \\
135\end{array}$ & $\begin{array}{r}43 \\
53 \\
102\end{array}$ & $\begin{array}{l}33 \\
39 \\
73\end{array}$ \\
\hline
\end{tabular}

Duration of daily flow for indicated periods

\begin{tabular}{l|r|r|r|r|r|r|r|r|r|r|r|r|r}
\hline \multirow{2}{*}{ Period } & \multicolumn{8}{|c|}{ Discharge $\left(\mathrm{ft}^{3} / \mathrm{s}\right)$ which was equaled or exceeded for indicated percent of time } \\
\cline { 2 - 13 } & 98 & 95 & 90 & 85 & 80 & 75 & 70 & 60 & 50 & 40 & 30 & 20 & 10 \\
\hline Apr. - Mar. & 6.5 & 12 & 20 & 30 & 41 & 53 & 72 & 120 & 190 & 290 & 460 & 720 & 1200 \\
May - Nov. & 4.5 & 8.5 & 14 & 19 & 25 & 32 & 40 & 60 & 90 & 140 & 210 & 330 & 630 \\
June - Aug. & 7.5 & 11 & 18 & 24 & 31 & 38 & 47 & 67 & 92 & 120 & 170 & 260 & 510 \\
Sept. - Nov. & 2.8 & 5.2 & 9.4 & 13 & 17 & 20 & 25 & 36 & 48 & 70 & 120 & 230 & 460 \\
Dec. - Feb. & 18 & 32 & 49 & 76 & 100 & 130 & 160 & 240 & 360 & 530 & 720 & 970 & 1400 \\
Mar. - May & 777 & 110 & 160 & 200 & 240 & 270 & 320 & 410 & 540 & 730 & 960 & 1200 & 1700 \\
\hline
\end{tabular}


MUSKINGUM RIVER BASIN

03128500 Little Stillwater Creek below Tappan Dam, at Tappan, Ohio

LOCATION:

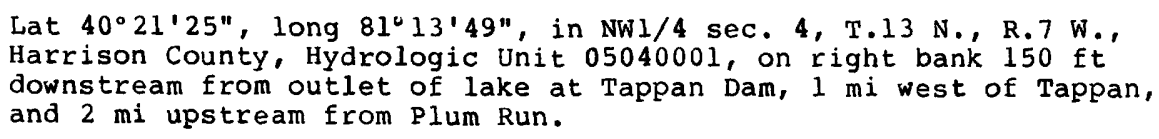

DRAINAGE AREA:

$71.1 \mathrm{mi}^{2}$

TRIBUTARY T0:

Stillwater Creek.

DISCHARGE DATA USED: Uctober 1938 to September 1978.

REMARKS:

Flow completely regulated by Tappan Lake.

SELECTED DISCHARGE CHARACTERISTICS: Average discharge: $75 \mathrm{ft}^{3} / \mathrm{s}$ (40 years).

Minimum daily discharge: No flow September, October 1939.

Magnitude and frequency of low flow for indicated periods

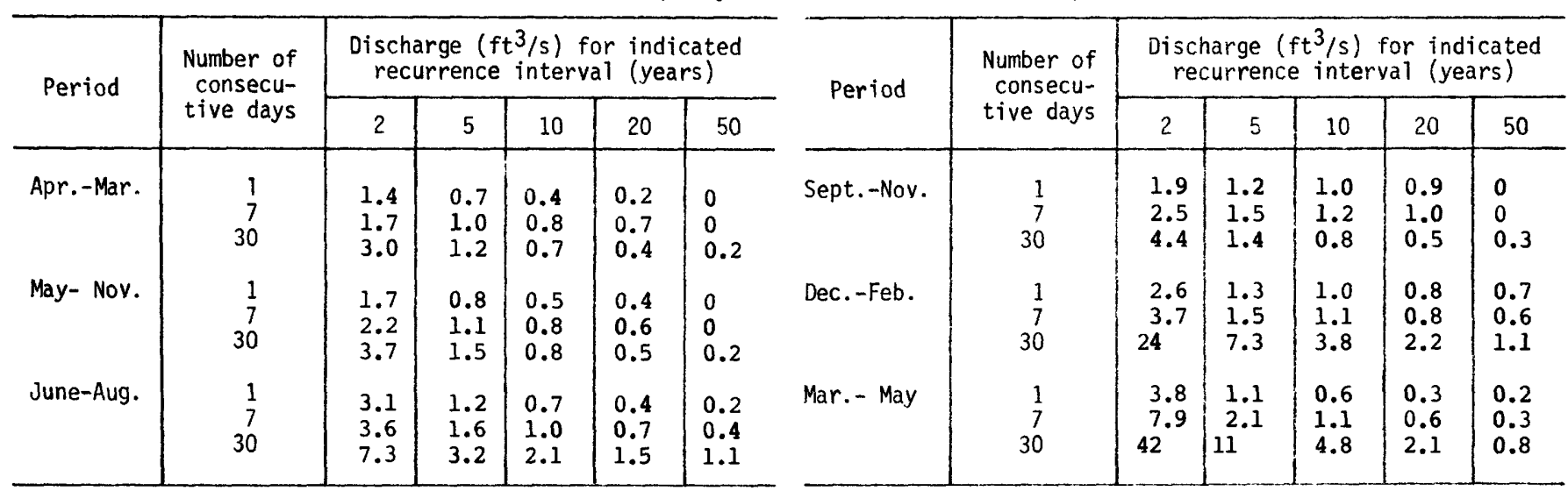

Duration of daily flow for indicated periods

\begin{tabular}{|c|c|c|c|c|c|c|c|c|c|c|c|c|c|c|}
\hline \multirow{2}{*}{\multicolumn{2}{|c|}{ Period }} & \multicolumn{13}{|c|}{ Discharge $\left(\mathrm{ft}^{3} / \mathrm{s}\right)$ which was equaled or exceeded for indicated percent of time } \\
\hline & & 98 & 95 & 90 & 85 & 80 & 75 & 70 & 60 & 50 & 40 & 30 & 20 & 10 \\
\hline $\begin{array}{l}\text { Apr. - } \\
\text { May - } \\
\text { June - } \\
\text { Sept. - } \\
\text { Dec. - } \\
\text { Mar. - }\end{array}$ & $\begin{array}{l}\text { Mar. } \\
\text { Nov. } \\
\text { Aug. } \\
\text { Nov. } \\
\text { Feb. } \\
\text { May }\end{array}$ & $\begin{array}{l}1.3 \\
1.2 \\
1.2 \\
1.2 \\
1.5 \\
1.3\end{array}$ & $\begin{array}{l}1.7 \\
1.7 \\
1.8 \\
1.5 \\
1.9 \\
1.8\end{array}$ & $\begin{array}{l}2.1 \\
2.1 \\
2.4 \\
1.8 \\
2.2 \\
2.4\end{array}$ & $\begin{array}{l}2.5 \\
2.4 \\
3.1 \\
2.1 \\
2.5 \\
6.2\end{array}$ & $\begin{array}{r}3.0 \\
2.9 \\
4.3 \\
2.3 \\
3.2 \\
19\end{array}$ & $\begin{array}{l}4.5 \\
3.9 \\
5.6 \\
2.5 \\
5.3 \\
30\end{array}$ & $\begin{array}{r}6.8 \\
5.3 \\
7.1 \\
2.8 \\
9.9 \\
40\end{array}$ & $\begin{array}{c}14 \\
9.7 \\
11 \\
4.5 \\
21 \\
54\end{array}$ & $\begin{array}{r}23 \\
15 \\
14 \\
8.2 \\
43 \\
69\end{array}$ & $\begin{array}{r}46 \\
22 \\
20 \\
15 \\
67 \\
110\end{array}$ & $\begin{array}{r}73 \\
41 \\
30 \\
24 \\
120 \\
150\end{array}$ & $\begin{array}{r}140 \\
70 \\
51 \\
75 \\
180 \\
210\end{array}$ & $\begin{array}{l}230 \\
150 \\
100 \\
170 \\
260 \\
320\end{array}$ \\
\hline
\end{tabular}


Appendix 3.--Low-flow data--Continued

MUSKINGUM RIVER BASIN

03128600 Little Stillwater Creek near Dennison, Ohio

LOCATION: Lat $40^{\circ} 24^{\prime \prime} 19^{\prime \prime}$, long $81^{\circ} 1^{\prime} 18^{\prime \prime}$, Tuscarawas County, Hydrologic Unit 05040001 , at county road bridge, $1.3 \mathrm{mi}$ upstream from

Irish Run, $2.5 \mathrm{mi}$ east of Dennison.

DRAINAGE AREA: $\quad 96.4 \mathrm{mi}^{2}$.

TRIBUTARY TO: Stillwater creek.

DISCHARGE DATA USED: Low-flow measurements 1974-1978, 1980 water years.

INDEX STATION: $\quad 03129000$ Tuscarawas River at Newcomerstown, Ohio.

REMARKS: $\quad$ None.

SELECTED DISCHARGE CHARACTERISTICS: Minimum discharge observed: $8.1 \mathrm{ft}^{3} / \mathrm{s}$ September 1976 .

\begin{tabular}{|c|c|c|c|}
\hline \multirow[t]{2}{*}{ Period } & \multirow{2}{*}{$\begin{array}{l}\text { Number of } \\
\text { consecu- } \\
\text { tive days }\end{array}$} & \multicolumn{2}{|c|}{$\begin{array}{l}\left.\text { Discharge ( } \mathrm{ft}^{3} / \mathrm{s}\right) \text { for } \\
\text { indicated recurrence } \\
\text { interval (years) }\end{array}$} \\
\hline & & 2 & 10 \\
\hline Apr. - Mar. & $\begin{array}{r}7 \\
30\end{array}$ & $\begin{array}{l}3.9 \\
5.2\end{array}$ & $\begin{array}{l}2.0 \\
2.6\end{array}$ \\
\hline May - Noy. & $\begin{array}{r}7 \\
30\end{array}$ & $\begin{array}{l}3.9 \\
5.3\end{array}$ & $\begin{array}{l}2.1 \\
2.6\end{array}$ \\
\hline June - Aug. & $\begin{array}{r}7 \\
30\end{array}$ & $12^{6.2}$ & $\begin{array}{l}2.8 \\
4.3\end{array}$ \\
\hline Sept.- Nov. & $\begin{array}{r}7 \\
30\end{array}$ & $\begin{array}{l}4.1 \\
6.1\end{array}$ & $\begin{array}{l}2.2 \\
2.6\end{array}$ \\
\hline
\end{tabular}

Duration of daily flow for indicated periods

\begin{tabular}{l|c|c|c|c|c}
\hline \multirow{2}{*}{ Period } & \multicolumn{4}{|c|}{$\begin{array}{c}\text { Discharge }\left(\mathrm{ft}^{3} / \mathrm{s}\right) \\
\text { exceeded } \text { which was equaled or } \\
\text { indicated percent of time }\end{array}$} \\
\cline { 2 - 6 } & 98 & 95 & 90 & 85 & 80 \\
\hline Apr. - Mar. & 2.8 & 3.6 & 5.0 & 6.5 & 8.1 \\
May - Nov. & 2.5 & 3.1 & 4.0 & 5.0 & 6.1 \\
June - Aug. & 2.9 & 3.8 & 5.2 & 6.3 & 7.7 \\
Sept. - Nov. & 2.2 & 2.6 & 3.3 & 3.8 & 4.4 \\
\hline
\end{tabular}


LOCATION:

Lat $40^{\circ} 15^{\prime} 41^{\prime \prime}$, long $81^{\circ} 36^{\prime} 33^{\prime \prime}$, in T.5 N., R.3 W. Tuscarawas County, Hydrologic Unit 05040001 , on right bank $150 \mathrm{ft}$ upstream from highway bridge, $0.2 \mathrm{mi}$ south of Newcomerstown, $2 \mathrm{mi}$ upstream from Buckhorn Creek, and $4 \mathrm{mi}$ downstream from Dunlap Creek.

DRAINAGE AREA: $\quad 2,443 \mathrm{mi}^{2}$.

TRIBUTARY TO: Head of Muskingum River.

DISCHARGE DATA USED: October 1937 to September 1978.

REMARKS: Diversion from basin at Portage. Flow regulated by eight flood-control reservoirs at points $40 \mathrm{mi}$ to $64 \mathrm{mi}$ upstream.

SELECTED DISCHARGE CHARACTERISTICS: Average discharge: $2,489 \mathrm{ft}^{3} / \mathrm{s}\{41$ years).

Minimum daily discharge: $216 \mathrm{ft} / \mathrm{s}$ August 1944.

Magnitude and frequency of low flow for indicated periods

\begin{tabular}{|c|c|c|c|c|c|c|c|c|c|c|c|c|c|}
\hline \multirow{2}{*}{ Period } & \multirow{2}{*}{$\begin{array}{l}\text { Number of } \\
\text { consecu- } \\
\text { tive days }\end{array}$} & \multicolumn{5}{|c|}{$\begin{array}{l}\text { Discharge ( } \mathrm{ft}^{3} / \mathrm{s} \text { ) for indicated } \\
\text { recurrence interval (years) }\end{array}$} & \multirow{2}{*}{ Period } & \multirow{2}{*}{$\begin{array}{l}\text { Number of } \\
\text { consecu- } \\
\text { tive days }\end{array}$} & \multicolumn{5}{|c|}{$\begin{array}{l}\text { Discharge }\left(\mathrm{ft}^{3} / \mathrm{s}\right) \text { for indicated } \\
\text { recurrence interval (years) }\end{array}$} \\
\hline & & 2 & 5 & 10 & 20 & 50 & & & 2 & 5 & 10 & 20 & 50 \\
\hline Apr.-Mar. & $\begin{array}{r}1 \\
7 \\
30\end{array}$ & $\begin{array}{l}343 \\
364 \\
433\end{array}$ & $\begin{array}{l}271 \\
285 \\
329\end{array}$ & $\begin{array}{l}242 \\
253 \\
291\end{array}$ & $\begin{array}{l}221 \\
232 \\
266\end{array}$ & $\begin{array}{l}201 \\
210 \\
242\end{array}$ & Sept.-Nov. & $\begin{array}{r}1 \\
7 \\
30\end{array}$ & $\begin{array}{l}344 \\
375 \\
471\end{array}$ & $\begin{array}{l}270 \\
287 \\
333\end{array}$ & $\begin{array}{l}247 \\
259 \\
289\end{array}$ & $\begin{array}{l}233 \\
242 \\
261\end{array}$ & $\begin{array}{l}222 \\
228 \\
237\end{array}$ \\
\hline May- Nov. & $\begin{array}{r}1 \\
7 \\
30\end{array}$ & $\begin{array}{l}344 \\
365 \\
435\end{array}$ & $\begin{array}{l}272 \\
285 \\
329\end{array}$ & $\begin{array}{l}242 \\
254 \\
290\end{array}$ & $\begin{array}{l}222 \\
232 \\
265\end{array}$ & $\begin{array}{l}201 \\
210 \\
241\end{array}$ & Dec.-Feb. & $\begin{array}{r}1 \\
7 \\
30\end{array}$ & $\begin{array}{r}679 \\
753 \\
1290\end{array}$ & $\begin{array}{l}430 \\
461 \\
666\end{array}$ & $\begin{array}{l}344 \\
364 \\
468\end{array}$ & $\begin{array}{l}289 \\
303 \\
349\end{array}$ & $\begin{array}{l}240 \\
249 \\
250\end{array}$ \\
\hline June-Aug. & $\begin{array}{r}1 \\
7 \\
30\end{array}$ & $\begin{array}{l}441 \\
483 \\
689\end{array}$ & $\begin{array}{l}332 \\
357 \\
465\end{array}$ & $\begin{array}{l}286 \\
306 \\
385\end{array}$ & $\begin{array}{l}252 \\
270 \\
332\end{array}$ & $\begin{array}{l}218 \\
235 \\
282\end{array}$ & Mar. - May & $\begin{array}{r}1 \\
7 \\
30\end{array}$ & $\begin{array}{l}1060 \\
1260 \\
2140\end{array}$ & $\begin{array}{r}779 \\
906 \\
1450\end{array}$ & $\begin{array}{r}668 \\
776 \\
1180\end{array}$ & $\begin{array}{l}591 \\
689 \\
990\end{array}$ & $\begin{array}{l}516 \\
608 \\
810\end{array}$ \\
\hline
\end{tabular}

Duration of daily flow for indicated periods

\begin{tabular}{|c|c|c|c|c|c|c|c|c|c|c|c|c|c|c|}
\hline \multirow{2}{*}{\multicolumn{2}{|c|}{ Period }} & \multicolumn{13}{|c|}{ Discharge $\left(\mathrm{ft}^{3} / \mathrm{s}\right)$ which was equaled or exceeded for indicated percent of time } \\
\hline & & 98 & 95 & 90 & 85 & 80 & 75 & 70 & 60 & 50 & 40 & 30 & 20 & 10 \\
\hline $\begin{array}{l}\text { Apr. - } \\
\text { May - } \\
\text { June - } \\
\text { Sept. - } \\
\text { Dec. - } \\
\text { Mar. - }\end{array}$ & $\begin{array}{l}\text { Mar. } \\
\text { Nov. } \\
\text { Aug. } \\
\text { Nov. } \\
\text { Feb. } \\
\text { May }\end{array}$ & $\begin{array}{l}300 \\
280 \\
310 \\
260 \\
350 \\
830\end{array}$ & $\begin{array}{r}350 \\
320 \\
360 \\
290 \\
420 \\
1000\end{array}$ & $\begin{array}{r}420 \\
370 \\
430 \\
330 \\
520 \\
1300\end{array}$ & $\begin{array}{r}490 \\
420 \\
480 \\
360 \\
620 \\
1500\end{array}$ & $\begin{array}{r}560 \\
470 \\
540 \\
390 \\
750 \\
1800\end{array}$ & $\begin{array}{r}660 \\
520 \\
600 \\
420 \\
880 \\
2000\end{array}$ & $\begin{array}{r}780 \\
580 \\
660 \\
450 \\
1000 \\
2200\end{array}$ & $\begin{array}{r}1000 \\
740 \\
800 \\
520 \\
1400 \\
2700\end{array}$ & $\begin{array}{r}1400 \\
930 \\
950 \\
630 \\
2000 \\
3400\end{array}$ & $\begin{array}{r}1900 \\
1200 \\
1100 \\
830 \\
2600 \\
4200\end{array}$ & $\begin{array}{l}2700 \\
1500 \\
1400 \\
1100 \\
3600 \\
5200\end{array}$ & $\begin{array}{l}3900 \\
2100 \\
1900 \\
1400 \\
5200 \\
6900\end{array}$ & $\begin{array}{l}6600 \\
3500 \\
3200 \\
2200 \\
8000 \\
9000\end{array}$ \\
\hline
\end{tabular}


Appendix 3.--Low-flow data--Continued

MUSKINGUM RIVER BASIN

03129100 White Eyes Creek near Fresno, Ohio

LOCATION:

Lat $40^{\circ} 18^{\prime} 17^{\prime \prime}$, long $81^{\circ} 45^{\prime} 01^{\prime \prime}$, Coshocton County, Hydrologic Unit 05040001 , at bridge on private road adjacent to state Route 93, $2 \mathrm{mi}$ south of Fresno.

DRAINAGE AREA: $\quad 52.1 \mathrm{mi}^{2}$

TRIBUTARY TO: Tuscarawas River.

DISCHARGE DATA USED: Low-flow measurements 1972-1977 water years.

INDEX STATION: $\quad 03140000$ Mill Creek near Coshocton, Ohio.

REMARKS: None.

SELECTED DISCHARGE CHARACTERISTICS: Minimum discharge observed: $2.9 \mathrm{ft}^{3} / \mathrm{s}$ June 1977.

Magnitude and frequency of low flow for indicated periods

\begin{tabular}{|c|c|c|c|}
\hline \multirow[t]{2}{*}{ Period } & \multirow{2}{*}{$\begin{array}{l}\text { Number of } \\
\text { consecu- } \\
\text { tive days }\end{array}$} & \multicolumn{2}{|c|}{$\begin{array}{l}\text { Discharge }\left(\mathrm{ft}^{3} / \mathrm{s}\right) \text { for } \\
\text { indicated recurrence } \\
\text { interval (years) }\end{array}$} \\
\hline & & 2 & 10 \\
\hline Apr. - Mar. & $\begin{array}{r}7 \\
30\end{array}$ & $\begin{array}{l}0.5 \\
1.2\end{array}$ & $\begin{array}{l}0.1 \\
0.2\end{array}$ \\
\hline May - Nov. & $\begin{array}{r}7 \\
30\end{array}$ & $\begin{array}{l}0.5 \\
1.2\end{array}$ & $\begin{array}{l}0.1 \\
0.2\end{array}$ \\
\hline June - Aug. & $\begin{array}{r}7 \\
30\end{array}$ & $\begin{array}{l}1.2 \\
4.7\end{array}$ & $\begin{array}{l}0.2 \\
0.8\end{array}$ \\
\hline Sept.- Nov. & $\begin{array}{r}7 \\
30\end{array}$ & $\begin{array}{l}0.7 \\
2.1\end{array}$ & $\begin{array}{l}0.1 \\
0.3\end{array}$ \\
\hline
\end{tabular}

Duration of daily flow for indicated periods

\begin{tabular}{l|c|c|c|c|c}
\hline \multirow{2}{*}{ Period } & \multicolumn{5}{|c}{$\begin{array}{c}\text { Discharge }\left(\mathrm{ft}^{3} / \mathrm{s} \text { ) which was equaled or }\right. \\
\text { exceeded for indicated percent of time }\end{array}$} \\
\cline { 2 - 6 } & 98 & 95 & 90 & 85 & 80 \\
\hline Apr. - Mar. & 0.2 & 0.5 & 1.4 & 2.3 & 3.6 \\
May - Nov. & 0.2 & 0.3 & 0.7 & 1.2 & 1.9 \\
June - Aug. & 0.2 & 0.5 & 1.0 & 1.6 & 2.3 \\
Sept. - Nov. & 0.1 & 0.2 & 0.3 & 0.7 & 1.0 \\
\hline
\end{tabular}


MUSKINGUM RIVER BASIN

03129150 Tuscarawas River at Coshocton, Ohio

LOCATION:

Lat $40^{\circ} 16^{\prime} 44^{\prime \prime}$, long $81^{\circ} 52^{\prime} 15^{\prime \prime}$, Coshocton County, Hydrologic Unit

05040001 , at bridge on Bridge Street at Coshocton city limits,

$0.3 \mathrm{mi}$ upstream from confluence with Walhonding River.

DRAINAGE AREA: $\quad 2,596 \mathrm{mi}^{2}$.

TRIBUTARY TO: Head of Muskingum River.

DISCHARGE DATA USED: Low-flow measurements 1974-1980 water years.

INDEX STATION: $\quad 03129000$ Tuscarawas River at Newcomerstown, Ohio.

REMARKS: $\quad$ Regulation and diversions may occur at various and sundry points upstream from site.

SELECTED DISCHARGE CHARACTERISTICS: Minimum discharge observed: $727 \mathrm{ft}^{3} / \mathrm{s}$ September 1976.

\begin{tabular}{l|c|c|c}
\multicolumn{4}{c}{$\begin{array}{l}\text { Magnitude and frequency of low flow for } \\
\text { indicated periods }\end{array}$} \\
\hline & $\begin{array}{c}\text { Number of } \\
\text { consecu- } \\
\text { tive days }\end{array}$ & $\begin{array}{c}\text { Discharge }\left(\mathrm{ft}^{3} / \mathrm{s}\right) \text { for } \\
\text { indicated recurrence } \\
\text { interval (years) }\end{array}$ \\
\cline { 3 - 4 } & 7 & 2 & 10 \\
\hline Apr. - Mar. & 30 & 420 & 300 \\
& 7 & 500 & 340 \\
May - Nov. & 30 & 520 & 300 \\
& 7 & 550 & 340 \\
June - Aug. & 30 & 780 & 350 \\
& 7 & 430 & 440 \\
Sept. - Nov. & 30 & 540 & 300 \\
& &
\end{tabular}

Duration of daily flow for indicated periods

\begin{tabular}{l|c|c|c|c|c}
\hline \multirow{2}{*}{ Period } & \multicolumn{5}{|c}{$\begin{array}{c}\text { Discharge ( } \mathrm{ft}^{3} / \mathrm{s} \text { ) which was equaled or } \\
\text { exceeded for indicated percent of time }\end{array}$} \\
\cline { 2 - 6 } & 98 & 95 & 90 & 85 & 80 \\
\hline Apr. - Mar. & 350 & 410 & 490 & 560 & 640 \\
May - Nov. & 330 & 370 & 430 & 490 & 540 \\
June - Aug. & 360 & 420 & 500 & 550 & 610 \\
Sept. - Nov. & 300 & 340 & 380 & 420 & 450 \\
\hline
\end{tabular}


MUSKINGUM RIVER BASIN

03129400 Black Fork above Charles Mill Dam, near Mifflin, Ohio

LOCATION: $\quad$ Lat $40^{\circ} 47^{\prime} 50^{\prime \prime}$, long $82^{\circ} 23^{\prime} 25^{\prime \prime}$, Ashland County, Hydrologic Unit $05040002,0.3 \mathrm{mi}$ downstream from Steigerwal Bridge, $2.1 \mathrm{mi}$ northwest of Miffiin.

DRAINAGE AREA: $\quad 193 \mathrm{mi}^{2}$.

TRIBUTARY TO: Tuscarawas River.

DISCHARGE DATA USED: Low-flow measurements 1944-1967, 1971 water years.

INDEX STATION: $\quad 03137000$ Kokosing River at Millwood, Ohio.

REMARKS: None.

SELECTED DISCHARGE CHARACTERISTICS: Minimum discharge observed: $2.9 \mathrm{ft}^{3} / \mathrm{s}$ September 1944.

Magnitude and frequency of low flow for indicated periods

\begin{tabular}{c|c|c|c}
\hline \multirow{2}{*}{ Period } & \multirow{2}{*}{$\begin{array}{c}\text { Number of } \\
\text { consecu- } \\
\text { tive days }\end{array}$} & \multicolumn{2}{|c}{$\begin{array}{c}\text { Discharge (ft } 3 / \mathrm{s} \text { ) for } \\
\text { indicated recurrence } \\
\text { interval (years) }\end{array}$} \\
\cline { 3 - 4 } & & 2 & 10 \\
\hline Apr. - Mar. & 7 & 6.4 & 4.7 \\
& 30 & 7.1 & 5.2 \\
May - Nov. & 7 & 6.4 & 4.7 \\
& 30 & 7.1 & 5.2 \\
June - Aug. & 7 & 7.3 & 5.0 \\
& 30 & 9.4 & 6.1 \\
Sept. - Nov. & 7 & 6.6 & 4.8 \\
& 30 & 7.5 & 5.4 \\
\hline
\end{tabular}

Duration of daily flow for indicated periods

\begin{tabular}{l|c|c|c|c|c}
\hline \multirow{2}{*}{ Period } & \multicolumn{5}{|c}{$\begin{array}{c}\text { Discharge }\left(\mathrm{ft}^{3} / \mathrm{s} \text { ) which was equaled or }\right. \\
\text { exceeded for indicated percent of time }\end{array}$} \\
\cline { 2 - 6 } & 98 & 95 & 90 & 85 & 80 \\
\hline Apr. - Mar. & 5.1 & 5.8 & 6.7 & 7.5 & 8.3 \\
May - Nov. & 4.8 & 5.4 & 6.2 & 6.8 & 7.3 \\
June - Aug. & 5.0 & 5.7 & 6.6 & 7.3 & 8.0 \\
Sept. - Nov. & 4.6 & 5.0 & 5.6 & 6.1 & 6.6 \\
\hline
\end{tabular}


MUSKINGUM RIVER BASIN

03130000 Black Fork below Charles Mill Dam, near Mifflin, Ohio

LOCATION:

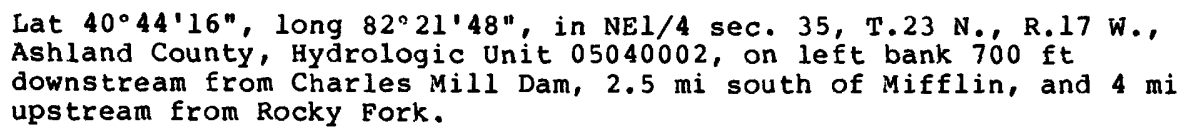

DRAINAGE AREA: $\quad 217 \mathrm{mi}^{2}$.

TRIBUTARY TO: TUscarawas River.

DISCHARGE DATA USED: October 1938 to september 1978.

REMARKS :

Flow regulated by Charles Mill Lake.

SELECTED DISCHARGE CHARACTERISTICS: Average discharge: $197 \mathrm{ft}^{3} / \mathrm{s}$ (49 years).

Minimum daily discharge: $0.9 \mathrm{ft} / \mathrm{s}$ April 1940.

Magnitude and frequency of low flow for indicated periods

\begin{tabular}{|c|c|c|c|c|c|c|c|c|c|c|c|c|c|}
\hline \multirow{2}{*}{ Period } & \multirow{2}{*}{$\begin{array}{l}\text { Number of } \\
\text { consecu- } \\
\text { tive days }\end{array}$} & \multicolumn{5}{|c|}{$\begin{array}{l}\text { Discharge }\left(\mathrm{ft}^{3} / \mathrm{s}\right) \text { for indicated } \\
\text { recurrence interval (years) }\end{array}$} & \multirow{2}{*}{ Period } & \multirow{2}{*}{$\begin{array}{l}\text { Number of } \\
\text { consecu- } \\
\text { tive days }\end{array}$} & \multicolumn{5}{|c|}{$\begin{array}{l}\text { Discharge }\left(\mathrm{ft}^{3} / \mathrm{s}\right) \text { for indicated } \\
\text { recurrence interval (years) }\end{array}$} \\
\hline & & 2 & 5 & 10 & 20 & 50 & & & 2 & 5 & 10 & 20 & 50 \\
\hline Apr.-Mar. & $\begin{array}{r}1 \\
7 \\
30\end{array}$ & $\begin{array}{l}9.8 \\
16 \\
17\end{array}$ & $\begin{array}{l}4.8 \\
8.3 \\
12\end{array}$ & $\begin{array}{l}2.8 \\
5.2 \\
9.9\end{array}$ & $\begin{array}{l}1.6 \\
3.3 \\
8.6\end{array}$ & $\begin{array}{l}0.6 \\
1.8 \\
7.4\end{array}$ & Sept.-Nov. & $\begin{array}{r}1 \\
7 \\
30\end{array}$ & $\begin{array}{l}15 \\
17 \\
20\end{array}$ & $\begin{array}{l}8.1 \\
9.6 \\
12\end{array}$ & $\begin{array}{l}5.4 \\
6.5 \\
10\end{array}$ & $\begin{array}{l}3.6 \\
4.5 \\
9.0\end{array}$ & $\begin{array}{l}2.2 \\
2.9 \\
8.0\end{array}$ \\
\hline June-Aug. & $\begin{array}{r}1 \\
7 \\
30\end{array}$ & $\begin{array}{l}15 \\
22 \\
28\end{array}$ & $\begin{array}{l}7.6 \\
12 \\
18\end{array}$ & $\begin{array}{r}4.9 \\
7.8 \\
15\end{array}$ & $\begin{array}{l}3.3 \\
5.2 \\
14\end{array}$ & $\begin{array}{r}2.0 \\
3.1 \\
12\end{array}$ & Mar. - May & $\begin{array}{r}1 \\
7 \\
30\end{array}$ & $\begin{array}{r}32 \\
62 \\
132\end{array}$ & $\begin{array}{l}13 \\
36 \\
75\end{array}$ & $\begin{array}{l}6.8 \\
27 \\
55\end{array}$ & $\begin{array}{l}3.8 \\
20 \\
42\end{array}$ & $\begin{array}{l}1.9 \\
15 \\
31\end{array}$ \\
\hline
\end{tabular}

Duration of daily flow for indicated periods

\begin{tabular}{|c|c|c|c|c|c|c|c|c|c|c|c|c|c|}
\hline \multirow{2}{*}{ Period } & \multicolumn{13}{|c|}{ Discharge $\left(\mathrm{ft}^{3} / \mathrm{s}\right)$ which was equaled or exceeded for indicated percent of time } \\
\hline & 98 & 95 & 90 & 85 & 80 & 75 & 70 & 60 & 50 & 40 & 30 & 20 & 10 \\
\hline $\begin{array}{l}\text { Apr. - Mar. } \\
\text { May - Nov. } \\
\text { June - Aug. } \\
\text { Sept. - Nov. } \\
\text { Dec. - Feb. } \\
\text { Mar. - May }\end{array}$ & $\begin{array}{l}9.4 \\
8.3 \\
11 \\
7.5 \\
13 \\
28\end{array}$ & $\begin{array}{l}14 \\
12 \\
15 \\
9.1 \\
18 \\
39\end{array}$ & $\begin{array}{l}18 \\
16 \\
19 \\
13 \\
22 \\
60\end{array}$ & $\begin{array}{l}22 \\
19 \\
21 \\
15 \\
28 \\
76\end{array}$ & $\begin{array}{l}25 \\
21 \\
24 \\
17 \\
36 \\
94\end{array}$ & $\begin{array}{r}29 \\
23 \\
26 \\
19 \\
47 \\
110\end{array}$ & $\begin{array}{r}34 \\
26 \\
28 \\
20 \\
66 \\
130\end{array}$ & $\begin{array}{r}50 \\
32 \\
34 \\
23 \\
110 \\
180\end{array}$ & $\begin{array}{r}78 \\
41 \\
43 \\
28 \\
150 \\
250\end{array}$ & $\begin{array}{r}120 \\
57 \\
56 \\
35 \\
190 \\
340\end{array}$ & $\begin{array}{r}190 \\
82 \\
75 \\
49 \\
250 \\
460\end{array}$ & $\begin{array}{r}310 \\
140 \\
120 \\
77 \\
410 \\
590\end{array}$ & $\begin{array}{l}570 \\
300 \\
300 \\
140 \\
710 \\
840\end{array}$ \\
\hline
\end{tabular}


Appendix 3.--Low-flow data--Continued

\author{
MUSKINGUM RIVER BASIN
}

03130500 Touby Run at Mansfield, Ohio

LOCATION:

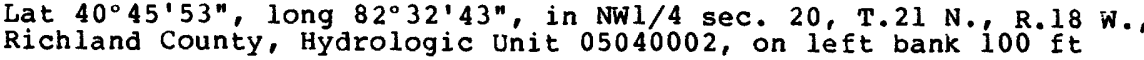
downstream from West 4 th Street Bridge at west edge of Mansfield, and $2 \mathrm{mi}$ upstream from mouth.
\end{abstract}

DRAINAGE AREA: $\quad 5.44 \mathrm{mi}^{2}$.

TRIBUTARY TO: ROCkY FOrK.

DISCHARGE DATA USED: October 1946 to September 1978.

REMARKS: $\quad$ None.

SELECTED DISCHARGE CHARACTERISTICS: Average discharge: $5.12 \mathrm{ft}^{3} / \mathrm{s}$ ( 32 years). Minimum daily discharge: No flow July 1975.

Magnitude and frequency of low flow for indicated periods

\begin{tabular}{|c|c|c|c|c|c|c|}
\hline \multirow{2}{*}{ Period } & \multirow{2}{*}{$\begin{array}{l}\text { Number of } \\
\text { consecu- } \\
\text { tive days }\end{array}$} & \multicolumn{5}{|c|}{$\begin{array}{l}\text { Discharge }\left(\mathrm{ft}^{3} / \mathrm{s}\right) \text { for indicated } \\
\text { recurrence interval (years) }\end{array}$} \\
\hline & & 2 & 5 & 10 & 20 & 50 \\
\hline Apr.-Mar. & $\begin{array}{r}1 \\
7 \\
30\end{array}$ & $\begin{array}{l}0.2 \\
0.3 \\
0.6\end{array}$ & $\begin{array}{l}0.1 \\
0.2 \\
0.3\end{array}$ & $\begin{array}{l}0 \\
0.1 \\
0.2\end{array}$ & $\begin{array}{l}0 \\
0.1 \\
0.2\end{array}$ & $\begin{array}{l}0 \\
0.1 \\
0.1\end{array}$ \\
\hline May- Nov. & $\begin{array}{r}1 \\
7 \\
30\end{array}$ & $\begin{array}{l}0.2 \\
0.3 \\
0.6\end{array}$ & $\begin{array}{l}0.1 \\
0.2 \\
0.3\end{array}$ & $\begin{array}{l}0 \\
0.1 \\
0.2\end{array}$ & $\begin{array}{l}0 \\
0.1 \\
0.2\end{array}$ & $\begin{array}{l}0 \\
0.1 \\
0.1\end{array}$ \\
\hline June-Aug. & $\begin{array}{r}1 \\
7 \\
30\end{array}$ & $\begin{array}{l}0.3 \\
0.4 \\
0.9\end{array}$ & $\begin{array}{l}0.2 \\
0.2 \\
0.5\end{array}$ & $\begin{array}{l}0.1 \\
0.2 \\
0.4\end{array}$ & $\begin{array}{l}0 \\
0.1 \\
0.3\end{array}$ & $\begin{array}{l}0 \\
0.1 \\
0.3\end{array}$ \\
\hline
\end{tabular}

\begin{tabular}{|c|c|c|c|c|c|c|}
\hline \multirow{2}{*}{ Period } & \multirow{2}{*}{$\begin{array}{l}\text { Number of } \\
\text { consecu- } \\
\text { tive day's }\end{array}$} & \multicolumn{5}{|c|}{$\begin{array}{l}\text { Discharge }\left(\mathrm{ft}^{3} / \mathrm{s}\right) \text { for indicated } \\
\text { recurrence interval (years) }\end{array}$} \\
\hline & & $i$ & 5 & 10 & 2.0 & 50 \\
\hline Sept.-Nov. & $\begin{array}{r}1 \\
7 \\
30\end{array}$ & $\begin{array}{l}0.3 \\
0.4 \\
0.7\end{array}$ & $\begin{array}{l}0.1 \\
0.2 \\
0.4\end{array}$ & $\begin{array}{l}0.1 \\
0.1 \\
0.3\end{array}$ & $\begin{array}{l}0.1 \\
0.1 \\
0.2\end{array}$ & $\begin{array}{l}0 \\
0.1 \\
0.1\end{array}$ \\
\hline Dec.-Feb. & $\begin{array}{r}1 \\
7 \\
30\end{array}$ & $\begin{array}{l}0.6 \\
0.7 \\
2.0\end{array}$ & $\begin{array}{l}0.2 \\
0.3 \\
0.8\end{array}$ & $\begin{array}{l}0.1 \\
0.2 \\
0.5\end{array}$ & $\begin{array}{l}0.1 \\
0.2 \\
0.3\end{array}$ & $\begin{array}{l}0.1 \\
0.1 \\
0.2\end{array}$ \\
\hline Mar. - May & $\begin{array}{r}1 \\
7 \\
30\end{array}$ & $\begin{array}{l}0.7 \\
1.0 \\
3.0\end{array}$ & $\begin{array}{l}0.5 \\
0.6 \\
1.7\end{array}$ & $\begin{array}{l}0.3 \\
0.5 \\
1.2\end{array}$ & $\begin{array}{l}0.3 \\
0.4 \\
0.9\end{array}$ & $\begin{array}{l}0.2 \\
0.3 \\
0.6\end{array}$ \\
\hline
\end{tabular}

Duration of daily flow for indicated periods

\begin{tabular}{l|c|c|c|c|c|c|c|c|c|c|c|c|c|}
\hline \multirow{2}{*}{ Period } & \multicolumn{8}{|c|}{ Discharge $\left(\mathrm{ft}^{3} / \mathrm{s}\right)$ which was equaled or exceeded for indicated percent of time } \\
\cline { 2 - 12 } & 98 & 95 & 90 & 85 & 80 & 75 & 70 & 60 & 50 & 40 & 30 & 20 & 10 \\
\hline Apr. - Mar. & 0.2 & 0.3 & 0.4 & 0.6 & 0.7 & 0.8 & 0.9 & 1.2 & 1.6 & 2.3 & 3.5 & 5.7 & 12 \\
May - Nov. & 0.2 & 0.2 & 0.3 & 0.4 & 0.5 & 0.6 & 0.7 & 0.8 & 1.0 & 1.4 & 1.8 & 2.8 & 5.8 \\
June - Aug. & 0.2 & 0.3 & 0.4 & 0.5 & 0.5 & 0.6 & 0.7 & 0.8 & 1.0 & 1.2 & 1.6 & 2.4 & 4.9 \\
Sept. - Nov. & 0.1 & 0.2 & 0.3 & 0.3 & 0.4 & 0.5 & 0.6 & 0.7 & 0.9 & 1.2 & 1.5 & 2.1 & 4.4 \\
Dec. - Feb. & 0.2 & 0.4 & 0.6 & 0.8 & 0.9 & 1.1 & 1.3 & 1.7 & 2.3 & 3.3 & 4.9 & 7.9 & 16 \\
Mar. - May & 0.5 & 0.8 & 1.1 & 1.5 & 1.8 & 2.1 & 2.4 & 3.0 & 4.0 & 5.3 & 7.1 & 11 & 21 \\
\hline
\end{tabular}




\section{MUSKINGUM RIVER BASIN \\ 03131500 Black Fork at Loudonville, Ohio}

LOCATION:

Lat 40.38'09", long $82^{\circ} 14^{\prime} 22^{\prime \prime}$, in NWI/4 sec. l, T.19 N., R.16 W.,

Ashland County, Hydrologic Unit 05040002, on right bank at upstream side of bridge on State Route 3 at Loudonville, $1.5 \mathrm{mi}$ downstream from Big Run.

DRAINAGE AREA: $\quad 349 \mathrm{mi}^{2}$.

TRIBITARY TO: TUScarawas River.

DISCHARGE DATA USED: October 1936 to September 1978.

REMARKS: $\quad$ Flow regulated since 1936 by Charles Mill Lake, 16 mi upstream from station. Records include diversion from Clear Fork Reservoir which enters the Black Fork drainage as sewage effluent from the city of Mansfield.

SELECTED DISCHARGE CHARACTERISTICS: Average discharge: $353 \mathrm{ft}^{3} / \mathrm{s}(42$ years). Minimum daily discharge: $34 \mathrm{ft} / \mathrm{s}$ September 1939, January 1940.

Magnitude and frequency of low flow for indicated periods

\begin{tabular}{|c|c|c|c|c|c|c|}
\hline \multirow{2}{*}{ Period } & \multirow{2}{*}{$\begin{array}{l}\text { Number of } \\
\text { consecu- } \\
\text { tive days }\end{array}$} & \multicolumn{5}{|c|}{$\begin{array}{l}\text { Discharge }\left(\mathrm{ft}^{3} / \mathrm{s}\right) \text { for indicated } \\
\text { recurrence interval (years) }\end{array}$} \\
\hline & & 2 & 5 & 10 & 20 & 50 \\
\hline Apr. -Mar. & $\begin{array}{r}7 \\
7 \\
30\end{array}$ & $\begin{array}{l}65 \\
70 \\
77\end{array}$ & $\begin{array}{l}51 \\
56 \\
62\end{array}$ & $\begin{array}{l}44 \\
50 \\
57\end{array}$ & $\begin{array}{l}39 \\
46 \\
54\end{array}$ & $\begin{array}{l}35 \\
42 \\
51\end{array}$ \\
\hline May- Nov. & $\begin{array}{r}1 \\
7 \\
30\end{array}$ & $\begin{array}{l}66 \\
71 \\
78\end{array}$ & $\begin{array}{l}51 \\
56 \\
63\end{array}$ & $\begin{array}{l}44 \\
50 \\
58\end{array}$ & $\begin{array}{l}40 \\
46 \\
54\end{array}$ & $\begin{array}{l}35 \\
41 \\
51\end{array}$ \\
\hline June-Aug. & $\begin{array}{r}1 \\
7 \\
30\end{array}$ & $\begin{array}{r}75 \\
81 \\
102\end{array}$ & $\begin{array}{l}59 \\
65 \\
78\end{array}$ & $\begin{array}{l}53 \\
60 \\
70\end{array}$ & $\begin{array}{l}48 \\
56 \\
65\end{array}$ & $\begin{array}{l}44 \\
53 \\
60\end{array}$ \\
\hline
\end{tabular}

\begin{tabular}{|c|c|c|c|c|c|c|}
\hline \multirow{2}{*}{ Period } & \multirow{2}{*}{$\begin{array}{l}\text { Number of } \\
\text { consecu- } \\
\text { tive days }\end{array}$} & \multicolumn{5}{|c|}{$\begin{array}{l}\text { Discharge }\left(\mathrm{ft}^{3} / \mathrm{s}\right) \text { for indicated } \\
\text { recurrence interval (years) }\end{array}$} \\
\hline & & 2 & 5 & 10 & 20 & 50 \\
\hline Sept.-Nov. & $\begin{array}{r}1 \\
7 \\
30\end{array}$ & $\begin{array}{l}67 \\
72 \\
81\end{array}$ & $\begin{array}{l}51 \\
56 \\
64\end{array}$ & $\begin{array}{l}45 \\
50 \\
59\end{array}$ & $\begin{array}{l}40 \\
46 \\
56\end{array}$ & $\begin{array}{l}35 \\
42 \\
53\end{array}$ \\
\hline Dec. -Feb. & $\begin{array}{r}1 \\
7 \\
30\end{array}$ & $\begin{array}{r}99 \\
113 \\
180\end{array}$ & $\begin{array}{l}66 \\
74 \\
99\end{array}$ & $\begin{array}{l}54 \\
60 \\
74\end{array}$ & $\begin{array}{l}47 \\
51 \\
58\end{array}$ & $\begin{array}{l}40 \\
44 \\
44\end{array}$ \\
\hline Mar. - May & $\begin{array}{r}1 \\
7 \\
30\end{array}$ & $\begin{array}{l}138 \\
169 \\
271\end{array}$ & $\begin{array}{l}104 \\
125 \\
183\end{array}$ & $\begin{array}{r}90 \\
107 \\
148\end{array}$ & $\begin{array}{r}79 \\
93 \\
124\end{array}$ & $\begin{array}{r}69 \\
80 \\
102\end{array}$ \\
\hline
\end{tabular}

Duration of daily flow for indicated periods

\begin{tabular}{l|r|r|r|r|r|r|r|r|r|r|r|r|r}
\hline \multirow{2}{*}{ Period } & \multicolumn{8}{|c}{ Discharge $\left(\mathrm{ft}^{3} / \mathrm{s}\right.$ ) which was equaled or exceeded for indicated percent of time } \\
\cline { 2 - 12 } & 98 & 95 & 90 & 85 & 80 & 75 & 70 & 60 & 50 & 40 & 30 & 20 & 10 \\
\hline Apr. - Mar. & 55 & 64 & 74 & 81 & 89 & 98 & 110 & 140 & 190 & 260 & 360 & 560 & 880 \\
May - Nov. & 53 & 60 & 68 & 75 & 80 & 85 & 91 & 110 & 130 & 160 & 200 & 280 & 540 \\
June - Aug. & 60 & 67 & 76 & 82 & 87 & 93 & 99 & 110 & 130 & 160 & 200 & 290 & 550 \\
Sept.- Nov. & 48 & 55 & 61 & 66 & 71 & 75 & 78 & 85 & 96 & 110 & 140 & 180 & 260 \\
Dec. - Feb. & 56 & 66 & 80 & 91 & 100 & 130 & 160 & 210 & 270 & 350 & 490 & 700 & 1100 \\
Mar. - May & 110 & 130 & 160 & 190 & 220 & 250 & 280 & 350 & 470 & 600 & 740 & 930 & 1200 \\
\hline
\end{tabular}




\section{MUSKINGUM RIVER BASIN}

03132000 Clear Fork at Butler, Ohio

LOCATION:

Lat $40^{\circ} 35^{\prime} 37^{\prime \prime}$, long $82^{\circ} 25^{\prime} 20^{\prime \prime}$, in NEl/4 sec.20, T.21 N., R.17 W., Richland County, Hydrologic unit 05040002 , on left bank at downstream side of bridge on state Route $95,0.3 \mathrm{mi}$ northeast of Butler.

DRAINAGE AREA: $\quad 136 \mathrm{mi}^{2}$.

TRIBUTARY TO: Black Fork.

DISCHARGE DATA USED: October 1953 to September 1975.

REMARKS:

Flow regulated by Clear Fork Reservoir, $16 \mathrm{mi}$ upstream from station since 1949. Water diverted from Clear Fork for municipal supply of city of Mansfield since 1953.

SELECTED DISCHARGE CHARACTERISTICS: Average discharge: $136 \mathrm{ft} 3 / \mathrm{s}$ ( 22 years).

Minimum daily discharge: $16 \mathrm{ft} 3 / \mathrm{s}$ September 1954.

Magnitude and frequency of low flow for indicated periods

\begin{tabular}{|c|c|c|c|c|c|c|}
\hline \multirow{2}{*}{ Period } & \multirow{2}{*}{$\begin{array}{l}\text { Number of } \\
\text { consecu- } \\
\text { tive days }\end{array}$} & \multicolumn{5}{|c|}{$\begin{array}{l}\text { Discharge }\left(\mathrm{ft}^{3} / \mathrm{s}\right) \text { for indicated } \\
\text { recurrence interval (years) }\end{array}$} \\
\hline & & 2 & 5 & 10 & 20 & 50 \\
\hline Apr.-Mar. & $\begin{array}{r}1 \\
7 \\
30\end{array}$ & $\begin{array}{l}24 \\
25 \\
27\end{array}$ & $\begin{array}{l}20 \\
20 \\
22\end{array}$ & $\begin{array}{l}18 \\
19 \\
20\end{array}$ & $\begin{array}{l}17 \\
17 \\
19\end{array}$ & $\begin{array}{l}15 \\
16 \\
17\end{array}$ \\
\hline May- Nov. & $\begin{array}{r}1 \\
7 \\
30\end{array}$ & $\begin{array}{l}24 \\
25 \\
27\end{array}$ & $\begin{array}{l}20 \\
20 \\
22\end{array}$ & $\begin{array}{l}18 \\
19 \\
20\end{array}$ & $\begin{array}{l}16 \\
17 \\
19\end{array}$ & $\begin{array}{l}15 \\
16 \\
17\end{array}$ \\
\hline June-Aug. & $\begin{array}{r}1 \\
7 \\
30\end{array}$ & $\begin{array}{l}30 \\
31 \\
38\end{array}$ & $\begin{array}{l}23 \\
24 \\
28\end{array}$ & $\begin{array}{l}20 \\
21 \\
25\end{array}$ & $\begin{array}{l}18 \\
20 \\
23\end{array}$ & $\begin{array}{l}16 \\
18 \\
21\end{array}$ \\
\hline
\end{tabular}

\begin{tabular}{l|c|c|c|c|c|c}
\hline \multirow{2}{*}{ Period } & \multirow{2}{*}{$\begin{array}{c}\text { Number of } \\
\text { consecu- } \\
\text { tive days }\end{array}$} & \multicolumn{4}{|c}{$\begin{array}{c}\text { Discharge } \\
\text { recurrence }{ }^{3} / \mathrm{s} \text { ) }\end{array}$ for interval indicated } \\
\cline { 3 - 7 } & 2 & 5 & 10 & 20 & 50 \\
\hline Sept.-Nov. & 1 & 24 & 20 & 18 & 17 & 16 \\
& 7 & 25 & 20 & 19 & 18 & 16 \\
& 30 & 28 & 22 & 21 & 20 & 19 \\
Dec.-Feb. & 1 & 35 & 25 & 22 & 20 & 19 \\
& 7 & 39 & 28 & 24 & 22 & 20 \\
& 30 & 58 & 35 & 28 & 24 & 20 \\
& 1 & 60 & 44 & 36 & 31 & 25 \\
& 7 & 70 & 53 & 46 & 41 & 36 \\
& 30 & 107 & 76 & 65 & 57 & 50 \\
\hline
\end{tabular}

Duration of daity flow for indicated periods

\begin{tabular}{l|c|c|c|c|c|c|c|c|c|c|c|c|c}
\hline \multirow{2}{*}{ Period } & \multicolumn{8}{|c|}{ Discharge $(\mathrm{ft} / \mathrm{s}$ ) which was equaled or exceeded for indicated percent of time } \\
\cline { 2 - 13 } & 98 & 95 & 90 & 85 & 80 & 75 & 70 & 60 & 50 & 40 & 30 & 20 & 10 \\
\hline Apr. - Mar. & 22 & 24 & 26 & 30 & 33 & 37 & 41 & 51 & 67 & 89 & 120 & 180 & 310 \\
May - Nov. & 20 & 23 & 25 & 26 & 28 & 31 & 34 & 39 & 46 & 56 & 71 & 100 & 170 \\
June - Aug. & 22 & 24 & 27 & 31 & 33 & 35 & 38 & 43 & 50 & 59 & 71 & 91 & 140 \\
Sept.- Nov. & 19 & 21 & 23 & 24 & 25 & 26 & 27 & 31 & 35 & 39 & 45 & 55 & 79 \\
Dec. - Feb. & 24 & 26 & 30 & 34 & 38 & 44 & 49 & 64 & 82 & 100 & 140 & 200 & 350 \\
Mar. - May & 47 & 58 & 69 & 79 & 89 & 98 & 110 & 130 & 160 & 190 & 240 & 330 & 530 \\
\hline
\end{tabular}


MUSKINGUM RIVER BASIN

03133500 Clear Fork below Pleasant Hill Dam, near Perrysville, Ohio

LOCATION:

Lat $40^{\circ} 37^{\prime} 13^{\prime \prime}$, long $82^{\circ} 19^{\prime} 28^{\prime \prime}$, in NEI/4 sec. 7, T.19 N., R.16 W., Ashland

County, Hydrologic Unit 05040002 , on right bank $0.2 \mathrm{mi}$ downstream from

pleasant Hill Dam, $2.8 \mathrm{mi}$ south of Perrysville, and $4.7 \mathrm{mi}$ upstream from

the confluence of Clear Fork and Black Fork.

DRAINAGE AREA: $\quad 198 \mathrm{mi}^{2}$.

TRIBLTARY TO: Black Fork.

DISCHARGE DATA USED: November 1938 to September 1978.

REMARKS:

Flow regulated by Pleasant Hill Lake. Water diverted from Clear Fork Reservoir (upstream from Pleasant Hill Lake) for municipal supply of city of Mansfield since 1953.

SELECTED DISCHARGE CHARACTERISTICS: Average discharge: $195 \mathrm{ft}^{3} / \mathrm{s}$ ( 39 years).

Minimum daily discharge: $0.6 \mathrm{ft} 3 / \mathrm{s}$ November, 1938.

Magnitude and frequency of low flow for indicated periods

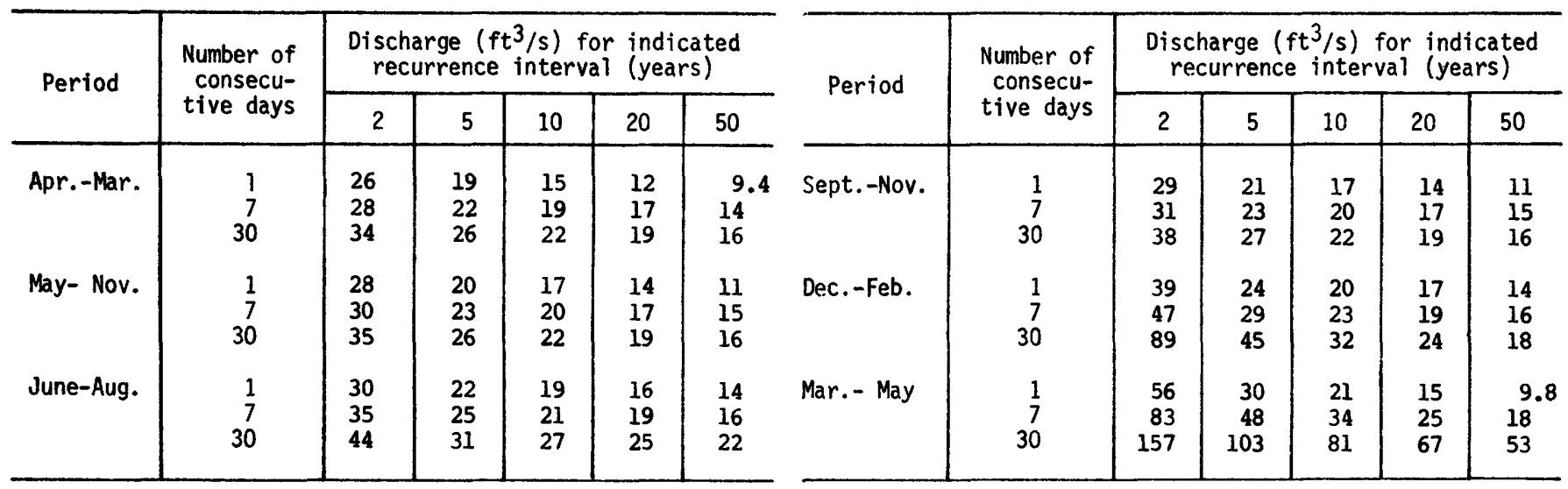

Duration of daily flow for indicated periods

\begin{tabular}{|c|c|c|c|c|c|c|c|c|c|c|c|c|c|}
\hline \multirow{2}{*}{ Period } & \multicolumn{13}{|c|}{ Discharge $\left(\mathrm{ft}^{3} / \mathrm{s}\right)$ which was equaled or exceeded for indicated percent of time } \\
\hline & 98 & 95 & 90 & 85 & 80 & 75 & 70 & 60 & 50 & 40 & 30 & 20 & 10 \\
\hline $\begin{array}{l}\text { Apr. - Mar. } \\
\text { May - Nov. } \\
\text { June - Aug. } \\
\text { Sept. - Nov. } \\
\text { Dec. - Feb. } \\
\text { Mar. - May }\end{array}$ & $\begin{array}{l}21 \\
19 \\
25 \\
16 \\
25 \\
29\end{array}$ & $\begin{array}{l}27 \\
26 \\
27 \\
21 \\
30 \\
62\end{array}$ & $\begin{array}{l}31 \\
29 \\
31 \\
26 \\
35 \\
84\end{array}$ & $\begin{array}{r}36 \\
32 \\
35 \\
28 \\
40 \\
100\end{array}$ & $\begin{array}{r}40 \\
36 \\
39 \\
31 \\
47 \\
120\end{array}$ & $\begin{array}{r}45 \\
39 \\
43 \\
33 \\
56 \\
140\end{array}$ & $\begin{array}{r}51 \\
42 \\
46 \\
35 \\
64 \\
150\end{array}$ & $\begin{array}{r}67 \\
51 \\
54 \\
40 \\
90 \\
190\end{array}$ & $\begin{array}{r}92 \\
63 \\
65 \\
46 \\
130 \\
240\end{array}$ & $\begin{array}{r}130 \\
80 \\
78 \\
54 \\
170 \\
300\end{array}$ & $\begin{array}{r}180 \\
110 \\
99 \\
68 \\
240 \\
420\end{array}$ & $\begin{array}{r}280 \\
150 \\
140 \\
95 \\
400 \\
540\end{array}$ & $\begin{array}{l}530 \\
270 \\
230 \\
150 \\
660 \\
720\end{array}$ \\
\hline
\end{tabular}




\section{MUSKINGUM RIVER BASIN}

03134000 Jerome Fork at Jeromeville, Ohio

LOCATION:

Lat $40^{\circ} 48^{\prime} 07^{\prime \prime}$, long $82^{\circ} 12^{\prime} 01^{\prime \prime}$, in SW1/4 sec. 5, T.2 N., R.15 W., Ashland County, Hydrologic Unit 05040002 , at highway bridge at Jeromeville, $1.0 \mathrm{mi}$ upstream from oldtown Run.

DRAINAGE AREA:

$120 \mathrm{mi}^{2}$

TR.IBUTARY TO:

Mohican River.

DISCHARGE DATA USED:

October 1925 to September 1949.

REMARKS :

None.

SELECTED DISCHARGE CHARACTERISTICS: Average discharge: $100 \mathrm{ft}^{3} / \mathrm{s}$ (24 years).

Minimum daily discharge: $2.0 \mathrm{ft}^{3} / \mathrm{s} \mathrm{July}$, August 1934, January 1935, October 1939 .

Magnitude and frequency of low flow for indicated periods

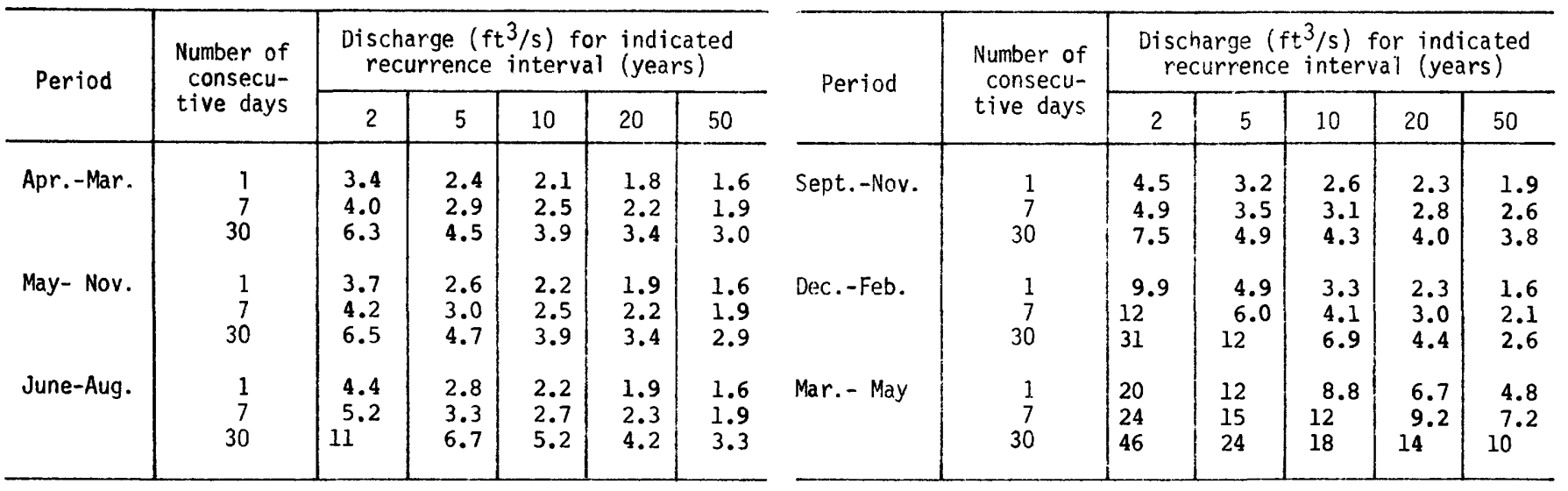

Duration of daily flow for indicated periods

\begin{tabular}{|c|c|c|c|c|c|c|c|c|c|c|c|c|c|c|}
\hline \multirow{2}{*}{\multicolumn{2}{|c|}{ Period }} & \multicolumn{13}{|c|}{ Discharge $\left(\mathrm{ft}^{3} / \mathrm{s}\right)$ which was equaled or exceeded for indicated percent of time } \\
\hline & & 98 & 95 & 90 & 85 & 80 & 75 & 70 & 60 & 50 & 40 & 30 & 20 & 10 \\
\hline $\begin{array}{l}\text { Apr. - } \\
\text { May - } \\
\text { June - } \\
\text { Sept. - } \\
\text { Dec. - } \\
\text { Mar. - }\end{array}$ & $\begin{array}{l}\text { Mar. } \\
\text { Nov. } \\
\text { Aug. } \\
\text { Nov. } \\
\text { Feb. } \\
\text { May }\end{array}$ & $\begin{array}{c}3.4 \\
3.2 \\
2.8 \\
3.3 \\
4.2 \\
13\end{array}$ & $\begin{array}{l}4.2 \\
3.8 \\
3.5 \\
3.9 \\
5.4 \\
18\end{array}$ & $\begin{array}{l}5.4 \\
4.6 \\
4.4 \\
4.4 \\
7.5 \\
23\end{array}$ & $\begin{array}{r}6.7 \\
5.4 \\
5.2 \\
5.0 \\
9.8 \\
28\end{array}$ & $\begin{array}{l}8.2 \\
6.2 \\
6.0 \\
5.5 \\
13 \\
33\end{array}$ & $\begin{array}{c}10 \\
7.3 \\
7.3 \\
6.1 \\
16 \\
39\end{array}$ & $\begin{array}{c}12 \\
8.5 \\
8.5 \\
6.8 \\
20 \\
44\end{array}$ & $\begin{array}{c}18 \\
11 \\
12 \\
8.4 \\
30 \\
57\end{array}$ & $\begin{array}{l}26 \\
15 \\
14 \\
11 \\
46 \\
75\end{array}$ & $\begin{array}{r}41 \\
20 \\
19 \\
13 \\
68 \\
100\end{array}$ & $\begin{array}{r}64 \\
29 \\
26 \\
18 \\
98 \\
140\end{array}$ & $\begin{array}{r}110 \\
45 \\
40 \\
28 \\
160 \\
210\end{array}$ & $\begin{array}{r}220 \\
98 \\
82 \\
64 \\
340 \\
390\end{array}$ \\
\hline
\end{tabular}




\section{MUSKINGUM RIVER BASIN}

03134300 Muddy Fork near Rowsburg, Ohio

LOCATION:

Lat 40 $50^{\prime} 10^{\prime \prime}$, long $82^{\circ} 08^{\prime} 16^{\prime \prime}$, Ashland County, Hydrologic Unit 05040002 , at bridge on Township Road 1550, $1.8 \mathrm{mi}$ southeast of Rowsburg.

DRAINAGE AREA: $\quad 66.2 \mathrm{mi}^{2}$.

TRIBUTARY TO: Lake Fork.

DISCHARGE DATA USED: Low-flow measurements 1959, 1972-1976, 1978 water years.

INDEX STATION: $\quad 03144000$ Wakatomika Creek near Frazeysburg, Ohio.

REMARKS: $\quad$ None.

SELECTED DISCHARGE CHARACTERISTICS: Minimum discharge observed: $0.6 \mathrm{ft}^{3} / \mathrm{s}$ september 1978 .

\begin{tabular}{l|c|c|c}
\multicolumn{4}{c}{$\begin{array}{c}\text { Magnitude and frequency of low flow for } \\
\text { indicated periods }\end{array}$} \\
\hline & $\begin{array}{c}\text { Number of } \\
\text { consecu- } \\
\text { tive days }\end{array}$ & $\begin{array}{c}\text { Discharge }\left(\mathrm{ft}^{3} / \mathrm{s}\right) \text { for } \\
\text { indicated recurrence } \\
\text { interval (years) }\end{array}$ \\
\cline { 3 - 4 } & 7 & 2 & 10 \\
\hline Apr. - Mar. & 30 & 3.0 & 1.7 \\
& 7 & 3.9 & 2.1 \\
May - Nov. & 30 & 3.9 & 1.7 \\
June - Aug. & 7 & 3.9 & 2.1 \\
& 30 & 6.0 & 2.2 \\
Sept. - Nov. & 7 & 3.3 & 3.0 \\
& 30 & 4.7 & 1.8 \\
\hline
\end{tabular}

Duration of daily flow for indicated periods

\begin{tabular}{l|c|c|c|c|c}
\hline \multirow{2}{*}{ Period } & \multicolumn{6}{|c}{$\begin{array}{c}\text { Discharge ( } \mathrm{ft}^{3} / \mathrm{s} \text { ) which was equaled or } \\
\text { exceeded for indicated percent of time }\end{array}$} \\
\cline { 2 - 6 } & 98 & 95 & 90 & 85 & 80 \\
\hline Apr. - Mar. & 2.2 & 2.9 & 3.9 & 4.7 & 5.4 \\
May - Nov. & 1.9 & 2.5 & 3.1 & 3.6 & 4.2 \\
June - Aug. & 2.2 & 2.8 & 3.5 & 4.2 & 4.7 \\
Sept. - Nov. & 1.6 & 2.1 & 2.7 & 3.0 & 3.4 \\
\hline
\end{tabular}




\section{MUSKINGUM RIVER BASIN}

03135000 Lake Fork below Mohicanville Dam, near Mohicanville, Ohio

LOCATION:

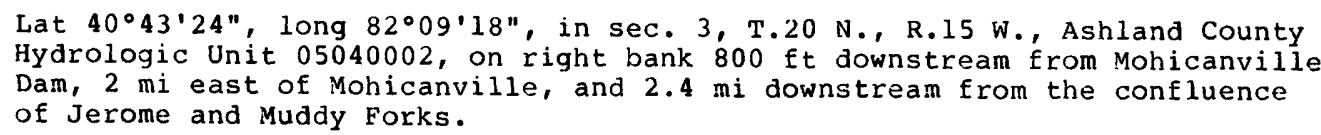

DRAINAGE AREA:

$271 \mathrm{mi}^{2}$.

TRIBUTARY TO:

Mohican River

DISCHARGE DATA USED: October 1938 to September 1978.

REMARKS:

Flow regulated by Mohicanville Reservoir.

SELECTED DISCHARGE CHARACTERISTICS: Average discharge: $234 \mathrm{ft}^{3} / \mathrm{s}$ (40 years)

Minimum daily discharge: $1 \mathrm{ft} 3 / \mathrm{s}$ June 1947, January 1959.

Magnitude and frequency of low flow for indicated periods

\begin{tabular}{|c|c|c|c|c|c|c|c|c|c|c|c|c|c|}
\hline \multirow{2}{*}{ Period } & \multirow{2}{*}{$\begin{array}{l}\text { Number of } \\
\text { consecu- } \\
\text { tive days }\end{array}$} & \multicolumn{5}{|c|}{$\begin{array}{l}\text { Discharge }\left(\mathrm{ft}^{3} / \mathrm{s}\right) \text { for indicated } \\
\text { recurrence interval (years) }\end{array}$} & \multirow{2}{*}{ Period } & \multirow{2}{*}{$\begin{array}{l}\text { Number of } \\
\text { consecu- } \\
\text { tive days }\end{array}$} & \multicolumn{5}{|c|}{$\begin{array}{l}\text { Discharge }\left(\mathrm{ft}^{3} / \mathrm{s}\right) \text { for indicated } \\
\text { recurrence interval (years) }\end{array}$} \\
\hline & & 2 & 5 & 10 & 20 & 50 & & & 2 & 5 & 10 & 20 & 50 \\
\hline Apr.-Mar. & $\begin{array}{r}7 \\
7 \\
30\end{array}$ & $\begin{array}{l}11 \\
14 \\
18\end{array}$ & $\begin{array}{l}5.6 \\
8.9 \\
12\end{array}$ & $\begin{array}{l}3.6 \\
7.2 \\
9.4\end{array}$ & $\begin{array}{l}2.3 \\
6.1 \\
8.0\end{array}$ & $\begin{array}{l}1.3 \\
5.1 \\
6.7\end{array}$ & Sept.-Nov. & $\begin{array}{r}1 \\
7 \\
30\end{array}$ & $\begin{array}{l}13 \\
14 \\
20\end{array}$ & $\begin{array}{l}8.0 \\
9.1 \\
12\end{array}$ & $\begin{array}{l}6.4 \\
7.3 \\
9.8\end{array}$ & $\begin{array}{l}5.4 \\
6.1 \\
8.6\end{array}$ & $\begin{array}{l}4.5 \\
5.1 \\
7.7\end{array}$ \\
\hline June-Aug. & $\begin{array}{r}1 \\
7 \\
30\end{array}$ & $\begin{array}{l}17 \\
17 \\
33\end{array}$ & $\begin{array}{l}9.1 \\
11 \\
19\end{array}$ & $\begin{array}{l}6.0 \\
9.4 \\
15\end{array}$ & $\begin{array}{l}4.0 \\
8.0 \\
12\end{array}$ & $\begin{array}{l}2.4 \\
6.8 \\
10\end{array}$ & Mar. - May & $\begin{array}{r}1 \\
7 \\
30\end{array}$ & $\begin{array}{r}48 \\
74 \\
158\end{array}$ & $\begin{array}{l}24 \\
52 \\
94\end{array}$ & $\begin{array}{l}15 \\
43 \\
71\end{array}$ & $\begin{array}{l}9.6 \\
37 \\
56\end{array}$ & $\begin{array}{l}5.4 \\
31 \\
43\end{array}$ \\
\hline
\end{tabular}

Duration of daily flow for indicated periods

\begin{tabular}{l|c|c|c|c|c|c|c|c|r|r|r|r|r}
\hline \multirow{2}{*}{ Period } & \multicolumn{8}{|c|}{ Discharge $\left(\mathrm{ft}^{3} / \mathrm{s}\right)$ which was equaled or exceeded for indicated percent of time } \\
\cline { 2 - 12 } & 98 & 95 & 90 & 85 & 80 & 75 & 70 & 60 & 50 & 40 & 30 & 20 & 10 \\
\hline Apr. - Mar. & 9.1 & 13 & 16 & 20 & 24 & 29 & 35 & 52 & 77 & 120 & 180 & 320 & 800 \\
May - Nov. & 8.2 & 11 & 14 & 16 & 19 & 21 & 24 & 32 & 44 & 59 & 82 & 130 & 300 \\
June - Aug. & 10 & 13 & 16 & 19 & 21 & 24 & 27 & 34 & 43 & 57 & 77 & 110 & 260 \\
Sept.- Nov. & 7.4 & 8.6 & 11 & 13 & 15 & 16 & 18 & 22 & 28 & 38 & 52 & 74 & 140 \\
Dec. - Feb. & 13 & 17 & 23 & 28 & 34 & 43 & 55 & 82 & 120 & 170 & 260 & 490 & 1000 \\
Mar. - May & 41 & 55 & 73 & 89 & 100 & 120 & 140 & 180 & 240 & 340 & 550 & 850 & 1100 \\
\hline
\end{tabular}


MUSKINGUM RIVER BASIN

03136000 Mohican River at Greer, Ohio

LOCATION:

Lat $40^{\circ} 30^{\prime} 53^{\prime \prime}$, long $82^{\circ} 11^{\prime} 44^{\prime \prime}$, in NW1/4 sec.10, T.8 N., R.10 W., Knox County, Hydrologic Unit 05040002, on left bank 3,000 ft downstream from bridge on State koute 514 at Greer, $5 \mathrm{mi}$ upstream from Negro Run, and $7 \mathrm{mi}$ downstream from Lake Fork.

DRAINAGE AREA:

$948 \mathrm{mi}^{2}$

TRIBUTARY TO:

Tuscarawas River.

DISCHARGE DATA USED:

October 1938 to september 1978 .

REMARKS:

Flow regulated by Charles Mill Lake on Black Fork, $30 \mathrm{mi}$ upstream, Pleasant Hill Lake on Clear Fork, $17 \mathrm{mi}$ upstream, and Mohicanville Reservoir on Lake Fork, $19 \mathrm{mi}$ upstream, beginning August 1936.

SELECTED DISCHARGE CHARACTERISTICS: Average discharge: $899 \mathrm{ft}^{3} / \mathrm{s}$ (40 years). Minimum daily discharge: $77 \mathrm{ft}^{3} / \mathrm{s}$ September 1941 .

Magnitude and frequency of low flow for indicated periods

\begin{tabular}{|c|c|c|c|c|c|c|}
\hline \multirow{2}{*}{ Period } & \multirow{2}{*}{$\begin{array}{l}\text { Number of } \\
\text { consecu- } \\
\text { tive days }\end{array}$} & \multicolumn{5}{|c|}{$\begin{array}{l}\text { Discharge }\left(\mathrm{ft}^{3} / \mathrm{s}\right) \text { for indicated } \\
\text { recurrence interval (years) }\end{array}$} \\
\hline & & 2 & 5 & 10 & 20 & 50 \\
\hline Apr.-Mar. & $\begin{array}{r}1 \\
7 \\
30\end{array}$ & $\begin{array}{l}128 \\
139 \\
153\end{array}$ & $\begin{array}{l}103 \\
112 \\
127\end{array}$ & $\begin{array}{r}93 \\
101 \\
119\end{array}$ & $\begin{array}{r}86 \\
93 \\
115\end{array}$ & $\begin{array}{r}79 \\
86 \\
112\end{array}$ \\
\hline May- Nov. & $\begin{array}{r}1 \\
7 \\
30\end{array}$ & $\begin{array}{l}132 \\
141 \\
155\end{array}$ & $\begin{array}{l}105 \\
114 \\
127\end{array}$ & $\begin{array}{r}95 \\
103 \\
118\end{array}$ & $\begin{array}{r}87 \\
95 \\
114\end{array}$ & $\begin{array}{r}80 \\
87 \\
110\end{array}$ \\
\hline June-Aug. & $\begin{array}{r}1 \\
7 \\
30\end{array}$ & $\begin{array}{l}148 \\
159 \\
221\end{array}$ & $\begin{array}{l}118 \\
127 \\
164\end{array}$ & $\begin{array}{l}107 \\
117 \\
146\end{array}$ & $\begin{array}{l}100 \\
110 \\
136\end{array}$ & $\begin{array}{r}94 \\
105 \\
127\end{array}$ \\
\hline
\end{tabular}

\begin{tabular}{|c|c|c|c|c|c|c|}
\hline \multirow{2}{*}{ Period } & \multirow{2}{*}{$\begin{array}{l}\text { Number of } \\
\text { consecu- } \\
\text { tive days }\end{array}$} & \multicolumn{5}{|c|}{$\begin{array}{l}\text { Discharge }\left(\mathrm{ft}^{3} / \mathrm{s}\right) \text { for indicated } \\
\text { recurrence interval (years) }\end{array}$} \\
\hline & & 2 & 5 & 10 & 20 & 50 \\
\hline Sept.-Nov. & $\begin{array}{r}1 \\
7 \\
30\end{array}$ & $\begin{array}{l}136 \\
145 \\
168\end{array}$ & $\begin{array}{l}107 \\
114 \\
130\end{array}$ & $\begin{array}{r}95 \\
102 \\
119\end{array}$ & $\begin{array}{r}86 \\
94 \\
113\end{array}$ & $\begin{array}{r}78 \\
85 \\
109\end{array}$ \\
\hline Dec.-Feb. & $\begin{array}{r}1 \\
7 \\
30\end{array}$ & $\begin{array}{l}212 \\
240 \\
405\end{array}$ & $\begin{array}{l}134 \\
151 \\
211\end{array}$ & $\begin{array}{l}108 \\
122 \\
154\end{array}$ & $\begin{array}{r}92 \\
105 \\
120\end{array}$ & $\begin{array}{l}79 \\
89 \\
92\end{array}$ \\
\hline Mar. - May & $\begin{array}{r}1 \\
7 \\
30\end{array}$ & $\begin{array}{l}343 \\
426 \\
730\end{array}$ & $\begin{array}{l}249 \\
306 \\
486\end{array}$ & $\begin{array}{l}211 \\
259 \\
389\end{array}$ & $\begin{array}{l}183 \\
225 \\
322\end{array}$ & $\begin{array}{l}156 \\
193 \\
259\end{array}$ \\
\hline
\end{tabular}

Duration of daily flow for indicated periods

\begin{tabular}{|c|c|c|c|c|c|c|c|c|c|c|c|c|c|}
\hline \multirow{2}{*}{ Period } & \multicolumn{13}{|c|}{ Discharge $\left(\mathrm{ft}^{3} / \mathrm{s}\right)$ which was equaled or exceeded for indicated percent of time } \\
\hline & 98 & 95 & 90 & 85 & 80 & 75 & 70 & 60 & 50 & 40 & 30 & 20 & 10 \\
\hline $\begin{array}{l}\text { Apr. - Mar. } \\
\text { May - Nov. } \\
\text { June - Aug. } \\
\text { Sept. - Nov. } \\
\text { Dec. - Feb. } \\
\text { Mar. - May }\end{array}$ & $\begin{array}{l}120 \\
110 \\
120 \\
100 \\
120 \\
270\end{array}$ & $\begin{array}{l}130 \\
130 \\
140 \\
120 \\
140 \\
330\end{array}$ & $\begin{array}{l}150 \\
140 \\
150 \\
130 \\
170 \\
420\end{array}$ & $\begin{array}{l}170 \\
150 \\
170 \\
140 \\
190 \\
500\end{array}$ & $\begin{array}{l}190 \\
170 \\
180 \\
150 \\
220 \\
580\end{array}$ & $\begin{array}{l}210 \\
180 \\
200 \\
150 \\
270 \\
670\end{array}$ & $\begin{array}{l}240 \\
200 \\
210 \\
160 \\
320 \\
760\end{array}$ & $\begin{array}{l}320 \\
240 \\
250 \\
180 \\
460 \\
980\end{array}$ & $\begin{array}{r}450 \\
280 \\
290 \\
210 \\
610 \\
1200\end{array}$ & $\begin{array}{r}630 \\
360 \\
370 \\
250 \\
840 \\
1500\end{array}$ & $\begin{array}{r}930 \\
490 \\
480 \\
310 \\
1200 \\
2000\end{array}$ & $\begin{array}{r}1400 \\
740 \\
720 \\
420 \\
1800 \\
2600\end{array}$ & $\begin{array}{r}2400 \\
1300 \\
1300 \\
660 \\
3000 \\
3300\end{array}$ \\
\hline
\end{tabular}


MUSKINGUM RIVER BASIN

03136500 Kokosing River at Mount Vernon, Ohio

LOCATION:

Lat $40^{\circ} 24^{\prime} 20^{\prime \prime}$, long $82^{\circ} 30^{\prime} 00^{\prime \prime}$, in sec.2, T.6 N., R.13 W., Knox County, Hydrologic Unit 05040003 , on right bank at downstream side of Tilden Avenue Bridge at Mount Vernon, $0.8 \mathrm{mi}$ downstream from North Branch, and $2.7 \mathrm{mi}$ upstream from Dry Creek.

DRAINAGE AREA: $202 \mathrm{mi}^{2}$.

TRIBUTARY T0: $\quad$ Mohican River.

DISCHARGE DATA USED: October 1954 to September 1978.

REMARKS:

Some regulation by Knox Lake, capacity 3,750 acre-ft, $8.2 \mathrm{mi}$ upstream on East Branch of North Branch Kokosing River beginning in 1954 and North Branch Kokosing River Lake $10.0 \mathrm{mi}$ upstream on North Branch Kokosing River, beginning in June 1972 .

SELECTED DISCHARGE CHARACTERISTICS: Average discharge: $211 \mathrm{ft}^{3} / \mathrm{s}$ (24 years).

Minimum daily discharge: $14 \mathrm{ft}^{3} / \mathrm{s}$ september, October 1963.

Magnitude and frequency of low flow for indicated periods

\begin{tabular}{|c|c|c|c|c|c|c|c|c|c|c|c|c|c|}
\hline \multirow{2}{*}{ Period } & \multirow{2}{*}{$\begin{array}{l}\text { Number of } \\
\text { consecu- } \\
\text { tive days }\end{array}$} & \multicolumn{5}{|c|}{$\begin{array}{l}\text { Discharge }\left(\mathrm{ft}^{3} / \mathrm{s}\right) \text { for indicated } \\
\text { recurrence interval (years) }\end{array}$} & \multirow{2}{*}{ Period } & \multirow{2}{*}{$\begin{array}{l}\text { Number of } \\
\text { consecu- } \\
\text { tive days }\end{array}$} & \multicolumn{5}{|c|}{$\begin{array}{l}\text { Discharge }\left(\mathrm{ft}^{3} / \mathrm{s}\right) \text { for indicated } \\
\text { recurrence interval (years) }\end{array}$} \\
\hline & & 2 & 5 & 10 & 20 & 50 & & & 2 & 5 & 10 & 20 & 50 \\
\hline Apr.-Mar. & $\begin{array}{r}1 \\
7 \\
30\end{array}$ & $\begin{array}{l}27 \\
29 \\
33\end{array}$ & $\begin{array}{l}20 \\
21 \\
24\end{array}$ & $\begin{array}{l}17 \\
18 \\
20\end{array}$ & $\begin{array}{l}15 \\
16 \\
18\end{array}$ & $\begin{array}{l}13 \\
14 \\
15\end{array}$ & Sept.-Nov. & $\begin{array}{r}1 \\
7 \\
30\end{array}$ & $\begin{array}{l}29 \\
31 \\
34\end{array}$ & $\begin{array}{l}21 \\
22 \\
24\end{array}$ & $\begin{array}{l}18 \\
18 \\
21\end{array}$ & $\begin{array}{l}15 \\
16 \\
19\end{array}$ & $\begin{array}{l}13 \\
13 \\
17\end{array}$ \\
\hline June-Aug. & $\begin{array}{r}1 \\
7 \\
30\end{array}$ & $\begin{array}{l}32 \\
34 \\
49\end{array}$ & $\begin{array}{l}24 \\
26 \\
32\end{array}$ & $\begin{array}{l}21 \\
23 \\
27\end{array}$ & $\begin{array}{l}19 \\
20 \\
24\end{array}$ & $\begin{array}{l}17 \\
18 \\
21\end{array}$ & Mar. - May & $\begin{array}{r}1 \\
7 \\
30\end{array}$ & $\begin{array}{r}75 \\
89 \\
150\end{array}$ & $\begin{array}{l}51 \\
60 \\
93\end{array}$ & $\begin{array}{l}41 \\
49 \\
72\end{array}$ & $\begin{array}{l}33 \\
41 \\
59\end{array}$ & $\begin{array}{l}26 \\
33 \\
46\end{array}$ \\
\hline
\end{tabular}

Duration of daily flow for indicated periods

\begin{tabular}{|c|c|c|c|c|c|c|c|c|c|c|c|c|c|c|}
\hline \multirow{2}{*}{\multicolumn{2}{|c|}{ Period }} & \multicolumn{13}{|c|}{ Discharge $\left(\mathrm{ft}^{3} / \mathrm{s}\right)$ which was equaled or exceeded for indicated percent of time } \\
\hline & & 98 & 95 & 90 & 85 & 80 & 75 & 70 & 60 & 50 & 40 & 30 & 20 & 10 \\
\hline $\begin{array}{l}\text { Apr. - } \\
\text { May - } \\
\text { June - } \\
\text { Sept. - } \\
\text { Dec. - } \\
\text { Mar. - }\end{array}$ & $\begin{array}{l}\text { Mar. } \\
\text { Nov. } \\
\text { Aug. } \\
\text { Nov. } \\
\text { Feb. } \\
\text { May }\end{array}$ & $\begin{array}{l}21 \\
19 \\
23 \\
17 \\
26 \\
53\end{array}$ & $\begin{array}{l}25 \\
23 \\
26 \\
19 \\
32 \\
70\end{array}$ & $\begin{array}{l}31 \\
27 \\
30 \\
23 \\
42 \\
90\end{array}$ & $\begin{array}{r}35 \\
31 \\
33 \\
26 \\
50 \\
100\end{array}$ & $\begin{array}{r}41 \\
34 \\
37 \\
29 \\
58 \\
120\end{array}$ & $\begin{array}{r}47 \\
37 \\
41 \\
31 \\
68 \\
130\end{array}$ & $\begin{array}{r}53 \\
41 \\
44 \\
33 \\
77 \\
150\end{array}$ & $\begin{array}{r}72 \\
50 \\
52 \\
39 \\
110 \\
180\end{array}$ & $\begin{array}{r}98 \\
61 \\
63 \\
45 \\
140 \\
220\end{array}$ & $\begin{array}{r}130 \\
78 \\
80 \\
54 \\
180 \\
280\end{array}$ & $\begin{array}{r}180 \\
100 \\
100 \\
68 \\
240 \\
350\end{array}$ & $\begin{array}{r}260 \\
150 \\
150 \\
89 \\
340 \\
470\end{array}$ & $\begin{array}{l}460 \\
240 \\
230 \\
140 \\
610 \\
760\end{array}$ \\
\hline
\end{tabular}


Appendix 3.--Low-flow data--Continued

MUSKINGUM RIVER BASIN

LOCATION:

031370000 Kokosing River at Millwood, Ohio

Lat $40^{\circ} 23^{\prime} 51^{\prime \prime}$, long $82^{\circ} 17^{\prime} 09^{\prime \prime}$, in SE1/4 T.7 N., R.11 W., Knox County, Hydrologic Unit 05040003 , on left bank $0.4 \mathrm{mi}$ west of Millwood, $1.5 \mathrm{mi}$ upstream from Honey Run, and $2.0 \mathrm{mi}$ downstream from Jelloway Creek.

DRAINAGE AREA: $\quad 455 \mathrm{mi}^{2}$.

TRIBUTARY TO: MOhican River.

DISCHARGE DATA USED: October 1921 to September 1974.

REMARKS:

Some regulation by North Branch Kokosing River Lake, $29.0 \mathrm{mi}$ upstream on North Branch Kokosing River, beginning June 1972 .

SELECTED DISCHARGE CHARACTERISTICS: Average discharge: $481 \mathrm{ft}^{3} / \mathrm{s}$ ( 53 years).

Minimum daily discharge: $34 \mathrm{ft}^{3} / \mathrm{s}$ August 1932, September 1954 .

Magnitude and frequency of low flow for indicated periods

\begin{tabular}{|c|c|c|c|c|c|c|c|c|c|c|c|c|c|}
\hline \multirow{2}{*}{ Period } & \multirow{2}{*}{$\begin{array}{l}\text { Number of } \\
\text { consecu- } \\
\text { tive days }\end{array}$} & \multicolumn{5}{|c|}{$\begin{array}{l}\text { Discharge }\left(\mathrm{ft}^{3} / \mathrm{s}\right) \text { for indicated } \\
\text { recurrence interval (years) }\end{array}$} & \multirow{2}{*}{ Period } & \multirow{2}{*}{$\begin{array}{l}\text { Number of } \\
\text { consecu- } \\
\text { tive days }\end{array}$} & \multicolumn{5}{|c|}{$\begin{array}{l}\text { Discharge }\left(\mathrm{ft}^{3} / \mathrm{s}\right) \text { for indicated } \\
\text { recurrence interval (years) }\end{array}$} \\
\hline & & 2 & 5 & 10 & 20 & 50 & & & 2 & 5 & 10 & 20 & 50 \\
\hline Apr.-Mar. & $\begin{array}{r}1 \\
7 \\
30\end{array}$ & $\begin{array}{l}59 \\
62 \\
71\end{array}$ & $\begin{array}{l}45 \\
48 \\
54\end{array}$ & $\begin{array}{l}40 \\
42 \\
48\end{array}$ & $\begin{array}{l}36 \\
38 \\
44\end{array}$ & $\begin{array}{l}32 \\
34 \\
40\end{array}$ & Sept.-Nov. & $\begin{array}{r}1 \\
7 \\
30\end{array}$ & $\begin{array}{l}62 \\
65 \\
75\end{array}$ & $\begin{array}{l}48 \\
50 \\
57\end{array}$ & $\begin{array}{l}41 \\
44 \\
50\end{array}$ & $\begin{array}{l}37 \\
40 \\
44\end{array}$ & $\begin{array}{l}32 \\
36 \\
38\end{array}$ \\
\hline May- Nov. & $\begin{array}{r}1 \\
7 \\
30\end{array}$ & $\begin{array}{l}60 \\
62 \\
71\end{array}$ & $\begin{array}{l}46 \\
48 \\
54\end{array}$ & $\begin{array}{l}40 \\
43 \\
48\end{array}$ & $\begin{array}{l}36 \\
38 \\
44\end{array}$ & $\begin{array}{l}32 \\
34 \\
39\end{array}$ & Dec.-Feb. & $\begin{array}{r}1 \\
7 \\
30\end{array}$ & $\begin{array}{l}102 \\
118 \\
214\end{array}$ & $\begin{array}{r}66 \\
73 \\
108\end{array}$ & $\begin{array}{l}53 \\
58 \\
76\end{array}$ & $\begin{array}{l}45 \\
47 \\
56\end{array}$ & $\begin{array}{l}37 \\
38 \\
41\end{array}$ \\
\hline June-Aug. & $\begin{array}{r}1 \\
7 \\
30\end{array}$ & $\begin{array}{r}68 \\
73 \\
101\end{array}$ & $\begin{array}{l}50 \\
54 \\
69\end{array}$ & $\begin{array}{l}43 \\
46 \\
59\end{array}$ & $\begin{array}{l}38 \\
41 \\
52\end{array}$ & $\begin{array}{l}33 \\
36 \\
46\end{array}$ & Mar. - May & $\begin{array}{r}1 \\
7 \\
30\end{array}$ & $\begin{array}{l}176 \\
209 \\
328\end{array}$ & $\begin{array}{l}121 \\
139 \\
203\end{array}$ & $\begin{array}{r}97 \\
111 \\
157\end{array}$ & $\begin{array}{r}79 \\
92 \\
127\end{array}$ & $\begin{array}{l}63 \\
73 \\
99\end{array}$ \\
\hline
\end{tabular}

Duration of daily flow for indicated periods

\begin{tabular}{l|c|c|c|c|c|c|c|c|c|c|c|c|c}
\hline \multirow{2}{*}{ Period } & \multicolumn{8}{|c|}{ Discharge $\left(\mathrm{ft}^{3} / \mathrm{s}\right)$ which was equaled or exceeded for indicated percent of time } \\
\cline { 2 - 13 } & 98 & 95 & 90 & 85 & 80 & 75 & 70 & 60 & 50 & 40 & 30 & 20 & 10 \\
\hline Apr. - Mar. & 47 & 55 & 66 & 75 & 85 & 94 & 110 & 150 & 200 & 290 & 410 & 620 & 1100 \\
May - Nov. & 44 & 51 & 60 & 67 & 73 & 80 & 86 & 100 & 120 & 160 & 220 & 310 & 560 \\
June - Aug. & 46 & 54 & 64 & 73 & 81 & 88 & 96 & 120 & 140 & 170 & 210 & 300 & 520 \\
Sept.- Nov. & 41 & 46 & 53 & 59 & 64 & 68 & 72 & 80 & 89 & 100 & 120 & 170 & 290 \\
Dec. - Feb. & 54 & 64 & 78 & 96 & 120 & 150 & 170 & 230 & 310 & 420 & 580 & 830 & 1500 \\
Mar. - May & 110 & 140 & 99 & 230 & 260 & 290 & 320 & 400 & 490 & 610 & 780 & 1100 & 1700 \\
\hline
\end{tabular}


Appendix 3.--Low-flow data--Continued

MUSKINGUM RIVER BASIN

03138500 Walhonding River below Mohawk Dam, at Nellie, Ohio

LOCATION: Lat $40^{\circ} 20^{\prime} 29^{\prime \prime}$, long $82^{\circ} 03^{\prime} 56^{\prime \prime}$, in T.6 N., R.8 W., Coshocton County, Hydrologic Unit 05040003 , on right bank at upstream side of bridge on U.S. Highway 36 at Nellie, $0.5 \mathrm{mi}$ upstream from Mohawk Creek, and $1.7 \mathrm{mi}$ downstream from Mohawk Dam.

DRAINAGE AREA: $\quad 1,505 \mathrm{mi}^{2}$.

TRIBUTARY TO: Muskingum River.

DISCHARGE DATA USED: October 1937 to September 1978.

REMARKS: $\quad$ Flow regulated beginning 1936 by 5 flood-control reservoirs at points $1.7 \mathrm{mi}$ to $54 \mathrm{mi}$ upstream.

SELECTED DISCHARGE CHARACTERISTICS: Average discharge: $1,486 \mathrm{ft}^{3} / \mathrm{s}$ (41 years). Minimum daily discharge: $19 \mathrm{ft}^{3} / \mathrm{s}$ February, 1954.

Magnitude and frequency of low flow for indicated periods

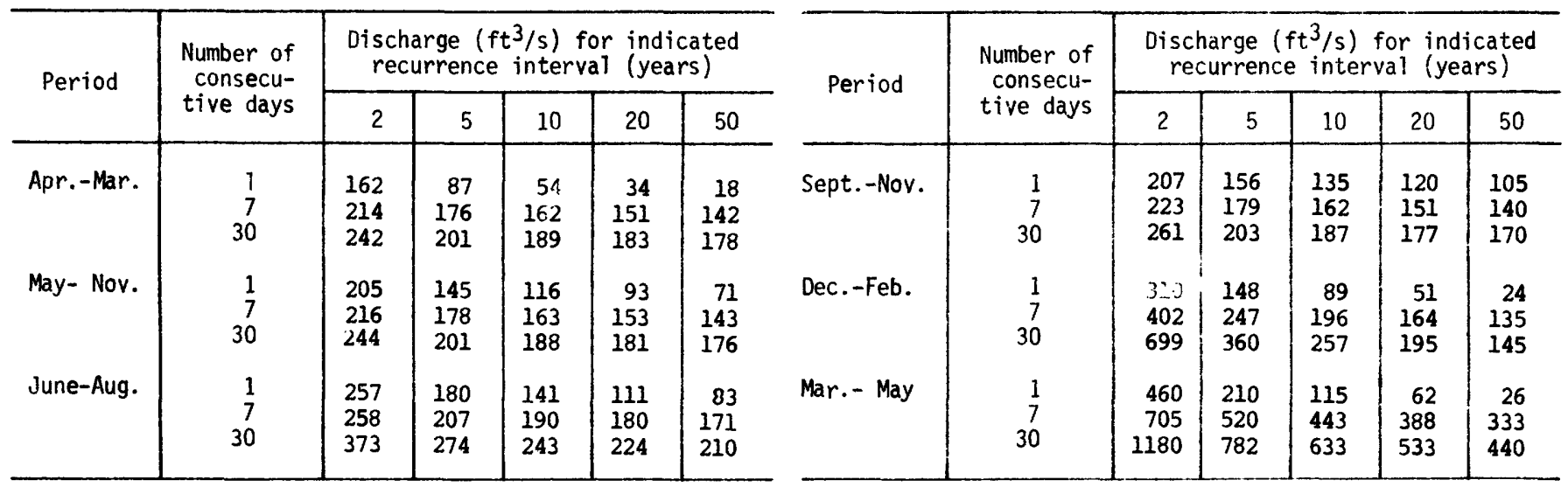

Duration of daily flow for indicated periods

\begin{tabular}{l|r|r|r|r|r|r|r|r|r|r|r|r|r}
\hline \multirow{2}{*}{ Period } & \multicolumn{8}{|c|}{ Discharge $\left(\mathrm{ft}^{3} / \mathrm{s}\right)$} & which was equaled or exceeded for indicated percent of time \\
\cline { 2 - 12 } & 98 & 95 & 90 & 85 & 80 & 75 & 70 & 60 & 50 & 40 & 30 & 20 & 10 \\
\hline Apr. - Mar. & 180 & 210 & 240 & 270 & 310 & 350 & 400 & 540 & 730 & 1000 & 1500 & 2200 & 4000 \\
May - Nov. & 180 & 200 & 220 & 250 & 270 & 290 & 320 & 380 & 470 & 610 & 800 & 1200 & 2100 \\
June - Aug. & 200 & 220 & 260 & 280 & 300 & 330 & 360 & 420 & 510 & 630 & 810 & 1200 & 2000 \\
Sept.- Nov. & 160 & 180 & 200 & 210 & 230 & 240 & 260 & 290 & 330 & 390 & 490 & 660 & 1000 \\
Dec. - Feb. & 190 & 230 & 280 & 330 & 400 & 480 & 570 & 760 & 1000 & 1400 & 1900 & 2900 & 5100 \\
Mar. - May & 420 & 550 & 690 & 820 & 940 & 1100 & 1200 & 1500 & 1900 & 2400 & 3200 & 4300 & 5900 \\
\hline
\end{tabular}


Appendix 3.--Low-flow data--Continued

MUSKINGUM RIVER BASIN

03138790 Killbuck Creek at Burbank, Ohio

LOCATION:

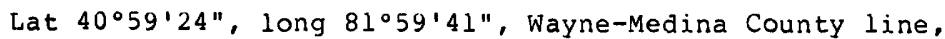
Hydrologic Unit 05040003 , at bridge on State koute 76 at at Burbank, Ohio.

DRAINAGE AREA: $\quad 42.4 \mathrm{mi}^{2}$.

TRIBUTARY TO: Walhonding River.

DISCHARGE DATA USED: Low-flow measurements 1975-current water years.

INDEX STATION: $\quad$ U3139000 Killbuck Creek at Killbuck.

REMARKS: Three medium-flow measurements and one low-flow measurement is taken annually.

SELECTED DISCHARGE CHARACTERISTICS: Minimum flow observed: $0.9 \mathrm{Et} 3 / \mathrm{s}$ september 1273.

\begin{tabular}{c|r|c|c}
\multicolumn{4}{c}{ Magnitude and frequency of low flow for } \\
indicated periods \\
\hline Period & $\begin{array}{c}\text { Number of } \\
\text { consecu- } \\
\text { tive days }\end{array}$ & $\begin{array}{c}\text { Discharge (ft } 3 / \mathrm{s} \text { ) for } \\
\text { indicated recurrence } \\
\text { interval (years) }\end{array}$ \\
\cline { 3 - 4 } & & 2 & 10 \\
\hline Apr. - Mar. & 7 & 0.4 & 0.1 \\
May - Nov. & 30 & 0.6 & 0.2 \\
June - Aug. & 7 & 0.4 & 0.1 \\
& 30 & 0.6 & 0.2 \\
Sept. - Nov. & 7 & 0.7 & 0.2 \\
& 7 & 1.8 & 0.4 \\
& 30 & 0.4 & 0.1 \\
\end{tabular}

Duration of daily flow for indicated periods

\begin{tabular}{|c|c|c|c|c|c|}
\hline \multirow{2}{*}{ Period } & \multicolumn{5}{|c|}{$\begin{array}{l}\text { Discharge }\left(\mathrm{ft}^{3} / \mathrm{s}\right) \text { which was equaled or } \\
\text { exceeded for indicated percent of time }\end{array}$} \\
\hline & 98 & 95 & 90 & 85 & 80 \\
\hline $\begin{array}{l}\text { Apr. - Mar. } \\
\text { May - Nov. } \\
\text { June - Aug. } \\
\text { Sept. - Nov. }\end{array}$ & $\begin{array}{l}0.2 \\
0.1 \\
0.2 \\
0.1\end{array}$ & $\begin{array}{l}0.3 \\
0.2 \\
0.3 \\
0.2\end{array}$ & $\begin{array}{l}0.5 \\
0.4 \\
0.4 \\
0.3\end{array}$ & $\begin{array}{l}0.7 \\
0.5 \\
0.6 \\
0.3\end{array}$ & $\begin{array}{l}1.0 \\
0.6 \\
0.8 \\
0.4\end{array}$ \\
\hline
\end{tabular}


Appendix 3.--Low-flow data--Continued

MUSKINGUM RIVER BASIN

03138800 Killbuck Creek at Wooster, Ohio

LOCATION:

Lat $40^{\circ} 48^{\prime} 05^{\prime \prime}$, long $81^{\circ} 58^{\prime} 30^{\prime \prime}$, Wayne County, Hydrologic Unit 05040003 , at bridge on old Mansfield Road, $2.0 \mathrm{mi}$ northwest of Wooster.

DRAINAGE AREA: $\quad 128 \mathrm{mi}^{2}$.

TRIBUTARY TO: Walhonding River.

DISCHARGE DATA USED: Low-flow measurements 1959, 1962-1978 water years.

INDEX STATION: $\quad 03139000$ Killbuck Creek at Killbuck, Ohio.

REMARKS: None.

SELECTED DISCHARGE CHARACTERISTICS: Minimum discharge observed: $1.5 \mathrm{ft}^{3} / \mathrm{s}$ September 1963.

\begin{tabular}{c|c|c|c}
\multicolumn{4}{c}{$\begin{array}{c}\text { Magnitude and frequency of low flow for } \\
\text { indicated periods }\end{array}$} \\
\hline & $\begin{array}{c}\text { Number of } \\
\text { consecu- } \\
\text { tive days }\end{array}$ & $\begin{array}{c}\text { Discharge (ft }{ }^{3} / \mathrm{s} \text { ) for } \\
\text { indicated recurrence } \\
\text { interval (years) }\end{array}$ \\
\cline { 3 - 4 } & 7 & 2 & 10 \\
\hline Apr. - Mar. & 30 & 3.8 & 2.0 \\
May - Nov. & 7 & 3.8 & 2.5 \\
& 30 & 4.9 & 2.0 \\
June - Aug. & 7 & 5.0 & 2.5 \\
& 30 & 8.8 & 2.4 \\
Sept. - Nov. & 7 & 4.0 & 3.7 \\
& 30 & 5.5 & 2.1 \\
\hline
\end{tabular}

Duration of daily flow for indicated periods

\begin{tabular}{l|c|c|c|c|c}
\hline \multirow{2}{*}{ Period } & \multicolumn{6}{|c}{$\begin{array}{c}\text { Discharge }\left(\mathrm{ft}^{3} / \mathrm{s} \text { ) which was equaled or }\right. \\
\text { exceeded for indicated percent of time }\end{array}$} \\
\cline { 2 - 6 } & 98 & 95 & 90 & 85 & 80 \\
\hline Apr. - Mar. & 2.6 & 3.3 & 4.2 & 5.3 & 6.5 \\
May - Nov. & 2.2 & 2.9 & 3.6 & 4.1 & 4.9 \\
June - Aug. & 2.6 & 3.2 & 3.9 & 4.7 & 5.6 \\
Sept. - Nov. & 2.0 & 2.5 & 3.1 & 3.5 & 3.9 \\
\hline
\end{tabular}




\section{MUSKINGUM RIVER BASIN}

03139000 Killbuck Creek at Killbuck, Ohio

LOCATION:

Lat $40^{\circ} 28^{\prime} 53^{\prime \prime}$, long $81^{\circ} 59^{\prime} 10^{\prime \prime}$, Holmes County, Hydrologic Unit 05040003 , on right bank at downstream side of U.S. Highway 62 bridge south of Killbuck, $1.2 \mathrm{mi}$ downstream from Black Creek. Prior to Oct. 5, 1976, at site $0.9 \mathrm{mi}$ upstream.

DRAINAGE AREA:

$464 \mathrm{mi}^{2}$.

TRIBUTARY TO:

Walhonding River.

DISCHARGE DATA USED:

October 1930 to September 1978.

REMARKS:

None.

SELECTED DISCHARGE CHARACTERISTICS: Average discharge: $402 \mathrm{ft}^{3} / \mathrm{s}$ (48 years).

Minimum daily discharge: $23 \mathrm{ft}^{3} / \mathrm{s}$ September 1954 .

Magnitude and frequency of low flow for indicated periods

\begin{tabular}{|c|c|c|c|c|c|c|}
\hline \multirow{2}{*}{ Period } & \multirow{2}{*}{$\begin{array}{l}\text { Number of } \\
\text { consecu- } \\
\text { tive days }\end{array}$} & \multicolumn{5}{|c|}{$\begin{array}{l}\text { Discharge }\left(\mathrm{ft}^{3} / \mathrm{s}\right) \text { for indicated } \\
\text { recurrence interval (years) }\end{array}$} \\
\hline & & 2 & 5 & 10 & 20 & 50 \\
\hline Apr. - Mar. & $\begin{array}{r}1 \\
7 \\
30\end{array}$ & $\begin{array}{l}44 \\
47 \\
57\end{array}$ & $\begin{array}{l}32 \\
34 \\
40\end{array}$ & $\begin{array}{l}28 \\
29 \\
34\end{array}$ & $\begin{array}{l}25 \\
26 \\
30\end{array}$ & $\begin{array}{l}22 \\
23 \\
26\end{array}$ \\
\hline May- Nov. & $\begin{array}{r}1 \\
7 \\
30\end{array}$ & $\begin{array}{l}44 \\
47 \\
57\end{array}$ & $\begin{array}{l}32 \\
34 \\
40\end{array}$ & $\begin{array}{l}28 \\
29 \\
34\end{array}$ & $\begin{array}{l}25 \\
26 \\
30\end{array}$ & $\begin{array}{l}22 \\
23 \\
26\end{array}$ \\
\hline June-Aug. & $\begin{array}{r}1 \\
7 \\
30\end{array}$ & $\begin{array}{l}52 \\
58 \\
88\end{array}$ & $\begin{array}{l}37 \\
40 \\
57\end{array}$ & $\begin{array}{l}31 \\
33 \\
46\end{array}$ & $\begin{array}{l}27 \\
29 \\
39\end{array}$ & $\begin{array}{l}23 \\
25 \\
33\end{array}$ \\
\hline
\end{tabular}

\begin{tabular}{|c|c|c|c|c|c|c|}
\hline \multirow{2}{*}{ Period } & \multirow{2}{*}{$\begin{array}{l}\text { Number of } \\
\text { consecu- } \\
\text { tive days }\end{array}$} & \multicolumn{5}{|c|}{$\begin{array}{l}\text { Discharge }\left(\mathrm{ft}^{3} / \mathrm{s}\right) \text { for indicated } \\
\text { recurrence interva1 (years) }\end{array}$} \\
\hline & & 2 & 5 & 10 & 20 & 50 \\
\hline Sept.-Nov. & $\begin{array}{r}1 \\
7 \\
30\end{array}$ & $\begin{array}{l}46 \\
49 \\
62\end{array}$ & $\begin{array}{l}33 \\
35 \\
41\end{array}$ & $\begin{array}{l}28 \\
30 \\
35\end{array}$ & $\begin{array}{l}25 \\
27 \\
30\end{array}$ & $\begin{array}{l}23 \\
24 \\
27\end{array}$ \\
\hline Dec.-Feb. & $\begin{array}{r}1 \\
7 \\
30\end{array}$ & $\begin{array}{r}81 \\
92 \\
156\end{array}$ & $\begin{array}{l}51 \\
56 \\
78\end{array}$ & $\begin{array}{l}41 \\
44 \\
56\end{array}$ & $\begin{array}{l}35 \\
37 \\
43\end{array}$ & $\begin{array}{l}30 \\
31 \\
32\end{array}$ \\
\hline Mar. - May & $\begin{array}{r}1 \\
7 \\
30\end{array}$ & $\begin{array}{l}149 \\
183 \\
313\end{array}$ & $\begin{array}{l}101 \\
122 \\
195\end{array}$ & $\begin{array}{r}81 \\
98 \\
149\end{array}$ & $\begin{array}{r}67 \\
80 \\
118\end{array}$ & $\begin{array}{l}53 \\
64 \\
91\end{array}$ \\
\hline
\end{tabular}

Duration of daily flow for indicated periods

\begin{tabular}{|c|c|c|c|c|c|c|c|c|c|c|c|c|c|c|}
\hline \multirow{2}{*}{\multicolumn{2}{|c|}{ Period }} & \multicolumn{13}{|c|}{ Discharge $\left(\mathrm{ft}^{3} / \mathrm{s}\right)$ which was equaled or exceeded for indicated percent of time } \\
\hline & & 98 & 95 & 90 & 85 & 80 & 75 & 70 & 60 & 50 & 40 & 30 & 20 & 10 \\
\hline $\begin{array}{l}\text { Apr. - } \\
\text { May - } \\
\text { June - } \\
\text { Sept. - } \\
\text { Dec. - } \\
\text { Mar. - }\end{array}$ & $\begin{array}{l}\text { Mar. } \\
\text { Nov. } \\
\text { Aug. } \\
\text { Nov. } \\
\text { Feb. } \\
\text { May }\end{array}$ & $\begin{array}{r}35 \\
31 \\
35 \\
29 \\
43 \\
100\end{array}$ & $\begin{array}{r}42 \\
38 \\
41 \\
34 \\
54 \\
130\end{array}$ & $\begin{array}{r}51 \\
45 \\
48 \\
40 \\
65 \\
180\end{array}$ & $\begin{array}{r}60 \\
50 \\
55 \\
44 \\
78 \\
210\end{array}$ & $\begin{array}{r}70 \\
57 \\
63 \\
48 \\
90 \\
250\end{array}$ & $\begin{array}{r}81 \\
63 \\
71 \\
52 \\
100 \\
280\end{array}$ & $\begin{array}{r}94 \\
71 \\
79 \\
56 \\
120 \\
320\end{array}$ & $\begin{array}{r}130 \\
89 \\
98 \\
66 \\
170 \\
390\end{array}$ & $\begin{array}{r}180 \\
110 \\
120 \\
78 \\
250 \\
490\end{array}$ & $\begin{array}{r}260 \\
150 \\
150 \\
94 \\
350 \\
610\end{array}$ & $\begin{array}{l}380 \\
200 \\
200 \\
120 \\
480 \\
800\end{array}$ & $\begin{array}{r}580 \\
290 \\
290 \\
160 \\
750 \\
1100\end{array}$ & $\begin{array}{r}1000 \\
510 \\
500 \\
270 \\
1300 \\
1500\end{array}$ \\
\hline
\end{tabular}


LOCATION:

03140000 Mill Creek near Coshocton, Ohio

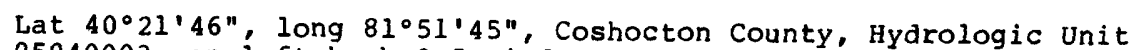

05040003 , on left bank $0.5 \mathrm{mi}$ downstream from Little Mili Creek and

$6 \mathrm{mi}$ north of Coshocton.

DRAINAGE AREA: $\quad 27.2 \mathrm{mi}^{2}$.

TRIBITARY TO:

Walhonding River.

DISCHARGE DATA USED: November 1936 to September 1978.

REMARKS:

None.

SELECTED DISCHARGE CHARACTERISTICS: Average discharge: $27.4 \mathrm{ft}^{3} / \mathrm{s}$ ( 41 years).

Minimum daily discharge: No flow September 1954 to August 1962.

Magnitude and frequency of low flow for indicated periods

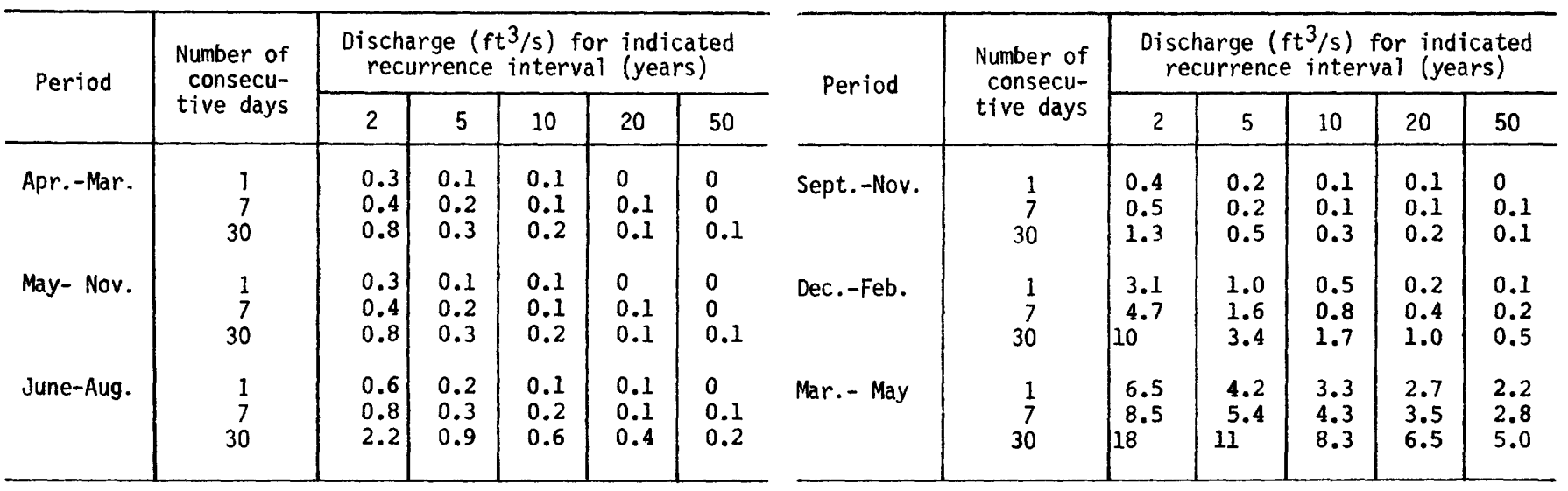

Duration of daily flow for indicated periods

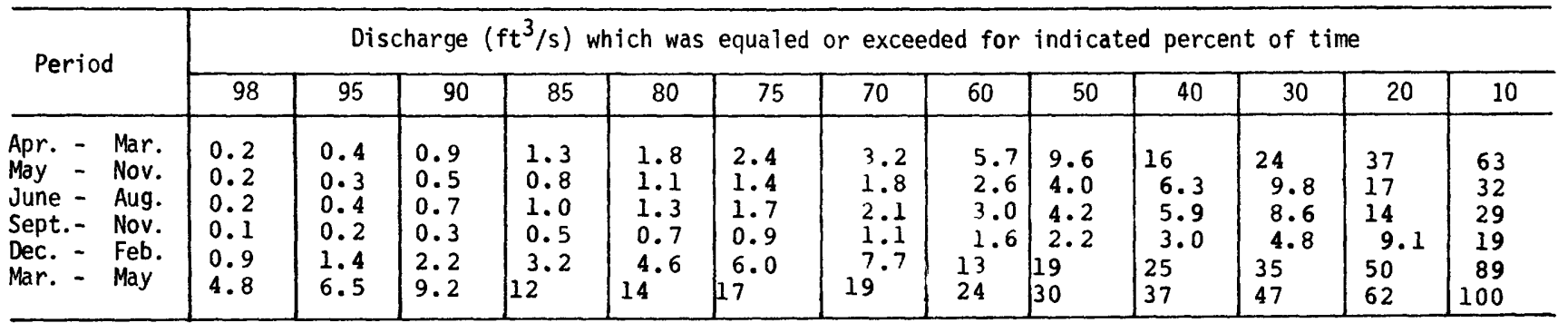


MUSKINGUM RIVER BASIN

03140500 Muskingum River near Coshocton, Ohio

LOCATION: Lat $40^{\circ} 14^{\prime} 54^{\prime \prime}$, long $81^{\circ} 52^{\prime} 23^{\prime \prime}$, in T.5 N., R.6 W., Coshocton County, Hydrologic Unit 05040004 , on right bank at upstream side of highway bridge, $1 \mathrm{mi}$ southwest of Coshocton, and $2 \mathrm{mi}$ downstream from confluence of Tuscarawas and Walhonding Rivers.

DRAIMAGE AREA: $\quad 4,859 \mathrm{mi}^{2}$.

TRIBUTARY TO: Ohio River.

DISCHARGE DATA USED: October 1937 to September 1978.

REY'ARKS:

Flow regulated by 13 flood-control reservoirs at points $19 \mathrm{mi}$ to $88 \mathrm{mi}$ upstream.

SELECTED DISCHARGE CHARACTERISTICS: Average discharge: $4,805 \mathrm{ft}^{3} / \mathrm{s}$ (41 years).

Minimum daily discharge: $420 \mathrm{ft} 3 / \mathrm{s}$ September, 1954.

Magnitude and frequency of low flow for indicated periods

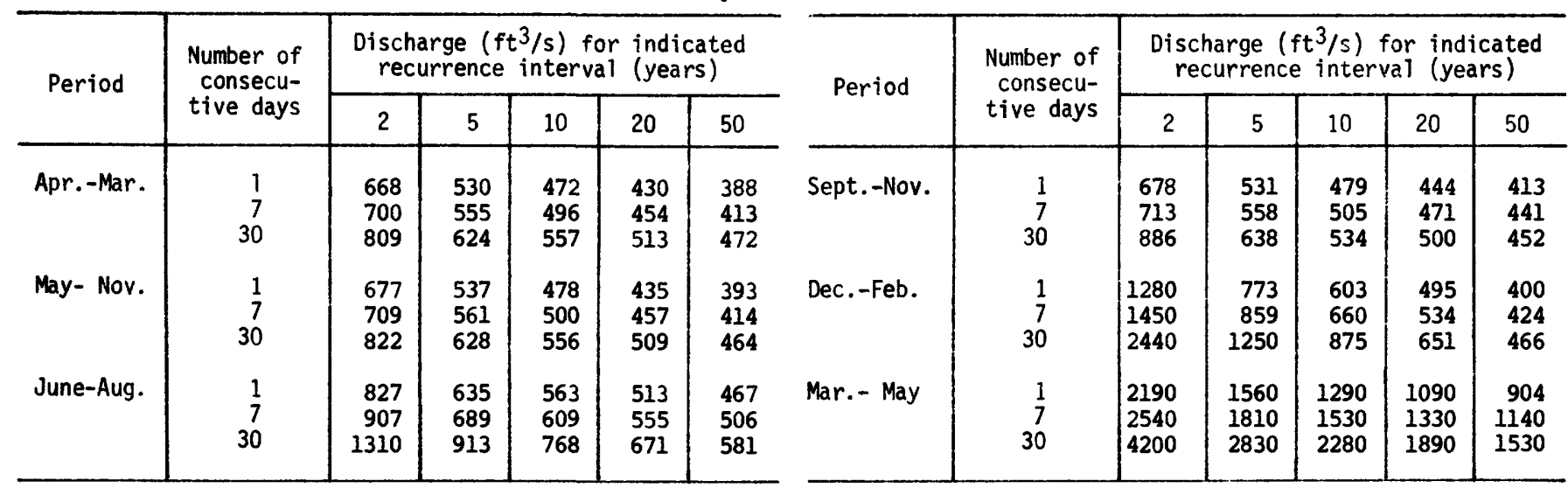

Duration of daily flow for indicated periods

\begin{tabular}{l|r|r|r|r|r|r|r|r|r|r|r|r|r}
\hline \multirow{2}{*}{ Period } & \multicolumn{8}{|c|}{ Discharge $\left(\mathrm{ft}^{3} / \mathrm{s}\right.$ ) which was equaled or exceeded for indicated percent of time } \\
\cline { 2 - 12 } & 98 & 95 & 90 & 85 & 80 & 75 & 70 & 60 & 50 & 40 & 30 & 20 & 10 \\
\hline Apr. - Mar. & 560 & 660 & 800 & 920 & 1100 & 1300 & 1500 & 2000 & 2700 & 3700 & 5100 & 7600 & 13000 \\
May - Nov. & 530 & 610 & 720 & 800 & 890 & 990 & 1100 & 1400 & 1700 & 2200 & 2900 & 4100 & 6700 \\
June - Aug. & 610 & 720 & 840 & 950 & 1100 & 1200 & 1300 & 1500 & 1900 & 2300 & 2900 & 3900 & 6100 \\
Sept.- Nov. & 500 & 540 & 610 & 680 & 740 & 790 & 840 & 960 & 1200 & 1500 & 1900 & 2500 & 3800 \\
Dec. - Feb. & 620 & 780 & 950 & 1200 & 1500 & 1800 & 2000 & 2700 & 3800 & 5000 & 6700 & 10000 & 16000 \\
Mar. - May & 1600 & 2000 & 2600 & 3100 & 3500 & 4000 & 4400 & 5500 & 6800 & 8300 & 10000 & 12000 & 17000 \\
\hline
\end{tabular}


LOCATION:

Lat $39^{\circ} 55^{\prime} 28^{\prime \prime}$, long $81^{\circ} 26^{\prime} 17^{\prime \prime}$, Guernsey County, Hydrologic

Unit 05040005 , on left bank $650 \mathrm{ft}$ downstream from Senecaville

Dam, and $1.5 \mathrm{mi}$ southeast of Senecaville.

DRAINAGE AREA: $\quad 118 \mathrm{mi}^{2}$.

TRIBUTARY T0: Wills Creek.

DISCHARGE DATA USED: September 1938 to september 1978.

REMARKS:

Flow regulated by Senecaville Lake. Water is diverted from Senecaville Lake for U.S. Fish Hatchery.

SELECTED DISCHARGE CHARACTERISTICS: Average discharge: $128 \mathrm{ft}^{3} / \mathrm{s}$ (40 years).

Minimum daily discharge: No flow January, February, April, 1952.

Magnitude and frequency of low flow for indicated periods

\begin{tabular}{|c|c|c|c|c|c|c|}
\hline \multirow{2}{*}{ Period } & \multirow{2}{*}{$\begin{array}{l}\text { Number of } \\
\text { consecu- } \\
\text { tive days }\end{array}$} & \multicolumn{5}{|c|}{$\begin{array}{l}\text { Discharge }\left(\mathrm{ft}^{3} / \mathrm{s}\right) \text { for indicated } \\
\text { recurrence interval (years) }\end{array}$} \\
\hline & & 2 & 5 & 10 & 20 & 50 \\
\hline Apr.-Mar. & $\begin{array}{r}1 \\
7 \\
30\end{array}$ & $\begin{array}{l}0.7 \\
2.2 \\
3.1\end{array}$ & $\begin{array}{l}0.2 \\
0.9 \\
1.7\end{array}$ & $\begin{array}{l}0.1 \\
0.5 \\
1.3\end{array}$ & $\begin{array}{l}0 \\
0.3 \\
1.0\end{array}$ & $\begin{array}{l}0 \\
0.2 \\
0.8\end{array}$ \\
\hline May- Nov. & $\begin{array}{r}1 \\
7 \\
30\end{array}$ & $\begin{array}{l}1.6 \\
2.5 \\
3.4\end{array}$ & $\begin{array}{l}0.6 \\
1.0 \\
1.8\end{array}$ & $\begin{array}{l}0.4 \\
0.6 \\
1.3\end{array}$ & $\begin{array}{l}0.2 \\
0.4 \\
1.0\end{array}$ & $\begin{array}{l}0.1 \\
0.2 \\
0.8\end{array}$ \\
\hline June-Aug. & $\begin{array}{r}1 \\
7 \\
30\end{array}$ & $\begin{array}{l}3.1 \\
5.2 \\
14\end{array}$ & $\begin{array}{l}1.1 \\
1.8 \\
5.3\end{array}$ & $\begin{array}{l}0.7 \\
1.0 \\
3.2\end{array}$ & $\begin{array}{l}0.5 \\
0.6 \\
2.2\end{array}$ & $\begin{array}{l}0.3 \\
0.3 \\
1.4\end{array}$ \\
\hline
\end{tabular}

\begin{tabular}{|c|c|c|c|c|c|c|}
\hline \multirow{2}{*}{ Period } & \multirow{2}{*}{$\begin{array}{l}\text { Number of } \\
\text { consecu- } \\
\text { tive days }\end{array}$} & \multicolumn{5}{|c|}{$\begin{array}{l}\text { Discharge }\left(\mathrm{ft}^{3} / \mathrm{s}\right) \text { for indicated } \\
\text { recurrence interval (years) }\end{array}$} \\
\hline & & 2 & 5 & 10 & 20 & 50 \\
\hline Sept.-Nov. & $\begin{array}{r}1 \\
7 \\
30\end{array}$ & $\begin{array}{l}2.1 \\
2.9 \\
4.0\end{array}$ & $\begin{array}{l}1.0 \\
1.3 \\
1.8\end{array}$ & $\begin{array}{l}0.7 \\
0.8 \\
1.3\end{array}$ & $\begin{array}{l}0.5 \\
0.6 \\
1.1\end{array}$ & $\begin{array}{l}0.3 \\
0.4 \\
0.9\end{array}$ \\
\hline Dec. -Feb. & $\begin{array}{r}1 \\
7 \\
30\end{array}$ & $\begin{array}{l}2.0 \\
7.4 \\
48\end{array}$ & $\begin{array}{l}0.4 \\
2.0 \\
11\end{array}$ & $\begin{array}{l}0.2 \\
1.0 \\
4.3\end{array}$ & $\begin{array}{l}0.1 \\
0.6 \\
1.8\end{array}$ & $\begin{array}{l}0 \\
0.3 \\
0.6\end{array}$ \\
\hline Mar. - May & $\begin{array}{r}1 \\
7 \\
30\end{array}$ & $\begin{array}{l}2.3 \\
14 \\
76\end{array}$ & $\begin{array}{r}0.6 \\
4.0 \\
21\end{array}$ & $\begin{array}{l}0.3 \\
2.0 \\
9.0\end{array}$ & $\begin{array}{l}0.1 \\
1.1 \\
4.0\end{array}$ & $\begin{array}{l}0 \\
0.6 \\
1.4\end{array}$ \\
\hline
\end{tabular}

Duration of daily flow for indicated periods

\begin{tabular}{l|c|c|c|c|c|c|c|c|c|c|c|c|c}
\hline \multirow{2}{*}{ Period } & \multicolumn{8}{|c}{ Discharge $\left(\mathrm{ft}^{3} / \mathrm{s}\right)$ which was equaled or exceeded for indicated percent of time } \\
\cline { 2 - 12 } & 98 & 95 & 90 & 85 & 80 & 75 & 70 & 60 & 50 & 40 & 30 & 20 & 10 \\
\hline Apr. - Mar. & 1.5 & 1.9 & 2.5 & 3.0 & 3.6 & 5.0 & 8.1 & 21 & 44 & 78 & 130 & 240 & 430 \\
May - Nov. & 1.5 & 1.9 & 2.3 & 2.7 & 3.2 & 3.8 & 5.0 & 11 & 22 & 39 & 64 & 110 & 260 \\
June - Aug. & 1.8 & 2.2 & 2.7 & 3.3 & 3.9 & 5.6 & 8.0 & 17 & 27 & 41 & 58 & 86 & 190 \\
Sept. - Nov. & 1.3 & 1.7 & 2.0 & 2.2 & 2.5 & 2.8 & 3.2 & 4.3 & 8.2 & 15 & 27 & 76 & 220 \\
Dec. - Feb. & 1.5 & 2.0 & 2.6 & 3.3 & 4.7 & 12 & 24 & 54 & 98 & 160 & 250 & 380 & 530 \\
Mar. - May & 1.8 & 2.5 & 3.9 & 7.2 & 26 & 45 & 58 & 88 & 120 & 160 & 260 & 400 & 550 \\
\hline
\end{tabular}


Appendix 3.--Low-flow data--Continued

MUSKINGUM RIVER BASIN

03141900 Leatherwood Creek near Cambridge, Ohio

LOCATION:

Lat $40^{\circ} 01^{\prime} 15^{\prime \prime}$, long $81^{\circ} 32^{\prime} 5^{\prime \prime}$, Guernsey County, Hydrologic Unit 05040005, at bridge on County Road 461, $2.3 \mathrm{mi}$ east of Cambridge, and $3.5 \mathrm{mi}$ upstream from mouth.

DRAINAGE AREA: $\quad 88 \mathrm{mi}^{2}$.

TRIBUTARY TO: Wills Creek.

DISCHARGE DATA USED: Low-flow measurements 1959, 1962-1967, 1969-1973 water years.

INDEX STATION: $\quad 03149500$ Salt Creek near Chandlersville, Ohio.

REMARKS: None.

SELECTED DISCHARGE CHARACTERISTICS: Minimum discharge observed: $0.4 \mathrm{ft}^{3} / \mathrm{s}$ October. 1963.

Magnitude and frequency of low flow for indicated periods

\begin{tabular}{c|c|c|c}
\hline \multirow{2}{*}{ Period } & \multirow{2}{*}{$\begin{array}{c}\text { Number of } \\
\text { consecu- } \\
\text { tive days }\end{array}$} & $\begin{array}{c}\text { Discharge }\left(\mathrm{ft}^{3} / \mathrm{s}\right) \text { for } \\
\text { ind icated recurrence } \\
\text { interval (years) }\end{array}$ \\
\cline { 3 - 4 } & & 2 & 10 \\
\hline Apr. - Mar. & 7 & 0.8 & 0.2 \\
& 30 & 2.5 & 0.8 \\
May - Nov. & 7 & 0.8 & 0.1 \\
& 30 & 2.5 & 0.8 \\
June - Aug. & 7 & 2.5 & 0.1 \\
& 30 & 10 & 2.4 \\
Sept. - Nov. & 7 & 1.0 & 0.2 \\
& 30 & 3.9 & 1.0 \\
\hline
\end{tabular}

Duration of daily flow for indicated periods

\begin{tabular}{l|c|c|c|c|c}
\hline \multirow{2}{*}{ Period } & \multicolumn{6}{|c}{$\begin{array}{c}\text { Discharge }\left(\mathrm{ft}^{3} / \mathrm{s} \text { ) which was equaled or }\right. \\
\text { exceeded for indicated percent of time }\end{array}$} \\
\cline { 2 - 6 } & 98 & 95 & 90 & 85 & 80 \\
\hline Apr. - Mar. & 0.7 & 1.7 & 3.1 & 4.4 & 5.9 \\
May - Nov. & 0.5 & 1.0 & 2.0 & 2.9 & 3.8 \\
June - Aug. & 0.6 & 1.3 & 2.4 & 3.3 & 4.4 \\
Sept. - Nov. & 0.4 & 0.7 & 1.3 & 2.0 & 2.7 \\
\hline
\end{tabular}


MUSKINGUM RIVER BASIN

03142000 Wills Creek at Cambridge, Ohio

LOCATION:

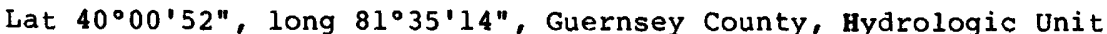

05040005 , on left bank at upstream side of bridge on Campbell Avenue in Cambridge, $0.9 \mathrm{mi}$ downstream from Leatherwood creek.

DRAINAGE AREA: $\quad 406 \mathrm{mi}^{2}$.

TRIBITARY TO: Muskingum River.

DISCHARGE DATA USED: October 1937 to September 1978.

REMARKS :

Flow regulated by Senecaville Lake on Seneca Fork, $22 \mathrm{mi}$ upstream, beginning in 1937. Water is diverted $2.7 \mathrm{mi}$ upstream from station for municipal supply of city of Cambridge.

SELECTED DISCHARGE CHARACTERISTICS: Average discharge: $431 \mathrm{ft}^{3} / \mathrm{s}$ (41 years).

Minimum daily discharge: $0.7 \mathrm{ft}^{3} / \mathrm{s}$ October, 1960.

Magnitude and frequency of low flow for indicated periods

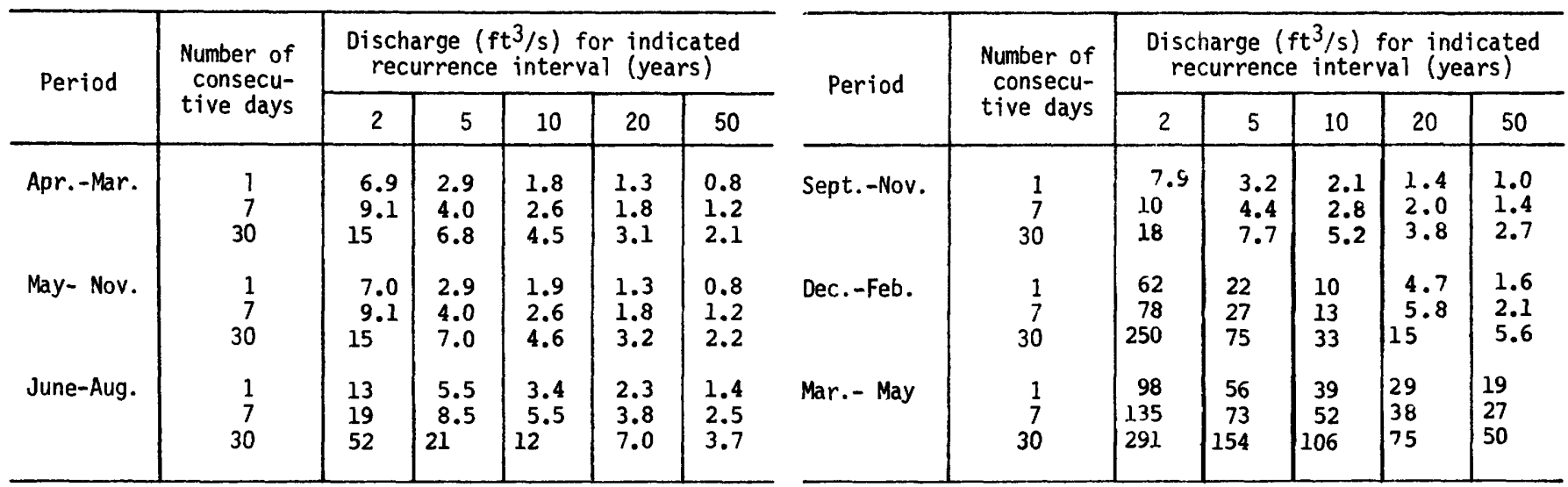

Duration of daily flow for indicated periods

\begin{tabular}{|c|c|c|c|c|c|c|c|c|c|c|c|c|c|c|}
\hline \multirow{2}{*}{\multicolumn{2}{|c|}{ Period }} & \multicolumn{13}{|c|}{ Discharge $\left(\mathrm{ft}^{3} / \mathrm{s}\right)$ which was equaled or exceeded for indicated percent of time } \\
\hline & & 98 & 95 & 90 & 85 & 80 & 75 & 70 & 60 & 50 & 40 & 30 & 20 & 10 \\
\hline $\begin{array}{l}\text { Apr. - } \\
\text { May - } \\
\text { June - } \\
\text { Sept. - } \\
\text { Dec. - } \\
\text { Mar. - }\end{array}$ & $\begin{array}{l}\text { Mar. } \\
\text { Nov. } \\
\text { Aug. } \\
\text { Nov. } \\
\text { Feb. } \\
\text { May }\end{array}$ & $\begin{array}{l}4.0 \\
3.3 \\
4.1 \\
2.9 \\
8.8 \\
56\end{array}$ & $\begin{array}{l}7.9 \\
5.3 \\
8.1 \\
3.8 \\
22 \\
90\end{array}$ & $\begin{array}{c}16 \\
11 \\
16 \\
6.0 \\
48 \\
130\end{array}$ & $\begin{array}{c}24 \\
16 \\
22 \\
9.0 \\
66 \\
160\end{array}$ & $\begin{array}{r}35 \\
20 \\
29 \\
13 \\
94 \\
200\end{array}$ & $\begin{array}{r}48 \\
26 \\
36 \\
16 \\
120 \\
240\end{array}$ & $\begin{array}{r}63 \\
33 \\
43 \\
19 \\
160 \\
280\end{array}$ & $\begin{array}{r}110 \\
52 \\
61 \\
26 \\
240 \\
380\end{array}$ & $\begin{array}{r}170 \\
79 \\
82 \\
38 \\
380 \\
520\end{array}$ & $\begin{array}{r}280 \\
120 \\
110 \\
58 \\
570 \\
690\end{array}$ & $\begin{array}{r}460 \\
180 \\
160 \\
99 \\
790 \\
890\end{array}$ & $\begin{array}{r}750 \\
320 \\
280 \\
190 \\
1100 \\
1200\end{array}$ & $\begin{array}{r}1200 \\
660 \\
600 \\
410 \\
1600 \\
1700\end{array}$ \\
\hline
\end{tabular}


LOCATION:

03143500 Wills Creek below Wills Creek Dam, at Wills Creek, Ohio

Lat $40^{\circ} 09^{\prime} 34^{\prime \prime}$, long $81^{\circ} 50^{\prime} 51^{\prime \prime}$, in sec.22, T.4 N., R.6 W., Coshocton

County, Hydrologic Unit 05040005 , on left bank 1,200 ft downstream

from Wills Creek Dam, $1.3 \mathrm{mi}$ southeast of town of Wills Creek, $2.7 \mathrm{mi}$ southeast of Conesville, and $6.2 \mathrm{mi}$ upstream from mouth.

DRAINAGE AREA:

$842 \mathrm{mi}^{2}$

TRIBUTARY TO: Muskingum River.

DISCHARGE DATA USED: October 1938 to September 1978.

REMARKS:

Flow regulated by Senecaville Lake on Seneca Fork, $80 \mathrm{mi}$ upstream, Salt Fork Reservoir, $43 \mathrm{mi}$ upstream, and Wills Creek Lake, $0.2 \mathrm{mi}$ upstream.

SELECTED DISCHARGE CHARACTERISTICS: Average discharge: $909 \mathrm{ft}^{3} / \mathrm{s}$ (40 years). Minimum daily discharge: $1.0 \mathrm{ft}^{3} / \mathrm{s}$ many times.

Magnitude and frequency of low flow for indicated periods

\begin{tabular}{|c|c|c|c|c|c|c|c|c|c|c|c|c|c|}
\hline \multirow{2}{*}{ Period } & \multirow{2}{*}{$\begin{array}{l}\text { Number of } \\
\text { consecu- } \\
\text { tive days }\end{array}$} & \multicolumn{5}{|c|}{$\begin{array}{l}\left.\text { Discharge ( } \mathrm{ft}^{3} / \mathrm{s}\right) \text { for indicated } \\
\text { recurrence interval (years) }\end{array}$} & \multirow{2}{*}{ Period } & \multirow{2}{*}{$\begin{array}{l}\text { Number of } \\
\text { consecu- } \\
\text { tive days }\end{array}$} & \multicolumn{5}{|c|}{$\begin{array}{l}\text { Discharge }\left(\mathrm{ft}^{3} / \mathrm{s}\right) \text { for indicated } \\
\text { recurrence interval (years) }\end{array}$} \\
\hline & & 2 & 5 & 10 & 20 & 50 & & & 2 & 5 & 10 & 20 & 50 \\
\hline Apr.-Mar. & $\begin{array}{r}1 \\
7 \\
30\end{array}$ & $\begin{array}{l}11 \\
33 \\
42\end{array}$ & $\begin{array}{l}3.2 \\
15 \\
25\end{array}$ & $\begin{array}{c}1.6 \\
9.3 \\
19\end{array}$ & $\begin{array}{l}0.8 \\
5.8 \\
16\end{array}$ & $\begin{array}{l}0.4 \\
3.2 \\
13\end{array}$ & Sept.-Nov. & $\begin{array}{r}1 \\
7 \\
30\end{array}$ & $\begin{array}{l}31 \\
34 \\
47\end{array}$ & $\begin{array}{l}12 \\
15 \\
26\end{array}$ & $\begin{array}{r}6.2 \\
9.2 \\
20\end{array}$ & $\begin{array}{l}3.5 \\
6.0 \\
17\end{array}$ & $\begin{array}{l}1.7 \\
3.7 \\
15\end{array}$ \\
\hline June-Aug. & $\begin{array}{r}1 \\
7 \\
30\end{array}$ & $\begin{array}{r}48 \\
57 \\
124\end{array}$ & $\begin{array}{l}17 \\
32 \\
59\end{array}$ & $\begin{array}{l}8.4 \\
24 \\
39\end{array}$ & $\begin{array}{l}4.1 \\
19 \\
28\end{array}$ & $\begin{array}{l}1.6 \\
14 \\
19\end{array}$ & Mar. - May & $\begin{array}{r}1 \\
7 \\
30\end{array}$ & $\begin{array}{l}151 \\
350 \\
713\end{array}$ & $\begin{array}{r}29 \\
217 \\
423\end{array}$ & $\begin{array}{c}9.2 \\
167 \\
316\end{array}$ & $\begin{array}{c}3.1 \\
134 \\
246\end{array}$ & $\begin{array}{c}0.8 \\
104 \\
183\end{array}$ \\
\hline
\end{tabular}

Duration of daily flow for indicated periods

\begin{tabular}{|c|c|c|c|c|c|c|c|c|c|c|c|c|c|c|}
\hline \multirow{2}{*}{\multicolumn{2}{|c|}{ Period }} & \multicolumn{13}{|c|}{ Discharge $\left(\mathrm{ft}^{3} / \mathrm{s}\right)$ which was equaled or exceeded for indicated percent of time } \\
\hline & & 98 & 95 & 90 & 85 & 80 & 75 & 70 & 60 & 50 & 40 & 30 & 20 & 10 \\
\hline $\begin{array}{l}\text { Apr. - } \\
\text { May - } \\
\text { June - } \\
\text { Sept. - } \\
\text { Dec. - } \\
\text { Mar. - }\end{array}$ & $\begin{array}{l}\text { Mar. } \\
\text { Nov. } \\
\text { Aug. } \\
\text { Nov. } \\
\text { Feb. } \\
\text { May }\end{array}$ & $\begin{array}{r}20 \\
17 \\
23 \\
14 \\
40 \\
160\end{array}$ & $\begin{array}{r}31 \\
24 \\
36 \\
18 \\
75 \\
250\end{array}$ & $\begin{array}{r}48 \\
36 \\
52 \\
25 \\
120 \\
350\end{array}$ & $\begin{array}{r}66 \\
46 \\
66 \\
32 \\
170 \\
430\end{array}$ & $\begin{array}{r}89 \\
57 \\
82 \\
38 \\
240 \\
520\end{array}$ & $\begin{array}{r}120 \\
69 \\
99 \\
44 \\
310 \\
600\end{array}$ & $\begin{array}{r}150 \\
86 \\
120 \\
50 \\
380 \\
700\end{array}$ & $\begin{array}{r}260 \\
130 \\
160 \\
66 \\
550 \\
900\end{array}$ & $\begin{array}{r}410 \\
190 \\
210 \\
89 \\
790 \\
1100\end{array}$ & $\begin{array}{r}640 \\
270 \\
270 \\
130 \\
1100 \\
1500\end{array}$ & $\begin{array}{r}970 \\
410 \\
390 \\
200 \\
1500 \\
1900\end{array}$ & $\begin{array}{r}1500 \\
700 \\
650 \\
340 \\
2100 \\
2700\end{array}$ & $\begin{array}{r}2600 \\
1300 \\
1200 \\
720 \\
3200 \\
3900\end{array}$ \\
\hline
\end{tabular}


MUSKINGUM RIVER BASIN

03144000 Wakatomika Creek near Frazeysburg, Ohio

LOCATION:

Lat $40^{\circ} 07^{\prime} 57^{\prime \prime}$, long $82^{\circ} 08^{\prime} 53^{\prime \prime}$, in NWl/4 sec.13, T.3 N., R.9 W., Muskingum County, Hydrologic Unit 05040004, on right bank $2.0 \mathrm{mi}$ northwest of Frazeysburg, $2.0 \mathrm{mi}$ downstream from Fivemile Run, and $2.5 \mathrm{mi}$ upstream from Black Run.

DRAINAGE AREA: $\quad 140 \mathrm{mi}^{2}$.

TRIBUTARY TO: Muskingum River

DISCHARGE DATA USED: October 1936 to September 1978.

REMARKS: $\quad$ None.

SELECTED DISCHARGE CHARACTERISTICS: Average discharge: $148 \mathrm{ft}^{3} / \mathrm{s}$ (42 years).

Minimum daily discharge: $2.6 \mathrm{ft}^{3} / \mathrm{s}$ October, 1963.

Magnitude and frequencs of low flow for indicated periods

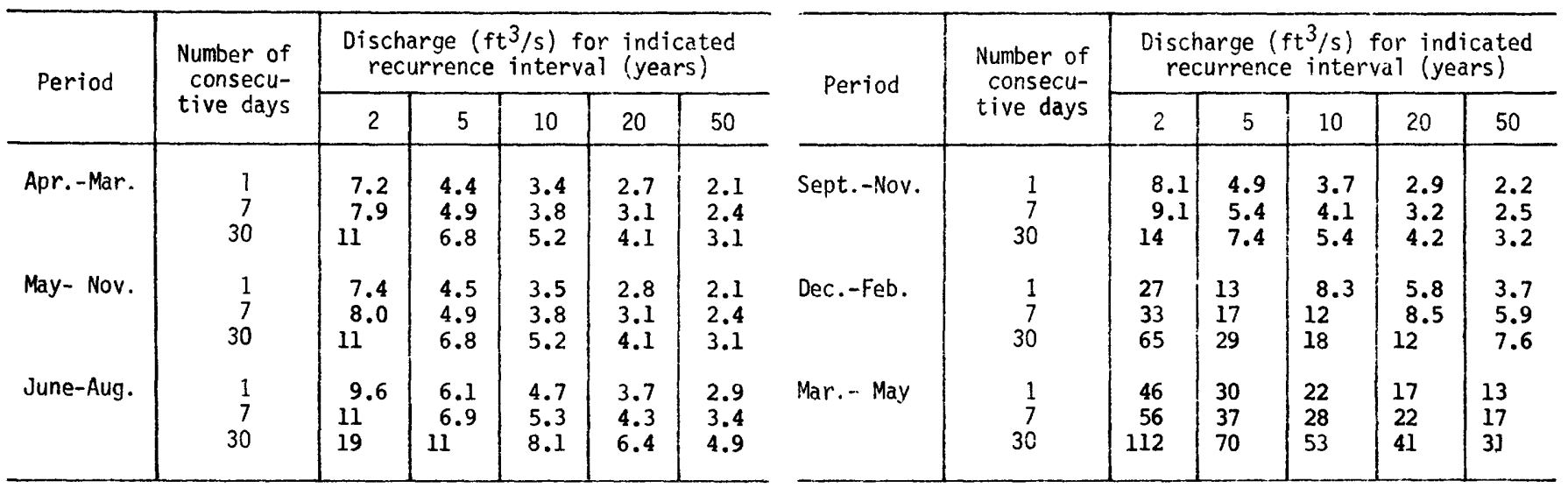

Duration of daily flow for indicated periods

\begin{tabular}{|c|c|c|c|c|c|c|c|c|c|c|c|c|c|}
\hline \multirow{2}{*}{ Period } & \multicolumn{13}{|c|}{ Discharge $\left(\mathrm{ft}^{3} / \mathrm{s}\right)$ which was equaled or exceeded for indicated percent of time } \\
\hline & 98 & 95 & 90 & 85 & 80 & 75 & 70 & 60 & 50 & 40 & 30 & 20 & 10 \\
\hline $\begin{array}{l}\text { Apr. - Mar. } \\
\text { May - Nov. } \\
\text { June - Aug. } \\
\text { Sept. - Nov. } \\
\text { Dec. - Feb. } \\
\text { Mar. - May }\end{array}$ & $\begin{array}{r}5.4 \\
4.5 \\
5.5 \\
3.7 \\
9.5 \\
32\end{array}$ & $\begin{array}{l}7.6 \\
6.3 \\
7.2 \\
5.0 \\
13 \\
45\end{array}$ & $\begin{array}{l}11 \\
8.3 \\
9.6 \\
6.9 \\
18 \\
58\end{array}$ & $\begin{array}{l}14 \\
10 \\
12 \\
8.1 \\
30 \\
72\end{array}$ & $\begin{array}{c}17 \\
12 \\
14 \\
9.4 \\
36 \\
85\end{array}$ & $\begin{array}{l}21 \\
14 \\
16 \\
11 \\
44 \\
97\end{array}$ & $\begin{array}{r}27 \\
17 \\
18 \\
12 \\
52 \\
110\end{array}$ & $\begin{array}{r}40 \\
23 \\
24 \\
16 \\
74 \\
140\end{array}$ & $\begin{array}{r}59 \\
30 \\
31 \\
21 \\
100 \\
170\end{array}$ & $\begin{array}{r}90 \\
43 \\
40 \\
26 \\
140 \\
210\end{array}$ & $\begin{array}{r}130 \\
60 \\
53 \\
36 \\
190 \\
250\end{array}$ & $\begin{array}{r}200 \\
93 \\
75 \\
56 \\
280 \\
350\end{array}$ & $\begin{array}{l}340 \\
170 \\
140 \\
100 \\
470 \\
520\end{array}$ \\
\hline
\end{tabular}


Appendix 3.--Low-flow data--Continued

\author{
MUSKINGUM RIVER BASIN \\ 03144500 Muskingum River at Dresden, Ohio
}

LOCATION:

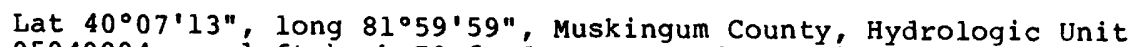

05040004 , on left bank $70 \mathrm{ft}$ downstream from bridge on state Route

$208,0.5 \mathrm{mi}$ east of Dresden, and $0.5 \mathrm{mi}$ downstream from Wakatomika Creek.

$\begin{array}{ll}\text { DRAINAGE AREA: } & 5,993 \mathrm{mi}^{2} . \\ \text { TRIBUTARY TO: } & \text { Ohio River. }\end{array}$

DISCHARGE DATA USED: October 1952 to September 1978.

REMARKS: Flow regulated by 16 flood-control reservoirs at points $15 \mathrm{mi}$ to $105 \mathrm{mi}$
upstream.

SELECTED DISCHARGE CHARACTERISTICS: Average discharge: $6,086 \mathrm{ft}^{3} / \mathrm{s}$ (26 years). Minimum daily discharge: 460 september, 1954.

Magnitude and frequency of low flow for indicated periods

\begin{tabular}{|c|c|c|c|c|c|c|c|c|c|c|c|c|c|}
\hline \multirow{2}{*}{ Period } & \multirow{2}{*}{$\begin{array}{l}\text { Number of } \\
\text { consecu- } \\
\text { tive days }\end{array}$} & \multicolumn{5}{|c|}{$\begin{array}{l}\text { Discharge }\left(\mathrm{ft}^{3} / \mathrm{s}\right) \text { for indicated } \\
\text { recurrence interval (years) }\end{array}$} & \multirow{2}{*}{ Period } & \multirow{2}{*}{$\begin{array}{l}\text { Number of } \\
\text { consecu- } \\
\text { tive days }\end{array}$} & \multicolumn{5}{|c|}{$\begin{array}{l}\text { Discharge }\left(\mathrm{ft}^{3} / \mathrm{s}\right) \text { for indicated } \\
\text { recurrence interval (years) }\end{array}$} \\
\hline & & 2 & 5 & 10 & 20 & 50 & & & 2 & 5 & 10 & 20 & 50 \\
\hline Apr.-Mar. & $\begin{array}{r}7 \\
7 \\
30\end{array}$ & $\begin{array}{r}835 \\
885 \\
1020\end{array}$ & $\begin{array}{l}617 \\
648 \\
718\end{array}$ & $\begin{array}{l}525 \\
549 \\
608\end{array}$ & $\begin{array}{l}459 \\
478 \\
533\end{array}$ & $\begin{array}{l}393 \\
409 \\
464\end{array}$ & Sept.-Nov. & $\begin{array}{r}1 \\
7 \\
30\end{array}$ & \begin{tabular}{r|}
860 \\
918 \\
1130
\end{tabular} & $\begin{array}{l}614 \\
650 \\
748\end{array}$ & $\begin{array}{l}524 \\
554 \\
616\end{array}$ & $\begin{array}{l}463 \\
490 \\
530\end{array}$ & $\begin{array}{l}406 \\
431 \\
453\end{array}$ \\
\hline May- Nov. & $\begin{array}{r}1 \\
7 \\
30\end{array}$ & $\begin{array}{r}842 \\
895 \\
1040\end{array}$ & $\begin{array}{l}619 \\
650 \\
724\end{array}$ & $\begin{array}{l}524 \\
548 \\
607\end{array}$ & $\begin{array}{l}457 \\
475 \\
527\end{array}$ & $\begin{array}{l}390 \\
405 \\
453\end{array}$ & Dec.-Feb. & $\begin{array}{r}1 \\
7 \\
30\end{array}$ & $\begin{array}{l}1740 \\
1990 \\
3040\end{array}$ & $\begin{array}{r}987 \\
1100 \\
1500\end{array}$ & $\begin{array}{r}743 \\
815 \\
1020\end{array}$ & $\begin{array}{l}590 \\
636 \\
742\end{array}$ & $\begin{array}{l}458 \\
482 \\
515\end{array}$ \\
\hline June-Aug. & $\begin{array}{r}1 \\
7 \\
30\end{array}$ & $\begin{array}{l}1080 \\
1180 \\
1710\end{array}$ & $\begin{array}{r}780 \\
848 \\
1120\end{array}$ & $\begin{array}{l}661 \\
717 \\
905\end{array}$ & $\begin{array}{l}577 \\
628 \\
762\end{array}$ & $\begin{array}{l}496 \\
543 \\
630\end{array}$ & Mar. - May & $\begin{array}{r}1 \\
7 \\
30\end{array}$ & $\begin{array}{l}2760 \\
3300 \\
5580\end{array}$ & $\begin{array}{l}1910 \\
2300 \\
3690\end{array}$ & $\begin{array}{l}1570 \\
1920 \\
2980\end{array}$ & $\begin{array}{l}1330 \\
1670 \\
2490\end{array}$ & $\begin{array}{l}1100 \\
1440 \\
2040\end{array}$ \\
\hline
\end{tabular}

Duration of daily flow for indicated periods

\begin{tabular}{|c|c|c|c|c|c|c|c|c|c|c|c|c|c|}
\hline \multirow{2}{*}{ Period } & \multicolumn{13}{|c|}{ Discharge $\left(\mathrm{ft}^{3} / \mathrm{s}\right)$ which was equaled or exceeded for indicated percent of time } \\
\hline & 98 & 95 & 90 & 85 & 80 & 75 & 70 & 60 & 50 & 40 & 30 & 20 & 10 \\
\hline \multirow[b]{2}{*}{$\begin{array}{l}\text { Apr. - Mar. } \\
\text { May - Nov. } \\
\text { June - Aug. } \\
\text { Sept. - Nov. } \\
\text { Dec. - Feb. } \\
\text { Mar. - May }\end{array}$} & 630 & 770 & 980 & 1200 & 1400 & 1600 & 1900 & 2600 & 3500 & 4800 & 6800 & 10000 & 16000 \\
\hline & $\begin{array}{r}600 \\
710 \\
540 \\
700 \\
2100\end{array}$ & $\begin{array}{r}690 \\
850 \\
610 \\
950 \\
2500\end{array}$ & $\begin{array}{r}860 \\
1000 \\
690 \\
1200 \\
3400\end{array}$ & $\begin{array}{r}980 \\
1100 \\
800 \\
1500 \\
4000\end{array}$ & $\begin{array}{r}1100 \\
1300 \\
890 \\
1900 \\
4600\end{array}$ & $\begin{array}{r}1200 \\
1400 \\
970 \\
2300 \\
5200\end{array}$ & $\begin{array}{l}1400 \\
1600 \\
1100 \\
2600 \\
5800\end{array}$ & $\begin{array}{l}1800 \\
1900 \\
1300 \\
3400 \\
7300\end{array}$ & $\begin{array}{l}2300 \\
2300 \\
1600 \\
4800 \\
9100\end{array}$ & $\begin{array}{r}2900 \\
2900 \\
2000 \\
6300 \\
11000\end{array}$ & $\begin{array}{r}3800 \\
3700 \\
2600 \\
8400 \\
14000\end{array}$ & $\begin{array}{r}5400 \\
4900 \\
3600 \\
12000 \\
18000\end{array}$ & $\begin{array}{r}9300 \\
8300 \\
5300 \\
18000 \\
23000\end{array}$ \\
\hline
\end{tabular}


Appendix 3.--Low-flow data--Continued

\section{MUSKINGUM RIVER BASIN}

03145000 South Fork Licking River near Hebron, Ohio

LOCATION:

Lat $39^{\circ} 59^{\prime} 19^{\prime \prime}$, long $82^{\circ} 28^{\prime} 30^{\prime \prime}$, in NWl/4 sec.3, T.I N., N.12 W.,

Licking County, Hydrologic Unit 05040006, on left bank at upstream

side of bridge on county road, $800 \mathrm{ft}$ downstream from Beaver Run,

$2.3 \mathrm{mi}$ north of Hebron, and $2.5 \mathrm{mi}$ upstream from Ramp Creek.

DRAINAGE AREA: $\quad 133 \mathrm{mi}^{2}$.

TRIBUTARY TO: Head of Licking River.

DISCHARGE DATA USED: October 1939 to September 1948, July 1968 to September 1978.

REMARKS:

Occasional regulation by Buckeye Lake, capacity, 27,300 acre-ft, on unnamed tributary $5.6 \mathrm{mi}$ upstream from station. Occasional diversion from Buckeye Lake into Jonathan Creek which bypasses station.

SELECTED DISCHARGE CHARACTERISTICS: Average discharge: $145 \mathrm{ft}^{3} / \mathrm{s}$ (19 years).

Minimum daily discharge: No flow August 1942.

Magnitude and frequency of low flow for indicated periods

\begin{tabular}{|c|c|c|c|c|c|c|}
\hline \multirow{2}{*}{ Period } & \multirow{2}{*}{$\begin{array}{l}\text { Number of } \\
\text { consecu- } \\
\text { tive days }\end{array}$} & \multicolumn{5}{|c|}{$\begin{array}{l}\text { Discharge }\left(\mathrm{ft}^{3} / \mathrm{s}\right) \text { for indicated } \\
\text { recurrence interval (years) }\end{array}$} \\
\hline & & 2 & 5 & 10 & 20 & 50 \\
\hline Apr.-Mar. & $\begin{array}{r}1 \\
7 \\
30\end{array}$ & $\begin{array}{l}3.9 \\
4.8 \\
7.9\end{array}$ & $\begin{array}{l}1.5 \\
2.2 \\
4.1\end{array}$ & $\begin{array}{l}0.7 \\
1.4 \\
2.8\end{array}$ & $\begin{array}{l}0 \\
1.0 \\
2.0\end{array}$ & $\begin{array}{l}0 \\
0.6 \\
1.4\end{array}$ \\
\hline May- Nov. & $\begin{array}{r}1 \\
7 \\
30\end{array}$ & $\begin{array}{l}3.9 \\
4.8 \\
8.2\end{array}$ & $\begin{array}{l}1.5 \\
2.2 \\
4.2\end{array}$ & $\begin{array}{l}0.7 \\
1.4 \\
2.8\end{array}$ & $\begin{array}{l}0 \\
1.0 \\
2.0\end{array}$ & $\begin{array}{l}0 \\
0.6 \\
1.4\end{array}$ \\
\hline June-Aug. & $\begin{array}{r}1 \\
7 \\
30\end{array}$ & $\begin{array}{l}5.7 \\
7.1 \\
16\end{array}$ & $\begin{array}{l}2.5 \\
3.3 \\
7.0\end{array}$ & $\begin{array}{l}1.4 \\
2.0 \\
4.3\end{array}$ & $\begin{array}{l}0 \\
1.3 \\
2.8\end{array}$ & $\begin{array}{l}0 \\
0.8 \\
1.7\end{array}$ \\
\hline
\end{tabular}

\begin{tabular}{|c|c|c|c|c|c|c|}
\hline \multirow{2}{*}{ Period } & \multirow{2}{*}{$\begin{array}{l}\text { Number of } \\
\text { consecu- } \\
\text { tive days }\end{array}$} & \multicolumn{5}{|c|}{$\begin{array}{l}\text { Discharge }\left(\mathrm{ft}^{3} / \mathrm{s}\right) \text { for indicated } \\
\text { recurrence interval (years) }\end{array}$} \\
\hline & & 2 & 5 & 10 & 20 & 50 \\
\hline Sept.-Nov. & $\begin{array}{r}1 \\
7 \\
30\end{array}$ & $\begin{array}{l}5.3 \\
6.8 \\
14\end{array}$ & $\begin{array}{l}2.2 \\
3.2 \\
7.2\end{array}$ & $\begin{array}{l}1.4 \\
2.1 \\
5.1\end{array}$ & $\begin{array}{l}0.9 \\
1.4 \\
3.9\end{array}$ & $\begin{array}{l}0.5 \\
0.9 \\
2.8\end{array}$ \\
\hline Dec. -Feb. & $\begin{array}{r}1 \\
7 \\
30\end{array}$ & $\begin{array}{l}15 \\
19 \\
59\end{array}$ & $\begin{array}{c}6.6 \\
7.7 \\
21\end{array}$ & $\begin{array}{l}4.0 \\
4.5 \\
12\end{array}$ & $\begin{array}{l}2.5 \\
2.7 \\
7.0\end{array}$ & $\begin{array}{l}1.5 \\
1.5 \\
3.7\end{array}$ \\
\hline Mar. - May & $\begin{array}{r}1 \\
7 \\
30\end{array}$ & $\begin{array}{l}21 \\
26 \\
77\end{array}$ & $\begin{array}{l}9.1 \\
12 \\
29\end{array}$ & $\begin{array}{l}4.7 \\
6.6 \\
16\end{array}$ & $\begin{array}{l}2.4 \\
3.6 \\
9.0\end{array}$ & $\begin{array}{l}1.0 \\
1.6 \\
4.4\end{array}$ \\
\hline
\end{tabular}

Duration of daily flow for indicated periods

\begin{tabular}{l|c|c|c|c|c|c|c|c|c|c|c|c|c}
\hline \multirow{2}{*}{ Period } & \multicolumn{8}{|c|}{ Discharge $\left(\mathrm{ft}^{3} / \mathrm{s}\right)$ which was equaled or exceeded for indicated percent of time } \\
\cline { 2 - 12 } & 98 & 95 & 90 & 85 & 80 & 75 & 70 & 60 & 50 & 40 & 30 & 20 & 10 \\
\hline Apr. - Mar. & 2.7 & 4.2 & 6.8 & 9.8 & 13 & 16 & 20 & 29 & 43 & 66 & 110 & 210 & 420 \\
May - Nov. & 2.1 & 3.1 & 4.9 & 6.6 & 8.6 & 11 & 13 & 18 & 24 & 35 & 53 & 100 & 250 \\
June - Aug. & 1.9 & 3.1 & 5.4 & 7.7 & 9.6 & 11 & 13 & 17 & 21 & 29 & 43 & 71 & 200 \\
Sept. - Nov. & 2.5 & 3.2 & 4.5 & 5.6 & 6.8 & 8.0 & 9.8 & 15 & 21 & 31 & 50 & 110 & 260 \\
Dec. - Feb. & 4.0 & 7.4 & 13 & 17 & 21 & 27 & 34 & 51 & 81 & 130 & 200 & 320 & 560 \\
Mar. - May & 5.7 & 16 & 23 & 29 & 35 & 41 & 48 & 65 & 93 & 140 & 220 & 350 & 610 \\
\hline
\end{tabular}




\section{MUSKINGUM RIVER BASIN}

03146000 North Fork Licking River at Utica, Ohio

LOCATION:

Lat $40^{\circ} 13^{\prime} 41^{\prime \prime}$, long $82^{\circ} 27^{\prime} 06^{\prime \prime}$, in T.4 N., R.12 W., Licking County, Hydrologic Unit 05040006 , on left bank at upstream side of bridge on State Route 13 at south edge of Utica, $0.2 \mathrm{mi}$ downstream from unnamed right bank tributary, and $2.0 \mathrm{mi}$ upstreait from lake Fork.

DRAINAGE AREA: $\quad 116 \mathrm{mi}^{2}$.

TRIBUTARY TO:

South Fork Licking River.

DISCHARGE DATA USED:

October 1939 to September 1948, October 1969 to September 1978.

REMARKS:

None.

SELECTED DISCHARGE CHARACTERISTICS: Average discharge: $133 \mathrm{ft}^{3} / \mathrm{s}$ (18 years). Minimum daily discharge: $0.7 \mathrm{ft}^{3} / \mathrm{s}$ August, 1944 .

Magnitude and frequency of low flow for indicated periods

\begin{tabular}{|c|c|c|c|c|c|c|}
\hline \multirow{2}{*}{ Period } & \multirow{2}{*}{$\begin{array}{l}\text { Number of } \\
\text { consecu- } \\
\text { tive days }\end{array}$} & \multicolumn{5}{|c|}{$\begin{array}{l}\text { Discharge }\left(\mathrm{ft}^{3} / \mathrm{s}\right) \text { for indicated } \\
\text { recurrence interval (years) }\end{array}$} \\
\hline & & 2 & 5 & 10 & 20 & 50 \\
\hline Apr.-Mar. & $\begin{array}{r}1 \\
7 \\
30\end{array}$ & $\begin{array}{l}3.7 \\
4.3 \\
5.9\end{array}$ & $\begin{array}{l}2.2 \\
2.8 \\
3.8\end{array}$ & $\begin{array}{l}1.5 \\
2.1 \\
3.0\end{array}$ & $\begin{array}{l}1.1 \\
1.6 \\
2.5\end{array}$ & $\begin{array}{l}0.7 \\
1.1 \\
2.0\end{array}$ \\
\hline May- Nov. & $\begin{array}{r}1 \\
7 \\
30\end{array}$ & $\begin{array}{l}3.7 \\
4.3 \\
5.9\end{array}$ & $\begin{array}{l}2.2 \\
2.8 \\
3.8\end{array}$ & $\begin{array}{l}1.5 \\
2.1 \\
3.0\end{array}$ & $\begin{array}{l}1.1 \\
1.6 \\
2.5\end{array}$ & $\begin{array}{l}0.8 \\
1.1 \\
2.0\end{array}$ \\
\hline June-Aug. & $\begin{array}{r}1 \\
7 \\
30\end{array}$ & $\begin{array}{l}4.6 \\
5.5 \\
9.3\end{array}$ & $\begin{array}{l}2.5 \\
3.1 \\
4.6\end{array}$ & $\begin{array}{l}1.7 \\
2.2 \\
3.3\end{array}$ & $\begin{array}{l}1.2 \\
1.7 \\
2.6\end{array}$ & $\begin{array}{l}0.8 \\
1.2 \\
2.0\end{array}$ \\
\hline
\end{tabular}

\begin{tabular}{|c|c|c|c|c|c|c|}
\hline \multirow{2}{*}{ Period } & \multirow{2}{*}{$\begin{array}{l}\text { Number of } \\
\text { consecu- } \\
\text { tive days }\end{array}$} & \multicolumn{5}{|c|}{$\begin{array}{l}\text { Discharge }\left(\mathrm{ft}^{3} / \mathrm{s}\right) \text { for indicated } \\
\text { recurrence interval (years) }\end{array}$} \\
\hline & & 2 & 5 & 10 & 20 & 50 \\
\hline Sept.-Nov. & $\begin{array}{r}1 \\
7 \\
30\end{array}$ & $\begin{array}{l}4.2 \\
4.9 \\
7.9\end{array}$ & $\begin{array}{l}2.3 \\
2.9 \\
4.1\end{array}$ & $\begin{array}{l}1.7 \\
2.2 \\
3.1\end{array}$ & $\begin{array}{l}1.2 \\
1.7 \\
2.6\end{array}$ & $\begin{array}{l}0.8 \\
1.4 \\
2.1\end{array}$ \\
\hline Dec.-Feb. & $\begin{array}{r}1 \\
7 \\
30\end{array}$ & $\begin{array}{l}15 \\
18 \\
57\end{array}$ & $\begin{array}{l}6.7 \\
8.2 \\
18\end{array}$ & $\begin{array}{l}4.4 \\
5.3 \\
9.0\end{array}$ & $\begin{array}{l}3.0 \\
3.7 \\
4.9\end{array}$ & $\begin{array}{l}2.0 \\
2.5 \\
2.4\end{array}$ \\
\hline Mar. - May & $\begin{array}{r}1 \\
7 \\
30\end{array}$ & $\begin{array}{l}19 \\
24 \\
60\end{array}$ & $\begin{array}{l}11 \\
14 \\
27\end{array}$ & $\begin{array}{l}8.2 \\
9.7 \\
17\end{array}$ & $\begin{array}{l}6.3 \\
7.1 \\
11\end{array}$ & $\begin{array}{l}4.5 \\
4.9 \\
6.5\end{array}$ \\
\hline
\end{tabular}

Duration of daily flow for indicated periods

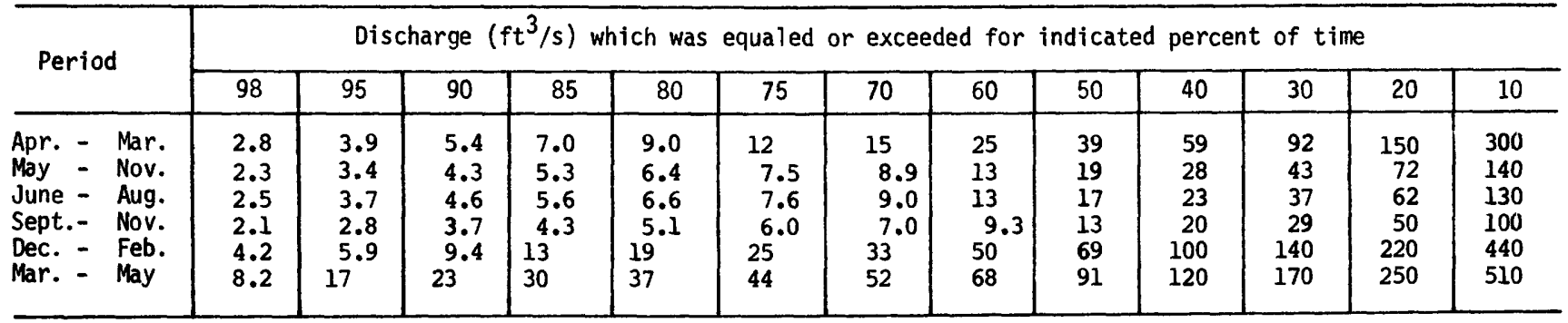


LOCATION:

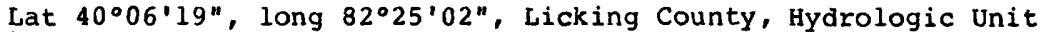
05040006 , at American Aggregates Plant, $1.3 \mathrm{mi}$ downstream from Dry Creek, and $1.5 \mathrm{mi}$ upstream from Newark water plant.

DRAINAGE AREA: $\quad 224 \mathrm{mi}^{2}$.

TRIBUTARY TO: South Fork Licking River.

DISCHARGE DATA USED: LOW-flow measurements 1944, 1964, 1972-1977 water years.

INDEX STATION: $\quad 03146500$ Licking River near Newark, Ohio.

REMARKS:

None.

SELECTED DISCHARGE CHARACTERISTICS: Minimum discharge observed: $19 \mathrm{ft}^{3} / \mathrm{s}$ October 1963.

Magnitude and frequency of low flow for indicated periods

\begin{tabular}{c|c|c|c}
\hline \multirow{2}{*}{ Period } & $\begin{array}{c}\text { Number of } \\
\text { consecu- } \\
\text { tive days }\end{array}$ & $\begin{array}{c}\text { Discharge (ft } 3 / \mathrm{s} \text { ) for } \\
\text { indicated recurrence } \\
\text { interval (years) }\end{array}$ \\
\cline { 3 - 4 } Apr. - Mar. & 7 & 2 & 10 \\
\hline May - Nov. & 30 & 26 & 18 \\
& 7 & 29 & 20 \\
June - Aug. & 30 & 30 & 19 \\
& 7 & 33 & 20 \\
Sept.- Nov. & 30 & 44 & 27 \\
& 30 & 27 & 19 \\
& 7 & 31 & 20 \\
\hline
\end{tabular}

Duration of daily flow for indicated periods

\begin{tabular}{l|c|c|c|c|c}
\hline \multirow{2}{*}{ Period } & \multicolumn{5}{|c}{$\begin{array}{c}\text { Discharge ( } \mathrm{ft}^{3} / \mathrm{s} \text { ) which was equaled or } \\
\text { exceeded for indicated percent of time }\end{array}$} \\
\cline { 2 - 6 } & 98 & 95 & 90 & 85 & 80 \\
\hline Apr. - Mar. & 19 & 23 & 27 & 31 & 36 \\
May - Nov. & 19 & 22 & 25 & 28 & 31 \\
June - Aug. & 23 & 27 & 30 & 33 & 37 \\
Sept. - Nov. & 17 & 19 & 21 & 23 & 25 \\
\hline
\end{tabular}


Appendix 3.--Low-flow data--Continued

MUSKINGUM RIVER BASIN

03146500 Licking River near Newark, Ohio

LOCATION:

DRAINAGE AREA:

TRIBUTARY TO:

DISCHARGE DATA USED:

REMARKS:

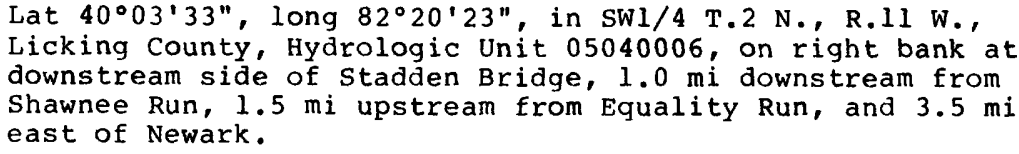

Muskingum River.

October 1939 to September 1978.

Occasional regulation by Buckeye Lake, capacity 27,300 acre-ft on South Fork $15.2 \mathrm{mi}$ upstream.

SELECTED DISCHARGE CHARACTERISTICS: Average discharge: $566 \mathrm{ft}^{3} / \mathrm{s}$ (39 years).

Minimum daily discharge: $28 \mathrm{ft}^{3} / \mathrm{s}$ September 1954.

Magnitude and frequency of low flow for indicated periods

\begin{tabular}{|c|c|c|c|c|c|c|c|c|c|c|c|c|c|}
\hline \multirow{2}{*}{ Period } & \multirow{2}{*}{$\begin{array}{l}\text { Number of } \\
\text { consecu- } \\
\text { tive days }\end{array}$} & \multicolumn{5}{|c|}{$\begin{array}{l}\text { Discharge }\left(\mathrm{ft}^{3} / \mathrm{s}\right) \text { for indicated } \\
\text { recurrence interval (years) }\end{array}$} & \multirow{2}{*}{ Period } & \multirow{2}{*}{$\begin{array}{l}\text { Number of } \\
\text { consecu- } \\
\text { tive days }\end{array}$} & \multicolumn{5}{|c|}{$\begin{array}{l}\text { Discharge }\left(\mathrm{ft}^{3} / \mathrm{s}\right) \text { for indicated } \\
\text { recurrence interval (years) }\end{array}$} \\
\hline & & 2 & 5 & 10 & 20 & 50 & & & 2 & 5 & 10 & 20 & 50 \\
\hline Apr.-Mar. & $\begin{array}{r}1 \\
7 \\
30\end{array}$ & $\begin{array}{l}57 \\
60 \\
70\end{array}$ & $\begin{array}{l}44 \\
47 \\
53\end{array}$ & $\begin{array}{l}39 \\
41 \\
46\end{array}$ & $\begin{array}{l}34 \\
37 \\
40\end{array}$ & $\begin{array}{l}30 \\
33 \\
35\end{array}$ & Sept.-Nov. & $\begin{array}{r}1 \\
7 \\
30\end{array}$ & $\begin{array}{l}59 \\
63 \\
74\end{array}$ & $\begin{array}{l}45 \\
48 \\
53\end{array}$ & $\begin{array}{l}39 \\
42 \\
46\end{array}$ & $\begin{array}{l}34 \\
37 \\
41\end{array}$ & $\begin{array}{l}30 \\
32 \\
37\end{array}$ \\
\hline June-Aug. & $\begin{array}{r}1 \\
7 \\
30\end{array}$ & $\begin{array}{r}74 \\
81 \\
112\end{array}$ & $\begin{array}{l}57 \\
62 \\
75\end{array}$ & $\begin{array}{l}50 \\
54 \\
64\end{array}$ & $\begin{array}{l}45 \\
49 \\
56\end{array}$ & $\begin{array}{l}40 \\
43 \\
50\end{array}$ & Mar. - May & $\begin{array}{r}1 \\
7 \\
30\end{array}$ & $\begin{array}{l}174 \\
202 \\
357\end{array}$ & $\begin{array}{l}124 \\
145 \\
222\end{array}$ & $\begin{array}{l}100 \\
120 \\
173\end{array}$ & $\begin{array}{r}81 \\
102 \\
140\end{array}$ & $\begin{array}{r}63 \\
84 \\
110\end{array}$ \\
\hline
\end{tabular}

Duration of daily flow for indicated periods

\begin{tabular}{|c|c|c|c|c|c|c|c|c|c|c|c|c|c|}
\hline \multirow{2}{*}{ Period } & \multicolumn{13}{|c|}{ Discharge $\left(\mathrm{ft}^{3} / \mathrm{s}\right)$ which was equaled or exceeded for indicated percent of time } \\
\hline & 98 & 95 & 90 & 85 & 80 & 75 & 70 & 60 & 50 & 40 & 30 & 20 & 10 \\
\hline $\begin{array}{l}\text { Apr. - Mar. } \\
\text { May - Nov. } \\
\text { June - Aug. } \\
\text { Sept. - Nov. } \\
\text { Dec. - Feb. } \\
\text { Mar. - May }\end{array}$ & $\begin{array}{r}44 \\
43 \\
54 \\
38 \\
45 \\
120\end{array}$ & $\begin{array}{r}53 \\
50 \\
63 \\
43 \\
53 \\
150\end{array}$ & $\begin{array}{r}64 \\
59 \\
72 \\
49 \\
66 \\
200\end{array}$ & $\begin{array}{r}75 \\
67 \\
81 \\
54 \\
87 \\
230\end{array}$ & $\begin{array}{r}88 \\
74 \\
90 \\
59 \\
120 \\
260\end{array}$ & $\begin{array}{r}100 \\
83 \\
99 \\
64 \\
150 \\
290\end{array}$ & $\begin{array}{r}120 \\
91 \\
110 \\
69 \\
180 \\
330\end{array}$ & $\begin{array}{r}170 \\
110 \\
130 \\
80 \\
260 \\
420\end{array}$ & $\begin{array}{r}230 \\
140 \\
150 \\
93 \\
370 \\
520\end{array}$ & $\begin{array}{l}320 \\
180 \\
180 \\
120 \\
520 \\
670\end{array}$ & $\begin{array}{l}470 \\
240 \\
240 \\
160 \\
740 \\
880\end{array}$ & $\begin{array}{r}730 \\
350 \\
350 \\
230 \\
1100 \\
1200\end{array}$ & $\begin{array}{r}1300 \\
640 \\
650 \\
420 \\
1900 \\
2100\end{array}$ \\
\hline
\end{tabular}


Appendix 3.--Low-flow data--Continued

MUSKINGUM RIVER BASIN

03147500 Licking River below Dillon Dam, near Dillon Falls, Ohio

LOCATION:

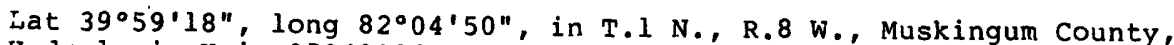
Hydrologic Unit 05040006 , on left bank $500 \mathrm{ft}$ downstream from Dillón Dam, $2.0 \mathrm{mi}$ northwest of Dillon Falls, and $5.8 \mathrm{mi}$ upstream from mouth.

DRAINAGE AREA: $\quad 742 \mathrm{mi}^{2}$.

TRIBUTARY TO: Muskingum River.

DISCHARGE DATA USED: October 1960 to September 1978.

REMARKS:

Flow regulated by Dillon Lake since December 1960.

SELECTED DISCHARGE CHARACTERISTICS: Average discharge: $823 \mathrm{ft}^{3} / \mathrm{s}$ (18 years).

Minimum daily discharge: $19 \mathrm{ft} / \mathrm{s}$ December 1960.

Magnitude and frequency of low flow for indicated periods

\begin{tabular}{|c|c|c|c|c|c|c|c|c|c|c|c|c|c|}
\hline \multirow{2}{*}{ Period } & \multirow{2}{*}{$\begin{array}{l}\text { Number of } \\
\text { consecu- } \\
\text { tive days }\end{array}$} & \multicolumn{5}{|c|}{$\begin{array}{l}\text { Discharge }\left(\mathrm{ft}^{3} / \mathrm{s}\right) \text { for indicated } \\
\text { recurrence interval (years) }\end{array}$} & \multirow{2}{*}{ Period } & \multirow{2}{*}{$\begin{array}{l}\text { Number of } \\
\text { consecu- } \\
\text { tive days }\end{array}$} & \multicolumn{5}{|c|}{$\begin{array}{l}\text { Discharge }\left(\mathrm{ft}^{3} / \mathrm{s}\right) \text { for indicated } \\
\text { recurrence interval (years) }\end{array}$} \\
\hline & & 2 & 5 & 10 & 20 & 50 & & & 2 & 5 & 10 & 20 & 50 \\
\hline Apr. -Mar. & $\begin{array}{r}1 \\
7 \\
30\end{array}$ & $\begin{array}{r}66 \\
80 \\
105\end{array}$ & $\begin{array}{l}56 \\
66 \\
77\end{array}$ & $\begin{array}{l}51 \\
59 \\
64\end{array}$ & $\begin{array}{l}48 \\
54 \\
55\end{array}$ & $\begin{array}{l}44 \\
48 \\
46\end{array}$ & Sept.-Nov. & $\begin{array}{r}1 \\
7 \\
30\end{array}$ & $\begin{array}{r}77 \\
92 \\
134\end{array}$ & $\begin{array}{l}62 \\
68 \\
82\end{array}$ & $\begin{array}{l}55 \\
58 \\
64\end{array}$ & $\begin{array}{l}50 \\
52 \\
53\end{array}$ & $\begin{array}{l}46 \\
46 \\
44\end{array}$ \\
\hline June-Aug. & $\begin{array}{r}1 \\
7 \\
30\end{array}$ & $\begin{array}{r}77 \\
97 \\
161\end{array}$ & $\begin{array}{r}62 \\
75 \\
107\end{array}$ & $\begin{array}{l}55 \\
67 \\
89\end{array}$ & $\begin{array}{l}51 \\
60 \\
76\end{array}$ & $\begin{array}{l}46 \\
55 \\
65\end{array}$ & Mar. - May & $\begin{array}{r}1 \\
7 \\
30\end{array}$ & $\begin{array}{l}104 \\
217 \\
535\end{array}$ & $\begin{array}{r}70 \\
112 \\
292\end{array}$ & $\begin{array}{r}59 \\
77 \\
209\end{array}$ & $\begin{array}{r}53 \\
56 \\
157\end{array}$ & $\begin{array}{r}47 \\
38 \\
113\end{array}$ \\
\hline
\end{tabular}

Duration of daily flow for indicated periods

\begin{tabular}{l|c|c|c|r|r|r|r|r|r|r|r|r|r|r}
\hline \multirow{2}{*}{ Period } & \multicolumn{8}{|c|}{ Discharge $\left(\mathrm{ft}^{3} / \mathrm{s}\right)$ which was equaled or exceeded for indicated percent of time } \\
\cline { 2 - 12 } & 98 & 95 & 90 & 85 & 80 & 75 & 70 & 60 & 50 & 40 & 30 & 20 & 10 \\
\hline Apr. - Mar. & 66 & 76 & 90 & 110 & 130 & 150 & 190 & 260 & 360 & 510 & 730 & 1200 & 2400 \\
May - Nov. & 64 & 71 & 82 & 92 & 100 & 120 & 130 & 170 & 220 & 290 & 400 & 590 & 1100 \\
June - Aug. & 66 & 77 & 90 & 100 & 110 & 120 & 140 & 170 & 210 & 250 & 320 & 470 & 920 \\
Sept.- Nov. & 59 & 67 & 75 & 82 & 88 & 97 & 110 & 130 & 180 & 240 & 350 & 520 & 870 \\
Dec. - Feb. & 70 & 89 & 130 & 170 & 220 & 260 & 300 & 420 & 560 & 740 & 1000 & 1500 & 2700 \\
Mar. - May & 80 & 120 & 230 & 300 & 360 & 430 & 500 & 660 & 840 & 1200 & 1700 & 2700 & 3900 \\
\hline
\end{tabular}


Appendix 3.--Low-flow data--Continued

MUSKINGUM RIVER BASIN

03148300 Moxanala Creek at Roseville, Ohio

LOCATION:

Lat $39^{\circ} 48^{\prime} 40^{\prime \prime}$, long $82^{\circ} 04^{\prime} 10^{\prime \prime}$, Muskingum County, Hydrologic Unit 05040004 , at pumping station about 2,500 ft downstream from First Street Bridge in Roseville.

DRAINAGE AREA: $\quad 80.6 \mathrm{mi}^{2}$.

TRIBUTARY TO: Muskingum River.

DISCHARGE DATA USED: Low-flow measurements 1961-1971 water years.

INDEX STATION: $\quad 03202000$ Raccoon Creek at Adamsville, Ohio.

REMARKS:

Flood flow controlled by levee on left bank.

SELECTED DISCHARGE CHARACTERISTICS: Minimum discharge observed: $3.3 \mathrm{ft}^{3} / \mathrm{s}$ August 1962 .

\begin{tabular}{l|c|c|c}
\multicolumn{4}{c}{ Magnitude and frequency of low flow for } \\
indicated periods \\
\hline \multirow{2}{*}{ Period } & $\begin{array}{c}\text { Number of } \\
\text { consecu- } \\
\text { tive days }\end{array}$ & $\begin{array}{c}\text { Discharge (ft } 3 / \mathrm{s} \text { ) for } \\
\text { indicated recurrence } \\
\text { interval (years) }\end{array}$ \\
\cline { 3 - 4 } & & 2 & 10 \\
\hline Apr. - Mar. & 7 & 8.2 & 3.6 \\
May - Nov. & 30 & 11 & 4.6 \\
June - Aug. & 7 & 8.2 & 3.6 \\
& 30 & 11 & 4.6 \\
Sept. - Nov. & 7 & 12 & 7.4 \\
& 30 & 18 & 9.7 \\
\hline
\end{tabular}

Ouration of daily flow for indicated periods

\begin{tabular}{|c|c|c|c|c|c|}
\hline \multirow{2}{*}{ Period } & \multicolumn{5}{|c|}{$\begin{array}{l}\text { Discharge }\left(\mathrm{ft}^{3} / \mathrm{s}\right) \text { which was equaled or } \\
\text { exceeded for indicated percent of time }\end{array}$} \\
\hline & 98 & 95 & 90 & 85 & 80 \\
\hline $\begin{array}{l}\text { Apr. - Mar. } \\
\text { May - Nov. } \\
\text { June - Aug. } \\
\text { Sept. - Nov. }\end{array}$ & $\begin{array}{l}5.0 \\
4.2 \\
7.4 \\
3.1\end{array}$ & $\begin{array}{l}6.7 \\
5.7 \\
9.1 \\
4.3\end{array}$ & $\begin{array}{l}10 \\
8.2 \\
11 \\
5.4\end{array}$ & $\begin{array}{l}13 \\
10 \\
13 \\
6.2\end{array}$ & $\begin{array}{l}16 \\
12 \\
14 \\
7.8\end{array}$ \\
\hline
\end{tabular}


Appendix 3.--Low-flow data--Continued

MUSKINGUM RIVER BASIN

03148400 Moxahala Creek at Roberts, Ohio

LOCATION: Lat $39^{\circ} 51^{\prime} 20^{\prime \prime}$, long $82^{\circ} 03^{\prime} 25^{\prime \prime}$, Muskingum County, Hydrologic Unit 05040004 , at bridge on county road, $0.5 \mathrm{mi}$ east of former location of Roberts, $2.5 \mathrm{mi}$ southeast of white Cottage, $2.6 \mathrm{mi}$ southeast of present (1965) Roberts, and $2.1 \mathrm{mi}$ upstream from Jonathan Creek.

DRAINAGE AREA: $\quad 98.1 \mathrm{mi}^{2}$

TRIBUTARY TO: Muskingum River.

DISCHARGE DATA USED: Low-flow measurements 1959, 1962-1971 water years.

INDEX STATION: $\quad 03157000$ Clear Creek near Rockbridge, Ohio.

REMARKS: $\quad$ None.

SELECTED DISCHARGE CHARACTERISTICS: Minimum discharge observed: $3.9 \mathrm{ft}^{3} / \mathrm{s}$ August 1962 .

Magnitude and frequency of low flow for indicated periods

\begin{tabular}{c|c|c|c}
\hline \multirow{2}{*}{ Period } & $\begin{array}{c}\text { Number of } \\
\text { consecu- } \\
\text { tive days }\end{array}$ & \multicolumn{2}{|c}{$\begin{array}{c}\text { Discharge (ft3/s) for } \\
\text { indicated recurrence } \\
\text { interval }\end{array}$} \\
\cline { 3 - 4 } (years)
\end{tabular}

Duration of daily flow for indicated periods

\begin{tabular}{|c|c|c|c|c|c|}
\hline \multirow{2}{*}{ Period } & \multicolumn{5}{|c|}{$\begin{array}{l}\text { Discharge }\left(\mathrm{ft}^{3} / \mathrm{s}\right) \text { which was equaled or } \\
\text { exceeded for indicated percent of time }\end{array}$} \\
\hline & 98 & 95 & 90 & 85 & 80 \\
\hline $\begin{array}{l}\text { Apr. - Mar. } \\
\text { May - Nov. } \\
\text { June - Aug. } \\
\text { Sept. - Nov. }\end{array}$ & $\begin{array}{l}5.3 \\
4.4 \\
4.4 \\
4.4\end{array}$ & $\begin{array}{l}7.6 \\
6.4 \\
6.4 \\
5.3\end{array}$ & $\begin{array}{l}12 \\
8.8 \\
8.8 \\
7.6\end{array}$ & $\begin{array}{l}15 \\
10 \\
12 \\
8.8\end{array}$ & $\begin{array}{l}19 \\
13 \\
13 \\
10\end{array}$ \\
\hline
\end{tabular}


Appendix 3.--Low-flow data--Continued

MUSKINGUM RIVER BASIN

03148450 Jonathan Creek at East Fultonham, Ohio

LOCATION: $\quad$ Lat $39^{\circ} 51^{\prime} 20^{\prime \prime}$, long $82^{\circ} 07^{\prime} 35^{\prime \prime}$, Muskingum County, Hydrologic

Unit 05040004 , at old U.S. Highway 22 bridge in East Fultonham $1.0 \mathrm{mi}$ upstream from Buckeye Fork.

DRAINAGE AREA: $\quad 125 \mathrm{mi}^{2}$.

TRIBUTARY TO: Moxahala Creek.

DISCHARGE DATA USED: Low-flow measurements 1942, 1959, 1965, 1972-1977 water years.

INDEX STATION: $\quad 03146500$ Licking River near Newark, Ohio.

REMARKS: $\quad$ None.

SELECTED DISCHARGE CHARACTERISTICS: Minimum discharge observed: $3.2 \mathrm{ft}^{3} / \mathrm{s}$ August 1972.

\begin{tabular}{|c|c|c|c|}
\hline \multicolumn{4}{|c|}{$\begin{array}{l}\text { Magnitude and frequency of low flow for } \\
\text { indicated periods }\end{array}$} \\
\hline \multirow[t]{2}{*}{ Period } & \multirow{2}{*}{$\begin{array}{l}\text { Number of } \\
\text { consecu- } \\
\text { tive days }\end{array}$} & \multicolumn{2}{|c|}{$\begin{array}{l}\text { Discharge }\left(\mathrm{ft}^{3} / \mathrm{s}\right) \text { for } \\
\text { indicated recurrence } \\
\text { interval (years) }\end{array}$} \\
\hline & & 2 & 10 \\
\hline Apr. - Mar. & $\begin{array}{r}7 \\
30\end{array}$ & $\begin{array}{l}5.4 \\
6.6\end{array}$ & $\begin{array}{l}3.3 \\
3.8\end{array}$ \\
\hline May - Nov. & $\begin{array}{r}7 \\
30\end{array}$ & $\begin{array}{l}5.6 \\
6.8\end{array}$ & $\begin{array}{l}3.4 \\
3.8\end{array}$ \\
\hline June - Aug. & $\begin{array}{r}7 \\
30\end{array}$ & $12^{8.0}$ & $\begin{array}{l}4.7 \\
5.9\end{array}$ \\
\hline Sept. - Nov. & $\begin{array}{r}7 \\
30\end{array}$ & $\begin{array}{l}5.8 \\
7.1\end{array}$ & $\begin{array}{l}3.4 \\
3.8\end{array}$ \\
\hline
\end{tabular}

Duration of daily flow for indicated periods

\begin{tabular}{|c|c|c|c|c|c|}
\hline \multirow{2}{*}{ Period } & \multicolumn{5}{|c|}{$\begin{array}{l}\text { Discharge }\left(\mathrm{ft}^{3} / \mathrm{s}\right) \text { which was equaled or } \\
\text { exceeded for indicated percent of time }\end{array}$} \\
\hline & 98 & 95 & 90 & 85 & 80 \\
\hline $\begin{array}{l}\text { Apr. - Mar. } \\
\text { May - Nov. } \\
\text { June - Aug. } \\
\text { Sept. - Nov. }\end{array}$ & $\begin{array}{l}3.6 \\
3.5 \\
4.7 \\
3.0\end{array}$ & $\begin{array}{l}4.6 \\
4.3 \\
5.8 \\
3.5\end{array}$ & $\begin{array}{l}5.9 \\
5.3 \\
6.8 \\
4.1\end{array}$ & $\begin{array}{l}7.2 \\
6.2 \\
8.0 \\
4.7\end{array}$ & $\begin{array}{l}8.9 \\
7.1 \\
9.2 \\
5.3\end{array}$ \\
\hline
\end{tabular}


Appendix 3.--Low-flow data--Continued

MUSKINGUM RIVER BASIN

03148600 Moxahala Creek near Zanesville, Ohio

LOCATION: $\quad$ Lat $39^{\circ} 53^{\prime} 45^{\prime \prime}$, long $82^{\circ} 00^{\prime} 20^{\prime \prime}$, Muskingum County, Hydrologic Unit $05040004,1.0 \mathrm{mi}$ east of South Zanesville, and $0.5 \mathrm{mi}$ upstream from mouth.

DRAINAGE AREA: $\quad 302 \mathrm{mi}^{2}$.

TRIBUTARY TO: Muskingum River.

DISCHARGE DATA USED: LOW-flOw measurements 1959, 1962-1973 water years.

INDEX STATION: $\quad 03157000$ Clear Creek near Rockbridge, Ohio.

REMARKS: None.

SELECTED DISCHARGE CHARACTERISTICS: Minimum discharge observed: $8.3 \mathrm{ft} \mathrm{t}^{3} / \mathrm{s}$ September 1964 .

\begin{tabular}{c|c|c|c}
\multicolumn{3}{c}{$\begin{array}{c}\text { Magnitude and frequency of low flow for } \\
\text { indicated periods }\end{array}$} \\
\hline Period & $\begin{array}{c}\text { Number of } \\
\text { consecu- } \\
\text { tive days }\end{array}$ & $\begin{array}{c}\text { Discharge (ft3/s) for } \\
\text { indicated recurrence } \\
\text { interval (years) }\end{array}$ \\
\cline { 2 - 4 } & 7 & 2 & 10 \\
\hline Apr. - Mar. & 30 & 14 & 5.2 \\
May - Nov. & 7 & 19 & 9.4 \\
& 30 & 14 & 5.2 \\
June - Aug. & 7 & 19 & 9.4 \\
& 30 & 19 & 6.6 \\
Sept.- Nov. & 7 & 40 & 14 \\
& 30 & 16 & 7.0 \\
\hline
\end{tabular}

Duration of daily flow for indicated periods

\begin{tabular}{|c|c|c|c|c|c|}
\hline \multirow{2}{*}{ Period } & \multicolumn{5}{|c|}{$\begin{array}{l}\text { Discharge }\left(\mathrm{ft}^{3} / \mathrm{s}\right) \text { which was equaled or } \\
\text { exceeded for indicated percent of time }\end{array}$} \\
\hline & 98 & 95 & 90 & 85 & 80 \\
\hline $\begin{array}{l}\text { Apr. - Mar. } \\
\text { May - Nov. } \\
\text { June - Aug. } \\
\text { Sept. - Nov. }\end{array}$ & $\begin{array}{l}9.4 \\
7.6 \\
7.6 \\
7.6\end{array}$ & $\begin{array}{l}14 \\
11 \\
11 \\
9.4\end{array}$ & $\begin{array}{l}22 \\
16 \\
16 \\
14\end{array}$ & $\begin{array}{l}29 \\
19 \\
22 \\
16\end{array}$ & $\begin{array}{l}36 \\
25 \\
25 \\
19\end{array}$ \\
\hline
\end{tabular}




\author{
MUSKINGUM RIVER BASIN \\ 03149500 Salt Creek near Chandlersville, Ohio
}

LOCATION:

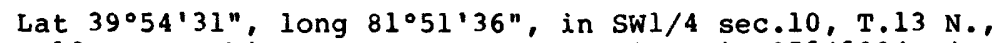
R.12 W., Muskingum County, Hydrologic Unit 05040004 , just upstream from highway bridge $1.0 \mathrm{mi}$ upstream from Buffalo Fork, and $2 \mathrm{mi}$ northwest of Chandlersville.

ORAINAGE AREA: $\quad 75.7 \mathrm{mi}^{2}$.

TRIBUTARY TO:

Muskingum River.

OISCHARGE DATA USED: October 1935 to september 1947.

REMARKS:

None.

SELECTED DISCHARGE CHARACTERISTICS: Average discharge: $89.2 \mathrm{ft}^{3} / \mathrm{s}$ (12 years)

Minimum daily discharge: No flow July 1936, August 1944, September. 1947.

Magnitude and frequency of low flow for indicated periods

\begin{tabular}{|c|c|c|c|c|c|c|c|c|c|c|c|c|c|}
\hline \multirow{2}{*}{ Period } & \multirow{2}{*}{$\begin{array}{l}\text { Number of } \\
\text { consecu- } \\
\text { tive days }\end{array}$} & \multicolumn{5}{|c|}{$\begin{array}{l}\text { Discharge }\left(\mathrm{ft}^{3} / \mathrm{s}\right) \text { for indicated } \\
\text { recurrence interval (years) }\end{array}$} & \multirow{2}{*}{ Period } & \multirow{2}{*}{$\begin{array}{l}\text { Number of } \\
\text { consecu- } \\
\text { tive days }\end{array}$} & \multicolumn{5}{|c|}{$\begin{array}{l}\text { Discharge }\left(\mathrm{ft}^{3} / \mathrm{s}\right) \text { for indicated } \\
\text { recurrence interval (years) }\end{array}$} \\
\hline & & 2 & 5 & 10 & 20 & 50 & & & 2 & 5 & 10 & 20 & 50 \\
\hline Apr.-Mar. & $\begin{array}{r}1 \\
7 \\
30\end{array}$ & $\begin{array}{l}0.6 \\
0.7 \\
2.2\end{array}$ & $\begin{array}{l}0.1 \\
0.1 \\
1.0\end{array}$ & $\begin{array}{l}0 \\
0.1 \\
0.7\end{array}$ & $\begin{array}{l}0 \\
0 \\
0.5\end{array}$ & $\begin{array}{l}0 \\
0 \\
0.3\end{array}$ & Sept.-Nov. & $\begin{array}{r}1 \\
7 \\
30\end{array}$ & $\begin{array}{l}0.8 \\
0.9 \\
3.5\end{array}$ & $\begin{array}{l}0.2 \\
0.3 \\
1.4\end{array}$ & $\begin{array}{l}0.1 \\
0.2 \\
0.9\end{array}$ & $\begin{array}{l}0.1 \\
0.1 \\
0.6\end{array}$ & $\begin{array}{l}0 \\
0.1 \\
0.4\end{array}$ \\
\hline June-Aug. & $\begin{array}{r}1 \\
7 \\
30\end{array}$ & $\begin{array}{l}1.6 \\
2.2 \\
9.4\end{array}$ & $\begin{array}{l}0.4 \\
0.4 \\
3.6\end{array}$ & $\begin{array}{l}0 \\
0.1 \\
2.1\end{array}$ & $\begin{array}{l}0 \\
0 \\
1.3\end{array}$ & $\begin{array}{l}0 \\
0 \\
0.7\end{array}$ & Mar. - May & $\begin{array}{r}1 \\
7 \\
30\end{array}$ & $\begin{array}{l}15 \\
22 \\
45\end{array}$ & $\begin{array}{l}8.1 \\
11 \\
24\end{array}$ & $\begin{array}{r}5.5 \\
7.5 \\
17\end{array}$ & $\begin{array}{c}3.8 \\
5.1 \\
13\end{array}$ & $\begin{array}{l}2.5 \\
3.1 \\
9.5\end{array}$ \\
\hline
\end{tabular}

Duration of daily flow for indicated periods

\begin{tabular}{|c|c|c|c|c|c|c|c|c|c|c|c|c|c|}
\hline \multirow{2}{*}{ Period } & \multicolumn{13}{|c|}{ Discharge $\left(\mathrm{ft}^{3} / \mathrm{s}\right)$ which was equaled or exceeded for indicated percent of time } \\
\hline & 98 & 95 & 90 & 85 & 80 & 75 & 70 & 60 & 50 & 40 & 30 & 20 & 10 \\
\hline $\begin{array}{l}\text { Apr. - Mar. } \\
\text { May - Nov. } \\
\text { June - Aug. } \\
\text { Sept. - Nov. } \\
\text { Dec. - Feb. } \\
\text { Mar. - May }\end{array}$ & $\begin{array}{l}0.6 \\
0.4 \\
0.5 \\
0.3 \\
2.7 \\
8.7\end{array}$ & $\begin{array}{l}1.5 \\
0.9 \\
1.1 \\
0.6 \\
3.9 \\
14\end{array}$ & $\begin{array}{r}2.8 \\
1.8 \\
2.1 \\
1.1 \\
6.3 \\
22\end{array}$ & $\begin{array}{l}4.0 \\
2.6 \\
3.0 \\
1.8 \\
10 \\
29\end{array}$ & $\begin{array}{l}5.4 \\
3.4 \\
4.0 \\
2.4 \\
18 \\
34\end{array}$ & $\begin{array}{l}7.1 \\
4.3 \\
5.1 \\
3.0 \\
23 \\
39\end{array}$ & $\begin{array}{l}9.7 \\
5.3 \\
6.5 \\
3.5 \\
28 \\
45\end{array}$ & $\begin{array}{l}19 \\
8.0 \\
9.9 \\
4.9 \\
39 \\
59\end{array}$ & $\begin{array}{c}30 \\
13 \\
16 \\
6.5 \\
52 \\
80\end{array}$ & $\begin{array}{c}44 \\
21 \\
24 \\
9.3 \\
71 \\
100\end{array}$ & $\begin{array}{r}66 \\
32 \\
35 \\
16 \\
99 \\
140\end{array}$ & $\begin{array}{r}100 \\
52 \\
56 \\
28 \\
150 \\
200\end{array}$ & $\begin{array}{r}200 \\
100 \\
120 \\
65 \\
260 \\
340\end{array}$ \\
\hline
\end{tabular}


Appendix 3.--Low-flow data--Continued

\section{MUSKINGUM RIVER BASIN}

03150000 Muskingum River at McConnelsville, Ohio

LOCATION:

Lat $39^{\circ} 38^{\prime} 4^{\prime \prime}$, long $81^{\circ} 51^{\prime} 00^{\prime \prime}$, in SEl/4 sec.11, T.10 N., R.12 W., Morgan County, Hydrologic Unit 05040004 , on left bank just upstream from Dam 7, at McConnelsville, and $3.5 \mathrm{mi}$ downstream from Oilspring Run.

DRAINAGE AREA:

$7,422 \mathrm{mi}^{2}$.

TRIBUTARY TO:

Ohio River.

DISCHARGE DATA USED:

REMARKS:

October 1937 to September 1978.

Flow regulated by 17 flood-control reservoirs $36.6 \mathrm{mi}$ to $148 \mathrm{mi}$ upstream from station. Some regulation at low flow by power plant $19 \mathrm{mi}$ upstream from station.

SELECTED DISCHARGE CHARACTERISTICS: Average discharge: $7,457 \mathrm{ft}^{3} / \mathrm{s}$ (41 years)

Minimum daily discharge: $385 \mathrm{ft}^{3} / \mathrm{s}$ September 1954.

Magnitude and frequency of low flow for indicated periods

\begin{tabular}{c|r|r|r|r|r|r}
\hline \multirow{2}{*}{ Period } & \multirow{2}{*}{$\begin{array}{c}\text { Number of } \\
\text { consecu- } \\
\text { tive days }\end{array}$} & \multicolumn{4}{|c|}{$\begin{array}{c}\text { Discharge (ft } 3 / \mathrm{s} \text { ) for indicated } \\
\text { recurrence interval }\end{array}$} \\
\cline { 3 - 7 } (years)
\end{tabular}

\begin{tabular}{|c|c|c|c|c|c|c|}
\hline \multirow{2}{*}{ Period } & \multirow{2}{*}{$\begin{array}{l}\text { Number of } \\
\text { consecu- } \\
\text { tive days }\end{array}$} & \multicolumn{5}{|c|}{$\begin{array}{l}\text { Discharge }\left(\mathrm{ft}^{3} / \mathrm{s}\right) \text { for indicated } \\
\text { recurrence interval (years) }\end{array}$} \\
\hline & & 2 & 5 & 10 & 20 & 50 \\
\hline Sept.-Nov. & $\begin{array}{r}1 \\
7 \\
30\end{array}$ & $\begin{array}{r}907 \\
987 \\
1260\end{array}$ & $\begin{array}{l}642 \\
738 \\
870\end{array}$ & $\begin{array}{l}544 \\
653 \\
738\end{array}$ & $\begin{array}{l}477 \\
598 \\
653\end{array}$ & $\begin{array}{l}415 \\
550 \\
577\end{array}$ \\
\hline Dec.-Feb. & $\begin{array}{r}1 \\
7 \\
30\end{array}$ & $\begin{array}{l}1960 \\
2220 \\
3870\end{array}$ & $\begin{array}{l}1120 \\
1260 \\
1900\end{array}$ & $\begin{array}{r}847 \\
945 \\
1290\end{array}$ & $\begin{array}{l}672 \\
746 \\
934\end{array}$ & $\begin{array}{l}520 \\
574 \\
641\end{array}$ \\
\hline Mar. - May & $\begin{array}{r}1 \\
7 \\
30\end{array}$ & $\begin{array}{l}3350 \\
3840 \\
6440\end{array}$ & $\begin{array}{l}2400 \\
2740 \\
4290\end{array}$ & $\begin{array}{l}1990 \\
2320 \\
3440\end{array}$ & $\begin{array}{l}1700 \\
2020 \\
2860\end{array}$ & $\begin{array}{l}1420 \\
1740 \\
2310\end{array}$ \\
\hline
\end{tabular}

Duration of daily flow for indicated periods

\begin{tabular}{l|r|r|r|r|r|r|r|r|r|r|r|r|r}
\hline \multirow{2}{*}{ Period } & \multicolumn{8}{|c}{ Discharge $\left(\mathrm{ft}^{3} / \mathrm{s}\right.$ ) which was equaled or exceeded for indicated percent of time } \\
\cline { 2 - 12 } & 98 & 95 & 90 & 85 & 80 & 75 & 70 & 60 & 50 & 40 & 30 & 20 & 10 \\
\hline Apr. - Mar. & 750 & 930 & 1100 & 1300 & 1600 & 1900 & 2200 & 3000 & 4100 & 5700 & 8100 & 12000 & 20000 \\
May - Nov. & 700 & 840 & 1000 & 1100 & 1300 & 1400 & 1600 & 2100 & 2600 & 3400 & 4500 & 6200 & 10000 \\
June - Aug. & 860 & 1000 & 1200 & 1400 & 1600 & 1800 & 2000 & 2400 & 2900 & 3500 & 4500 & 6100 & 9700 \\
Sept.- Nov. & 630 & 710 & 840 & 940 & 1000 & 1100 & 1200 & 1400 & 1700 & 2100 & 2700 & 3700 & 5800 \\
Dec. - Feb. & 890 & 1100 & 1400 & 1900 & 2300 & 2800 & 3200 & 4300 & 6100 & 8200 & 11000 & 16000 & 24000 \\
Mar. - May & 2500 & 3000 & 3900 & 4600 & 5300 & 6000 & 6700 & 8500 & 11000 & 13000 & 17000 & 21000 & 27000 \\
& & & & & & & & & & & & & \\
\hline
\end{tabular}


MUSKINGUM RIVER BASIN

03150250 Meigs Creek near Beverly, Ohio

LOCATION:

Lat $39^{\circ} 36^{\prime} 00^{\prime \prime}$, long $81^{\circ} 42^{\prime} 42^{\prime \prime}$; Morgan County, Hydrologic

Unit 05040004 , on right bank $400 \mathrm{ft}$ downstream from county road bridge at Mill Giane, $0.4 \mathrm{mi}$ downstream from Perry Run, $0.5 \mathrm{mi}$ upstream from onion Run, $2.2 \mathrm{mi}$ upstream from mouth, and $5.3 \mathrm{mi}$ northwest of Beverly.

DRAINAGE AREA: $136 \mathrm{mi}^{2}$.

TRIBUTARY TO: Muskingum River.

DISCHARGE DATA USED: Low-flow measurements 1972-1974 water years.

INDEX STATION: $\quad 03115400$ Little Muskingum River at Bloomfield, Ohio.

REMARKS: None.

SELECTED DISCHARGE CHARACTERISTICS: Minimum discharge observed: $4.5 \mathrm{ft}^{3} / \mathrm{s}$ September 1972 .

\begin{tabular}{|c|c|c|c|}
\hline \multirow[t]{2}{*}{ Period } & \multirow{2}{*}{$\begin{array}{l}\text { Number of } \\
\text { consecu- } \\
\text { tive days }\end{array}$} & \multicolumn{2}{|c|}{$\begin{array}{l}\text { Discharge }\left(\mathrm{ft}^{3} / \mathrm{s}\right) \text { for } \\
\text { indicated recurrence } \\
\text { interval (years) }\end{array}$} \\
\hline & & 2 & 10 \\
\hline Apr. - Mar. & $\begin{array}{r}7 \\
30\end{array}$ & $\begin{array}{l}3.3 \\
6.8\end{array}$ & $\begin{array}{l}0.8 \\
1.8\end{array}$ \\
\hline May - Nov. & $\begin{array}{r}7 \\
30\end{array}$ & $\begin{array}{l}3.3 \\
6.8\end{array}$ & $\begin{array}{l}0.8 \\
1.8\end{array}$ \\
\hline June - Aug. & $\begin{array}{r}7 \\
30\end{array}$ & ${ }^{82.2}$ & $\begin{array}{l}2.3 \\
5.2\end{array}$ \\
\hline Sept. - Nov. & $\begin{array}{r}7 \\
30\end{array}$ & $\begin{array}{l}4.4 \\
10\end{array}$ & $\begin{array}{l}0.8 \\
2.1\end{array}$ \\
\hline
\end{tabular}

Duration of daily flow for indicated periods

\begin{tabular}{|c|c|c|c|c|c|}
\hline \multirow{2}{*}{ Period } & \multicolumn{5}{|c|}{$\begin{array}{l}\text { Discharge }\left(\mathrm{ft}^{3} / \mathrm{s}\right) \text { which was equaled or } \\
\text { exceeded for indicated percent of time }\end{array}$} \\
\hline & 98 & 95 & 90 & 85 & 80 \\
\hline $\begin{array}{l}\text { Apr. - Mar. } \\
\text { May - Nov. } \\
\text { June - Aug. } \\
\text { Sept. - Nov. }\end{array}$ & $\begin{array}{l}1.8 \\
1.4 \\
2.3 \\
1.1\end{array}$ & $\begin{array}{l}3.5 \\
2.3 \\
3.5 \\
1.6\end{array}$ & $\begin{array}{l}7.2 \\
4.0 \\
5.7 \\
2.5\end{array}$ & $\begin{array}{r}12 \\
6.2 \\
7.9 \\
3.5\end{array}$ & $\begin{array}{l}18 \\
8.7 \\
10 \\
5.0\end{array}$ \\
\hline
\end{tabular}


MUSKINGUM RIVER BASIN

03150480 West Branch Wolf Creek near Waterford, Ohio

LOCATION:

Lat $39^{\circ} 31^{\prime} 43^{\prime \prime}$, long $81^{\circ} 39^{\prime} 22^{\prime \prime}$, Washington County, Hydrologic Unit 05040004, $400 \mathrm{ft}$ upstream from South Branch adjacent to State Route 76 and 1.2 mi southwest of Waterford.

DRAINAGE AREA: $\quad 144 \mathrm{mi}^{2}$.

TRIBUTARY TO: Muskingum River.

DISCHARGE DATA USED: Low-flow measurements 1959, 1972-1977 water years.

INDEX STATION: $\quad 03159500$ Hocking River at Athens.

REMARKS: $\quad$ None.

SELECTED DISCHARGE CHARACTERISTICS: Minimum flow observed: $0.3 \mathrm{ft}^{3} / \mathrm{s}$ September 1957.

\begin{tabular}{l|c|c|c}
\multicolumn{4}{c}{$\begin{array}{c}\text { Magnitude and frequency of low flow for } \\
\text { indicated periods }\end{array}$} \\
\hline & $\begin{array}{c}\text { Number of } \\
\text { consecu- } \\
\text { tive days }\end{array}$ & $\begin{array}{c}\text { Discharge }\left(\mathrm{ft}^{3} / \mathrm{s} \text { ) for }\right. \\
\text { indicated recurrence } \\
\text { interval (years) }\end{array}$ \\
\cline { 3 - 4 } & & 2 & 10 \\
\hline Apr. - Mar. & 7 & 0.6 & 0.1 \\
& 30 & 1.1 & 0.2 \\
May - Nov. & 7 & 0.6 & 0.1 \\
& 30 & 1.1 & 0.2 \\
Sune - Aug. & 7 & 1.4 & 0.3 \\
& 30 & 5.9 & 0.7 \\
Sept.- Nov. & 7 & 0.7 & 0.1 \\
& 30 & 1.4 & 0.2 \\
\hline
\end{tabular}

Duration of daily flow for indicated periods

\begin{tabular}{l|c|l|l|l|l}
\hline \multirow{2}{*}{ Period } & \multicolumn{5}{|c}{$\begin{array}{c}\text { Discharge }\left(\mathrm{ft}^{3} / \mathrm{s}\right) \\
\text { exceeded for }\end{array}$} \\
\cline { 2 - 6 } & 98 & 95 & 90 & 85 & 80 \\
\hline Apr. - Mar. & 0.3 & 0.5 & 0.9 & 1.5 & 2.5 \\
May - Nov. & 0.2 & 0.4 & 0.6 & 0.9 & 1.3 \\
June - Aug. & 0.3 & 0.6 & 1.0 & 1.5 & 1.9 \\
Sept. - Nov. & 0.1 & 0.2 & 0.4 & 0.6 & 0.7 \\
\hline
\end{tabular}


Appendix 3.--Low-flow data--Continued

MUSKINGUM RIVER BASIN

03150490 South Branch Wolf Creek near Waterford, Ohio

LOCATION:

Lat 39031'28", long 81०39'31", Washington County, Hydrologic Unit $05040004,4,000 \mathrm{ft}$ upstream from mouth, at State Route 76 bridge, $1.5 \mathrm{mi}$. southwest of Waterford.

DRAINAGE AREA: $\quad 79.3 \mathrm{mi}^{2}$.

TRIBUTARY TO: Wolf Creek.

DISCHARGE DATA USED: Low-flow measurements 1972-1977 water years.

INDEX STATION: $\quad 03115400 \mathrm{Little}$ Muskingum River at Bloomfield.

REMARKS: Three medium to low-flow discharge were obtained each year to correlate with Little Muskingum River at Bloomfield.

SELECTED DISCHARGE CHARACTERISTICS: Minimum flow observed: $0.7 \mathrm{ft} / 3 / \mathrm{s}$ August 1973.

\begin{tabular}{c|c|c|c}
\multicolumn{4}{c}{$\begin{array}{c}\text { Magnitude and frequency of low flow for } \\
\text { indicated periods }\end{array}$} \\
\hline & $\begin{array}{c}\text { Number of } \\
\text { consecu- } \\
\text { tive days }\end{array}$ & $\begin{array}{c}\text { Discharge }\left(\mathrm{ft}^{3} / \mathrm{s}\right) \text { for } \\
\text { indicated recurrence } \\
\text { interval }\end{array}$ \\
\cline { 3 - 4 } & & 2 & 10 \\
\hline Apr. - Mar. & 7 & 0.3 & 0.1 \\
& 30 & 0.8 & 0.2 \\
May - Noy. & 7 & 0.3 & 0.1 \\
& 30 & 0.8 & 0.2 \\
June - Aug. & 7 & 1.1 & 0.2 \\
& 30 & 3.5 & 0.6 \\
Sept. - Nov. & 7 & 0.5 & 0.1 \\
& 30 & 1.4 & 0.2 \\
\hline
\end{tabular}

Duration of daily flow for indicated periods

\begin{tabular}{c|c|c|c|c|c}
\hline \multirow{2}{*}{ Period } & \multicolumn{5}{|c}{$\begin{array}{c}\text { Discharge ( } \mathrm{ft}^{3} / \mathrm{s} \text { ) which was equaled or } \\
\text { exceeded for indicated percent of time }\end{array}$} \\
\cline { 2 - 6 } & 98 & 95 & 90 & 85 & 80 \\
\hline Apr. - Mar. & 0.2 & 0.4 & 0.9 & 1.6 & 2.7 \\
May - Nov. & 0.1 & 0.2 & 0.4 & 0.7 & 1.1 \\
June - Aug. & 0.2 & 0.4 & 0.7 & 1.0 & 1.4 \\
Sept. - Nov. & 0.1 & 0.1 & 0.2 & 0.4 & 0.6 \\
\hline
\end{tabular}


Appendix 3.--Low-flow data--Continued

LITTLE HOCKING RIVER BASIN

03155800 Little Hocking River near Little Hocking, Onio

LOCATION:

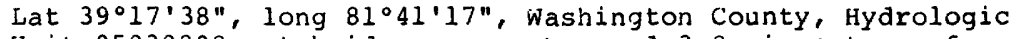
Unit 05030202 , at bridge on county road $3.2 \mathrm{mi}$ upstream from mouth and $2.2 \mathrm{mi}$ north-northeast of Little Hocking.

DRAINAGE AREA: $\quad 47.9 \mathrm{mi}^{2}$.

TRIBUTARY TO: Ohio River.

DISCHARGE DATA USED: LOW-flow measurements 1959-1969, 1971-1974 water years.

INDEX STATION: $\quad 03159500$ Hocking River at Athens, Ohio.

REMARKS:

None.

SELECTED DISCHARGE CHARACTERISTICS: Minimum discharqe observed: No flow September 1559, October 1963.

\begin{tabular}{c|c|c|c}
\multicolumn{4}{c}{$\begin{array}{c}\text { Magnitude and frequency of low flow for } \\
\text { indicated periods }\end{array}$} \\
\hline \multirow{2}{*}{ Period } & $\begin{array}{c}\text { Number of } \\
\text { consecu- } \\
\text { tive days }\end{array}$ & $\begin{array}{c}\text { Discharge (ft } 3 / \mathrm{s} \text { ) for } \\
\text { indicated recurrence } \\
\text { interval (years) }\end{array}$ \\
\cline { 3 - 4 } & 7 & 2 & 10 \\
\hline Apr. - Mar. & 30 & 0 & 0 \\
May - Nov. & 7 & 0.1 & 0 \\
& 30 & 0.1 & 0 \\
June - Aug. & 7 & 0.1 & 0 \\
& 30 & 0.8 & 0 \\
Sept.- Nov. & 7 & 0 & 0 \\
& 30 & 0.1 & 0 \\
\hline
\end{tabular}

Duration of daily flow for indicated periods

\begin{tabular}{c|c|c|c|c|c}
\hline \multirow{2}{*}{ Period } & \multicolumn{5}{|c}{$\begin{array}{c}\text { Discharge ( } \mathrm{ft}^{3 / \mathrm{s}} \text { ) which was equaled or } \\
\text { exceeded for indicated percent of time }\end{array}$} \\
\cline { 2 - 6 } & 98 & 95 & 90 & 85 & 80 \\
\hline Apr. - Mar. & 0 & 0 & 0 & 0.1 & 0.2 \\
May - Nov. & 0 & 0 & 0 & 0 & 0.1 \\
June - Aug. & 0 & 0 & 0 & 0.1 & 0.1 \\
Sept. - Nov. & 0 & 0 & 0 & 0 & 0 \\
\hline
\end{tabular}


HOCKING RIVER BASIN

03156000 Hunters Run at Lancaster, Ohio

LOCATION:

Lat $39^{\circ} 41^{\prime} 57^{\prime \prime}$, long $82^{\circ} 37^{\prime} 18^{\prime \prime}$, in NEl/4 sec. 11, T.14 N., R.19 W., Fairfield County, Hydrologic Unit 05030204, on rignt bank at downstream side of bridge on U.S. Highway $22,1.0 \mathrm{mi}$ southwest of Lancaster, and $1.5 \mathrm{mi}$ unstream from mouth.

DRAINAGE AREA: $\quad 10.0 \mathrm{mi}^{2}$.

TRIBUTARY TO: Hocking River.

DISCHARGE DATA USED: January 1956 to September 1978.

REMARKS:

Flood peaks affected by temporary retention in four retaraing basins upstream from station, combined capacity, 2,800 acre-ft. Controlled drainage area is $8.49 \mathrm{mi}^{2}$.

SELECTED DISCHARGE CHARACTERISTICS: Average discharge: $10 \mathrm{ft}^{3} / \mathrm{s}$ (22 years)

Minimum daily discharge: $0.08 \mathrm{ft} 3 / \mathrm{s}$ July 1977.

Magnitude and frequency of low flow for indicated periods

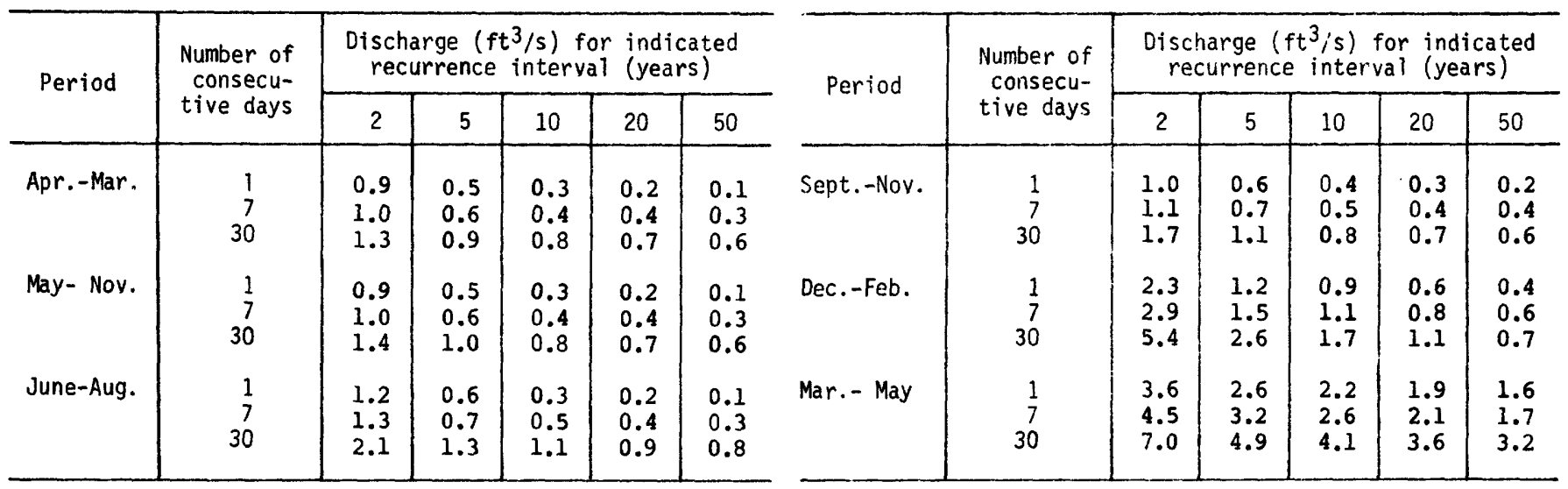

Duration of daily flow for indicated periods

\begin{tabular}{|c|c|c|c|c|c|c|c|c|c|c|c|c|c|c|}
\hline \multirow{2}{*}{\multicolumn{2}{|c|}{ Period }} & \multicolumn{13}{|c|}{ Discharge $\left(\mathrm{ft}^{3} / \mathrm{s}\right)$ which was equaled or exceeded for indicated percent of time } \\
\hline & & 98 & 95 & 90 & 85 & 80 & 75 & 70 & 60 & 50 & 40 & 30 & 20 & 10 \\
\hline $\begin{array}{l}\text { Apr. - } \\
\text { May - } \\
\text { June - } \\
\text { Sept. - } \\
\text { Dec. - } \\
\text { Mar. - }\end{array}$ & $\begin{array}{l}\text { Mar. } \\
\text { Nov. } \\
\text { Aug. } \\
\text { Nov. } \\
\text { Feb. } \\
\text { May }\end{array}$ & $\begin{array}{l}0.7 \\
0.7 \\
0.7 \\
0.6 \\
0.8 \\
2.9\end{array}$ & $\begin{array}{l}1.0 \\
0.9 \\
0.9 \\
0.8 \\
1.2 \\
3.6\end{array}$ & $\begin{array}{l}1.3 \\
1 \cdot 1 \\
1.2 \\
1.0 \\
1.9 \\
4.5\end{array}$ & $\begin{array}{l}1.5 \\
1.3 \\
1.4 \\
1.1 \\
2.5 \\
5.0\end{array}$ & $\begin{array}{l}1.9 \\
1.4 \\
1.7 \\
1.2 \\
3.0 \\
5.6\end{array}$ & $\begin{array}{l}2.2 \\
1.7 \\
1.9 \\
1.3 \\
3.6 \\
6.3\end{array}$ & $\begin{array}{l}2.6 \\
1.9 \\
2.2 \\
1.4 \\
4.2 \\
6.9\end{array}$ & $\begin{array}{l}3.6 \\
2.4 \\
2.5 \\
1.7 \\
5.5 \\
8.4\end{array}$ & $\begin{array}{r}4.9 \\
3.0 \\
3.0 \\
2.1 \\
6.9 \\
10\end{array}$ & $\begin{array}{r}6.5 \\
3.8 \\
3.7 \\
2.5 \\
8.9 \\
12\end{array}$ & $\begin{array}{l}8.8 \\
5.1 \\
4.7 \\
3.4 \\
12 \\
15\end{array}$ & $\begin{array}{c}13 \\
7.2 \\
6.9 \\
4.9 \\
16 \\
20\end{array}$ & $\begin{array}{l}20 \\
12 \\
12 \\
7.6 \\
25 \\
31\end{array}$ \\
\hline
\end{tabular}


LOCATION:

Lat $39^{\circ} 42^{\prime} 24^{\prime \prime}$, long $82^{\circ} 36^{\prime} 03^{\prime \prime}$, in NE1/4 sec. 12, T.14 N., R.19 W.,

Fairfield County, Hydrologic Unit 05030204 , on right bank $25 \mathrm{ft}$ upstream from Columbus Street Bridge in Lancaster, and $0.5 \mathrm{mi}$ downstream from Hunters Run.

DRAINAGE AREA: $\quad 48.2 \mathrm{mi}^{2}$.

TRIBUTARY TO: Ohio River.

DISCHARGE DATA USED: June 1956 to September 1974.

REMARKS: $\quad$ Some diurnal fluctuation caused by industrial plants upstream from

station. Water supply for city of Lancaster is pumped from wells adjacent to the ${ }_{3}$ Hocking River $1.1 \mathrm{mi}$ upstream from station. The pumpage averaged

$8.5 \mathrm{ft}^{3} / \mathrm{s}$ in 1974 and is returned as sewage $0.8 \mathrm{mi}$ downstream from the station. Flood flow affected by temporary retention in eight retarding basins, combined capacity 8,710 acre-ft upstream from station. Controlled drainage area is $24.4 \mathrm{mi}^{2}$. SELECTED DISCHARGE CHARACTERISTICS: Average discharge: $41.4 \mathrm{ft} / \mathrm{s}$ ( 38 years). Minimum daily discharge: $1.4 \mathrm{ft} / \mathrm{s}$ September 1964.

Magnitude and frequency of low flow for indicated periods

\begin{tabular}{|c|c|c|c|c|c|c|c|c|c|c|c|c|c|}
\hline \multirow{2}{*}{ Period } & \multirow{2}{*}{$\begin{array}{l}\text { Number of } \\
\text { consecu- } \\
\text { tive days }\end{array}$} & \multicolumn{5}{|c|}{$\begin{array}{l}\text { Discharge }\left(\mathrm{ft}^{3} / \mathrm{s}\right) \text { for indicated } \\
\text { recurrence interval (years) }\end{array}$} & \multirow{2}{*}{ Period } & \multirow{2}{*}{$\begin{array}{l}\text { Number of } \\
\text { consecu- } \\
\text { tive days }\end{array}$} & \multicolumn{5}{|c|}{$\begin{array}{l}\text { Discharge }\left(\mathrm{ft}^{3} / \mathrm{s}\right) \text { for indicated } \\
\text { recurrence interval (years) }\end{array}$} \\
\hline & & 2 & 5 & 10 & 20 & 50 & & & 2 & 5 & 10 & 20 & 50 \\
\hline Apr.-Mar. & $\begin{array}{r}1 \\
7 \\
30\end{array}$ & $\begin{array}{l}4.0 \\
5.1 \\
6.6\end{array}$ & $\begin{array}{l}2.4 \\
3.1 \\
4.3\end{array}$ & $\begin{array}{l}1.8 \\
2.4 \\
3.5\end{array}$ & $\begin{array}{l}1.5 \\
1.9 \\
3.0\end{array}$ & $\begin{array}{l}1.1 \\
1.5 \\
2.5\end{array}$ & Sept.-Nov. & $\begin{array}{r}1 \\
7 \\
30\end{array}$ & $\begin{array}{l}4.8 \\
5.6 \\
8.1\end{array}$ & $\begin{array}{l}2.9 \\
3.4 \\
4.9\end{array}$ & $\begin{array}{l}2.1 \\
2.6 \\
3.8\end{array}$ & $\begin{array}{l}1.7 \\
2.1 \\
3.1\end{array}$ & $\begin{array}{l}1.2 \\
1.6 \\
2.4\end{array}$ \\
\hline June-Aug. & $\begin{array}{r}1 \\
7 \\
30\end{array}$ & $\begin{array}{r}5.9 \\
7.3 \\
11\end{array}$ & $\begin{array}{l}3.6 \\
5.0 \\
7.0\end{array}$ & $\begin{array}{l}2.8 \\
4.1 \\
5.9\end{array}$ & $\begin{array}{l}2.2 \\
3.6 \\
5.1\end{array}$ & $\begin{array}{l}1.7 \\
3.0 \\
4.5\end{array}$ & Mar. - May & $\begin{array}{r}1 \\
7 \\
30\end{array}$ & $\begin{array}{l}16 \\
19 \\
28\end{array}$ & $\begin{array}{l}13 \\
15 \\
21\end{array}$ & $\begin{array}{l}12 \\
13 \\
19\end{array}$ & $\begin{array}{l}11 \\
12 \\
17\end{array}$ & $\begin{array}{l}9.5 \\
11 \\
16\end{array}$ \\
\hline
\end{tabular}

Duration of daily flow for indicated periods

\begin{tabular}{l|c|c|c|c|c|c|c|c|c|c|c|c|c}
\hline \multirow{2}{*}{ Period } & \multicolumn{8}{|c|}{ Discharge $\left(\mathrm{ft}^{3} / \mathrm{s}\right)$} & which was equaled or exceeded for indicated percent of time \\
\cline { 2 - 14 } & 98 & 95 & 90 & 85 & 80 & 75 & 70 & 60 & 50 & 40 & 30 & 20 & 10 \\
\hline Apr. - Mar. & 3.4 & 4.7 & 6.2 & 7.5 & 8.8 & 10 & 12 & 16 & 21 & 26 & 35 & 50 & 83 \\
May - Nov. & 3.0 & 4.0 & 5.2 & 6.1 & 7.0 & 7.8 & 8.6 & 11 & 14 & 17 & 21 & 28 & 48 \\
June - Aug. & 4.0 & 5.0 & 6.3 & 7.1 & 8.0 & 8.7 & 9.5 & 12 & 14 & 17 & 21 & 28 & 50 \\
Sept. - Nov. & 2.5 & 3.2 & 4.2 & 4.9 & 5.6 & 6.2 & 6.9 & 8.2 & 9.8 & 12 & 15 & 20 & 31 \\
Dec. - Feb. & 4.9 & 7.0 & 10 & 13 & 15 & 17 & 19 & 23 & 29 & 36 & 46 & 60 & 100 \\
Mar. - May & 15 & 17 & 19 & 21 & 23 & 26 & 28 & 33 & 39 & 47 & 57 & 79 & 130 \\
\hline
\end{tabular}


Appendix 3.--Low-flow data--Continued

\section{HOCKING RIVER BASIN}

03156700 Rush Creek near Sugar Grove, Ohio

LOCATION:

Lat 39०38'15", long $82^{\circ} 30^{\prime} 40^{\prime \prime}$, Fairfield County, Hydrologic Unit 05020304 , at highway bridge on Berne Township Road 294, $2 \mathrm{mi}$ northeast of Sugar Grove.

DRAINAGE AREA: $\quad 229 \mathrm{mi}^{2}$.

TRIBUTARY TO: Hocking River.

DISCHARGE DATA USED: Low-flow measurements 1962-1973 water years.

INDEX STATION: $\quad 03157500$ Hocking River at Enterprise, Ohio. REMARKS: None.

SELECTED DISCHARGE CHARACTERISTICS: None.

\begin{tabular}{c|c|c|c}
\multicolumn{4}{c}{$\begin{array}{c}\text { Magnitude and frequency of low flow for } \\
\text { indicated periods }\end{array}$} \\
\hline \multirow{3}{*}{ Period } & $\begin{array}{c}\text { Number of } \\
\text { consecu- } \\
\text { tive days }\end{array}$ & $\begin{array}{c}\text { Discharge (ft3/s) for } \\
\text { indicated recurrence } \\
\text { interval (years) }\end{array}$ \\
\cline { 3 - 4 } & 7 & 2 & 10 \\
\hline Apr. - Mar. & 30 & 14 & 3.7 \\
May - Nov. & 7 & 9.5 & 5.2 \\
& 30 & 14 & 3.7 \\
June - Aug. & 7 & 14 & 5.2 \\
Sept. - Nov. & 30 & 32 & 5.2 \\
& 7 & 10 & 9.8 \\
& 30 & 17 & 3.9 \\
\hline
\end{tabular}

Duration of daily flow for indicated periods

\begin{tabular}{c|c|c|c|c|c}
\hline \multirow{2}{*}{ Period } & \multicolumn{5}{|c}{$\begin{array}{c}\text { Discharge ( } \mathrm{ft}^{3} / \mathrm{s} \text { ) which was equa led or } \\
\text { exceeded for ind icated percent of time }\end{array}$} \\
\cline { 2 - 6 } & 98 & 95 & 90 & 85 & 80 \\
\hline Apr. - Mar. & 5.2 & 7.8 & 12 & 16 & 21 \\
May - Nov. & 4.7 & 6.4 & 9.0 & 12 & 14 \\
June - Aug. & 5.8 & 8.2 & 11 & 14 & 19 \\
Sept. - Nov. & 3.9 & 5.0 & 6.9 & 8.6 & 10 \\
\hline
\end{tabular}


Appendix 3.--Low-flow data--Continued

HOCKING RIVER BASIN

03157000 Clear Creek near Rockbridge, Ohio

LOCATION:

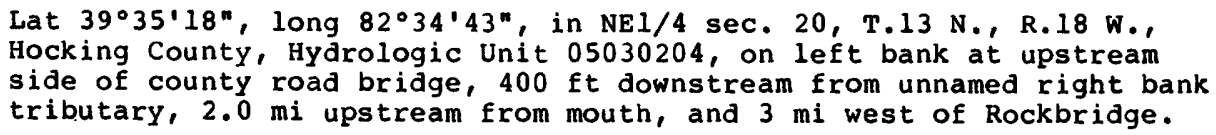

DRAINAGE AREA: $\quad 89.0 \mathrm{mi}^{2}$.

TRIBUTARY TO: Hocking River.

DISCHARGE DATA USED: October 1939 to September 1978.

REMARKS:

None.

SELECTED DISCHARGE CHARACTERISTICS: Average discharge: $86.7 \mathrm{ft}^{3} / \mathrm{s}$ (39 years).

Minimum daily discharge: $3.5 \mathrm{ft} 3 / \mathrm{s}$ August 1942.

Magnitude and frequency of low flow for indicated periods

\begin{tabular}{|c|c|c|c|c|c|c|c|c|c|c|c|c|c|}
\hline \multirow{2}{*}{ Period } & \multirow{2}{*}{$\begin{array}{l}\text { Number of } \\
\text { consecu- } \\
\text { tive days }\end{array}$} & \multicolumn{5}{|c|}{$\begin{array}{l}\text { Discharge }\left(\mathrm{ft}^{3} / \mathrm{s}\right) \text { for indicated } \\
\text { recurrence interval (years) }\end{array}$} & \multirow{2}{*}{ Period } & \multirow{2}{*}{$\begin{array}{l}\text { Number of } \\
\text { consecu- } \\
\text { tive days }\end{array}$} & \multicolumn{5}{|c|}{$\begin{array}{l}\text { Discharge }\left(\mathrm{ft}^{3} / \mathrm{s}\right) \text { for indicated } \\
\text { recurrence interval (years) }\end{array}$} \\
\hline & & 2 & 5 & 10 & 20 & 50 & & & 2 & 5 & 10 & 20 & 50 \\
\hline Apr.-Mar. & $\begin{array}{r}1 \\
7 \\
30\end{array}$ & $\begin{array}{l}13 \\
13 \\
15\end{array}$ & $\begin{array}{c}9.1 \\
9.8 \\
12\end{array}$ & $\begin{array}{l}7.4 \\
8.5 \\
11\end{array}$ & $\begin{array}{c}6.1 \\
7.6 \\
10\end{array}$ & $\begin{array}{l}4.9 \\
6.7 \\
9.1\end{array}$ & Sept.-Nov. & $\begin{array}{r}1 \\
7 \\
30\end{array}$ & $\begin{array}{l}13 \\
14 \\
18\end{array}$ & $\begin{array}{l}9.7 \\
11 \\
14\end{array}$ & $\begin{array}{r}8.4 \\
9.7 \\
12\end{array}$ & $\begin{array}{r}7.4 \\
9.0 \\
11\end{array}$ & $\begin{array}{l}6.4 \\
8.4 \\
10\end{array}$ \\
\hline June-Aug. & $\begin{array}{r}1 \\
7 \\
30\end{array}$ & $\begin{array}{l}14 \\
15 \\
21\end{array}$ & $\begin{array}{l}10 \\
11 \\
15\end{array}$ & $\begin{array}{l}8.0 \\
9.4 \\
13\end{array}$ & $\begin{array}{l}6.5 \\
8.2 \\
11\end{array}$ & $\begin{array}{l}5.0 \\
7.0 \\
9.9\end{array}$ & Mar.- May & $\begin{array}{r}1 \\
7 \\
30\end{array}$ & $\begin{array}{l}35 \\
41 \\
62\end{array}$ & $\begin{array}{l}27 \\
32 \\
44\end{array}$ & $\begin{array}{l}24 \\
28 \\
37\end{array}$ & $\begin{array}{l}21 \\
25 \\
32\end{array}$ & $\begin{array}{l}18 \\
22 \\
28\end{array}$ \\
\hline
\end{tabular}

Duration of daily flow for indicated periods

\begin{tabular}{|c|c|c|c|c|c|c|c|c|c|c|c|c|c|}
\hline \multirow{2}{*}{ Period } & \multicolumn{13}{|c|}{ Discharge $\left(\mathrm{ft}^{3} / \mathrm{s}\right)$ which was equaled or exceeded for indicated percent of time } \\
\hline & 98 & 95 & 90 & 85 & 80 & 75 & 70 & 60 & 50 & 40 & 30 & 20 & 10 \\
\hline $\begin{array}{l}\text { Apr. - Mar. } \\
\text { May - Nov. } \\
\text { June - Aug. } \\
\text { Sept. - Nov. } \\
\text { Dec. - Feb. } \\
\text { Mar. - May }\end{array}$ & $\begin{array}{l}11 \\
10 \\
10 \\
10 \\
15 \\
28\end{array}$ & $\begin{array}{l}13 \\
12 \\
12 \\
11 \\
18 \\
34\end{array}$ & $\begin{array}{l}16 \\
14 \\
14 \\
13 \\
21 \\
40\end{array}$ & $\begin{array}{l}18 \\
15 \\
16 \\
14 \\
25 \\
45\end{array}$ & $\begin{array}{l}20 \\
17 \\
17 \\
15 \\
30 \\
50\end{array}$ & $\begin{array}{l}22 \\
18 \\
19 \\
16 \\
34 \\
55\end{array}$ & $\begin{array}{l}25 \\
19 \\
21 \\
17 \\
38 \\
60\end{array}$ & $\begin{array}{l}32 \\
23 \\
24 \\
19 \\
49 \\
72\end{array}$ & $\begin{array}{l}41 \\
27 \\
27 \\
21 \\
63 \\
86\end{array}$ & $\begin{array}{r}55 \\
33 \\
32 \\
24 \\
80 \\
100\end{array}$ & $\begin{array}{r}74 \\
41 \\
40 \\
28 \\
100 \\
130\end{array}$ & $\begin{array}{r}110 \\
57 \\
55 \\
36 \\
140 \\
170\end{array}$ & $\begin{array}{r}180 \\
93 \\
94 \\
53 \\
240 \\
280\end{array}$ \\
\hline
\end{tabular}


HOCKING RIVER BASIN

03157500 Hocking River at Enterprise, Ohio

LOCATION:

Lat $39^{\circ} 33^{\prime} 54^{\prime \prime}$, long $82^{\circ} 28^{\prime} 29^{\prime \prime}$, in NWl/4 sec. 5, T.14 N., R.17 W., Hocking

County, Hydrologic Unit 05030204, at right bank at upstream side of

abandoned bridge at Enterprise, $4.0 \mathrm{mi}$ downstream from Buck Run, and

$4.3 \mathrm{mi}$ upstream from Scott Creek.

DRAINAGE AREA:

$459 \mathrm{mi}^{2}$.

TRIBUTARY TO:

Ohio River.

DISCHARGE DATA USED:

May 1931 to September 1978.

REMARKS:

Flood flow affected by temporary retention in eight retarding basins. upstream from station.

SELECTED DISCHARGE CHARACTERISTICS: Average discharge: $450 \mathrm{ft}^{3} / \mathrm{s}$ ( 47 years).

Minimum daily discharge: $23 \mathrm{ft} 3 / \mathrm{s}$ August 1944.

Magnitude and frequency of low flow for indicated periods

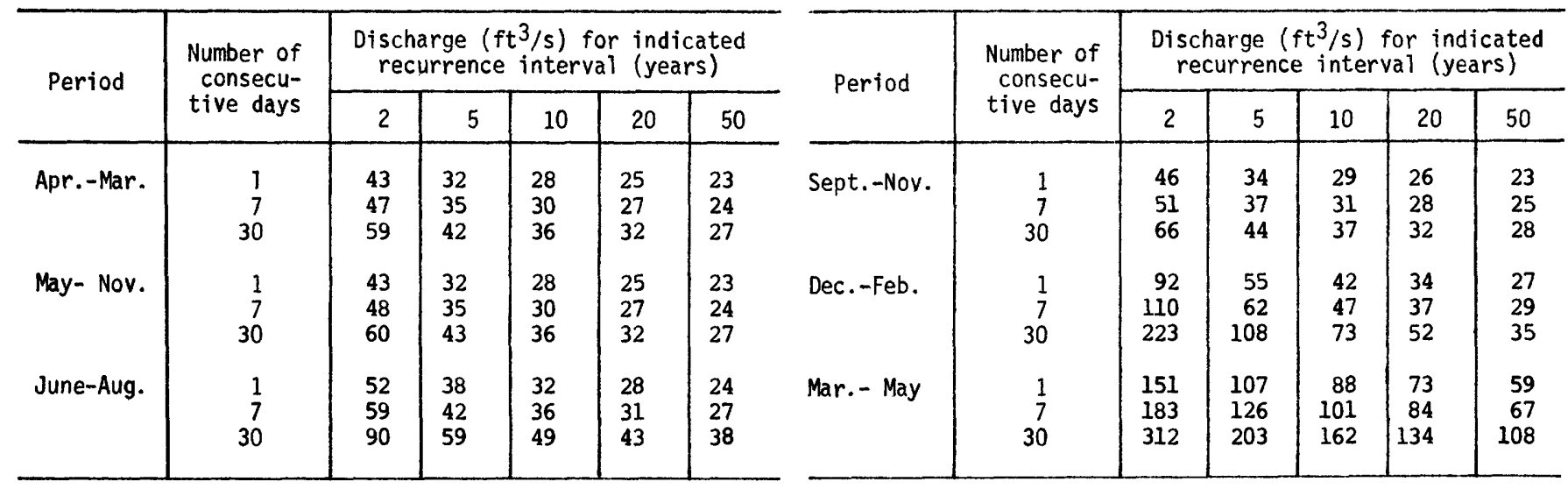

Duration of daily flow for indicated periods

\begin{tabular}{l|r|r|r|r|r|r|r|r|r|r|r|r|r}
\hline \multirow{2}{*}{ Period } & \multicolumn{8}{|c|}{ Discharge $\left(\mathrm{ft}^{3} / \mathrm{s}\right)$ which was equaled or exceeded for indicated percent of time } \\
\cline { 2 - 12 } & 98 & 95 & 90 & 85 & 80 & 75 & 70 & 60 & 50 & 40 & 30 & 20 & 10 \\
\hline Apr. - Mar. & 36 & 44 & 54 & 63 & 73 & 86 & 100 & 140 & 190 & 270 & 390 & 590 & 1000 \\
May - Nov. & 34 & 40 & 47 & 54 & 60 & 66 & 74 & 92 & 120 & 150 & 200 & 290 & 510 \\
June - Aug. & 38 & 45 & 53 & 60 & 68 & 76 & 84 & 100 & 120 & 150 & 200 & 290 & 520 \\
Sept. - Nov. & 31 & 35 & 41 & 46 & 50 & 54 & 58 & 67 & 80 & 97 & 130 & 170 & 290 \\
Dec. - Feb. & 46 & 58 & 75 & 100 & 120 & 150 & 170 & 240 & 320 & 430 & 570 & 810 & 1400 \\
Mar. - May & 110 & 150 & 180 & 210 & 240 & 270 & 310 & 380 & 460 & 580 & 730 & 1000 & 1700 \\
\hline
\end{tabular}




\section{HOCKING RIVER BASIN \\ 03159000 Sunday Creek at Glouster, Ohio}

LOCATION:

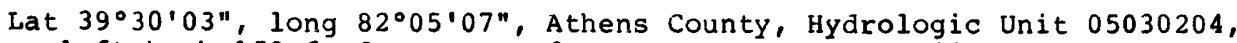
on left bank $150 \mathrm{ft}$ downstream from West Branch and $200 \mathrm{ft}$ upstream from bridge on State Route 78 at Glouster.

DRAINAGE AREA: $\quad 104 \mathrm{mi}^{2}$.

TRIBUTARY TO: Hocking River.

DISCHARGE DATA USED: October 1951 to September 1978.

REMARKS:

Flow partly regulated by Burr Oak Reservoir $5.2 \mathrm{mi}$ upstream. Most of small diversion downstream from Burr Oak Reservoir, average discharge $0.90 \mathrm{ft} / \mathrm{s}$ is returned to stream upstream from station.

SELECTED DISCHARGE CHARACTERISTICS: Average discharge: $101 \mathrm{ft}^{3} / \mathrm{s}$ ( $2 \xi$ years).

Minimum daily discharge: $0.5 \mathrm{ft}^{3} / \mathrm{s}$ November, December 1953.

Magnitude and frequency of low flow for indicated periods

\begin{tabular}{|c|c|c|c|c|c|c|c|c|c|c|c|c|c|}
\hline \multirow{2}{*}{ Period } & \multirow{2}{*}{$\begin{array}{l}\text { Number of } \\
\text { consecu- } \\
\text { tive days }\end{array}$} & \multicolumn{5}{|c|}{$\begin{array}{l}\text { Discharge }\left(\mathrm{ft}^{3} / \mathrm{s}\right) \text { for indicated } \\
\text { recurrence interval (years) }\end{array}$} & \multirow{2}{*}{ Period } & \multirow{2}{*}{$\begin{array}{l}\text { Number of } \\
\text { consecu- } \\
\text { tive days }\end{array}$} & \multicolumn{5}{|c|}{$\begin{array}{l}\text { Discharge }\left(\mathrm{ft}^{3} / \mathrm{s}\right) \text { for indicated } \\
\text { recurrence interval (years) }\end{array}$} \\
\hline & & 2 & 5 & 10 & 20 & 50 & & & 2 & 5 & 10 & 20 & 50 \\
\hline Apr.-Mar. & $\begin{array}{r}1 \\
7 \\
30\end{array}$ & $\begin{array}{l}4.3 \\
5.0 \\
6.2\end{array}$ & $\begin{array}{l}2.3 \\
2.7 \\
3.2\end{array}$ & $\begin{array}{l}1.6 \\
1.8 \\
2.2\end{array}$ & $\begin{array}{l}1.1 \\
1.3 \\
1.6\end{array}$ & $\begin{array}{l}0.7 \\
0.8 \\
1.1\end{array}$ & Sept.-Nov. & $\begin{array}{r}1 \\
7 \\
30\end{array}$ & $\begin{array}{l}4.8 \\
5.6 \\
7.4\end{array}$ & $\begin{array}{l}2.5 \\
2.8 \\
3.3\end{array}$ & $\begin{array}{l}1.6 \\
1.8 \\
2.1\end{array}$ & $\begin{array}{l}1.1 \\
1.2 \\
1.5\end{array}$ & $\begin{array}{l}0.7 \\
0.8 \\
1.0\end{array}$ \\
\hline June-Aug. & $\begin{array}{r}1 \\
7 \\
30\end{array}$ & $\begin{array}{r}5.7 \\
6.7 \\
11\end{array}$ & $\begin{array}{l}3.1 \\
3.7 \\
5.3\end{array}$ & $\begin{array}{l}2.0 \\
2.5 \\
3.6\end{array}$ & $\begin{array}{l}1.4 \\
1.7 \\
2.5\end{array}$ & $\begin{array}{l}0.9 \\
1.1 \\
1.7\end{array}$ & Mar. - May & $\begin{array}{r}1 \\
7 \\
30\end{array}$ & $\begin{array}{l}22 \\
27 \\
66\end{array}$ & $\begin{array}{l}14 \\
18 \\
41\end{array}$ & $\begin{array}{l}11 \\
14 \\
32\end{array}$ & $\begin{array}{l}9.5 \\
12 \\
27\end{array}$ & $\begin{array}{r}7.6 \\
9.5 \\
22\end{array}$ \\
\hline
\end{tabular}

Duration of daily flow for indicated periods

\begin{tabular}{|c|c|c|c|c|c|c|c|c|c|c|c|c|c|c|}
\hline \multirow{2}{*}{\multicolumn{2}{|c|}{ Period }} & \multicolumn{13}{|c|}{ Discharge $\left(\mathrm{ft}^{3} / \mathrm{s}\right)$ which was equaled or exceeded for indicated percent of time } \\
\hline & & 98 & 95 & 90 & 85 & 80 & 75 & 70 & 60 & 50 & 40 & 30 & 20 & 10 \\
\hline $\begin{array}{l}\text { Apr. - } \\
\text { May - } \\
\text { June - } \\
\text { Sept. - } \\
\text { Dec. - } \\
\text { Mar. - }\end{array}$ & $\begin{array}{l}\text { Mar. } \\
\text { Nov. } \\
\text { Aug. } \\
\text { Nov. } \\
\text { Feb. } \\
\text { May }\end{array}$ & $\begin{array}{l}2.1 \\
1.8 \\
2.5 \\
1.1 \\
3.5 \\
16\end{array}$ & $\begin{array}{r}3.7 \\
3.0 \\
3.9 \\
2.3 \\
5.8 \\
22\end{array}$ & $\begin{array}{l}5.1 \\
4.2 \\
5.1 \\
3.2 \\
11 \\
30\end{array}$ & $\begin{array}{c}6.4 \\
5.1 \\
6.1 \\
3.9 \\
14 \\
38\end{array}$ & $\begin{array}{l}8.1 \\
5.9 \\
7.0 \\
4.6 \\
18 \\
46\end{array}$ & $\begin{array}{l}11 \\
6.9 \\
7.8 \\
5.1 \\
24 \\
54\end{array}$ & $\begin{array}{c}13 \\
8.0 \\
8.8 \\
5.6 \\
30 \\
61\end{array}$ & $\begin{array}{l}19 \\
11 \\
11 \\
7.2 \\
45 \\
78\end{array}$ & $\begin{array}{r}31 \\
15 \\
14 \\
11 \\
65 \\
100\end{array}$ & $\begin{array}{r}51 \\
20 \\
18 \\
14 \\
89 \\
130\end{array}$ & $\begin{array}{r}83 \\
29 \\
23 \\
19 \\
130 \\
170\end{array}$ & $\begin{array}{r}140 \\
52 \\
33 \\
30 \\
210 \\
280\end{array}$ & $\begin{array}{r}300 \\
130 \\
81 \\
88 \\
400 \\
490\end{array}$ \\
\hline
\end{tabular}


HOCKING RIVER BASIN

03159500 Hocking River at Athens, Ohio

LOCATION:

Lat $39^{\circ} 19^{\prime} 44^{\prime \prime}$, long $82^{\circ} 05^{\prime} 16^{\prime \prime}$, in T.9 N., R.14 W., Athens County, Hydrologic Unit 05030204 , on right bank $0.8 \mathrm{mi}$ east of business section of Athens, $1.4 \mathrm{mi}$ downstream from Coats Run, and $3.0 \mathrm{mi}$ downstream from Margaret Creek.

DRAINAGE AREA: $\quad 943 \mathrm{mi}^{2}$.

TRIBUTARY TO: Ohio River.

DISCHARGE DATA USED: October 1915 to September 1976.

REMARKS:

Some regulation by Burr Oak Reservoir on East Branch Sunday Creek 29 mi upstream beginning 1952; by Hocking Lake, capactiy 3,080 acre-ft, on Clear Fork $39.4 \mathrm{mi}$ upstream beginning in 1949; and by temporary retention in eight retarding basins, combined capacity, 8,710 acre-ft, constructed between 1955 and 1961 upstream from Lancaster. Diurnal fluctuation at low flow caused by mill $3.2 \mathrm{mi}$ upstream from station. Channel work has destroyed stage-discharge relationship that existed prior to June 1970 .

SELECTED DISCHARGE CHARACTERISTICS: Average discharge: $986 \mathrm{ft}^{3} / \mathrm{s}$ (61 years). Minimum daily discharge: $10 \mathrm{ft} / \mathrm{s}$ October 1930.

Magnitude and frequency of low flow for indicated periods

\begin{tabular}{|c|c|c|c|c|c|c|}
\hline \multirow{2}{*}{ Period } & \multirow{2}{*}{$\begin{array}{l}\text { Number of } \\
\text { consecu- } \\
\text { tive days }\end{array}$} & \multicolumn{5}{|c|}{$\begin{array}{l}\text { Discharge }\left(\mathrm{ft}^{3} / \mathrm{s}\right) \text { for indicated } \\
\text { recurrence interval (years) }\end{array}$} \\
\hline & & 2 & 5 & io & 20 & 50 \\
\hline Apr.-Mar. & $\begin{array}{r}1 \\
7 \\
30\end{array}$ & $\begin{array}{l}65 \\
73 \\
90\end{array}$ & $\begin{array}{l}40 \\
50 \\
61\end{array}$ & $\begin{array}{l}30 \\
41 \\
50\end{array}$ & $\begin{array}{l}23 \\
34 \\
43\end{array}$ & $\begin{array}{l}17 \\
28 \\
36\end{array}$ \\
\hline May- Nov. & $\begin{array}{r}1 \\
7 \\
30\end{array}$ & $\begin{array}{l}65 \\
73 \\
91\end{array}$ & $\begin{array}{l}40 \\
50 \\
61\end{array}$ & $\begin{array}{l}30 \\
41 \\
50\end{array}$ & $\begin{array}{l}23 \\
34 \\
43\end{array}$ & $\begin{array}{l}17 \\
28 \\
36\end{array}$ \\
\hline June-Aug. & $\begin{array}{r}1 \\
7 \\
30\end{array}$ & $\begin{array}{r}85 \\
97 \\
164\end{array}$ & $\begin{array}{l}53 \\
66 \\
98\end{array}$ & $\begin{array}{l}39 \\
55 \\
76\end{array}$ & $\begin{array}{l}30 \\
46 \\
62\end{array}$ & $\begin{array}{l}22 \\
39 \\
49\end{array}$ \\
\hline
\end{tabular}

\begin{tabular}{c|c|c|c|c|c|c}
\hline \multirow{2}{*}{ Period } & \multirow{2}{*}{$\begin{array}{c}\text { Number of } \\
\text { consecu- } \\
\text { tive days }\end{array}$} & \multicolumn{3}{|c}{$\begin{array}{c}\text { Discharge } \\
\left.\text { recurrence } \mathrm{ft}^{3} / \mathrm{s}\right)\end{array}$ for interval $\begin{array}{c}\text { indicated } \\
\text { (years) }\end{array}$} \\
\cline { 3 - 7 } Sept.-Nov. & 1 & 71 & 43 & 33 & 26 & 19 \\
& 7 & 76 & 50 & 41 & 35 & 29 \\
& 30 & 96 & 61 & 50 & 43 & 38 \\
Dec.-Feb. & 1 & 166 & 87 & 62 & 47 & 35 \\
& 7 & 205 & 105 & 73 & 54 & 39 \\
& 30 & 473 & 206 & 128 & 84 & 51 \\
& 1 & 303 & 201 & 157 & 126 & 96 \\
& 7 & 372 & 238 & 183 & 145 & 110 \\
& 30 & 662 & 401 & 305 & 242 & 185 \\
\hline
\end{tabular}

Duration of daily flow for indicated periods

\begin{tabular}{l|r|r|r|r|r|r|r|r|r|r|r|r|r}
\hline \multirow{2}{*}{ Period } & \multicolumn{8}{|c|}{ Discharge $\left(\mathrm{ft}^{3} / \mathrm{s}\right)$} & which was equaled or exceeded for indicated percent of time \\
\cline { 2 - 12 } & 98 & 95 & 90 & 85 & 80 & 75 & 70 & 60 & 50 & 40 & 30 & 20 & 10 \\
\hline Apr. - Mar. & 52 & 66 & 83 & 100 & 120 & 140 & 180 & 270 & 400 & 590 & 850 & 1300 & 2400 \\
May - Nov. & 46 & 58 & 72 & 83 & 94 & 110 & 120 & 150 & 200 & 280 & 390 & 610 & 1100 \\
June - Aug. & 55 & 69 & 85 & 100 & 110 & 130 & 140 & 180 & 230 & 290 & 380 & 570 & 1100 \\
Sept.- Nov. & 40 & 49 & 60 & 59 & 75 & 82 & 88 & 100 & 120 & 160 & 210 & 320 & 660 \\
Dec. - Feb. & 66 & 87 & 130 & 180 & 250 & 310 & 380 & 530 & 710 & 950 & 1300 & 1900 & 3400 \\
Mar. - May & 200 & 270 & 350 & 430 & 510 & 570 & 050 & 790 & 970 & 1200 & 1600 & 2300 & 3900 \\
\hline
\end{tabular}


Appendix 3.--Low-flow data--Continued

SHADE RIVER BASIN

03159540 Shade River near Chester, Ohio

LOCATION:

Lat $39^{\circ} 03^{\prime} 49^{\prime \prime}$, long $81^{\circ} 52^{\prime} 55^{\prime \prime}$, in NEl/4 sec. 10, T.3 N., R.12 W.,

Meigs County, Hydrologic Unit 05030202, on right bank at downstream

side of bridge on Oak Hill Road, $200 \mathrm{ft}$ upstream from Sugar Run,

$2.8 \mathrm{mi}$ southeast of Chester, and $8.5 \mathrm{mi}$ northeast of Pomeroy.

DRAINAGE AREA: $\quad 156 \mathrm{mi}^{2}$.

TRIBUTARY TO: Ohio River.

DISCHARGE DATA USED: June 1965 to September 1978.

REMARKS: None.

SELECTED DISCHARGE CHARACTERISTICS: Average discharge: $168 \mathrm{ft}^{3} / \mathrm{s}$ ( 13 years).

Minimum daily discharge: $0.3 \mathrm{ft} / \mathrm{s}$ September 1966.

Magnitude and frequency of low flow for indicated periods

\begin{tabular}{|c|c|c|c|c|c|c|c|c|c|c|c|c|c|}
\hline \multirow{2}{*}{ Period } & \multirow{2}{*}{$\begin{array}{l}\text { Number of } \\
\text { consecu- } \\
\text { tive days }\end{array}$} & \multicolumn{5}{|c|}{$\begin{array}{l}\text { Discharge }\left(\mathrm{ft}^{3} / \mathrm{s}\right) \text { for indicated } \\
\text { recurrence interval (years) }\end{array}$} & \multirow{2}{*}{ Period } & \multirow{2}{*}{$\begin{array}{l}\text { Number of } \\
\text { consecu- } \\
\text { tive days }\end{array}$} & \multicolumn{5}{|c|}{$\begin{array}{l}\text { Discharge }\left(\mathrm{ft}^{3} / \mathrm{s}\right) \text { for indicated } \\
\text { recurrence interval (years) }\end{array}$} \\
\hline & & 2 & 5 & 10 & 20 & 50 & & & 2 & 5 & 10 & 20 & 50 \\
\hline Apr. -Mar. & $\begin{array}{r}1 \\
7 \\
30\end{array}$ & $\begin{array}{l}2.9 \\
3.6 \\
8.9\end{array}$ & $\begin{array}{l}1.4 \\
1.6 \\
3.9\end{array}$ & $\begin{array}{l}0.8 \\
1.0 \\
2.3\end{array}$ & $\begin{array}{l}0.5 \\
0.6 \\
1.3\end{array}$ & $\begin{array}{l}0.2 \\
0.3 \\
0.7\end{array}$ & Sept.-Nov. & $\begin{array}{r}1 \\
7 \\
30\end{array}$ & $\begin{array}{r}3.5 \\
5.2 \\
15\end{array}$ & $\begin{array}{l}1.5 \\
2.3 \\
7.0\end{array}$ & $\begin{array}{l}0.9 \\
1.2 \\
4.6\end{array}$ & $\begin{array}{l}0.5 \\
0.7 \\
3.2\end{array}$ & $\begin{array}{l}0.3 \\
0.3 \\
2.1\end{array}$ \\
\hline June-Aug. & $\begin{array}{r}1 \\
7 \\
30\end{array}$ & $\begin{array}{l}3.3 \\
4.6 \\
15\end{array}$ & $\begin{array}{l}1.5 \\
2.1 \\
6.4\end{array}$ & $\begin{array}{l}1.0 \\
1.3 \\
4.0\end{array}$ & $\begin{array}{l}0.7 \\
0.9 \\
2.7\end{array}$ & $\begin{array}{l}0.4 \\
0.6 \\
1.7\end{array}$ & Mar. - May & $\begin{array}{r}1 \\
7 \\
30\end{array}$ & $\begin{array}{l}23 \\
29 \\
95\end{array}$ & $\begin{array}{l}15 \\
19 \\
58\end{array}$ & $\begin{array}{l}12 \\
16 \\
46\end{array}$ & $\begin{array}{l}10 \\
14 \\
38\end{array}$ & $\begin{array}{l}9.0 \\
12 \\
31\end{array}$ \\
\hline
\end{tabular}

Duration of daily flow for indicated periods

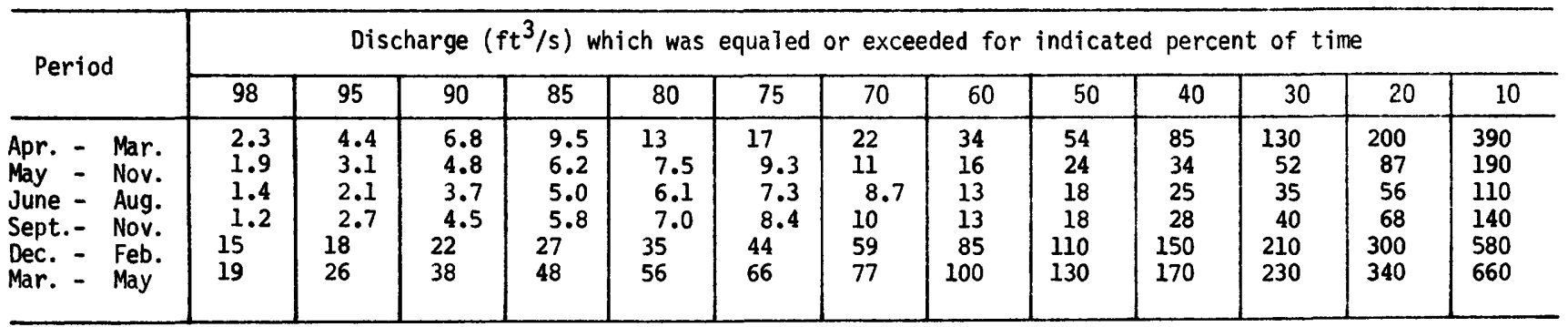


Appendix 3.--Low-flow data--Continued

SHADE RIVER BASIN

03159550 Shade River at Keno, Ohio

LOCATION:

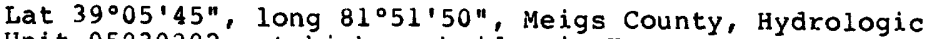

Unit 05030202 , at highway bridge in keno. To reach

station from Chester, follow state route 248 east $4.1 \mathrm{mi}$

to intersection on small road on right. Turn sharp right

and proceed $0.5 \mathrm{mi}$ to bridge in Keno. Portland quadrangle.

DRAINAGE AREA :

$160 \mathrm{mi}^{2}$.

TRIBUTARY TO:

Ohio River.

DISCHARGE DATA USED: LOW-flow measurements 1955-1964 water years.

INDEX STATION: $\quad 03202000$ Raccoon Creek at Adamsville.

REMARKS:

sone.

SELECTED DISCHARGE CHARACTERISTICS: None.

\begin{tabular}{c|c|c|c}
\multicolumn{4}{c}{ Magnitude and frequency of low flow for } \\
indicated periods \\
\hline \multirow{2}{*}{ Period } & $\begin{array}{c}\text { Number of } \\
\text { consecu- } \\
\text { tive days }\end{array}$ & $\begin{array}{c}\text { Discharge (ft } 3 / \mathrm{s} \text { ) for } \\
\text { indicated recurrence } \\
\text { interval (years) }\end{array}$ \\
\cline { 3 - 4 } & & 2 & 10 \\
\hline Apr. - Mar. & 7 & 1.3 & 0.1 \\
May - Nov. & 30 & 1.3 & 0.2 \\
June - Aug. & 7 & 1.3 & 0.1 \\
& 30 & 1.3 & 0.2 \\
Sept. - Nov. & 7 & 5.4 & 1.0 \\
& 7 & 20 & 2.5 \\
\hline
\end{tabular}

Duration of daily flow for indicated periods

\begin{tabular}{c|c|c|c|c|c}
\hline \multirow{2}{*}{ Period } & \multicolumn{6}{|c|}{$\begin{array}{c}\text { Discharge ( } \mathrm{ft}^{3} / \mathrm{s} \text { ) which was equaled or } \\
\text { exceeded for indicated percent of time }\end{array}$} \\
\cline { 2 - 6 } & 98 & 95 & 90 & 85 & 80 \\
\hline Apr. - Mar. & 0.2 & 0.7 & 2.7 & 6.6 & 10 \\
May - Nov. & 0.1 & 0.4 & 1.3 & 2.7 & 4.6 \\
June - Aug. & 1.0 & 2.0 & 4.0 & 6.6 & 9.2 \\
Sept. - Nov. & 0.1 & 0.1 & 0.3 & 0.5 & 1.1 \\
\hline
\end{tabular}




\section{RACCOON CREEK BASIN}

03201800 Sandy Run near Lake Hope, Ohio

LOCATION:

Lat $39^{\circ} 20^{\prime} 01^{\prime \prime}$, long $82^{\circ} 19^{\prime} 56^{\prime \prime}$, in T.11 N., R.16 W., Vinton County, Hydrologic Unit 05090101, on right bank at upstream side on bridge of King Hollow Trail, 1,200 ft downstream from Harbargar Hollow, $2.6 \mathrm{mi}$ upstream from spillway of Lake Hope, and $5.0 \mathrm{mi}$ northeast of $\mathrm{Zaleski.}$

DRAINAGE AREA:

$4.99 \mathrm{mi}^{2}$.

TRIBUTARY TO:

Raccoon Creek.

DISCHARGE DATA USED: October 1957 to September 1978.

REMARKS:

None.

SELECTED DISCHARGE CHARACTERISTICS: Average discharge: $573 \mathrm{ft}^{3} / \mathrm{s}$ (21 years).

Minimum daily discharge: No flow at times most years.

Magnitude and frequency of low flow for indicated periods

\begin{tabular}{|c|c|c|c|c|c|c|}
\hline \multirow{2}{*}{ Period } & \multirow{2}{*}{$\begin{array}{l}\text { Number of } \\
\text { consecu- } \\
\text { tive days }\end{array}$} & \multicolumn{5}{|c|}{$\begin{array}{l}\text { Discharge }\left(\mathrm{ft}^{3} / \mathrm{s}\right) \text { for indicated } \\
\text { recurrence interval (years) }\end{array}$} \\
\hline & & 2 & 5 & 10 & 20 & 50 \\
\hline Apr.-Mar. & $\begin{array}{r}1 \\
7 \\
30\end{array}$ & $\begin{array}{l}0 \\
0 \\
0.1\end{array}$ & $\begin{array}{l}0 \\
0 \\
0\end{array}$ & $\begin{array}{l}0 \\
0 \\
0\end{array}$ & $\begin{array}{l}0 \\
0 \\
0\end{array}$ & $\begin{array}{l}0 \\
0 \\
0\end{array}$ \\
\hline May- Nov. & $\begin{array}{r}1 \\
7 \\
30\end{array}$ & $\begin{array}{l}0 \\
0 \\
0.1\end{array}$ & $\begin{array}{l}0 \\
0 \\
0\end{array}$ & $\begin{array}{l}0 \\
0 \\
0\end{array}$ & $\begin{array}{l}0 \\
0 \\
0\end{array}$ & $\begin{array}{l}0 \\
0 \\
0\end{array}$ \\
\hline June-Aug. & $\begin{array}{r}1 \\
7 \\
30\end{array}$ & $\begin{array}{l}0 \\
0 \\
0.2\end{array}$ & $\begin{array}{l}0 \\
0 \\
0.1\end{array}$ & $\begin{array}{l}0 \\
0 \\
0\end{array}$ & $\begin{array}{l}0 \\
0 \\
0\end{array}$ & $\begin{array}{l}0 \\
0 \\
0\end{array}$ \\
\hline
\end{tabular}

\begin{tabular}{|c|c|c|c|c|c|c|}
\hline \multirow{2}{*}{ Period } & \multirow{2}{*}{$\begin{array}{l}\text { Number of } \\
\text { consecu- } \\
\text { tive days }\end{array}$} & \multicolumn{5}{|c|}{$\begin{array}{l}\text { Discharge }\left(\mathrm{ft}^{3} / \mathrm{s}\right) \text { for indicated } \\
\text { recurrence interval (years) }\end{array}$} \\
\hline & & 2 & 5 & 10 & 20 & 50 \\
\hline Sept. -Nov. & $\begin{array}{r}1 \\
7 \\
30\end{array}$ & $\begin{array}{l}0 \\
0 \\
0.1\end{array}$ & $\begin{array}{l}0 \\
0 \\
0\end{array}$ & $\begin{array}{l}0 \\
0 \\
0\end{array}$ & $\begin{array}{l}0 \\
0 \\
0\end{array}$ & $\begin{array}{l}0 \\
0 \\
0\end{array}$ \\
\hline Dec. -Feb. & $\begin{array}{r}1 \\
7 \\
30\end{array}$ & $\begin{array}{l}0.5 \\
0.7 \\
2.3\end{array}$ & $\begin{array}{l}0.2 \\
0.3 \\
0.8\end{array}$ & $\begin{array}{l}0 \\
0.1 \\
0.4\end{array}$ & $\begin{array}{l}0 \\
0 \\
0.2\end{array}$ & $\begin{array}{l}0 \\
0 \\
0.1\end{array}$ \\
\hline Mar. - May & $\begin{array}{r}1 \\
7 \\
30\end{array}$ & $\begin{array}{l}0.9 \\
1.2 \\
4.3\end{array}$ & $\begin{array}{l}0.4 \\
0.6 \\
2.5\end{array}$ & $\begin{array}{l}0.3 \\
0.3 \\
1.9\end{array}$ & $\begin{array}{l}0.2 \\
0.2 \\
1.4\end{array}$ & $\begin{array}{l}0.1 \\
0.1 \\
1.0\end{array}$ \\
\hline
\end{tabular}

Duration of daily flow for indicated periods

\begin{tabular}{|c|c|c|c|c|c|c|c|c|c|c|c|c|c|}
\hline \multirow{2}{*}{ Period } & \multicolumn{13}{|c|}{ Discharge $\left(\mathrm{ft}^{3} / \mathrm{s}\right)$ which was equaled or exceeded for indicated percent of time } \\
\hline & 98 & 95 & 90 & 85 & 80 & 75 & 70 & 60 & 50 & 40 & 30 & 20 & 10 \\
\hline $\begin{array}{l}\text { Apr. - Mar. } \\
\text { May - Nov. } \\
\text { June - Aug. } \\
\text { Sept. - Nov. } \\
\text { Dec. - Feb. } \\
\text { Mar. - May }\end{array}$ & $\begin{array}{l}0 \\
0 \\
0 \\
0 \\
0.1 \\
0.4\end{array}$ & $\begin{array}{l}0.0 \\
0 \\
0 \\
0 \\
0.3 \\
0.9\end{array}$ & $\begin{array}{l}0.0 \\
0 \\
0 \\
0 \\
0.4 \\
1.4\end{array}$ & $\begin{array}{l}0.1 \\
0 \\
0 \\
0 \\
0.7 \\
2.0\end{array}$ & $\begin{array}{l}0.2 \\
0.1 \\
0.1 \\
0 \\
0.9 \\
2.5\end{array}$ & $\begin{array}{l}0.3 \\
0.1 \\
0.1 \\
0.1 \\
1.2 \\
2.9\end{array}$ & $\begin{array}{l}0.4 \\
0.2 \\
0.2 \\
0.1 \\
1.5 \\
3.3\end{array}$ & $\begin{array}{l}0.8 \\
0.3 \\
0.3 \\
0.2 \\
2.2 \\
4.4\end{array}$ & $\begin{array}{l}1.4 \\
0.5 \\
0.4 \\
0.4 \\
3.2 \\
5.7\end{array}$ & $\begin{array}{l}2.5 \\
0.8 \\
0.6 \\
0.6 \\
4.4 \\
7.4\end{array}$ & $\begin{array}{c}4.0 \\
1.3 \\
0.9 \\
0.9 \\
6.2 \\
10\end{array}$ & $\begin{array}{l}6.6 \\
2.4 \\
1.3 \\
1.5 \\
9.3 \\
15\end{array}$ & $\begin{array}{r}14 \\
5.1 \\
3.1 \\
3.2 \\
17 \\
24\end{array}$ \\
\hline
\end{tabular}


03201900 Raccoon Creek near Prattsville, Ohio

LOCATION: Lat $39^{\circ} 14^{\prime} 20^{\prime \prime}$, long $82^{\circ} 17^{\prime} 10^{\prime \prime}$, Vinton County, Hydrologic Unit 05090101 , at bridge on U.S. Highway 50, five miles east of Prattsville, $11 / 2$ miles above Russell Run.

DRAINAGE AREA: $200 \mathrm{mi}^{2}$.

TRIBUTARY TO: Ohio River.

DISCHARGE DATA USED: Low-flow measurements 1951-1971 water years.

INDEX STATION: 03202000 Raccoon Creek at Adamsville.

REMARKS:

Discontinued in 1971 as sufficient length of record had been obtained to define low-flow characteristics.

SELECTED OISCHARGE CHARACTERISTICS: Minimum flow observed: $0 \mathrm{ft}^{3} / \mathrm{s}$ october 1963.

\begin{tabular}{|c|c|c|c|}
\hline \multirow[t]{2}{*}{ Period } & \multirow{2}{*}{$\begin{array}{l}\text { Number of } \\
\text { consecu- } \\
\text { tive days }\end{array}$} & \multicolumn{2}{|c|}{$\begin{array}{l}\text { Discharge }\left(\mathrm{ft}^{3} / \mathrm{s}\right) \text { for } \\
\text { indicated recurrence } \\
\text { interval (years) }\end{array}$} \\
\hline & & 2 & 10 \\
\hline Apr. - Mar. & $\begin{array}{r}7 \\
30\end{array}$ & $\begin{array}{l}1.0 \\
2.2\end{array}$ & $\begin{array}{l}0.1 \\
0.2\end{array}$ \\
\hline May - Nov. & $\begin{array}{r}7 \\
30\end{array}$ & $\begin{array}{l}1.0 \\
2.2\end{array}$ & $\begin{array}{l}0.1 \\
0.2\end{array}$ \\
\hline June - Aug. & $\begin{array}{r}7 \\
30\end{array}$ & $10^{3.3}$ & $\begin{array}{l}0.8 \\
1.7\end{array}$ \\
\hline Sept. - Nov. & $\begin{array}{r}7 \\
30\end{array}$ & $\begin{array}{l}1.1 \\
2.9\end{array}$ & $\begin{array}{l}0.1 \\
0.2\end{array}$ \\
\hline
\end{tabular}

Duration of daily flow for indicated periods

\begin{tabular}{l|c|c|c|c|c}
\hline \multirow{2}{*}{ Period } & \multicolumn{5}{|c}{$\begin{array}{c}\text { Discharge ( } \mathrm{ft} 3 / \mathrm{s} \text { ) which was equaled or } \\
\text { exceeded for indicated percent of time }\end{array}$} \\
\cline { 2 - 6 } & 98 & 95 & 90 & 85 & 80 \\
\hline Apr. - Mar. & 0.2 & 0.6 & 1.9 & 4.0 & 7.4 \\
May - Nov. & 0.1 & 0.4 & 1.0 & 1.9 & 2.9 \\
June - Aug. & 0.8 & 1.4 & 2.6 & 4.0 & 5.2 \\
Sept. - Nov. & 0.1 & 0.1 & 0.3 & 0.4 & 0.9 \\
\hline
\end{tabular}


Appendix 3.--Low-flow data--Continued

RACCOON CREEK BASIN

03201990 Little Raccoon Creek near Vinton, Ohio

LOCATION:

Lat $38^{\circ} 57^{\prime} 12^{\prime \prime}$, long $82^{\circ} 21^{\prime} 57^{\prime \prime}$, Gallia County, Hydrologic Unit $05090101,1.2 \mathrm{mi}$ upstream from mouth at bridge on state Route 325 , $2.0 \mathrm{mi}$ southwest of vinton.

DRAINAGE AREA: $\quad 154 \mathrm{mi}^{2}$.

TRIBUTARY TO: Kaccoon Creek.

DISCHARGE DATA USED: LOW-flow measurements 1951-1953, 1959, 1965, 1972-1975 water years.

INDEX STATION: $\quad 03202000$ Raccoon Creek at Adamsville, Ohio.

REMARKS: None.

SELECTED DISCHARGE CHARACTERISTICS: Minimum discharge observed: $4.6 \mathrm{ft}^{3} / \mathrm{s}$ September 1959 .

Magnitude and frequency of low flow for indicated periods

\begin{tabular}{c|c|c|c}
\hline \multirow{2}{*}{ Period } & $\begin{array}{c}\text { Number of } \\
\text { consecu- } \\
\text { tive days }\end{array}$ & $\begin{array}{c}\text { Discharge }\left(\mathrm{ft}^{3} / \mathrm{s}\right) \text { for } \\
\text { indicated } \\
\text { interval }\end{array}$ \\
\cline { 3 - 4 } (years)
\end{tabular}

Duration of daily flow for indicated periods

\begin{tabular}{c|c|c|c|c|c}
\hline \multirow{2}{*}{ Period } & \multicolumn{6}{|c|}{$\begin{array}{c}\text { Discharge } \\
\left.\text { exceeded for } \mathrm{ft}^{3} \mathrm{~s}\right)\end{array}$ which was equaled or } \\
\cline { 2 - 6 } & 98 & 95 & 90 & 85 & 80 \\
\hline Apr. - Mar. & 2.1 & 3.4 & 6.3 & 9.4 & 13 \\
May - Nov. & 1.6 & 2.6 & 4.6 & 6.3 & 8.0 \\
June - Aug. & 4.0 & 5.4 & 7.4 & 9.4 & 11 \\
Sept. - Nov. & 1.0 & 1.6 & 2.4 & 3.0 & 4.3 \\
\hline
\end{tabular}


Appendix 3.--Low-flow data--Continued

RACCOON CREEK BASIN

$032020 \mathrm{ju}$ Raccoon Creek at Adamsville, Ohio

LOCATION: Lat $38^{\circ} 52^{\prime} 25^{\prime \prime}$, long $82^{\circ} 21^{\prime} 22^{\prime \prime}$, in SEl/4 sec. $26, T .6$ N., R.16 W., Gallia County, Hydrologic Unit 05090101, on left bank at downstream side of U.S. Highway 35 bridge at Adamsville, $1.3 \mathrm{mi}$ upstream from Ryan Run, and $1.4 \mathrm{mi}$ downstream from Indian Creek.

DRAINAGE AREA: $585 \mathrm{mi}^{2}$.

TRIBUTARY TO: Ohio River.

DISCHARGE DATA USED: October 1938 to September 1978.

REMARKS :

None.

SELECTED DISCHARGE CHARACTERISTICS: Average discharge: $620 \mathrm{ft}^{3} / \mathrm{s}$ (40 years).

Minimum daily discharge: $1.1 \mathrm{ft} / \mathrm{s}$ October 1964.

Magnitude and frequency of low flow for indicated periods

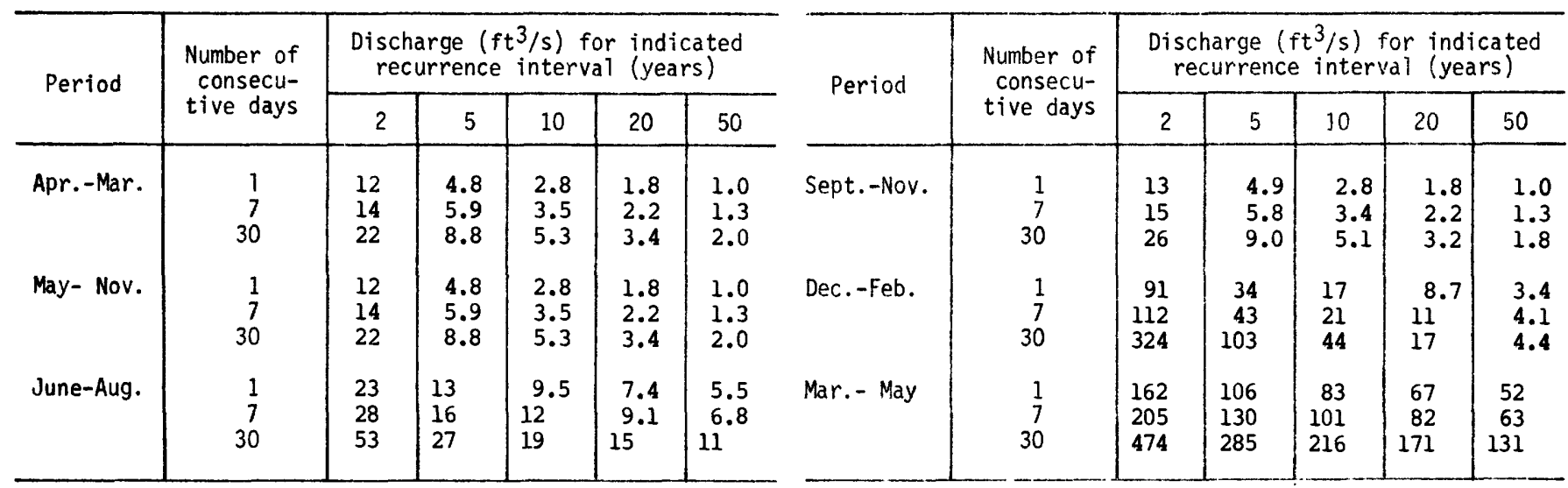

Duration of daity flow for indicated periods

\begin{tabular}{|c|c|c|c|c|c|c|c|c|c|c|c|c|c|c|}
\hline \multirow{2}{*}{\multicolumn{2}{|c|}{ Period }} & \multicolumn{13}{|c|}{ Discharge $\left(\mathrm{ft}^{3} / \mathrm{s}\right)$ which was equaled or exceeded for indicated percent of time } \\
\hline & & 98 & 95 & 90 & 85 & 80 & 75 & 70 & 60 & 50 & 40 & 30 & 20 & 10 \\
\hline $\begin{array}{l}\text { Apr. - } \\
\text { May - } \\
\text { June - } \\
\text { Sept. - } \\
\text { Dec. - } \\
\text { Mar. - }\end{array}$ & $\begin{array}{l}\text { Mar. } \\
\text { Nov. } \\
\text { Aug. } \\
\text { Nov. } \\
\text { Feb. } \\
\text { May }\end{array}$ & $\begin{array}{r}6.0 \\
4.5 \\
12 \\
2.7 \\
11 \\
110\end{array}$ & $\begin{array}{c}10 \\
7.5 \\
17 \\
4.6 \\
35 \\
150\end{array}$ & $\begin{array}{c}20 \\
14 \\
24 \\
0.9 \\
b y \\
80\end{array}$ & $\begin{array}{c}31 \\
20 \\
31 \\
8.7 \\
96 \\
280\end{array}$ & $\begin{array}{r}44 \\
26 \\
36 \\
13 \\
140 \\
330\end{array}$ & $\begin{array}{l}59 \\
33 \\
44 \\
17 \\
180 \\
390\end{array}$ & $\begin{array}{r}76 \\
41 \\
21 \\
21 \\
200 \\
440\end{array}$ & $\begin{array}{r}120 \\
61 \\
69 \\
32 \\
340 \\
570\end{array}$ & $\begin{array}{r}210 \\
86 \\
89 \\
47 \\
490 \\
730\end{array}$ & $\begin{array}{r}350 \\
120 \\
120 \\
66 \\
680 \\
940\end{array}$ & $\begin{array}{r}550 \\
190 \\
160 \\
96 \\
930 \\
1300\end{array}$ & $\begin{array}{r}910 \\
320 \\
230 \\
150 \\
1400 \\
1800\end{array}$ & $\begin{array}{r}1700 \\
660 \\
460 \\
310 \\
2300 \\
2900\end{array}$ \\
\hline
\end{tabular}


Appendix 3.--Low-flow data--Continued

INDIAN GUYAN CREEK BASIN

03205210 Indian Guyan Creek near Bradrick, Ohio

LOCATION:

Lat $38^{\circ} 28^{\prime} 4^{\prime \prime}$, long $82^{\circ} 23^{\prime} 54^{\prime \prime}$, Lawrence County, Hydrologic Unit

$05090101,200 \mathrm{ft}$ upstream from relocated Fourmile Creek at bridge

on Indian Guyan Road (Township Road C-69) $2.5 \mathrm{mi}$ north of Bradrick.

DRAINAGE AREA: $\quad 67.5 \mathrm{mi}^{2}$.

TRIBUTARY TO: Ohio River.

DISCHARGE DATA USED: Low-flow measurements 1959, 1972-1977 water years.

INDEX STATION: $\quad 03202000$ Raccoon Creek at Adamsville, Ohio.

REMARKS: $\quad$ None.

SELECTED DISCHARGE CHARACTERISTICS: Minimum discharge observed: $0.8 \mathrm{ft}^{3} / \mathrm{s}$ September 1976.

Magnitude and frequency of low flow for indicated periods

\begin{tabular}{|c|c|c|c|}
\hline \multirow[t]{2}{*}{ Period } & \multirow{2}{*}{$\begin{array}{l}\text { Number of } \\
\text { consecu- } \\
\text { tive days }\end{array}$} & \multicolumn{2}{|c|}{$\begin{array}{l}\text { Discharge }\left(\mathrm{ft}^{3} / \mathrm{s}\right) \text { for } \\
\text { indicated recurrence } \\
\text { interval (years) }\end{array}$} \\
\hline & & 2 & 10 \\
\hline Apr. - Mar. & $\begin{array}{r}7 \\
30\end{array}$ & $\begin{array}{l}0.2 \\
0.4\end{array}$ & $\begin{array}{l}0 \\
0\end{array}$ \\
\hline May - Nov. & $\begin{array}{r}7 \\
30\end{array}$ & $\begin{array}{l}0.2 \\
0.4\end{array}$ & $\begin{array}{l}0 \\
0\end{array}$ \\
\hline June - Aug. & $\begin{array}{r}7 \\
30\end{array}$ & $\begin{array}{l}0.6 \\
1.8\end{array}$ & $\begin{array}{l}0.2 \\
0.3\end{array}$ \\
\hline Sept.- Nov. & $\begin{array}{r}7 \\
30\end{array}$ & $\begin{array}{l}0.2 \\
0.5\end{array}$ & $\begin{array}{l}0 \\
0\end{array}$ \\
\hline
\end{tabular}

Duration of daily flow for indicated periods

\begin{tabular}{l|l|l|l|l|l}
\hline \multirow{2}{*}{ Period } & \multicolumn{6}{|c}{$\begin{array}{c}\text { Discharge ( } \mathrm{ft}^{3} / \mathrm{s} \text { ) which was equaled or } \\
\text { exceeded for indicated percent of time }\end{array}$} \\
\cline { 2 - 6 } & 98 & 95 & 90 & 85 & 80 \\
\hline Apr. - Mar. & 0.1 & 0.1 & 0.4 & 0.7 & 1.3 \\
May - Nov. & 0 & 0.1 & 0.2 & 0.4 & 0.5 \\
June - Aug. & 0.2 & 0.3 & 0.5 & 0.7 & 0.9 \\
Sept. - Nov. & 0 & 0 & 0.1 & 0.1 & 0.2 \\
\hline
\end{tabular}


Appendix 3.--Low-flow data--Continued

SCIOTO RIVER BASIN

03217400 Scioto River near Kenton, Ohio

LOCATION: Lat $40^{\circ} 38^{\prime} 20^{\prime \prime}$, long $83^{\circ} 38^{\prime} 20^{\prime \prime}$, Hardin County, Hydrologic

Unit 05060001 , at bridge on County Road $130,11 / 2 \mathrm{mi}$ west of court house in Kenton.

DRAINAGE AREA: $129 \mathrm{mi}^{2}$.

TRIBUTARY TO: Ohio River.

DISCHARGE DATA USED: LOW-flow measurements 1961-1973 water years.

INDEX STATION: $\quad 03217500$ Scioto River at La Rue.

REMARKS: $\quad$ Discontinued in 1973 as sufficient record had been obtained to define low-flow characteristics.

SELECTED DISCHARGE CHARACTERISTICS: Minimum flow observed: $3.3 \mathrm{ft}^{3} / \mathrm{s}$ october 1963.

\begin{tabular}{|c|c|c|c|}
\hline \multirow[t]{2}{*}{ Period } & \multirow{2}{*}{$\begin{array}{l}\text { Number of } \\
\text { consecu- } \\
\text { tive days }\end{array}$} & \multicolumn{2}{|c|}{$\begin{array}{l}\text { Discharge }\left(\mathrm{ft}^{3} / \mathrm{s}\right) \text { for } \\
\text { indicated recurrence } \\
\text { interval (years) }\end{array}$} \\
\hline & & 2 & 10 \\
\hline Apr. - Mar. & $\begin{array}{r}7 \\
30\end{array}$ & $\begin{array}{l}3.2 \\
4.2\end{array}$ & $\begin{array}{l}2.0 \\
2.4\end{array}$ \\
\hline May - Nov. & $\begin{array}{r}7 \\
30\end{array}$ & $\begin{array}{l}3.2 \\
4.3\end{array}$ & $\begin{array}{l}2.0 \\
2.5\end{array}$ \\
\hline June - Aug. & $\begin{array}{r}7 \\
30\end{array}$ & $\begin{array}{l}4.4 \\
7.3\end{array}$ & $\begin{array}{l}2.2 \\
2.8\end{array}$ \\
\hline Sept. - Nov. & $\begin{array}{r}7 \\
30\end{array}$ & $\begin{array}{l}3.6 \\
5.4\end{array}$ & $\begin{array}{l}2.1 \\
2.6\end{array}$ \\
\hline
\end{tabular}

Duration of daily flow for indicated periods

\begin{tabular}{c|c|c|c|c|c}
\hline \multirow{2}{*}{ Period } & \multicolumn{5}{|c}{$\begin{array}{c}\text { Discharge ( } \mathrm{ft}^{3} / \mathrm{s} \text { ) which was equaled or } \\
\text { exceeded for indicated percent of time }\end{array}$} \\
\cline { 2 - 6 } & 98 & 95 & 90 & 85 & 80 \\
\hline Apr. - Mar. & 2.4 & 2.9 & 4.0 & 5.0 & 6.4 \\
May - Nov. & 2.4 & 2.6 & 3.2 & 4.0 & 4.7 \\
June - Aug. & 2.4 & 2.8 & 3.3 & 4.1 & 4.9 \\
Sept. - Nov. & 2.2 & 2.4 & 2.7 & 3.3 & 3.9 \\
\hline
\end{tabular}


SCIOTO RIVER BASIN

03217500 Scioto River at LaRue, Ohio

LOCATION:

Lat $40^{\circ} 34^{\prime} 28^{\prime \prime}$, long $83^{\circ} 23^{\prime} 1^{\prime \prime}$, Marion County, Hydrologic Unit 05060001 , on right bank $200 \mathrm{ft}$ downstream from county highway bridge at LaRue $500 \mathrm{ft}$ downstream from Cleveland, Cincinnati, Chicago and St. Louis Railway bridge and $3.5 \mathrm{mi}$ upstream from Rush creek.

DRAINAGE AREA:

$257 \mathrm{mi}^{2}$.

TRIBUTARY TO:

Ohio River.

DISCHARGE DATA USED: October 1926 to September 1935, October 1938 to September 1951.

REMARKS:

None.

SELECTED DISCHARGE CHARACTERISTICS: Average discharge: $214 \mathrm{ft}^{3} / \mathrm{s}$ (22 years).

Minimum daily discharge: $2.8 \mathrm{ft}^{3} / \mathrm{s}$ October 1941, 1942, 1946.

Magnitude and frequency of low flow for indicated periods

\begin{tabular}{|c|c|c|c|c|c|c|c|c|c|c|c|c|c|}
\hline \multirow{2}{*}{ Period } & \multirow{2}{*}{$\begin{array}{l}\text { Number of } \\
\text { consecu- } \\
\text { tive days }\end{array}$} & \multicolumn{5}{|c|}{$\begin{array}{l}\text { Discharge }\left(\mathrm{ft}^{3} / \mathrm{s}\right) \text { for indicated } \\
\text { recurrence interval (years) }\end{array}$} & \multirow{2}{*}{ Period } & \multirow{2}{*}{$\begin{array}{l}\text { Number of } \\
\text { consecu- } \\
\text { tive days }\end{array}$} & \multicolumn{5}{|c|}{$\begin{array}{l}\text { Discharge }\left(\mathrm{ft}^{3} / \mathrm{s}\right) \text { for indicated } \\
\text { recurrence interval (years) }\end{array}$} \\
\hline & & 2 & 5 & 10 & 20 & 50 & & & 2 & 5 & 10 & 20 & 50 \\
\hline Apr.-Mar. & $\begin{array}{r}1 \\
7 \\
30\end{array}$ & $\begin{array}{l}4.6 \\
5.3 \\
7.3\end{array}$ & $\begin{array}{l}3.1 \\
3.6 \\
4.7\end{array}$ & $\begin{array}{l}2.6 \\
3.1 \\
3.9\end{array}$ & $\begin{array}{l}2.3 \\
2.8 \\
3.4\end{array}$ & $\begin{array}{l}2.1 \\
2.5 \\
3.0\end{array}$ & Sept.-Nov. & $\begin{array}{r}1 \\
7 \\
30\end{array}$ & $\begin{array}{l}4.8 \\
6.1 \\
9.9\end{array}$ & $\begin{array}{l}3.1 \\
3.9 \\
5.4\end{array}$ & $\begin{array}{l}2.7 \\
3.3 \\
4.2\end{array}$ & $\begin{array}{l}2.4 \\
2.9 \\
3.4\end{array}$ & $\begin{array}{l}2.2 \\
2.5 \\
2.8\end{array}$ \\
\hline June-Aug. & $\begin{array}{r}1 \\
7 \\
30\end{array}$ & $\begin{array}{r}6.3 \\
7.7 \\
14\end{array}$ & $\begin{array}{l}3.9 \\
4.5 \\
6.5\end{array}$ & $\begin{array}{l}3.1 \\
3.4 \\
4.6\end{array}$ & $\begin{array}{l}2.6 \\
2.8 \\
3.6\end{array}$ & $\begin{array}{l}2.1 \\
2.2 \\
2.7\end{array}$ & Mar. - May & $\begin{array}{r}1 \\
7 \\
30\end{array}$ & $\begin{array}{l}29 \\
35 \\
83\end{array}$ & $\begin{array}{l}18 \\
21 \\
39\end{array}$ & $\begin{array}{l}14 \\
16 \\
27\end{array}$ & $\begin{array}{l}11 \\
13 \\
19\end{array}$ & $\begin{array}{r}8.9 \\
9.6 \\
13\end{array}$ \\
\hline
\end{tabular}

Duration of daily flow for indicated periods

\begin{tabular}{l|c|c|c|c|c|c|c|c|c|c|c|c|c}
\hline \multirow{2}{*}{ Period } & \multicolumn{8}{|c|}{ Discharge $\left(\mathrm{ft}^{3} / \mathrm{s}\right)$ which was equaled or exceeded for indicated percent of time } \\
\cline { 2 - 13 } & 98 & 95 & 90 & 85 & 80 & 75 & 70 & 60 & 50 & 40 & 30 & 20 & 10 \\
\hline Apr. - Mar. & 3.9 & 4.7 & 6.9 & 8.9 & 12 & 14 & 17 & 28 & 47 & 78 & 130 & 230 & 530 \\
May - Nov. & 3.8 & 4.2 & 5.4 & 7.0 & 8.4 & 10 & 12 & 16 & 22 & 32 & 53 & 95 & 220 \\
June - Aug. & 3.9 & 4.5 & 5.5 & 7.1 & 8.8 & 12 & 14 & 19 & 25 & 35 & 54 & 96 & 230 \\
Sept.- Nov. & 3.5 & 3.9 & 4.4 & 5.5 & 6.7 & 7.8 & 8.6 & 11 & 14 & 17 & 24 & 49 & 140 \\
Dec. - Feb. & 4.4 & 6.8 & 9.8 & 13 & 18 & 25 & 35 & 58 & 90 & 140 & 220 & 390 & 940 \\
Mar. - May & 18 & 24 & 34 & 42 & 51 & 62 & 73 & 100 & 140 & 200 & 290 & 440 & 840 \\
\hline
\end{tabular}




\section{SCIOTO RIVER BASIN}

03218000 Little Scioto River above Marion, Ohio

LOCATION :

Lat $40^{\circ} 37^{\prime} 43^{\prime \prime}$, long $83^{\circ} 10^{\prime} 11^{\prime \prime}$, in NEl/4 sec. 7, T.5 S., R.15 E., Marion County, Hydrologic Unit 05060001 , on left bank at downstream side of Chesapeake \& Ohio Railway bridge, $1.0 \mathrm{mi}$ downstream from Rock Fork, $3.5 \mathrm{mi}$ northwest of Marion, and $7.2 \mathrm{mi}$ upstream from Honey Creek.

DRAINAGE AREA:

$72.4 \mathrm{mi}^{2}$

TRIBUTARY TO:

Scioto River.

DISCHARGE DATA USED:

October 1938 to September 1971.

REMARKS:

None.

SELECTED DISCHARGE CHARACTERISTICS: Average discharge: $50.2 \mathrm{ft}^{3} / \mathrm{s}$ ( 33 years).

Minimum daily discharge: No flow many times.

Magnitude and frequency of low flow for indicated periods

\begin{tabular}{|c|c|c|c|c|c|c|}
\hline \multirow{2}{*}{ Period } & \multirow{2}{*}{$\begin{array}{l}\text { Number of } \\
\text { consecu- } \\
\text { tive days }\end{array}$} & \multicolumn{5}{|c|}{$\begin{array}{l}\text { Discharge }\left(\mathrm{ft}^{3} / \mathrm{s}\right) \text { for indicated } \\
\text { recurrence interval (years) }\end{array}$} \\
\hline & & 2 & 5 & 10 & 20 & 50 \\
\hline Apr.-Mar. & $\begin{array}{r}1 \\
7 \\
30\end{array}$ & $\begin{array}{l}0 \\
0.1 \\
0.2\end{array}$ & $\begin{array}{l}0 \\
0 \\
0\end{array}$ & $\begin{array}{l}0 \\
0 \\
0\end{array}$ & $\begin{array}{l}0 \\
0 \\
0\end{array}$ & $\begin{array}{l}0 \\
0 \\
0\end{array}$ \\
\hline May- Nov. & $\begin{array}{r}1 \\
7 \\
30\end{array}$ & $\begin{array}{l}0 \\
0.1 \\
0.2\end{array}$ & $\begin{array}{l}0 \\
0 \\
0\end{array}$ & $\begin{array}{l}0 \\
0 \\
0\end{array}$ & $\begin{array}{l}0 \\
0 \\
0\end{array}$ & $\begin{array}{l}0 \\
0 \\
0\end{array}$ \\
\hline June-Aug. & $\begin{array}{r}1 \\
7 \\
30\end{array}$ & $\begin{array}{l}0.2 \\
0.4 \\
1.1\end{array}$ & $\begin{array}{l}0 \\
0.1 \\
0.4\end{array}$ & $\begin{array}{l}0 \\
0 \\
0.2\end{array}$ & $\begin{array}{l}0 \\
0 \\
0.1\end{array}$ & $\begin{array}{l}0 \\
0 \\
0\end{array}$ \\
\hline
\end{tabular}

\begin{tabular}{|c|c|c|c|c|c|c|}
\hline \multirow{2}{*}{ Period } & \multirow{2}{*}{$\begin{array}{l}\text { Number of } \\
\text { consecu- } \\
\text { tive days }\end{array}$} & \multicolumn{5}{|c|}{$\begin{array}{l}\text { Discharge }\left(\mathrm{ft}^{3} / \mathrm{s}\right) \text { for indicated } \\
\text { recurrence interval (years) }\end{array}$} \\
\hline & & 2 & 5 & 10 & 20 & 50 \\
\hline Sept.-Nov. & $\begin{array}{r}1 \\
7 \\
30\end{array}$ & $\begin{array}{l}0 \\
0.1 \\
0.4\end{array}$ & $\begin{array}{l}0 \\
0 \\
0\end{array}$ & $\begin{array}{l}0 \\
0 \\
0\end{array}$ & $\begin{array}{l}0 \\
0 \\
0\end{array}$ & $\begin{array}{l}0 \\
0 \\
0\end{array}$ \\
\hline Dec. -Feb. & $\begin{array}{r}1 \\
7 \\
30\end{array}$ & $\begin{array}{l}2.3 \\
3.3 \\
11\end{array}$ & $\begin{array}{l}0.3 \\
0.4 \\
0.9\end{array}$ & $\begin{array}{l}0 \\
0 \\
0.1\end{array}$ & $\begin{array}{l}0 \\
0 \\
0\end{array}$ & $\begin{array}{l}0 \\
0 \\
0\end{array}$ \\
\hline Mar. - May & $\begin{array}{r}1 \\
7 \\
30\end{array}$ & $\begin{array}{l}8.0 \\
10 \\
22\end{array}$ & $\begin{array}{r}4.5 \\
6.2 \\
12\end{array}$ & $\begin{array}{l}3.2 \\
4.7 \\
8.7\end{array}$ & $\begin{array}{l}2.4 \\
3.7 \\
6.6\end{array}$ & $\begin{array}{l}1.7 \\
2.8 \\
4.8\end{array}$ \\
\hline
\end{tabular}

Duration of daily flow for indicated periods

\begin{tabular}{l|l|l|l|l|l|l|l|l|l|l|l|l|l|r}
\hline \multirow{2}{*}{ Period } & \multicolumn{8}{|c}{ Discharge $\left(\mathrm{ft}^{3} / \mathrm{s}\right)$ which was equaled or exceeded for indicated percent of time } \\
\cline { 2 - 12 } & 98 & 95 & 90 & 85 & 80 & 75 & 70 & 60 & 50 & 40 & 30 & 20 & 10 \\
\hline Apr. - Mar. & 0 & 0.1 & 0.2 & 0.4 & 0.7 & 1.2 & 2.0 & 5.2 & 11 & 20 & 33 & 61 & 130 \\
May - Nov. & 0 & 0 & 0.1 & 0.2 & 0.4 & 0.5 & 0.7 & 1.5 & 3.2 & 6.1 & 11 & 22 & 51 \\
June - Aug. & 0.1 & 0.1 & 0.3 & 0.5 & 0.7 & 0.9 & 1.3 & 2.4 & 3.9 & 6.5 & 11 & 22 & 52 \\
Sept. - Nov. & 0 & 0 & 0 & 0.1 & 0.1 & 0.1 & 0.3 & 0.5 & 0.8 & 1.5 & 3.1 & 6.3 & 18 \\
Dec. - Feb. & 0 & 0.1 & 0.5 & 1.2 & 2.5 & 4.8 & 7.3 & 14 & 21 & 33 & 54 & 91 & 200 \\
Mar. - May & 5.6 & 7.6 & 11 & 14 & 17 & 21 & 24 & 34 & 47 & 64 & 89 & 130 & 230 \\
\hline
\end{tabular}


SCIOTO RIVER BASIN

03218500 Little Scioto River at Sewage Treatment Plant, near Marion, Ohio

LOCATION:

Lat $40^{\circ} 35^{\prime} 31^{\prime \prime}$, long $83^{\circ} 11^{\prime} 04^{\prime \prime}$, in SWl/4 sec. 19, T.5 S., R.15 E.,

Marion County, Hydrologic Unit 05060001 , at outfall of sewage treatment plant, $300 \mathrm{ft}$ downstream from Erie Railroad crossing, $2.0 \mathrm{mi}$ west of Marion, and $5 \mathrm{mi}$ upstream from Honey Creek.

DRAINAGE AREA: $\quad 85.8 \mathrm{mi}^{2}$.

TRIBLTTARY TO: $\quad$ Scioto River.

DISCHARGE DATA USED: August 1925 to December 1935, January 1938 to March 1939.

REMARKS :

City of Marion pumps from well field in basin upstream from station and return as sewage.

SELECTED DISCHARGE CHARACTERISTICS: Average discharge: $75 \mathrm{ft}^{3} / \mathrm{s}$ ( $10_{3}$ years)

Minimum daily discharge: $0.1 \mathrm{ft}^{3} / \mathrm{s}$ October, December 1934.

Magnitude and frequency of low flow for indicated periods

\begin{tabular}{|c|c|c|c|c|c|c|c|c|c|c|c|c|c|}
\hline \multirow{2}{*}{ Period } & \multirow{2}{*}{$\begin{array}{l}\text { Number of } \\
\text { consecu- } \\
\text { tive days }\end{array}$} & \multicolumn{5}{|c|}{$\begin{array}{l}\text { Discharge }\left(\mathrm{ft}^{3} / \mathrm{s}\right) \text { for indicated } \\
\text { recurrence interval (years) }\end{array}$} & \multirow{2}{*}{ Period } & \multirow{2}{*}{$\begin{array}{l}\text { Number of } \\
\text { consecu- } \\
\text { tive days }\end{array}$} & \multicolumn{5}{|c|}{$\begin{array}{l}\text { Discharge }\left(\mathrm{ft}^{3} / \mathrm{s}\right) \text { for indicated } \\
\text { recurrence interval (years) }\end{array}$} \\
\hline & & 2 & 5 & 10 & 20 & 50 & & & 2 & 5 & 10 & 20 & 50 \\
\hline Apr.-Mar. & $\begin{array}{r}1 \\
7 \\
30\end{array}$ & $\begin{array}{l}1.1 \\
1.2 \\
1.6\end{array}$ & $\begin{array}{l}0.4 \\
0.6 \\
0.8\end{array}$ & $\begin{array}{l}0.3 \\
0.4 \\
0.5\end{array}$ & $\begin{array}{l}0.1 \\
0.3 \\
0.4\end{array}$ & $\begin{array}{l}0.1 \\
0.2 \\
0.3\end{array}$ & Sept.-Nov. & $\begin{array}{r}1 \\
7 \\
30\end{array}$ & $\begin{array}{l}1.3 \\
1.4 \\
2.5\end{array}$ & $\begin{array}{l}0.5 \\
0.6 \\
0.9\end{array}$ & $\begin{array}{l}0.3 \\
0.5 \\
0.6\end{array}$ & $\begin{array}{l}0.2 \\
0.4 \\
0.5\end{array}$ & $\begin{array}{l}0.1 \\
0.3 \\
0.4\end{array}$ \\
\hline June-Aug. & $\begin{array}{r}1 \\
7 \\
30\end{array}$ & $\begin{array}{l}1.7 \\
2.2 \\
4.1\end{array}$ & $\begin{array}{l}0.8 \\
1.1 \\
1.9\end{array}$ & $\begin{array}{l}0.5 \\
0.7 \\
1.2\end{array}$ & $\begin{array}{l}0.4 \\
0.5 \\
0.8\end{array}$ & $\begin{array}{l}0.2 \\
0.3 \\
0.5\end{array}$ & Mar. - May & $\begin{array}{r}1 \\
7 \\
30\end{array}$ & $\begin{array}{l}9.9 \\
12 \\
23\end{array}$ & $\begin{array}{l}3.9 \\
5.5 \\
9.7\end{array}$ & $\begin{array}{l}2.1 \\
3.4 \\
6.4\end{array}$ & $\begin{array}{l}1.1 \\
2.2 \\
4.6\end{array}$ & $\begin{array}{l}0.5 \\
1.3 \\
3.3\end{array}$ \\
\hline
\end{tabular}

Duration of daily flow for indicated periods

\begin{tabular}{|c|c|c|c|c|c|c|c|c|c|c|c|c|c|}
\hline \multirow{2}{*}{ Period } & \multicolumn{13}{|c|}{ Discharge $\left(\mathrm{ft}^{3} / \mathrm{s}\right)$ which was equaled or exceeded for indicated percent of time } \\
\hline & 98 & 95 & 90 & 85 & 80 & 75 & 70 & 60 & 50 & 40 & 30 & 20 & 10 \\
\hline $\begin{array}{l}\text { Apr. - Mar. } \\
\text { May - Nov. } \\
\text { June - Aug. } \\
\text { Sept. - Nov. } \\
\text { Dec. - Feb. } \\
\text { Mar. - May }\end{array}$ & $\begin{array}{l}0.5 \\
0.5 \\
0.7 \\
0.3 \\
0.4 \\
3.6\end{array}$ & $\begin{array}{l}0.8 \\
0.8 \\
0.9 \\
0.5 \\
0.6 \\
6.6\end{array}$ & $\begin{array}{l}1.2 \\
1.1 \\
1.2 \\
0.9 \\
1.8 \\
9.7\end{array}$ & $\begin{array}{l}1.7 \\
1.4 \\
1.6 \\
1.2 \\
4.1 \\
13\end{array}$ & $\begin{array}{r}2.3 \\
1.8 \\
2.1 \\
1.4 \\
5.6 \\
17\end{array}$ & $\begin{array}{l}3.3 \\
2.2 \\
2.8 \\
1.6 \\
7.5 \\
20\end{array}$ & $\begin{array}{l}4.4 \\
2.7 \\
3.6 \\
1.9 \\
10 \\
23\end{array}$ & $\begin{array}{l}8.3 \\
4.1 \\
5.1 \\
2.4 \\
20 \\
33\end{array}$ & $\begin{array}{l}15 \\
6.4 \\
6.8 \\
3.4 \\
30 \\
43\end{array}$ & $\begin{array}{l}27 \\
10 \\
9.3 \\
5.1 \\
50 \\
61\end{array}$ & $\begin{array}{l}45 \\
18 \\
14 \\
12 \\
77 \\
86\end{array}$ & $\begin{array}{r}80 \\
33 \\
22 \\
37 \\
140 \\
150\end{array}$ & $\begin{array}{r}190 \\
75 \\
47 \\
90 \\
310 \\
300\end{array}$ \\
\hline
\end{tabular}


LOCATION:

Lat $40^{\circ} 25^{\prime} 10^{\prime \prime}$, long $83^{\circ} 11^{\prime} 50^{\prime \prime}$, Delaware County, Hydrologic Unit 05060001 , on downstream side of pier of Hoskins Bridge, $1.5 \mathrm{mi}$ upstream from Ottawa Creek, $2.0 \mathrm{mi}$ south of Prospect, and $2.5 \mathrm{mi}$ downstream from Patton Run.

DRAINAGE AREA: $\quad 567 \mathrm{mi}^{2}$.

TRIBUTARY TO: Ohio River.

DISCHARGE DATA USED: October 1925 to September 1932, October 1939 to September 1978.

REMARKS: None.

SELECTED DISCHARGE CHARACTERISTICS: Average discharge: $453 \mathrm{ft}^{3} / \mathrm{s}$ ( 46 years).

Minimum daily discharge: $4.5 \mathrm{ft} 3 / \mathrm{s}$ September 1953.

Magnitude and frequency of low flow for indicated periods

\begin{tabular}{|c|c|c|c|c|c|c|c|c|c|c|c|c|c|}
\hline \multirow{2}{*}{ Period } & \multirow{2}{*}{$\begin{array}{l}\text { Number of } \\
\text { consecu- } \\
\text { tive days }\end{array}$} & \multicolumn{5}{|c|}{$\begin{array}{l}\text { Discharge }\left(\mathrm{ft}^{3} / \mathrm{s}\right) \text { for indicated } \\
\text { recurrence interval (years) }\end{array}$} & \multirow{2}{*}{ Period } & \multirow{2}{*}{$\begin{array}{l}\text { Number of } \\
\text { consecu- } \\
\text { tive days }\end{array}$} & \multicolumn{5}{|c|}{$\begin{array}{l}\text { Discharge }\left(\mathrm{ft}^{3} / \mathrm{s}\right) \text { for indicated } \\
\text { recurrence interval (years) }\end{array}$} \\
\hline & & 2 & 5 & 10 & 20 & 50 & & & 2 & 5 & 10 & 20 & 50 \\
\hline Apr.-Mar. & $\begin{array}{r}7 \\
7 \\
30\end{array}$ & $\begin{array}{l}13 \\
16 \\
20\end{array}$ & $\begin{array}{l}8.5 \\
11 \\
13\end{array}$ & $\begin{array}{r}6.9 \\
9.3 \\
11\end{array}$ & $\begin{array}{l}5.8 \\
8.1 \\
10\end{array}$ & $\begin{array}{l}4.9 \\
7.0 \\
8.9\end{array}$ & Sept.-Nov. & $\begin{array}{r}1 \\
7 \\
30\end{array}$ & $\begin{array}{l}14 \\
17 \\
21\end{array}$ & $\begin{array}{l}9.0 \\
11 \\
13\end{array}$ & $\begin{array}{l}7.1 \\
9.6 \\
12\end{array}$ & $\begin{array}{l}5.8 \\
8.3 \\
11\end{array}$ & $\begin{array}{r}4.7 \\
7.1 \\
10\end{array}$ \\
\hline June-Aug. & $\begin{array}{r}1 \\
7 \\
30\end{array}$ & $\begin{array}{l}17 \\
21 \\
33\end{array}$ & $\begin{array}{l}12 \\
14 \\
18\end{array}$ & $\begin{array}{l}9.5 \\
11 \\
15\end{array}$ & $\begin{array}{r}8.2 \\
9.9 \\
13\end{array}$ & $\begin{array}{l}6.9 \\
8.6 \\
11\end{array}$ & Mar.- May & $\begin{array}{r}1 \\
7 \\
30\end{array}$ & $\begin{array}{r}78 \\
92 \\
193\end{array}$ & $\begin{array}{r}53 \\
64 \\
111\end{array}$ & $\begin{array}{l}43 \\
54 \\
85\end{array}$ & $\begin{array}{l}37 \\
47 \\
69\end{array}$ & $\begin{array}{l}31 \\
41 \\
54\end{array}$ \\
\hline
\end{tabular}

Duration of daily flow for indicated periods

\begin{tabular}{|c|c|c|c|c|c|c|c|c|c|c|c|c|c|c|}
\hline \multirow{2}{*}{\multicolumn{2}{|c|}{ Period }} & \multicolumn{13}{|c|}{ Discharge $\left(\mathrm{ft}^{3} / \mathrm{s}\right)$ which was equaled or exceeded for indicated percent of time } \\
\hline & & 98 & 95 & 90 & 85 & 80 & 75 & 70 & 60 & 50 & 40 & 30 & 20 & 10 \\
\hline $\begin{array}{l}\text { Apr. - } \\
\text { May - } \\
\text { June - } \\
\text { Sept. - } \\
\text { Dec. - } \\
\text { Mar. - }\end{array}$ & $\begin{array}{l}\text { Mar. } \\
\text { Nov. } \\
\text { Aug. } \\
\text { Nov. } \\
\text { Feb. } \\
\text { May }\end{array}$ & $\begin{array}{c}12 \\
11 \\
13 \\
9.3 \\
16 \\
60\end{array}$ & $\begin{array}{l}16 \\
14 \\
16 \\
11 \\
19 \\
74\end{array}$ & $\begin{array}{l}20 \\
17 \\
19 \\
14 \\
25 \\
93\end{array}$ & $\begin{array}{r}23 \\
20 \\
22 \\
16 \\
32 \\
120\end{array}$ & $\begin{array}{r}28 \\
22 \\
25 \\
18 \\
44 \\
140\end{array}$ & $\begin{array}{r}34 \\
25 \\
29 \\
20 \\
62 \\
160\end{array}$ & $\begin{array}{r}44 \\
29 \\
34 \\
21 \\
84 \\
190\end{array}$ & $\begin{array}{r}73 \\
39 \\
46 \\
26 \\
140 \\
260\end{array}$ & $\begin{array}{r}120 \\
56 \\
62 \\
31 \\
200 \\
360\end{array}$ & $\begin{array}{r}190 \\
83 \\
88 \\
40 \\
310 \\
500\end{array}$ & $\begin{array}{r}310 \\
130 \\
130 \\
56 \\
510 \\
720\end{array}$ & $\begin{array}{r}560 \\
220 \\
220 \\
110 \\
900 \\
1200\end{array}$ & $\begin{array}{r}1300 \\
490 \\
500 \\
280 \\
2000 \\
2100\end{array}$ \\
\hline
\end{tabular}


LOCATION:

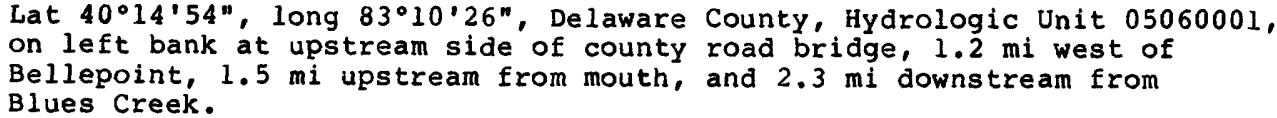

DRAINAGE AREA: $\quad 178 \mathrm{mi}^{2}$.

TRIBITARY TO: Scioto River.

DISCHARGE DATA USED: October 1943 to september 1978.

REMARKS :

Diurnal fluctuation caused by stone quarry upstream from station.

SELECTED DISCHARGE CHARACTERISTICS: Average discharge: $150 \mathrm{ft}^{3} / \mathrm{s}$ (35 years).

Minimum daily discharge: No flow September 1944.

Magnitude and frequency of low flow for indicated periods

\begin{tabular}{|c|c|c|c|c|c|c|c|c|c|c|c|c|c|}
\hline \multirow{2}{*}{ Period } & \multirow{2}{*}{$\begin{array}{l}\text { Number of } \\
\text { consecu- } \\
\text { tive days }\end{array}$} & \multicolumn{5}{|c|}{$\begin{array}{l}\text { Discharge }\left(\mathrm{ft}^{3} / \mathrm{s}\right) \text { for indicated } \\
\text { recurrence interval (years) }\end{array}$} & \multirow{2}{*}{ Period } & \multirow{2}{*}{$\begin{array}{l}\text { Number of } \\
\text { consecu- } \\
\text { tive days }\end{array}$} & \multicolumn{5}{|c|}{$\begin{array}{l}\text { Discharge }\left(\mathrm{ft}^{3} / \mathrm{s}\right) \text { for indicated } \\
\text { recurrence interval (years) }\end{array}$} \\
\hline & & 2 & 5 & 10 & 20 & 50 & & & 2 & 5 & 10 & 20 & 50 \\
\hline Apr.-Mar. & $\begin{array}{r}1 \\
7 \\
30\end{array}$ & $\begin{array}{l}1.3 \\
1.8 \\
2.9\end{array}$ & $\begin{array}{l}0.4 \\
0.7 \\
1.3\end{array}$ & $\begin{array}{l}0.2 \\
0.4 \\
0.9\end{array}$ & $\begin{array}{l}0.1 \\
0.2 \\
0.7\end{array}$ & $\begin{array}{l}0 \\
0.1 \\
0.5\end{array}$ & Sept.-Nov. & $\begin{array}{r}1 \\
7 \\
30\end{array}$ & $\begin{array}{l}1.5 \\
2.3 \\
4.0\end{array}$ & $\begin{array}{l}0.5 \\
0.8 \\
1.7\end{array}$ & $\begin{array}{l}0.2 \\
0.5 \\
1.1\end{array}$ & $\begin{array}{l}0.1 \\
0.3 \\
0.8\end{array}$ & $\begin{array}{l}0 \\
0.2 \\
0.5\end{array}$ \\
\hline June-Aug. & $\begin{array}{r}1 \\
7 \\
30\end{array}$ & $\begin{array}{l}2.3 \\
3.0 \\
6.8\end{array}$ & $\begin{array}{l}0.9 \\
1.3 \\
2.9\end{array}$ & $\begin{array}{l}0.4 \\
0.8 \\
1.9\end{array}$ & $\begin{array}{l}0.2 \\
0.5 \\
1.4\end{array}$ & $\begin{array}{l}0.1 \\
0.3 \\
1.0\end{array}$ & Mar.- May & $\begin{array}{r}1 \\
7 \\
30\end{array}$ & $\begin{array}{l}16 \\
20 \\
55\end{array}$ & $\begin{array}{l}10 \\
12 \\
27\end{array}$ & $\begin{array}{c}8.0 \\
9.4 \\
18\end{array}$ & $\begin{array}{l}6.5 \\
7.5 \\
14\end{array}$ & $\begin{array}{l}5.1 \\
5.8 \\
9.8\end{array}$ \\
\hline
\end{tabular}

Duration of daily flow for indicated periods

\begin{tabular}{l|c|c|c|c|c|c|c|c|c|c|c|c|c}
\hline \multirow{2}{*}{ Period } & \multicolumn{8}{|c|}{ Discharge $\left(\mathrm{ft}^{3} / \mathrm{s}\right.$ ) which was equaled or exceeded for indicated percent of time } \\
\cline { 2 - 13 } & 98 & 95 & 90 & 85 & 80 & 75 & 70 & 60 & 50 & 40 & 30 & 20 & 10 \\
\hline Apr. - Mar. & 1.0 & 1.7 & 3.3 & 4.4 & 5.8 & 7.3 & 9.1 & 15 & 25 & 41 & 70 & 140 & 330 \\
May - Nov. & 0.8 & 1.2 & 2.0 & 3.0 & 3.9 & 4.7 & 5.7 & 8.0 & 11 & 17 & 25 & 44 & 110 \\
June - Aug. & 0.9 & 1.7 & 2.6 & 3.5 & 4.2 & 5.0 & 6.0 & 8.0 & 11 & 15 & 22 & 38 & 100 \\
Sept.- Nov. & 0.7 & 0.9 & 1.3 & 1.8 & 2.6 & 3.4 & 4.1 & 5.6 & 7.4 & 9.6 & 14 & 23 & 53 \\
Dec. - Feb. & 3.4 & 4.1 & 5.8 & 8.1 & 11 & 15 & 20 & 33 & 53 & 79 & 130 & 250 & 640 \\
Mar. - May & 10 & 16 & 22 & 26 & 31 & 37 & 43 & 59 & 81 & 120 & 180 & 300 & 700 \\
\hline
\end{tabular}




\section{SCIOTO RIVER BASIN}

03221000 Scioto River below O'Shaughnessy Dam, near Dublin, Ohio

LOCATION:

Lat $40^{\circ} 08^{\prime} 36^{\prime \prime}$, long $83^{\circ} 07^{\prime} 14^{\prime \prime}$, Delaware County, Hydrologic Unit 05060001 , on left bank, $0.2 \mathrm{mi}$ north of county line, $0.8 \mathrm{mi}$ downstream from $O$ 'Shaughnessy Dam, and $3.0 \mathrm{mi}$ north of Dublin.

DRAINAGE AREA: $\quad 980 \mathrm{mi}^{2}$.

TRIBUTARY TO: Ohio River.

DISCHARGE DATA USED: October 1924 to september 1978.

REMARKS:

Flow regulated since 1924 by O'Shaughnessy Reservoir, $0.8 \mathrm{mi}$ upstream.

SELECTED DISCHARGE CHARACTERISTICS: Average discharge: $775 \mathrm{ft}^{3} / \mathrm{s}$ ( 54 years).

Minimum daily discharge: $0.4 \mathrm{ft} 3 / \mathrm{s}$ November 1924.

Magnitude and frequency of low flow for indicated periods

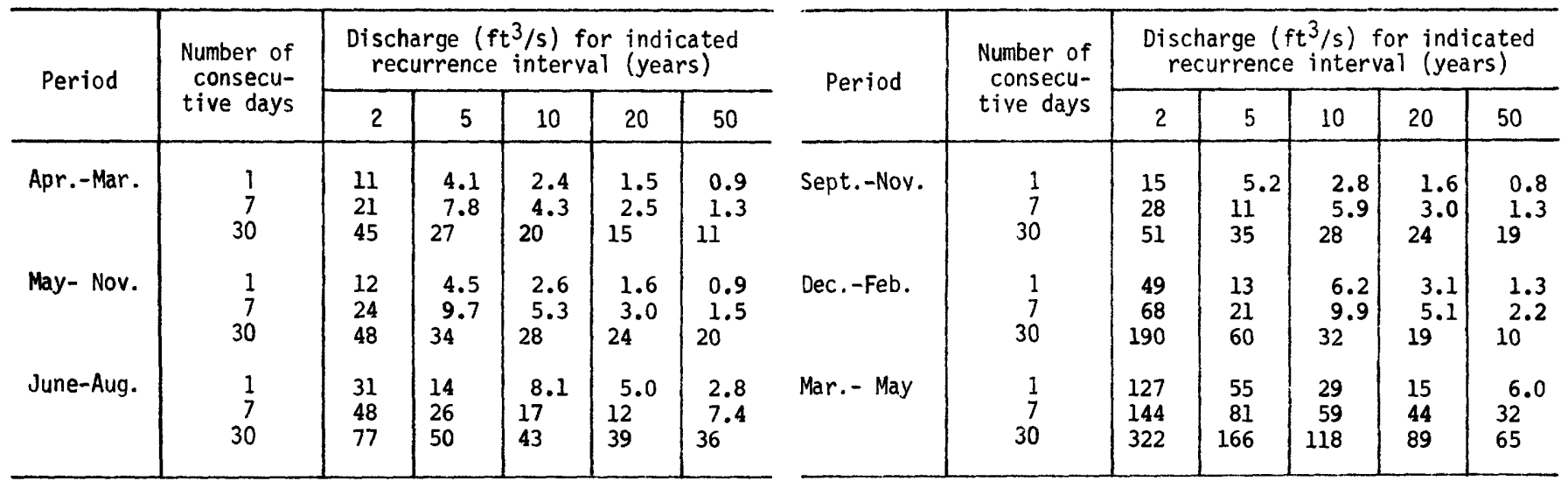

Duration of daily flow for indicated periods

\begin{tabular}{|c|c|c|c|c|c|c|c|c|c|c|c|c|c|c|}
\hline \multirow{2}{*}{\multicolumn{2}{|c|}{ Period }} & \multicolumn{13}{|c|}{ Discharge $\left(\mathrm{ft}^{3} / \mathrm{s}\right)$ which was equaled or exceeded for indicated percent of time } \\
\hline & & 98 & 95 & 90 & 85 & 80 & 75 & 70 & 60 & 50 & 40 & 30 & 20 & 10 \\
\hline $\begin{array}{l}\text { Apr. - } \\
\text { May - } \\
\text { June - } \\
\text { Sept. - } \\
\text { Dec. - } \\
\text { Mar. - }\end{array}$ & $\begin{array}{l}\text { Mar. } \\
\text { Nov. } \\
\text { Aug. } \\
\text { Nov. } \\
\text { Feb.7 } \\
\text { May }\end{array}$ & $\begin{array}{l}14 \\
12 \\
23 \\
6.3 \\
10 \\
66\end{array}$ & $\begin{array}{r}32 \\
29 \\
35 \\
18 \\
28 \\
100\end{array}$ & $\begin{array}{r}43 \\
39 \\
46 \\
32 \\
43 \\
140\end{array}$ & $\begin{array}{r}52 \\
46 \\
52 \\
37 \\
54 \\
180\end{array}$ & $\begin{array}{r}59 \\
52 \\
59 \\
42 \\
66 \\
220\end{array}$ & $\begin{array}{r}69 \\
58 \\
66 \\
47 \\
83 \\
260\end{array}$ & $\begin{array}{r}79 \\
63 \\
73 \\
51 \\
120 \\
310\end{array}$ & $\begin{array}{r}110 \\
78 \\
89 \\
58 \\
200 \\
430\end{array}$ & $\begin{array}{r}180 \\
97 \\
110 \\
68 \\
310 \\
590\end{array}$ & $\begin{array}{r}290 \\
130 \\
150 \\
79 \\
500 \\
840\end{array}$ & $\begin{array}{r}480 \\
200 \\
220 \\
95 \\
830 \\
1200\end{array}$ & $\begin{array}{r}900 \\
340 \\
370 \\
140 \\
1500 \\
2000\end{array}$ & $\begin{array}{r}2200 \\
800 \\
840 \\
370 \\
3400 \\
3600\end{array}$ \\
\hline
\end{tabular}




\section{SCIOTO RIVER BASIN}

03223000 Olentangy River at Claridon, Ohio

LOCATION:

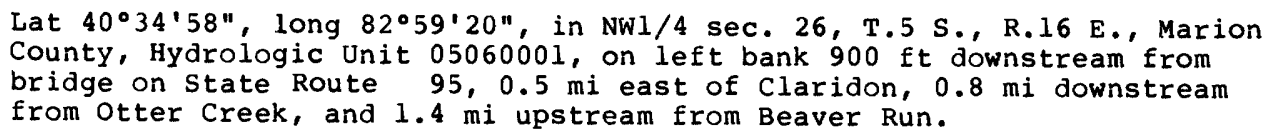

DRAINAGE AREA: $\quad 157 \mathrm{mi}^{2}$.

TRIBUTARY TO: Scioto River.

DISCHARGE DATA USED: October 1946 to september 1978.

REMARKS:

None.

SELECTED DISCHARGE CHARACTERISTICS: Average discharge: $150 \mathrm{ft}^{3} / \mathrm{s}$ (32 years).

Minimum daily discharge: No flow October 1953, september 1955.

Magnitude and frequency of low flow for indicated periods

\begin{tabular}{|c|c|c|c|c|c|c|c|c|c|c|c|c|c|}
\hline \multirow{2}{*}{ Period } & \multirow{2}{*}{$\begin{array}{l}\text { Number of } \\
\text { consecu- } \\
\text { tive days }\end{array}$} & \multicolumn{5}{|c|}{$\begin{array}{l}\text { Discharge }\left(\mathrm{ft}^{3} / \mathrm{s}\right) \text { for indicated } \\
\text { recurrence interval (years) }\end{array}$} & \multirow{2}{*}{ Period } & \multirow{2}{*}{$\begin{array}{l}\text { Number of } \\
\text { consecu- } \\
\text { tive days }\end{array}$} & \multicolumn{5}{|c|}{$\begin{array}{l}\text { Discharge }\left(\mathrm{ft}^{3} / \mathrm{s}\right) \text { for indicated } \\
\text { recurrence interva] (years) }\end{array}$} \\
\hline & & 2 & 5 & 10 & 20 & 50 & & & 2 & 5 & 10 & 20 & 50 \\
\hline Apr.-Mar. & $\begin{array}{r}1 \\
7 \\
30\end{array}$ & $\begin{array}{l}1.8 \\
2.4 \\
4.5\end{array}$ & $\begin{array}{l}0.6 \\
0.7 \\
1.3\end{array}$ & $\begin{array}{l}0.2 \\
0.3 \\
0.5\end{array}$ & $\begin{array}{l}0 \\
0 \\
0.2\end{array}$ & $\begin{array}{l}0 \\
0 \\
0\end{array}$ & Sept.-Nov. & $\begin{array}{r}1 \\
7 \\
30\end{array}$ & $\begin{array}{l}2.0 \\
2.7 \\
6.2\end{array}$ & $\begin{array}{l}0.6 \\
0.8 \\
1.5\end{array}$ & $\begin{array}{l}0.2 \\
0.3 \\
0.6\end{array}$ & $\begin{array}{l}0 \\
0 \\
0.2\end{array}$ & $\begin{array}{l}0 \\
0 \\
0.1\end{array}$ \\
\hline June-Aug. & $\begin{array}{r}1 \\
7 \\
30\end{array}$ & $\begin{array}{l}3.0 \\
4.2 \\
10\end{array}$ & $\begin{array}{l}1.2 \\
1.7 \\
4.6\end{array}$ & $\begin{array}{l}0.7 \\
1.0 \\
3.0\end{array}$ & $\begin{array}{l}0.4 \\
0.6 \\
2.2\end{array}$ & $\begin{array}{l}0.2 \\
0.3 \\
1.5\end{array}$ & Mar. - May & $\begin{array}{r}1 \\
7 \\
30\end{array}$ & $\begin{array}{l}20 \\
27 \\
71\end{array}$ & $\begin{array}{l}14 \\
18 \\
38\end{array}$ & $\begin{array}{l}12 \\
15 \\
27\end{array}$ & $\begin{array}{l}10 \\
12 \\
20\end{array}$ & $\begin{array}{l}8.6 \\
10^{\circ} \\
15\end{array}$ \\
\hline
\end{tabular}

Duration of daily flow for indicated periods

\begin{tabular}{|c|c|c|c|c|c|c|c|c|c|c|c|c|c|c|}
\hline \multirow{2}{*}{\multicolumn{2}{|c|}{ Period }} & \multicolumn{13}{|c|}{ Discharge $\left(\mathrm{ft}^{3} / \mathrm{s}\right)$ which was equaled or exceeded for indicated percent of time } \\
\hline & & 98 & 95 & 90 & 85 & 80 & 75 & 70 & 60 & 50 & 40 & 30 & 20 & 10 \\
\hline $\begin{array}{l}\text { Apr. - } \\
\text { May - } \\
\text { June - } \\
\text { Sept. - } \\
\text { Dec. - } \\
\text { Mar. - }\end{array}$ & $\begin{array}{l}\text { Mar. } \\
\text { Nov. } \\
\text { Aug. } \\
\text { Nov. } \\
\text { Feb. } \\
\text { May }\end{array}$ & $\begin{array}{c}0.9 \\
0.6 \\
1.2 \\
0.3 \\
3.4 \\
16\end{array}$ & $\begin{array}{c}2.1 \\
1.2 \\
2.3 \\
0.7 \\
5.6 \\
22\end{array}$ & $\begin{array}{l}3.8 \\
2.5 \\
3.4 \\
1.3 \\
9.0 \\
29\end{array}$ & $\begin{array}{c}5.5 \\
3.6 \\
4.6 \\
2.1 \\
13 \\
37\end{array}$ & $\begin{array}{l}7.3 \\
4.7 \\
5.8 \\
2.9 \\
19 \\
46\end{array}$ & $\begin{array}{c}9.5 \\
5.9 \\
6.8 \\
3.8 \\
26 \\
54\end{array}$ & $\begin{array}{l}13 \\
7.0 \\
8.0 \\
4.5 \\
34 \\
64\end{array}$ & $\begin{array}{l}22 \\
10 \\
11 \\
6.2 \\
50 \\
85\end{array}$ & $\begin{array}{c}37 \\
16 \\
15 \\
8.4 \\
71 \\
120\end{array}$ & $\begin{array}{r}60 \\
23 \\
22 \\
12 \\
110 \\
160\end{array}$ & $\begin{array}{r}96 \\
36 \\
34 \\
19 \\
170 \\
230\end{array}$ & $\begin{array}{r}170 \\
65 \\
58 \\
32 \\
290 \\
360\end{array}$ & $\begin{array}{r}380 \\
150 \\
130 \\
81 \\
680 \\
660\end{array}$ \\
\hline
\end{tabular}


Appendix 3.---Low-flow data--Continued

WABASH RIVER BASIN

03322480 Wabash River above Beaver Creek at Wabash, Ohio

LOCATION:

Lat $40^{\circ} 32^{\prime} 44^{\prime \prime}$, long $84^{\circ} 44^{\prime} 29^{\prime \prime}$, Mercer County, Hydrologic Unit 05120101 , at bridge on State Route $29,0.5 \mathrm{mi}$ east of Wabash and $0.2 \mathrm{mi}$ upstream from Crab Branch.

DRAINAGE AREA: $\quad 119 \mathrm{mi}^{2}$.

TRIBUTARY TO:

Ohio River.

DISCHARGE DATA USED: LOW-flow measurements 1959, 1972-1974 water years.

INDEX STATION: $\quad 03264000$ Greenville Creek near Bradford, Ohio.

REMARKS:

Three U.S. Soil Conservation Service retarding structures in headwaters are assumed to have sinall effect on low flow. Fort Recovery sewage discharges into wabash River and a new plant is being constructed. Fort Recovery water system pumps 115,000 to $150,000 \mathrm{gal} / \mathrm{d}$ from ground water wells.

SELECTED DISCHARGE CHARACTERISTICS: Minimum discharae observed: $1.1 \mathrm{ft}^{3} / \mathrm{s}$ September 1959.

Magnitude and frequency of low flow for indicated periods

\begin{tabular}{c|c|c|c}
\hline \multirow{2}{*}{ Period } & \multirow{2}{*}{$\begin{array}{c}\text { Number of } \\
\text { consecu- } \\
\text { tive days }\end{array}$} & $\begin{array}{c}\text { Discharge }\left(\mathrm{ft}^{3} / \mathrm{s} \text { ) for }\right. \\
\text { indicated recurrence } \\
\text { interval (years) }\end{array}$ \\
\cline { 3 - 4 } & & 2 & 10 \\
\hline Apr. - Mar. & 7 & 0.8 & 0.3 \\
May - Nov. & 30 & 1.1 & 0.4 \\
& 7 & 0.8 & 0.3 \\
June - Aug. & 30 & 1.1 & 0.4 \\
& 7 & 1.2 & 0.4 \\
Sept. - Nov. & 70 & 2.4 & 0.5 \\
& 30 & 0.8 & 0.3 \\
& & 1.5 & 0.4 \\
\hline
\end{tabular}

Duration of daily flow for indicated periods

\begin{tabular}{|c|c|c|c|c|c|}
\hline \multirow{2}{*}{ Period } & \multicolumn{5}{|c|}{$\begin{array}{l}\text { Discharge }\left(\mathrm{ft}^{3} / \mathrm{s}\right) \text { which was equaled or } \\
\text { exceeded for indicated percent of time }\end{array}$} \\
\hline & 98 & 95 & 90 & 85 & 80 \\
\hline $\begin{array}{l}\text { Apr. - Mar. } \\
\text { May - Nov. } \\
\text { June - Aug. } \\
\text { Sept. - Nov. }\end{array}$ & $\begin{array}{l}0.4 \\
0.4 \\
0.4 \\
0.3\end{array}$ & $\begin{array}{l}0.5 \\
0.5 \\
0.5 \\
0.4\end{array}$ & $\begin{array}{l}1.0 \\
0.7 \\
0.9 \\
0.5\end{array}$ & $\begin{array}{l}1.5 \\
1.0 \\
1.2 \\
0.7\end{array}$ & $\begin{array}{l}1.9 \\
1.4 \\
1.6 \\
0.9\end{array}$ \\
\hline
\end{tabular}




\section{SCIOTO RIVER BASIN}

03224500 Whetstone Creek near Ashley, Ohio

LOCATION:

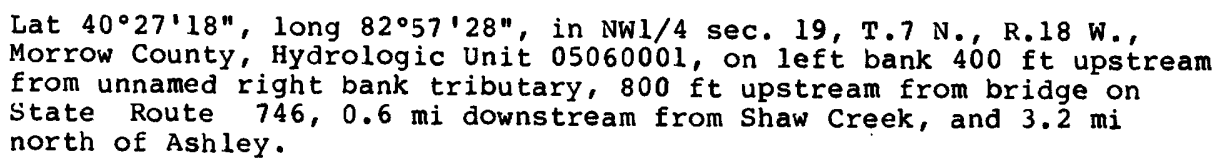

DRAINAGE AREA:

$98.7 \mathrm{mi}^{2}$.

TRIBUTARY TO:

Olentangy River.

DISCHARGE DATA USED:

October 1954 to September 1974.

REMARKS :

None.

SELECTED DISCHARGE CHARACTERISTICS: Average discharge: $97.9 \mathrm{ft}^{3} / \mathrm{s}$ (20 years).

Minimum daily discharge: No flow October 1954, September 1955.

Magnitude and frequency of low flow for indicated periods

\begin{tabular}{|c|c|c|c|c|c|c|c|c|c|c|c|c|c|}
\hline \multirow{2}{*}{ Period } & \multirow{2}{*}{$\begin{array}{l}\text { Number of } \\
\text { consecu- } \\
\text { tive days }\end{array}$} & \multicolumn{5}{|c|}{$\begin{array}{l}\text { Discharge }\left(\mathrm{ft}^{3} / \mathrm{s}\right) \text { for indicated } \\
\text { recurrence interval (years) }\end{array}$} & \multirow{2}{*}{ Period } & \multirow{2}{*}{$\begin{array}{l}\text { Number of } \\
\text { consecu- } \\
\text { tive days }\end{array}$} & \multicolumn{5}{|c|}{$\begin{array}{l}\text { Discharge }\left(\mathrm{ft}^{3} / \mathrm{s}\right) \text { for indicated } \\
\text { recurrence interval (years) }\end{array}$} \\
\hline & & 2 & 5 & 10 & 20 & 50 & & & 2 & 5 & 10 & 20 & 50 \\
\hline Apr.-Mar. & $\begin{array}{r}7 \\
7 \\
30\end{array}$ & $\begin{array}{l}1.9 \\
2.5 \\
3.7\end{array}$ & $\begin{array}{l}0.5 \\
0.6 \\
1.2\end{array}$ & $\begin{array}{l}0.2 \\
0.2 \\
0.6\end{array}$ & $\begin{array}{l}0 \\
0 \\
0.4\end{array}$ & $\begin{array}{l}0 \\
0 \\
0.2\end{array}$ & Sept.-Nov. & $\begin{array}{r}1 \\
7 \\
30\end{array}$ & $\begin{array}{l}2.3 \\
3.0 \\
5.1\end{array}$ & $\begin{array}{l}0.6 \\
0.8 \\
2.0\end{array}$ & $\begin{array}{l}0.2 \\
0.3 \\
1.1\end{array}$ & $\begin{array}{l}0 \\
0 \\
0.6\end{array}$ & $\begin{array}{l}0 \\
0 \\
0.3\end{array}$ \\
\hline June-Aug. & $\begin{array}{r}1 \\
7 \\
30\end{array}$ & $\begin{array}{l}3.0 \\
3.6 \\
8.4\end{array}$ & $\begin{array}{l}1.0 \\
1.4 \\
3.6\end{array}$ & $\begin{array}{l}0.5 \\
0.8 \\
2.4\end{array}$ & $\begin{array}{l}0.3 \\
0.5 \\
1.8\end{array}$ & $\begin{array}{l}0.1 \\
0.3 \\
1.3\end{array}$ & Mar. - May & $\begin{array}{r}1 \\
7 \\
30\end{array}$ & $\begin{array}{l}16 \\
22 \\
50\end{array}$ & $\begin{array}{l}9.5 \\
13 \\
26\end{array}$ & $\begin{array}{c}7.0 \\
9.0 \\
19\end{array}$ & $\begin{array}{l}5.3 \\
6.7 \\
14\end{array}$ & $\begin{array}{r}3.9 \\
4.7 \\
10\end{array}$ \\
\hline
\end{tabular}

Duration of daily flow for indicated periods

\begin{tabular}{|c|c|c|c|c|c|c|c|c|c|c|c|c|c|}
\hline \multirow{2}{*}{ Period } & \multicolumn{13}{|c|}{ Discharge $\left(\mathrm{ft}^{3} / \mathrm{s}\right)$ which was equaled or exceeded for indicated percent of time } \\
\hline & 98 & 95 & 90 & 85 & 80 & 75 & 70 & 60 & 50 & 40 & 30 & 20 & 10 \\
\hline $\begin{array}{l}\text { Apr. - Mar. } \\
\text { May - Nov. } \\
\text { June - Aug. } \\
\text { Sept. - Nov. } \\
\text { Dec. - Feb. } \\
\text { Mar. - May }\end{array}$ & $\begin{array}{l}1.2 \\
0.8 \\
1.2 \\
0.4 \\
3.9 \\
11\end{array}$ & $\begin{array}{l}2.3 \\
1.6 \\
2.0 \\
1.0 \\
6.1 \\
17\end{array}$ & $\begin{array}{r}3.7 \\
2.6 \\
2.8 \\
2.0 \\
9.4 \\
23\end{array}$ & $\begin{array}{l}5.2 \\
3.5 \\
3.6 \\
2.7 \\
12 \\
28\end{array}$ & $\begin{array}{l}6.9 \\
4.4 \\
4.5 \\
3.4 \\
15 \\
33\end{array}$ & $\begin{array}{l}9.1 \\
5.4 \\
5.6 \\
4.1 \\
19 \\
38\end{array}$ & $\begin{array}{l}12 \\
6.6 \\
6.8 \\
4.8 \\
23 \\
45\end{array}$ & $\begin{array}{l}19 \\
9.5 \\
9.9 \\
6.4 \\
32 \\
60\end{array}$ & $\begin{array}{c}29 \\
14 \\
14 \\
8.5 \\
43 \\
78\end{array}$ & $\begin{array}{r}44 \\
21 \\
19 \\
12 \\
58 \\
100\end{array}$ & $\begin{array}{r}66 \\
33 \\
29 \\
18 \\
85 \\
140\end{array}$ & $\begin{array}{r}110 \\
56 \\
49 \\
31 \\
140 \\
200\end{array}$ & $\begin{array}{r}220 \\
120 \\
110 \\
70 \\
320 \\
400\end{array}$ \\
\hline
\end{tabular}


LOCATION:

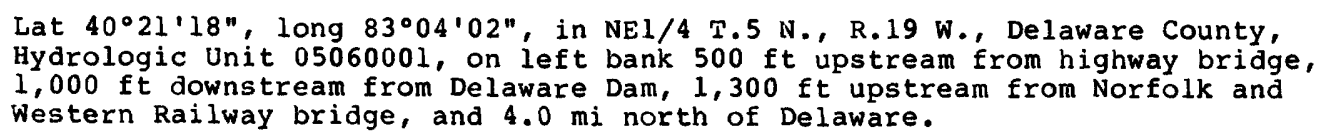

DRAINAGE AREA:

$393 \mathrm{mi}^{2}$.

TRIBUTARY TO:

Scioto River.

DISCHARGE DATA USED:

October 1950 to September 1978.

REMARKS:

Flow completely regulated by Delaware Lake since 1951.

SELECTED DISCHARGE CHARACTERISTICS: Average discharge: $335 \mathrm{ft}^{3} / \mathrm{s} 28$ years).

Minimum daily discharge: $4.1 \mathrm{ft} 3 / \mathrm{s}$ April 1951, February 1954.

Magnitude and frequency of low flow for indicated periods

\begin{tabular}{|c|c|c|c|c|c|c|c|c|c|c|c|c|c|}
\hline \multirow{2}{*}{ Period } & \multirow{2}{*}{$\begin{array}{l}\text { Number of } \\
\text { consecu- } \\
\text { tive days }\end{array}$} & \multicolumn{5}{|c|}{$\begin{array}{l}\text { Discharge }\left(\mathrm{ft}^{3} / \mathrm{s}\right) \text { for indicated } \\
\text { recurrence interval (years) }\end{array}$} & \multirow{2}{*}{ Period } & \multirow{2}{*}{$\begin{array}{l}\text { Number of } \\
\text { consecu- } \\
\text { tive days }\end{array}$} & \multicolumn{5}{|c|}{$\begin{array}{l}\text { Discharge }\left(\mathrm{ft}^{3} / \mathrm{s}\right) \text { for indicated } \\
\text { recurrence interval (years) }\end{array}$} \\
\hline & & 2 & 5 & 10 & 20 & 50 & & & 2 & 5 & 10 & 20 & 50 \\
\hline Apr.-Mar. & $\begin{array}{r}1 \\
7 \\
30\end{array}$ & $\begin{array}{l}8.4 \\
11 \\
17\end{array}$ & $\begin{array}{l}5.4 \\
6.7 \\
12\end{array}$ & $\begin{array}{l}4.4 \\
5.2 \\
9.6\end{array}$ & $\begin{array}{l}3.8 \\
4.3 \\
8.0\end{array}$ & $\begin{array}{l}3.2 \\
3.4 \\
6.4\end{array}$ & Sept.-Nov. & $\begin{array}{r}1 \\
7 \\
30\end{array}$ & $\begin{array}{l}15 \\
16 \\
20\end{array}$ & $\begin{array}{l}9.9 \\
11 \\
14\end{array}$ & $\begin{array}{l}8.2 \\
9.3 \\
12\end{array}$ & $\begin{array}{l}7.0 \\
8.0 \\
10\end{array}$ & $\begin{array}{l}6.0 \\
6.7 \\
9.0\end{array}$ \\
\hline June-Aug. & $\begin{array}{r}1 \\
7 \\
30\end{array}$ & $\begin{array}{l}14 \\
19 \\
34\end{array}$ & $\begin{array}{l}8.2 \\
10 \\
19\end{array}$ & $\begin{array}{l}6.0 \\
7.4 \\
15\end{array}$ & $\begin{array}{l}4.6 \\
5.5 \\
12\end{array}$ & $\begin{array}{l}3.4 \\
3.8 \\
11\end{array}$ & Mar. - May & $\begin{array}{r}1 \\
7 \\
30\end{array}$ & $\begin{array}{r}14 \\
32 \\
145\end{array}$ & $\begin{array}{l}7.5 \\
13 \\
63\end{array}$ & $\begin{array}{l}5.4 \\
8.1 \\
40\end{array}$ & $\begin{array}{r}4.1 \\
5.2 \\
27\end{array}$ & $\begin{array}{r}3.1 \\
3.1 \\
17\end{array}$ \\
\hline
\end{tabular}

Duration of daily flow for indicated periods

\begin{tabular}{|c|c|c|c|c|c|c|c|c|c|c|c|c|c|}
\hline \multirow{2}{*}{ Period } & \multicolumn{13}{|c|}{ Discharge $\left(\mathrm{ft}^{3} / \mathrm{s}\right)$ which was equaled or exceeded for indicated percent of time } \\
\hline & 98 & 95 & 90 & 85 & 80 & 75 & 70 & 60 & 50 & 40 & 30 & 20 & 10 \\
\hline $\begin{array}{l}\text { Apr. - Mar. } \\
\text { May - Nov. } \\
\text { June - Aug. } \\
\text { Sept. - Nov. } \\
\text { Dec. - Feb. } \\
\text { Mar. - May }\end{array}$ & $\begin{array}{c}9.0 \\
9.1 \\
8.5 \\
9.4 \\
11 \\
6.5\end{array}$ & $\begin{array}{l}13 \\
12 \\
13 \\
11 \\
17 \\
15\end{array}$ & $\begin{array}{l}18 \\
15 \\
21 \\
13 \\
21 \\
28\end{array}$ & $\begin{array}{l}21 \\
19 \\
24 \\
15 \\
27 \\
50\end{array}$ & $\begin{array}{l}23 \\
21 \\
27 \\
18 \\
42 \\
65\end{array}$ & $\begin{array}{l}28 \\
23 \\
30 \\
19 \\
55 \\
91\end{array}$ & $\begin{array}{r}34 \\
26 \\
33 \\
20 \\
71 \\
120\end{array}$ & $\begin{array}{r}46 \\
33 \\
39 \\
22 \\
120 \\
160\end{array}$ & $\begin{array}{r}76 \\
42 \\
43 \\
26 \\
160 \\
240\end{array}$ & $\begin{array}{r}130 \\
53 \\
50 \\
34 \\
230 \\
330\end{array}$ & $\begin{array}{r}210 \\
89 \\
74 \\
53 \\
360 \\
480\end{array}$ & $\begin{array}{l}370 \\
170 \\
150 \\
110 \\
620 \\
840\end{array}$ & $\begin{array}{r}880 \\
360 \\
340 \\
230 \\
1400 \\
1700\end{array}$ \\
\hline
\end{tabular}


LOCATION:

Lat $40^{\circ} 06^{\prime} 37^{\prime \prime}$, long $83^{\circ} 01^{\prime} 55^{\prime \prime}$, in NWl/4 T.2 N., R.18 W., Franklin County, Hydrologic Unit 05060001 , on left bank $350 \mathrm{ft}$ downstream from Interstate Highway 270 bridge, $1.5 \mathrm{mi}$ northwest of worthington and $2.8 \mathrm{mi}$ upstream from Rush Run.

DRAINAGE AREA: $\quad 497 \mathrm{mi}^{2}$.

TRIBITARY TO: Scioto River.

DISCHARGE DATA USED: October 1955 to September 1978.

REMARKS:

Flow regulated by Delaware Lake $21 \mathrm{mi}$ upstream.

SELECTED DISCHARGE CHARACTERISTICS: Average discharge: $444 \mathrm{ft}^{3} / \mathrm{s}$ ( 23 years).

Minimum daily discharge: $8.5 \mathrm{ft} 3 / \mathrm{s}$ September 1967.

Magnitude and frequency of low flow for indicated periods

\begin{tabular}{|c|c|c|c|c|c|c|c|c|c|c|c|c|c|}
\hline \multirow{2}{*}{ Period } & \multirow{2}{*}{$\begin{array}{l}\text { Number of } \\
\text { consecu- } \\
\text { tive days }\end{array}$} & \multicolumn{5}{|c|}{$\begin{array}{l}\text { Discharge }\left(\mathrm{ft}^{3} / \mathrm{s}\right) \text { for indicated } \\
\text { recurrence interval (years) }\end{array}$} & \multirow{2}{*}{ Period } & \multirow{2}{*}{$\begin{array}{l}\text { Number of } \\
\text { consecu- } \\
\text { tive days }\end{array}$} & \multicolumn{5}{|c|}{$\begin{array}{l}\text { Discharge }\left(\mathrm{ft}^{3} / \mathrm{s}\right) \text { for indicated } \\
\text { recurrence interval (years) }\end{array}$} \\
\hline & & 2 & 5 & 10 & 20 & 50 & & & 2 & 5 & 10 & 20 & 50 \\
\hline Apr.-Mar. & $\begin{array}{r}1 \\
7 \\
30\end{array}$ & $\begin{array}{l}15 \\
17 \\
24\end{array}$ & $\begin{array}{l}10 \\
12 \\
16\end{array}$ & $\begin{array}{l}8.9 \\
13\end{array}$ & $\begin{array}{r}7.9 \\
9.7 \\
12\end{array}$ & $\begin{array}{l}7.1 \\
8.9 \\
11\end{array}$ & Sept.-Nov. & $\begin{array}{r}1 \\
7 \\
30\end{array}$ & $\begin{array}{l}17 \\
20 \\
29\end{array}$ & $\begin{array}{l}11 \\
14 \\
18\end{array}$ & $\begin{array}{l}9.4 \\
11\end{array}$ & $\begin{array}{l}8.1 \\
9.9 \\
12\end{array}$ & $\begin{array}{l}6.9 \\
8.6 \\
9.5\end{array}$ \\
\hline June-Aug. & $\begin{array}{r}1 \\
7 \\
30\end{array}$ & $\begin{array}{l}24 \\
29 \\
52\end{array}$ & $\begin{array}{l}16 \\
19 \\
29\end{array}$ & $\begin{array}{l}13 \\
15 \\
23\end{array}$ & $\begin{array}{l}10 \\
13 \\
20\end{array}$ & $\begin{array}{l}8.1 \\
10 \\
17\end{array}$ & Mar. - May & $\begin{array}{r}1 \\
7 \\
30\end{array}$ & $\begin{array}{r}43 \\
65 \\
223\end{array}$ & $\begin{array}{r}26 \\
37 \\
101\end{array}$ & $\begin{array}{l}20 \\
28 \\
65\end{array}$ & $\begin{array}{l}16 \\
22 \\
45\end{array}$ & $\begin{array}{l}13 \\
17 \\
29\end{array}$ \\
\hline
\end{tabular}

Duration of daily flow for indicated periods

\begin{tabular}{|c|c|c|c|c|c|c|c|c|c|c|c|c|c|}
\hline \multirow{2}{*}{ Period } & \multicolumn{13}{|c|}{ Discharge $\left(\mathrm{ft}^{3} / \mathrm{s}\right)$ which was equaled or exceeded for indicated percent of time } \\
\hline & 98 & 95 & 90 & 85 & 80 & 75 & 70 & 60 & 50 & 40 & 30 & 20 & 10 \\
\hline $\begin{array}{l}\text { Apr. - Mar. } \\
\text { May - Nov. } \\
\text { June - Aug. } \\
\text { Sept. - Nov. } \\
\text { Dec. - Feb. } \\
\text { Mar. - May }\end{array}$ & $\begin{array}{l}14 \\
13 \\
15 \\
11 \\
14 \\
28\end{array}$ & $\begin{array}{l}18 \\
16 \\
23 \\
13 \\
20 \\
39\end{array}$ & $\begin{array}{l}25 \\
22 \\
30 \\
16 \\
31 \\
66\end{array}$ & $\begin{array}{l}31 \\
26 \\
35 \\
20 \\
51 \\
90\end{array}$ & $\begin{array}{r}37 \\
31 \\
38 \\
22 \\
66 \\
120\end{array}$ & $\begin{array}{r}44 \\
35 \\
42 \\
25 \\
86 \\
150\end{array}$ & $\begin{array}{r}52 \\
39 \\
45 \\
27 \\
110 \\
170\end{array}$ & $\begin{array}{r}77 \\
48 \\
51 \\
33 \\
170 \\
240\end{array}$ & $\begin{array}{r}130 \\
62 \\
63 \\
41 \\
230 \\
350\end{array}$ & $\begin{array}{r}200 \\
92 \\
85 \\
55 \\
330 \\
500\end{array}$ & $\begin{array}{r}320 \\
160 \\
150 \\
90 \\
530 \\
720\end{array}$ & $\begin{array}{r}550 \\
270 \\
260 \\
160 \\
880 \\
1200\end{array}$ & $\begin{array}{r}1200 \\
560 \\
530 \\
300 \\
1800 \\
2100\end{array}$ \\
\hline
\end{tabular}


SCIOTO RIVER BASIN

03227500 Scioto River at Columbus, Ohio

LOCATION:

DRAINAGE AREA:

TRIBUTARY TO:

DISCHARGE DATA USED:

REMARKS :

\begin{tabular}{|c|c|c|c|c|c|c|}
\hline \multirow{2}{*}{ Period } & \multirow{2}{*}{$\begin{array}{l}\text { Number of } \\
\text { consecu- } \\
\text { tive days }\end{array}$} & \multicolumn{5}{|c|}{$\begin{array}{l}\text { Discharge }\left(\mathrm{ft}^{3} / \mathrm{s}\right) \text { for indicated } \\
\text { recurrence interval (years) }\end{array}$} \\
\hline & & 2 & 5 & 10 & 20 & 50 \\
\hline Apr.-Mar. & $\begin{array}{r}7 \\
7 \\
30\end{array}$ & $\begin{array}{l}122 \\
140 \\
157\end{array}$ & $\begin{array}{r}96 \\
109 \\
118\end{array}$ & $\begin{array}{r}84 \\
95 \\
105\end{array}$ & $\begin{array}{l}75 \\
86 \\
97\end{array}$ & $\begin{array}{l}66 \\
76 \\
90\end{array}$ \\
\hline May- Nov. & $\begin{array}{r}1 \\
7 \\
30\end{array}$ & $\begin{array}{l}124 \\
144 \\
164\end{array}$ & $\begin{array}{r}98 \\
112 \\
123\end{array}$ & $\begin{array}{r}86 \\
98 \\
109\end{array}$ & $\begin{array}{r}77 \\
87 \\
100\end{array}$ & $\begin{array}{r}69 \\
77 \\
-92\end{array}$ \\
\hline June-Aug. & $\begin{array}{r}1 \\
7 \\
30\end{array}$ & $\begin{array}{l}154 \\
183 \\
239\end{array}$ & $\begin{array}{l}121 \\
142 \\
168\end{array}$ & $\begin{array}{l}107 \\
124 \\
150\end{array}$ & $\begin{array}{r}96 \\
111 \\
141\end{array}$ & $\begin{array}{r}86 \\
97 \\
135\end{array}$ \\
\hline
\end{tabular}

Duration of daily flow for indicated periods

\begin{tabular}{|c|c|c|c|c|c|c|c|c|c|c|c|c|c|}
\hline \multirow{2}{*}{ Period } & \multicolumn{13}{|c|}{ Discharge $\left(\mathrm{ft}^{3} / \mathrm{s}\right)$ which was equaled or exceeded for indicated percent of time } \\
\hline & 98 & 95 & 90 & 85 & 80 & 75 & 70 & 60 & 50 & 40 & 30 & 20 & 10 \\
\hline $\begin{array}{l}\text { Apr. - Mar. } \\
\text { May - Nov. } \\
\text { June - Aug. } \\
\text { Sept. - Nov. } \\
\text { Dec. - Feb. } \\
\text { Mar. - May }\end{array}$ & $\begin{array}{r}100 \\
99 \\
110 \\
89 \\
92 \\
220\end{array}$ & $\begin{array}{l}120 \\
110 \\
130 \\
100 \\
120 \\
290\end{array}$ & $\begin{array}{l}140 \\
130 \\
150 \\
120 \\
140 \\
370\end{array}$ & $\begin{array}{l}160 \\
150 \\
170 \\
130 \\
180 \\
430\end{array}$ & $\begin{array}{l}180 \\
160 \\
180 \\
140 \\
220 \\
510\end{array}$ & $\begin{array}{l}210 \\
180 \\
200 \\
150 \\
270 \\
610\end{array}$ & $\begin{array}{l}240 \\
190 \\
220 \\
160 \\
330 \\
710\end{array}$ & $\begin{array}{l}320 \\
230 \\
250 \\
180 \\
500 \\
950\end{array}$ & $\begin{array}{r}450 \\
280 \\
310 \\
200 \\
720 \\
1300\end{array}$ & $\begin{array}{r}660 \\
370 \\
400 \\
240 \\
1000 \\
1800\end{array}$ & $\begin{array}{r}1000 \\
510 \\
540 \\
300 \\
1600 \\
2500\end{array}$ & $\begin{array}{r}1700 \\
790 \\
810 \\
440 \\
2800 \\
4000\end{array}$ & $\begin{array}{r}3800 \\
1500 \\
1500 \\
730 \\
5500 \\
6700\end{array}$ \\
\hline
\end{tabular}

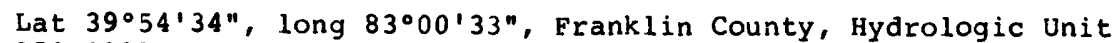
05060001 , on right bank at sewage-treatment plant of city of Columbus, $0.4 \mathrm{mi}$ downstream from bridge on Frank Road, $2.8 \mathrm{mi}$ upstream from Scioto Big Run, and $5 \mathrm{mi}$ downstream from Olentangy River.

$1,629 \mathrm{mi}^{2}$.

Ohio River.

Flow regulated by Griggs Reservoir $10.4 \mathrm{mi}$ upstream, D'Shaughnessy Reservoir $20.4 \mathrm{mi}$ upstream, and Delaware Lake $35 \mathrm{mi}$ upstream from station. Records include sewage return flow from Frank Road Treatment Plant. Shadeville Treatment plant flow enters downstream. Water supply for city of Columbus is obtained from Scioto River downstream from Griggs Dam. Big Walnut Creek downstream from Central College, and from well field in Alum Creek basin.

SELECTED DISCHARGE CHARACTERISTICS: Average discharge: $1,392 \mathrm{ft}^{3} / \mathrm{s}_{3}(28$ years).

Minimum daily discharge: $68 \mathrm{ft}^{3} / \mathrm{s}$ December 1954.

\begin{tabular}{|c|c|c|c|c|c|c|}
\hline \multirow{2}{*}{ Period } & \multirow{2}{*}{$\begin{array}{l}\text { Number of } \\
\text { consecu- } \\
\text { tive days }\end{array}$} & \multicolumn{5}{|c|}{$\begin{array}{l}\text { Discharge }\left(\mathrm{ft}^{3} / \mathrm{s}\right) \text { for indicated } \\
\text { recurrence interval (years) }\end{array}$} \\
\hline & & 2 & 5 & 10 & 20 & 50 \\
\hline Sept.-Nov. & $\begin{array}{r}1 \\
7 \\
30\end{array}$ & $\begin{array}{l}125 \\
144 \\
161\end{array}$ & $\begin{array}{r}97 \\
112 \\
122\end{array}$ & $\begin{array}{r}85 \\
98 \\
112\end{array}$ & $\begin{array}{r}77 \\
87 \\
106\end{array}$ & $\begin{array}{r}69 \\
77 \\
102\end{array}$ \\
\hline Dec.-Feb. & $\begin{array}{r}1 \\
7 \\
30\end{array}$ & $\begin{array}{l}208 \\
248 \\
477\end{array}$ & $\begin{array}{l}123 \\
143 \\
199\end{array}$ & $\begin{array}{r}95 \\
108 \\
129\end{array}$ & $\begin{array}{l}77 \\
87 \\
91\end{array}$ & $\begin{array}{l}61 \\
69 \\
61\end{array}$ \\
\hline Mar. - Mav & $\begin{array}{r}1 \\
7 \\
30\end{array}$ & $\begin{array}{l}277 \\
349 \\
741\end{array}$ & $\begin{array}{l}189 \\
240 \\
442\end{array}$ & $\begin{array}{l}153 \\
200 \\
341\end{array}$ & $\begin{array}{l}128 \\
172 \\
276\end{array}$ & $\begin{array}{l}104 \\
147 \\
219\end{array}$ \\
\hline
\end{tabular}


LOCATION:

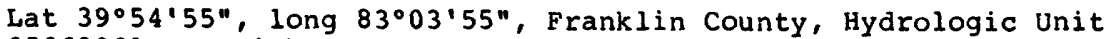
05060001 , on right bank at downstream side of bridge on U.S. Highway 62 at Briggsdale, $2.8 \mathrm{mi}$ northeast of Grove City, and $4.0 \mathrm{mi}$ upstream from station.

DRAINAGE AREA:

$11.0 \mathrm{mi}^{2}$

TRIBUTARY TO:

Scioto River.

DISCHARGE DATA USED: October 1946 to September 1958.

REMARKS:

None.

SELECTED DISCHARGE CHARACTERISTICS: Average discharge: $10.7 \mathrm{ft}^{3} / \mathrm{s}$ (12 years).

Minimum daily discharge: No flow many times each year.

Magnitude and frequency of low flow for indicated periods

\begin{tabular}{|c|c|c|c|c|c|c|}
\hline \multirow{2}{*}{ Period } & \multirow{2}{*}{$\begin{array}{l}\text { Number of } \\
\text { consecu- } \\
\text { tive days }\end{array}$} & \multicolumn{5}{|c|}{$\begin{array}{l}\text { Discharge }\left(\mathrm{ft}^{3} / \mathrm{s}\right) \text { for indicated } \\
\text { recurrence interval (years) }\end{array}$} \\
\hline & & 2 & 5 & 10 & 20 & 50 \\
\hline Apr.-Mar. & $\begin{array}{r}1 \\
7 \\
30\end{array}$ & $\begin{array}{l}0 \\
0 \\
0\end{array}$ & $\begin{array}{l}0 \\
0 \\
0\end{array}$ & $\begin{array}{l}0 \\
0 \\
0\end{array}$ & $\begin{array}{l}0 \\
0 \\
0\end{array}$ & $\begin{array}{l}0 \\
0 \\
0\end{array}$ \\
\hline May - Nov. & $\begin{array}{r}1 \\
7 \\
30\end{array}$ & $\begin{array}{l}0 \\
0 \\
0\end{array}$ & $\begin{array}{l}0 \\
0 \\
0\end{array}$ & $\begin{array}{l}0 \\
0 \\
0\end{array}$ & $\begin{array}{l}0 \\
0 \\
0\end{array}$ & $\begin{array}{l}0 \\
0 \\
0\end{array}$ \\
\hline June-Aug. & $\begin{array}{r}1 \\
7 \\
30\end{array}$ & $\begin{array}{l}0 \\
0 \\
0.1\end{array}$ & $\begin{array}{l}0 \\
0 \\
0\end{array}$ & $\begin{array}{l}0 \\
0 \\
0\end{array}$ & $\begin{array}{l}0 \\
0 \\
0\end{array}$ & $\begin{array}{l}0 \\
0 \\
0\end{array}$ \\
\hline
\end{tabular}

\begin{tabular}{|c|c|c|c|c|c|c|}
\hline \multirow{2}{*}{ Period } & \multirow{2}{*}{$\begin{array}{l}\text { Number of } \\
\text { consecu- } \\
\text { tive days }\end{array}$} & \multicolumn{5}{|c|}{$\begin{array}{l}\text { Discharge }\left(\mathrm{ft}^{3} / \mathrm{s}\right) \text { for indicated } \\
\text { recurrence interval (years) }\end{array}$} \\
\hline & & 2 & 5 & 10 & 20 & 50 \\
\hline Sept.-Nov. & $\begin{array}{r}1 \\
7 \\
30\end{array}$ & $\begin{array}{l}0 \\
0 \\
0\end{array}$ & $\begin{array}{l}0 \\
0 \\
0\end{array}$ & $\begin{array}{l}0 \\
0 \\
0\end{array}$ & $\begin{array}{l}0 \\
0 \\
0\end{array}$ & $\begin{array}{l}0 \\
0 \\
0\end{array}$ \\
\hline Dec. -Feb. & $\begin{array}{r}1 \\
7 \\
30\end{array}$ & $\begin{array}{l}0.2 \\
0.4 \\
2.3\end{array}$ & $\begin{array}{l}0 \\
0 \\
0.2\end{array}$ & $\begin{array}{l}0 \\
0 \\
0\end{array}$ & $\begin{array}{l}0 \\
0 \\
0\end{array}$ & $\begin{array}{l}0 \\
0 \\
0\end{array}$ \\
\hline Mar. - May & $\begin{array}{r}1 \\
7 \\
30\end{array}$ & $\begin{array}{l}0.4 \\
0.8 \\
4.4\end{array}$ & $\begin{array}{l}0.2 \\
0.5 \\
2.2\end{array}$ & $\begin{array}{l}0.1 \\
0.3 \\
1.4\end{array}$ & $\begin{array}{l}0.1 \\
0.3 \\
0.9\end{array}$ & $\begin{array}{l}0.1 \\
0.2 \\
0.6\end{array}$ \\
\hline
\end{tabular}

Duration of daily flow for indicated periods

\begin{tabular}{|c|c|c|c|c|c|c|c|c|c|c|c|c|c|}
\hline \multirow{2}{*}{ Period } & \multicolumn{13}{|c|}{ Discharge $\left(\mathrm{ft}^{3} / \mathrm{s}\right)$ which was equaled or exceeded for indicated percent of time } \\
\hline & 98 & 95 & 90 & 85 & 80 & 75 & 70 & 60 & 50 & 40 & 30 & 20 & 10 \\
\hline $\begin{array}{l}\text { Apr. - Mar. } \\
\text { May - Nov. } \\
\text { June - Aug. } \\
\text { Sept. - Nov. } \\
\text { Dec. - Feb. } \\
\text { Mar. - May }\end{array}$ & $\begin{array}{l}0 \\
0 \\
0 \\
0 \\
0 \\
0.4\end{array}$ & $\begin{array}{l}0 \\
0 \\
0 \\
0 \\
0.1 \\
0.8\end{array}$ & $\begin{array}{l}0 \\
0 \\
0 \\
0 \\
0.2 \\
1.2\end{array}$ & $\begin{array}{l}0.1 \\
0 \\
0 \\
0 \\
0.3 \\
1.6\end{array}$ & $\begin{array}{l}0.1 \\
0 \\
0 \\
0 \\
0.5 \\
2.2\end{array}$ & $\begin{array}{l}0.1 \\
0.1 \\
0.1 \\
0 \\
0.9 \\
2.7\end{array}$ & $\begin{array}{l}0.1 \\
0.1 \\
0.1 \\
0.1 \\
1.3 \\
3.3\end{array}$ & $\begin{array}{l}0.4 \\
0.1 \\
0.1 \\
0.1 \\
2.4 \\
4.5\end{array}$ & $\begin{array}{l}1.2 \\
0.2 \\
0.2 \\
0.1 \\
4.7 \\
6.3\end{array}$ & $\begin{array}{l}2.6 \\
0.4 \\
0.4 \\
0.1 \\
8.2 \\
8.6\end{array}$ & $\begin{array}{l}5.2 \\
1.0 \\
0.9 \\
0.3 \\
14 \\
12\end{array}$ & $\begin{array}{l}9.9 \\
2.2 \\
2.0 \\
0.9 \\
24 \\
18\end{array}$ & $\begin{array}{l}24 \\
5.7 \\
7.4 \\
3.1 \\
46 \\
35\end{array}$ \\
\hline
\end{tabular}


Appendix 3.--Low-flow data--Continued

SCIOTO RIVER BASIN

03228200 Big Walnut creek above Sunbury, Ohio

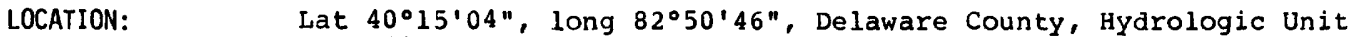
05060001 , at U.S. Highway 36 bridge at north edge of Sunbury, $0.5 \mathrm{mi}$ downstream from Perfect Creek.

DRAINAGE AREA: $\quad 77.8 \mathrm{mi}^{2}$.

TRIBUTARY TO:

DISCHARGE DATA USED: Low-flow measurements 1972-1977 water years.

INDEX STATION: $\quad 03220000$ Mill Creek near Bellepoint, Ohio.

REMARKS: None upstream. 400,000 to $500,000 \mathrm{gal} / \mathrm{d}$ are pumped through Sunbury water supply and intake pumps at dam; can pump a maximum of $1,550 \mathrm{gal} / \mathrm{d}$ of water directly from Big walnut Creek.

SELECTED DISCHARGE CHARACTERISTICS: Minimum discharge observed: $0.0 \mathrm{ft}^{3} / \mathrm{s}$ August 1974.

\begin{tabular}{l|r|c|c}
\multicolumn{4}{c}{$\begin{array}{c}\text { Magnitude and frequency of low flow for } \\
\text { indicated periods }\end{array}$} \\
\hline \multirow{2}{*}{ Period } & $\begin{array}{c}\text { Number of } \\
\text { consecu- } \\
\text { tive days }\end{array}$ & $\begin{array}{c}\text { Discharge (ft } 3 / \mathrm{s} \text { ) for } \\
\text { indicated recurrence } \\
\text { interval (years) }\end{array}$ \\
\cline { 3 - 4 } & 7 & 2 & 10 \\
\hline Apr. - Mar. & 30 & 0.1 & 0 \\
May - Nov. & 7 & 0.2 & 0 \\
June - Aug. & 30 & 0.2 & 0 \\
& 7 & 0.3 & 0 \\
Sept. - Nov. & 30 & 1.1 & 0.1 \\
& 30 & 0.2 & 0 \\
\hline
\end{tabular}

Duration of daily flow for indicated periods

\begin{tabular}{c|c|c|c|c|c}
\hline \multirow{2}{*}{ Period } & \multicolumn{5}{|c}{$\begin{array}{c}\text { Discharge }\left(\mathrm{ft}^{3} / \mathrm{s}\right) \text { which was equaled or } \\
\text { exceeded for indicated percent of time }\end{array}$} \\
\cline { 2 - 6 } & 98 & 95 & 90 & 85 & 80 \\
\hline Apr. - Mar. & 0 & 0.1 & 0.3 & 0.5 & 0.8 \\
May - Nov. & 0 & 0.1 & 0.1 & 0.3 & 0.4 \\
June - Aug. & 0 & 0.1 & 0.2 & 0.3 & 0.5 \\
Sept. - Nov. & 0 & 0 & 0.1 & 0.1 & 0.2 \\
\hline
\end{tabular}


Appendix 3.--Low-flow data--Continued

\section{SCIOTO RIVER BASIN}

03228700 Blacklick Creek near Groveport, Ohio

LOCATION:

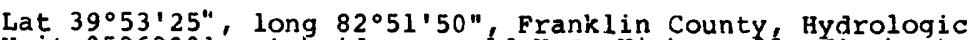
Unit 05060001 , at bridge on old U.S. Highway 33 , Winchester Pike, $2.0 \mathrm{mi}$ upstream from mouth and $2.5 \mathrm{mi}$ northeast of Groveport.

DRAINAGE AREA: $\quad 57.4 \mathrm{mi}^{2}$.

TRIBUTARY TO: Big Walnut Creek.

DISCHARGE DATA USED: Low-flow measurements 1950, 1961-1967, 1969, 1971-1972

water years.

INDEX STATION: $\quad 03146500$ Licking River near Newark, Ohio.

REMARKS: None.

SELECTED DISCHARGE CHARACTERISTICS: Minimum discharge observed: $0.8 \mathrm{ft}^{3} / \mathrm{s}$ October 1963 .

Magnitude and frequency of low flow for indicated periods

\begin{tabular}{c|c|c|c}
\hline \multirow{2}{*}{ Period } & \multirow{2}{*}{$\begin{array}{c}\text { Number of } \\
\text { consecu- } \\
\text { tive days }\end{array}$} & \multicolumn{2}{|c}{$\begin{array}{c}\text { Discharge }\left(\mathrm{ft}^{3} / \mathrm{s}\right) \text { for } \\
\text { indicated recurrence } \\
\text { interval (years) }\end{array}$} \\
\cline { 3 - 4 } Apr. - Mar. & 7 & 2 & 10 \\
\hline May - Nov. & 30 & 2.4 & 1.0 \\
& 7 & 2.5 & 1.3 \\
June - Aug. & 30 & 3.8 & 1.0 \\
& 7 & 5.0 & 1.3 \\
Sept.- Nov. & 7 & 11 & 1.9 \\
& 30 & 2.7 & 1.0 \\
& & 4.0 & 1.3 \\
\hline
\end{tabular}

Duration of daily flow for indicated periods

\begin{tabular}{l|c|c|c|c|c}
\hline \multirow{2}{*}{ Period } & \multicolumn{5}{|c}{$\begin{array}{c}\text { Discharge ( } \mathrm{ft}^{3} / \mathrm{s} \text { ) which was equaled or } \\
\text { exceeded for indicated percent of time }\end{array}$} \\
\cline { 2 - 6 } & 98 & 95 & 90 & 85 & 80 \\
\hline Apr. - Mar. & 1.2 & 1.8 & 2.8 & 4.2 & 6.2 \\
May - Nov. & 1.1 & 1.6 & 2.3 & 3.2 & 4.0 \\
June - Aug. & 1.9 & 2.7 & 3.8 & 5.0 & 6.5 \\
Sept. - Nov. & 0.8 & 1.1 & 1.5 & 1.9 & 2.3 \\
\hline
\end{tabular}


Appendix 3.--Low-flow data--Continued

SCIOTO RIVER BASIN

03229800 Walnut Creek near Ashville, Ohio

LOCATION:

Lat $39^{\circ} 40^{\prime} 56^{\prime \prime}$, long $82^{\circ} 58^{\prime} 30^{\prime \prime}$, Pickaway County, Hydrologic

Unit 05060001 , at bridge on U.S. Highway $23,2.5 \mathrm{mi}$ southeast

of South Bloomfield and $2.5 \mathrm{mi}$ southwest of Ashville.

DRAINAGE AREA: $\quad 285 \mathrm{mi}^{2}$.

TRIBUTARY TO: Scioto River.

DISCHARGE DATA USED:

Low-flow measurements 1952, 1954, 1961-1973 water years.

INDEX STATION: $\quad 03157500$ Hocking River at Enterprise, Ohio.

REMARKS: $\quad$ None.

SELECTED DISCHARGE CHARACTERISTICS: Minimum discharge observed: $11 \mathrm{ft}^{3} / \mathrm{s}$ October 1953.

Magnitude and frequency of low flow for indicated periods

\begin{tabular}{c|c|c|c}
\hline \multirow{2}{*}{ Period } & \multirow{2}{*}{$\begin{array}{c}\text { Number of } \\
\text { consecu- } \\
\text { tive days }\end{array}$} & \multicolumn{2}{|c}{$\begin{array}{c}\text { Discharge }\left(\mathrm{ft}^{3} / \mathrm{s}\right) \text { for } \\
\text { indicated recurrence } \\
\text { interval (years) }\end{array}$} \\
\cline { 3 - 4 } & & 2 & 10 \\
\hline Apr. - Mar. & 7 & 22 & 11 \\
May - Nov. & 30 & 31 & 15 \\
& 7 & 23 & 11 \\
June - Aug. & 30 & 32 & 15 \\
& 7 & 31 & 15 \\
Sept. - Nov. & 30 & 58 & 24 \\
& 30 & 25 & 12 \\
& & 37 & 15 \\
\hline
\end{tabular}

Duration of daily flow for indicated periods

\begin{tabular}{l|c|c|c|c|c}
\hline \multirow{2}{*}{ Period } & \multicolumn{5}{|c|}{$\begin{array}{c}\text { Discharge }(\mathrm{ft} / \mathrm{s} \text { ) which was equaled or } \\
\text { exceeded for indicated percent of time }\end{array}$} \\
\cline { 2 - 6 } & 98 & 95 & 90 & 85 & 80 \\
\hline Apr. - Mar. & 15 & 20 & 27 & 34 & 42 \\
May - Nov. & 13 & 17 & 22 & 27 & 32 \\
June - Aug. & 16 & 20 & 26 & 32 & 39 \\
Sept. - Nov. & 12 & 14 & 18 & 21 & 24 \\
\hline
\end{tabular}


Appendix 3.--Low-flow data--Continued

SCIOTO RIVER BASIN

03230200 Big Darby Creek at Plain City, Ohio

LOCATION:

Lat $40^{\circ} 06^{\prime} 25^{\prime \prime}$, long $83^{\circ} 15^{\prime} 20^{\prime \prime}$, Union County, Hydrologic

Unit 05060001 , at bridge on state Route 161 at Plain City.

DRAINAGE AREA: $\quad 151 \mathrm{mi}^{2}$.

TRIBUTARY TO: Scioto River.

DISCHARGE DATA USED: LOW-flow measurements 1950, 1961-1970 water years.

INDEX STATION: $\quad 03230500$ Big Darby Creek at Darbyville, Ohio.

REMARKS: None.

SELECTED DISCHARGE CHARACTERISTICS: Minimum discharge observed: $0.2 \mathrm{ft}^{3} / \mathrm{s}$ October 1963.

\begin{tabular}{l|c|c|c}
\multicolumn{4}{c}{ Magnitude and frequency of low flow for } \\
indicated periods \\
\hline & $\begin{array}{c}\text { Number of } \\
\text { consecu- } \\
\text { Period }\end{array}$ & $\begin{array}{c}\text { Discharge }\left(\mathrm{ft}^{3} / \mathrm{s}\right) \text { for } \\
\text { indicated recurrence } \\
\text { interval (years) }\end{array}$ \\
\cline { 3 - 4 } & & 2 & 10 \\
\hline Apr. - Mar. & 7 & 2.5 & 0.6 \\
& 30 & 3.5 & 1.0 \\
May - Nov. & 7 & 2.5 & 0.6 \\
& 30 & 3.5 & 1.0 \\
Sune - Aug. & 7 & 4.4 & 1.2 \\
& 30 & 8.3 & 2.3 \\
Sept. - Nov. & 7 & 2.7 & 0.6 \\
& 30 & 3.9 & 1.0 \\
\hline
\end{tabular}

Duration of daily flow for indicated periods

\begin{tabular}{l|c|c|c|c|c}
\hline \multirow{2}{*}{ Period } & \multicolumn{6}{|c}{$\begin{array}{c}\text { Discharge }\left(\mathrm{ft}^{3} / \mathrm{s} \text { ) which was equaled or }\right. \\
\text { exceeded for indicated percent of time }\end{array}$} \\
\cline { 2 - 6 } & 98 & 95 & 90 & 85 & 80 \\
\hline Apr. - Mar. & 1.1 & 2.0 & 3.2 & 4.4 & 5.7 \\
May - Nov. & 0.8 & 1.5 & 2.3 & 3.2 & 4.0 \\
June - Aug. & 1.4 & 2.2 & 3.4 & 4.6 & 5.7 \\
Sept. - Nov. & 0.6 & 0.9 & 1.5 & 2.2 & 2.7 \\
\hline
\end{tabular}


Appendix 3.--Low-flow data--Continued

SCIOTO RIVER BASIN

03230300 Little Darby Creek at Chuckery, Ohio

LOCATION:

Lat $40^{\circ} 06^{\prime} 40^{\prime \prime}$, long $83^{\circ} 23^{\prime} 30^{\prime \prime}$, Union County, Hydrologic Unit 05060001 , at bridge on State Route 161 at Chuckery, Ohio.

DRAINAGE AREA: $\quad 71.4 \mathrm{mi}^{2}$.

TRIBUTARY TO:

Big Darby Creek.

DISCHARGE DATA USED:

Low-flow measurements 1950, 1962-1971 water years.

INDEX STATION:

03230500 Big Darby Creek at Darbyville, Ohio

REMARKS:

None.

SELECTED DISCHARGE CHARACTERISTICS: Minimum discnarge observed: $1.7 \mathrm{ft}^{3} / \mathrm{s}$ October 1963 .

Magnitude and frequency of low flow for indicated periods

\begin{tabular}{c|c|c|c}
\hline \multirow{2}{*}{ Period } & \multirow{2}{*}{$\begin{array}{c}\text { Number of } \\
\text { consecu- } \\
\text { tive days }\end{array}$} & \multicolumn{2}{|c}{$\begin{array}{c}\text { Discharge (ft3/s) for } \\
\text { indicated recurrence } \\
\text { interval (years) }\end{array}$} \\
\cline { 3 - 4 } (ypr. - Mar. & 7 & 2 & 10 \\
\hline May - Nov. & 30 & 4.4 & 0.3 \\
& 7 & 2.4 & 0.6 \\
June - Aug. & 30 & 4.0 & 0.3 \\
& 7 & 5.7 & 0.6 \\
Sept. - Nov. & 70 & 15 & 2.2 \\
& 30 & 2.6 & 0.3 \\
& & 4.7 & 0.6 \\
\hline
\end{tabular}

Duration of daily flow for indicated periods

\begin{tabular}{l|c|c|c|c|c}
\hline \multirow{2}{*}{ Period } & \multicolumn{5}{|c}{$\begin{array}{c}\text { Discharge }(\mathrm{ft} 3 / \mathrm{s}) \text { which was equaled or } \\
\text { exceeded for indicated percent of time }\end{array}$} \\
\cline { 2 - 6 } & 98 & 95 & 90 & 85 & 80 \\
\hline Apr. - Mar. & 0.7 & 1.7 & 3.4 & 5.7 & 8.3 \\
May - Nov. & 0.4 & 1.2 & 2.2 & 3.4 & 5.0 \\
June - Aug. & 1.0 & 2.0 & 3.7 & 6.0 & 8.3 \\
Sept. - Nov. & 0.3 & 0.5 & 1.2 & 2.0 & 2.6 \\
\hline
\end{tabular}


LOCATION: Lat $39^{\circ} 42^{\prime} 02^{\prime \prime}$, long 83०06'37", Pickaway County, Hydrologic Unit 05060001, on right iank on downstream side of bridge on state Route $316,0.4 \mathrm{mi}$ northeast of Darbyville, $0.4 \mathrm{mi}$ upstream from Lizzard Run, and $3 \mathrm{mi}$ downstream from Greenbrier Creek.

DRAINAGE AREA: $\quad 534 \mathrm{mi}^{2}$.

TRIBUTARY TO: $\quad$ Scioto River.

DISCHARGE DATA USED: October 1921 to September 1935, October 1938 to september 1978.

REMARKS: $\quad$ None.

SELECTED DISCHARGE CHARACTERISTICS: Average discharge: $443 \mathrm{ft}^{3} / \mathrm{s}$ ( 54 years).

Minimum daily discharge: $1.4 \mathrm{ft} / \mathrm{s}$ September 1932.

Magnitude and frequency of low flow for indicated periods

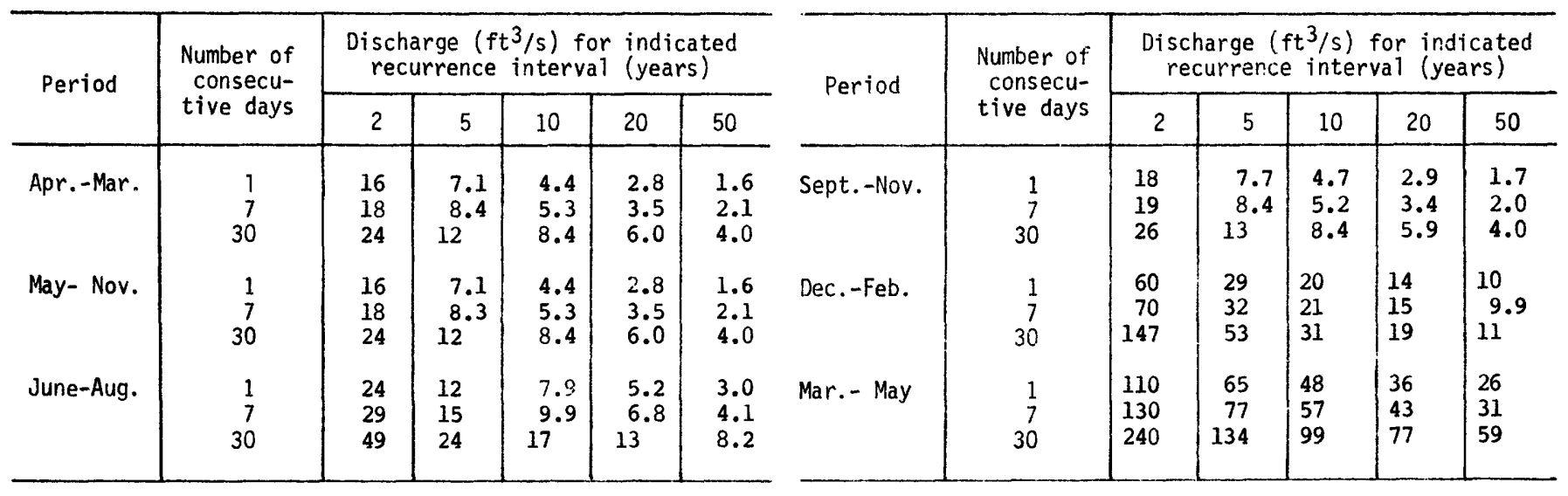

Duration of daily flow for indicated periods

\begin{tabular}{|c|c|c|c|c|c|c|c|c|c|c|c|c|c|}
\hline \multirow{2}{*}{ Period } & \multicolumn{13}{|c|}{ Discharge $\left(\mathrm{ft}^{3} / \mathrm{s}\right)$ which was equaled or exceeded for indicated percent of time } \\
\hline & 98 & 95 & 90 & 85 & 80 & 75 & 70 & 60 & 50 & 40 & 30 & 20 & 10 \\
\hline $\begin{array}{l}\text { Apr. - Mar. } \\
\text { May - Nov. } \\
\text { June - Aug. } \\
\text { Sept. - Nov. } \\
\text { Dec. - Feb. } \\
\text { Mar. - May }\end{array}$ & $\begin{array}{l}9.3 \\
7.0 \\
11 \\
5.1 \\
19 \\
60\end{array}$ & $\begin{array}{l}15 \\
12 \\
16 \\
7.9 \\
24 \\
86\end{array}$ & $\begin{array}{r}22 \\
17 \\
23 \\
12 \\
34 \\
120\end{array}$ & $\begin{array}{r}29 \\
22 \\
30 \\
16 \\
43 \\
140\end{array}$ & $\begin{array}{r}36 \\
27 \\
36 \\
19 \\
56 \\
170\end{array}$ & $\begin{array}{r}44 \\
32 \\
41 \\
21 \\
77 \\
190\end{array}$ & $\begin{array}{r}55 \\
37 \\
48 \\
24 \\
100 \\
220\end{array}$ & $\begin{array}{r}89 \\
51 \\
65 \\
31 \\
180 \\
300\end{array}$ & $\begin{array}{r}140 \\
72 \\
87 \\
39 \\
260 \\
380\end{array}$ & $\begin{array}{r}210 \\
100 \\
120 \\
50 \\
380 \\
510\end{array}$ & $\begin{array}{r}330 \\
150 \\
160 \\
72 \\
550 \\
690\end{array}$ & $\begin{array}{l}540 \\
240 \\
240 \\
110 \\
860 \\
990\end{array}$ & $\begin{array}{r}1100 \\
460 \\
450 \\
220 \\
1600 \\
1700\end{array}$ \\
\hline
\end{tabular}


Appendix 3.--Low-flow data--Continued

SCIOTO RIVER BASIN

03230600 Hominy Creek at Circleville, Ohio

LOCATION:

Lat $39^{\circ} 36^{\prime} 25^{\prime \prime}$, long $82^{\circ} 55^{\prime} 25^{\prime \prime}$, Pickaway County, Hydrologic Unit 05060002 , in sec. 29, T.11 N., R.21 W., on left upstream

wingwall of private farm bridge across creek. Gage is reached

by driving southeast $0.4 \mathrm{mi}$ on state Route 56 from railroad crossing at east edge of Circleville and turning left on farm road to bridge.

DRAINAGE AREA:

$5.66 \mathrm{mi}^{2}$.

TRIBUTARY TO:

Hargus Creek.

DISCHARGE DATA USED: LOW-flow measurements 1962-1973 water years.

INDEX STATION: $\quad 03157500$ Hocking River at Enterprise, Ohio.

REMARKS: None.

SELECTED DISCHARGE CHARACTERISTICS: Minimum discharge observed: $0.2 \mathrm{ft}^{3} / \mathrm{s}$ August 1964 .

Magnitude and frequency of low flow for indicated periods

\begin{tabular}{c|c|c|c}
\hline \multirow{2}{*}{ Period } & \multirow{2}{*}{$\begin{array}{c}\text { Number of } \\
\text { consecu- } \\
\text { tive days }\end{array}$} & \multicolumn{2}{|c}{$\begin{array}{c}\text { Discharge }\left(\mathrm{ft}^{3} / \mathrm{s}\right) \text { for } \\
\text { indicated recurrence } \\
\text { interval (years) }\end{array}$} \\
\cline { 3 - 4 } Apr. - Mar. & 7 & 2 & 10 \\
\hline May - Nov. & 30 & 0.3 & 0.1 \\
& 7 & 0.4 & 0.2 \\
June - Aug. & 30 & 0.4 & 0.1 \\
& 7 & 0.4 & 0.2 \\
Sept. - Nov. & 30 & 0.6 & 0.2 \\
& 7 & 0.3 & 0.2 \\
& 30 & 0.4 & 0.2 \\
\hline
\end{tabular}

Duration of daily flow for indicated periods

\begin{tabular}{|c|c|c|c|c|c|}
\hline \multirow{2}{*}{ Period } & \multicolumn{5}{|c|}{$\begin{array}{l}\text { Discharge }\left(\mathrm{ft}^{3} / \mathrm{s}\right) \text { which was equaled or } \\
\text { exceeded for indicated percent of time }\end{array}$} \\
\hline & 98 & 95 & 90 & 85 & 80 \\
\hline $\begin{array}{l}\text { Apr. - Mar. } \\
\text { May - Nov. } \\
\text { June - Aug. } \\
\text { Sept. - Nov. }\end{array}$ & $\begin{array}{l}0.2 \\
0.2 \\
0.2 \\
0.2\end{array}$ & $\begin{array}{l}0.2 \\
0.2 \\
0.2 \\
0.2\end{array}$ & $\begin{array}{l}0.3 \\
0.3 \\
0.3 \\
0.2\end{array}$ & $\begin{array}{l}0.4 \\
0.3 \\
0.4 \\
0.3\end{array}$ & $\begin{array}{l}0.5 \\
0.4 \\
0.4 \\
0.3\end{array}$ \\
\hline
\end{tabular}


SCIOTO RIVER BASTIN

03230800 Deer Creek at Mount Sterling, Ohio

LOCATION:

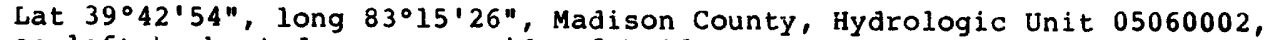
on left bank at downstream side of bridge on State Route $56,0.2 \mathrm{mi}$ downstream from unnamed right bank tributary, $0.6 \mathrm{mi}$ southeast of Mount Sterling, and $4.9 \mathrm{mi}$ upstream from Duffs Fork.

DRAINAGE AREA:

$228 \mathrm{mi}^{2}$

TRIBUTARY TO:

Scioto River.

DISCHARGE DATA USED:

REMARKS :

October 1966 to September 1978.

None.

SELECTED DISCHARGE CHARACTERISTICS: Average discharge: $224 \mathrm{ft}^{3} / \mathrm{s}$ (12 years). Minimum daily discharge: $5.4 \mathrm{ft}^{3} / \mathrm{s}$ July 1977.

Magnitude and frequency of low flow for indicated periods

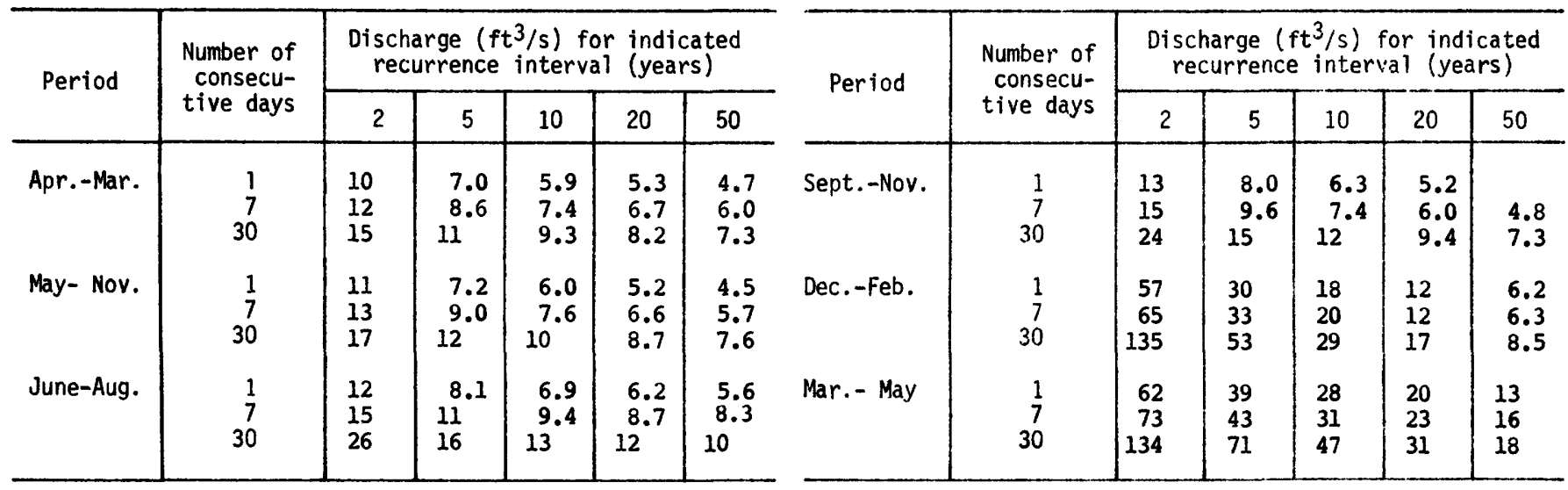

Duration of daily flow for indicated periods

\begin{tabular}{|c|c|c|c|c|c|c|c|c|c|c|c|c|c|}
\hline \multirow{2}{*}{ Period } & \multicolumn{13}{|c|}{ Discharge $\left(\mathrm{ft}^{3} / \mathrm{s}\right)$ which was equaled or exceeded for indicated percent of time } \\
\hline & 98 & 95 & 90 & 85 & 80 & 75 & 70 & 60 & 50 & 40 & 30 & 20 & 10 \\
\hline $\begin{array}{l}\text { Apr. - Mar. } \\
\text { May - Nov. } \\
\text { June - Aug. } \\
\text { Sept. - Nov. } \\
\text { Dec. - Feb. } \\
\text { Mar. - May }\end{array}$ & $\begin{array}{c}9.7 \\
9.1 \\
10 \\
8.4 \\
9.9 \\
34\end{array}$ & $\begin{array}{l}13 \\
12 \\
13 \\
10 \\
14 \\
47\end{array}$ & $\begin{array}{l}18 \\
15 \\
16 \\
13 \\
43 \\
62\end{array}$ & $\begin{array}{l}23 \\
18 \\
19 \\
15 \\
54 \\
78\end{array}$ & $\begin{array}{l}29 \\
21 \\
21 \\
17 \\
67 \\
94\end{array}$ & $\begin{array}{r}37 \\
24 \\
24 \\
20 \\
81 \\
110\end{array}$ & $\begin{array}{r}44 \\
28 \\
28 \\
230 \\
97 \\
120\end{array}$ & $\begin{array}{r}62 \\
38 \\
37 \\
29 \\
140 \\
150\end{array}$ & $\begin{array}{r}91 \\
49 \\
49 \\
37 \\
180 \\
180\end{array}$ & $\begin{array}{r}130 \\
66 \\
66 \\
45 \\
240 \\
240\end{array}$ & $\begin{array}{r}180 \\
93 \\
91 \\
57 \\
310 \\
330\end{array}$ & $\begin{array}{r}290 \\
130 \\
130 \\
81 \\
440 \\
470\end{array}$ & $\begin{array}{l}530 \\
250 \\
240 \\
130 \\
740 \\
800\end{array}$ \\
\hline
\end{tabular}


Appendix 3.--Low-flow data--Continued

SCIOTO RIVER BASIN

03231300 Kinnikinnick Creek near Kinnikinnick, Ohio

LOCATION:

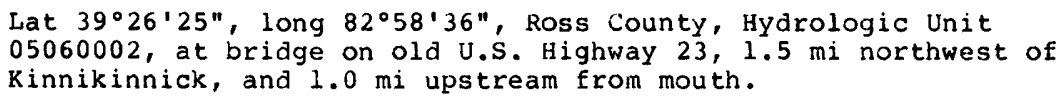

DRAINAGE AREA: $\quad 36.2 \mathrm{mi}^{2}$.

TRIBUTARY TO: Scioto River.

DISCHARGE DATA USED: LOW-flow measurements 1953, 1958, 1961-1973 water years.

INDEX STATION: $\quad 03157000$ Clear Creek near Rockbridge, Ohio.

REMARKS: $\quad$ None.

SELECTED DISCHARGE CHARACTERISTICS: Minimum discharge observed: $5.5 \mathrm{ft}^{3} / \mathrm{s}^{\mathrm{oct}}$ ober 1953 .

\begin{tabular}{l|c|c|c}
\multicolumn{3}{c}{$\begin{array}{c}\text { Magnitude and frequency of low flow for } \\
\text { indicated periods }\end{array}$} \\
\hline Period & $\begin{array}{r}\text { Number of } \\
\text { consecu- } \\
\text { tive days }\end{array}$ & $\begin{array}{c}\text { Discharge (ft } 3 / 5) \text { for } \\
\text { indicated recurrence } \\
\text { interval (years) }\end{array}$ \\
\cline { 2 - 4 } & & 2 & 10 \\
\hline Apr. - Mar. & 7 & 7.9 & 5.8 \\
May - Nov. & 30 & 8.8 & 7.0 \\
& 7 & 7.9 & 5.8 \\
June - Aug. & 30 & 8.8 & 7.0 \\
& 7 & 8.8 & 6.2 \\
Sept. - Nov. & 30 & 11 & 7.9 \\
& 7 & 8.3 & 6.4 \\
\hline
\end{tabular}

Duration of daily flow for indicated periods

\begin{tabular}{l|c|c|c|c|c}
\hline \multirow{2}{*}{ Period } & \multicolumn{5}{|c|}{$\begin{array}{l}\text { Discharge ( } \mathrm{ft}^{3} / \mathrm{s} \text { ) which was equaled or } \\
\text { exceeded for indicated percent of time }\end{array}$} \\
\cline { 2 - 6 } & 98 & 95 & 90 & 85 & 80 \\
\hline Apr. - Mar. & 7.0 & 7.9 & 9.3 & 10 & 11 \\
May - Nov. & 6.5 & 7.4 & 8.3 & 8.8 & 9.7 \\
June - Aug. & 6.5 & 7.4 & 8.3 & 9.3 & 9.7 \\
Sept. - Nov. & 6.5 & 7.0 & 7.9 & 8.3 & 8.8 \\
\hline
\end{tabular}


SCIOTO RIVER BASIN

03231500 Scioto River at Chillicothe, Ohio

LOCATION:

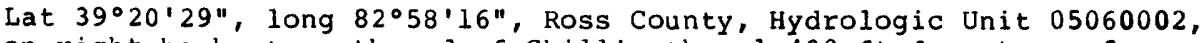
on right bank at north end of Chillicothe, $1,400 \mathrm{ft}$ downstream from Bridge Street bridge, $7.4 \mathrm{mi}$ upstream from Paint Creek, and $15.4 \mathrm{mi}$ downstream from Deer Creek.

DRAINAGE AREA: $\quad 3,849 \mathrm{mi}^{2}$.

TRIBUTARY TO: $\quad$ Ohio River.

DISCHARGE DATA USED: April 1921 to September 1978.

REMARKS :

Flow regulated by $\mathrm{six}$ reservoirs $36 \mathrm{mi}$ to $91 \mathrm{mi}$ upstream from station.

SELECTED DISCHARGE CHARACTERISTICS: Average discharge: $3,360 \mathrm{ft}^{3} / \mathrm{s}(57$ years). Minimum daily discharge: $166 \mathrm{ft}^{3} / \mathrm{s}$ September 1944.

Magnitude and frequency of low flow for indicated periods

\begin{tabular}{c|c|c|c|c|c|c}
\hline \multirow{2}{*}{ Period } & \multirow{2}{*}{$\begin{array}{c}\text { Number of } \\
\text { consecu- } \\
\text { tive days }\end{array}$} & \multicolumn{4}{|c|}{$\begin{array}{c}\text { Discharge }\left(\mathrm{ft}^{3} / \mathrm{s} \text { ) for indicated }\right. \\
\text { recurrence interval (years) }\end{array}$} \\
\cline { 3 - 7 } & & 2 & 5 & 10 & 20 & 50 \\
\hline \multirow{2}{*}{ Apr.-Mar. } & 1 & 291 & 223 & 196 & 177 & 159 \\
& 7 & 311 & 235 & 204 & 183 & 163 \\
& 30 & 359 & 265 & 230 & 207 & 186 \\
& 1 & 293 & 224 & 196 & 177 & 158 \\
May- Nov. & 7 & 314 & 235 & 204 & 183 & 162 \\
& 30 & 363 & 265 & 230 & 206 & 184 \\
& 1 & 381 & 277 & 236 & 208 & 181 \\
& 7 & 426 & 304 & 257 & 224 & 194 \\
& 30 & 626 & 406 & 341 & 302 & 270 \\
\hline
\end{tabular}

\begin{tabular}{|c|c|c|c|c|c|c|}
\hline \multirow{2}{*}{ Period } & \multirow{2}{*}{$\begin{array}{l}\text { Number of } \\
\text { consecu- } \\
\text { tive days }\end{array}$} & \multicolumn{5}{|c|}{$\begin{array}{l}\text { Discharge }\left(\mathrm{ft}^{3} / \mathrm{s}\right) \text { for indicated } \\
\text { recurrence interval (years) }\end{array}$} \\
\hline & & 2 & 5 & 10 & 20 & 50 \\
\hline Sept.-Nov. & $\begin{array}{r}1 \\
7 \\
30\end{array}$ & $\begin{array}{l}303 \\
313 \\
356\end{array}$ & $\begin{array}{l}223 \\
230 \\
260\end{array}$ & $\begin{array}{l}194 \\
204 \\
238\end{array}$ & $\begin{array}{l}175 \\
188 \\
228\end{array}$ & $\begin{array}{l}157 \\
175 \\
216\end{array}$ \\
\hline Dec. -Feb. & $\begin{array}{r}1 \\
7 \\
30\end{array}$ & $\begin{array}{r}576 \\
644 \\
1260\end{array}$ & $\begin{array}{l}333 \\
359 \\
555\end{array}$ & $\begin{array}{l}255 \\
271 \\
362\end{array}$ & $\begin{array}{l}207 \\
217 \\
254\end{array}$ & $\begin{array}{l}165 \\
171 \\
170\end{array}$ \\
\hline Mar. - May & $\begin{array}{r}1 \\
7 \\
30\end{array}$ & $\begin{array}{l}991 \\
1140 \\
1950\end{array}$ & $\begin{array}{r}650 \\
741 \\
114 C\end{array}$ & $\begin{array}{l}508 \\
584 \\
861\end{array}$ & $\begin{array}{l}409 \\
477 \\
684\end{array}$ & $\begin{array}{l}315 \\
377 \\
529\end{array}$ \\
\hline
\end{tabular}

Duration of daily flow for indicated periods

\begin{tabular}{|c|c|c|c|c|c|c|c|c|c|c|c|c|c|}
\hline \multirow{2}{*}{ Period } & \multicolumn{13}{|c|}{ Discharge $\left(\mathrm{ft}^{3} / \mathrm{s}\right)$ which was equaled or exceeded for indicated percent of time } \\
\hline & 98 & 95 & 90 & 85 & 80 & 75 & 70 & 60 & 50 & 40 & 30 & 20 & 10 \\
\hline $\begin{array}{l}\text { Apr. - Mar. } \\
\text { May - Nov. } \\
\text { June - Aug. } \\
\text { Sept. - Nov. } \\
\text { Dec. - Feb. } \\
\text { Mar. - May }\end{array}$ & $\begin{array}{l}220 \\
210 \\
250 \\
190 \\
250 \\
600\end{array}$ & $\begin{array}{l}270 \\
250 \\
310 \\
220 \\
310 \\
810\end{array}$ & $\begin{array}{r}330 \\
290 \\
380 \\
250 \\
380 \\
1000\end{array}$ & $\begin{array}{r}390 \\
340 \\
430 \\
270 \\
480 \\
1200\end{array}$ & $\begin{array}{r}450 \\
380 \\
480 \\
300 \\
610 \\
1400\end{array}$ & $\begin{array}{r}530 \\
420 \\
540 \\
320 \\
780 \\
1700\end{array}$ & $\begin{array}{r}640 \\
470 \\
600 \\
350 \\
960 \\
1900\end{array}$ & $\begin{array}{r}910 \\
610 \\
730 \\
400 \\
1500 \\
2400\end{array}$ & $\begin{array}{r}1300 \\
780 \\
910 \\
470 \\
2100 \\
3100\end{array}$ & $\begin{array}{r}1900 \\
1000 \\
1200 \\
590 \\
3000 \\
4100\end{array}$ & $\begin{array}{r}2800 \\
1400 \\
1600 \\
770 \\
4300 \\
5600\end{array}$ & $\begin{array}{l}4400 \\
2100 \\
2300 \\
1100 \\
6800 \\
8300\end{array}$ & $\begin{array}{r}8600 \\
3900 \\
4000 \\
2000 \\
13000 \\
14000\end{array}$ \\
\hline
\end{tabular}


LOCATION:

Lat $39^{\circ} 13^{\prime} 06^{\prime \prime}$, long $83^{\circ} 23^{\prime} 08^{\prime \prime}$, Highland County, Hydrologic Unit 05060003, on left bank at downstream side of highway bridge, $1.1 \mathrm{mi}$ north of Barretts Mills, $2 \mathrm{mi}$ east of Rainsboro, $2.8 \mathrm{mi}$ upstream from mouth, and $6 \mathrm{mi}$ downstream from Rocky Fork Lake.

DRAINAGE AREA: $\quad 140 \mathrm{mi}^{2}$.

TRIBUTARY T0: Paint Creek.

DISCHARGE DATA USED: October 1952 to September 1978.

REMARKS:

Some diurnal fluctuation caused by mill $6 \mathrm{mi}$ upstream from station. Flow regulated by Rocky Fork Lake 6 mi upstream, since 1952, capacity, 34,100 acre-ft.

SELECTED DISCHARGE CHARACTERISTICS: Average discharge: $149 \mathrm{ft}^{3} / \mathrm{s}$ (26 years). Minimum daily discharge: $0.5 \mathrm{ft}^{3} / \mathrm{s}$ October 1964.

Magnitude and frequency of low flow for indicated periods

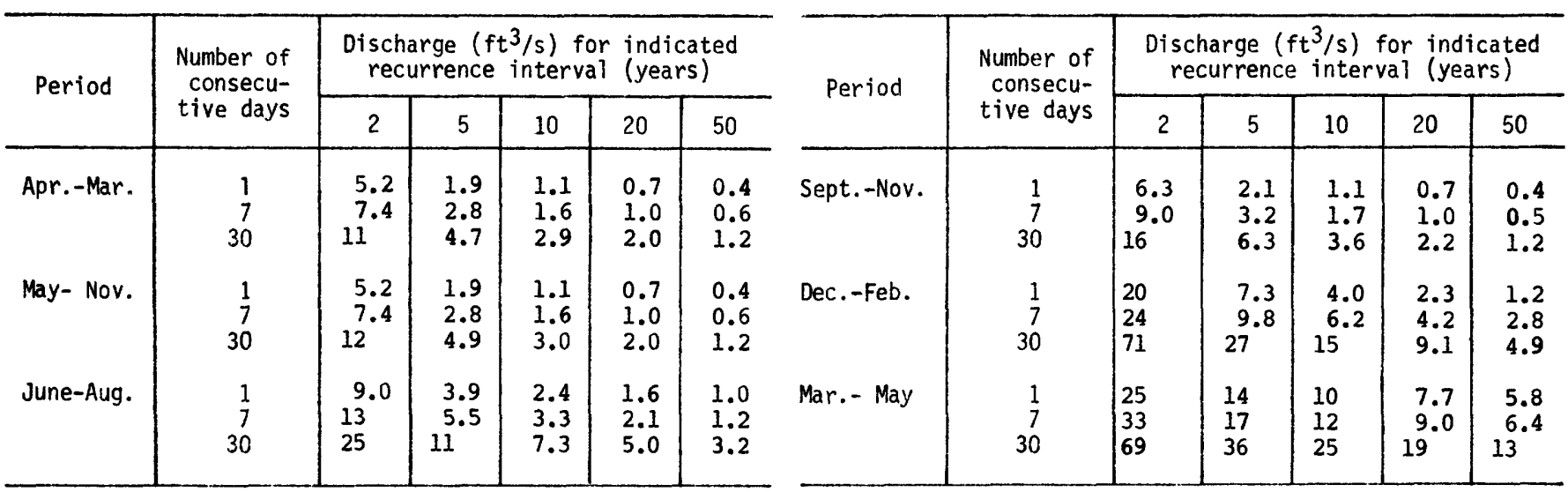

Duration of daily flow for indicated periods

\begin{tabular}{l|c|c|c|c|c|c|c|c|c|r|r|r|r}
\hline \multirow{2}{*}{ Period } & \multicolumn{8}{|c|}{ Discharge $\left(\mathrm{ft}^{3} / \mathrm{s}\right)$ which was equaled or exceeded for indicated percent of time } \\
\cline { 2 - 12 } & 98 & 95 & 90 & 85 & 80 & 75 & 70 & 60 & 50 & 40 & 30 & 20 & 10 \\
\hline Apr. - Mar. & 2.9 & 4.8 & 9.2 & 14 & 20 & 23 & 27 & 39 & 61 & 91 & 130 & 210 & 370 \\
May - Nov. & 2.1 & 3.5 & 5.7 & 9.0 & 14 & 19 & 22 & 27 & 34 & 49 & 73 & 110 & 210 \\
June - Aug. & 2.9 & 4.8 & 7.9 & 13 & 18 & 20 & 22 & 27 & 33 & 45 & 63 & 95 & 170 \\
Sept.- Nov. & 1.6 & 2.7 & 3.8 & 5.1 & 7.1 & 10 & 14 & 22 & 27 & 33 & 51 & 90 & 200 \\
Dec. - Feb. & 6.0 & 8.8 & 12 & 17 & 25 & 32 & 47 & 74 & 110 & 160 & 210 & 300 & 450 \\
Mar. - May & 13 & 18 & 30 & 40 & 49 & 60 & 73 & 98 & 130 & 160 & 220 & 320 & 540 \\
\hline
\end{tabular}


LOCATION:

Lat $39^{\circ} 12^{\prime} 44^{\prime \prime}$, long $82^{\circ} 51^{\prime} 50^{\prime \prime}$, in sec. 6, T.7 N., R. 20 W., Ross

County, Hydrologic Unit 05060002 , on left bank at downstream side of highway bridge, $0.8 \mathrm{mi}$ downstream from walnut creek, $1.2 \mathrm{mi}$ north of Higby, $3 \mathrm{mi}$ northwest of Richmondale and $5.0 \mathrm{mi}$ upstream from Salt Creek.

DRAINAGE AREA: $\quad 5,131 \mathrm{mi}^{2}$.

TRIBITARY TO: Ohio River.

DISCHARGE DATA USED: October 1930 to september 1978.

REMARKS:

Flow slightly regulated by 7 reservoirs $45 \mathrm{mi}$ to $105 \mathrm{mi}$ upstream from station and since 1952 by Rocky Fork Lake 31 mi upstream.

SELECTED DISCHARGE CHARACTERISTICS: Average discharge: $4,490 \mathrm{ft}^{3} / \mathrm{s}$ (48 years).

Minimum daily discharge: $244 \mathrm{ft}^{3} / \mathrm{s}$ October 1930.

Magnitude and frequency of low flow for indicated periods

\begin{tabular}{|c|c|c|c|c|c|c|c|c|c|c|c|c|c|}
\hline \multirow{2}{*}{ Period } & \multirow{2}{*}{$\begin{array}{l}\text { Number of } \\
\text { consecu- } \\
\text { tive days }\end{array}$} & \multicolumn{5}{|c|}{$\begin{array}{l}\text { Discharge }\left(\mathrm{ft}^{3} / \mathrm{s}\right) \text { for indicated } \\
\text { recurrence interval (years) }\end{array}$} & \multirow{2}{*}{ Period } & \multirow{2}{*}{$\begin{array}{l}\text { Number of } \\
\text { consecu- } \\
\text { tive days }\end{array}$} & \multicolumn{5}{|c|}{$\begin{array}{l}\text { Discharge }\left(\mathrm{ft}^{3} / \mathrm{s}\right) \text { for indicated } \\
\text { recurrence interva1 (years) }\end{array}$} \\
\hline & & 2 & 5 & 10 & 20 & 50 & & & 2 & 5 & 10 & 20 & 50 \\
\hline Apr.-Mar. & $\begin{array}{r}1 \\
7 \\
30\end{array}$ & $\begin{array}{l}422 \\
446 \\
518\end{array}$ & $\begin{array}{l}323 \\
339 \\
377\end{array}$ & $\begin{array}{l}282 \\
296 \\
323\end{array}$ & $\begin{array}{l}253 \\
266 \\
287\end{array}$ & $\begin{array}{l}225 \\
237 \\
253\end{array}$ & Sept.-Nov. & $\begin{array}{r}1 \\
7 \\
30\end{array}$ & $\begin{array}{l}451 \\
475 \\
558\end{array}$ & $\begin{array}{l}337 \\
348 \\
377\end{array}$ & $\begin{array}{l}290 \\
299 \\
315\end{array}$ & $\begin{array}{l}258 \\
266 \\
276\end{array}$ & $\begin{array}{l}226 \\
235 \\
241\end{array}$ \\
\hline June-Aug. & $\begin{array}{r}1 \\
7 \\
30\end{array}$ & $\begin{array}{l}572 \\
638 \\
953\end{array}$ & $\begin{array}{l}419 \\
458 \\
623\end{array}$ & $\begin{array}{l}359 \\
390 \\
523\end{array}$ & $\begin{array}{l}318 \\
343 \\
464\end{array}$ & $\begin{array}{l}278 \\
299 \\
414\end{array}$ & Mar. - May & $\begin{array}{r}1 \\
7 \\
30\end{array}$ & $\begin{array}{l}1490 \\
1700 \\
2870\end{array}$ & $\begin{array}{r}981 \\
1130 \\
1740\end{array}$ & $\begin{array}{r}756 \\
895 \\
1350\end{array}$ & $\begin{array}{r}596 \\
731 \\
1090\end{array}$ & $\begin{array}{l}445 \\
576 \\
867\end{array}$ \\
\hline
\end{tabular}

Duration of daily flow for indicated periods

\begin{tabular}{l|r|r|r|r|r|r|r|r|r|r|r|r|r}
\hline \multirow{2}{*}{ Period } & \multicolumn{8}{|c|}{ Discharge $\left(\mathrm{ft}^{3} / \mathrm{s}\right)$ which was equaled or exceeded for indicated percent of time } \\
\cline { 2 - 12 } & 98 & 95 & 90 & 85 & 80 & 75 & 70 & 60 & 50 & 40 & 30 & 20 & 10 \\
\hline Apr. - Mar. & 320 & 390 & 480 & 560 & 660 & 800 & 960 & 1300 & 1900 & 2700 & 3900 & 6200 & 11000 \\
May - Nov. & 300 & 350 & 440 & 490 & 550 & 620 & 700 & 910 & 1200 & 1600 & 2100 & 3000 & 5200 \\
June - Aug. & 430 & 490 & 580 & 660 & 740 & 830 & 930 & 1100 & 1400 & 1800 & 2200 & 3200 & 5500 \\
Sept. - Nov. & 280 & 310 & 340 & 390 & 430 & 460 & 490 & 570 & 680 & 860 & 1100 & 1600 & 2600 \\
Dec. - Feb. & 340 & 400 & 490 & 600 & 790 & 1100 & 1300 & 1900 & 2900 & 4000 & 6000 & 9000 & 17000 \\
Mar. - May & 900 & 1200 & 1600 & 1900 & 2200 & 2500 & 2800 & 3600 & 4700 & 6100 & 8100 & 11000 & 18000 \\
\hline
\end{tabular}




\author{
SCIOTO RIVER BASIN \\ 03235000 Salt Creek at Tarlton, Ohio
}

LOCATION:

Lat $39^{\circ} 33^{\prime} 20^{\prime \prime}$, long $82^{\circ} 46^{\prime} 50^{\prime \prime}$, in NWl/4 sec. 3, T.11 N., R.20 W.,

Pickaway County, Hydrologic Unit 05060002 , on left bank at bridge

on State koute 159 in Tarlton, and $5.7 \mathrm{mi}$ upstream from Plum Run.

DRAINAGE AREA: $\quad 11.5 \mathrm{mi}^{2}$.

TRIBUTARY TO: Scioto River.

DISCHARGE DATA USED: October 1946 to September 1961.

REMARKS: $\quad$ None.

SELECTED DISCHARGE CHARACTERISTICS: Average discharge: $10.4 \mathrm{ft}^{3} / \mathrm{s}$ (15 years).

Minimum daily discharge: No flow at times each year.

Magnitude and frequency of low flow for indicated periods

\begin{tabular}{|c|c|c|c|c|c|c|c|c|c|c|c|c|c|}
\hline \multirow{2}{*}{ Period } & \multirow{2}{*}{$\begin{array}{l}\text { Number of } \\
\text { consecu- } \\
\text { tive days }\end{array}$} & \multicolumn{5}{|c|}{$\begin{array}{l}\text { Discharge }\left(\mathrm{ft}^{3} / \mathrm{s}\right) \text { for indicated } \\
\text { recurrence interval (years) }\end{array}$} & \multirow{2}{*}{ Period } & \multirow{2}{*}{$\begin{array}{l}\text { Number of } \\
\text { consecu- } \\
\text { tive days }\end{array}$} & \multicolumn{5}{|c|}{$\begin{array}{l}\left.\text { Discharge ( } \mathrm{ft}^{3} / \mathrm{s}\right) \text { for indicated } \\
\text { recurrence interval (years) }\end{array}$} \\
\hline & & 2 & 5 & 10 & 20 & 50 & & & 2 & 5 & 10 & 20 & 50 \\
\hline Apr.-Mar. & $\begin{array}{r}1 \\
7 \\
30\end{array}$ & $\begin{array}{l}0 \\
0 \\
0.1\end{array}$ & $\begin{array}{l}0 \\
0 \\
0\end{array}$ & $\begin{array}{l}0 \\
0 \\
0\end{array}$ & $\begin{array}{l}0 \\
0 \\
0\end{array}$ & $\begin{array}{l}0 \\
0 \\
0\end{array}$ & Sept.-Nov. & $\begin{array}{r}1 \\
7 \\
30\end{array}$ & $\begin{array}{l}0.1 \\
0.1 \\
0.2\end{array}$ & $\begin{array}{l}0 \\
0 \\
0\end{array}$ & $\begin{array}{l}0 \\
0 \\
0\end{array}$ & $\begin{array}{l}0 \\
0 \\
0\end{array}$ & $\begin{array}{l}0 \\
0 \\
0\end{array}$ \\
\hline June-Aug. & $\begin{array}{r}1 \\
7 \\
30\end{array}$ & $\begin{array}{l}0 \\
0.1 \\
0.4\end{array}$ & $\begin{array}{l}0 \\
0 \\
0.1\end{array}$ & $\begin{array}{l}0 \\
0 \\
0.1\end{array}$ & $\begin{array}{l}0 \\
0 \\
0\end{array}$ & $\begin{array}{l}0 \\
0 \\
0\end{array}$ & Mar. - May & $\begin{array}{r}1 \\
7 \\
30\end{array}$ & $\begin{array}{l}1.1 \\
1.7 \\
4.3\end{array}$ & $\begin{array}{l}0.8 \\
1.2 \\
2.5\end{array}$ & $\begin{array}{l}0.6 \\
1.0 \\
1.9\end{array}$ & $\begin{array}{l}0.5 \\
0.9 \\
1.5\end{array}$ & $\begin{array}{l}0.4 \\
0.8 \\
1.2\end{array}$ \\
\hline
\end{tabular}

Duration of daily flow for indicated periods

\begin{tabular}{l|l|l|l|l|l|l|l|l|l|l|r|r|r|r}
\hline \multirow{2}{*}{ Period } & \multicolumn{8}{|c|}{ Discharge $\left(\mathrm{ft}^{3} / \mathrm{s}\right)$ which was equaled or exceeded for indicated percent of time } \\
\cline { 2 - 12 } & 98 & 95 & 90 & 85 & 80 & 75 & 70 & 60 & 50 & 40 & 30 & 20 & 10 \\
\hline Apr. - Mar. & 0 & 0.1 & 0.2 & 0.2 & 0.3 & 0.5 & 0.6 & 1.3 & 2.2 & 3.9 & 6.4 & 11 & 22 \\
May - Nov. & 0 & 0.1 & 0.1 & 0.1 & 0.2 & 0.2 & 0.3 & 0.4 & 0.7 & 1.2 & 1.8 & 3.0 & 7.0 \\
June - Aug. & 0 & 0.1 & 0.1 & 0.1 & 0.2 & 0.2 & 0.3 & 0.4 & 0.6 & 0.9 & 1.5 & 2.8 & 8.9 \\
Sept. - Nov. & 0 & 0 & 0.1 & 0.1 & 0.1 & 0.2 & 0.2 & 0.3 & 0.4 & 0.7 & 1.1 & 1.8 & 3.3 \\
Dec. - Feb. & 0.3 & 0.5 & 0.9 & 1.3 & 1.7 & 2.3 & 2.9 & 4.6 & 6.9 & 9.6 & 14 & 21 & 46 \\
Mar. - May & 1.1 & 1.5 & 2.1 & 2.6 & 3.1 & 3.6 & 4.1 & 5.2 & 6.6 & 8.7 & 12 & 17 & 32 \\
\hline
\end{tabular}




\section{SCIOTO RIVER BASIN}

03235500 Tar Hollow Creek at Tar Hollow State Park, Ohio

LOCATION:

Lat $39^{\circ} 23^{\prime} 22^{\prime \prime}$, long $82^{\circ} 45^{\prime} 03^{\prime \prime}$, in NEl/4 sec. 36, T.10 N., R. 20 W.

Ross County, Hydrologic Unit 05060002, in Tar Hollow State Park, on

left bank $2.0 \mathrm{mi}$ upstream from mouth and $5.2 \mathrm{mi}$ south of Adelphi.

DRAINAGE AREA: $\quad 1.35 \mathrm{mi}^{2}$.

TRIBUTARY TO: Pike Run.

DISCHARGE DATA USED: October 1946 to September 1978.

REMARKS:

None.

SELECTED DISCHARGE CHARACTERISTICS: Average discharge: $1.24 \mathrm{ft}^{3} / \mathrm{s}$ ( 32 years).

Minimum daily discharge: No flow many days each year.

Magnitude and frequency of low flow for indicated periods

\begin{tabular}{|c|c|c|c|c|c|c|}
\hline \multirow{2}{*}{ Period } & \multirow{2}{*}{$\begin{array}{l}\text { Number of } \\
\text { consecu- } \\
\text { tive days }\end{array}$} & \multicolumn{5}{|c|}{$\begin{array}{l}\text { Discharge }\left(\mathrm{ft}^{3} / \mathrm{s}\right) \text { for indicated } \\
\text { recurrence interval (years) }\end{array}$} \\
\hline & & 2 & 5 & 10 & 20 & 50 \\
\hline Apr.-Mar. & $\begin{array}{r}1 \\
7 \\
30\end{array}$ & $\begin{array}{l}0 \\
0 \\
0\end{array}$ & $\begin{array}{l}0 \\
0 \\
0\end{array}$ & $\begin{array}{l}0 \\
0 \\
0\end{array}$ & $\begin{array}{l}0 \\
0 \\
0\end{array}$ & $\begin{array}{l}0 \\
0 \\
0\end{array}$ \\
\hline May-Nov. & $\begin{array}{r}1 \\
7 \\
30\end{array}$ & $\begin{array}{l}0 \\
0 \\
0\end{array}$ & $\begin{array}{l}0 \\
0 \\
0\end{array}$ & $\begin{array}{l}0 \\
0 \\
0\end{array}$ & $\begin{array}{l}0 \\
0 \\
0\end{array}$ & $\begin{array}{l}0 \\
0 \\
0\end{array}$ \\
\hline June-Aug. & $\begin{array}{r}1 \\
7 \\
30\end{array}$ & $\begin{array}{l}0 \\
0 \\
0\end{array}$ & $\begin{array}{l}0 \\
0 \\
0\end{array}$ & $\begin{array}{l}0 \\
0 \\
0\end{array}$ & $\begin{array}{l}0 \\
0 \\
0\end{array}$ & $\begin{array}{l}0 \\
0 \\
0\end{array}$ \\
\hline
\end{tabular}

\begin{tabular}{|c|c|c|c|c|c|c|}
\hline \multirow{2}{*}{ Period } & \multirow{2}{*}{$\begin{array}{l}\text { Number of } \\
\text { consecu- } \\
\text { tive days }\end{array}$} & \multicolumn{5}{|c|}{$\begin{array}{l}\text { Discharge }\left(\mathrm{ft}^{3} / \mathrm{s}\right) \text { for indicated } \\
\text { recurrence interval (years) }\end{array}$} \\
\hline & & 2 & 5 & 10 & 20 & 50 \\
\hline Sept.-Nov. & $\begin{array}{r}1 \\
7 \\
30\end{array}$ & $\begin{array}{l}0 \\
0 \\
0\end{array}$ & $\begin{array}{l}0 \\
0 \\
0\end{array}$ & $\begin{array}{l}0 \\
0 \\
0\end{array}$ & $\begin{array}{l}0 \\
0 \\
0\end{array}$ & $\begin{array}{l}0 \\
0 \\
0\end{array}$ \\
\hline Dec. -Feb. & $\begin{array}{r}1 \\
7 \\
30\end{array}$ & $\begin{array}{l}0 \\
0 \\
0.4\end{array}$ & $\begin{array}{l}0 \\
0 \\
0.1\end{array}$ & $\begin{array}{l}0 \\
0 \\
0\end{array}$ & $\begin{array}{l}0 \\
0 \\
0\end{array}$ & $\begin{array}{l}0 \\
0 \\
0\end{array}$ \\
\hline Mar. - May & $\begin{array}{r}1 \\
7 \\
30\end{array}$ & $\begin{array}{l}0.1 \\
0.2 \\
0.9\end{array}$ & $\begin{array}{l}0 \\
0 \\
0.4\end{array}$ & $\begin{array}{l}0 \\
0 \\
0.3\end{array}$ & $\begin{array}{l}0 \\
0 \\
0.2\end{array}$ & $\begin{array}{l}0 \\
0 \\
0.1\end{array}$ \\
\hline
\end{tabular}

Duration of daily flow for indicated periods

\begin{tabular}{l|l|l|l|l|l|l|l|l|l|l|l|l|l|l|}
\hline \multirow{2}{*}{ Period } & \multicolumn{8}{|c}{ Discharge $\left(\mathrm{ft}^{3} / \mathrm{s}\right.$ ) which was equaled or exceeded for indicated percent of time } \\
\cline { 2 - 13 } & 98 & 95 & 90 & 85 & 80 & 75 & 70 & 60 & 50 & 40 & 30 & 20 & 10 \\
\hline Apr. - Mar. & 0 & 0 & 0 & 0 & 0 & 0 & 0 & 0 & 0.2 & 0.5 & 0.9 & 1.6 & 3.2 \\
May - Nov. & 0 & 0 & 0 & 0 & 0 & 0 & 0 & 0 & 0 & 0.1 & 0.2 & 0.4 & 1.1 \\
June - Aug. & 0 & 0 & 0 & 0 & 0 & 0 & 0 & 0 & 0 & 0 & 0.1 & 0.2 & 0.5 \\
Sept. - Nov. & 0 & 0 & 0 & 0 & 0 & 0 & 0 & 0 & 0 & 0 & 0.1 & 0.2 & 0.4 \\
Dec. - Feb. & 0 & 0 & 0 & 0 & 0 & 0.1 & 0.2 & 0.4 & 0.6 & 1.0 & 1.5 & 2.2 & 3.8 \\
Mar. - May & 0 & 0.1 & 0.3 & 0.4 & 0.6 & 0.7 & 0.8 & 1.1 & 1.5 & 1.9 & 2.6 & 3.7 & 6.2 \\
\hline
\end{tabular}




\section{SCIOTO RIVER BASIN}

03236000 Salt Creek near Londonderry, Ohio

LOCATION:

Lat $39^{\circ} 15^{\prime} 22^{\prime \prime}$, long $82^{\circ} 46^{\prime} 12^{\prime \prime}$, in SW $1 / 4$ sec. 13, T.8 N., R. 20 W., Ross County, Hydrologic Unit 05060002, at bridge on U.S. Highway 20 , $1.3 \mathrm{mi}$ southeast of Londonderry, $2.3 \mathrm{mi}$ downstream from Poe Run, and $4.3 \mathrm{mi}$ upstream from Little Salt creek.

DRAINAGE AREA:

$286 \mathrm{mi}^{2}$.

TRIBUTARY TO: Scioto River.

DISCHARGE DATA USED: October 1938 to september 1950.

REMARKS :

None.

SELECTED DISCHARGE CHARACTERISTICS: Average discharge: $299 \mathrm{ft}^{3} / \mathrm{s}$ (12 years).

Minimum daily discharge: $4.9 \mathrm{ft}^{3} / \mathrm{s}$ September 1939.

Magnitude and frequency of low flow for indicated periods

\begin{tabular}{|c|c|c|c|c|c|c|c|c|c|c|c|c|c|}
\hline \multirow{2}{*}{ Period } & \multirow{2}{*}{$\begin{array}{l}\text { Number of } \\
\text { consecu- } \\
\text { tive days }\end{array}$} & \multicolumn{5}{|c|}{$\begin{array}{l}\text { Discharge }\left(\mathrm{ft}^{3} / \mathrm{s}\right) \text { for indicated } \\
\text { recurrence interval (years) }\end{array}$} & \multirow{2}{*}{ Period } & \multirow{2}{*}{$\begin{array}{l}\text { Number of } \\
\text { consecu- } \\
\text { tive days }\end{array}$} & \multicolumn{5}{|c|}{$\begin{array}{l}\text { Discharge }\left(\mathrm{ft}^{3} / \mathrm{s}\right) \text { for indicated } \\
\text { recurrence interval (years) }\end{array}$} \\
\hline & & 2 & 5 & 10 & 20 & 50 & & & 2 & 5 & 10 & 20 & 50 \\
\hline Apr.-Mar. & $\begin{array}{r}1 \\
7 \\
30\end{array}$ & $\begin{array}{r}8.2 \\
8.7 \\
13\end{array}$ & $\begin{array}{l}6.2 \\
6.7 \\
9.1\end{array}$ & $\begin{array}{l}5.6 \\
6.1 \\
8.0\end{array}$ & $\begin{array}{l}5.3 \\
5.8 \\
7.3\end{array}$ & $\begin{array}{l}5.1 \\
5.6 \\
6.8\end{array}$ & Sept.-Nov. & $\begin{array}{r}1 \\
7 \\
30\end{array}$ & $\begin{array}{r}8.9 \\
9.2 \\
15\end{array}$ & $\begin{array}{l}6.5 \\
6.9 \\
9.7\end{array}$ & $\begin{array}{l}5.8 \\
6.2 \\
7.9\end{array}$ & $\begin{array}{l}5.4 \\
5.9 \\
6.8\end{array}$ & $\begin{array}{l}5.1 \\
5.6 \\
5.9\end{array}$ \\
\hline June-Aug. & $\begin{array}{r}1 \\
7 \\
30\end{array}$ & $\begin{array}{l}13 \\
15 \\
29\end{array}$ & $\begin{array}{l}9.0 \\
10^{\circ} \\
18\end{array}$ & $\begin{array}{r}7.5 \\
8.4 \\
14\end{array}$ & $\begin{array}{r}6.5 \\
7.1 \\
12\end{array}$ & $\begin{array}{r}5.7 \\
5.9 \\
10\end{array}$ & Mar. - May & $\begin{array}{r}1 \\
7 \\
30\end{array}$ & $\begin{array}{r}65 \\
96 \\
183\end{array}$ & $\begin{array}{r}43 \\
65 \\
108\end{array}$ & $\begin{array}{l}34 \\
49 \\
85\end{array}$ & $\begin{array}{l}27 \\
38 \\
70\end{array}$ & $\begin{array}{l}21 \\
27 \\
57\end{array}$ \\
\hline
\end{tabular}

Duration of daily flow for indicated periods

\begin{tabular}{|c|c|c|c|c|c|c|c|c|c|c|c|c|c|}
\hline \multirow{2}{*}{ Period } & \multicolumn{13}{|c|}{ Discharge $\left(\mathrm{ft}^{3} / \mathrm{s}\right)$ which was equaled or exceeded for indicated percent of time } \\
\hline & 98 & 95 & 90 & 85 & 80 & 75 & 70 & 60 & 50 & 40 & 30 & 20 & 10 \\
\hline $\begin{array}{l}\text { Apr. - Mar. } \\
\text { May - Nov. } \\
\text { June - Aug. } \\
\text { Sept. - Nov. } \\
\text { Dec. - Feb. } \\
\text { Mar. - May }\end{array}$ & $\begin{array}{l}8.5 \\
7.8 \\
8.8 \\
6.7 \\
17 \\
50\end{array}$ & $\begin{array}{l}11 \\
9.3 \\
12 \\
8.1 \\
21 \\
69\end{array}$ & $\begin{array}{l}14 \\
12 \\
16 \\
9.7 \\
27 \\
91\end{array}$ & $\begin{array}{r}18 \\
14 \\
19 \\
11 \\
32 \\
110\end{array}$ & $\begin{array}{r}22 \\
16 \\
23 \\
12 \\
43 \\
130\end{array}$ & $\begin{array}{r}28 \\
18 \\
27 \\
13 \\
58 \\
150\end{array}$ & $\begin{array}{r}34 \\
21 \\
31 \\
14 \\
73 \\
170\end{array}$ & $\begin{array}{r}55 \\
30 \\
40 \\
17 \\
110 \\
230\end{array}$ & $\begin{array}{r}85 \\
43 \\
51 \\
20 \\
150 \\
310\end{array}$ & $\begin{array}{r}140 \\
60 \\
65 \\
25 \\
230 \\
410\end{array}$ & $\begin{array}{r}230 \\
85 \\
84 \\
38 \\
350 \\
530\end{array}$ & $\begin{array}{r}390 \\
150 \\
130 \\
58 \\
530 \\
760\end{array}$ & $\begin{array}{r}710 \\
350 \\
280 \\
120 \\
910 \\
1200\end{array}$ \\
\hline
\end{tabular}


UPPER TWIN CREEK BASIN

03237280 Upper Twin Creek at McGaw, Ohio

LOCATION:

Lat $38^{\circ} 38^{\prime} 37^{\prime \prime}$, long $83^{\circ} 12^{\prime} 57^{\prime \prime}$, Scioto County, Hydrologic Unit 05090201, on right bank, $0.3 \mathrm{mi}$ downstream from Brown Run, $0.3 \mathrm{mi}$ upstream from Tucker Run, $0.7 \mathrm{mi}$ upstream from bridge on U.S. Highway 52 at McGaw, $2.7 \mathrm{mi}$ northeast of Buena Vista, and $3.2 \mathrm{mi}$ upstream from mouth.

DRAINAGE AREA: $\quad 12.2 \mathrm{mi}^{2}$.

TRIBUTARY TO: Ohio River.

DISCHARGE DATA USED: July 1963 to September 1978.

REMARKS:

None.

SELECTED DISCHARGE CHARACTERISTICS: Average discharge: $13.2 \mathrm{ft}^{3} / \mathrm{s}$ (15 years). Minimum daily discharge: No flow for many days most years.

Magnitude and frequency of low flow for indicated periods

\begin{tabular}{|c|c|c|c|c|c|c|c|c|c|c|c|c|c|}
\hline \multirow{2}{*}{ Period } & \multirow{2}{*}{$\begin{array}{l}\text { Number of } \\
\text { consecu- } \\
\text { tive days }\end{array}$} & \multicolumn{5}{|c|}{$\begin{array}{l}\text { Discharge }\left(\mathrm{ft}^{3} / \mathrm{s}\right) \text { for indicated } \\
\text { recurrence interval (years) }\end{array}$} & \multirow{2}{*}{ Period } & \multirow{2}{*}{$\begin{array}{l}\text { Number of } \\
\text { consecu- } \\
\text { tive days }\end{array}$} & \multicolumn{5}{|c|}{$\begin{array}{l}\text { Discharge }\left(\mathrm{ft}^{3} / \mathrm{s}\right) \text { for indicated } \\
\text { recurrence interval (years) }\end{array}$} \\
\hline & & 2 & 5 & 10 & 20 & 50 & & & 2 & 5 & 10 & 20 & 50 \\
\hline Apr.-Mar. & $\begin{array}{r}1 \\
7 \\
30\end{array}$ & $\begin{array}{l}0 \\
0.1 \\
0.2\end{array}$ & $\begin{array}{l}0 \\
0 \\
0\end{array}$ & $\begin{array}{l}0 \\
0 \\
0\end{array}$ & $\begin{array}{l}0 \\
0 \\
0\end{array}$ & $\begin{array}{l}0 \\
0 \\
0\end{array}$ & Sept.-Nov. & $\begin{array}{r}1 \\
7 \\
30\end{array}$ & $\begin{array}{l}0.1 \\
0.1 \\
0.4\end{array}$ & $\begin{array}{l}0 \\
0 \\
0\end{array}$ & $\begin{array}{l}0 \\
0 \\
0\end{array}$ & $\begin{array}{l}0 \\
0 \\
0\end{array}$ & $\begin{array}{l}0 \\
0 \\
0\end{array}$ \\
\hline June-Aug. & $\begin{array}{r}1 \\
7 \\
30\end{array}$ & $\begin{array}{l}0.1 \\
0.1 \\
0.5\end{array}$ & $\begin{array}{l}0 \\
0 \\
0.2\end{array}$ & $\begin{array}{l}0 \\
0 \\
0.1\end{array}$ & $\begin{array}{l}0 \\
0 \\
0.1\end{array}$ & $\begin{array}{l}0 \\
0 \\
0.1\end{array}$ & Mar. - May & $\begin{array}{r}1 \\
7 \\
30\end{array}$ & $\begin{array}{l}1.4 \\
2.1 \\
8.5\end{array}$ & $\begin{array}{l}0.6 \\
0.6 \\
4.0\end{array}$ & $\begin{array}{l}0.3 \\
0.3 \\
2.6\end{array}$ & $\begin{array}{l}0 \\
0.1 \\
1.8\end{array}$ & $\begin{array}{l}0 \\
0 \\
1.1\end{array}$ \\
\hline
\end{tabular}

Duration of daily flow for indicated periods

\begin{tabular}{|c|c|c|c|c|c|c|c|c|c|c|c|c|c|c|}
\hline \multirow{2}{*}{\multicolumn{2}{|c|}{ Period }} & \multicolumn{13}{|c|}{ Discharge $\left(\mathrm{ft}^{3} / \mathrm{s}\right)$ which was equaled or exceeded for indicated percent of time } \\
\hline & & 98 & 95 & 90 & 85 & 80 & 75 & 70 & 60 & 50 & 40 & 30 & 20 & 10 \\
\hline $\begin{array}{l}\text { Apr. - } \\
\text { May - } \\
\text { June - } \\
\text { Sept. - } \\
\text { Dec. - } \\
\text { Mar. - }\end{array}$ & $\begin{array}{l}\text { Mar. } \\
\text { Nov. } \\
\text { Aug. } \\
\text { Nov. } \\
\text { Feb. } \\
\text { May }\end{array}$ & $\begin{array}{l}0 \\
0 \\
0 \\
0 \\
0 \\
0.7\end{array}$ & $\begin{array}{l}0 \\
0 \\
0 \\
0 \\
0.6 \\
1.2\end{array}$ & $\begin{array}{l}0.2 \\
0 \\
0.1 \\
0 \\
1.2 \\
2.2\end{array}$ & $\begin{array}{l}0.3 \\
0.1 \\
0.2 \\
0 \\
1.7 \\
3.6\end{array}$ & $\begin{array}{l}0.4 \\
0.2 \\
0.2 \\
0 \\
2.3 \\
5.0\end{array}$ & $\begin{array}{l}0.6 \\
0.3 \\
0.3 \\
0.1 \\
2.8 \\
6.2\end{array}$ & $\begin{array}{l}0.9 \\
0.4 \\
0.3 \\
0.2 \\
3.7 \\
7.3\end{array}$ & $\begin{array}{r}1.7 \\
0.6 \\
0.5 \\
0.4 \\
5.5 \\
11\end{array}$ & $\begin{array}{l}3.1 \\
0.9 \\
0.7 \\
0.7 \\
8.6 \\
14\end{array}$ & $\begin{array}{l}5.8 \\
1.5 \\
1.0 \\
1.1 \\
12 \\
19\end{array}$ & $\begin{array}{c}10 \\
2.6 \\
1.6 \\
1.8 \\
17 \\
25\end{array}$ & $\begin{array}{c}17 \\
5.1 \\
2.6 \\
3.2 \\
23 \\
36\end{array}$ & $\begin{array}{l}31 \\
11 \\
6.8 \\
6.9 \\
37 \\
60\end{array}$ \\
\hline
\end{tabular}


Appendix 3.--Low-flow data--Continued

OHIO BRUSH CREEK BASIN

03237500 Ohio Brush Creek near West Union, Ohio

LOCATION:

Lat $38^{\circ} 48^{\prime} 13^{\prime \prime}$, long $83^{\circ} 25^{\prime} 16^{\prime \prime}$, Adams County, Hydrologic Unit 05090201, on right bank at downstream side of bridge on State Route $348,0.3 \mathrm{mi}$ downstream from Cedar Run, $7.0 \mathrm{mi}$ east of West Union, and $7.1 \mathrm{mi}$ upstream from Beasley Fork.

DRAINAGE AREA: $\quad 387 \mathrm{mi}^{2}$.

TRIBUTARY TO: Ohio River.

DISCHARGE DATA USED: October 1926 to September 1935, October 1940 to September 1978.

REMARKS:

None.

SELECTED DISCHARGE CHARACTERISTICS: Average discharge: $446 \mathrm{ft}^{3} / \mathrm{s}$ (47 years).

Minimum daily discharge: No flow September 1955.

Magnitude and frequency of low flow for indicated periods

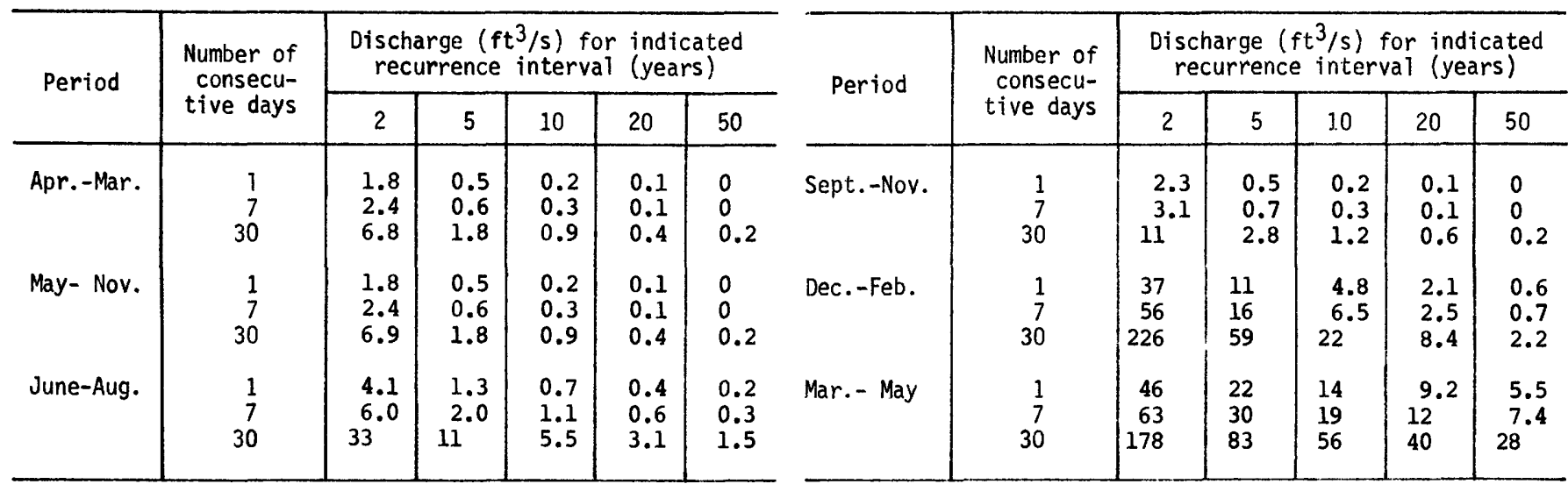

Duration of daily flow for indicated periods

\begin{tabular}{|c|c|c|c|c|c|c|c|c|c|c|c|c|c|}
\hline \multirow{2}{*}{ Period } & \multicolumn{13}{|c|}{ Discharge $\left(\mathrm{ft}^{3} / \mathrm{s}\right)$ which was equaled or exceeded for indicated percent of time } \\
\hline & 98 & 95 & 90 & 85 & 80 & 75 & 70 & 60 & 50 & 40 & 30 & 20 & 10 \\
\hline $\begin{array}{l}\text { Apr. - Mar. } \\
\text { May - Nov. } \\
\text { June - Aug. } \\
\text { Sept. - Nov. } \\
\text { Dec. - Feb. } \\
\text { Mar. - May }\end{array}$ & $\begin{array}{l}0.9 \\
0.5 \\
1.1 \\
0.3 \\
4.1 \\
26\end{array}$ & $\begin{array}{l}2.4 \\
1.4 \\
2.6 \\
0.6 \\
12 \\
44\end{array}$ & $\begin{array}{l}5.3 \\
3.0 \\
5.2 \\
1.6 \\
32 \\
65\end{array}$ & $\begin{array}{l}9.1 \\
4.9 \\
7.6 \\
2.5 \\
51 \\
86\end{array}$ & $\begin{array}{l}15 \\
7.1 \\
10 \\
3.6 \\
67 \\
110\end{array}$ & $\begin{array}{c}23 \\
9.7 \\
13 \\
4.9 \\
86 \\
130\end{array}$ & $\begin{array}{c}33 \\
13 \\
17 \\
6.3 \\
110 \\
150\end{array}$ & $\begin{array}{r}60 \\
24 \\
26 \\
11 \\
170 \\
200\end{array}$ & $\begin{array}{r}100 \\
38 \\
39 \\
19 \\
240 \\
270\end{array}$ & $\begin{array}{r}160 \\
60 \\
59 \\
34 \\
340 \\
370\end{array}$ & $\begin{array}{r}260 \\
94 \\
91 \\
58 \\
490 \\
530\end{array}$ & $\begin{array}{l}450 \\
170 \\
160 \\
100 \\
770 \\
820\end{array}$ & $\begin{array}{r}970 \\
390 \\
380 \\
250 \\
1600 \\
1700\end{array}$ \\
\hline
\end{tabular}


Appendix 3.--Low-flow data--Continued

EAGLE CREEK BASIN

03238200 Eagle Creek near Ripley, Ohio

LOCATION:

Lat $38^{\circ} 43^{\prime} 35^{\prime \prime}$, long $83^{\circ} 47^{\prime} 15^{\prime \prime}$, Brown County, Hydrologic Unit 05090201 , at highway bridge $3.3 \mathrm{mi}$ southeast of Ripley and $0.4 \mathrm{mi}$ upstream from Beetle Creek.

DRAINAGE AREA: $\quad 137 \mathrm{mi}^{2}$.

TRIBUTARY TO:

Ohio River.

DISCHARGE DATA USED:

Low-flow measurements 1959-1973 water years.

INDEX STATION: $\quad 03237500$ Ohio Brush creek near west Union, Ohio.

REMARKS:

None.

SELECTED DISCHARGE CHARACTERISTICS: Minimum discharge observed: $0 \mathrm{ft}^{3} / \mathrm{s}$ September 1964 .

\begin{tabular}{|c|c|c|c|}
\hline \multirow[t]{2}{*}{ Period } & \multirow{2}{*}{$\begin{array}{l}\text { Number of } \\
\text { consecu- } \\
\text { tive days }\end{array}$} & \multicolumn{2}{|c|}{$\begin{array}{l}\text { Discharge }\left(\mathrm{ft}^{3} / \mathrm{s}\right) \text { for } \\
\text { indicated recurrence } \\
\text { interval (years) }\end{array}$} \\
\hline & & 2 & 10 \\
\hline Apr. - Mar. & $\begin{array}{r}7 \\
30\end{array}$ & $\begin{array}{l}0.2 \\
0.4\end{array}$ & $\begin{array}{l}0 \\
0.1\end{array}$ \\
\hline May - Nov. & $\begin{array}{r}7 \\
30\end{array}$ & $\begin{array}{l}0.2 \\
0.4\end{array}$ & $\begin{array}{l}0 \\
0.1\end{array}$ \\
\hline June - Aug. & $\begin{array}{r}7 \\
30\end{array}$ & $\begin{array}{l}0.3 \\
1.0\end{array}$ & $\begin{array}{l}0.1 \\
0.3\end{array}$ \\
\hline Sept. - Nov. & $\begin{array}{r}7 \\
30\end{array}$ & $\begin{array}{l}0.2 \\
0.5\end{array}$ & $\begin{array}{l}0 \\
0.1\end{array}$ \\
\hline
\end{tabular}

Duration of daily flow for indicated periods

\begin{tabular}{l|c|c|c|c|c}
\hline \multirow{2}{*}{ Period } & \multicolumn{4}{|c|}{$\begin{array}{c}\text { Discharge ( } \mathrm{ft}^{3} / \mathrm{s} \text { ) which was equaled or } \\
\text { exceeded for indicated percent of time }\end{array}$} \\
\cline { 2 - 6 } & 98 & 95 & 90 & 85 & 80 \\
\hline Apr. - Mar. & 0.1 & 0.2 & 0.3 & 0.4 & 0.6 \\
May - Nov. & 0.1 & 0.1 & 0.2 & 0.3 & 0.4 \\
June - Aug. & 0.1 & 0.2 & 0.3 & 0.4 & 0.4 \\
Sept. - Nov. & 0 & 0.1 & 0.1 & 0.2 & 0.2 \\
\hline
\end{tabular}


WHITEOAK CREEK BASIN

03238500 Whiteoak Creek near Georgetown, Ohio

LOCATION:

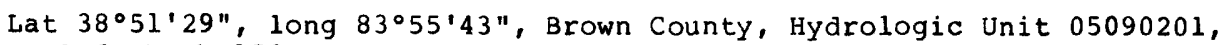
on left bank $150 \mathrm{ft}$ upstream from diversion dam for Georgetown water treatment plant, $0.7 \mathrm{mi}$ upstream from Town Run, $1.4 \mathrm{mi}$ southwest of Georgetown, and $7.2 \mathrm{mi}$ upstream from mouth.

DRAINAGE AREA:

$218 \mathrm{mi}^{2}$

TRIBUTARY TO:

Ohio River.

DISCHARGE DATA USED: October 1939 to September 1978.

REMARKS:

None.

SELECTED DISCHARGE CHARACTERISTICS: Average discharge: $253 \mathrm{ft}^{3} / \mathrm{s}$ (39 years).

Minimum daily discharge: No flow at times many years.

Magnitude and frequency of low flow for indicated periods

\begin{tabular}{|c|c|c|c|c|c|c|c|c|c|c|c|c|c|}
\hline \multirow{2}{*}{ Period } & \multirow{2}{*}{$\begin{array}{l}\text { Number of } \\
\text { consecu- } \\
\text { tive days }\end{array}$} & \multicolumn{5}{|c|}{$\begin{array}{l}\text { Discharge }\left(\mathrm{ft}^{3} / \mathrm{s}\right) \text { for indicated } \\
\text { recurrence interval (years) }\end{array}$} & \multirow{2}{*}{ Period } & \multirow{2}{*}{$\begin{array}{l}\text { Number of } \\
\text { consecu- } \\
\text { tive days }\end{array}$} & \multicolumn{5}{|c|}{$\begin{array}{l}\text { Discharge }\left(\mathrm{ft}^{3} / \mathrm{s}\right) \text { for indicated } \\
\text { recurrence interval (years) }\end{array}$} \\
\hline & & 2 & 5 & 10 & 20 & 50 & & & 2 & 5 & 10 & 20 & 50 \\
\hline Apr.-Mar. & $\begin{array}{r}1 \\
7 \\
30\end{array}$ & $\begin{array}{l}0.4 \\
0.7 \\
2.7\end{array}$ & $\begin{array}{l}0 \\
0.2 \\
0.6\end{array}$ & $\begin{array}{l}0 \\
0 \\
0.2\end{array}$ & $\begin{array}{l}0 \\
0 \\
0.1\end{array}$ & $\begin{array}{l}0 \\
0 \\
0\end{array}$ & Sept.-Nov. & $\begin{array}{r}1 \\
7 \\
30\end{array}$ & $\begin{array}{l}0.6 \\
1.0 \\
4.9\end{array}$ & $\begin{array}{l}0.1 \\
0.2 \\
0.8\end{array}$ & $\begin{array}{l}0 \\
0 \\
0.3\end{array}$ & $\begin{array}{l}0 \\
0 \\
0.1\end{array}$ & $\begin{array}{l}0 \\
0 \\
0\end{array}$ \\
\hline June-Aug. & $\begin{array}{r}1 \\
7 \\
30\end{array}$ & $\begin{array}{r}1.1 \\
2.0 \\
17\end{array}$ & $\begin{array}{l}0.2 \\
0.6 \\
5.8\end{array}$ & $\begin{array}{l}0 \\
0.3 \\
3.0\end{array}$ & $\begin{array}{l}0 \\
0 \\
1.7\end{array}$ & $\begin{array}{l}0 \\
0 \\
0.8\end{array}$ & Mar. - May & $\begin{array}{r}1 \\
7 \\
30\end{array}$ & $\begin{array}{l}17 \\
23 \\
75\end{array}$ & $\begin{array}{l}8.8 \\
12 \\
38\end{array}$ & $\begin{array}{r}5.4 \\
7.8 \\
26\end{array}$ & $\begin{array}{r}3.2 \\
5.2 \\
19\end{array}$ & $\begin{array}{r}1.6 \\
3.0 \\
13\end{array}$ \\
\hline
\end{tabular}

Duration of daily flow for indicated periods

\begin{tabular}{l|c|c|c|c|c|c|c|c|c|c|c|r|r}
\hline \multirow{2}{*}{ Period } & \multicolumn{8}{|c|}{ Discharge $\left(\mathrm{ft}^{3} / \mathrm{s}\right)$ which was equaled or exceeded for indicated percent of time } \\
\cline { 2 - 12 } & 98 & 95 & 90 & 85 & 80 & 75 & 70 & 60 & 50 & 40 & 30 & 20 & 10 \\
\hline Apr. - Mar. & 0.3 & 0.8 & 2.4 & 4.3 & 6.7 & 10 & 15 & 25 & 42 & 68 & 110 & 210 & 550 \\
May - Nov. & 0.1 & 0.4 & 1.1 & 2.2 & 3.2 & 4.6 & 6.2 & 10 & 17 & 26 & 41 & 74 & 180 \\
June - Aug. & 0.4 & 1.0 & 2.1 & 3.2 & 4.3 & 5.6 & 7.1 & 11 & 17 & 25 & 40 & 72 & 170 \\
Sept. - Nov. & 0.1 & 0.2 & 0.4 & 0.8 & 1.4 & 2.3 & 3.0 & 5.4 & 9.1 & 17 & 26 & 48 & 120 \\
Dec. - Feb. & 1.9 & 4.8 & 13 & 19 & 25 & 32 & 41 & 66 & 99 & 150 & 230 & 420 & 1000 \\
Mar. - May & 11 & 17 & 25 & 32 & 40 & 48 & 56 & 76 & 100 & 150 & 230 & 420 & 960 \\
\hline
\end{tabular}


LOCATION:

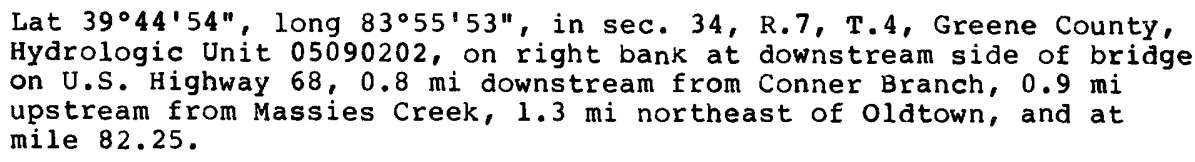

DRAINAGE AREA: $\quad 129 \mathrm{mi}^{2}$.

TRIBUTARY TO: Ohio River.

DISCHARGE DATA USED: August 1952 to September 1978.

REMARKS: $\quad$ None.

SELECTED DISCHARGE CHARACTERISTICS: Average discharge: $108 \mathrm{ft}^{3} / \mathrm{s}$ ( 26 years).

Minimum daily discharge: $6.9 \mathrm{ft}^{3} / \mathrm{s}$ September 1955.

Magnitude and frequency of low flow for indicated periods

\begin{tabular}{|c|c|c|c|c|c|c|c|c|c|c|c|c|c|}
\hline \multirow{2}{*}{ Period } & \multirow{2}{*}{$\begin{array}{l}\text { Number of } \\
\text { consecu- } \\
\text { tive days }\end{array}$} & \multicolumn{5}{|c|}{$\begin{array}{l}\text { Discharge }\left(\mathrm{ft}^{3} / \mathrm{s}\right) \text { for indicated } \\
\text { recurrence interval (years) }\end{array}$} & \multirow{2}{*}{ Period } & \multirow{2}{*}{$\begin{array}{l}\text { Number of } \\
\text { consecu- } \\
\text { tive days }\end{array}$} & \multicolumn{5}{|c|}{$\begin{array}{l}\text { Discharge }\left(\mathrm{ft}^{3} / \mathrm{s}\right) \text { for indicated } \\
\text { recurrence interval (years) }\end{array}$} \\
\hline & & 2 & 5 & 10 & 20 & 50 & & & 2 & 5 & 10 & 20 & 50 \\
\hline Apr.-Mar. & $\begin{array}{r}1 \\
7 \\
30\end{array}$ & $\begin{array}{l}13 \\
15 \\
16\end{array}$ & $\begin{array}{r}8.8 \\
9.8 \\
11\end{array}$ & $\begin{array}{l}7.3 \\
8.1 \\
9.0\end{array}$ & $\begin{array}{l}6.4 \\
7.0 \\
7.7\end{array}$ & $\begin{array}{l}5.5 \\
6.0 \\
6.6\end{array}$ & Sept.-Nov. & $\begin{array}{r}1 \\
7 \\
30\end{array}$ & $\begin{array}{l}14 \\
15 \\
19\end{array}$ & $\begin{array}{l}9.1 \\
10 \\
12\end{array}$ & $\begin{array}{l}7.5 \\
8.2 \\
9.5\end{array}$ & $\begin{array}{l}6.4 \\
7.0 \\
8.0\end{array}$ & $\begin{array}{l}5.4 \\
6.0 \\
6.5\end{array}$ \\
\hline June-Aug. & $\begin{array}{r}1 \\
7 \\
30\end{array}$ & $\begin{array}{l}17 \\
19 \\
25\end{array}$ & $\begin{array}{l}11 \\
13 \\
16\end{array}$ & $\begin{array}{l}9.4 \\
11 \\
13\end{array}$ & $\begin{array}{r}8.2 \\
9.1 \\
12\end{array}$ & $\begin{array}{r}7.1 \\
7.8 \\
10^{-8}\end{array}$ & Mar. - May & $\begin{array}{r}1 \\
7 \\
30\end{array}$ & $\begin{array}{l}50 \\
58 \\
84\end{array}$ & $\begin{array}{l}35 \\
40 \\
55\end{array}$ & $\begin{array}{l}29 \\
34 \\
44\end{array}$ & $\begin{array}{l}24 \\
29 \\
37\end{array}$ & $\begin{array}{l}19 \\
24 \\
30\end{array}$ \\
\hline
\end{tabular}

Duration of daily flow for indicated periods

\begin{tabular}{l|c|c|c|c|c|c|c|c|c|c|r|r|r}
\hline \multirow{2}{*}{ Period } & \multicolumn{8}{|c|}{ Discharge $\left(\mathrm{ft}^{3} / \mathrm{s}\right)$ which was equaled or exceeded for indicated percent of time } \\
\cline { 2 - 13 } & 98 & 95 & 90 & 85 & 80 & 75 & 70 & 60 & 50 & 40 & 30 & 20 & 10 \\
\hline Apr. - Mar. & 9.8 & 12 & 16 & 19 & 22 & 26 & 30 & 41 & 53 & 72 & 100 & 140 & 240 \\
May - Nov. & 8.9 & 11 & 14 & 16 & 19 & 21 & 23 & 29 & 37 & 46 & 58 & 81 & 130 \\
June - Aug. & 10 & 14 & 17 & 20 & 22 & 24 & 27 & 32 & 39 & 48 & 61 & 82 & 130 \\
Sept.- Nov. & 8.1 & 9.7 & 11 & 13 & 14 & 16 & 17 & 20 & 23 & 28 & 37 & 47 & 65 \\
Dec. - Feb. & 11 & 13 & 18 & 23 & 29 & 35 & 41 & 56 & 76 & 96 & 130 & 190 & 300 \\
Mar. - May & 33 & 41 & 50 & 58 & 65 & 74 & 83 & 100 & 120 & 140 & 180 & 240 & 360 \\
& & & & & & & & & & & &
\end{tabular}


LOCATION:

DRAINAGE AREA:

TRIBUTTARY TO:

DISCHARGE DATA USED:

REMARKS :
Lat $39^{\circ} 45^{\prime} 25^{\prime \prime}$, long $83^{\circ} 47^{\prime} 25^{\prime \prime}$, Greene County, Hydrologic Unit 05090202, on left bank at downstream side of bridge on James Barber Road, $1.0 \mathrm{mi}$ upstream from confluence with South Fork and $1.0 \mathrm{mi}$ northeast of Cedarville.

$28.9 \mathrm{mi}^{2}$

Little Miami River.

July 1954 to September 1968.

None.

SELECTED DISCHARGE CHARACTERISTICS: Average discharge: $26.1 \mathrm{ft}^{3} / \mathrm{s}$ (14 years).

Minimum daily discharge: No flow at times in 1954-55, 1964.

Magnitude and frequency of low flow for indicated periods

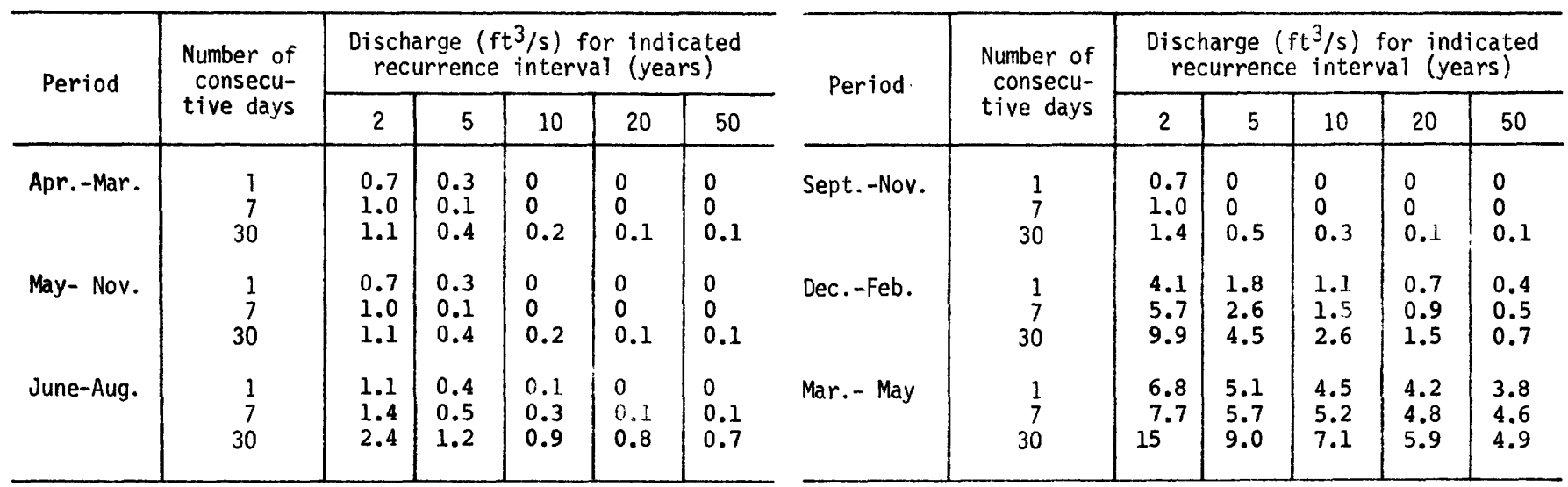

Duration of daily flow for indicated periods

\begin{tabular}{|c|c|c|c|c|c|c|c|c|c|c|c|c|c|}
\hline \multirow{2}{*}{ Period } & \multicolumn{13}{|c|}{ Discharge $\left(\mathrm{ft}^{3} / \mathrm{s}\right)$ which was equaled or exceeded for indicated percent of time } \\
\hline & 98 & 95 & 90 & 85 & 80 & 75 & 70 & 60 & 50 & 40 & 30 & 20 & 10 \\
\hline $\begin{array}{l}\text { Apr. - Mar. } \\
\text { May - Nov. } \\
\text { June - Aug. } \\
\text { Sept. - Nov. } \\
\text { Dec. - Feb. } \\
\text { Mar. - May }\end{array}$ & $\begin{array}{l}0.4 \\
0.3 \\
0.3 \\
0.1 \\
1.5 \\
5.2\end{array}$ & $\begin{array}{l}0.9 \\
0.6 \\
0.7 \\
0.3 \\
2.2 \\
6.4\end{array}$ & $\begin{array}{l}1.4 \\
1.0 \\
1.3 \\
0.7 \\
3.5 \\
8.4\end{array}$ & $\begin{array}{l}1.8 \\
1.3 \\
1.8 \\
0.9 \\
5.1 \\
11\end{array}$ & $\begin{array}{l}2.5 \\
1.6 \\
2.2 \\
1.1 \\
6.6 \\
12\end{array}$ & $\begin{array}{l}3.4 \\
1.9 \\
2.6 \\
1.3 \\
7.9 \\
14\end{array}$ & $\begin{array}{r}4.3 \\
2.4 \\
3.0 \\
1.5 \\
9.3 \\
16\end{array}$ & $\begin{array}{l}6.7 \\
3.5 \\
4.0 \\
1.8 \\
12 \\
22\end{array}$ & $\begin{array}{l}9.9 \\
4.8 \\
5.2 \\
2.5 \\
17 \\
29\end{array}$ & $\begin{array}{l}15 \\
6.7 \\
6.7 \\
3.6 \\
22 \\
37\end{array}$ & $\begin{array}{l}22 \\
9.7 \\
9.5 \\
4.9 \\
30 \\
50\end{array}$ & $\begin{array}{l}35 \\
16 \\
15 \\
7.6 \\
43 \\
68\end{array}$ & $\begin{array}{r}65 \\
33 \\
32 \\
16 \\
76 \\
100\end{array}$ \\
\hline
\end{tabular}




\section{LITTLE MIAMI RIVER BASIN}

U32410u0 South Fork Massies Creek nuar Cedarvi.1le, Ohio

LOCATION: Lat $3 y^{\circ} 44^{\prime} 20^{\prime \prime}$, long $8^{\circ} 3^{\circ} 45^{\prime} 50^{\prime \prime}$, Greene County, Hydrologic Unit U509U202, on right bank at downstream side of bridge on iveimer koad, $2.3 \mathrm{mi}$ east of Cedarville and $2.4 \mathrm{ini}$ uostrean troil confluence with ivorth Fork.

DRAINAGE AREA:

$17.1 \mathrm{mi}^{2}$

TRIBLITARY TO:

Massies Creek.

DISCHARGE DATA USED:

July 1954 to September 1968.

REMARKS:

none.

SELECTED DISCHARGE CHARACTERISTICS: Average discharge: $18.1 \mathrm{ft}^{3} / \mathrm{s}$ (14 years).

Minimum daily discharge: No flow at times in 1954-1955, 1957, 1964-1967.

Magnitude and frequency of low flow for indicated periods

\begin{tabular}{|c|c|c|c|c|c|c|c|c|c|c|c|c|c|}
\hline \multirow{2}{*}{ Period } & \multirow{2}{*}{$\begin{array}{l}\text { Number of } \\
\text { consecu- } \\
\text { tive days }\end{array}$} & \multicolumn{5}{|c|}{$\begin{array}{l}\text { Discharge }\left(\mathrm{ft}^{3} / \mathrm{s}\right) \text { for indicated } \\
\text { recurrence interval (years) }\end{array}$} & \multirow{2}{*}{ Period } & \multirow{2}{*}{$\begin{array}{l}\text { Number of } \\
\text { consecu- } \\
\text { tive days }\end{array}$} & \multicolumn{5}{|c|}{$\begin{array}{l}\text { Discharge }\left(\mathrm{ft}^{3} / \mathrm{s}\right) \text { for indicated } \\
\text { recurrence interval (years) }\end{array}$} \\
\hline & & 2 & 5 & 10 & 20 & 50 & & & 2 & 5 & 10 & 20 & 50 \\
\hline Apr.-Mar. & $\begin{array}{r}1 \\
7 \\
30\end{array}$ & $\begin{array}{l}0.2 \\
0.2 \\
0.3\end{array}$ & $\begin{array}{l}0 \\
0 \\
0.1\end{array}$ & $\begin{array}{l}0 \\
0 \\
0\end{array}$ & $\begin{array}{l}0 \\
0 \\
0\end{array}$ & $\begin{array}{l}0 \\
0 \\
0\end{array}$ & Sept.-Nov. & $\begin{array}{r}1 \\
7 \\
30\end{array}$ & $\begin{array}{l}0.2 \\
0.2 \\
0.3\end{array}$ & $\begin{array}{l}0 \\
0 \\
0.1\end{array}$ & $\begin{array}{l}0 \\
0 \\
0\end{array}$ & $\begin{array}{l}0 \\
0 \\
0\end{array}$ & $\begin{array}{l}0 \\
0 \\
0\end{array}$ \\
\hline June-Aug. & $\begin{array}{r}1 \\
7 \\
30\end{array}$ & $\begin{array}{l}0.3 \\
0.5 \\
1.6\end{array}$ & $\begin{array}{l}0.1 \\
0.1 \\
0.4\end{array}$ & $\begin{array}{l}0 \\
0 \\
0.1\end{array}$ & $\begin{array}{l}0 \\
0 \\
0.1\end{array}$ & $\begin{array}{l}0 \\
0 \\
0\end{array}$ & Mar.- May & $\begin{array}{r}1 \\
7 \\
30\end{array}$ & $\begin{array}{l}4.1 \\
4.9 \\
9.1\end{array}$ & $\begin{array}{l}2.9 \\
3.4 \\
5.5\end{array}$ & $\begin{array}{l}2.5 \\
2.9 \\
4.3\end{array}$ & $\begin{array}{l}2.1 \\
2.6 \\
3.6\end{array}$ & $\begin{array}{l}1.8 \\
2.2 \\
3.0\end{array}$ \\
\hline
\end{tabular}

Duration of daily flow for indicated periods

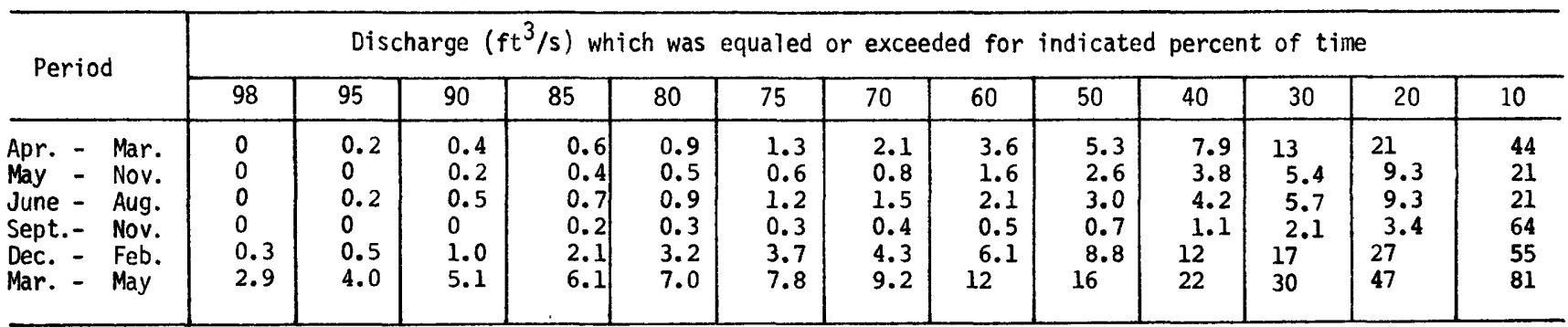


LITTLE MIAMI RIVER BASIN

03241500 Massies Creek at Wilberforce, Ohio

LOCATION :

Lat $39^{\circ} 43^{\prime} 22^{\prime \prime}$, long $83^{\circ} 52^{\prime} 58^{\prime \prime}$, Greene County, Hydrologic Unit 05090202 , on right bank $200 \mathrm{ft}$ downstream from bridge on Wilberforce-Clifton Road, $0.5 \mathrm{mi}$ northwest of Wilberforce $0.6 \mathrm{mi}$ downstream from unnamed right bank tributary and $1.7 \mathrm{mi}$ upstream from Clark Run.

DRAINAGE AREA: $63.2 \mathrm{mi}^{2}$.

TRIBUTARY TO:

Little Miami River.

DISCHARGE DATA USED: September 1952 to september 1978.

REMARKS:

None.

SELECTED DISCHARGE CHARACTERISTICS: Average discharge: $59.1 \mathrm{ft}^{3} / \mathrm{s}$ (26 years). Minimum daily discharge: $0.3 \mathrm{ft}^{3} / \mathrm{s}$ September 1954 .

Magnitude and frequency of low flow for indicated periods

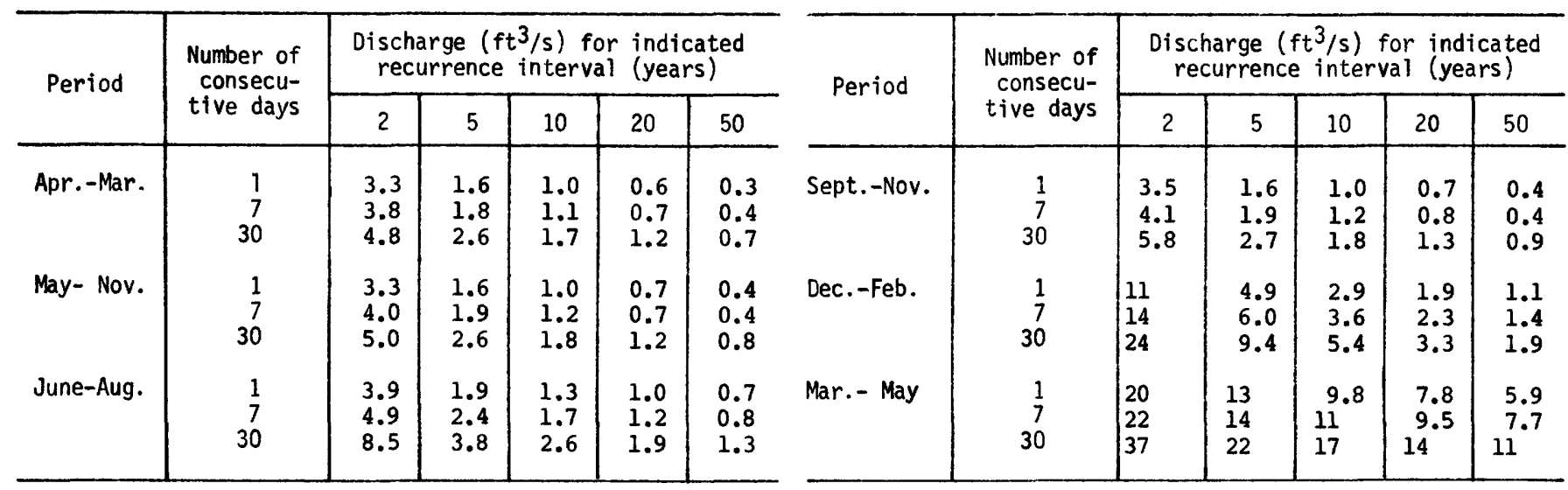

Duration of daily flow for indicated periods

\begin{tabular}{|c|c|c|c|c|c|c|c|c|c|c|c|c|c|}
\hline \multirow{2}{*}{ Period } & \multicolumn{13}{|c|}{ Discharge $\left(\mathrm{ft}^{3} / \mathrm{s}\right)$ which was equaled or exceeded for indicated percent of time } \\
\hline & 98 & 95 & 90 & 85 & 80 & 75 & 70 & 60 & 50 & 40 & 30 & 20 & 10 \\
\hline $\begin{array}{l}\text { Apr. - Mar. } \\
\text { May - Nov. } \\
\text { June - Aug. } \\
\text { Sept. - Nov. } \\
\text { Dec. - Feb. } \\
\text { Mar. - May }\end{array}$ & $\begin{array}{l}1.7 \\
1.2 \\
1.2 \\
1.1 \\
2.5 \\
12\end{array}$ & $\begin{array}{l}2.9 \\
2.3 \\
2.5 \\
1.8 \\
4.4 \\
16\end{array}$ & $\begin{array}{l}4.3 \\
3.3 \\
3.8 \\
2.6 \\
6.5 \\
20\end{array}$ & $\begin{array}{r}5.7 \\
4.3 \\
5.0 \\
3.3 \\
9.9 \\
24\end{array}$ & $\begin{array}{l}7.2 \\
5.3 \\
6.0 \\
3.9 \\
14 \\
28\end{array}$ & $\begin{array}{l}9.0 \\
6.3 \\
7.1 \\
4.5 \\
18 \\
32\end{array}$ & $\begin{array}{c}12 \\
7.4 \\
8.3 \\
5.0 \\
21 \\
36\end{array}$ & $\begin{array}{l}18 \\
10 \\
11 \\
6.5 \\
30 \\
46\end{array}$ & $\begin{array}{l}25 \\
14 \\
15 \\
8.4 \\
42 \\
57\end{array}$ & $\begin{array}{l}35 \\
19 \\
20 \\
11 \\
55 \\
72\end{array}$ & $\begin{array}{l}51 \\
26 \\
27 \\
15 \\
75 \\
96\end{array}$ & $\begin{array}{r}78 \\
40 \\
39 \\
22 \\
110 \\
140\end{array}$ & $\begin{array}{r}140 \\
74 \\
72 \\
36 \\
180 \\
230\end{array}$ \\
\hline
\end{tabular}




\section{LITTLE MIAMI RIVER BASIN}

03242050 Lxttle Miami River near Spring Valley, Ohio

LOCATION:

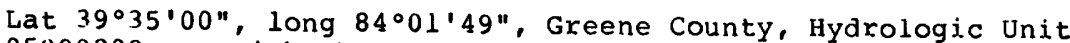

05090202 , on right bank at downstream side of bridge on New

Burlington Road, $0.3 \mathrm{mi}$ upstream from unnamed right bank

tributary, $2.2 \mathrm{mi}$ southwest of Spring Valley, $2.8 \mathrm{mi}$ downstream

from Glady Run, and at mile 61.95.

DRAINAGE AREA:

$366 \mathrm{mi}^{2}$.

TRIBUTARY TO:

Ohio River.

DISCHARGE DATA USED: October 1925 to September 1935, October 1939 to September 1951,

REMARKS :

July 1968 to September 1978.

None

SELECTED DISCHARGE CHARACTERISTICS: Average discharge: $379 \mathrm{ft}^{3} / \mathrm{s}$ (332 years).

Minimum daily discharge: $24 \mathrm{ft} / \mathrm{s}$ July, 1934.

Magnitude and frequency of low flow for indicated periods

\begin{tabular}{c|c|c|c|c|c|c}
\hline \multirow{2}{*}{ Period } & \multirow{2}{*}{$\begin{array}{c}\text { Number of } \\
\text { consecu- } \\
\text { tive days }\end{array}$} & \multicolumn{4}{|c}{$\begin{array}{c}\text { Discharge (ft } 3 / \mathrm{s} \text { ) for indicated } \\
\text { recurrence interval }\end{array}$} \\
\cline { 3 - 7 } (years)
\end{tabular}

\begin{tabular}{|c|c|c|c|c|c|c|}
\hline \multirow{2}{*}{ Period } & \multirow{2}{*}{$\begin{array}{l}\text { Number of } \\
\text { consecu- } \\
\text { tive days }\end{array}$} & \multicolumn{5}{|c|}{$\begin{array}{l}\text { Discharge }\left(\mathrm{ft}^{3} / \mathrm{s}\right) \text { for indicated } \\
\text { recurrence interval (years) }\end{array}$} \\
\hline & & 2 & 5 & 10 & 20 & 50 \\
\hline Sept. -Nov. & $\begin{array}{r}1 \\
7 \\
30\end{array}$ & $\begin{array}{l}65 \\
68 \\
84\end{array}$ & $\begin{array}{l}43 \\
45 \\
52\end{array}$ & $\begin{array}{l}35 \\
36 \\
42\end{array}$ & $\begin{array}{l}29 \\
31 \\
35\end{array}$ & $\begin{array}{l}24 \\
26 \\
30\end{array}$ \\
\hline Dec. -Feb. & $\begin{array}{r}1 \\
7 \\
30\end{array}$ & $\begin{array}{l}105 \\
119 \\
206\end{array}$ & $\begin{array}{l}60 \\
65 \\
99\end{array}$ & $\begin{array}{l}45 \\
48 \\
66\end{array}$ & $\begin{array}{l}36 \\
37 \\
47\end{array}$ & $\begin{array}{l}28 \\
27 \\
31\end{array}$ \\
\hline Mar. - May & $\begin{array}{r}1 \\
7 \\
30\end{array}$ & $\begin{array}{l}161 \\
178 \\
258\end{array}$ & $\begin{array}{r}98 \\
106 \\
149\end{array}$ & $\begin{array}{r}71 \\
77 \\
110\end{array}$ & $\begin{array}{l}54 \\
58 \\
84\end{array}$ & $\begin{array}{l}38 \\
41 \\
61\end{array}$ \\
\hline
\end{tabular}

Duration of daily flow for indicated periods

\begin{tabular}{|c|c|c|c|c|c|c|c|c|c|c|c|c|c|}
\hline \multirow{2}{*}{ Period } & \multicolumn{13}{|c|}{ Discharge $\left(\mathrm{ft}^{3} / \mathrm{s}\right)$ which was equaled or exceeded for indicated percent of time } \\
\hline & 98 & 95 & 90 & 85 & 80 & 75 & 70 & 60 & 50 & 40 & 30 & 20 & 10 \\
\hline $\begin{array}{l}\text { Apr. - Mar. } \\
\text { May - Nov. } \\
\text { June - Aug. } \\
\text { Sept. - Nov. } \\
\text { Dec. - Feb. } \\
\text { Mar. - May }\end{array}$ & $\begin{array}{l}38 \\
36 \\
36 \\
35 \\
42 \\
68\end{array}$ & $\begin{array}{l}46 \\
42 \\
46 \\
39 \\
52 \\
98\end{array}$ & $\begin{array}{r}60 \\
51 \\
61 \\
45 \\
67 \\
140\end{array}$ & $\begin{array}{r}72 \\
62 \\
74 \\
51 \\
81 \\
160\end{array}$ & $\begin{array}{r}84 \\
72 \\
84 \\
59 \\
97 \\
180\end{array}$ & $\begin{array}{r}97 \\
81 \\
94 \\
66 \\
130 \\
210\end{array}$ & $\begin{array}{r}110 \\
91 \\
100 \\
73 \\
160 \\
240\end{array}$ & $\begin{array}{r}150 \\
110 \\
120 \\
88 \\
220 \\
300\end{array}$ & $\begin{array}{l}190 \\
140 \\
150 \\
100 \\
300 \\
360\end{array}$ & $\begin{array}{l}260 \\
170 \\
180 \\
120 \\
380 \\
420\end{array}$ & $\begin{array}{l}350 \\
220 \\
220 \\
150 \\
490 \\
510\end{array}$ & $\begin{array}{l}480 \\
300 \\
290 \\
200 \\
670 \\
660\end{array}$ & $\begin{array}{r}780 \\
470 \\
460 \\
330 \\
1100 \\
1100\end{array}$ \\
\hline
\end{tabular}


LITTLE MIAMI RIVER BASIN

03242150 Caesar Creek near Xenia, Ohio

LOCATION:

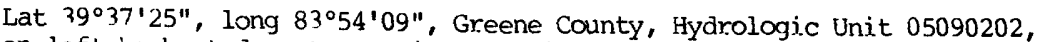
on left bank at downstream side of bridge on Winchester. Road, $0.2 \mathrm{mi}$. downstream from unnamed left bank tributary, $4.5 \mathrm{mi}$ south of Xenia, $7.4 \mathrm{mi}$ upstream from Anderson Fork, and at mile 22.1.

DRAINAGE AREA: $\quad 71.4 \mathrm{mi}^{2}$.

TRIBUTARY TO: Little Miami. River.

DISCHARCE DATA USED: Aujust 1968 to september 1979.

REMARKS:

Since 1964, some regulation by seasonal changes in storage in Lake Shawnee, $7.2 \mathrm{mi}$ upstream, drainage area $10.9 \mathrm{mi} 2$. Sumner storage is about 1,100 acre-ft more than wintcr.

SELECTED DISCHARGE CHARACTERISTICS: Average discharge: $81 \mathrm{ft}^{3} / \mathrm{s}(11$,years). Minimum daily discharge: $0.42 \mathrm{ft} / \mathrm{s}$ July 1977.

Magnitude and frequency of low flow for indicated periods

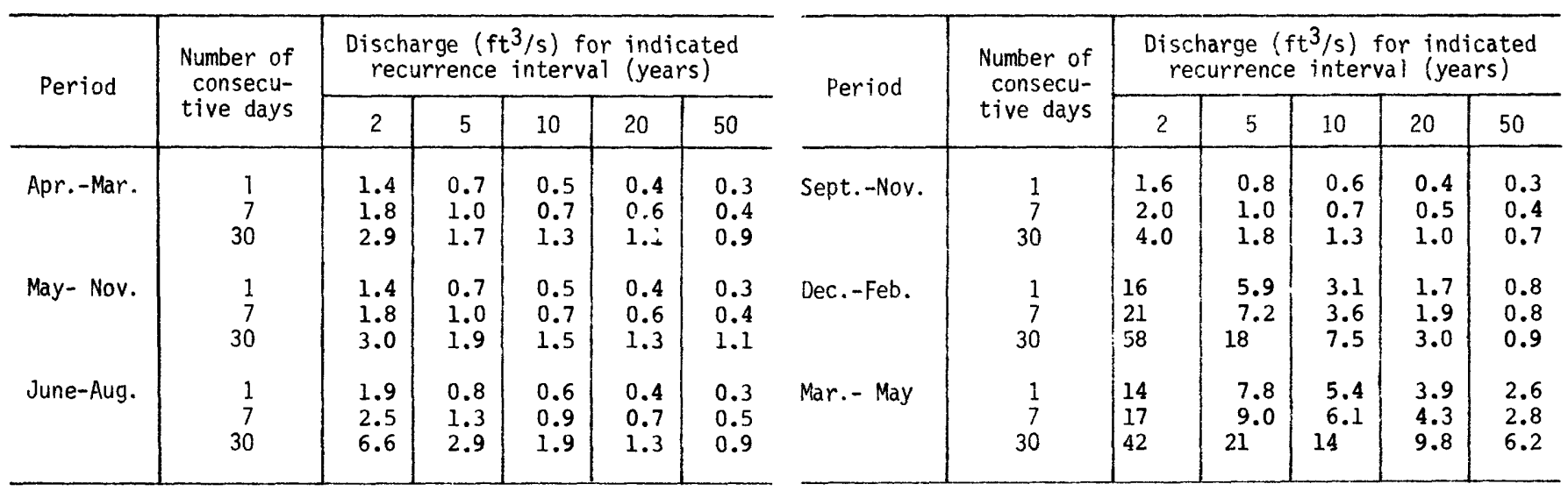

Duration of daily flow for indicated periods

\begin{tabular}{|c|c|c|c|c|c|c|c|c|c|c|c|c|c|}
\hline \multirow{2}{*}{ Period } & \multicolumn{13}{|c|}{ Discharge $\left(\mathrm{ft}^{3} / \mathrm{s}\right)$ which was equaled or exceeded for indicated percent of time } \\
\hline & 98 & 95 & 90 & 85 & 80 & 75 & 70 & 60 & 50 & 40 & 30 & 20 & 10 \\
\hline $\begin{array}{l}\text { Apr. - Mar. } \\
\text { May - Nov. } \\
\text { June - Aug. } \\
\text { Sept. - Nov. } \\
\text { Dec. - Feb. } \\
\text { Mar. - May }\end{array}$ & $\begin{array}{l}1.3 \\
1.0 \\
1.0 \\
0.8 \\
1.4 \\
6.4\end{array}$ & $\begin{array}{l}1.7 \\
1.5 \\
1.5 \\
1.3 \\
2.1 \\
12\end{array}$ & $\begin{array}{l}2.6 \\
2.1 \\
2.2 \\
1.6 \\
12 \\
16\end{array}$ & $\begin{array}{l}4.0 \\
2.7 \\
2.8 \\
1.9 \\
19 \\
21\end{array}$ & $\begin{array}{l}5.8 \\
3.5 \\
3.7 \\
2.3 \\
25 \\
26\end{array}$ & $\begin{array}{l}7.9 \\
4.5 \\
4.9 \\
2.8 \\
30 \\
30\end{array}$ & $\begin{array}{l}11 \\
5.7 \\
6.0 \\
3.4 \\
36 \\
35\end{array}$ & $\begin{array}{l}19 \\
8.4 \\
8.7 \\
5.3 \\
48 \\
43\end{array}$ & $\begin{array}{l}29 \\
13 \\
13 \\
8.1 \\
63 \\
52\end{array}$ & $\begin{array}{l}42 \\
19 \\
18 \\
12 \\
85 \\
71\end{array}$ & $\begin{array}{r}61 \\
29 \\
25 \\
21 \\
110 \\
100\end{array}$ & $\begin{array}{r}99 \\
45 \\
38 \\
38 \\
170 \\
140\end{array}$ & $\begin{array}{r}180 \\
91 \\
77 \\
91 \\
280 \\
290\end{array}$ \\
\hline
\end{tabular}


Appendix 3.--Low-flow data--Continued

LITTLE MIAMI RIVER BASIN

03242200 Anderson Fork near New Burlington, Ohio

LOCATION:

Lat 39033'59", long 83०54'10", Greene County, Hydrologic Unit 05090202, on right bank at downstream side of bridge on old winchester Trail, 1.0 $\mathrm{mi}$ downstream Irom rainters kun, $3.4 \mathrm{mi}$ cast of New Burlington, $5.0 \mathrm{mi}$ upstream from mouth, and at mile 29.7 .

DRAINAGE AREA:

$77.8 \mathrm{mi}^{2}$.

TRIBLITARY TO:

Caesar Creek.

DISCHARGE DATA USED:

July 1968 to September 1979.

REMARKS:

None.

SELECTED DISCHARGE CHARACTERISTICS: Average discharge: $87.7 \mathrm{ft}^{3} / \mathrm{s}$ (11 years)

Minimum daily discharge: $0.16 \mathrm{ft}^{3} / \mathrm{s}$ september 1970 .

Magnitude and frequency of low flow for indicated periods

\begin{tabular}{|c|c|c|c|c|c|c|c|c|c|c|c|c|c|}
\hline \multirow{2}{*}{ Period } & \multirow{2}{*}{$\begin{array}{l}\text { Number of } \\
\text { consecu- } \\
\text { tive days }\end{array}$} & \multicolumn{5}{|c|}{$\begin{array}{l}\text { Discharge }\left(\mathrm{ft}^{3} / \mathrm{s}\right) \text { for indicated } \\
\text { recurrence interval (years) }\end{array}$} & \multirow{2}{*}{ Period } & \multirow{2}{*}{$\begin{array}{l}\text { Number of } \\
\text { consecu- } \\
\text { tive days }\end{array}$} & \multicolumn{5}{|c|}{$\begin{array}{l}\text { Discharge }\left(\mathrm{ft}^{3} / \mathrm{s}\right) \text { for indicated } \\
\text { recurrence interval (years) }\end{array}$} \\
\hline & & 2 & 5 & 10 & 20 & 50 & & & 2 & 5 & 10 & 20 & 50 \\
\hline Apr.-Mar. & $\begin{array}{r}1 \\
7 \\
30\end{array}$ & $\begin{array}{l}1.2 \\
1.6 \\
3.1\end{array}$ & $\begin{array}{l}0.5 \\
0.6 \\
1.1\end{array}$ & $\begin{array}{l}0.3 \\
0.4 \\
0.6\end{array}$ & $\begin{array}{l}0.2 \\
0.3 \\
0.4\end{array}$ & $\begin{array}{l}0.1 \\
0.2 \\
0.2\end{array}$ & Sept.-Nov. & $\begin{array}{r}1 \\
7 \\
30\end{array}$ & $\begin{array}{l}1.7 \\
2.2 \\
5.2\end{array}$ & $\begin{array}{l}0.6 \\
0.8 \\
1.9\end{array}$ & $\begin{array}{l}0.3 \\
0.5 \\
1.0\end{array}$ & $\begin{array}{l}0.2 \\
0.3 \\
0.6\end{array}$ & $\begin{array}{l}0.1 \\
0.2 \\
0.3\end{array}$ \\
\hline June-Aug. & $\begin{array}{r}1 \\
7 \\
30\end{array}$ & $\begin{array}{l}1.9 \\
2.5 \\
6.4\end{array}$ & $\begin{array}{l}0.7 \\
1.1 \\
2.4\end{array}$ & $\begin{array}{l}0.5 \\
0.7 \\
1.5\end{array}$ & $\begin{array}{l}0.3 \\
0.5 \\
1.0\end{array}$ & $\begin{array}{l}0.2 \\
0.4 \\
0.6\end{array}$ & Mar. - May & $\begin{array}{r}1 \\
7 \\
30\end{array}$ & $\begin{array}{l}16 \\
20 \\
47\end{array}$ & $\begin{array}{l}9.7 \\
23\end{array}$ & $\begin{array}{r}6.7 \\
7.7 \\
14\end{array}$ & $\begin{array}{l}4.7 \\
5.3 \\
8.8\end{array}$ & $\begin{array}{l}3.0 \\
3.3 \\
4.7\end{array}$ \\
\hline
\end{tabular}

Duration of daily flow for indicated periods

\begin{tabular}{|c|c|c|c|c|c|c|c|c|c|c|c|c|c|}
\hline \multirow{2}{*}{ Period } & \multicolumn{13}{|c|}{ Discharge $\left(\mathrm{ft}^{3} / \mathrm{s}\right)$ which was equaled or exceeded for indicated percent of time } \\
\hline & 98 & 95 & 90 & 85 & 80 & 75 & 70 & 60 & 50 & 40 & 30 & 20 & 10 \\
\hline $\begin{array}{l}\text { Apr. - Mar. } \\
\text { May - Nov. } \\
\text { June - Aug. } \\
\text { Sept. - Nov. } \\
\text { Dec. - Feb. } \\
\text { Mar. - May }\end{array}$ & $\begin{array}{l}0.6 \\
0.6 \\
0.8 \\
0.4 \\
0.6 \\
5.8\end{array}$ & $\begin{array}{l}1.1 \\
1.0 \\
1.5 \\
0.8 \\
1.0 \\
13\end{array}$ & $\begin{array}{l}2.3 \\
1.8 \\
2.4 \\
1.2 \\
10 \\
18\end{array}$ & $\begin{array}{l}4.2 \\
2.7 \\
3.3 \\
1.5 \\
16 \\
22\end{array}$ & $\begin{array}{l}6.2 \\
3.8 \\
4.3 \\
1.9 \\
22 \\
25\end{array}$ & $\begin{array}{l}8.4 \\
5.0 \\
5.3 \\
2.4 \\
29 \\
30\end{array}$ & $\begin{array}{l}11 \\
6.2 \\
6.5 \\
3.2 \\
34 \\
35\end{array}$ & $\begin{array}{c}19 \\
9.0 \\
9.3 \\
6.0 \\
46 \\
43\end{array}$ & $\begin{array}{l}29 \\
13 \\
13 \\
8.3 \\
65 \\
54\end{array}$ & $\begin{array}{l}43 \\
20 \\
18 \\
13 \\
90 \\
71\end{array}$ & $\begin{array}{r}63 \\
30 \\
26 \\
21 \\
130 \\
100\end{array}$ & $\begin{array}{r}110 \\
48 \\
43 \\
37 \\
180 \\
160\end{array}$ & $\begin{array}{r}200 \\
92 \\
84 \\
84 \\
320 \\
300\end{array}$ \\
\hline
\end{tabular}


Appendix 3.--Low-flow data--Continued

LITTLE MIAMI RIVER BASIN

03243400 Cowan Creek at Clinton County A.F.B., Ohio

LOCATION:

Lat $39^{\circ} 24^{\prime} 25^{\prime \prime}$, long $83^{\circ} 47^{\prime} 55^{\prime \prime}$, Clinton County, Hydrologic

Unit 05090202, at bridge on Jenkins Road, at Clinton County

Air Force Base, $500 \mathrm{ft}$ upstream from Indian Run and $3.0 \mathrm{mi}$

southeast of Wilmington.

DRAINAGE AREA: $\quad 29.7 \mathrm{mi}^{2}$.

TRIBUTARY T0: Todd Fork

DISCHARGE DATA USED: Low-flow measurements 1959-1964 water years.

INDEX STATION: $\quad 03240000$ Little Miami River near Oldtown, Ohio.

REMARKS: None.

SELECTED DISCHARGE CHARACTERISTICS: Minimum discharge observed: No flow several times.

\begin{tabular}{c|c|c|c}
\multicolumn{3}{c}{ Magnitude and frequency of low flow for } \\
indicated periods \\
\hline Period & $\begin{array}{r}\text { Number of } \\
\text { consecu- } \\
\text { tive days }\end{array}$ & $\begin{array}{c}\text { Discharge (ft } 3 / \mathrm{s} \text { ) for } \\
\text { indicated recurrence } \\
\text { interval (years) }\end{array}$ \\
\cline { 3 - 4 } & 7 & 2 & 10 \\
\hline Apr. - Mar. & 30 & 0.1 & 0 \\
May - Nov. & 7 & 0.1 & 0 \\
June - Aug. & 30 & 0.1 & 0 \\
& 7 & 0.1 & 0 \\
Sept. - Nov. & 7 & 0.4 & 0 \\
& 30 & 0.1 & 0 \\
\hline
\end{tabular}

Duration of daily flow for indicated periods

\begin{tabular}{c|c|l|l|l|l}
\hline \multirow{2}{*}{ Period } & \multicolumn{5}{|c}{$\begin{array}{c}\text { Discharge ( } \mathrm{ft}^{3} / \mathrm{s} \text { ) which was equaled or } \\
\text { exceeded for ind icated percent of time }\end{array}$} \\
\cline { 2 - 6 } & 98 & 95 & 90 & 85 & 80 \\
\hline Apr. - Mar. & 0 & 0 & 0.1 & 0.1 & 0.2 \\
May - Nov. & 0 & 0 & 0 & 0.1 & 0.1 \\
June - Aug. & 0 & 0 & 0.1 & 0.2 & 0.2 \\
Sept. - Nov. & 0 & 0 & 0 & 0 & 0 \\
\hline
\end{tabular}


LOCATION:

Lat 39.03'09", long 84.03'02", Clermont County, Hydrologic Unit 05090202 , on right bank at downstream side of Main Street Bridge in 'williamsburg, $1.1 \mathrm{mi}$ upstream from Todd Run, and $2.4 \mathrm{mi}$ downstream from Crane kun.

DRAINAGE AREA: $\quad 237 \mathrm{mi}^{2}$.

TRIBUTARY TO: Little Miami River.

DISCHARGE DATA USED: March 1949 to September 1953, October 1960 to September 1974.

REMARKS: None.

SELECTED DISCHARGE CHARACTERISTICS: Average discharge: $277 \mathrm{ft} \mathrm{t}^{3} \mathrm{~s}$ (18 years). Minimum daily discharge: No flow for many days in 1951-1953, 1963-1965.

Magnitude and frequency of low flow for indicated periods

\begin{tabular}{|c|c|c|c|c|c|c|c|c|c|c|c|c|c|}
\hline \multirow{2}{*}{ Period } & \multirow{2}{*}{$\begin{array}{l}\text { Number of } \\
\text { consecu- } \\
\text { tive days }\end{array}$} & \multicolumn{5}{|c|}{$\begin{array}{l}\text { Discharge }\left(\mathrm{ft}^{3} / \mathrm{s}\right) \text { for indicated } \\
\text { recurrence interval (years) }\end{array}$} & \multirow{2}{*}{ Period } & \multirow{2}{*}{$\begin{array}{l}\text { Number of } \\
\text { consecu- } \\
\text { tive days }\end{array}$} & \multicolumn{5}{|c|}{$\begin{array}{l}\text { Discharge }\left(\mathrm{ft}^{3} / \mathrm{s}\right) \text { for indicated } \\
\text { recurrence interval (years) }\end{array}$} \\
\hline & & 2 & 5 & 10 & 20 & 50 & & & 2 & 5 & 10 & 20 & 50 \\
\hline Apr.-Mar. & $\begin{array}{r}1 \\
7 \\
30\end{array}$ & $\begin{array}{l}0.5 \\
0.8 \\
1.7\end{array}$ & $\begin{array}{l}0 \\
0.1 \\
0.3\end{array}$ & $\begin{array}{l}0 \\
0 \\
0.1\end{array}$ & $\begin{array}{l}0 \\
0 \\
0.1\end{array}$ & $\begin{array}{l}0 \\
0 \\
0\end{array}$ & Sept.-Nov. & $\begin{array}{r}1 \\
7 \\
30\end{array}$ & $\begin{array}{l}0.6 \\
1.1 \\
2.5\end{array}$ & $\begin{array}{l}0 \\
0.1 \\
0.3\end{array}$ & $\begin{array}{l}0 \\
0 \\
0.1\end{array}$ & $\begin{array}{l}0 \\
0 \\
0\end{array}$ & $\begin{array}{l}0 \\
0 \\
0\end{array}$ \\
\hline May- Nov. & $\begin{array}{r}1 \\
7 \\
30\end{array}$ & $\begin{array}{l}0.5 \\
0.8 \\
1.7\end{array}$ & $\begin{array}{l}0 \\
0.1 \\
0.3\end{array}$ & $\begin{array}{l}0 \\
0 \\
0.1\end{array}$ & $\begin{array}{l}0 \\
0 \\
0.1\end{array}$ & $\begin{array}{l}0 \\
0 \\
0\end{array}$ & Dec.-Feb. & $\begin{array}{r}1 \\
7 \\
30\end{array}$ & $\begin{array}{r}19 \\
29 \\
118\end{array}$ & $\begin{array}{l}5.8 \\
8.5 \\
36\end{array}$ & $\begin{array}{l}2.3 \\
3.5 \\
16\end{array}$ & $\begin{array}{l}0.8 \\
1.5 \\
6.6\end{array}$ & $\begin{array}{l}0.2 \\
0.4 \\
2.1\end{array}$ \\
\hline June-Aug. & $\begin{array}{r}1 \\
7 \\
30\end{array}$ & $\begin{array}{c}1.3 \\
2.2 \\
12\end{array}$ & $\begin{array}{l}0.2 \\
0.7 \\
3.7\end{array}$ & $\begin{array}{l}0 \\
0.4 \\
2.0\end{array}$ & $\begin{array}{l}0 \\
0 \\
1.2\end{array}$ & $\begin{array}{l}0 \\
0 \\
0.7\end{array}$ & Mar. - May & $\begin{array}{r}1 \\
7 \\
30\end{array}$ & $\begin{array}{l}22 \\
29 \\
92\end{array}$ & $\begin{array}{l}15 \\
18 \\
42\end{array}$ & $\begin{array}{l}12 \\
14 \\
27\end{array}$ & $\begin{array}{l}9.9 \\
11 \\
19\end{array}$ & $\begin{array}{r}7.9 \\
8.7 \\
12\end{array}$ \\
\hline
\end{tabular}

Duration of daily flow for indicated periods

\begin{tabular}{|c|c|c|c|c|c|c|c|c|c|c|c|c|c|c|}
\hline \multirow{2}{*}{\multicolumn{2}{|c|}{ Period }} & \multicolumn{13}{|c|}{ Discharge $\left(\mathrm{ft}^{3} / \mathrm{s}\right)$ which was equaled or exceeded for indicated percent of time } \\
\hline & & 98 & 95 & 90 & 85 & 80 & 75 & 70 & 60 & 50 & 40 & 30 & 20 & 10 \\
\hline $\begin{array}{l}\text { Apr. - } \\
\text { May - } \\
\text { June - } \\
\text { Sept. - } \\
\text { Dec. - } \\
\text { Mar. - }\end{array}$ & $\begin{array}{l}\text { Mar. } \\
\text { Nov. } \\
\text { Aug. } \\
\text { Nov. } \\
\text { Feb. } \\
\text { May }\end{array}$ & $\begin{array}{l}0.2 \\
0.1 \\
0.4 \\
0 \\
3.3 \\
15\end{array}$ & $\begin{array}{c}0.6 \\
0.2 \\
1.2 \\
0.1 \\
16 \\
22\end{array}$ & $\begin{array}{l}1.7 \\
0.8 \\
2.2 \\
0.2 \\
21 \\
32\end{array}$ & $\begin{array}{c}3.3 \\
1.5 \\
3.1 \\
0.4 \\
25 \\
42\end{array}$ & $\begin{array}{l}5.5 \\
2.3 \\
4.2 \\
0.7 \\
32 \\
50\end{array}$ & $\begin{array}{c}10 \\
3.3 \\
5.3 \\
1.1 \\
39 \\
58\end{array}$ & $\begin{array}{c}16 \\
4.7 \\
6.9 \\
1.5 \\
50 \\
68\end{array}$ & $\begin{array}{c}29 \\
9.7 \\
11 \\
2.9 \\
77 \\
91\end{array}$ & $\begin{array}{c}47 \\
18 \\
16 \\
5.3 \\
110 \\
130\end{array}$ & $\begin{array}{r}77 \\
28 \\
24 \\
15 \\
170 \\
180\end{array}$ & $\begin{array}{r}130 \\
43 \\
37 \\
29 \\
270 \\
270\end{array}$ & $\begin{array}{r}240 \\
74 \\
62 \\
51 \\
470 \\
500\end{array}$ & $\begin{array}{r}610 \\
190 \\
160 \\
140 \\
1200 \\
1200\end{array}$ \\
\hline
\end{tabular}


MILL CREEK BASIN

\author{
03255500 Mill Creek at Reading, Ohio
}

LOCATION:

Lat $39^{\circ} 13^{\prime} 14^{\prime \prime}$, long $84^{\circ} 26^{\prime} 49^{\prime \prime}$, in sec. 32, R.1, T.4, Hamilton County, Hydrologic Unit 05090203, on right bank at upstream side of Koehler street Bridge at Reading, $1.0 \mathrm{mi}$ upstream from West Fork Mill Creek, and $13.0 \mathrm{mi}$ upstream from mouth.

DRAINAGE AREA: $\quad 73.0 \mathrm{mi}^{2}$.

TRIBUTARY TO: Ohio River.

DISCHARGE DATA USED: April 1953 to september 1978.

REMARKS: $\quad$ Some diversion and ground water pumpage from Mill Creek and Great Miami

River basin by industrial plants of the greater Cincinnati area upstream trom station.

SELECTED DISCHARGE CHARACTERISTICS: Average discharge: $66.4 \mathrm{ft}^{3} / \mathrm{s}$ (25 years).

Minimum daily discharge: $1.8 \mathrm{ft}^{3} / \mathrm{s}$ September 1970 .

Magnitude and frequency of low flow for indicated periods

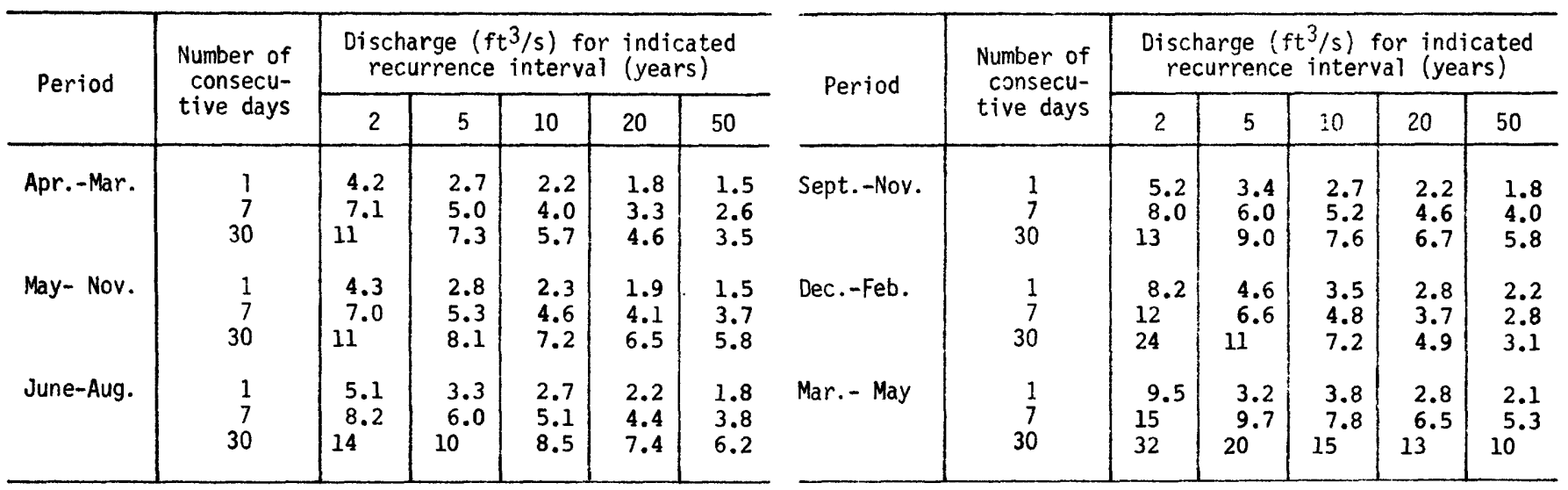

Duration of daily flow for indicated periods

\begin{tabular}{l|c|c|c|c|c|c|c|c|c|c|c|c|c}
\hline \multirow{2}{*}{ Period } & \multicolumn{8}{|c|}{ Discharge $(\mathrm{ft} / \mathrm{s}$ ) which was equaled or exceeded for indicated percent of time } \\
\cline { 2 - 13 } & 98 & 95 & 90 & 85 & 80 & 75 & 70 & 60 & 50 & 40 & 30 & 20 & 10 \\
\hline Apr. - Mar. & 4.7 & 6.0 & 7.4 & 8.6 & 10 & 12 & 13 & 16 & 21 & 29 & 42 & 66 & 140 \\
May - Nov. & 4.5 & 5.7 & 6.8 & 7.7 & 8.6 & 9.8 & 11 & 13 & 15 & 18 & 24 & 36 & 74 \\
June - Aug. & 4.2 & 5.4 & 6.6 & 7.6 & 8.6 & 9.8 & 11 & 13 & 15 & 19 & 26 & 35 & 76 \\
Sept.- Nov. & 4.6 & 5.8 & 6.7 & 7.5 & 8.2 & 8.9 & 9.9 & 12 & 14 & 16 & 19 & 26 & 51 \\
Dec. - Feb. & 4.7 & 6.2 & 7.8 & 9.6 & 12 & 14 & 17 & 23 & 30 & 42 & 57 & 91 & 170 \\
Mar. - May & 8.0 & 11 & 14 & 17 & 20 & 24 & 27 & 34 & 44 & 56 & 77 & 120 & 230 \\
\hline
\end{tabular}


MILL CREEK BASIN

03257500 West Fork Mill Creek at Woodland, Ohio

LOCATION:

Lat $39^{\circ} 15^{\prime} 14^{\prime \prime}$, long $84^{\circ} 28^{\prime} 13^{\prime \prime}$, in NEl/4 sec. 10, R.1, T.3, Hamilton County, Hydrologic Unit 05090203 , on left bank at upstream side of Riddle Road Bridge in woodlawn, $0.5 \mathrm{mi}$ upstream from small left bank tributary, $1.9 \mathrm{mi}$ downstream from West Fork Mill Creek Dam, and $4.0 \mathrm{mi}$ upstream from mouth.

DRAINAGE AREA: $\quad 32.2 \mathrm{mi}^{2}$.

TRIBITARY TO: Mill Creek.

DISCHARGE DATA USED: December 1952 to September 1978.

REMARKS:

Flow regulated by West Fork Mill Creek Reservoir $1.9 \mathrm{mi}$ upstream beginning 1953 .

SELECTED DISCHARGE CHARACTERISTICS: Average discharge: $32.8 \mathrm{ft}^{3} / \mathrm{s}$ (25 years).

Minimum daily discharge: No flow for many days most years.

Magnitude and frequency of low flow for indicated periods

\begin{tabular}{|c|c|c|c|c|c|c|c|c|c|c|c|c|c|}
\hline \multirow{2}{*}{ Period } & \multirow{2}{*}{$\begin{array}{l}\text { Number of } \\
\text { consecu- } \\
\text { tive days }\end{array}$} & \multicolumn{5}{|c|}{$\begin{array}{l}\text { Discharge }\left(\mathrm{ft}^{3} / \mathrm{s}\right) \text { for indicated } \\
\text { recurrence interval (years) }\end{array}$} & \multirow{2}{*}{ Periad } & \multirow{2}{*}{$\begin{array}{l}\text { Number of } \\
\text { consecu- } \\
\text { tive days }\end{array}$} & \multicolumn{5}{|c|}{$\begin{array}{l}\text { Discharge }\left(\mathrm{ft}^{3} / \mathrm{s}\right) \text { for indicated } \\
\text { recurrence interval (years) }\end{array}$} \\
\hline & & 2 & 5 & 10 & 20 & 50 & & & 2 & 5 & 10 & 20 & 50 \\
\hline Apr.-Mar. & $\begin{array}{r}1 \\
7 \\
30\end{array}$ & $\begin{array}{l}0 \\
0 \\
0.7\end{array}$ & $\begin{array}{l}0 \\
0 \\
0\end{array}$ & $\begin{array}{l}0 \\
0 \\
0\end{array}$ & $\begin{array}{l}0 \\
0 \\
0\end{array}$ & $\begin{array}{l}0 \\
0 \\
0\end{array}$ & Sept.-Nov. & $\begin{array}{r}1 \\
7 \\
30\end{array}$ & $\begin{array}{l}0 \\
0 \\
2.0\end{array}$ & $\begin{array}{l}0 \\
0 \\
0.2\end{array}$ & $\begin{array}{l}0 \\
0 \\
0\end{array}$ & $\begin{array}{l}0 \\
0 \\
0\end{array}$ & $\begin{array}{l}0 \\
0 \\
0\end{array}$ \\
\hline May- Nov. & $\begin{array}{r}1 \\
7 \\
30\end{array}$ & $\begin{array}{l}0 \\
0 \\
0.7\end{array}$ & $\begin{array}{l}0 \\
0 \\
0\end{array}$ & $\begin{array}{l}0 \\
0 \\
0\end{array}$ & $\begin{array}{l}0 \\
0 \\
0\end{array}$ & $\begin{array}{l}0 \\
0 \\
0\end{array}$ & Dec. -Feb. & $\begin{array}{r}1 \\
7 \\
30\end{array}$ & $\begin{array}{l}0.5 \\
1.4 \\
9.0\end{array}$ & $\begin{array}{l}0 \\
0.2 \\
3.7\end{array}$ & $\begin{array}{l}0 \\
0 \\
0.8\end{array}$ & $\begin{array}{l}0 \\
0 \\
0\end{array}$ & $\begin{array}{l}0 \\
0 \\
0\end{array}$ \\
\hline June-Aug. & $\begin{array}{r}1 \\
7 \\
30\end{array}$ & $\begin{array}{l}0 \\
0.1 \\
3.6\end{array}$ & $\begin{array}{l}0 \\
0 \\
1.3\end{array}$ & $\begin{array}{l}0 \\
0 \\
0.6\end{array}$ & $\begin{array}{l}0 \\
0 \\
0.2\end{array}$ & $\begin{array}{l}0 \\
0 \\
0\end{array}$ & Mar. - May & $\begin{array}{r}1 \\
7 \\
30\end{array}$ & $\begin{array}{c}0.8 \\
1.8 \\
10\end{array}$ & $\begin{array}{l}0.1 \\
0.5 \\
4.6\end{array}$ & $\begin{array}{l}0 \\
0.2 \\
2.9\end{array}$ & $\begin{array}{l}0 \\
0.1 \\
1.9\end{array}$ & $\begin{array}{l}0 \\
0 \\
1.2\end{array}$ \\
\hline
\end{tabular}

Duration of daily flow for indicated periods

\begin{tabular}{l|l|l|l|l|l|l|l|l|l|l|r|r|r|r}
\hline \multirow{2}{*}{ Period } & \multicolumn{8}{|c|}{ Discharge $\left(\mathrm{ft}^{3} / \mathrm{s}\right.$ ) which was equaled or exceeded for indicated percent of time } \\
\cline { 2 - 12 } & 98 & 95 & 90 & 85 & 80 & 75 & 70 & 60 & 50 & 40 & 30 & 20 & 10 \\
\hline Apr. - Mar. & 0 & 0 & 0.1 & 0.3 & 0.6 & 1.0 & 1.7 & 3.3 & 5.8 & 9.7 & 17 & 29 & 71 \\
May - Nov. & 0 & 0 & 0 & 0.1 & 0.2 & 0.3 & 0.5 & 1.2 & 2.4 & 4.0 & 7.6 & 15 & 34 \\
June - Aug. & 0 & 0 & 0 & 0.1 & 0.2 & 0.3 & 0.5 & 1.0 & 2.0 & 3.3 & 7.0 & 15 & 33 \\
Sept. - Nov. & 0 & 0 & 0 & 0 & 0 & 0.1 & 0.3 & 0.6 & 1.5 & 2.8 & 5.5 & 11 & 27 \\
Dec. - Feb. & 0 & 0.1 & 0.6 & 1.0 & 2.0 & 2.9 & 3.7 & 5.7 & 8.9 & 15 & 24 & 42 & 110 \\
Mar. - May & 0.2 & 0.7 & 2.1 & 3.2 & 4.1 & 5.4 & 6.8 & 10 & 15 & 22 & 32 & 48 & 140 \\
\hline
\end{tabular}


Appendix 3.--Low-flow data--Continued

MILL CREEK BASIN

03259000 Mill Creek at Carthage, Ohio

LOCATION: $\quad$ Lat $39^{\circ} 12^{\prime} 07^{\prime \prime}$, long $84^{\circ} 28^{\prime} 16^{\prime \prime}$, in SWl/4 sec.1, R.1, T.3, Hamilton County Hydrologic Unit 05090203 , on right bank $100 \mathrm{ft}$ downstream from Anthony Wayne Avenue Bridge in Carthage, $1.0 \mathrm{mi}$ downstream from West Fork Mill Creek, and $11.0 \mathrm{~m} \dot{i}$ upstream from mouth.

DRAINAGE AREA: $\quad 115 \mathrm{mi}^{2}$.

TRIBUTARY TO: Ohio River.

DISCHARGE DATA USED: April 1952 to September 1978.

REMARKS :

Some inter-basin transfers of water between Mill Creek and Great Miami River basins by industrial and municipal operations. Flow regulated by West Fork Mill Creek Reservoix, $6.9 \mathrm{mi}$ upstream, beginning 1953.

SELECTED DISCHARGE CHARACTERISTICS: Average discharge: $102 \mathrm{ft}^{3} / \mathrm{s}$ (26.years).

Minimum daily discharge: $0.3 \mathrm{ft} / \mathrm{s}$ November 1951.

Magnitude and frequency of low flow for indicated periods

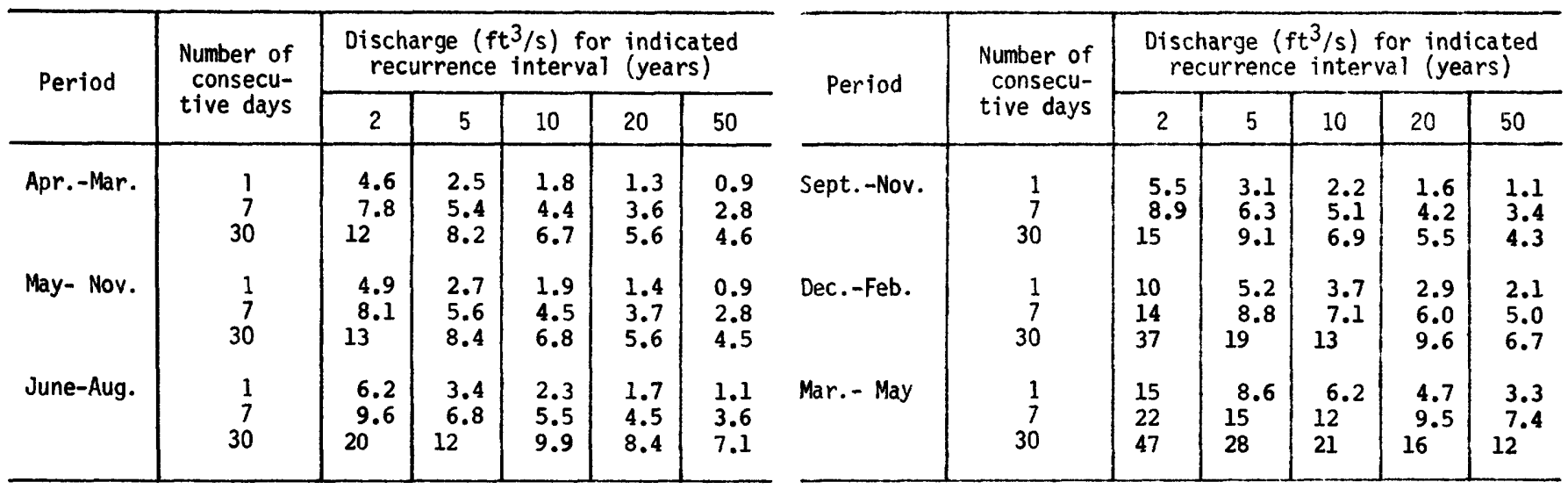

Duration of daily flow for indicated periods

\begin{tabular}{|c|c|c|c|c|c|c|c|c|c|c|c|c|c|}
\hline \multirow{2}{*}{ Period } & \multicolumn{13}{|c|}{ Discharge $\left(\mathrm{ft}^{3} / \mathrm{s}\right)$ which was equaled or exceeded for indicated percent of time } \\
\hline & 98 & 95 & 90 & 85 & 80 & 75 & 70 & 60 & 50 & 40 & 30 & 20 & 10 \\
\hline $\begin{array}{l}\text { Apr. - Mar. } \\
\text { May - Nov. } \\
\text { June - Aug. } \\
\text { Sept. - Nov. } \\
\text { Dec. - Feb. } \\
\text { Mar. - May }\end{array}$ & $\begin{array}{r}5.2 \\
4.4 \\
4.5 \\
4.0 \\
7.0 \\
11\end{array}$ & $\begin{array}{l}7.1 \\
6.0 \\
6.2 \\
5.4 \\
8.7 \\
15\end{array}$ & $\begin{array}{l}9.0 \\
7.7 \\
8.0 \\
7.0 \\
12 \\
20\end{array}$ & $\begin{array}{l}11 \\
9.1 \\
9.5 \\
8.0 \\
14 \\
25\end{array}$ & $\begin{array}{l}12 \\
10 \\
11 \\
9.0 \\
17 \\
29\end{array}$ & $\begin{array}{l}14 \\
11 \\
12 \\
10 \\
20 \\
33\end{array}$ & $\begin{array}{l}16 \\
13 \\
13 \\
11 \\
23 \\
37\end{array}$ & $\begin{array}{l}21 \\
15 \\
16 \\
13 \\
33 \\
48\end{array}$ & $\begin{array}{l}29 \\
19 \\
20 \\
15 \\
46 \\
63\end{array}$ & $\begin{array}{l}41 \\
24 \\
25 \\
19 \\
66 \\
85\end{array}$ & $\begin{array}{r}62 \\
34 \\
33 \\
25 \\
91 \\
110\end{array}$ & $\begin{array}{r}100 \\
52 \\
51 \\
38 \\
140 \\
190\end{array}$ & $\begin{array}{r}220 \\
120 \\
120 \\
84 \\
330 \\
440\end{array}$ \\
\hline
\end{tabular}


Appendix 3.--Low-flow data--Continued

GREAT MIAMI RIVER BASIN

03260600 Great Miami River at Russells Point, Ohio

LOCATION:

Lat $40^{\circ} 27^{\prime} 02^{\prime \prime}$, long $83^{\circ} 54^{\prime} 25^{\prime \prime}$, Logan County, Hydrologic Unit

05080001 , in $\mathrm{N} 1 / 2 \mathrm{sec}$. $11, \mathrm{~T} .7 \mathrm{~S} ., \mathrm{R} .8 \mathrm{E}$. , on a concrete bridge $2.7 \mathrm{mi}$ upstream from Muchinippi Creek.

DRAINAGE AREA: $\quad 133 \mathrm{mi}^{2}$.

TRIBUTARY TO: Ohio River.

DISCHARGE DATA USED: Low-flow measurements 1947-1949, 1957-1963 water years.

INDEX STATION: $\quad 03261500$ Great Miami River at Sidney, Ohio.

REMARKS:

Flow regulated by Indian Lake.

SELECTED DISCHARGE CHARACTERISTICS: Minimum discharge observed: $2.9 \mathrm{ft}^{3} / \mathrm{s}$ October 1963.

\begin{tabular}{l|c|c|c}
\multicolumn{4}{c}{$\begin{array}{c}\text { Magnitude and frequency of low flow for } \\
\text { indicated periods }\end{array}$} \\
\hline & $\begin{array}{c}\text { Number of } \\
\text { consecu- } \\
\text { tive days }\end{array}$ & $\begin{array}{c}\text { Discharge }\left(\mathrm{ft}^{3} / \mathrm{s}\right) \text { for } \\
\text { indicated } \\
\text { interval (yecurrence }\end{array}$ \\
\cline { 3 - 4 } & & 2 & 10 \\
\hline Apr. - Mar. & 7 & 5.0 & 3.2 \\
& 30 & 5.6 & 3.8 \\
May - Nov. & 7 & 5.0 & 3.2 \\
& 30 & 5.6 & 3.8 \\
June - Aug. & 7 & 5.8 & 3.8 \\
& 30 & 7.7 & 4.3 \\
Sept. - Nov. & 7 & 5.1 & 3.4 \\
& 30 & 6.1 & 4.0 \\
\hline
\end{tabular}

Duration of daily flow for indicated periods

\begin{tabular}{|c|c|c|c|c|c|}
\hline \multirow{2}{*}{ Period } & \multicolumn{5}{|c|}{$\begin{array}{l}\text { Discharge }\left(\mathrm{ft}^{3} / \mathrm{s}\right) \text { which was equaled or } \\
\text { exceeded for indicated percent of time }\end{array}$} \\
\hline & 98 & 95 & 90 & 85 & 80 \\
\hline $\begin{array}{l}\text { Apr. - Mar. } \\
\text { May - Nov. } \\
\text { June - Aug. } \\
\text { Sept. - Nov. }\end{array}$ & $\begin{array}{l}4.0 \\
3.7 \\
3.9 \\
3.5\end{array}$ & $\begin{array}{l}4.7 \\
4.3 \\
4.5 \\
3.9\end{array}$ & $\begin{array}{l}5.5 \\
5.0 \\
5.1 \\
4.5\end{array}$ & $\begin{array}{l}6.2 \\
5.4 \\
5.7 \\
5.0\end{array}$ & $\begin{array}{l}7.0 \\
5.9 \\
6.2 \\
5.3\end{array}$ \\
\hline
\end{tabular}


Appendix 3.--Low-flow data--Continued

GREAT MIAMI RIVER BASIN

03260620 Muchinippi Creek near Russells Point, Ohio

LOCATION: $\quad$ Lat $40^{\circ} 26^{\prime} 21^{\prime \prime}$, long $8^{\circ} 56^{\prime} 28^{\prime \prime}$, Logan County, Hydrologic Unit $05080001,2.3 \mathrm{mi}$ upstream from mouth at state Route 274 bridge, $3.5 \mathrm{mi}$ southwest of kussells Point.

DRAINAGE AREA: $\quad 86.2 \mathrm{mi}^{2}$.

TRIBUTARY TO:

Great Miami River.

DISCHARGE DATA USED:

Low-flow measurements 1959, 1972-1974 water years.

INDEX STATION:

03262000 Loramie Creek at Lockington, Ohio

REMARKS:

None.

SELECTED DISCHARGE CHARACTERISTICS: Minimum discharge observed: $1.8 \mathrm{ft}^{3} / \mathrm{s}$. September 1959.

Magnitude and frequency of low flow for indicated periods

\begin{tabular}{c|c|c|c}
\hline \multirow{2}{*}{ Period } & \multirow{2}{*}{$\begin{array}{c}\text { Number of } \\
\text { consecu- } \\
\text { tive days }\end{array}$} & \multicolumn{2}{|c}{$\begin{array}{c}\text { Discharge }\left(\mathrm{ft}^{3} / \mathrm{s} \text { ) for }\right. \\
\text { indicated recurrence } \\
\text { interval (years) }\end{array}$} \\
\cline { 3 - 4 } Apr. - Mar. & 7 & 2 & 10 \\
\hline May - Nov. & 30 & 1.3 & 0.7 \\
& 7 & 1.7 & 0.8 \\
June - Aug. & 30 & 1.3 & 0.7 \\
& 7 & 1.7 & 0.8 \\
Sept. - Nov. & 30 & 2.0 & 0.9 \\
& 7 & 3.6 & 1.3 \\
& 30 & 1.4 & 0.7 \\
\hline
\end{tabular}

Duration of daily flow for indicated periods

\begin{tabular}{|c|c|c|c|c|c|}
\hline \multirow{2}{*}{ Period } & \multicolumn{5}{|c|}{$\begin{array}{l}\text { Discharge }\left(\mathrm{ft}^{3} / \mathrm{s}\right) \text { which was equaled or } \\
\text { exceeded for indicated percent of time }\end{array}$} \\
\hline & 98 & 95 & 90 & 85 & 80 \\
\hline $\begin{array}{l}\text { Apr. - Mar. } \\
\text { May - Nov. } \\
\text { June - Aug. } \\
\text { Sept. - Nov. }\end{array}$ & $\begin{array}{l}0.9 \\
0.8 \\
0.9 \\
0.7\end{array}$ & $\begin{array}{l}1.2 \\
1.0 \\
1.3 \\
0.9\end{array}$ & $\begin{array}{l}1.7 \\
1.3 \\
1.7 \\
1.0\end{array}$ & $\begin{array}{l}2.1 \\
1.7 \\
2.1 \\
1.3\end{array}$ & $\begin{array}{l}2.6 \\
2.0 \\
2.5 \\
1.5\end{array}$ \\
\hline
\end{tabular}


LOCATION:

Lat $40^{\circ} 20^{\prime} 50^{\prime \prime}$, long $83^{\circ} 53^{\prime} 28^{\prime \prime}$, in El/2 sec. 3, R.14, T.2, Logan County, Hydrologic Unit 05080001 , on right bank at downstream side of county road bridge, $2 \mathrm{mi}$ downstream from Bluejacket Creek, $2.8 \mathrm{mi}$ northeast of DeGraff, and $4 \mathrm{mi}$ upstream from mouth.

DRAINAGE AREA: $\quad 36.3 \mathrm{mi}^{2}$.

TRIBUTARY TO: Great Miami River.

DISCHARGE DATA USED: October 1957 to September 1978.

REMARKS: Diurnal fluctuation caused by municipal plant operation in Bellefontaine, $9.8 \mathrm{mi}$ upstream; since storage capacity is small, daily flows are not affected appreciably.

SELECTED DISCHARGE CHARACTERISTICS: Average discharge: $32.3 \mathrm{ft}^{3} / \mathrm{s}$ ( 2$\}$ years).

Minimum daily discharge: $2.2 \mathrm{ft}^{3} / \mathrm{s}$ September, October 1963.

Magnitude and frequency of low flow for indicated periods

\begin{tabular}{|c|c|c|c|c|c|c|c|c|c|c|c|c|c|}
\hline \multirow{2}{*}{ Period } & \multirow{2}{*}{$\begin{array}{l}\text { Number of } \\
\text { consecu- } \\
\text { tive days }\end{array}$} & \multicolumn{5}{|c|}{$\begin{array}{l}\text { Discharge }\left(\mathrm{ft}^{3} / \mathrm{s}\right) \text { for indicated } \\
\text { recurrence interval (years) }\end{array}$} & \multirow{2}{*}{ Period } & \multirow{2}{*}{$\begin{array}{l}\text { Number of } \\
\text { consecu- } \\
\text { tive days }\end{array}$} & \multicolumn{5}{|c|}{$\begin{array}{l}\text { Discharge }\left(\mathrm{ft}^{3} / \mathrm{s}\right) \text { for indicated } \\
\text { recurrence interval (years) }\end{array}$} \\
\hline & & 2 & 5 & 10 & 20 & 50 & & & 2 & 5 & 10 & 20 & 50 \\
\hline Apr.-Mar. & $\begin{array}{r}1 \\
7 \\
30\end{array}$ & $\begin{array}{l}5.4 \\
5.9 \\
6.6\end{array}$ & $\begin{array}{l}3.8 \\
4.1 \\
4.7\end{array}$ & $\begin{array}{l}3.1 \\
3.4 \\
4.0\end{array}$ & $\begin{array}{l}2.7 \\
3.0 \\
3.5\end{array}$ & $\begin{array}{l}2.3 \\
2.6 \\
3.1\end{array}$ & Sept.-Nov. & $\begin{array}{r}1 \\
7 \\
30\end{array}$ & $\begin{array}{l}5.6 \\
6.2 \\
7.0\end{array}$ & $\begin{array}{l}3.8 \\
4.2 \\
4.8\end{array}$ & $\begin{array}{l}3.1 \\
3.5 \\
4.0\end{array}$ & $\begin{array}{l}2.7 \\
3.0 \\
3.6\end{array}$ & $\begin{array}{l}2.3 \\
2.6 \\
3.1\end{array}$ \\
\hline June-Aug. & $\begin{array}{r}1 \\
7 \\
30\end{array}$ & $\begin{array}{l}6.3 \\
7.0 \\
8.9\end{array}$ & $\begin{array}{l}4.7 \\
5.1 \\
6.1\end{array}$ & $\begin{array}{l}4.1 \\
4.5 \\
5.3\end{array}$ & $\begin{array}{l}3.7 \\
4.1 \\
4.9\end{array}$ & $\begin{array}{l}3.4 \\
3.7 \\
4.5\end{array}$ & Mar. - May & $\begin{array}{r}1 \\
7 \\
30\end{array}$ & $\begin{array}{l}14 \\
17 \\
26\end{array}$ & $\begin{array}{l}9.2 \\
11\end{array}$ & $\begin{array}{r}7.3 \\
9.4 \\
14\end{array}$ & $\begin{array}{l}6.0 \\
8.1 \\
11\end{array}$ & $\begin{array}{l}4.7 \\
6.8 \\
9.2\end{array}$ \\
\hline
\end{tabular}

Duration of daily flow for indicated periods

\begin{tabular}{|c|c|c|c|c|c|c|c|c|c|c|c|c|c|c|}
\hline \multirow{2}{*}{\multicolumn{2}{|c|}{ Period }} & \multicolumn{13}{|c|}{ Discharge $\left(\mathrm{ft}^{3} / \mathrm{s}\right)$ which was equaled or exceeded for indicated percent of time } \\
\hline & & 98 & 95 & 90 & 85 & 80 & 75 & 70 & 60 & 50 & 40 & 30 & 20 & 10 \\
\hline $\begin{array}{l}\text { Apr. } \\
\text { May } \\
\text { June } \\
\text { Sept. } \\
\text { Dec. - } \\
\text { Mar. - }\end{array}$ & $\begin{array}{l}\text { Mar. } \\
\text { Nov. } \\
\text { Aug. } \\
\text { Nov. } \\
\text { Feb. } \\
\text { May }\end{array}$ & $\begin{array}{c}4.2 \\
4.0 \\
4.8 \\
3.4 \\
4.2 \\
10\end{array}$ & $\begin{array}{r}4.9 \\
4.7 \\
5.5 \\
4.1 \\
4.8 \\
13\end{array}$ & $\begin{array}{r}5.7 \\
5.4 \\
6.5 \\
4.7 \\
5.6 \\
15\end{array}$ & $\begin{array}{r}6.6 \\
6.0 \\
6.8 \\
5.1 \\
7.2 \\
18\end{array}$ & $\begin{array}{r}7.4 \\
6.6 \\
7.3 \\
5.5 \\
9.0 \\
21\end{array}$ & $\begin{array}{l}8.4 \\
7.2 \\
7.8 \\
5.9 \\
12 \\
24\end{array}$ & $\begin{array}{l}9.8 \\
7.8 \\
8.4 \\
6.3 \\
14 \\
26\end{array}$ & $\begin{array}{c}13 \\
9.5 \\
9.9 \\
7.2 \\
18 \\
32\end{array}$ & $\begin{array}{c}17 \\
12 \\
13 \\
8.5 \\
23 \\
37\end{array}$ & $\begin{array}{l}23 \\
15 \\
16 \\
10 \\
29 \\
44\end{array}$ & $\begin{array}{l}31 \\
19 \\
19 \\
13 \\
37 \\
54\end{array}$ & $\begin{array}{l}43 \\
26 \\
25 \\
16 \\
52 \\
72\end{array}$ & $\begin{array}{r}70 \\
41 \\
39 \\
22 \\
83 \\
110\end{array}$ \\
\hline
\end{tabular}


GREAT MIAMI RIVER BASIN

03260800 Stony Creek near DeGraff, Ohio

LOCATION:

Lat $40^{\circ} 17^{\prime} 27^{\prime \prime}$, long $83^{\circ} 54^{\prime} 36^{\prime \prime}$, in NWl/4 sec.5, R.13, T.3, Lojän County, Hydrologic Unit ij 5 uouuvi, on right bank at downstream side of county road bridge, $0.6 \mathrm{mi}$ downstream from Lee Creek, $1.5 \mathrm{mi}$ south of DeGraff, and $1.5 \mathrm{mi}$ upstream from mouth.

DRAINAGE AREA: $\quad 59.1 \mathrm{mi}^{2}$.

TRIBUTARY TO: Great Miami River.

DISCHARGE DATA USED: October 1957 to September 1975.

REMARKS: None.

SELECTED DISCHARGE CHARACTERISTICS: Average discharge: $53.2 \mathrm{ft}^{3} / \mathrm{s}_{3}$ (18 years).

Minimum daily discharge: $5 \mathrm{ft}^{3} / \mathrm{s}$ september 1963.

Magnitude and frequency of low flow for indicated periods

\begin{tabular}{|c|c|c|c|c|c|c|c|c|c|c|c|c|c|}
\hline \multirow{2}{*}{ Period } & \multirow{2}{*}{$\begin{array}{l}\text { Number of } \\
\text { consecu- } \\
\text { tive days }\end{array}$} & \multicolumn{5}{|c|}{$\begin{array}{l}\text { Discharge }\left(\mathrm{ft}^{3} / \mathrm{s}\right) \text { for indicated } \\
\text { recurrence interval (years) }\end{array}$} & \multirow{2}{*}{ Period } & \multirow{2}{*}{$\begin{array}{l}\text { Number of } \\
\text { consecu- } \\
\text { tive days }\end{array}$} & \multicolumn{5}{|c|}{$\begin{array}{l}\text { Discharge }\left(\mathrm{ft}^{3} / \mathrm{s}\right) \text { for indicated } \\
\text { recurrence intervai (years) }\end{array}$} \\
\hline & & 2 & 5 & 10 & 20 & 50 & & & 2 & 5 & 10 & 20 & 50 \\
\hline Apr.-Mar. & $\begin{array}{r}1 \\
7 \\
30\end{array}$ & $\begin{array}{l}11 \\
11 \\
12\end{array}$ & $\begin{array}{l}7.4 \\
7.7 \\
8.5\end{array}$ & $\begin{array}{l}6.1 \\
6.4 \\
7.1\end{array}$ & $\begin{array}{l}5.2 \\
5.5 \\
6.2\end{array}$ & $\begin{array}{l}4.3 \\
4.6 \\
5.4\end{array}$ & Sept.-Nov. & $\begin{array}{r}1 \\
7 \\
30\end{array}$ & $\begin{array}{l}11 \\
12 \\
14\end{array}$ & $\begin{array}{l}7.6 \\
8.0 \\
8.9\end{array}$ & $\begin{array}{l}6.2 \\
6.6 \\
7.3\end{array}$ & $\begin{array}{l}5.3 \\
5.6 \\
6.2\end{array}$ & $\begin{array}{l}4.4 \\
4.7 \\
5.3\end{array}$ \\
\hline June-Aug. & $\begin{array}{r}1 \\
7 \\
30\end{array}$ & $\begin{array}{l}12 \\
13 \\
16\end{array}$ & $\begin{array}{r}8.5 \\
8.9 \\
10\end{array}$ & $\begin{array}{l}7.2 \\
7.4 \\
8.1\end{array}$ & $\begin{array}{l}6.2 \\
6.4 \\
6.9\end{array}$ & $\begin{array}{l}5.4 \\
5.5 \\
5.9\end{array}$ & Mar. - May & $\begin{array}{r}1 \\
7 \\
30\end{array}$ & $\begin{array}{l}25 \\
29 \\
40\end{array}$ & $\begin{array}{l}17 \\
21 \\
28\end{array}$ & $\begin{array}{l}14 \\
18 \\
23\end{array}$ & $\begin{array}{l}12 \\
15 \\
19\end{array}$ & $\begin{array}{l}9.6 \\
13 \\
15\end{array}$ \\
\hline
\end{tabular}

Duration of daily flow for indicated periods

\begin{tabular}{l|c|c|c|c|c|c|c|c|c|c|c|c|c|c}
\hline \multirow{2}{*}{ Period } & \multicolumn{8}{|c|}{ Discharge $\left(\mathrm{ft}^{3} / \mathrm{s}\right)$ which was equaled or exceeded for indicated percent of time } \\
\cline { 2 - 13 } & 98 & 95 & 90 & 85 & 80 & 75 & 70 & 60 & 50 & 40 & 30 & 20 & 10 \\
\hline Apr. - Mar. & 7.3 & 8.7 & 10 & 12 & 14 & 16 & 18 & 22 & 28 & 36 & 48 & 66 & 110 \\
May - Nov. & 6.7 & 8.0 & 9.2 & 10 & 12 & 13 & 14 & 17 & 21 & 25 & 31 & 42 & 62 \\
June - Aug. & $\mathbf{7 . 4}$ & $\mathbf{8 . 5}$ & 9.6 & 11 & 13 & 14 & 15 & 18 & 22 & 26 & 33 & 42 & 62 \\
Sept. - Nov. & $\mathbf{6 . 0}$ & $\mathbf{7 . 1}$ & $\mathbf{8 . 6}$ & 9.2 & 9.8 & 11 & 12 & 14 & 16 & 19 & 23 & 29 & 40 \\
Dec. - Feb. & 8.1 & 10 & 12 & 14 & 17 & 20 & 24 & 30 & 36 & 46 & 60 & 84 & 140 \\
Mar. - May & 19 & 22 & 24 & 28 & 32 & 36 & 40 & 48 & 55 & 65 & 81 & 110 & 170 \\
\hline
\end{tabular}


GREAT MIAMI RIVER BASIN

03261500 Great Miami River at Sidney, Ohio

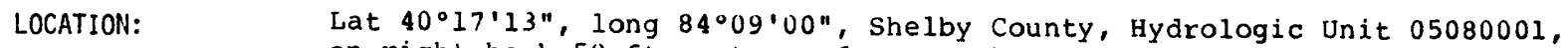
on right bank $50 \mathrm{ft}$ upstream from North street Bridge in Sidney, and 0.5 mi downstream from Tawawa Creek.

DRAINAGE AREA: $\quad 5 \div 1 \mathrm{mi}^{2}$.

TRIBUTARY TO: Ohio River.

DISCHARGE DATA USED: October 1926 to September. 1978.

REMARKS: Water supply for city of Sidney is pumped from the Great Miami River $1,200 \mathrm{ft}$ upstream and from wells adjacent to Great Miami River upstream from station. The pumpage averaged $4.6 \mathrm{ft}^{3} / \mathrm{s}$ in 1978 and is returned as sewage $1.2 \mathrm{mi}$ downstream from the station. Some regulation by Indian Lake, $28 \mathrm{mi}$ upstream, capacity 45,900 acre-ft prior to 1926 ; water diverted into Miami and Erie Canal at Port Jefferson, $2.8 \mathrm{mi}$ upstream, prior to 1926; amount of diversion not

SELECTED DISCHARGE CHARACTERISTICS: Average discharge: $471 \mathrm{ft}^{3} / \mathrm{s}(52$ years). Minimum daily discharge: $8.0 \mathrm{ft} / \mathrm{s}$ September 1935.

Magnitude and frequency of low flow for indicated periods

\begin{tabular}{|c|c|c|c|c|c|c|}
\hline \multirow{2}{*}{ Period } & \multirow{2}{*}{$\begin{array}{l}\text { Number of } \\
\text { consecu- } \\
\text { tive days }\end{array}$} & \multicolumn{5}{|c|}{$\begin{array}{l}\text { Discharge }\left(\mathrm{ft}^{3} / \mathrm{s}\right) \text { for indicated } \\
\text { recurrence interval (years) }\end{array}$} \\
\hline & & 2 & 5 & 10 & 20 & 50 \\
\hline Apr.-Mar. & $\begin{array}{r}1 \\
7 \\
30\end{array}$ & $\begin{array}{l}33 \\
37 \\
44\end{array}$ & $\begin{array}{l}21 \\
25 \\
31\end{array}$ & $\begin{array}{l}16 \\
21 \\
26\end{array}$ & $\begin{array}{l}13 \\
18 \\
23\end{array}$ & $\begin{array}{l}10 \\
16 \\
20\end{array}$ \\
\hline May- Nov. & $\begin{array}{r}1 \\
7 \\
30\end{array}$ & $\begin{array}{l}33 \\
37 \\
44\end{array}$ & $\begin{array}{l}21 \\
26 \\
31\end{array}$ & $\begin{array}{l}16 \\
21 \\
26\end{array}$ & $\begin{array}{l}13 \\
18 \\
23\end{array}$ & $\begin{array}{l}10 \\
16 \\
20\end{array}$ \\
\hline June-Aug. & $\begin{array}{r}1 \\
7 \\
30\end{array}$ & $\begin{array}{l}40 \\
46 \\
68\end{array}$ & $\begin{array}{l}26 \\
32 \\
40\end{array}$ & $\begin{array}{l}21 \\
26 \\
31\end{array}$ & $\begin{array}{l}18 \\
23 \\
27\end{array}$ & $\begin{array}{l}15 \\
20 \\
22\end{array}$ \\
\hline
\end{tabular}

\begin{tabular}{c|c|c|c|c|c|c}
\hline \multirow{2}{*}{ Period } & \multirow{2}{*}{$\begin{array}{c}\text { Number of } \\
\text { consecu- } \\
\text { tive days }\end{array}$} & \multicolumn{3}{|c}{$\begin{array}{c}\text { Discharge } \\
\text { recurrence } 3 / \mathrm{s} \text { ) }\end{array}$ for interval $\begin{array}{c}\text { indicated } \\
\text { (years) }\end{array}$} \\
\cline { 3 - 7 } & 2 & 5 & 10 & 20 & 50 \\
\hline Sept.-Nov. & 1 & 35 & 22 & 17 & 13 & 10 \\
& 7 & 39 & 27 & 22 & 19 & 16 \\
Dec.-Feb. & 30 & 49 & 33 & 28 & 25 & 23 \\
& 1 & 74 & 45 & 35 & 29 & 23 \\
& 7 & 86 & 51 & 39 & 32 & 25 \\
& 30 & 175 & 80 & 54 & 39 & 27 \\
& 1 & 119 & 75 & 58 & 46 & 34 \\
& 7 & 138 & 90 & 72 & 61 & 50 \\
& 30 & 245 & 145 & 112 & 91 & 72 \\
\hline
\end{tabular}

Duration of daily flow for indicated periods

\begin{tabular}{|c|c|c|c|c|c|c|c|c|c|c|c|c|c|}
\hline \multirow{2}{*}{ Period } & \multicolumn{13}{|c|}{ Discharge $\left(\mathrm{ft}^{3} / \mathrm{s}\right)$ which was equaled or exceeded for indicated percent of time } \\
\hline & 98 & 95 & 90 & 85 & 80 & 75 & 70 & 60 & 50 & 40 & 30 & 20 & 10 \\
\hline $\begin{array}{l}\text { Apr. - Mar. } \\
\text { May - Nov. } \\
\text { June - Aug. } \\
\text { Sept. - Nov. } \\
\text { Dec. - Feb. } \\
\text { Mar. - May }\end{array}$ & $\begin{array}{l}28 \\
25 \\
27 \\
23 \\
40 \\
79\end{array}$ & $\begin{array}{r}35 \\
31 \\
33 \\
27 \\
46 \\
100\end{array}$ & $\begin{array}{r}43 \\
37 \\
39 \\
33 \\
57 \\
130\end{array}$ & $\begin{array}{r}50 \\
42 \\
45 \\
37 \\
68 \\
150\end{array}$ & $\begin{array}{r}59 \\
47 \\
51 \\
41 \\
83 \\
180\end{array}$ & $\begin{array}{r}70 \\
54 \\
58 \\
44 \\
110 \\
210\end{array}$ & $\begin{array}{r}82 \\
60 \\
65 \\
48 \\
130 \\
240\end{array}$ & $\begin{array}{r}120 \\
77 \\
82 \\
57 \\
190 \\
320\end{array}$ & $\begin{array}{r}170 \\
100 \\
100 \\
69 \\
270 \\
430\end{array}$ & $\begin{array}{r}240 \\
130 \\
140 \\
88 \\
380 \\
570\end{array}$ & $\begin{array}{l}360 \\
180 \\
190 \\
120 \\
570 \\
780\end{array}$ & $\begin{array}{r}590 \\
280 \\
280 \\
170 \\
910 \\
1200\end{array}$ & $\begin{array}{r}1200 \\
510 \\
540 \\
290 \\
1800 \\
1900\end{array}$ \\
\hline
\end{tabular}


GREAT MIAMI RIVER BASIN

03261950 Loramie Creek near Newport, Ohio

LOCATION:

Lat $40^{\circ} 18^{\prime} 25^{\prime \prime}$; long $84^{\circ} 23^{\prime} 02^{\prime \prime}$, in SEl/4 sec.24, T.11 N., R.4 E., Shelby County, Hydrologic Unit 05080001, right bank at downstream side of bridge on Cardo Roman Road, $1.1 \mathrm{mi}$ northwest of Newport, $3 \mathrm{mi}$ south of Fort Loramie, $3 \mathrm{mi}$ downstream from Mile Creek, and at mile 16.5 .

DRAINAGE AREA: $\quad 152 \mathrm{mi}^{2}$.

TRIBITARY TO: Great Miami River.

DISCHARGE DATA USED: October 1964 to September 1978.

REMARKS:

Some regulation by Lake Loramie $5 \mathrm{mi}$ upstream.

SELECTED DISCHARGE CHARACTERISTICS: Average discharge: $128 \mathrm{ft}^{3} / \mathrm{s}$ ( 14 years).

Minimum daily discharge: $0.1 \mathrm{ft}^{3} / \mathrm{s}$ August 1965 , September 1966.

Magnitude and frequency of low flow for indicated periods

\begin{tabular}{|c|c|c|c|c|c|c|c|c|c|c|c|c|c|}
\hline \multirow{2}{*}{ Period } & \multirow{2}{*}{$\begin{array}{l}\text { Number of } \\
\text { consecu- } \\
\text { tive days }\end{array}$} & \multicolumn{5}{|c|}{$\begin{array}{l}\text { Discharge }\left(\mathrm{ft}^{3} / \mathrm{s}\right) \text { for indicated } \\
\text { recurrence interval (years) }\end{array}$} & \multirow{2}{*}{ Period } & \multirow{2}{*}{$\begin{array}{l}\text { Number of } \\
\text { consecu- } \\
\text { tive days }\end{array}$} & \multicolumn{5}{|c|}{$\begin{array}{l}\text { Discharge }\left(\mathrm{ft}^{3} / \mathrm{s}\right) \text { for indicated } \\
\text { recurrence interval (years) }\end{array}$} \\
\hline & & 2 & 5 & 10 & 20 & 50 & & & 2 & 5 & 10 & 20 & 50 \\
\hline Apr. -Mar. & $\begin{array}{r}1 \\
7 \\
30\end{array}$ & $\begin{array}{l}0.5 \\
0.7 \\
1.4\end{array}$ & $\begin{array}{l}0.2 \\
0.3 \\
0.6\end{array}$ & $\begin{array}{l}0.1 \\
0.2 \\
0.3\end{array}$ & $\begin{array}{l}0.1 \\
0.1 \\
0.1\end{array}$ & $\begin{array}{l}0 \\
0 \\
0.1\end{array}$ & Sept.-Nov. & $\begin{array}{r}1 \\
7 \\
30\end{array}$ & $\begin{array}{l}0.6 \\
1.0 \\
2.2\end{array}$ & $\begin{array}{l}0.3 \\
0.4 \\
1.0\end{array}$ & $\begin{array}{l}0.2 \\
0.3 \\
0.8\end{array}$ & $\begin{array}{l}0.1 \\
0.1 \\
0.7\end{array}$ & $\begin{array}{l}0 \\
0.1 \\
0.6\end{array}$ \\
\hline June-Aug. & $\begin{array}{r}1 \\
7 \\
30\end{array}$ & $\begin{array}{l}0.8 \\
1.0 \\
3.0\end{array}$ & $\begin{array}{l}0.3 \\
0.4 \\
0.9\end{array}$ & $\begin{array}{l}0.2 \\
0.3 \\
0.5\end{array}$ & $\begin{array}{l}0.1 \\
0.2 \\
0.3\end{array}$ & $\begin{array}{l}0 \\
0.1 \\
0.1\end{array}$ & Mar.- May & $\begin{array}{r}1 \\
7 \\
30\end{array}$ & $\begin{array}{l}8.0 \\
12 \\
36\end{array}$ & $\begin{array}{r}3.1 \\
4.1 \\
17\end{array}$ & $\begin{array}{r}1.4 \\
1.8 \\
11\end{array}$ & $\begin{array}{l}0.5 \\
0.8 \\
8.3\end{array}$ & $\begin{array}{l}0.1 \\
0.2 \\
5.8\end{array}$ \\
\hline
\end{tabular}

Duration of daily flow for indicated periods

\begin{tabular}{|c|c|c|c|c|c|c|c|c|c|c|c|c|c|}
\hline \multirow{2}{*}{ Period } & \multicolumn{13}{|c|}{ Discharge $\left(\mathrm{ft}^{3} / \mathrm{s}\right)$ which was equaled or exceeded for indicated percent of time } \\
\hline & 98 & 95 & 90 & 85 & 80 & 75 & 70 & 60 & 50 & 40 & 30 & 20 & 10 \\
\hline $\begin{array}{l}\text { Apr. - Mar. } \\
\text { May - Nov. } \\
\text { June - Aug. } \\
\text { Sept. - Nov. } \\
\text { Dec. - Feb. } \\
\text { Mar. - May }\end{array}$ & $\begin{array}{l}0.4 \\
0.3 \\
0.3 \\
0.3 \\
0.6 \\
4.0\end{array}$ & $\begin{array}{l}0.7 \\
0.6 \\
0.6 \\
0.5 \\
1.1 \\
6.9\end{array}$ & $\begin{array}{l}1.2 \\
0.9 \\
0.8 \\
0.8 \\
2.0 \\
12\end{array}$ & $\begin{array}{l}1.7 \\
1.1 \\
1.1 \\
1.1 \\
3.7 \\
16\end{array}$ & $\begin{array}{l}2.5 \\
1.5 \\
1.4 \\
1.3 \\
7.3 \\
21\end{array}$ & $\begin{array}{l}3.4 \\
1.9 \\
1.8 \\
1.6 \\
11 \\
25\end{array}$ & $\begin{array}{r}4.7 \\
2.4 \\
2.2 \\
2.0 \\
17 \\
31\end{array}$ & $\begin{array}{l}10 \\
3.7 \\
3.2 \\
2.8 \\
26 \\
45\end{array}$ & $\begin{array}{l}20 \\
5.9 \\
5.1 \\
3.9 \\
44 \\
68\end{array}$ & $\begin{array}{c}34 \\
11 \\
9.0 \\
5.9 \\
83 \\
110\end{array}$ & $\begin{array}{r}66 \\
21 \\
17 \\
11 \\
150 \\
180\end{array}$ & $\begin{array}{r}150 \\
41 \\
30 \\
27 \\
270 \\
320\end{array}$ & $\begin{array}{r}360 \\
110 \\
85 \\
96 \\
580 \\
660\end{array}$ \\
\hline
\end{tabular}


LOCATION:

Lat $40^{\circ} 12^{\prime} 35^{\prime \prime}$, long $84^{\circ} 14^{\prime} 32^{\prime \prime}$, in NE1/4 sec.30, T.7 N., R.6 E., Shelby County, Hydrologic Unit 05080001 , on left bank at downstream side of county road bridge, $1,300 \mathrm{ft}$ downstream from Lockington Dam, $0.5 \mathrm{mi}$ northwest of Lockington, and at mile 1.9 .

DRAINAGE AREA: $\quad 257 \mathrm{mi}^{2}$.

TRIBUTARY TO: Great Miami River.

DISCHARGE DATA USED: October $\perp$ y 15 to September 1978.

REMARKS: Slight regulation by Lake Loramie $18 \mathrm{mi}$ upstream. Flood flow regulated by Lockington retarding basin beginning 1921 .

SELECTED DISCHARGE CHARACTERISTICS: Average discharge: $206 \mathrm{ft}^{3} / \mathrm{s}$ (63 years). Minimum daily discharge: $1.7 \mathrm{ft}^{3} / \mathrm{s}$ September 1977.

Magnitude and frequency of low flow for indicated periods

\begin{tabular}{|c|c|c|c|c|c|c|c|c|c|c|c|c|c|}
\hline \multirow{2}{*}{ Period } & \multirow{2}{*}{$\begin{array}{l}\text { Number of } \\
\text { consecu- } \\
\text { tive days }\end{array}$} & \multicolumn{5}{|c|}{$\begin{array}{l}\text { Discharge }\left(\mathrm{ft}^{3} / \mathrm{s}\right) \text { for indicated } \\
\text { recurrence interva1 (years) }\end{array}$} & \multirow{2}{*}{ Period } & \multirow{2}{*}{$\begin{array}{l}\text { Number of } \\
\text { consecu- } \\
\text { tive days }\end{array}$} & \multicolumn{5}{|c|}{$\begin{array}{l}\left.\text { Discharge ( } \mathrm{ft}^{3} / \mathrm{s}\right) \text { for indicated } \\
\text { recurrence interval (years) }\end{array}$} \\
\hline & & 2 & 5 & 10 & 20 & 50 & & & 2 & 5 & 10 & 20 & 50 \\
\hline Apr.-Mar. & $\begin{array}{r}1 \\
7 \\
30\end{array}$ & $\begin{array}{l}5.2 \\
5.6 \\
6.9\end{array}$ & $\begin{array}{l}3.4 \\
3.8 \\
4.6\end{array}$ & $\begin{array}{l}2.8 \\
3.3 \\
3.8\end{array}$ & $\begin{array}{l}2.4 \\
2.9 \\
3.4\end{array}$ & $\begin{array}{l}2.1 \\
2.6 \\
3.0\end{array}$ & Sept.-Nov. & $\begin{array}{r}1 \\
7 \\
30\end{array}$ & $\begin{array}{l}5.5 \\
6.0 \\
8.0\end{array}$ & $\begin{array}{l}3.6 \\
3.9 \\
4.7\end{array}$ & $\begin{array}{l}3.0 \\
3.3 \\
3.9\end{array}$ & $\begin{array}{l}2.6 \\
3.0 \\
3.5\end{array}$ & $\begin{array}{l}2.2 \\
2.7 \\
3.2\end{array}$ \\
\hline June-Aug. & $\begin{array}{r}1 \\
7 \\
30\end{array}$ & $\begin{array}{l}7.3 \\
8.2 \\
13\end{array}$ & $\begin{array}{l}4.7 \\
5.3 \\
7.2\end{array}$ & $\begin{array}{l}3.8 \\
4.3 \\
5.6\end{array}$ & $\begin{array}{l}3.2 \\
3.7 \\
4.6\end{array}$ & $\begin{array}{l}2.7 \\
3.2 \\
3.9\end{array}$ & Mar. - May & $\begin{array}{r}1 \\
7 \\
30\end{array}$ & $\begin{array}{l}25 \\
30 \\
72\end{array}$ & $\begin{array}{l}15 \\
19 \\
37\end{array}$ & $\begin{array}{l}12 \\
15 \\
26\end{array}$ & $\begin{array}{l}9.2 \\
12 \\
19\end{array}$ & $\begin{array}{r}7.0 \\
9.1 \\
14\end{array}$ \\
\hline
\end{tabular}

Duration of daily flow for indicated periods

\begin{tabular}{l|c|c|c|c|c|c|c|c|c|c|c|c|c}
\hline \multirow{2}{*}{ Period } & \multicolumn{8}{|c|}{ Discharge $\left(\mathrm{ft}^{3} / \mathrm{s}\right)$ which was equaled or exceeded for indicated percent of time } \\
\cline { 2 - 13 } & 98 & 95 & 90 & 85 & 80 & 75 & 70 & 60 & 50 & 40 & 30 & 20 & 10 \\
\hline Apr. - Mar. & 4.0 & 5.3 & 6.9 & 8.4 & 9.9 & 12 & 15 & 24 & 39 & 61 & 100 & 200 & 520 \\
May - Nov. & 3.7 & 4.6 & 5.8 & 6.9 & 8.0 & 9.1 & 10 & 14 & 19 & 27 & 42 & 69 & 170 \\
June - Aug. & 4.3 & 5.6 & 7.1 & 8.5 & 9.8 & 11 & 13 & 17 & 21 & 29 & 42 & 68 & 160 \\
Sept.- Nov. & 3.3 & 4.0 & 4.7 & 5.5 & 6.2 & 6.9 & 7.6 & 9.1 & 11 & 15 & 21 & 39 & 100 \\
Dec. - Feb. & 5.5 & 7.3 & 9.2 & 13 & 18 & 24 & 31 & 50 & 74 & 120 & 210 & 390 & 860 \\
Mar. - May & 17 & 22 & 29 & 37 & 45 & 53 & 62 & 85 & 120 & 180 & 270 & 470 & 960 \\
& & & & & & & & & & & & &
\end{tabular}


GREAT MIAMI RIVER BASIW

03262500 Great Miami River at Piqua, Ohio

LOCATION:

Lat $40^{\circ} 08^{\prime} 58^{\prime \prime}$, long $84^{\circ} 13^{\prime} 48^{\prime \prime}$, Miami County, Hydrologic Unit

05080001 , on North Main Street (U.S. Highway 36) bridge in

Piqua, and $3.0 \mathrm{mi}$ downstream from Loramie Creek.

DRAINAGE AREA: $\quad 866 \mathrm{mi}^{2}$.

TRIBUTARY TO: Ohio River.

DISCHARGE DATA USED: LOW-flow measurements 1948, 1956-1970 water years.

INDEX STATION: $\quad 03263000$ Great Miami River at Taylorsville, ohio.

REMARKS: None.

SELECTED DISCHARGE CHARACTERISTICS: Minimum discharge observed: $11 \mathrm{ft}^{3} / \mathrm{s}$ Detober 1963.

Magnitude and frequency of low flow for indicated periods

\begin{tabular}{|c|c|c|c|}
\hline \multirow[t]{2}{*}{ Period } & \multirow{2}{*}{$\begin{array}{l}\text { Number of } \\
\text { consecu- } \\
\text { tive days }\end{array}$} & \multicolumn{2}{|c|}{$\begin{array}{l}\text { Discharge }\left(\mathrm{ft}^{3} / \mathrm{s}\right) \text { for } \\
\text { indicated recurrence } \\
\text { interval (years) }\end{array}$} \\
\hline & & 2 & 10 \\
\hline Apr. - Mar. & $\begin{array}{r}7 \\
30\end{array}$ & $\begin{array}{l}40 \\
49\end{array}$ & $\begin{array}{l}24 \\
28\end{array}$ \\
\hline May - Nov. & $\begin{array}{r}7 \\
30\end{array}$ & $\begin{array}{l}40 \\
50\end{array}$ & $\begin{array}{l}25 \\
29\end{array}$ \\
\hline June - Aug. & $\begin{array}{r}7 \\
30\end{array}$ & $\begin{array}{l}54 \\
82\end{array}$ & $\begin{array}{l}31 \\
38\end{array}$ \\
\hline Sept. - Nov. & $\begin{array}{r}7 \\
30\end{array}$ & $\begin{array}{l}40 \\
54\end{array}$ & $\begin{array}{l}26 \\
30\end{array}$ \\
\hline
\end{tabular}

Duration of daily flow for indicated periods

\begin{tabular}{l|c|c|c|c|c}
\hline \multirow{2}{*}{ Period } & \multicolumn{5}{|c}{$\begin{array}{c}\text { Discharge }\left(\mathrm{ft}^{3} / \mathrm{s} \text { ) which was equaled or }\right. \\
\text { exceeded for indicated percent of time }\end{array}$} \\
\cline { 2 - 6 } & 98 & 95 & 90 & 85 & 80 \\
\hline Apr. - Mar. & 30 & 37 & 46 & 57 & 68 \\
May - Nov. & 29 & 33 & 40 & 46 & 52 \\
June - Aug. & 32 & 39 & 46 & 52 & 63 \\
Sept. - Nov. & 25 & 30 & 34 & 38 & 42 \\
\hline
\end{tabular}


GREAT MIAMI RIVER BASIN

03262700 Great Miami. River at Troy, Ohio

LOCATION:

Lat 40.02'25", long $84^{\circ} 11^{\prime} 52^{\prime \prime}$, Miami County, Hydrologic Unit 05080001, $400 \mathrm{ft}$ downstream from B.\& O. Railroad bridge, $1,300 \mathrm{ft}$ downstream from bridge on State Route 55 at Troy, $1.2 \mathrm{mi}$ upstream from small left bank tributary, 2.3 mi downstream from Spring Creek, and at mile 105.

DRAINAGE AREA: $\quad 926 \mathrm{mi}^{2}$.

TRIBUTARY TO: Ohio River.

DISCHARGE DATA USED: October 1962 to September 1978.

REMARKS: Flood flow regulated by retarding basin on Loramie Creek, $18 \mathrm{mi}$ upstream. Low and medium flow slightly regulated by Indian Lake; capacity, 45,900 acre-ft $54 \mathrm{mi}$ upstream. Water supply for city of Troy is pumped from wells adjacent to the Great Miami River upstream from the station. The pumpage averaged $5.0 \mathrm{ft}^{3} / \mathrm{s}$ in 1978 and is returned as sewage $1 \mathrm{mi}$ downstream from the station.

SELECTED DISCHARGE CHARACTERISTICS: Average discharge: $776 \mathrm{ft}^{3} / \mathrm{s}$ (16 years). Minimum daily discharge: $4.3 \mathrm{ft}^{3} / \mathrm{s}$ July 1977 result of dam closure upstream.

Magnitude and frequency of low flow for indicated periods

\begin{tabular}{|c|c|c|c|c|c|c|c|c|c|c|c|c|c|}
\hline \multirow{2}{*}{ Period } & \multirow{2}{*}{$\begin{array}{l}\text { Number of } \\
\text { consecu- } \\
\text { tive days }\end{array}$} & \multicolumn{5}{|c|}{$\begin{array}{l}\text { Discharge }\left(\mathrm{ft}^{3} / \mathrm{s}\right) \text { for indicated } \\
\text { recurrence interval (years) }\end{array}$} & \multirow{2}{*}{ Period } & \multirow{2}{*}{$\begin{array}{l}\text { Number of } \\
\text { conisecu- } \\
\text { tive days }\end{array}$} & \multicolumn{5}{|c|}{$\begin{array}{l}\text { Discharge }\left(\mathrm{ft}^{3} / \mathrm{s}\right) \text { for indicated } \\
\text { recurrence interval (years) }\end{array}$} \\
\hline & & 2 & 5 & 10 & 20 & 50 & & & 2 & 5 & 10 & 20 & 50 \\
\hline Apr.-Mar. & $\begin{array}{r}1 \\
7 \\
30\end{array}$ & $\begin{array}{l}48 \\
56 \\
68\end{array}$ & $\begin{array}{l}23 \\
34 \\
41\end{array}$ & $\begin{array}{l}13 \\
27 \\
31\end{array}$ & $\begin{array}{l}8.3 \\
22 \\
25\end{array}$ & $\begin{array}{l}4.5 \\
17 \\
19\end{array}$ & Sept.-Nov. & $\begin{array}{r}1 \\
7 \\
30\end{array}$ & $\begin{array}{l}52 \\
61 \\
77\end{array}$ & $\begin{array}{l}32 \\
39 \\
45\end{array}$ & $\begin{array}{l}25 \\
30 \\
37\end{array}$ & $\begin{array}{l}21 \\
25 \\
32\end{array}$ & $\begin{array}{l}17 \\
19 \\
28\end{array}$ \\
\hline June-Aug. & $\begin{array}{r}1 \\
7 \\
30\end{array}$ & $\begin{array}{r}63 \\
72 \\
102\end{array}$ & $\begin{array}{l}27 \\
47 \\
59\end{array}$ & $\begin{array}{l}15 \\
38 \\
47\end{array}$ & $\begin{array}{l}9.0 \\
33 \\
40\end{array}$ & $\begin{array}{l}4.6 \\
29 \\
34\end{array}$ & Mar. - May & $\begin{array}{r}1 \\
7 \\
30\end{array}$ & $\begin{array}{l}177 \\
215 \\
427\end{array}$ & $\begin{array}{l}105 \\
140 \\
262\end{array}$ & $\begin{array}{r}78 \\
115 \\
207\end{array}$ & $\begin{array}{r}60 \\
99 \\
172\end{array}$ & $\begin{array}{r}43 \\
85 \\
141\end{array}$ \\
\hline
\end{tabular}

Duration of daily flow for indicated periods

\begin{tabular}{l|r|r|r|r|r|r|r|r|r|r|r|r|r}
\hline \multirow{2}{*}{ Period } & \multicolumn{8}{|c|}{ Discharge $\left(\mathrm{ft}^{3} / \mathrm{s}\right)$ which was equaled or exceeded for indicated percent of time } \\
\cline { 2 - 12 } & 98 & 95 & 90 & 85 & 80 & 75 & 70 & 60 & 50 & 40 & 30 & 20 & 10 \\
\hline Apr. - Mar. & 34 & 47 & 61 & 74 & 88 & 110 & 130 & 180 & 260 & 360 & 560 & 950 & 2100 \\
May - Nov. & 32 & 44 & 54 & 63 & 72 & 80 & 90 & 120 & 150 & 200 & 280 & 400 & 790 \\
June - Aug. & 39 & 48 & 60 & 70 & 79 & 89 & 100 & 130 & 160 & 210 & 280 & 370 & 670 \\
Sept.- Nov. & 24 & 35 & 46 & 54 & 59 & 65 & 71 & 84 & 100 & 130 & 170 & 230 & 410 \\
Dec. - Feb. & 31 & 50 & 72 & 100 & 130 & 160 & 190 & 290 & 390 & 550 & 850 & 1400 & 2800 \\
Mar. - May & 140 & 180 & 230 & 270 & 310 & 360 & 410 & 540 & 700 & 950 & 1400 & 2100 & 3800 \\
\hline
\end{tabular}


Appendix 3.--Low-flow data--Continued

GREAT MIAMI RIVER BASIN

03262800 Lost Creek near Troy, Ohio

LOCATION:

Lat $40^{\circ} 01,05^{\prime \prime}$, long $84^{\circ} 09^{\prime} 25^{\prime \prime}$, Miami County, ifydrologic Unit

05080001 , at Knoop Road bridge, $0.2 \mathrm{mi}$ south of State Route 70 , $2.8 \mathrm{mi}$ southeast of Troy, $2.8 \mathrm{mi}$ southwest of Casstown, and $4.3 \mathrm{mi}$ upstream from mouth.

DRAINAGE AREA: $\quad 55.3 \mathrm{mi}^{2}$.

TRIBUTARY TO:

Great Miami River.

DISCHARGE DATA USED:

Low-flow measurements 1959, 1961-1970 water years.

INDEX STATION:

03263000 Great Miami River at Taylorsville, Ohio.

REMARKS:

None.

SELECTED DISCHARGE CHARACTERISTICS: Minimum discharge observed: $0.5 \mathrm{ft} / \mathrm{s}$ September 1963.

Magnitude and frequency of low flow for indicated periods

\begin{tabular}{c|c|c|c}
\hline \multirow{2}{*}{ Period } & \multirow{2}{*}{$\begin{array}{c}\text { Number of } \\
\text { consecu- } \\
\text { tive days }\end{array}$} & \multicolumn{2}{|c}{$\begin{array}{c}\text { Discharge }\left(\mathrm{ft}^{3} / \mathrm{s} \text { ) for }\right. \\
\text { indicated recurrence } \\
\text { interval (years) }\end{array}$} \\
\cline { 3 - 4 } & & 2 & 10 \\
\hline Apr. - Mar. & 7 & 1.7 & 0.7 \\
May - Nov. & 30 & 2.5 & 0.9 \\
& 7 & 1.7 & 0.8 \\
June - Aug. & 30 & 2.5 & 1.0 \\
& 7 & 2.9 & 1.1 \\
Sept. - Nov. & 30 & 6.2 & 1.5 \\
& 7 & 1.7 & 0.8 \\
& 30 & 2.9 & 1.1 \\
\hline
\end{tabular}

Duration of daily flow for indicated periods

\begin{tabular}{l|c|c|c|c|c}
\hline \multirow{2}{*}{ Period } & \multicolumn{5}{|c}{$\begin{array}{c}\text { Discharge ( } \mathrm{ft} 3 / \mathrm{s} \text { ) which was equaled or } \\
\text { exceeded for ind icated percent of time }\end{array}$} \\
\cline { 2 - 6 } & 98 & 95 & 90 & 85 & 80 \\
\hline Apr. - Mar. & 1.1 & 1.5 & 2.2 & 3.2 & 4.3 \\
May - Nov. & 1.0 & 1.2 & 1.7 & 2.2 & 2.7 \\
June - Aug. & 1.2 & 1.6 & 2.2 & 2.7 & 3.8 \\
Sept. - Nov. & 0.8 & 1.0 & 1.3 & 1.6 & 1.9 \\
\hline
\end{tabular}


Appendix 3.--Low-flow data--Continued

GREAT MIAMI RIVER BASIN

03262900 Honey Creek near New Carlisle, Ohio

LOCATION:

Lat $39^{\circ} 58^{\prime} 11^{\prime \prime}$, long $84^{\circ} 06^{\prime} 33^{\prime \prime}$, Miami County, Hydrologic Unit

05080001 , at bridge on Rudy Road, $0.5 \mathrm{mi}$ downstream from

Indian Creek and $5.0 \mathrm{mi}$ northwest of New Carlisle.

DRAINAGE AREA: $\quad 72.8 \mathrm{mi}^{2}$.

TRIBUTARY TO: Great Miami River.

DISCHARGE DATA USED: LOW-flow measurements 1969-1974 water years.

INDEX STATION: $\quad 03264000$ Greenville Creek near Bradford, Ohio.

REMARKS:

New Carlisle ground water supply is discharged as sewage into Honey Creek, $5.0 \mathrm{mi}$ upstream from station. Average sewage discharge was $377,000 \mathrm{gal} / \mathrm{d}$ in 1971 .

SELECTED DISCHARGE CHARACTERISTICS: Minimum discharge observed: $7.8 \mathrm{Et}^{3} / \mathrm{s}$ June 1971.

Magnitude and frequency of low flow for indicated periods

\begin{tabular}{c|c|c|c}
\hline \multirow{2}{*}{ Period } & \multirow{2}{*}{$\begin{array}{c}\text { Number of } \\
\text { consecu- } \\
\text { tive days }\end{array}$} & \multicolumn{2}{|c}{$\begin{array}{c}\text { Discharge }\left(\mathrm{ft}^{3} / \mathrm{s}\right) \text { for } \\
\text { indicated recurrence } \\
\text { interval (years) }\end{array}$} \\
\cline { 3 - 4 } & & 2 & 10 \\
\hline Apr. - Mar. & 7 & 7.0 & 3.8 \\
May - Nov. & 30 & 8.1 & 4.7 \\
& 7 & 7.0 & 3.8 \\
June - Aug. & 30 & 8.1 & 4.7 \\
& 7 & 8.6 & 4.7 \\
Sept.- Nov. & 7 & 12 & 5.8 \\
& 30 & 7.0 & 3.9 \\
& & 9.3 & 5.0 \\
\hline
\end{tabular}

Duration of daily flow for indicated periods

\begin{tabular}{l|c|c|c|c|c}
\hline \multirow{2}{*}{ Period } & \multicolumn{5}{|c}{$\begin{array}{c}\text { Discharge }\left(\mathrm{ft}^{3} / \mathrm{s} \text { ) which was equaled or }\right. \\
\text { exceeded for indicated percent of time }\end{array}$} \\
\cline { 2 - 6 } & 98 & 95 & 90 & 85 & 80 \\
\hline Apr. - Mar. & 5.0 & 5.8 & 7.8 & 9.3 & 11.0 \\
May - Nov. & 4.7 & 5.4 & 6.6 & 7.8 & 9.0 \\
June - Aug. & 5.0 & 5.8 & 7.4 & 8.6 & 9.7 \\
Sept. - Nov. & 4.3 & 5.0 & 5.8 & 6.6 & 7.4 \\
\hline
\end{tabular}


Appendix 3.--Low-flow data--Continued

\section{GREAT MIAMI RIVER BASIN}

03263000 Great Miami River at Taylorsville, Ohio

LOCATION:

Lat 39.52'22", long $84^{\circ} 09^{\prime} 51^{\prime \prime}$, in SWl/4 sec.36, R.8, T.2, Montgomery County, Hydrologic Unit 05080001 , on left bank 600 ft downstream from Taylorsville Dam, $0.8 \mathrm{mi}$ north of Taylorsville, $2.1 \mathrm{mi}$ east of Vandalia, $9.5 \mathrm{mi}$ upstream from stillwater. River, and at mile 90.8 .

DRAINAGE AREA: $\quad 1,149 \mathrm{mi}^{2}$.

TRIBUTARY TO: $\quad$ Ohio River.

DISCHARGE DATA USED: January 1922 to September. 1978.

REMARKS:

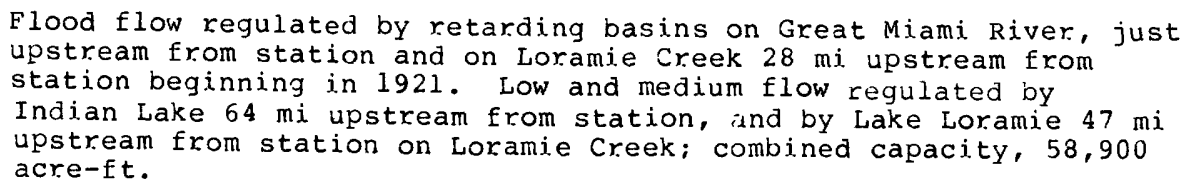

SELECTED DISCHARGE CHARACTERISTICS: Average discharge: $966 \mathrm{ft}^{3} / \mathrm{s}$ (56 years).

Minimum aaily ciscuarye. $45 \mathrm{ft} 3 / \mathrm{s}$ July 1977.

Magnitude and frequency of low flow for indicated periods

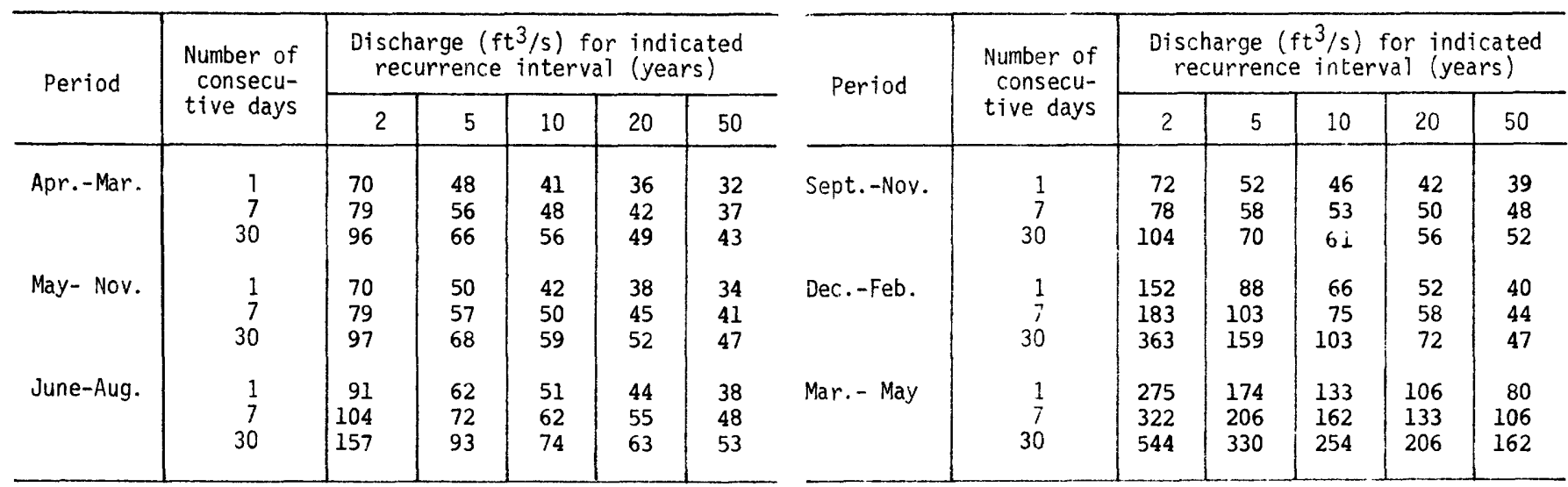

Duration of daily flow for indicated periods

\begin{tabular}{|c|c|c|c|c|c|c|c|c|c|c|c|c|c|}
\hline \multirow{2}{*}{ Period } & \multicolumn{13}{|c|}{ Discharge $\left(\mathrm{ft}^{3} / \mathrm{s}\right)$ which was equaled or exceeded for indicated percent of time } \\
\hline & 98 & 95 & 90 & 85 & 80 & 75 & 70 & 60 & 50 & 40 & 30 & 20 & 10 \\
\hline $\begin{array}{l}\text { Apr. - Mar. } \\
\text { May - Nov. } \\
\text { June - Aug. } \\
\text { Sept. - Nov. } \\
\text { Dec. - Feb. } \\
\text { Mar. - May }\end{array}$ & $\begin{array}{r}61 \\
58 \\
64 \\
51 \\
67 \\
170\end{array}$ & $\begin{array}{r}73 \\
66 \\
76 \\
60 \\
87 \\
230\end{array}$ & $\begin{array}{r}90 \\
79 \\
90 \\
67 \\
110 \\
300\end{array}$ & $\begin{array}{r}110 \\
90 \\
100 \\
75 \\
140 \\
350\end{array}$ & $\begin{array}{r}130 \\
100 \\
120 \\
83 \\
170 \\
400\end{array}$ & $\begin{array}{r}150 \\
120 \\
140 \\
91 \\
210 \\
460\end{array}$ & $\begin{array}{r}180 \\
130 \\
150 \\
99 \\
260 \\
520\end{array}$ & $\begin{array}{l}260 \\
170 \\
190 \\
120 \\
380 \\
690\end{array}$ & $\begin{array}{l}360 \\
220 \\
250 \\
140 \\
550 \\
870\end{array}$ & $\begin{array}{r}510 \\
300 \\
330 \\
180 \\
770 \\
1100\end{array}$ & $\begin{array}{r}750 \\
400 \\
430 \\
240 \\
1200 \\
1500\end{array}$ & $\begin{array}{r}1200 \\
580 \\
600 \\
330 \\
1800 \\
2300\end{array}$ & $\begin{array}{r}2400 \\
1100 \\
1100 \\
580 \\
3500 \\
3900\end{array}$ \\
\hline
\end{tabular}


Appendix 3.--Low-flow data--Continued

GREAT MIAMI RIVER BASIN

03264000 Greenville Creek near Bradford, Ohio

LOCATION:

Lat $40^{\circ} 06^{\prime} 08^{\prime \prime}$, long $84^{\circ} 25^{\prime} 48^{\prime \prime}$, in SWl/4 NWl/4 sec.34, T.9 N., R.4 E., Miami County, Hydrologic Unit 05080001 , on left bank at downstream side of bridge on state Route $721,0.8 \mathrm{mi}$ downstream from small left bank tributary, $1.8 \mathrm{mi}$ south of Bradford, and $6 \mathrm{mi}$ upstream from mouth.

DRAINAGE AREA: $193 \mathrm{mi}^{2}$.

TRIBUTARY TO: Stillwater River.

DISCHARGE DATA USED: March 1931 to Serteruber 1978.

REMARKS:

Some diurnal fluctuation caused by mill $8 \mathrm{mi}$ upstream from station, daily flows are not affected appreciably.

SELECTED OISCHARGE CHARACTERISTICS: Average discharge: $171 \mathrm{ft}^{3} / \mathrm{s}$ (47 years).

Minimum daily discharge: $5.3 \mathrm{ft} / \mathrm{s}$ September 1963.

Magnitude and frequency of low flow for indicated periods

\begin{tabular}{c|c|c|c|c|c|c}
\hline \multirow{2}{*}{ Period } & \multirow{2}{*}{$\begin{array}{c}\text { Number of } \\
\text { consecu- } \\
\text { tive days }\end{array}$} & \multicolumn{4}{|c|}{$\begin{array}{c}\text { Discharge ( } \mathrm{ft}^{3} / \mathrm{s} \text { ) for indicated } \\
\text { recurrence interval (years) }\end{array}$} \\
\cline { 3 - 7 } Apr.-Mar. & 1 & 14 & 9.2 & 7.4 & 6.2 & 5.1 \\
& 7 & 18 & 12 & 9.9 & 8.5 & 7.1 \\
& 30 & 21 & 15 & 12 & 10 & 8.7 \\
May- Nov. & 1 & 14 & 9.2 & 7.4 & 6.2 & 5.1 \\
& 7 & 18 & 12 & 9.9 & 8.5 & 7.1 \\
& 30 & 21 & 15 & 12 & 10 & 8.8 \\
& 1 & 18 & 12 & 9.4 & 7.9 & 6.5 \\
& 7 & 22 & 15 & 12 & 11 & 9.1 \\
& 30 & 30 & 19 & 15 & 13 & 12 \\
\hline
\end{tabular}

\begin{tabular}{|c|c|c|c|c|c|c|}
\hline \multirow{2}{*}{ Period } & \multirow{2}{*}{$\begin{array}{l}\text { Number of } \\
\text { consecu- } \\
\text { tive days }\end{array}$} & \multicolumn{5}{|c|}{$\begin{array}{l}\left.\text { Discharge ( } \mathrm{ft}^{3} / \mathrm{s}\right) \text { for indicated } \\
\text { recurrence interval (years) }\end{array}$} \\
\hline & & 2 & 5 & 10 & 20 & 50 \\
\hline Sept.-Nov. & $\begin{array}{r}1 \\
7 \\
30\end{array}$ & $\begin{array}{l}15 \\
18 \\
24\end{array}$ & $\begin{array}{l}10 \\
13 \\
16\end{array}$ & $\begin{array}{l}8.2 \\
10 \\
13\end{array}$ & $\begin{array}{r}6.9 \\
9.0 \\
11\end{array}$ & $\begin{array}{l}5.7 \\
7.7 \\
9.4\end{array}$ \\
\hline Dec.-Feb. & $\begin{array}{r}1 \\
7 \\
30\end{array}$ & $\begin{array}{l}33 \\
39 \\
68\end{array}$ & $\begin{array}{l}21 \\
23 \\
33\end{array}$ & $\begin{array}{l}16 \\
17 \\
22\end{array}$ & $\begin{array}{l}12 \\
14 \\
16\end{array}$ & $\begin{array}{l}9.6 \\
10 \\
11\end{array}$ \\
\hline Mar. - May & $\begin{array}{r}1 \\
7 \\
30\end{array}$ & $\begin{array}{r}62 \\
69 \\
106\end{array}$ & $\begin{array}{l}40 \\
46 \\
68\end{array}$ & $\begin{array}{l}31 \\
36 \\
54\end{array}$ & $\begin{array}{l}24 \\
29 \\
44\end{array}$ & $\begin{array}{l}18 \\
22 \\
35\end{array}$ \\
\hline
\end{tabular}

Duration of daily flow for indicated periods

\begin{tabular}{|c|c|c|c|c|c|c|c|c|c|c|c|c|c|}
\hline \multirow{2}{*}{ Period } & \multicolumn{13}{|c|}{ Discharge $\left(\mathrm{ft}^{3} / \mathrm{s}\right)$ which was equaled or exceeded for indicated percent of time } \\
\hline & 98 & 95 & 90 & 85 & 80 & 75 & 70 & 60 & 50 & 40 & 30 & 20 & 10 \\
\hline $\begin{array}{l}\text { Apr. - Mar. } \\
\text { May - Nov. } \\
\text { June - Aug. } \\
\text { Sept. - Nov. } \\
\text { Dec. - Feb. } \\
\text { Mar. - May }\end{array}$ & $\begin{array}{l}13 \\
12 \\
13 \\
11 \\
14 \\
37\end{array}$ & $\begin{array}{l}15 \\
14 \\
15 \\
13 \\
18 \\
49\end{array}$ & $\begin{array}{l}20 \\
17 \\
19 \\
15 \\
24 \\
60\end{array}$ & $\begin{array}{l}24 \\
20 \\
22 \\
17 \\
29 \\
70\end{array}$ & $\begin{array}{l}27 \\
23 \\
25 \\
19 \\
37 \\
82\end{array}$ & $\begin{array}{l}32 \\
26 \\
28 \\
21 \\
43 \\
92\end{array}$ & $\begin{array}{r}37 \\
28 \\
31 \\
23 \\
51 \\
100\end{array}$ & $\begin{array}{r}49 \\
35 \\
38 \\
27 \\
71 \\
120\end{array}$ & $\begin{array}{r}66 \\
43 \\
47 \\
31 \\
94 \\
150\end{array}$ & $\begin{array}{r}92 \\
55 \\
59 \\
37 \\
130 \\
190\end{array}$ & $\begin{array}{r}130 \\
75 \\
77 \\
45 \\
180 \\
240\end{array}$ & $\begin{array}{r}200 \\
110 \\
110 \\
59 \\
280 \\
350\end{array}$ & $\begin{array}{r}380 \\
180 \\
190 \\
96 \\
550 \\
620\end{array}$ \\
\hline
\end{tabular}


GREAT MIAMI RIVER BASIN

03265000 Stillwater River at Pleasant Hill, Ohio

LOCATION:

Lat $40^{\circ} 03^{\prime} 28^{\prime \prime}$, long $84^{\circ} 21^{\prime} 22^{\prime \prime}$, in SWl/4 sec.18, T.7 N., R.5 E., Miami County Hydrologic Unit 05080001 , on left bank at downstream side of bridge on Laurer Road, $0.8 \mathrm{mi}$ northwest of Pleasant Hill, $2 \mathrm{mi}$ downstream from Painter. Creek, $2 \mathrm{mi}$ upstream from Canyon Run, and at mile 28.35 .

DRAINAGE AREA:

$503 \mathrm{mi}^{2}$

TRIBUTARY TO:

Great Miami River.

DISCHARGE DATA USED:

October 1916 to September 1928, December 1934 to September 1978.

REMARKS :

None.

SELECTED DISCHARGE CHARACTERISTICS: Average discharge: $440 \mathrm{ft}^{3} / \mathrm{s}$ ( 55 years).

Minimum daily discharge: $4 \mathrm{ft}^{3} / \mathrm{s}$ October 1920, July, August, 1921.

Magnitude and frequency of low flow for indicated periods

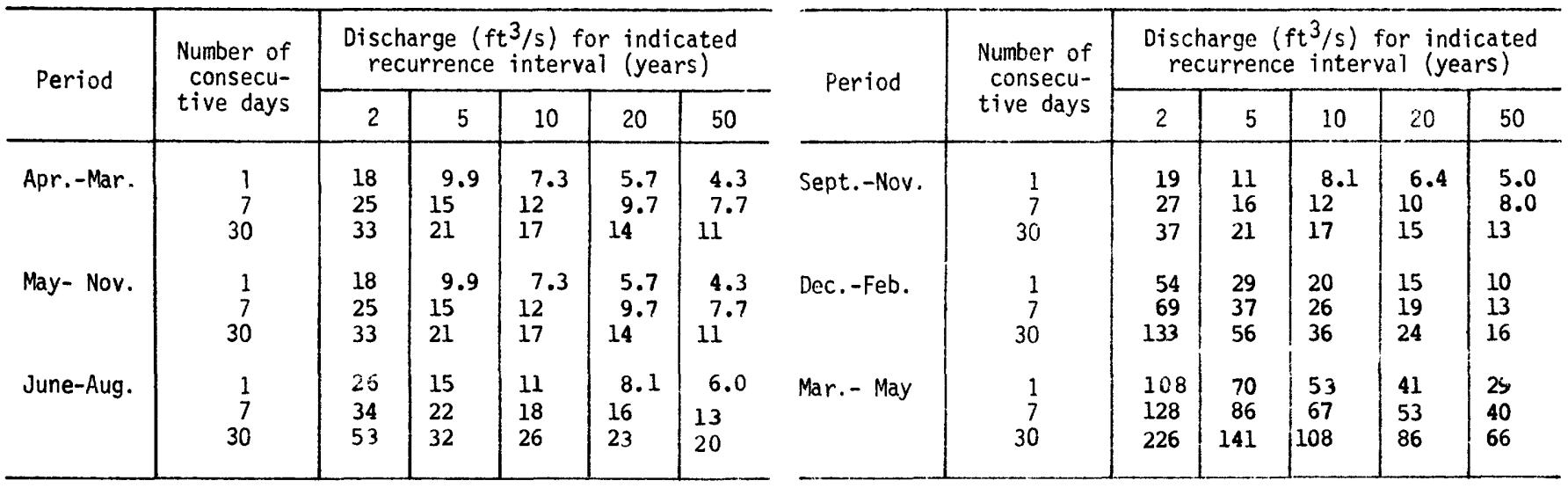

Duration of daily flow for indicated periods

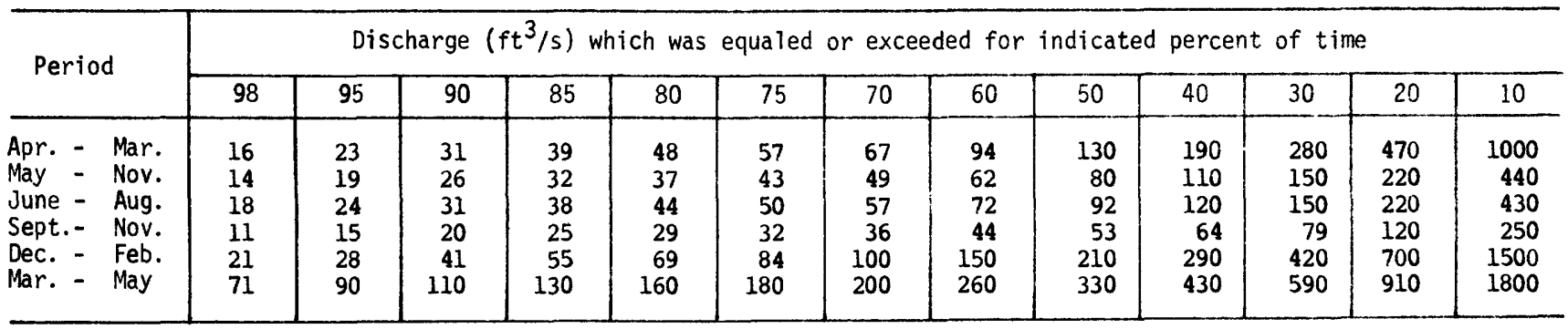


GREAT MIAMI RIVER BASIN

03266000 Stillwater River at Englewood, Ohio

LOCATION: Lat $39^{\circ} 52^{\prime} 10^{\prime \prime}$, long $84^{\circ} 16^{\prime} 57^{\prime \prime}$, in NWl/4 sec.23, T.5 N., R.5 E., Montgomery County, Hydrologic Unit 05080001, on right bank 1,000 ft downstream from Englewood Dam, $1 \mathrm{mi}$ southeast of Englewood, and at mile 8.9.

DRAINAGE AREA: $\quad 650 \mathrm{mi}^{2}$.

TRIBUTARY TO: Great Miami River.

DISCHARGE DATA USED: November 1925 to September 1978.

REMARKS: $\quad$ None.

SELECTED DISCHARGE CHARACTERISTICS: Average discharge: $563 \mathrm{ft}^{3} / \mathrm{s}$ ( 52 years).

Minimum daily discharge: $4.8 \mathrm{ft}^{3} / \mathrm{s}$ September 1955.

Magnitude and frequency of low flow for indicated periods

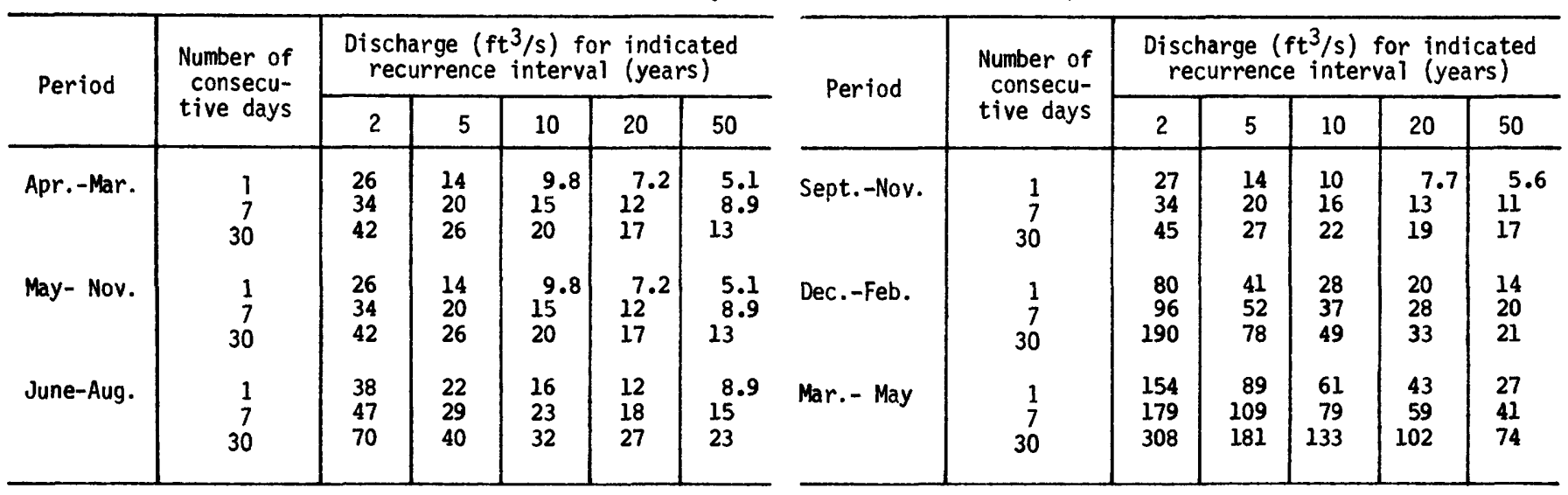

Duration of daily flow for indicated periods

\begin{tabular}{|c|c|c|c|c|c|c|c|c|c|c|c|c|c|c|}
\hline \multirow{2}{*}{\multicolumn{2}{|c|}{ Period }} & \multicolumn{13}{|c|}{ Discharge $\left(\mathrm{ft}^{3} / \mathrm{s}\right)$ which was equaled or exceeded for indicated percent of time } \\
\hline & & 98 & 95 & 90 & 85 & 80 & 75 & 70 & 60 & 50 & 40 & 30 & 20 & 10 \\
\hline $\begin{array}{l}\text { Apr. - } \\
\text { May - } \\
\text { June - } \\
\text { Sept. - } \\
\text { Dec. - } \\
\text { Mar. - }\end{array}$ & $\begin{array}{l}\text { Mar. } \\
\text { Nov. } \\
\text { Aug. } \\
\text { Nov. } \\
\text { Feb. } \\
\text { May }\end{array}$ & $\begin{array}{l}21 \\
18 \\
22 \\
15 \\
28 \\
82\end{array}$ & $\begin{array}{r}29 \\
25 \\
31 \\
20 \\
39 \\
120\end{array}$ & $\begin{array}{r}40 \\
33 \\
41 \\
26 \\
52 \\
160\end{array}$ & $\begin{array}{r}50 \\
41 \\
49 \\
31 \\
69 \\
190\end{array}$ & $\begin{array}{r}60 \\
47 \\
57 \\
36 \\
87 \\
220\end{array}$ & $\begin{array}{r}72 \\
54 \\
66 \\
41 \\
110 \\
250\end{array}$ & $\begin{array}{r}86 \\
62 \\
75 \\
45 \\
130 \\
280\end{array}$ & $\begin{array}{r}120 \\
80 \\
96 \\
55 \\
200 \\
350\end{array}$ & $\begin{array}{r}180 \\
110 \\
120 \\
67 \\
270 \\
450\end{array}$ & $\begin{array}{r}260 \\
140 \\
160 \\
82 \\
380 \\
590\end{array}$ & $\begin{array}{l}380 \\
200 \\
210 \\
100 \\
570 \\
810\end{array}$ & $\begin{array}{r}620 \\
300 \\
300 \\
150 \\
980 \\
1200\end{array}$ & $\begin{array}{r}1400 \\
580 \\
590 \\
290 \\
2300 \\
2500\end{array}$ \\
\hline
\end{tabular}


Appendix 3.--Low-flow data--Continued

GREAT MIAMI RIVER BASIN

03266500 Mad River at Zanesfield, Ohio

LOCATION:

Lat $40^{\circ} 21^{\prime} 01^{\prime \prime}$, long $83^{\circ} 40^{\prime} 28^{\prime \prime}$, Logan County, Hydrologic Unit 05080001, on left bank at upstream side of bridge on County Road No. 5 (adjacent to former U.S. Highway 33), $0.8 \mathrm{mi}$ upstream from Sugar Creek, l $\mathrm{mi}$ north of Zanesfield, and at mile 61.45 .

DRAINAGE AREA: $\quad 7.31 \mathrm{mi}^{2}$.

TRIBLITARY TO: Great Miami River.

DISCHARGE DATA USED: October 1946 to September 1978.

REMARKS:

None.

SELECTED DISCHARGE CHARACTERISTICS: Average discharge: $7.73 \mathrm{ft}^{3} / \mathrm{s}$ (32 years).

Minimum daily discharge: $0.5 \mathrm{ft}^{3} / \mathrm{s}$ December 1963.

Magnitude and frequency of low flow for indicated periods

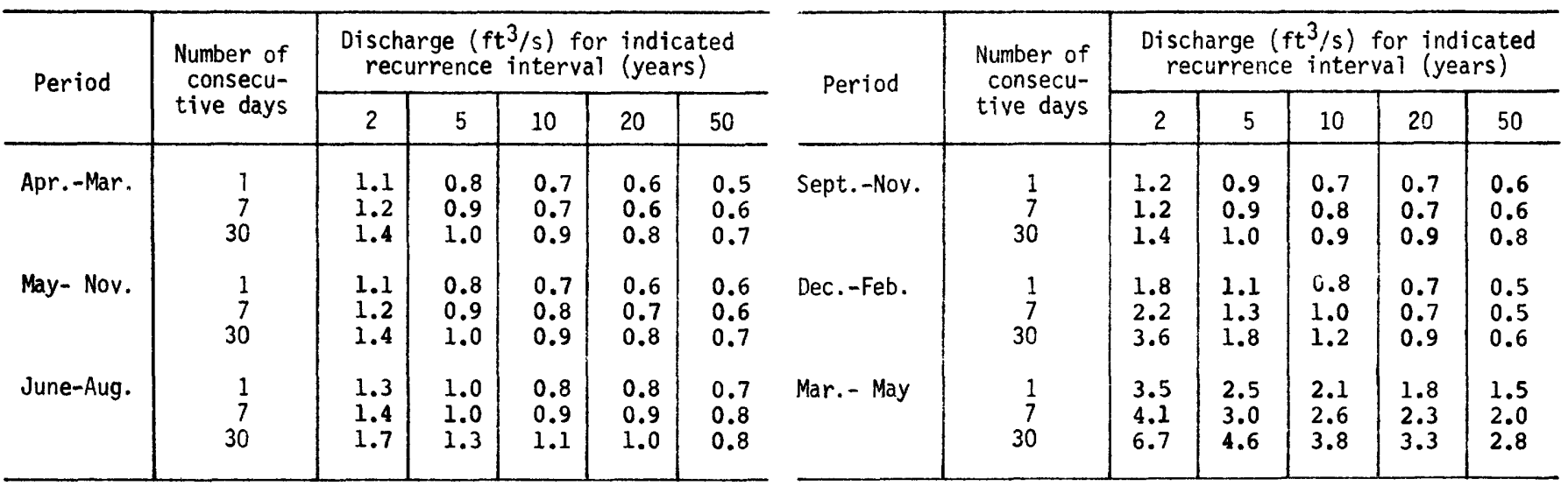

Duration of daily flow for indicated periods

\begin{tabular}{l|r|r|r|r|r|r|r|r|r|r|r|r|r}
\hline \multirow{2}{*}{ Period } & \multicolumn{8}{|c|}{ Discharge $\left(\mathrm{ft}^{3} / \mathrm{s}\right.$ ) which was equaled or exceeded for indicated percent of time } \\
\cline { 2 - 13 } & 98 & 95 & 90 & 85 & 80 & 75 & 70 & 60 & 50 & 40 & 30 & 20 & 10 \\
\hline Apr. - Mar. & 0.9 & 1.0 & 1.2 & 1.4 & 1.6 & 1.8 & 2.1 & 2.7 & 3.7 & 4.9 & 6.9 & 10 & 17 \\
May - Nov. & 0.9 & 1.0 & 1.2 & 1.3 & 1.4 & 1.6 & 1.7 & 2.0 & 2.4 & 3.0 & 4.0 & 5.5 & 9.3 \\
June - Aug. & 1.0 & 1.1 & 1.3 & 1.4 & 1.6 & 1.7 & 1.8 & 2.1 & 2.4 & 2.9 & 3.7 & 4.8 & 7.4 \\
Sept. - Nov. & 0.8 & 0.9 & 1.0 & 1.1 & 1.2 & 1.3 & 1.4 & 1.6 & 1.8 & 2.1 & 2.4 & 3.3 & 4.9 \\
Dec. - Feb. & 0.8 & 1.0 & 1.3 & 1.6 & 1.9 & 2.4 & 2.8 & 3.7 & 4.8 & 6.3 & 8.8 & 13 & 21 \\
Mar. - May & 2.9 & 3.4 & 4.0 & 4.6 & 5.2 & 5.8 & 6.5 & 7.9 & 9.5 & 11 & 14 & 17 & 27 \\
\hline
\end{tabular}


Appendix 3.--Low-flow data--Continued

GREAT MIAMI RIVER BASIN

03267000 Mad River near Urbana, Ohio

LOCATION:

Lat $40^{\circ} 06^{\prime} \mathbf{2 7}^{\prime \prime}$, long $83^{\circ} 47^{\prime} 57^{\prime \prime}$, on west line of sec.35, T.5 E., R.11 N., Champaign Coutny, Hydrologic Unit 05080001, on left bank at downstream side of bridge on U.S. Highway $36,1.8 \mathrm{mi}$ upstream from Dugan Run, 1.8 $\mathrm{mi}$ downstream from Muddy Creek, $2.5 \mathrm{mi}$ west of Urbana, and at mile 39.7 .

DRAINAGE AREA: $\quad 162 \mathrm{mi}^{2}$.

TRIBUTARY TO: Great Miami River.

DISCHARGE DATA USED: October 1925 to September 1931 , October 1939 to September 1978.

REMARKS:

None.

SELECTED DISCHARGE CHARACTERISTICS: Average discharge: $141 \mathrm{ft}^{3} / \mathrm{s}\{45$ years). Minimum daily discharge: $24 \mathrm{ft}^{3} / \mathrm{s}$ February 1945, January 1964.

Magnitude and frequency of low flow for indicated periods

\begin{tabular}{c|c|c|c|c|c|c}
\hline \multirow{2}{*}{ Period } & \multirow{2}{*}{$\begin{array}{c}\text { Number of } \\
\text { consecu- } \\
\text { tive days }\end{array}$} & \multicolumn{4}{|c|}{$\begin{array}{c}\text { Discharge } \\
\text { recurrence }\end{array}$ interval for indicated } \\
\cline { 3 - 7 } (years)
\end{tabular}

\begin{tabular}{|c|c|c|c|c|c|c|}
\hline \multirow{2}{*}{ Period } & \multirow{2}{*}{$\begin{array}{l}\text { Number of } \\
\text { consecu- } \\
\text { tive days }\end{array}$} & \multicolumn{5}{|c|}{$\begin{array}{l}\text { Discharge }\left(\mathrm{ft}^{3} / \mathrm{s}\right) \text { for indicated } \\
\text { recurrence interval (years) }\end{array}$} \\
\hline & & 2 & 5 & 10 & 20 & 50 \\
\hline Sept.-Nov. & $\begin{array}{r}1 \\
7 \\
30\end{array}$ & $\begin{array}{l}52 \\
55 \\
57\end{array}$ & $\begin{array}{l}40 \\
41 \\
43\end{array}$ & $\begin{array}{l}35 \\
36 \\
37\end{array}$ & $\begin{array}{l}31 \\
32 \\
34\end{array}$ & $\begin{array}{l}28 \\
28 \\
31\end{array}$ \\
\hline Dec. -Feb. & $\begin{array}{r}1 \\
7 \\
30\end{array}$ & $\begin{array}{l}59 \\
64 \\
82\end{array}$ & $\begin{array}{l}40 \\
43 \\
52\end{array}$ & $\begin{array}{l}32 \\
35 \\
41\end{array}$ & $\begin{array}{l}27 \\
29 \\
34\end{array}$ & $\begin{array}{l}22 \\
24 \\
27\end{array}$ \\
\hline Mar. - May & $\begin{array}{r}1 \\
7 \\
30\end{array}$ & $\begin{array}{r}92 \\
103 \\
128\end{array}$ & $\begin{array}{l}65 \\
75 \\
92\end{array}$ & $\begin{array}{l}54 \\
64 \\
77\end{array}$ & $\begin{array}{l}45 \\
56 \\
67\end{array}$ & $\begin{array}{l}37 \\
47 \\
57\end{array}$ \\
\hline
\end{tabular}

Duration of daily flow for indicated periods

\begin{tabular}{|c|c|c|c|c|c|c|c|c|c|c|c|c|c|}
\hline \multirow{2}{*}{ Period } & \multicolumn{13}{|c|}{ Discharge $\left(\mathrm{ft}^{3} / \mathrm{s}\right)$ which was equaled or exceeded for indicated percent of time } \\
\hline & 98 & 95 & 90 & 85 & 80 & 75 & 70 & 60 & 50 & 40 & 30 & 20 & 10 \\
\hline $\begin{array}{l}\text { Apr. - Mar. } \\
\text { May - Nov. } \\
\text { June - Aug. } \\
\text { Sept. - Nov. } \\
\text { Dec. - Feb. } \\
\text { Mar. - May }\end{array}$ & $\begin{array}{l}36 \\
36 \\
40 \\
32 \\
33 \\
62\end{array}$ & $\begin{array}{l}41 \\
41 \\
46 \\
37 \\
40 \\
72\end{array}$ & $\begin{array}{l}47 \\
46 \\
53 \\
41 \\
44 \\
84\end{array}$ & $\begin{array}{l}53 \\
51 \\
59 \\
44 \\
48 \\
93\end{array}$ & $\begin{array}{r}59 \\
56 \\
64 \\
47 \\
54 \\
100\end{array}$ & $\begin{array}{r}64 \\
60 \\
69 \\
51 \\
61 \\
110\end{array}$ & $\begin{array}{r}71 \\
64 \\
74 \\
54 \\
69 \\
120\end{array}$ & $\begin{array}{r}85 \\
74 \\
85 \\
59 \\
86 \\
140\end{array}$ & $\begin{array}{r}100 \\
86 \\
97 \\
64 \\
100 \\
160\end{array}$ & $\begin{array}{r}120 \\
100 \\
110 \\
73 \\
120 \\
190\end{array}$ & $\begin{array}{r}150 \\
120 \\
130 \\
84 \\
150 \\
210\end{array}$ & $\begin{array}{r}180 \\
140 \\
150 \\
98 \\
200 \\
260\end{array}$ & $\begin{array}{l}260 \\
190 \\
190 \\
130 \\
310 \\
340\end{array}$ \\
\hline
\end{tabular}


GREAT MIAMI RIVER BASIN

03267400 Cedar Run near Tremont City, Ohio

LOCATION:

Lat $40^{\circ} 0 \mathrm{i}^{\prime} 49^{\prime \prime}$, long $83^{\circ} 48^{\prime} 59^{\prime \prime}$, Champaign County, Hydrologic

Unit 05080001 , at private road bridge, 1,500 ft upstream from

mouth, $900 \mathrm{ft}$ north of County Line Road and $1.6 \mathrm{mi}$ northeast of

Tremont City.

DRAINAGE AREA: $\quad 2.08 \mathrm{mi}^{2}$.

TRIBUTARY TO: Mad kiver.

DISCHARGE DATA USED: Low-flow measurements 1972-1974 water years.

INDEX STATION: $\quad 03267000$ Mad River near Urbana, Ohio

REMARKS: $\quad$ None.

SELECTED DISCHARGE CHARACTERISTICS: Minimum discharge observed: $5.4 \mathrm{ft}^{3} / \mathrm{s}$. August 1972 .

Magnitude and frequency of low flow for indicated periods

\begin{tabular}{c|c|c|c}
\hline \multirow{2}{*}{ Period } & \multirow{2}{*}{$\begin{array}{c}\text { Number of } \\
\text { consecu- } \\
\text { tive days }\end{array}$} & \multicolumn{2}{|c}{$\begin{array}{c}\text { Discharge (ft } 3 / \mathrm{s} \text { ) for } \\
\text { indicated recurrence } \\
\text { interval (years) }\end{array}$} \\
\cline { 3 - 4 } Apr. - Mar. & 7 & 2 & 10 \\
\hline May - Nov. & 30 & 3.3 & 2.1 \\
& 7 & 3.5 & 2.3 \\
June - Aug. & 30 & 3.7 & 2.4 \\
& 7 & 4.1 & 2.4 \\
Sept. - Nov. & 30 & 4.7 & 2.7 \\
& 3 & 3.5 & 2.4 \\
& 30 & 3.6 & 2.4 \\
\hline
\end{tabular}

Duration of daily flow for indicated periods

\begin{tabular}{|c|c|c|c|c|c|}
\hline \multirow{2}{*}{ Period } & \multicolumn{5}{|c|}{$\begin{array}{l}\text { Discharge }\left(\mathrm{ft}^{3} / \mathrm{s}\right) \text { which was equaled or } \\
\text { exceeded for indicated percent of time }\end{array}$} \\
\hline & 98 & 95 & 90 & 85 & 80 \\
\hline $\begin{array}{l}\text { Apr. - Mar. } \\
\text { May - Nov. } \\
\text { June - Aug. } \\
\text { Sept. - Nov. }\end{array}$ & $\begin{array}{l}2.4 \\
2.4 \\
2.6 \\
2.1\end{array}$ & $\begin{array}{l}2.7 \\
2.7 \\
3.0 \\
2.4\end{array}$ & $\begin{array}{l}3.0 \\
3.0 \\
3.4 \\
2.7\end{array}$ & $\begin{array}{l}3.4 \\
3.3 \\
3.7 \\
2.9\end{array}$ & $\begin{array}{l}3.7 \\
3.5 \\
4.0 \\
3.0\end{array}$ \\
\hline
\end{tabular}


Appendix 3.--Low-flow data--Continued

GKEAT MIAMI RIVEK BASIN

03267600 Chapman Creek at Tremont City, Ohio

LOCATION:

Lat $40^{\circ} 00^{\prime} 38^{\prime \prime}$, long $8^{\circ} 50^{\prime} 08^{\prime \prime}$, Clark County, Hydrologic Unit $05080001,0.8 \mathrm{mi}$ upstream from mouth and at bridge on Upper Valley Pike in Tremont City.

DRAINAGE AREA: $\quad 24 \mathrm{mi}^{2}$.

TRIBUTARY TO: Mad River.

DISCHARGE DATA USED: Low-flow measurements 1944, 1948, 1968, 1969, 1972-1974 water years.

INDEX STATION: $\quad 03267000$ Mad River near Urbana, Ohio.

REMARKS: $\quad$ None.

SELECTED DISCHARGE CHARACTERISTICS: Minimum discharge observed: $1.4 \mathrm{it} / \mathrm{s}$ October 1943.

Magnitude and frequency of low flow for indicated periods

\begin{tabular}{c|c|c|c}
\hline \multirow{2}{*}{ Period } & \multirow{2}{*}{$\begin{array}{c}\text { Number of } \\
\text { consecu- } \\
\text { tive days }\end{array}$} & \multicolumn{2}{|c}{$\begin{array}{c}\text { Discharge }\left(\mathrm{ft}^{3} / \mathrm{s}\right) \text { for } \\
\text { indicated recurrence } \\
\text { interval (years) }\end{array}$} \\
\cline { 3 - 4 } Apr. - Mar. & 7 & 2 & 10 \\
\hline May - Nov. & 30 & 0.9 & 0.4 \\
& 7 & 1.1 & 0.5 \\
June - Aug. & 30 & 1.2 & 0.5 \\
& 7 & 1.6 & 0.5 \\
Sept. - Nov. & 70 & 2.0 & 0.6 \\
& 30 & 1.1 & 0.5 \\
& & 1.2 & 0.5 \\
\hline
\end{tabular}

Duration of daily flow for indicated periods

\begin{tabular}{l|c|c|c|c|c}
\hline \multirow{2}{*}{ Period } & \multicolumn{6}{|c}{$\begin{array}{c}\text { Discharge }(\mathrm{ft} 3 / \mathrm{s} \text { ) which was equaled or } \\
\text { exceeded for indicated percent of time }\end{array}$} \\
\cline { 2 - 6 } & 98 & 95 & 90 & 85 & 80 \\
\hline Apr. - Mar. & 0.5 & 0.6 & 0.8 & 1.0 & 1.2 \\
May - Nov. & 0.5 & 0.6 & 0.8 & 0.9 & 1.1 \\
June - Aug. & 0.6 & 0.8 & 1.0 & 1.2 & 1.5 \\
Sept. - Nov. & 0.4 & 0.5 & 0.6 & 0.7 & 0.8 \\
\hline
\end{tabular}


GREAT MIAMI RIVER BASIN

03267900 Mad River at St. Paris Pike at Eagle City, Ohio

LOCATION: $\quad$ Lat $39^{\circ} 57^{\prime} 51^{\prime \prime}$, long $83^{\circ} 49^{\prime} 54^{\prime \prime}$, in Wl/2 sec.1, R.10, T.4, Clark County, Hydrologic Unit 05080001 , on left bank at downstream side of bridge on St. Paris Pike, $0.8 \mathrm{mi}$ southeast of Eagle City, $1.1 \mathrm{mi}$ downstream from Moore Run, $3.1 \mathrm{mi}$ upstream from Buck Creek, $3.3 \mathrm{mi}$ south of Tremont City, and at mile 29.5 .

DRAINAGE AREA: $\quad 310 \mathrm{mi}^{2}$.

TRIBUTARY TO: Great Miami River.

DISCHARGE DATA USED: October 1965 to september 1978.

REMARKS: Water supply for city of springfield is pumped from wells, adjacent to Mad River, just upstream from station. Recharge to the well field is largely by induced infiltration from Mad River and Moore Run. Pumpage, averaging $24.5 \mathrm{ft}^{3} / \mathrm{s}$ in 1978 , is returned as sewage $1.4 \mathrm{mi}$ upstream from Buck Creek, $3.3 \mathrm{mi}$ south of Tremont City, and at mile 29.5 .

SELECTED DISCHARGE CHARACTERISTICS: Average discharge: $296 \mathrm{ft}^{3} / \mathrm{s}$ ( 13 years). Minimum daily discharge: $60 \mathrm{ft} / \mathrm{s}$ January 1972 (result of freezup).

Magnitude and frequency of low flow for indicated periods

\begin{tabular}{|c|c|c|c|c|c|c|c|c|c|c|c|c|c|}
\hline \multirow{2}{*}{ Period } & \multirow{2}{*}{$\begin{array}{l}\text { Number of } \\
\text { consecu- } \\
\text { tive days }\end{array}$} & \multicolumn{5}{|c|}{$\begin{array}{l}\text { Discharge }\left(\mathrm{ft}^{3} / \mathrm{s}\right) \text { for indicated } \\
\text { recurrence interval (years) }\end{array}$} & \multirow{2}{*}{ Period } & \multirow{2}{*}{$\begin{array}{l}\text { Number of } \\
\text { consecu- } \\
\text { tive days }\end{array}$} & \multicolumn{5}{|c|}{$\begin{array}{l}\text { Discharge }\left(\mathrm{ft}^{3} / \mathrm{s}\right) \text { for indicated } \\
\text { recurrence interval (years) }\end{array}$} \\
\hline & & 2 & 5 & 10 & 20 & 50 & & & 2 & 5 & 10 & 20 & 50 \\
\hline Apr.-Mar. & $\begin{array}{r}1 \\
7 \\
30\end{array}$ & $\begin{array}{l}109 \\
114 \\
121\end{array}$ & $\begin{array}{l}85 \\
89 \\
98\end{array}$ & $\begin{array}{l}74 \\
77 \\
88\end{array}$ & $\begin{array}{l}67 \\
70 \\
31\end{array}$ & $\begin{array}{l}59 \\
60 \\
74\end{array}$ & Sept.-Nov. & $\begin{array}{r}1 \\
7 \\
30\end{array}$ & $\begin{array}{l}111 \\
115 \\
126\end{array}$ & $\begin{array}{r}93 \\
98 \\
105\end{array}$ & $\begin{array}{l}87 \\
91 \\
97\end{array}$ & $\begin{array}{l}82 \\
86 \\
92\end{array}$ & $\begin{array}{l}78 \\
82 \\
87\end{array}$ \\
\hline June-Aug. & $\begin{array}{r}1 \\
7 \\
30\end{array}$ & $\begin{array}{l}129 \\
138 \\
161\end{array}$ & $\begin{array}{l}103 \\
109 \\
122\end{array}$ & $\begin{array}{r}93 \\
98 \\
108\end{array}$ & $\begin{array}{l}86 \\
89 \\
98\end{array}$ & $\begin{array}{l}79 \\
81 \\
89\end{array}$ & Mar.-May & $\begin{array}{r}1 \\
7 \\
30\end{array}$ & $\begin{array}{l}182 \\
203 \\
274\end{array}$ & $\begin{array}{l}147 \\
159 \\
205\end{array}$ & $\begin{array}{l}133 \\
140 \\
172\end{array}$ & $\begin{array}{l}122 \\
126 \\
147\end{array}$ & $\begin{array}{l}110 \\
112 \\
121\end{array}$ \\
\hline
\end{tabular}

Duration of daily flow for indicated periods

\begin{tabular}{|c|c|c|c|c|c|c|c|c|c|c|c|c|c|}
\hline \multirow{2}{*}{ Period } & \multicolumn{13}{|c|}{ Discharge $\left(\mathrm{ft}^{3} / \mathrm{s}\right)$ which was equaled or exceeded for indicated percent of time } \\
\hline & 98 & 95 & 90 & 85 & 80 & 75 & 70 & 60 & 50 & 40 & 30 & 20 & 10 \\
\hline $\begin{array}{l}\text { Apr. - Mar. } \\
\text { May - Nov. } \\
\text { June - Aug. } \\
\text { Sept. - Nov. } \\
\text { Dec. - Feb. } \\
\text { Mar. - May }\end{array}$ & $\begin{array}{r}94 \\
94 \\
99 \\
91 \\
85 \\
140\end{array}$ & $\begin{array}{r}100 \\
100 \\
110 \\
98 \\
100 \\
160\end{array}$ & $\begin{array}{l}110 \\
110 \\
120 \\
100 \\
120 \\
170\end{array}$ & $\begin{array}{l}120 \\
110 \\
130 \\
110 \\
130 \\
190\end{array}$ & $\begin{array}{l}140 \\
120 \\
140 \\
110 \\
150 \\
210\end{array}$ & $\begin{array}{l}150 \\
130 \\
150 \\
110 \\
170 \\
230\end{array}$ & $\begin{array}{l}160 \\
140 \\
160 \\
120 \\
180 \\
250\end{array}$ & $\begin{array}{l}180 \\
150 \\
180 \\
130 \\
210 \\
290\end{array}$ & $\begin{array}{l}210 \\
170 \\
200 \\
140 \\
250 \\
320\end{array}$ & $\begin{array}{l}250 \\
200 \\
220 \\
150 \\
310 \\
360\end{array}$ & $\begin{array}{l}310 \\
230 \\
250 \\
170 \\
360 \\
430\end{array}$ & $\begin{array}{l}380 \\
280 \\
290 \\
190 \\
450 \\
550\end{array}$ & $\begin{array}{l}550 \\
370 \\
360 \\
240 \\
660 \\
760\end{array}$ \\
\hline
\end{tabular}




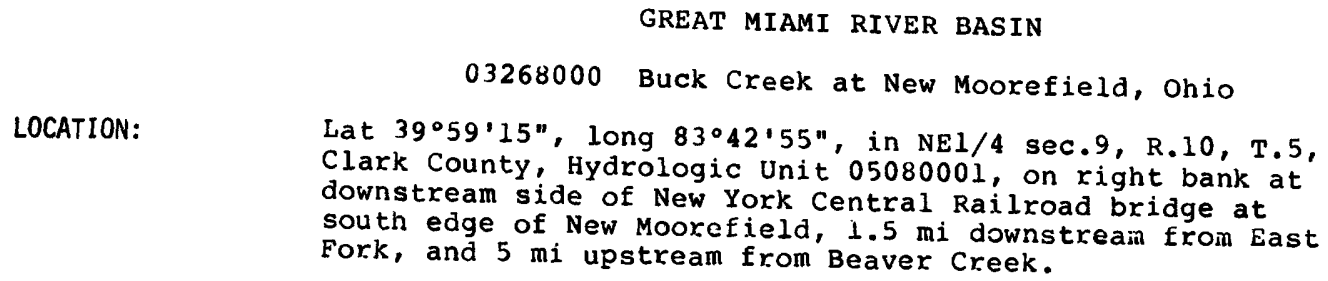

DRAINAGE AREA:

$65.3 \mathrm{mi}^{2}$.

TRIBUTARY TO:

Mad River.

DISCHARGE DATA USED: October 1942 to September 1958.

REMARKS :

None.

SELECTED DISCHARGE CHARACTERISTICS: Average discharge: $65.6 \mathrm{ft}^{3} / \mathrm{s}$ (16 years).

Minimum daily discharge: $13 \mathrm{ft}^{3} / \mathrm{s}$ August 1944, September 1955.

Magnitude and frequency of low flow for indicated periods

\begin{tabular}{c|c|c|c|c|c|c}
\hline \multirow{2}{*}{ Period } & \multirow{2}{*}{$\begin{array}{c}\text { Number of } \\
\text { consecu- } \\
\text { tive days }\end{array}$} & \multicolumn{4}{|c}{$\begin{array}{c}\text { Discharge }\left(\mathrm{ft}^{3} / \mathrm{s} \text { ) for indicated }\right. \\
\text { recurrence } \\
\text { interval }\end{array}$} \\
\cline { 3 - 7 } (years)
\end{tabular}

\begin{tabular}{c|c|c|c|c|c|c}
\hline \multirow{2}{*}{ Period } & \multirow{2}{*}{$\begin{array}{c}\text { Number of } \\
\text { consecu- } \\
\text { tive days }\end{array}$} & \multicolumn{4}{|c}{$\begin{array}{c}\text { Discharge } \\
\text { recurrence }{ }^{3} / \mathrm{s} \text { ) for interval } \begin{array}{c}\text { indicated } \\
\text { (years) }\end{array}\end{array}$} \\
\cline { 3 - 7 } Sept.-Nov. & 2 & 5 & 10 & 20 & 50 \\
\hline \multirow{5}{*}{ Dec.-Feb. } & 7 & 24 & 18 & 15 & 14 & 12 \\
& 30 & 25 & 19 & 16 & 14 & 12 \\
& 1 & 27 & 20 & 18 & 16 & 14 \\
& 7 & 29 & 21 & 18 & 16 & 14 \\
& 30 & 39 & 26 & 21 & 18 & 16 \\
& 1 & 43 & 31 & 27 & 23 & 20 \\
& 7 & 45 & 33 & 28 & 25 & 21 \\
& 30 & 56 & 40 & 33 & 28 & 23 \\
\hline
\end{tabular}

Duration of daily flow for indicated periods

\begin{tabular}{|c|c|c|c|c|c|c|c|c|c|c|c|c|c|}
\hline \multirow{2}{*}{ Period } & \multicolumn{13}{|c|}{ Discharge $\left(\mathrm{ft}^{3} / \mathrm{s}\right)$ which was equaled or exceeded for indicated percent of time } \\
\hline & 98 & 95 & 90 & 85 & 80 & 75 & 70 & 60 & 50 & 40 & 30 & 20 & 10 \\
\hline $\begin{array}{l}\text { Apr. - Mar. } \\
\text { May - Nov. } \\
\text { June - Aug. } \\
\text { Sept. - Nov. } \\
\text { Dec. - Feb. } \\
\text { Mar. - May }\end{array}$ & $\begin{array}{l}18 \\
16 \\
16 \\
16 \\
20 \\
27\end{array}$ & $\begin{array}{l}21 \\
19 \\
19 \\
18 \\
22 \\
31\end{array}$ & $\begin{array}{l}23 \\
22 \\
23 \\
21 \\
24 \\
37\end{array}$ & $\begin{array}{l}25 \\
23 \\
26 \\
22 \\
25 \\
43\end{array}$ & $\begin{array}{l}27 \\
25 \\
29 \\
23 \\
27 \\
47\end{array}$ & $\begin{array}{l}29 \\
27 \\
32 \\
24 \\
29 \\
51\end{array}$ & $\begin{array}{l}32 \\
30 \\
35 \\
25 \\
31 \\
54\end{array}$ & $\begin{array}{l}37 \\
33 \\
39 \\
28 \\
40 \\
61\end{array}$ & $\begin{array}{l}44 \\
37 \\
44 \\
30 \\
47 \\
70\end{array}$ & $\begin{array}{l}51 \\
43 \\
50 \\
33 \\
55 \\
81\end{array}$ & $\begin{array}{l}61 \\
50 \\
56 \\
36 \\
70 \\
92\end{array}$ & $\begin{array}{r}79 \\
59 \\
67 \\
40 \\
100 \\
110\end{array}$ & $\begin{array}{r}110 \\
78 \\
93 \\
50 \\
150 \\
140\end{array}$ \\
\hline
\end{tabular}


LOCATION:

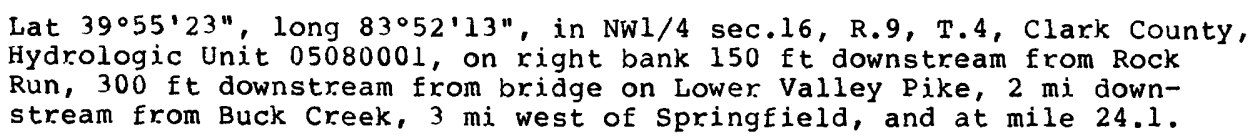

DRAINAGE AREA: $\quad 490 \mathrm{mi}^{2}$.

TRIBUTARY TO: Great Miami River.

DISCHARGE DATA USED: October 1914 to september. 1978.

REMARKS:

Some regulation by C.J. Brown Reservoir, $8.3 \mathrm{mi}$ upstream on Buck Creek, since 1972. Occasional low-flow regulation by powerplant $2.3 \mathrm{mi}$ upstream; daily flows are not affected appreciably.

SELECTED DISCHARGE CHARACTERISTICS: Average discharge: $483 \mathrm{ft}^{3} / \mathrm{s}$ (64 years).

Minimum daily discharge: $86 \mathrm{ft}^{3} / \mathrm{s}$ July 1934.

Magnitude and frequency of low flow for indicated periods

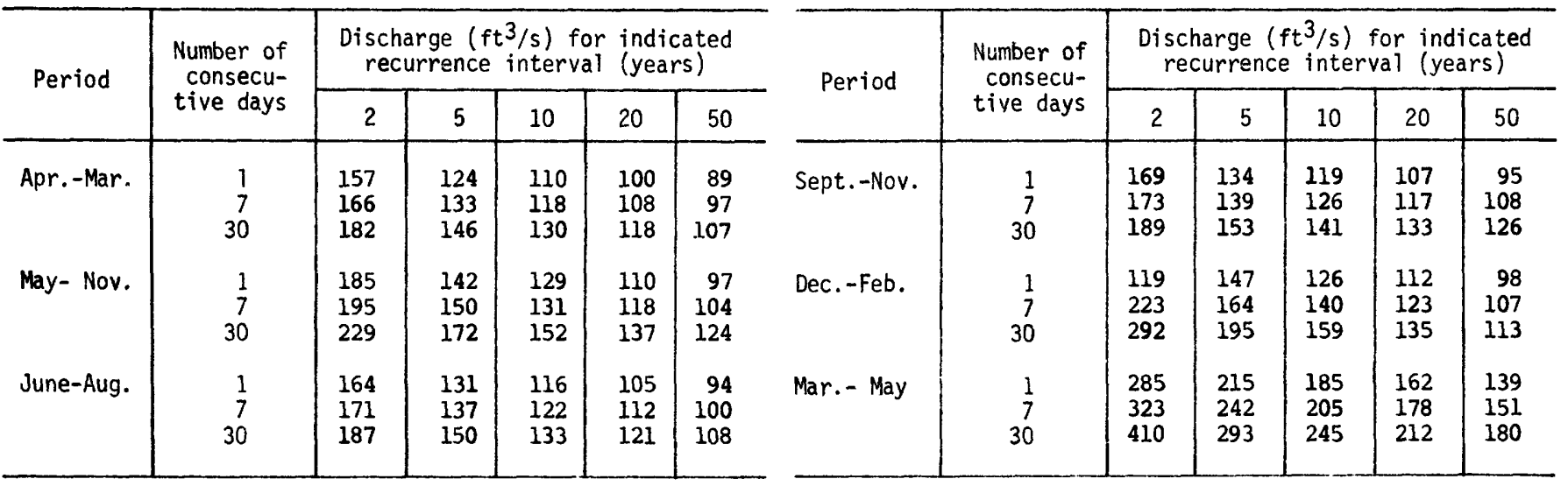

Duration of daily flow for indicated periods

\begin{tabular}{|c|c|c|c|c|c|c|c|c|c|c|c|c|c|}
\hline \multirow{2}{*}{ Period } & \multicolumn{13}{|c|}{ Discharge $\left(\mathrm{ft}^{3} / \mathrm{s}\right)$ which was equaled or exceeded for indicated percent of time } \\
\hline & 98 & 95 & 90 & 85 & 80 & 75 & 70 & 60 & 50 & 40 & 30 & 20 & 10 \\
\hline $\begin{array}{l}\text { Apr. - Mar. } \\
\text { May - Nov. } \\
\text { June - Aug. } \\
\text { Sept. - Nov. } \\
\text { Dec. - Feb. } \\
\text { Mar. - May }\end{array}$ & $\begin{array}{l}130 \\
120 \\
120 \\
120 \\
130 \\
190\end{array}$ & $\begin{array}{l}150 \\
140 \\
140 \\
130 \\
150 \\
230\end{array}$ & $\begin{array}{l}170 \\
160 \\
170 \\
150 \\
170 \\
260\end{array}$ & $\begin{array}{l}180 \\
170 \\
180 \\
160 \\
190 \\
290\end{array}$ & $\begin{array}{l}200 \\
180 \\
200 \\
170 \\
210 \\
320\end{array}$ & $\begin{array}{l}210 \\
200 \\
210 \\
170 \\
230 \\
350\end{array}$ & $\begin{array}{l}230 \\
210 \\
230 \\
180 \\
250 \\
380\end{array}$ & $\begin{array}{l}270 \\
230 \\
260 \\
200 \\
300 \\
450\end{array}$ & $\begin{array}{l}320 \\
260 \\
290 \\
220 \\
360 \\
510\end{array}$ & $\begin{array}{l}380 \\
300 \\
330 \\
240 \\
440 \\
580\end{array}$ & $\begin{array}{l}470 \\
360 \\
380 \\
260 \\
560 \\
680\end{array}$ & $\begin{array}{l}600 \\
440 \\
460 \\
310 \\
730 \\
840\end{array}$ & $\begin{array}{r}880 \\
620 \\
620 \\
410 \\
1100 \\
1200\end{array}$ \\
\hline
\end{tabular}


GREAT MIAMI RIVER BASIN

03270000 Mad River near Dayton, Ohio

LOCATION:

39.47'50", long 84.05'19", in SWl/4 sec.7, R.8, T.2, Green County, Hydrologic Unit 05080001 , on left bank in retarding basin $300 \mathrm{ft}$ upstream from Huffman Dam, $2.3 \mathrm{mi}$ downstream from Mud Run, $6.2 \mathrm{mi}$ northeast of Dayton and at mile 6.1.

DRAINAGE AREA: $\quad 635 \mathrm{mi}^{2}$.

TRIBUTARY TO: Great Miami River.

DISCHARGE DATA USED: October 1914 to September 1921, October 1924 to September. 1978.

REMARKS:

Flood flows affected by backwater from Huffman retarding dam beginning in 1921, some regulation by C.J. Brown Reservoir $26 \mathrm{mi}$ upstream on Buck Creek since 1972 .

SELECTED DISCHARGE CHARACTERISTICS: Average discharge: $614 \mathrm{ft}^{3} / \mathrm{s}(61$ years).

Minimum daily discharge: $94 \mathrm{ft}^{3} / \mathrm{s}$ August 1934 .

Magnitude and frequency of low flow for indicated periods

\begin{tabular}{|c|c|c|c|c|c|c|c|c|c|c|c|c|c|}
\hline \multirow{2}{*}{ Period } & \multirow{2}{*}{$\begin{array}{l}\text { Number of } \\
\text { consecu- } \\
\text { tive days }\end{array}$} & \multicolumn{5}{|c|}{$\begin{array}{l}\text { Discharge }\left(\mathrm{ft}^{3} / \mathrm{s}\right) \text { for indicated } \\
\text { recurrence interval (years) }\end{array}$} & \multirow{2}{*}{ Period } & \multirow{2}{*}{$\begin{array}{l}\text { Number of } \\
\text { consecu- } \\
\text { tive days }\end{array}$} & \multicolumn{5}{|c|}{$\begin{array}{l}\text { Discharge }\left(\mathrm{ft}^{3} / \mathrm{s}\right) \text { for indicated } \\
\text { recurrence interval (years) }\end{array}$} \\
\hline & & 2 & 5 & 10 & 20 & 50 & & & 2 & 5 & 10 & 20 & 50 \\
\hline Apr.-Mar. & $\begin{array}{r}1 \\
7 \\
30\end{array}$ & $\begin{array}{l}186 \\
195 \\
216\end{array}$ & $\begin{array}{l}144 \\
150 \\
165\end{array}$ & $\begin{array}{l}125 \\
131 \\
145\end{array}$ & $\begin{array}{l}112 \\
118 \\
130\end{array}$ & $\begin{array}{r}99 \\
104 \\
115\end{array}$ & Sept.-Nov. & $\begin{array}{r}1 \\
7 \\
30\end{array}$ & $\begin{array}{l}196 \\
204 \\
227\end{array}$ & $\begin{array}{l}148 \\
154 \\
174\end{array}$ & $\begin{array}{l}129 \\
135 \\
154\end{array}$ & $\begin{array}{l}115 \\
121 \\
140\end{array}$ & $\begin{array}{l}101 \\
108 \\
128\end{array}$ \\
\hline June-Aug. & $\begin{array}{r}1 \\
7 \\
30\end{array}$ & $\begin{array}{l}219 \\
232 \\
278\end{array}$ & $\begin{array}{l}163 \\
172 \\
200\end{array}$ & $\begin{array}{l}140 \\
148 \\
171\end{array}$ & $\begin{array}{l}123 \\
130 \\
151\end{array}$ & $\begin{array}{l}107 \\
113 \\
134\end{array}$ & Mar. - May & $\begin{array}{r}1 \\
7 \\
30\end{array}$ & $\begin{array}{l}356 \\
402 \\
526\end{array}$ & $\begin{array}{l}261 \\
293 \\
368\end{array}$ & $\begin{array}{l}219 \\
244 \\
302\end{array}$ & $\begin{array}{l}187 \\
207 \\
255\end{array}$ & $\begin{array}{l}156 \\
170 \\
209\end{array}$ \\
\hline
\end{tabular}

Duration of daily flow for indicated periods

\begin{tabular}{|c|c|c|c|c|c|c|c|c|c|c|c|c|c|}
\hline \multirow{2}{*}{ Period } & \multicolumn{13}{|c|}{ Discharge $\left(\mathrm{ft}^{3} / \mathrm{s}\right)$ which was equaled or exceeded for indicated percent of time } \\
\hline & 98 & 95 & 90 & 85 & 80 & 75 & 70 & 60 & 50 & 40 & 30 & 20 & 10 \\
\hline $\begin{array}{l}\text { Apr. - Mar. } \\
\text { May - Nov. } \\
\text { June - Aug. } \\
\text { Sept. - Nov. } \\
\text { Dec. - Feb. } \\
\text { Mar. - May }\end{array}$ & $\begin{array}{l}150 \\
140 \\
140 \\
130 \\
160 \\
220\end{array}$ & $\begin{array}{l}170 \\
160 \\
160 \\
150 \\
170 \\
270\end{array}$ & $\begin{array}{l}190 \\
180 \\
190 \\
160 \\
200 \\
330\end{array}$ & $\begin{array}{l}220 \\
200 \\
220 \\
180 \\
230 \\
370\end{array}$ & $\begin{array}{l}240 \\
220 \\
240 \\
190 \\
250 \\
410\end{array}$ & $\begin{array}{l}260 \\
230 \\
250 \\
200 \\
290 \\
450\end{array}$ & $\begin{array}{l}280 \\
250 \\
270 \\
210 \\
320 \\
500\end{array}$ & $\begin{array}{l}330 \\
280 \\
310 \\
240 \\
310 \\
570\end{array}$ & $\begin{array}{l}400 \\
320 \\
360 \\
260 \\
460 \\
660\end{array}$ & $\begin{array}{l}490 \\
380 \\
410 \\
300 \\
570 \\
750\end{array}$ & $\begin{array}{l}600 \\
450 \\
470 \\
330 \\
730 \\
890\end{array}$ & $\begin{array}{r}780 \\
570 \\
580 \\
400 \\
960 \\
1100\end{array}$ & $\begin{array}{r}1200 \\
790 \\
800 \\
540 \\
1400 \\
1500\end{array}$ \\
\hline
\end{tabular}


Appendix 3.--Low-flow data--Continued

GREAT MIAMI RIVER BASIN

03270500 Great Miami River at Dayton, Ohio

LOCATION: Lat $39^{\circ} 45^{\prime} 55^{\prime \prime}$, long $84^{\circ} 11^{\prime} 51^{\prime \prime}$, in sec.10, R.7, T.1, Montgomery County, Hydrologic Unit 05080002 , on left bank 1,000 ft downstream from Main Street Bridge in Dayton, $0.7 \mathrm{mi}$ upstream from Wolf Creek, $0.8 \mathrm{mi}$ downstream from Mad River, and at mile 80.0 .

DRAINAGE AREA: $\quad 2,511 \mathrm{mi}^{2}$.

TRIBUTARY TO: Ohio River

DISCHARGE DATA USED: April 1913 to september 1978.

REMARKS: $\quad$ Flood flow regulated by four retarding basins upstream from station beginning in 1920 on Mad River $6.5 \mathrm{mi}$ upstream, on Stillwater River $10.5 \mathrm{mi}$ upstream, on Great Miami Kiver $11.5 \mathrm{mi}$ upstream, and on Loramie Creek $40 \mathrm{mi}$ upstream. Water is diverted $6 \mathrm{mi}$ upstream from station for use in Dayton; most of return flow from diversions bypasses station in Dayton sewer systems.

SELECTED DISCHARGE CHARACTERISTICS: Average discharge: $2,190 \mathrm{ft}^{3} / \mathrm{s}\{65$ years). Minimum daily discharge: $109 \mathrm{ft}^{3} / \mathrm{s}$ August 1934 .

Magnitude and frequency of low flow for indicated periods

\begin{tabular}{|c|c|c|c|c|c|c|c|c|c|c|c|c|c|}
\hline \multirow{2}{*}{ Period } & \multirow{2}{*}{$\begin{array}{l}\text { Number of } \\
\text { consecu- } \\
\text { tive days }\end{array}$} & \multicolumn{5}{|c|}{$\begin{array}{l}\text { Discharge }\left(\mathrm{ft}^{3} / \mathrm{s}\right) \text { for indicated } \\
\text { recurrence interval (years) }\end{array}$} & \multirow{2}{*}{ Period } & \multirow{2}{*}{$\begin{array}{l}\text { Number of } \\
\text { consecu- } \\
\text { tive days }\end{array}$} & \multicolumn{5}{|c|}{$\begin{array}{l}\text { Discharge }\left(\mathrm{ft}^{3} / \mathrm{s}\right) \text { for indicated } \\
\text { recurrence interval (years) }\end{array}$} \\
\hline & & 2 & 5 & 10 & 20 & 50 & & & 2 & 5 & 10 & 20 & 50 \\
\hline Apr.-Mar. & $\begin{array}{r}1 \\
7 \\
30\end{array}$ & $\begin{array}{l}277 \\
299 \\
344\end{array}$ & $\begin{array}{l}190 \\
207 \\
241\end{array}$ & $\begin{array}{l}154 \\
169 \\
199\end{array}$ & $\begin{array}{l}129 \\
143 \\
170\end{array}$ & $\begin{array}{l}105 \\
117 \\
142\end{array}$ & Sept.-Nov. & $\begin{array}{r}1 \\
7 \\
30\end{array}$ & $\begin{array}{l}290 \\
303 \\
365\end{array}$ & $\begin{array}{l}197 \\
210 \\
250\end{array}$ & $\begin{array}{l}161 \\
176 \\
214\end{array}$ & $\begin{array}{l}135 \\
154 \\
192\end{array}$ & $\begin{array}{l}111 \\
134 \\
173\end{array}$ \\
\hline June-Aug. & $\begin{array}{r}1 \\
7 \\
30\end{array}$ & $\begin{array}{l}335 \\
375 \\
493\end{array}$ & $\begin{array}{l}227 \\
252 \\
313\end{array}$ & $\begin{array}{l}185 \\
205 \\
256\end{array}$ & $\begin{array}{l}155 \\
173 \\
221\end{array}$ & $\begin{array}{l}127 \\
143 \\
191\end{array}$ & Mar. - May & $\begin{array}{r}1 \\
7 \\
30\end{array}$ & $\begin{array}{r}813 \\
933 \\
1450\end{array}$ & $\begin{array}{l}543 \\
627 \\
914\end{array}$ & $\begin{array}{l}427 \\
501 \\
710\end{array}$ & $\begin{array}{l}344 \\
414 \\
573\end{array}$ & $\begin{array}{l}265 \\
330 \\
448\end{array}$ \\
\hline
\end{tabular}

Duration of daily flow for indicated periods

\begin{tabular}{|c|c|c|c|c|c|c|c|c|c|c|c|c|c|}
\hline \multirow{2}{*}{ Period } & \multicolumn{13}{|c|}{ Discharge $\left(\mathrm{ft}^{3} / \mathrm{s}\right)$ which was equaled or exceeded for indicated percent of time } \\
\hline & 98 & 95 & 90 & 85 & 80 & 75 & 70 & 60 & 50 & 40 & 30 & 20 & 10 \\
\hline $\begin{array}{l}\text { Apr. - Mar. } \\
\text { May - Nov. } \\
\text { June - Aug. } \\
\text { Sept. - Nov. } \\
\text { Dec. - Feb. } \\
\text { Mar. - May }\end{array}$ & $\begin{array}{l}200 \\
180 \\
210 \\
160 \\
250 \\
500\end{array}$ & $\begin{array}{l}260 \\
230 \\
250 \\
200 \\
290 \\
650\end{array}$ & $\begin{array}{l}320 \\
280 \\
310 \\
240 \\
370 \\
810\end{array}$ & $\begin{array}{l}370 \\
320 \\
360 \\
280 \\
450 \\
950\end{array}$ & $\begin{array}{r}420 \\
360 \\
400 \\
310 \\
540 \\
1100\end{array}$ & $\begin{array}{r}480 \\
400 \\
450 \\
330 \\
640 \\
1200\end{array}$ & $\begin{array}{r}560 \\
440 \\
490 \\
360 \\
760 \\
1400\end{array}$ & $\begin{array}{r}740 \\
530 \\
600 \\
410 \\
1100 \\
1700\end{array}$ & $\begin{array}{r}980 \\
660 \\
740 \\
470 \\
1400 \\
2100\end{array}$ & $\begin{array}{r}1300 \\
830 \\
900 \\
560 \\
1900 \\
2600\end{array}$ & $\begin{array}{r}1900 \\
11.00 \\
11.100 \\
680 \\
2600 \\
3300\end{array}$ & $\begin{array}{r}2700 \\
1500 \\
1500 \\
870 \\
3800 \\
4700\end{array}$ & $\begin{array}{l}5000 \\
2600 \\
2500 \\
1500 \\
7200 \\
8200\end{array}$ \\
\hline
\end{tabular}


Appendix 3.--Low-flow data--Continued

GREAT MIAMI RIVER BASIN

03270800 Wolf Creek at Trotwood, Ohio

LOCATION:

Lat $39^{\circ} 47^{\prime} 39^{\prime \prime}$, long $84^{\circ} 18^{\prime} 36^{\prime \prime}$, Montgomery County, Hydrologic Unit 05080002 , on right bank $350 \mathrm{ft}$ downstream from Union Road Bridge, $700 \mathrm{ft}$ downstream from unnamed right bank tributary, $0.2 \mathrm{mi}$ south of Trotwood, and $0.3 \mathrm{mi}$ upstream from North Branch.

DRAINAGE AREA: $\quad 22.7 \mathrm{mi}^{2}$.

TRIBUTARY TO: Great Miami River.

DISCHARGE DATA USED: October 1962 to September 1978.

REMARKS:

None.

SELECTED DISCHARGE CHARACTERISTICS: Average discharge: $20.5 \mathrm{ft}^{3} / \mathrm{s}$ (16 years).

Minimum daily discharge: No flow september 1964, 1967.

Magnitude and frequency of low flow for indicated periods

\begin{tabular}{|c|c|c|c|c|c|c|c|c|c|c|c|c|c|}
\hline \multirow{2}{*}{ Period } & \multirow{2}{*}{$\begin{array}{l}\text { Number of } \\
\text { consecu- } \\
\text { tive days }\end{array}$} & \multicolumn{5}{|c|}{$\begin{array}{l}\text { Discharge }\left(\mathrm{ft}^{3} / \mathrm{s}\right) \text { for indicated } \\
\text { recurrence interval (years) }\end{array}$} & \multirow{2}{*}{ Period } & \multirow{2}{*}{$\begin{array}{l}\text { Number of } \\
\text { consecu- } \\
\text { tive days }\end{array}$} & \multicolumn{5}{|c|}{$\begin{array}{l}\text { Discharge }\left(\mathrm{ft}^{3} / \mathrm{s}\right) \text { for indicated } \\
\text { recurrence interval (years) }\end{array}$} \\
\hline & & 2 & 5 & 10 & 20 & 50 & & & 2 & 5 & 10 & 20 & 50 \\
\hline Apr.-Mar. & $\begin{array}{r}1 \\
7 \\
30\end{array}$ & $\begin{array}{l}0.2 \\
0.4 \\
0.7\end{array}$ & $\begin{array}{l}0 \\
0.1 \\
0.3\end{array}$ & $\begin{array}{l}(: \\
0 \\
0.2\end{array}$ & $\begin{array}{l}0 \\
0 \\
0.1\end{array}$ & $\begin{array}{l}0 \\
0 \\
0.1\end{array}$ & Sept.-Nov. & $\begin{array}{r}1 \\
7 \\
30\end{array}$ & $\begin{array}{l}0.4 \\
0.5 \\
1.0\end{array}$ & $\begin{array}{l}0.1 \\
0.1 \\
0.4\end{array}$ & $\begin{array}{l}0 \\
0 \\
0.2\end{array}$ & $\begin{array}{l}0 \\
0 \\
0.1\end{array}$ & $\begin{array}{l}0 \\
0 \\
0.1\end{array}$ \\
\hline June-Aug. & $\begin{array}{r}1 \\
7 \\
30\end{array}$ & $\begin{array}{l}0.4 \\
0.5 \\
1.4\end{array}$ & $\begin{array}{l}0.2 \\
0.3 \\
0.6\end{array}$ & $\begin{array}{l}.01 \\
0.2 \\
0.4\end{array}$ & $\begin{array}{l}0 \\
0.2 \\
0.3\end{array}$ & $\begin{array}{l}0 \\
0.1 \\
0.2\end{array}$ & Mar. - May & $\begin{array}{r}1 \\
7 \\
30\end{array}$ & $\begin{array}{l}3.4 \\
4.5 \\
10\end{array}$ & $\begin{array}{l}2.4 \\
2.9 \\
6.3\end{array}$ & $\begin{array}{l}2.0 \\
2.3 \\
5.0\end{array}$ & $\begin{array}{l}1.8 \\
2.0 \\
4.3\end{array}$ & $\begin{array}{l}1.5 \\
1.7 \\
3.6\end{array}$ \\
\hline
\end{tabular}

Duration of daily flow for indicated periods

\begin{tabular}{l|c|c|c|c|c|c|c|c|c|c|r|r|r}
\hline \multirow{2}{*}{ Period } & \multicolumn{8}{|c|}{ Discharge $\left(\mathrm{ft}^{3} / \mathrm{s}\right)$ which was equaled or exceeded for indicated percent of time } \\
\cline { 2 - 12 } & 98 & 95 & 90 & 85 & 80 & 75 & 70 & 60 & 50 & 40 & 30 & 20 & 10 \\
\hline Apr. - Mar. & 0.2 & 0.4 & 0.5 & 0.8 & 1.2 & 1.6 & 2.2 & 3.6 & 5.6 & 8.7 & 13 & 22 & 45 \\
May - Nov. & 0.2 & 0.3 & 0.4 & 0.5 & 0.7 & 0.9 & 1.2 & 1.8 & 2.7 & 3.8 & 5.5 & 9.0 & 19 \\
June - Aug. & 0.2 & 0.3 & 0.4 & 0.6 & 0.8 & 1.0 & 1.3 & 1.8 & 2.6 & 3.7 & 5.3 & 7.6 & 15 \\
Sept. - Nov. & 0.1 & 0.2 & 0.3 & 0.4 & 0.5 & 0.6 & 0.8 & 1.2 & 1.7 & 2.3 & 3.3 & 4.8 & 10 \\
Dec. - Feb. & 0.4 & 0.5 & 1.1 & 1.8 & 2.6 & 3.5 & 4.6 & 7.4 & 9.9 & 14 & 21 & 33 & 65 \\
Mar. - May & 3.0 & 3.7 & 5.0 & 6.2 & 7.3 & 8.7 & 10 & 13 & 17 & 22 & 30 & 44 & 83 \\
\hline
\end{tabular}


Appendix 3.--Low-flow data--Continued

GREAT MIAMI RIVER BASIN

03271300 Holes Creek near Kettering, Ohio

LOCATION:

Lat $39^{\circ} 39^{\prime} 15^{\prime \prime}$, long $84^{\circ} 11^{\prime} 45^{\prime \prime}$, Montgomery County, Hydrologic

Unit 05080002 , at Mad River Road bridge, $200 \mathrm{ft}$ south of

Alexandria-Bellbrook Road, $2.8 \mathrm{mi}$ southwest of Kettering.

DRAINAGE AREA: $\quad 18.7 \mathrm{mi}^{2}$.

TRIBUTARY TO:

Great Miami River.

DISCHARGE DATA USED:

Low-flow measurements 1959-1972 water years.

INDEX STATION: $\quad 03272000$ Twin Creek near Germantown, Ohio.

REMARKS:

None.

SELECTED DISCHARGE CHARACTERISTICS: Minimum discharge observed: $1.0 \mathrm{ft} / \mathrm{s}$ November 1964.

Magnitude and frequency of low flow for indicated periods

\begin{tabular}{c|c|c|c}
\hline \multirow{2}{*}{ Period } & \multirow{2}{*}{$\begin{array}{c}\text { Number of } \\
\text { consecu- } \\
\text { tive days }\end{array}$} & \multicolumn{2}{|c}{$\begin{array}{c}\text { Discharge }\left(\mathrm{ft}^{3} / \mathrm{s}\right) \text { for } \\
\text { indicated recurrence } \\
\text { interval (years) }\end{array}$} \\
\cline { 3 - 4 } Apr. - Mar. & 7 & 2 & 10 \\
\hline May - Nov. & 30 & 1.2 & 0.3 \\
& 7 & 1.5 & 0.8 \\
June - Aug. & 30 & 1.4 & 0.7 \\
& 7 & 1.6 & 0.8 \\
Sept. - Nov. & 30 & 2.3 & 0.9 \\
& 7 & 1.2 & 0.7 \\
& 30 & 1.6 & 0.9 \\
\hline
\end{tabular}

Duration of daily flow for indicated periods

\begin{tabular}{l|c|c|c|c|c}
\hline \multirow{2}{*}{ Period } & \multicolumn{5}{|c|}{$\begin{array}{c}\text { Discharge }(\mathrm{ft} 3 / \mathrm{s} \text { ) which was equaled or } \\
\text { exceeded for indicated percent of time }\end{array}$} \\
\cline { 2 - 6 } & 98 & 95 & 90 & 85 & 80 \\
\hline Apr. - Mar. & 0.8 & 1.1 & 1.4 & 1.6 & 2.0 \\
May - Nov. & 0.8 & 1.0 & 1.2 & 1.4 & 1.6 \\
June - Aug. & 0.9 & 1.1 & 1.4 & 1.6 & 1.8 \\
Sept. - Nov. & 0.7 & 0.8 & 1.0 & 1.2 & 1.3 \\
\hline
\end{tabular}


Appendix 3.--Low-flow data--Continued

GREAT MIAMI RIVER BASIN

03271620 Great Miami River at Franklin, Ohio

LOCATION:

Lat $39^{\circ} 33^{\prime} 44^{\prime \prime}$, long $8^{\circ} 18^{\prime} 18^{\prime \prime}$, Warren County, Hydrologic Unit 05080002, at bridge on state Route 123 in Franklin.

DRAINAGE AREA: $\quad 2,727 \mathrm{mi}^{2}$.

TRIBUTARY TO: Ohio River.

DISCHARGE DATA USED:

Low-flow measurements 1965-1969 water years.

INDEX STATION: $\quad 03271500$ Great Miami River at Miamisburg, Ohio.

REMARKS:

Diurnal fluctuation caused by upstream power plant.

SELECTED DISCHARGE CHARACTERISTICS: Minimum discharge observed: $311 \mathrm{ft}^{3} / \mathrm{s}$ August 1965.

Magnitude and frequency of low flow for indicated periods

\begin{tabular}{c|c|c|c}
\hline \multirow{2}{*}{ Period } & \multirow{2}{*}{$\begin{array}{c}\text { Number of } \\
\text { consecu- } \\
\text { tive days }\end{array}$} & \multicolumn{2}{|c}{$\begin{array}{c}\text { Discharge (ft3/s) for } \\
\text { ind icated recurrence } \\
\text { interval (years) }\end{array}$} \\
\cline { 3 - 4 } & & 2 & 10 \\
\hline Apr. - Mar. & 7 & 380 & 235 \\
& 30 & 435 & 270 \\
May - Nov. & 7 & 390 & 240 \\
& 30 & 445 & 270 \\
June - Aug. & 7 & 490 & 285 \\
& 30 & 605 & 350 \\
Sept. - Nov. & 7 & 385 & 255 \\
& 30 & 460 & 285 \\
\hline
\end{tabular}

Duration of daily flow for indicated periods

\begin{tabular}{|c|c|c|c|c|c|}
\hline \multirow{2}{*}{ Period } & \multicolumn{5}{|c|}{$\begin{array}{l}\text { Discharge }\left(\mathrm{ft}^{3} / \mathrm{s}\right) \text { which was equaled or } \\
\text { exceeded for indicated percent of time }\end{array}$} \\
\hline & 98 & 95 & 90 & 85 & 80 \\
\hline $\begin{array}{l}\text { Apr. - Mar. } \\
\text { May - Nov. } \\
\text { June - Aug. } \\
\text { Sept. - Nov. }\end{array}$ & $\begin{array}{l}260 \\
240 \\
290 \\
220\end{array}$ & $\begin{array}{l}320 \\
290 \\
350 \\
250\end{array}$ & $\begin{array}{l}400 \\
360 \\
410 \\
290\end{array}$ & $\begin{array}{l}450 \\
410 \\
670 \\
350\end{array}$ & $\begin{array}{l}520 \\
450 \\
520 \\
390\end{array}$ \\
\hline
\end{tabular}


Appendix 3.--Low-flow data--Continued

GREAT MIAMI RIVER BASIN

03271700 Clear Creek at Franklin, Ohio

LOCATION:

Lat 39०33'05", long $84^{\circ} 17^{\prime} 55^{\prime \prime}$, Warren County, Hydrologic

Unit 05080002 , at bridge on Shaker Road at south edge of

Franklin, and $1.6 \mathrm{mi}$ upstream from mouth.

DRAINAGE AREA: $\quad 51.5 \mathrm{mi}^{2}$.

TRIBUTARY TO:

Great Miami River.

DISCHARGE DATA USED: Low-flow measurements 1959-1969 water years.

INDEX STATION: $\quad 03272000$ Twin Creek near Germantown, Ohio.

REMARKS:

None.

SELECTED DISCHARGE CHARACTERISTICS: Minimum discharge observed: No ijow August 1965.

\begin{tabular}{c|c|c|c}
\multicolumn{4}{c}{$\begin{array}{c}\text { Magnitude and frequency of low flow for } \\
\text { indicated periods }\end{array}$} \\
\hline \multirow{3}{*}{ Period } & $\begin{array}{c}\text { Number of } \\
\text { consecu- } \\
\text { tive days }\end{array}$ & $\begin{array}{c}\left.\text { Discharge (ft }{ }^{3} / \mathrm{s}\right) \text { for } \\
\text { indicated recurrence } \\
\text { interval (years) }\end{array}$ \\
\cline { 3 - 4 } & 7 & 2 & 10 \\
\hline Apr. - Mar. & 30 & 0.8 & 0.2 \\
May - Nov. & 7 & 1.2 & 0.1 \\
& 30 & 1.1 & 0.3 \\
June - Aug. & 7 & 1.3 & 0.4 \\
& 30 & 2.8 & 0.4 \\
Sept. - Nov. & 7 & 0.9 & 0.3 \\
& 30 & 1.4 & 0.4 \\
\hline
\end{tabular}

Duration of daily flow for indicated periods

\begin{tabular}{l|c|c|c|c|c}
\hline \multirow{2}{*}{ Period } & \multicolumn{5}{|c}{$\begin{array}{c}\text { Discharge (ft } 3 / \mathrm{s} \text { ) which was equaled or } \\
\text { exceeded for indicated percent of time }\end{array}$} \\
\cline { 2 - 6 } & 98 & 95 & 90 & 85 & 80 \\
\hline Apr. - Mar. & 0.4 & 0.6 & 1.1 & 1.4 & 2.1 \\
May - Nov. & 0.3 & 0.5 & 0.8 & 1.1 & 1.3 \\
June - Aug. & 0.4 & 0.7 & 1.1 & 1.4 & 1.8 \\
Sept. - Nov. & 0.2 & 0.3 & 0.5 & 0.7 & 0.9 \\
\hline
\end{tabular}


LOCATION:

DRAINAGE AREA:

TRIBUTARY TO:

DISCHARGE DATA USED:

REMARKS :

None.

SELECTED DISCHARGE CHARACTERISTICS:

Average discharge: $181 \mathrm{ft}^{3} / \mathrm{s}$ ( 16 years)

Minimum daily discharge: $2.5 \mathrm{ft}^{3} / \mathrm{s}$ September 1964 .

Magnitude and frequency of low fluw for indicated periods

\begin{tabular}{c|c|c|c|c|c|c}
\hline \multirow{2}{*}{ Period } & \multirow{2}{*}{$\begin{array}{c}\text { Number of } \\
\text { consecu- } \\
\text { tive days }\end{array}$} & \multicolumn{4}{|c|}{$\begin{array}{c}\text { Discharge }(\mathrm{ft} / \mathrm{s} \text { ) for indicated } \\
\text { recurrence interva1 (years) }\end{array}$} \\
\cline { 3 - 7 } Apr.-Mar. & 1 & 6.4 & 5 & 10 & 20 & 50 \\
\hline \multirow{4}{*}{ May- Nov. } & 7 & 7.1 & 4.4 & 3.1 & 2.6 & 2.1 \\
& 30 & 8.9 & 5.5 & 4.3 & 2.9 & 2.3 \\
& 1 & 6.4 & 4.0 & 3.1 & 2.6 & 2.7 \\
& 7 & 7.1 & 4.4 & 3.5 & 2.9 & 2.3 \\
& 30 & 8.9 & 5.5 & 4.3 & 3.5 & 2.7 \\
& 1 & 8.6 & 5.6 & 4.6 & 4.0 & 3.5 \\
& 7 & 10 & 6.6 & 5.4 & 4.7 & 4.1 \\
& 30 & 15 & 8.8 & 7.1 & 6.1 & 5.2 \\
\hline
\end{tabular}

\begin{tabular}{|c|c|c|c|c|c|c|}
\hline \multirow{2}{*}{ Period } & \multirow{2}{*}{$\begin{array}{l}\text { Number of } \\
\text { consecu- } \\
\text { tive days }\end{array}$} & \multicolumn{5}{|c|}{$\begin{array}{l}\text { Discharge }\left(\mathrm{ft}^{3} / \mathrm{s}\right) \text { for indicated } \\
\text { recurrence interval (years) }\end{array}$} \\
\hline & & 2 & 5 & 10 & 20 & 50 \\
\hline Sept.-Nov. & $\begin{array}{r}1 \\
7 \\
30\end{array}$ & $\begin{array}{l}6.6 \\
7.5 \\
10\end{array}$ & $\begin{array}{l}4.0 \\
4.6 \\
6.2\end{array}$ & $\begin{array}{l}3.2 \\
3.6 \\
4.6\end{array}$ & $\begin{array}{l}2.6 \\
2.9 \\
3.5\end{array}$ & $\begin{array}{l}2.1 \\
2.4 \\
2.6\end{array}$ \\
\hline Dec.-Feb. & $\begin{array}{r}1 \\
7 \\
30\end{array}$ & $\begin{array}{l}25 \\
30 \\
64\end{array}$ & $\begin{array}{l}11 \\
14 \\
21\end{array}$ & $\begin{array}{l}7.0 \\
8.0 \\
11\end{array}$ & $\begin{array}{l}4.6 \\
4.8 \\
5.8\end{array}$ & $\begin{array}{l}2.7 \\
2.5 \\
2.8\end{array}$ \\
\hline Mar. - May & $\begin{array}{r}1 \\
7 \\
30\end{array}$ & $\begin{array}{l}39 \\
47 \\
92\end{array}$ & $\begin{array}{l}26 \\
31 \\
58\end{array}$ & $\begin{array}{l}21 \\
26 \\
46\end{array}$ & $\begin{array}{l}17 \\
22 \\
39\end{array}$ & $\begin{array}{l}14 \\
18 \\
31\end{array}$ \\
\hline
\end{tabular}

Duration of daily flow for indicated periods

\begin{tabular}{|c|c|c|c|c|c|c|c|c|c|c|c|c|c|}
\hline \multirow{2}{*}{ Period } & \multicolumn{13}{|c|}{ Discharge $\left(\mathrm{ft}^{3} / \mathrm{s}\right)$ which was equaled or exceeded for indicated percent of time } \\
\hline & 98 & 95 & 90 & 85 & 80 & 75 & 70 & 60 & 50 & 40 & 30 & 20 & 10 \\
\hline $\begin{array}{l}\text { Apr. - Mar. } \\
\text { May - Nov. } \\
\text { June - Aug. } \\
\text { Sept. - Nov. } \\
\text { Dec. - Feb. } \\
\text { Mar. - May }\end{array}$ & $\begin{array}{l}4.8 \\
4.2 \\
5.9 \\
3.5 \\
5.8 \\
29\end{array}$ & $\begin{array}{l}6.1 \\
5.5 \\
7.3 \\
4.2 \\
7.2 \\
40\end{array}$ & $\begin{array}{l}8.2 \\
6.9 \\
9.2 \\
5.4 \\
15 \\
50\end{array}$ & $\begin{array}{l}11 \\
8.5 \\
11 \\
6.2 \\
21 \\
58\end{array}$ & $\begin{array}{l}14 \\
10 \\
13 \\
7.1 \\
28 \\
67\end{array}$ & $\begin{array}{l}17 \\
12 \\
15 \\
8.1 \\
36 \\
77\end{array}$ & $\begin{array}{l}21 \\
14 \\
17 \\
9.5 \\
44 \\
88\end{array}$ & $\begin{array}{r}32 \\
18 \\
21 \\
13 \\
61 \\
110\end{array}$ & $\begin{array}{r}50 \\
24 \\
27 \\
16 \\
82 \\
140\end{array}$ & $\begin{array}{r}75 \\
32 \\
36 \\
19 \\
110 \\
190\end{array}$ & $\begin{array}{r}110 \\
48 \\
48 \\
23 \\
180 \\
250\end{array}$ & $\begin{array}{r}190 \\
79 \\
72 \\
29 \\
280 \\
390\end{array}$ & $\begin{array}{r}410 \\
150 \\
130 \\
72 \\
640 \\
810\end{array}$ \\
\hline
\end{tabular}


GREAT MIAMI RIVER BASIN

03272000 Twin Creek near Germantown, Ohio

LOCATION:

Lat $3^{\circ} 38^{\prime} 1^{\prime \prime}$, long $84^{\circ} 23^{\prime} 48^{\prime \prime}$, in NW1/4 sec. 11, T.3 N., R.4 E., Montgomery

County, Hydrologic Unit 05080002 , on right bank $0.3 \mathrm{mi}$ downstream from

Germantown Dam, $1.4 \mathrm{mi}$ northwest of Germantown, and $3 \mathrm{mi}$ upstream from Little Twin Creek.

DRAINAGE AREA: $\quad 275 \mathrm{mi}^{2}$.

TRIBUTARY TO: Great Miami River.

DISCHARGE DATA USED: May 1914 to December 1923, January 1927 to September 1978.

REMARKS:

Flood flow regulated by Germantown retarding basin $0.3 \mathrm{mi}$ upstream beginning 1920 .

SELECTED DISCHARGE CHARACTERISTICS: Average discharge: $259 \mathrm{ft}^{3} / \mathrm{s}$ ( 60 years). Minimum daily discharge: $2.0 \mathrm{ft}^{3} / \mathrm{s}$ September 1941.

Magnitude and frequency of low flow for indicated periods

\begin{tabular}{|c|c|c|c|c|c|c|c|c|c|c|c|c|c|}
\hline \multirow{2}{*}{ Period } & \multirow{2}{*}{$\begin{array}{l}\text { Number of } \\
\text { consecu- } \\
\text { tive days }\end{array}$} & \multicolumn{5}{|c|}{$\begin{array}{l}\text { Discharge }\left(\mathrm{ft}^{3} / \mathrm{s}\right) \text { for indicated } \\
\text { recurrence interval (years) }\end{array}$} & \multirow{2}{*}{ Period } & \multirow{2}{*}{$\begin{array}{l}\text { Number of } \\
\text { consecu- } \\
\text { tive days }\end{array}$} & \multicolumn{5}{|c|}{$\begin{array}{l}\text { Discharge }\left(\mathrm{ft}^{3} / \mathrm{s}\right) \text { for indicated } \\
\text { recurrence interval (years) }\end{array}$} \\
\hline & & 2 & 5 & 10 & 20 & 50 & & & 2 & 5 & 10 & 20 & 50 \\
\hline Apr.-Mar. & $\begin{array}{r}1 \\
7 \\
30\end{array}$ & $\begin{array}{l}9.3 \\
10 \\
14\end{array}$ & $\begin{array}{l}5.2 \\
5.9 \\
7.8\end{array}$ & $\begin{array}{l}3.8 \\
4.4 \\
5.9\end{array}$ & $\begin{array}{l}2.9 \\
3.4 \\
4.7\end{array}$ & $\begin{array}{l}2.2 \\
2.6 \\
3.7\end{array}$ & Sept.-Nov. & $\begin{array}{r}1 \\
7 \\
30\end{array}$ & $\begin{array}{l}9.8 \\
11 \\
16\end{array}$ & $\begin{array}{l}5.5 \\
6.2 \\
8.6\end{array}$ & $\begin{array}{l}4.1 \\
4.7 \\
6.5\end{array}$ & $\begin{array}{l}3.2 \\
3.8 \\
5.3\end{array}$ & $\begin{array}{l}2.4 \\
3.0 \\
4.2\end{array}$ \\
\hline June-Aug. & $\begin{array}{r}1 \\
7 \\
30\end{array}$ & $\begin{array}{l}13 \\
15 \\
26\end{array}$ & $\begin{array}{l}7.6 \\
8.8 \\
14\end{array}$ & $\begin{array}{l}5.8 \\
6.7 \\
10\end{array}$ & $\begin{array}{l}4.6 \\
5.3 \\
8.0\end{array}$ & $\begin{array}{l}3.6 \\
4.2 \\
6.2\end{array}$ & Mar. - May & $\begin{array}{r}1 \\
7 \\
30\end{array}$ & $\begin{array}{r}59 \\
70 \\
126\end{array}$ & $\begin{array}{l}36 \\
43 \\
72\end{array}$ & $\begin{array}{l}27 \\
32 \\
53\end{array}$ & $\begin{array}{l}21 \\
25 \\
41\end{array}$ & $\begin{array}{l}15 \\
18 \\
31\end{array}$ \\
\hline
\end{tabular}

Duration of daily flow for indicated periods

\begin{tabular}{|c|c|c|c|c|c|c|c|c|c|c|c|c|c|c|}
\hline \multirow{2}{*}{\multicolumn{2}{|c|}{ Period }} & \multicolumn{13}{|c|}{ Discharge $\left(\mathrm{ft}^{3} / \mathrm{s}\right)$ which was equaled or exceeded for indicated percent of time } \\
\hline & & 98 & 95 & 90 & 85 & 80 & 75 & 70 & 60 & 50 & 40 & 30 & 20 & 10 \\
\hline $\begin{array}{l}\text { Apr. - } \\
\text { May - } \\
\text { June - } \\
\text { Sept. - } \\
\text { Dec. - } \\
\text { Mar. - }\end{array}$ & $\begin{array}{l}\text { Mar. } \\
\text { Nov. } \\
\text { Aug. } \\
\text { Nov. } \\
\text { Feb. } \\
\text { May }\end{array}$ & $\begin{array}{r}5.9 \\
5.1 \\
6.6 \\
4.2 \\
9.1 \\
34\end{array}$ & $\begin{array}{l}8.7 \\
7.3 \\
9.2 \\
5.6 \\
13 \\
48\end{array}$ & $\begin{array}{c}13 \\
10 \\
13 \\
7.6 \\
19 \\
63\end{array}$ & $\begin{array}{c}16 \\
13 \\
16 \\
9.4 \\
28 \\
76\end{array}$ & $\begin{array}{l}21 \\
15 \\
19 \\
11 \\
39 \\
89\end{array}$ & $\begin{array}{r}26 \\
18 \\
23 \\
13 \\
53 \\
100\end{array}$ & $\begin{array}{r}32 \\
21 \\
26 \\
15 \\
68 \\
120\end{array}$ & $\begin{array}{r}49 \\
28 \\
34 \\
18 \\
100 \\
150\end{array}$ & $\begin{array}{r}77 \\
38 \\
44 \\
22 \\
140 \\
190\end{array}$ & $\begin{array}{r}120 \\
53 \\
58 \\
29 \\
200 \\
250\end{array}$ & $\begin{array}{r}180 \\
80 \\
83 \\
37 \\
300 \\
340\end{array}$ & $\begin{array}{r}290 \\
130 \\
130 \\
58 \\
470 \\
500\end{array}$ & $\begin{array}{l}590 \\
270 \\
270 \\
130 \\
940 \\
930\end{array}$ \\
\hline
\end{tabular}


Appendix 3.--Low-flow data--Continued

GREATT MIAMI RIVER BASIN

03272200 Elk Creek at Miltonville, Ohio

LOCATION:

Lat $39^{\circ} 30^{\prime} 05^{\prime \prime}$. long $84^{\circ} 27^{\prime} 3^{\prime \prime}$, Butler County, Hydrologic

Unit 05080002 , at county road bridge at east edge of Miltonville and $1.5 \mathrm{mi}$ upstream from mouth.

DRAINAGE AREA: $\quad 46.2 \mathrm{mi}^{2}$.

TRIBUTARY TO: Great Miami River.

DISCHARGE DATA USED: LOW-flow measurements 1960-67 water years.

INDEX STATION: $\quad 03272000$ Twin Creek near Germantown, Ohio

REMARKS:

None.

SELECTED DISCHARGE CHARACTERISTIICS: Minimum discharge observed: $0.2 \mathrm{ft}^{3} / \mathrm{s}$ September 1964 .

Magnitude and frequency of low flow for indicated periods

\begin{tabular}{c|c|c|c}
\hline \multirow{2}{*}{ Period } & \multirow{2}{*}{$\begin{array}{c}\text { Number of } \\
\text { consecu- } \\
\text { tive days }\end{array}$} & $\begin{array}{c}\text { Discharge }\left(\mathrm{ft}^{3} / \mathrm{s} \text { ) for }\right. \\
\text { indicated } \\
\text { interval }\end{array}$ \\
\cline { 3 - 4 } (yecurs)
\end{tabular}

Duration of daily flow for indicated periods

\begin{tabular}{l|c|c|c|c|c}
\hline \multirow{2}{*}{ Period } & \multicolumn{5}{|c}{$\begin{array}{c}\text { Discharge }\left(\mathrm{ft}^{3} / \mathrm{s} \text { ) which was equaled or }\right. \\
\text { exceeded for indicated percent of time }\end{array}$} \\
\cline { 2 - 6 } & 98 & 95 & 90 & 85 & 80 \\
\hline Apr. - Mar. & 0.4 & 0.6 & 1.1 & 1.4 & 2.1 \\
May - Nov. & 0.3 & 0.5 & 0.8 & 1.1 & 1.3 \\
June - Aug. & 0.5 & 0.7 & 1.1 & 1.4 & 1.8 \\
Sept. - Nov. & 0.2 & 0.4 & 0.5 & 0.7 & 0.9 \\
\hline
\end{tabular}


Appendix 3.--Low-Elow data--Continued

GREAT MIAMI RIVER BASIN

03272300 Dicks Creek near Exce110, Ohio

LOCATION: Lat $39^{\circ} 28^{\prime} 25^{\prime \prime}$, long $84^{\circ} 23^{\prime} 50^{\prime \prime}$, Butler County, Hydrologic Unit 05080002, at Yankee Road Bridge, $0.2 \mathrm{mi}$ south of the city limits of Middletown, $1.3 \mathrm{mi}$ southeast of Excello, and $2.5 \mathrm{mi}$ upstream from mouth.

DRAINAGE AREA:

$44.7 \mathrm{mi}^{2}$

TRIBUTARY TO:

Great Miami River.

DISCHARGE DATA USED: Low-flow measurements 1960-1969 water years.

INDEX STATION: $\quad 03272000$ Twin Creek near Germantown, Ohio.

REMARKS: $\quad$ None.

SELECTED DISCHARGE CHARACTERISTICS: Minimum discharge observed: $1.4 \mathrm{ft} \mathrm{t}^{3} / \mathrm{s}$ september 1964 .

Magnitude and frequency of low flow for indicated periods

\begin{tabular}{c|c|c|c}
\hline \multirow{2}{*}{ Period } & $\begin{array}{r}\text { Number of } \\
\text { consecu- } \\
\text { tive days }\end{array}$ & \multicolumn{2}{|c}{$\begin{array}{c}\text { Discharge (ft } 3 / \mathrm{s} \text { ) for } \\
\text { indicated recurrence } \\
\text { interval (years) }\end{array}$} \\
\cline { 3 - 4 } Apr. - Mar. & 7 & 2 & 10 \\
\hline May - Nov. & 30 & 2.0 & 1.0 \\
& 7 & 2.5 & 1.3 \\
June - Aug. & 30 & 2.0 & 1.1 \\
& 7 & 2.7 & 1.3 \\
Sept. - Nov. & 30 & 4.1 & 1.4 \\
& 7 & 2.1 & 2.0 \\
\hline
\end{tabular}

Duration of daily flow for indicated periods

\begin{tabular}{|c|c|c|c|c|c|}
\hline \multirow{2}{*}{ Period } & \multicolumn{5}{|c|}{$\begin{array}{l}\text { Discharge }\left(\mathrm{ft}^{3} / \mathrm{s}\right) \text { which was equaled or } \\
\text { exceeded for indicated percent of time }\end{array}$} \\
\hline & 98 & 95 & 90 & 85 & 80 \\
\hline $\begin{array}{l}\text { Apr. - Mar. } \\
\text { May - Nov. } \\
\text { June - Aug. } \\
\text { Sept. - Nov. }\end{array}$ & $\begin{array}{l}1.3 \\
1.2 \\
1.4 \\
1.0\end{array}$ & $\begin{array}{l}1.8 \\
1.5 \\
1.8 \\
1.3\end{array}$ & $\begin{array}{l}2.4 \\
2.0 \\
2.4 \\
1.6\end{array}$ & $\begin{array}{l}2.8 \\
2.4 \\
2.8 \\
2.0\end{array}$ & $\begin{array}{l}3.5 \\
2.7 \\
3.2 \\
2.1\end{array}$ \\
\hline
\end{tabular}


Appendix 3.--Low-flow data--Continued

GREAT MIAMI RIVER BASIN

03272800 Sevenmile Creek at Collinsville, Ohio

LOCATION:

Lat 39031'23", long $84^{\circ} 36^{\prime} 39^{\prime \prime}$, in SEl/4 sec.14, T.5 N., R.2 E., Butlex County, Hydxologic Unit 05080002 , on left bank at downstxeam side of bridge, $0.3 \mathrm{mi}$ north of Collinsville, $1.0 \mathrm{mi}$ downstream from Ninemile creek, and $5.5 \mathrm{mi}$ upstream from mouth.

DRAINAGE AREA: $\quad 120 \mathrm{mi}^{2}$.

TRIBUTARY TO: $\quad$ Fourmile Creek.

DISCHARGE DATA USED: July 1960 to September 1972.

REMARKS: $\quad$ None.

SELECTED DISCHARGE CHARACTERISTICS: Average discharge: $103 \mathrm{ft}^{3} / \mathrm{s}$ (42 years).

Minimum daily discharge: $0.8 \mathrm{ft} / \mathrm{s}$ September 1960.

Magnitude and frequency of low flow for indicated periods

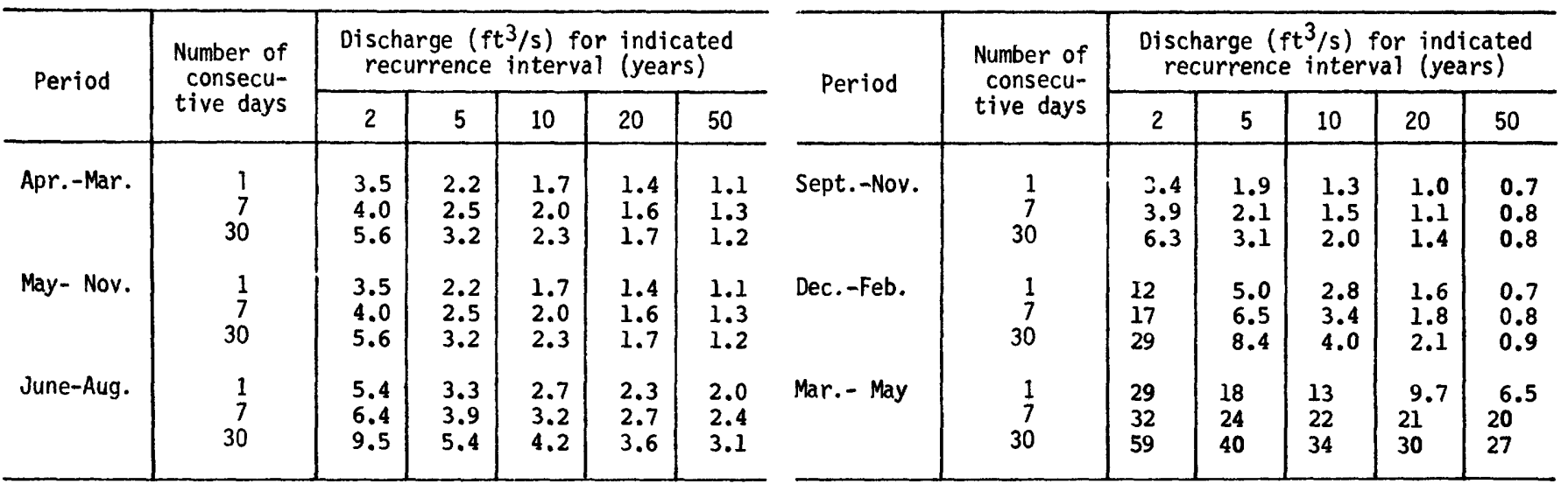

Duration of daily flow for indicated periods

\begin{tabular}{l|c|c|c|c|c|c|c|c|r|r|r|r|r}
\hline \multirow{2}{*}{ Period } & \multicolumn{8}{|c}{ Discharge $\left(\mathrm{ft}^{3} / \mathrm{s}\right)$ which was equaled or exceeded for indicated percent of time } \\
\cline { 2 - 13 } & 98 & 95 & 90 & 85 & 80 & 75 & 70 & 60 & 50 & 40 & 30 & 20 & 10 \\
\hline Apr. - Mar. & 2.2 & 3.1 & 5.2 & 7.0 & 8.5 & 10 & 13 & 21 & 32 & 47 & 71 & 120 & 210 \\
May - Nov. & 2.1 & 2.6 & 3.9 & 5.2 & 6.3 & 7.4 & 8.6 & 11 & 15 & 22 & 32 & 50 & 95 \\
June - Aug. & 3.4 & 4.4 & 5.6 & 6.6 & 7.5 & 8.6 & 9.7 & 13 & 17 & 23 & 31 & 43 & 72 \\
Sept. - Nov. & 1.6 & 2.0 & 2.3 & 2.7 & 3.3 & 4.1 & 4.9 & 6.6 & 8.4 & 11 & 14 & 19 & 32 \\
Dec. - Feb. & 2.2 & 4.7 & 7.2 & 8.6 & 12 & 19 & 25 & 35 & 51 & 67 & 87 & 140 & 260 \\
Mar. - May & 25 & 30 & 36 & 42 & 49 & 58 & 67 & 88 & 110 & 140 & 190 & 260 & 470 \\
\hline
\end{tabular}




\title{
GREAT MIAMI RIVER BASIN
}

\author{
03274000 Great Miami River at Hamilton, Ohio
}

LOCATION :

Lat $39^{\circ} 23^{\prime} 28^{\prime \prime}$, long $84^{\circ} 34^{\prime} 20^{\prime \prime}$, in NEl/4 sec.6, T.l N., R.3 E., Butler. County, Hydrologic Unit 05080002 , on right bank 1,000 ft downstream from Columbia Bridge at Hamilton, $3 \mathrm{mi}$ downstream from Four Mile Creek, $4.3 \mathrm{mi}$ upstream from pleasant Run, and at mile 34.8 .

DRAINAGE AREA:

$3,030 \mathrm{mi}^{2}$

TRIBUTARY TO: Ohio River.

DISCHARGE DATA USED: October 1930 to September 1976.

REMARKS :

Some regulation at low flow by industrial plants upstream from station. Flood flow regulated by five retarding basins upstream from station beginning in 1920 . Small diversion about $6 \mathrm{mi}$ upstream from gage for municipal supply of Hamilton. Diversion averaged $0.89 \mathrm{ft}^{3} / \mathrm{s}$ in 1978 and is returned as sewage $1.4 \mathrm{mi}$ downstream from the station. The Miami and Erie Canal diverted water from the basin $1.7 \mathrm{mi}$ upstream from station until Nov. 1, 1930, when the canal was abandoned; amount of diversion not known.

SELECTED DISCHARGE CHARACTERISTICS.-- Average discharge: $3,158 \mathrm{ft}^{3} / \mathrm{s}$ (48 years)

Minimimum daily discharge: $155 \mathrm{ft} 3 / \mathrm{s}$ September, 1941. Magnitude and frequency of low flow for indicated periods

\begin{tabular}{c|c|c|c|c|c|c}
\hline \multirow{2}{*}{ Period } & \multirow{2}{*}{$\begin{array}{c}\text { Number of } \\
\text { consecu- } \\
\text { tive days }\end{array}$} & \multicolumn{4}{|c|}{\begin{tabular}{c} 
Discharge (ft $3 / \mathrm{s}$ ) for indicated \\
recurrence interval \\
\cline { 3 - 7 } (years)
\end{tabular}} \\
\cline { 3 - 7 } Apr.-Mar. & 2 & 5 & 10 & 20 & 50 \\
\hline \multirow{3}{*}{ May- Nov. } & 7 & 405 & 287 & 239 & 206 & 173 \\
& 30 & 458 & 335 & 284 & 249 & 214 \\
& 1 & 416 & 380 & 327 & 291 & 255 \\
& 7 & 465 & 294 & 244 & 208 & 174 \\
& 30 & 525 & 385 & 330 & 249 & 213 \\
& 1 & 521 & 361 & 298 & 254 & 213 \\
& 7 & 597 & 419 & 350 & 302 & 256 \\
& 30 & 787 & 516 & 435 & 387 & 348 \\
\hline
\end{tabular}

\begin{tabular}{|c|c|c|c|c|c|c|}
\hline \multirow{2}{*}{ Period } & \multirow{2}{*}{$\begin{array}{l}\text { Number of } \\
\text { consecu- } \\
\text { tive days }\end{array}$} & \multicolumn{5}{|c|}{$\begin{array}{l}\text { Discharge }\left(\mathrm{ft}^{3} / \mathrm{s}\right) \text { for indicated } \\
\text { recurrence interval (years) }\end{array}$} \\
\hline & & 2 & 5 & 10 & 20 & 50 \\
\hline Sept.-Nov. & $\begin{array}{r}1 \\
7 \\
30\end{array}$ & $\begin{array}{l}423 \\
472 \\
561\end{array}$ & $\begin{array}{l}300 \\
345 \\
403\end{array}$ & $\begin{array}{l}251 \\
294 \\
344\end{array}$ & $\begin{array}{l}216 \\
258 \\
304\end{array}$ & $\begin{array}{l}183 \\
223 \\
268\end{array}$ \\
\hline Dec. -Feb. & $\begin{array}{r}1 \\
7 \\
30\end{array}$ & $\begin{array}{r}723 \\
841 \\
1380\end{array}$ & $\begin{array}{l}448 \\
518 \\
687\end{array}$ & $\begin{array}{l}350 \\
404 \\
478\end{array}$ & $\begin{array}{l}286 \\
331 \\
356\end{array}$ & $\begin{array}{l}229 \\
264 \\
256\end{array}$ \\
\hline Mar. - May & $\begin{array}{r}1 \\
7 \\
30\end{array}$ & $\begin{array}{l}1220 \\
1420 \\
2150\end{array}$ & $\begin{array}{r}793 \\
930 \\
1370\end{array}$ & $\begin{array}{r}607 \\
727 \\
1070\end{array}$ & $\begin{array}{l}476 \\
586 \\
874\end{array}$ & $\begin{array}{l}353 \\
453 \\
691\end{array}$ \\
\hline
\end{tabular}

Duration of daily flow for indicated periods

\begin{tabular}{|c|c|c|c|c|c|c|c|c|c|c|c|c|c|}
\hline \multirow{2}{*}{ Period } & \multicolumn{13}{|c|}{ Discharge $\left(\mathrm{ft}^{3} / \mathrm{s}\right)$ which was equaled or exceeded for indicated percent of time } \\
\hline & 98 & 95 & 90 & 85 & 80 & 75 & 70 & 60 & 50 & 40 & 30 & 20 & 10 \\
\hline $\begin{array}{l}\text { Apr. - Mar. } \\
\text { May - Nov. } \\
\text { June - Aug. } \\
\text { Sept. - Nov. } \\
\text { Dec. - Feb. } \\
\text { Mar. - May }\end{array}$ & $\begin{array}{l}320 \\
300 \\
340 \\
280 \\
350 \\
700\end{array}$ & $\begin{array}{l}390 \\
360 \\
420 \\
320 \\
420 \\
940\end{array}$ & $\begin{array}{r}480 \\
430 \\
500 \\
360 \\
500 \\
1200\end{array}$ & $\begin{array}{r}550 \\
500 \\
570 \\
410 \\
610 \\
1500\end{array}$ & $\begin{array}{r}630 \\
550 \\
640 \\
460 \\
750 \\
1700\end{array}$ & $\begin{array}{r}730 \\
600 \\
720 \\
500 \\
900 \\
1900\end{array}$ & $\begin{array}{r}840 \\
660 \\
790 \\
530 \\
1100 \\
2100\end{array}$ & $\begin{array}{r}1100 \\
810 \\
950 \\
600 \\
1500 \\
2700\end{array}$ & $\begin{array}{r}1400 \\
990 \\
1100 \\
680 \\
2000 \\
3300\end{array}$ & $\begin{array}{r}2000 \\
1200 \\
1400 \\
810 \\
2800 \\
4000\end{array}$ & $\begin{array}{l}2800 \\
1600 \\
1700 \\
980 \\
3900 \\
5100\end{array}$ & $\begin{array}{l}4100 \\
2200 \\
2300 \\
1200 \\
5800 \\
7200\end{array}$ & $\begin{array}{r}7300 \\
3700 \\
3700 \\
1800 \\
11000 \\
12000\end{array}$ \\
\hline
\end{tabular}


Appendix 3.--Low-flow data--Continued

GEEAT MIAMI RIVER BASIN

03274200 Indian Creek near Millville, Ohio

LOCATION:

Lat $39^{\circ} 21^{\prime} 45^{\prime \prime}$, long $84^{\circ} 38^{\prime} 3^{\prime \prime}$, Butler County, Hydrologic Unit

05080002 , at hamilton-New London Road Bridge, $1.9 \mathrm{mi}$ south of Millville, and $4.3 \mathrm{mi}$ upstream from mouth.

DRAINAGE AREA: $\quad 102 \mathrm{mi}^{2}$.

TRIBUTARY TO:

Great Miami River.

DISCHARGE DATA USED:

Low-flow measurements 1961-19us water years.

INDEX STATION:

03272000 Twin Creek near Germantown, Ohio.

REMARKS:

None.

SELECTED DISCHARGE CHARACTERISTICS: Minimum discharge observed: $0.4 \mathrm{ft}^{3} / \mathrm{s}$ September 1965 .

\begin{tabular}{c|c|c|c}
\multicolumn{3}{c}{$\begin{array}{c}\text { Magnitude and frequency of low flow for } \\
\text { indicated periods }\end{array}$} \\
\hline & $\begin{array}{c}\text { Number of } \\
\text { consecu- } \\
\text { tive days }\end{array}$ & $\begin{array}{c}\text { Discharge (ft3/s) for } \\
\text { indicated recurrence } \\
\text { interval }\end{array}$ \\
\cline { 3 - 4 } (years)
\end{tabular}

Duration of daily flow for indicated periods

\begin{tabular}{|c|c|c|c|c|c|}
\hline \multirow{2}{*}{ Period } & \multicolumn{5}{|c|}{$\begin{array}{l}\text { Discharge }\left(\mathrm{ft}^{3} / \mathrm{s}\right) \text { which was equaled or } \\
\text { exceeded for indicated percent of time }\end{array}$} \\
\hline & 98 & 95 & 90 & 85 & 80 \\
\hline $\begin{array}{l}\text { Apr. - Mar. } \\
\text { May - Nov. } \\
\text { June - Aug. } \\
\text { Sept. - Nov. }\end{array}$ & $\begin{array}{l}0.2 \\
0.1 \\
0.2 \\
0.1\end{array}$ & $\begin{array}{l}0.4 \\
0.3 \\
0.5 \\
0.2\end{array}$ & $\begin{array}{l}0.9 \\
0.6 \\
0.9 \\
0.3\end{array}$ & $\begin{array}{l}1.5 \\
0.9 \\
1.5 \\
0.5\end{array}$ & $\begin{array}{l}2.5 \\
1.3 \\
2.0 \\
0.7\end{array}$ \\
\hline
\end{tabular}


Appendix 3.--Low-flow data--Continued

GREAT MIAMI RIVER BASIN

03274600 Great Miami River at New Baltimore, Ohio

LOCATION:

Lat $39^{\circ} 15^{\prime} 50^{\prime \prime}$, long $84^{\circ} 40^{\prime} 04^{\prime \prime}$, Hamilton County, Hydrologic

Unit 05080002 , at bridge on Blue Rock Road at iNew Baltimore.

DRAINAGE AREA: $\quad 3,814 \mathrm{mi}^{2}$.

TRIBUTARY TO: Ohio River.

DISCHARGE DATA USED: Low-flow measurements 1955, 1956, 1959, 1961-1970 water years.

INDEX STATION: $\quad 03274000$ Great Miami River at Hamilton, Ohio.

REMARKS: None.

SELECTED DISCHARGE CHARACTERISTICS: Minimum discharge observed: $263 \mathrm{ft}^{3} / \mathrm{s}$ September 1955.

\begin{tabular}{l|c|c|c}
\multicolumn{4}{c}{$\begin{array}{c}\text { Magnitude and frequency of low flow for } \\
\text { indicated periods }\end{array}$} \\
\hline \multirow{3}{*}{ Period } & $\begin{array}{c}\text { Number of } \\
\text { consecu- } \\
\text { tive days }\end{array}$ & $\begin{array}{c}\text { Discharge (ft } 3 / \mathrm{s} \text { ) for } \\
\text { indicated recurrence } \\
\text { interval (years) }\end{array}$ \\
\cline { 3 - 4 } & & 2 & 10 \\
\hline Apr. - Mar. & 7 & 465 & 270 \\
& 30 & 530 & 320 \\
May - Nov. & 7 & 475 & 275 \\
& 30 & 545 & 320 \\
June - Aug. & 7 & 630 & 345 \\
& 30 & 860 & 440 \\
Sept.- Nov. & 7 & 480 & 280 \\
& 30 & 585 & 335 \\
\hline
\end{tabular}

Duration of daily flow for indicated periods

\begin{tabular}{l|c|c|c|c|c}
\hline \multirow{2}{*}{ Period } & \multicolumn{6}{|c}{$\begin{array}{c}\text { Discharge }\left(\mathrm{ft}^{3} / \mathrm{s}\right) \\
\text { exceeded for } \text { wich was equaled or }\end{array}$} \\
\cline { 2 - 6 } & 98 & 95 & 90 & 85 & 80 \\
\hline Apr. - Mar. & 310 & 390 & 490 & 570 & 670 \\
May - Nov. & 290 & 350 & 430 & 510 & 570 \\
June - Aug. & 330 & 420 & $5 ! 0$ & 600 & 630 \\
Sept. - Nov. & 265 & 310 & 350 & 410 & 470 \\
\hline
\end{tabular}


Appendix 3.--Low-flow data--Continued

\section{MAUMEE RIVER BASIN}

04177100 East Branch St. Joseph River near Pioneer, Ohio

LOCATION:

Lat $41^{\circ} 39^{\prime} 55^{\prime \prime}$, long $84^{\circ} 32^{\prime} 30^{\prime}$, williams County, Hydrologic Unit 04100003 , at bridge on U.S. Highway $20,0.6 \mathrm{mi}$ east of State Route 15 , and $1.3 \mathrm{mi}$ southeast of $\mathrm{Pioneer.}$

DRAINAGE AREA: $\quad 158 \mathrm{mi}^{2}$.

TRIBUTARY TO: Head of Maumee River.

DISCHARGE DATA USED:

Low-flow measurements 1955-1956, 1962-1974 water years.

INDEX STATION:

04184500 Bean Creek at Powers, Ohio.

REMARKS:

None.

SELECTED DISCHARGE CHARACTERISTICS: Minimum discharge observed: $4.1 \mathrm{ft}^{3} / \mathrm{s}$ October 1963.

Magnitude and frequency of low flow for indicated periods

\begin{tabular}{c|c|c|c}
\hline \multirow{2}{*}{ Period } & \multirow{2}{*}{$\begin{array}{c}\text { Number of } \\
\text { consecu- } \\
\text { tive days }\end{array}$} & \multicolumn{2}{|c}{$\begin{array}{c}\text { Discharge }\left(\mathrm{ft}^{3} / \mathrm{s} \text { ) for }\right. \\
\text { indicated recurrence } \\
\text { interval (years) }\end{array}$} \\
\cline { 3 - 4 } & & 2 & 10 \\
\hline Apr. - Mar. & 7 & 9.0 & 4.1 \\
& 30 & 12 & 5.6 \\
May - Nov. & 7 & 9.0 & 4.1 \\
& 30 & 12 & 5.6 \\
June - Aug. & 7 & 12 & 6.2 \\
& 30 & 19 & 7.0 \\
Sept.- Nov. & 7 & 11 & 6.2 \\
& 30 & 16 & 6.0 \\
\hline
\end{tabular}

Duration of daily flow for indicated periods

\begin{tabular}{|c|c|c|c|c|c|}
\hline \multirow{2}{*}{ Period } & \multicolumn{5}{|c|}{$\begin{array}{l}\text { Discharge }\left(\mathrm{ft}^{3} / \mathrm{s}\right) \text { which was equaled or } \\
\text { exceeded for indicated percent of time }\end{array}$} \\
\hline & 98 & 95 & 90 & 85 & 80 \\
\hline $\begin{array}{l}\text { Apr. - Mar. } \\
\text { May - Nov. } \\
\text { June - Aug. } \\
\text { Sept. - Nov. }\end{array}$ & $\begin{array}{l}5.5 \\
4.6 \\
4.6 \\
4.0\end{array}$ & $\begin{array}{l}9.0 \\
7.0 \\
7.0 \\
5.8\end{array}$ & $\begin{array}{l}12 \\
10 \\
10 \\
8.0\end{array}$ & $\begin{array}{l}16 \\
12 \\
12 \\
10\end{array}$ & $\begin{array}{l}21 \\
15 \\
15 \\
12\end{array}$ \\
\hline
\end{tabular}




\section{MAUMEE RIVER BASIN}

04183500 Maumee River at Antwerp, Ohio

LOCATION:

Lat $41^{\circ} 11^{\prime} 56^{\prime \prime}$, long $84^{\circ} 44^{\prime} 40^{\prime \prime}$, in sec. 22, T.3 N., R.1 E., Paulding County, Hydrologic Unit 04100005 , on left bank $425 \mathrm{ft}$ downstream from bridge on State Route $49,1 \mathrm{mi}$ north of Antwerp, $7 \mathrm{mi}$ downstream from Indiana State line and $10 \mathrm{mi}$ upstream from Marie DeLarme Creek.

DRAINAGE AREA: $\quad 2,129 \mathrm{mi}^{2}$.

TRIBLTARY TO: Lake Erie.

DISCHARGE DATA USED: October 1921 to September 1935, April 1939 to September 1978.

REMARKS :

Low flow slightly regulated by power plant at Fort Wayne, Indiana, $32 \mathrm{mi}$ upstream. Flow slightly regulated by upstream reservoirs.

SELECTED DISCHARGE CHARACTERISTICS: Average discharge: $1,684 \mathrm{ft}^{3} / \mathrm{s}_{3}(53 \mathrm{years}$ ). Minimum daily discharge: $26 \mathrm{ft}^{3} / \mathrm{s}$ July 1933 .

Magnitude and frequency of low flow for indicated periods

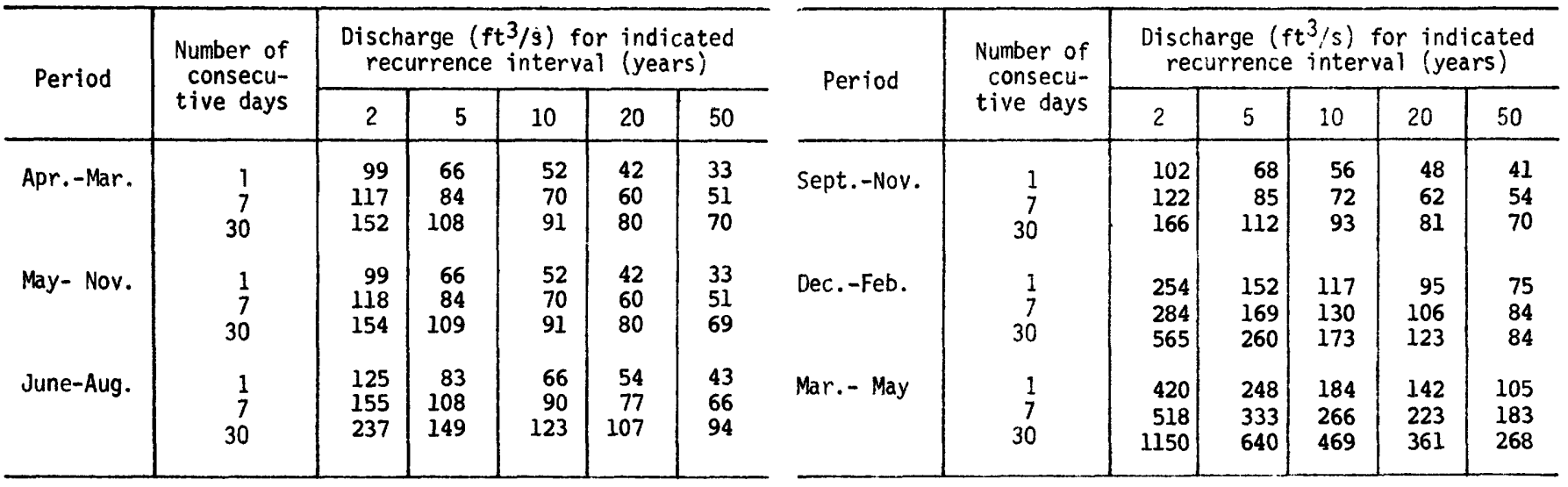

Duration of daily flow for indicated periods

\begin{tabular}{|c|c|c|c|c|c|c|c|c|c|c|c|c|c|}
\hline \multirow{2}{*}{ Period } & \multicolumn{13}{|c|}{ Discharge $\left(\mathrm{ft}^{3} / \mathrm{s}\right)$ which was equaled or exceeded for indicated percent of time } \\
\hline & 98 & 95 & 90 & 85 & 80 & 75 & 70 & 60 & 50 & 40 & 30 & 20 & 10 \\
\hline $\begin{array}{l}\text { Apr. - Mar. } \\
\text { May - Nov. } \\
\text { June - Aug. } \\
\text { Sept. - Nov. } \\
\text { Dec. - Feb. } \\
\text { Mar. - May }\end{array}$ & $\begin{array}{r}92 \\
81 \\
93 \\
73 \\
130 \\
290\end{array}$ & $\begin{array}{r}120 \\
100 \\
120 \\
88 \\
150 \\
390\end{array}$ & $\begin{array}{l}150 \\
120 \\
140 \\
110 \\
190 \\
510\end{array}$ & $\begin{array}{l}170 \\
140 \\
160 \\
120 \\
220 \\
640\end{array}$ & $\begin{array}{l}200 \\
160 \\
190 \\
130 \\
280 \\
780\end{array}$ & $\begin{array}{l}240 \\
190 \\
210 \\
150 \\
360 \\
930\end{array}$ & $\begin{array}{r}290 \\
210 \\
240 \\
160 \\
440 \\
1100\end{array}$ & $\begin{array}{r}420 \\
270 \\
300 \\
190 \\
660 \\
1400\end{array}$ & $\begin{array}{r}620 \\
360 \\
380 \\
240 \\
920 \\
1900\end{array}$ & $\begin{array}{r}910 \\
470 \\
490 \\
300 \\
1300 \\
2600\end{array}$ & $\begin{array}{r}1500 \\
710 \\
670 \\
400 \\
2100 \\
3600\end{array}$ & $\begin{array}{r}2400 \\
1100 \\
1000 \\
640 \\
3400 \\
5000\end{array}$ & $\begin{array}{l}4700 \\
2200 \\
1800 \\
1400 \\
6400 \\
7300\end{array}$ \\
\hline
\end{tabular}


MAUMEE RIVER BASIN

04184500 Bean Creek at Powers, Ohio

LOCATION:

Lat $41^{\circ} 40^{\prime} 39^{\prime \prime}$, long $84^{\circ} 13^{\prime} 56^{\prime \prime}$, in NE1/4 sec. 24, T.9 S., R.1 E., Fulton

County, Hydrologic Unit 04100006, on right bank at downstream side of

bridge on U.S. Highway $20,1 \mathrm{mi}$ east of Powers, $2.2 \mathrm{mi}$ upstream from

Iron Creek, $3 \mathrm{mi}$ downstream from Silver Creek, and $5.2 \mathrm{mi}$ east of

Fayette.

DRAINAGE AREA: $206 \mathrm{mi}^{2}$.

TRIBUTARY T0: $\quad$ Maumee River.

DISCHARGE DATA USED: October 1940 to September 1978.

REMARKS:

None.

SELECTED DISCHARGE CHARACTERISTICS: Average discharge: $163 \mathrm{ft}^{3} / \mathrm{s}$ ( 38 years).

Minimum daily discharge: $5.2 \mathrm{ft}^{3} / \mathrm{s}$ August 1964.

Magnitude and frequency of low flow for indicated periods

\begin{tabular}{c|c|c|c|c|c|c}
\hline \multirow{2}{*}{ Period } & \multirow{2}{*}{$\begin{array}{c}\text { Number of } \\
\text { consecu- } \\
\text { tive days }\end{array}$} & \multicolumn{4}{|c|}{$\begin{array}{c}\text { Discharge }\left(\mathrm{ft}^{3} / \mathrm{s}\right) \text { for indicated } \\
\text { recurrence interval (years) }\end{array}$} \\
\cline { 3 - 7 } Apr.-Mar. & 2 & 5 & 10 & 20 & 50 \\
\hline \multirow{3}{*}{ May-Nov. } & 7 & 12 & 8.4 & 6.9 & 5.9 & 4.9 \\
& 30 & 13 & 9.2 & 7.6 & 6.5 & 5.5 \\
& 16 & 11 & 9.4 & 8.0 & 6.7 \\
& 1 & 12 & 8.5 & 6.9 & 5.9 & 4.9 \\
& 3 & 13 & 9.3 & 7.6 & 6.5 & 5.4 \\
& 30 & 16 & 11 & 9.4 & 8.0 & 6.7 \\
& 1 & 15 & 9.5 & 7.6 & 6.3 & 5.1 \\
& 7 & 16 & 10 & 8.5 & 7.2 & 6.0 \\
& 30 & 21 & 13 & 11 & 9.1 & 7.6 \\
\hline
\end{tabular}

\begin{tabular}{|c|c|c|c|c|c|c|}
\hline \multirow{2}{*}{ Period } & \multirow{2}{*}{$\begin{array}{l}\text { Number of } \\
\text { consecu- } \\
\text { tive days }\end{array}$} & \multicolumn{5}{|c|}{$\begin{array}{l}\text { Discharge }\left(\mathrm{ft}^{3} / \mathrm{s}\right) \text { for indicated } \\
\text { recurrence interval (years) }\end{array}$} \\
\hline & & 2 & 5 & 10 & 20 & 50 \\
\hline Sept.-Nov. & $\begin{array}{r}1 \\
7 \\
30\end{array}$ & $\begin{array}{l}14 \\
15 \\
19\end{array}$ & $\begin{array}{l}9.2 \\
10 \\
12\end{array}$ & $\begin{array}{l}7.4 \\
8.1 \\
9.9\end{array}$ & $\begin{array}{l}6.2 \\
6.8 \\
8.4\end{array}$ & $\begin{array}{l}5.0 \\
5.6 \\
7.0\end{array}$ \\
\hline Dec.-Feb. & $\begin{array}{r}1 \\
7 \\
30\end{array}$ & $\begin{array}{l}32 \\
36 \\
58\end{array}$ & $\begin{array}{l}19 \\
21 \\
29\end{array}$ & $\begin{array}{l}14 \\
16 \\
21\end{array}$ & $\begin{array}{l}12 \\
13 \\
15\end{array}$ & $\begin{array}{l}9.0 \\
10 \\
11\end{array}$ \\
\hline Mar. - May & $\begin{array}{r}1 \\
7 \\
30\end{array}$ & $\begin{array}{r}58 \\
68 \\
133\end{array}$ & $\begin{array}{l}37 \\
45 \\
85\end{array}$ & $\begin{array}{l}28 \\
36 \\
66\end{array}$ & $\begin{array}{l}23 \\
30 \\
54\end{array}$ & $\begin{array}{l}17 \\
24 \\
42\end{array}$ \\
\hline
\end{tabular}

Duration of daily flow for indicated periods

\begin{tabular}{|c|c|c|c|c|c|c|c|c|c|c|c|c|c|c|}
\hline \multirow{2}{*}{\multicolumn{2}{|c|}{ Period }} & \multicolumn{13}{|c|}{ Discharge $\left(\mathrm{ft}^{3} / \mathrm{s}\right)$ which was equaled or exceeded for indicated percent of time } \\
\hline & & 98 & 95 & 90 & 85 & 80 & 75 & 70 & 60 & 50 & 40 & 30 & 20 & 10 \\
\hline $\begin{array}{l}\text { Apr. - } \\
\text { May - } \\
\text { June - } \\
\text { Sept. - } \\
\text { Dec. - } \\
\text { Mar. - }\end{array}$ & $\begin{array}{l}\text { Mar. } \\
\text { Nov. } \\
\text { Aug. } \\
\text { Nov. } \\
\text { Feb. } \\
\text { May }\end{array}$ & $\begin{array}{l}9.3 \\
8.2 \\
8.4 \\
7.5 \\
15 \\
39\end{array}$ & $\begin{array}{l}13 \\
11 \\
11 \\
9.6 \\
18 \\
53\end{array}$ & $\begin{array}{l}16 \\
14 \\
14 \\
12 \\
22 \\
70\end{array}$ & $\begin{array}{l}1.9 \\
16 \\
16 \\
14 \\
28 \\
84\end{array}$ & $\begin{array}{l}23 \\
18 \\
18 \\
16 \\
32 \\
98\end{array}$ & $\begin{array}{r}27 \\
20 \\
21 \\
17 \\
38 \\
110\end{array}$ & $\begin{array}{r}31 \\
23 \\
23 \\
19 \\
48 \\
130\end{array}$ & $\begin{array}{r}43 \\
29 \\
29 \\
23 \\
66 \\
160\end{array}$ & $\begin{array}{r}63 \\
37 \\
35 \\
28 \\
92 \\
200\end{array}$ & $\begin{array}{r}94 \\
48 \\
44 \\
34 \\
120 \\
250\end{array}$ & $\begin{array}{r}140 \\
67 \\
56 \\
44 \\
180 \\
330\end{array}$ & $\begin{array}{r}220 \\
100 \\
81 \\
62 \\
280 \\
440\end{array}$ & $\begin{array}{l}400 \\
190 \\
140 \\
120 \\
540 \\
690\end{array}$ \\
\hline
\end{tabular}




\section{MAUMEE RIVER BASIN}

04185000 Tiffin River at Stryker, Ohio

LOCATION:

Lat $41^{\circ} 30^{\prime} 16^{\prime \prime}$, long $84^{\circ} 25^{\prime} 47^{\prime \prime}$, in swl $/ 4$ sec. 5, T.6 N., R.4 E., Williams County, Hydrologic Unit 04100006 , on left bank $0.5 \mathrm{mi}$ downstream from bridge on State Route 191 at west edge of Stryker, $0.6 \mathrm{mi}$ upstream from Penn Central bridge, and $1.6 \mathrm{mi}$ downstream from Leatherwood Creek.

DRAINAGE AREA:

$410 \mathrm{mi}^{2}$

TRIBUTARY TO:

Maumee River.

DISCHARGE DATA USED:

REMARKS :

October 1921 to September 1928, October 1940 to September 1978.

Small diversion about $12.5 \mathrm{mi}$ upstream from gage for municipal supply of Archbold. Diversion averaged $1.58 \mathrm{ft} 3 / \mathrm{s}$ in 1978 and is returned as sewage to Brush Creek which flows into Tiffin River about $15 \mathrm{mi}$ downstream from station.

SELECTED DISCHARGE CHARACTERISTICS: Average discharge: $312 \mathrm{ft}^{3} / \mathrm{s}$ ( 45 years). Minimum daily discharge: $3.9 \mathrm{ft}^{3} / \mathrm{s}$ August, September 1953.

Magnitude and frequency of low flow for indicated periods

\begin{tabular}{c|c|c|c|c|c|c}
\hline \multirow{2}{*}{ Period } & \multirow{2}{*}{$\begin{array}{c}\text { Number of } \\
\text { consecu- } \\
\text { tive days }\end{array}$} & \multicolumn{4}{|c|}{$\begin{array}{c}\text { Discharge }\left(\mathrm{ft}^{3} / \mathrm{s} \text { ) }\right. \\
\text { recurrence interval indicated }\end{array}$} \\
\cline { 3 - 7 } (years)
\end{tabular}

\begin{tabular}{|c|c|c|c|c|c|c|}
\hline \multirow{2}{*}{ Period } & \multirow{2}{*}{$\begin{array}{l}\text { Number of } \\
\text { consecu- } \\
\text { tive days }\end{array}$} & \multicolumn{5}{|c|}{$\begin{array}{l}\text { Discharge }\left(\mathrm{ft}^{3} / \mathrm{s}\right) \text { for indicated } \\
\text { recurrence interval (years) }\end{array}$} \\
\hline & & 2 & 5 & 10 & 20 & 50 \\
\hline Sept.-Nov. & $\begin{array}{r}1 \\
7 \\
30\end{array}$ & $\begin{array}{l}17 \\
18 \\
25\end{array}$ & $\begin{array}{l}10 \\
11 \\
15\end{array}$ & $\begin{array}{l}7.5 \\
8.8 \\
12\end{array}$ & $\begin{array}{l}5.9 \\
7.2 \\
9.7\end{array}$ & $\begin{array}{l}4.5 \\
5.8 \\
8.1\end{array}$ \\
\hline Dec.-Feb. & $\begin{array}{r}1 \\
7 \\
30\end{array}$ & $\begin{array}{r}46 \\
54 \\
100\end{array}$ & $\begin{array}{l}25 \\
29 \\
44\end{array}$ & $\begin{array}{l}18 \\
21 \\
29\end{array}$ & $\begin{array}{l}14 \\
17 \\
20\end{array}$ & $\begin{array}{l}11 \\
13 \\
14\end{array}$ \\
\hline Mar. - May & $\begin{array}{r}1 \\
7 \\
30\end{array}$ & $\begin{array}{r}87 \\
102 \\
209\end{array}$ & $\begin{array}{r}55 \\
68 \\
124\end{array}$ & $\begin{array}{l}43 \\
55 \\
95\end{array}$ & $\begin{array}{l}34 \\
46 \\
75\end{array}$ & $\begin{array}{l}26 \\
38 \\
58\end{array}$ \\
\hline
\end{tabular}

Duration of daily flow for indicated periods

\begin{tabular}{|c|c|c|c|c|c|c|c|c|c|c|c|c|c|}
\hline \multirow{2}{*}{ Period } & \multicolumn{13}{|c|}{ Discharge $\left(\mathrm{ft}^{3} / \mathrm{s}\right)$ which was equaled or exceeded for indicated percent of time } \\
\hline & 98 & 95 & 90 & 85 & 80 & 75 & 70 & 60 & 50 & 40 & 30 & 20 & 10 \\
\hline $\begin{array}{l}\text { Apr. - Mar. } \\
\text { May - Nov. } \\
\text { June - Aug. } \\
\text { Sept. - Nov. } \\
\text { Dec. - Feb. } \\
\text { Mar. - May }\end{array}$ & $\begin{array}{l}12 \\
9.9 \\
11 \\
8.7 \\
19 \\
61\end{array}$ & $\begin{array}{l}16 \\
13 \\
15 \\
12 \\
24 \\
78\end{array}$ & $\begin{array}{r}22 \\
18 \\
19 \\
15 \\
31 \\
100\end{array}$ & $\begin{array}{r}27 \\
21 \\
22 \\
18 \\
41 \\
130\end{array}$ & $\begin{array}{r}32 \\
25 \\
26 \\
21 \\
53 \\
150\end{array}$ & $\begin{array}{r}39 \\
29 \\
29 \\
23 \\
66 \\
180\end{array}$ & $\begin{array}{r}47 \\
33 \\
32 \\
26 \\
81 \\
210\end{array}$ & $\begin{array}{r}70 \\
43 \\
41 \\
33 \\
120 \\
270\end{array}$ & $\begin{array}{r}110 \\
56 \\
52 \\
42 \\
170 \\
350\end{array}$ & $\begin{array}{r}160 \\
78 \\
68 \\
53 \\
240 \\
460\end{array}$ & $\begin{array}{r}250 \\
110 \\
92 \\
75 \\
360 \\
620\end{array}$ & $\begin{array}{l}420 \\
180 \\
140 \\
110 \\
600 \\
930\end{array}$ & $\begin{array}{r}890 \\
350 \\
260 \\
240 \\
1200 \\
1400\end{array}$ \\
\hline
\end{tabular}


04187500 Ottawa River at Allentown, Ohio

LOCATION: Lat $40^{\circ} 45^{\prime} 18^{\prime}$, long $84^{\circ} 11^{\prime} 41^{\prime \prime}$, in NW1/4 sec. 29, T.3 S., R.6 E., Allen County, Hydrologic Unit 04100007 , on left bank at upstream side of bridge on State Route 81 at Allen town, $0.3 \mathrm{mi}$ downstream from Kessler Run, and $1.5 \mathrm{mi}$ upstream from McBride Ditch.

DRAINAGE AREA: $\quad 160 \mathrm{mi}^{2}$.

TRIBITARY TO: Auglaize River.

DISCHARGE DATA USED: October 1943 to September 1978.

REMARKS: $\quad$ Diurnal fluctuation and some regulation caused by operation of water-supply and sewage-treatment plants of city of Lima upstream from station.

SELECTED DISCHARGE CHARACTERISTICS: Average discharge: $128 \mathrm{ft}^{3} / \mathrm{s}$ ( 35 years). Minimum daily discharge: $9.0 \mathrm{ft}^{3} / \mathrm{s}$ January, February 1945.

Magnitude and frequency of low flow for indicated periods

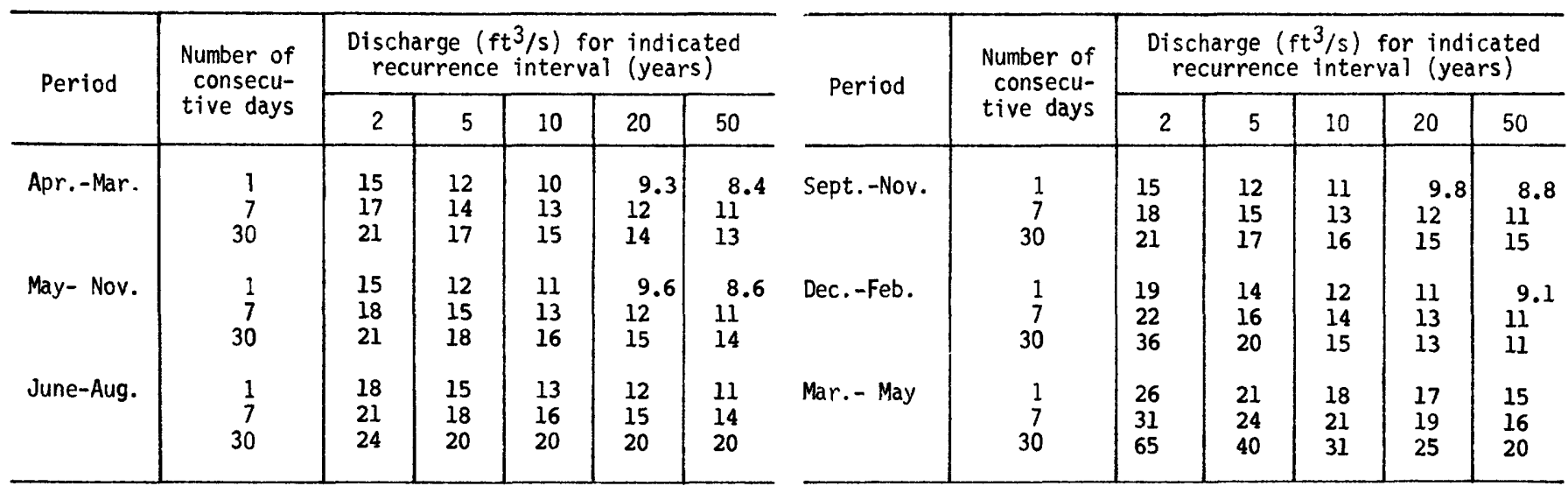

Duration of daily flow for indicated periods

\begin{tabular}{|c|c|c|c|c|c|c|c|c|c|c|c|c|c|}
\hline \multirow{2}{*}{ Period } & \multicolumn{13}{|c|}{ Discharge $\left(\mathrm{ft}^{3} / \mathrm{s}\right)$ which was equaled or exceeded for indicated percent of time } \\
\hline & 98 & 95 & 90 & 85 & 80 & 75 & 70 & 60 & 50 & 40 & 30 & 20 & 10 \\
\hline $\begin{array}{l}\text { Apr. - Mar. } \\
\text { May - Nov. } \\
\text { June - Aug. } \\
\text { Sept. - Nov. } \\
\text { Dec. - Feb. } \\
\text { Mar. - May }\end{array}$ & $\begin{array}{l}15 \\
15 \\
16 \\
14 \\
15 \\
22\end{array}$ & $\begin{array}{l}17 \\
17 \\
18 \\
15 \\
17 \\
25\end{array}$ & $\begin{array}{l}19 \\
18 \\
19 \\
17 \\
19 \\
30\end{array}$ & $\begin{array}{l}20 \\
19 \\
20 \\
18 \\
20 \\
35\end{array}$ & $\begin{array}{l}22 \\
20 \\
21 \\
18 \\
22 \\
40\end{array}$ & $\begin{array}{l}23 \\
21 \\
22 \\
19 \\
23 \\
45\end{array}$ & $\begin{array}{l}25 \\
23 \\
23 \\
20 \\
25 \\
50\end{array}$ & $\begin{array}{l}29 \\
25 \\
26 \\
22 \\
31 \\
65\end{array}$ & $\begin{array}{l}35 \\
29 \\
29 \\
25 \\
40 \\
84\end{array}$ & $\begin{array}{r}45 \\
33 \\
33 \\
28 \\
55 \\
120\end{array}$ & $\begin{array}{r}66 \\
40 \\
39 \\
32 \\
86 \\
170\end{array}$ & $\begin{array}{r}110 \\
55 \\
50 \\
39 \\
170 \\
280\end{array}$ & $\begin{array}{r}290 \\
100 \\
92 \\
62 \\
460 \\
580\end{array}$ \\
\hline
\end{tabular}


MAUMEE RIVER BASIN

04188300 Blanchard River at Mt. Blanchard, Ohio

LOCATION:

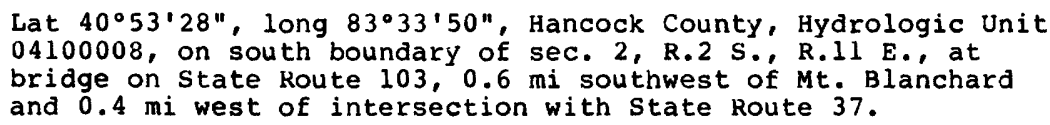

DRAINAGE AREA:

$109 \mathrm{mi}^{2}$.

TRIBUTARY TO:

Auglaize River.

DISCHARGE DATA USED: Low-flow measurements 1970-1977 water years.

INDEX STATION: $\quad 04189000$ Blanchard River near Findlay, Ohio.

REMARKS:

Prior to 1970 measured at site at bridge on Brooklyn street, northwest edge of Mt. Blanchard.

SELECTED DISCHARGE CHARACTERISTICS: Minimum discharge observed: $0 \mathrm{ft}^{3} / \mathrm{s}$ september $1 j 70$.

\begin{tabular}{|c|c|c|c|}
\hline \multirow[t]{2}{*}{ Period } & \multirow{2}{*}{$\begin{array}{l}\text { Number of } \\
\text { consecu- } \\
\text { tive days }\end{array}$} & \multicolumn{2}{|c|}{$\begin{array}{l}\text { Discharge }\left(\mathrm{ft}^{3} / \mathrm{s}\right) \text { for } \\
\text { indicated recurrence } \\
\text { interval (years) }\end{array}$} \\
\hline & & 2 & 10 \\
\hline Apr. - Mar. & $\begin{array}{r}7 \\
30\end{array}$ & $\begin{array}{l}0.2 \\
0.3\end{array}$ & ${ }_{.1}^{0}$ \\
\hline May - Nov. & $\begin{array}{r}7 \\
30\end{array}$ & $\begin{array}{l}0.2 \\
0.4\end{array}$ & ${ }^{0} .1$ \\
\hline June - Aug. & $\begin{array}{r}7 \\
30\end{array}$ & $\begin{array}{l}0.5 \\
1.2\end{array}$ & $\begin{array}{l}0.1 \\
0.2\end{array}$ \\
\hline Sept. - Nov. & $\begin{array}{r}7 \\
30\end{array}$ & $\begin{array}{l}0.2 \\
0.5\end{array}$ & $\begin{array}{l}0 \\
0.1\end{array}$ \\
\hline
\end{tabular}

Duration of daily flow for indicated periods

\begin{tabular}{|c|c|c|c|c|c|}
\hline \multirow{2}{*}{ Period } & \multicolumn{5}{|c|}{$\begin{array}{l}\text { Discharge }\left(\mathrm{ft}^{3} / \mathrm{s}\right) \text { which was equaled or } \\
\text { exceeded for indicated percent of time }\end{array}$} \\
\hline & 98 & 95 & 90 & 85 & 80 \\
\hline $\begin{array}{l}\text { Apr. - Mar. } \\
\text { May - Nov. } \\
\text { June - Aug. } \\
\text { Sept. - Nov. }\end{array}$ & $\begin{array}{l}0.1 \\
0.0 \\
0.1 \\
0.0\end{array}$ & $\begin{array}{l}0.1 \\
0.1 \\
0.1 \\
0.1\end{array}$ & $\begin{array}{l}0.3 \\
0.2 \\
0.3 \\
0.1\end{array}$ & $\begin{array}{l}0.4 \\
0.3 \\
0.5 \\
0.2\end{array}$ & $\begin{array}{l}0.7 \\
0.4 \\
0.6 \\
0.2\end{array}$ \\
\hline
\end{tabular}


LOCATION:

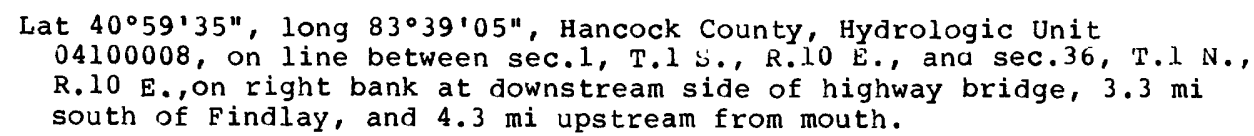

DRAINAGE AREA: $\quad 55 \mathrm{mi}^{2}$.

TRIBUTARY TO: $\quad$ Blanchard River

DISCHARGE DATA USED: January 1947 to July 1957.

REMARKS: $\quad$ None.

SELECTED DISCHARGE CHARACTERISTICS: Average discharge: $44.7 \mathrm{ft}^{3} / \mathrm{s}$ (10 years).

Minimum daily discharge: No flow for many days.

Magnitude and frequency of low flow for indicated periods

\begin{tabular}{|c|c|c|c|c|c|c|c|c|c|c|c|c|c|}
\hline \multirow{2}{*}{ Period } & \multirow{2}{*}{$\begin{array}{l}\text { Number of } \\
\text { consecu- } \\
\text { tive days }\end{array}$} & \multicolumn{5}{|c|}{$\begin{array}{l}\text { Discharge }\left(\mathrm{ft}^{3} / \mathrm{s}\right) \text { for indicated } \\
\text { recurrence interval (years) }\end{array}$} & \multirow{2}{*}{ Period } & \multirow{2}{*}{$\begin{array}{l}\text { Number of } \\
\text { consecu- } \\
\text { tive days }\end{array}$} & \multicolumn{5}{|c|}{$\begin{array}{l}\text { Discharge }\left(\mathrm{ft}^{3} / \mathrm{s}\right) \text { for indicated } \\
\text { recurrence interval (years) }\end{array}$} \\
\hline & & 2 & 5 & 10 & 20 & 50 & & & 2 & 5 & 10 & 20 & 50 \\
\hline Apr. -Mar. & $\begin{array}{r}1 \\
7 \\
30\end{array}$ & $\begin{array}{l}0 \\
0 \\
0\end{array}$ & $\begin{array}{l}0 \\
0 \\
0\end{array}$ & $\begin{array}{l}0 \\
0 \\
0\end{array}$ & $\begin{array}{l}0 \\
0 \\
0\end{array}$ & $\begin{array}{l}0 \\
0 \\
0\end{array}$ & Sept.-Nov. & $\begin{array}{r}1 \\
7 \\
30\end{array}$ & $\begin{array}{l}0 \\
0 \\
0\end{array}$ & $\begin{array}{l}0 \\
0 \\
0\end{array}$ & $\begin{array}{l}0 \\
0 \\
0\end{array}$ & $\begin{array}{l}0 \\
0 \\
0\end{array}$ & $\begin{array}{l}0 \\
0 \\
0\end{array}$ \\
\hline June-Aug. & $\begin{array}{r}1 \\
7 \\
30\end{array}$ & $\begin{array}{l}0 \\
0 \\
0.8\end{array}$ & $\begin{array}{l}0 \\
0 \\
0.1\end{array}$ & $\begin{array}{l}0 \\
0 \\
0\end{array}$ & $\begin{array}{l}0 \\
0 \\
0\end{array}$ & $\begin{array}{l}0 \\
0 \\
0\end{array}$ & Mar. - May & $\begin{array}{r}1 \\
7 \\
30\end{array}$ & $\begin{array}{r}3.6 \\
4.9 \\
21\end{array}$ & $\begin{array}{l}2.2 \\
3.0 \\
9.5\end{array}$ & $\begin{array}{l}1.5 \\
2.1 \\
6.0\end{array}$ & $\begin{array}{l}1.1 \\
1.5 \\
4.1\end{array}$ & $\begin{array}{l}0.7 \\
1.0 \\
2.6\end{array}$ \\
\hline
\end{tabular}

Duration of daily flow for indicated periods

\begin{tabular}{l|l|l|l|l|l|l|l|l|r|r|r|r|r|r}
\hline \multirow{2}{*}{ Period } & \multicolumn{8}{|c|}{ Discharge $\left(\mathrm{ft}^{3} / \mathrm{s}\right)$ which was equaled or exceeded for indicated percent of time } \\
\cline { 2 - 13 } & 98 & 95 & 90 & 85 & 80 & 75 & 70 & 60 & 50 & 40 & 30 & 20 & 10 \\
\hline Apr. - Mar. & 0 & 0 & 0.1 & 0.1 & 0.2 & 0.6 & 1.1 & 2.8 & 6.0 & 11 & 21 & 41 & 100 \\
May - Nov. & 0 & 0 & 0 & 0.1 & 0.1 & 0.1 & 0.2 & 0.7 & 1.6 & 3.2 & 5.9 & 12 & 33 \\
June - Aug. & 0 & 0 & 0.1 & 0.1 & 0.1 & 0.2 & 0.4 & 0.8 & 1.5 & 2.6 & 4.0 & 7.2 & 17 \\
Sept. - Nov. & 0 & 0 & 0 & 0 & 0.1 & 0.1 & 0.1 & 0.1 & 0.3 & 1.0 & 2.5 & 6.4 & 22 \\
Dec. - Feb. & 0.1 & 0.2 & 0.7 & 1.1 & 2.0 & 2.8 & 4.9 & 7.7 & 14 & 23 & 42 & 84 & 190 \\
Mar. - May & 2.5 & 4.6 & 6.1 & 7.7 & 9.5 & 12 & 15 & 20 & 28 & 39 & 60 & 98 & 190 \\
\hline
\end{tabular}


LOCATION :

DRAINAGE AREA:

TRIBUTARY TO:

DISCHARGE DATA USED:

REMARKS :

$346 \mathrm{mi}^{2}$.

SELECTED DISCHARGE CHARACTERISTICS: Average discharge: $244 \mathrm{ft}^{3} / \mathrm{s}$ (5q years).

Lat $41^{\circ} 03^{\prime} 21^{\prime \prime}$, long $83^{\circ} 41^{\prime} 17^{\prime \prime}$, on east line of sec. 10, T.1 N., R.10 E. Hancock County, Hydrologic Unit 04100008 , on left bank at upstream side of county road bridge, $2 \mathrm{mi}$ west of Findlay, $3 \mathrm{mi}$ downstream from Eagle Creek, and $3 \mathrm{mi}$ upstream from Aurand Run.

Auglaize River.

October 1923 to December 1935, October 1940 to September 1978.

Water is diverted upstream from station into Findlay Reservoir. Storage in Findlay Reservoir used for water supply of city of Findlay, and is available for low-flow augmentation. All water returns to stream upstream from station. No releases have been made for low-flow augmentation. Minimum daily discharge: $0.4 \mathrm{ft}^{3} / \mathrm{s}$ August, September 1934.

Magnitude and frequency of low flow for indicated periods

\begin{tabular}{c|c|c|c|c|c|c}
\hline \multirow{2}{*}{ Period } & \multirow{2}{*}{$\begin{array}{c}\text { Number of } \\
\text { consecu- } \\
\text { tive days }\end{array}$} & \multicolumn{4}{|c|}{$\begin{array}{c}\text { Discharge }\left(\mathrm{ft}^{3} / \mathrm{s}\right) \text { for indicated } \\
\text { recurrence interval }\end{array}$} \\
\cline { 3 - 7 } (years)
\end{tabular}

\begin{tabular}{|c|c|c|c|c|c|c|}
\hline \multirow{2}{*}{ Period } & \multirow{2}{*}{$\begin{array}{l}\text { Number of } \\
\text { consecu- } \\
\text { tive days }\end{array}$} & \multicolumn{5}{|c|}{$\begin{array}{l}\text { Discharge }\left(\mathrm{ft}^{3} / \mathrm{s}\right) \text { for indicated } \\
\text { recurrence interval (years) }\end{array}$} \\
\hline & & 2 & 5 & 10 & 20 & 50 \\
\hline Sept.-Nov. & $\begin{array}{r}1 \\
7 \\
30\end{array}$ & $\begin{array}{r}5.6 \\
6.7 \\
11\end{array}$ & $\begin{array}{l}2.6 \\
3.4 \\
5.3\end{array}$ & $\begin{array}{l}1.7 \\
2.4 \\
3.7\end{array}$ & $\begin{array}{l}1.2 \\
1.8 \\
2.7\end{array}$ & $\begin{array}{l}0.7 \\
1.4 \\
1.9\end{array}$ \\
\hline Dec. -Feb. & $\begin{array}{r}1 \\
7 \\
30\end{array}$ & $\begin{array}{l}16 \\
20 \\
51\end{array}$ & $\begin{array}{r}7.9 \\
9.6 \\
17\end{array}$ & $\begin{array}{l}5.4 \\
6.5 \\
9.2\end{array}$ & $\begin{array}{l}3.9 \\
4.6 \\
5.7\end{array}$ & $\begin{array}{l}2.7 \\
3.2 \\
3.3\end{array}$ \\
\hline Mar. - May & $\begin{array}{r}1 \\
7 \\
30\end{array}$ & $\begin{array}{r}35 \\
43 \\
105\end{array}$ & $\begin{array}{l}21 \\
27 \\
54\end{array}$ & $\begin{array}{l}15 \\
21 \\
38\end{array}$ & $\begin{array}{l}11 \\
17 \\
28\end{array}$ & $\begin{array}{l}7.7 \\
13 \\
20\end{array}$ \\
\hline
\end{tabular}

Duration of daily flow for indicated periods

\begin{tabular}{l|c|c|c|c|c|c|c|c|r|r|r|r|r}
\hline \multirow{2}{*}{ Period } & \multicolumn{8}{|c|}{ Discharge $\left(\mathrm{ft}^{3} / \mathrm{s}\right)$ which was equaled or exceeded for indicated percent of tine } \\
\cline { 2 - 13 } & 98 & 95 & 90 & 85 & 80 & 75 & 70 & 60 & 50 & 40 & 30 & 20 & 10 \\
\hline Apr. - Mar. & 3.2 & 5.3 & 7.7 & 10 & 14 & 17 & 21 & 33 & 50 & 77 & 130 & 240 & 600 \\
May - Nov. & 2.6 & 4.0 & 6.0 & 7.6 & 9.5 & 12 & 14 & 20 & 28 & 39 & 59 & 99 & 230 \\
June - Aug. & 2.8 & 5.3 & 7.9 & 11 & 13 & 15 & 18 & 24 & 31 & 40 & 57 & 89 & 190 \\
Sept. - Nov. & 2.3 & 3.1 & 4.4 & 5.5 & 6.6 & 7.5 & 8.5 & 12 & 16 & 21 & 30 & 52 & 140 \\
Dec. - Feb. & 5.9 & 7.6 & 11 & 15 & 20 & 25 & 32 & 49 & 74 & 120 & 200 & 390 & 990 \\
Mar. - May & 23 & 31 & 43 & 52 & 61 & 72 & 84 & 120 & 160 & 230 & 340 & 580 & 1100 \\
\hline
\end{tabular}


Appendix 3.--Low-flow data--Continued

MAUMEE RIVER BASIN

04189500 Blanchard River at Glandorf, Ohio

LOCATION: $\quad$ Lat $41^{\circ} 02^{\prime} 40^{\prime \prime}$, long $84^{\circ} 04^{\prime} 55^{\prime \prime}$, Putnam County, Hydrologic Unit 04100008 , in NEl/4 sec.17, T.1 N., R.7 E., near center of span on upstream side of highway bridge $0.5 \mathrm{mi}$ upstream from Pike Run and $0.8 \mathrm{mi}$ north of Glandorf.

DRAINAGE AREA: $\quad 644 \mathrm{mi}^{2}$.

TRIOLTARY TO: Auglaize River.

DISCHARGE DATA USED: September 1921 to July 1928, February 1947 to December 1951.

REMARKS:

None.

SELECTED DISCHARGE CHARACTERISTICS: Average discharge: $595 \mathrm{ft}^{3} / \mathrm{s}$ (10 years).

Minimum daily discharge: $0.3 \mathrm{ft}^{3} / \mathrm{s}$ October 1951.

Magnitude and frequency of low flow for indicated periods

\begin{tabular}{|c|c|c|c|c|c|c|c|c|c|c|c|c|c|}
\hline \multirow{2}{*}{ Period } & \multirow{2}{*}{$\begin{array}{l}\text { Number of } \\
\text { consecu- } \\
\text { tive days }\end{array}$} & \multicolumn{5}{|c|}{$\begin{array}{l}\text { Discharge }\left(\mathrm{ft}^{3} / \mathrm{s}\right) \text { for indicated } \\
\text { recurrence interval (years) }\end{array}$} & \multirow{2}{*}{ Period } & \multirow{2}{*}{$\begin{array}{l}\text { Number of } \\
\text { consecu- } \\
\text { tive days }\end{array}$} & \multicolumn{5}{|c|}{$\begin{array}{l}\text { Discharge }\left(\mathrm{ft}^{3} / \mathrm{s}\right) \text { for indicated } \\
\text { recurrence interval (years) }\end{array}$} \\
\hline & & 2 & 5 & 10 & 20 & 50 & & & $?$ & 5 & 10 & 20 & 50 \\
\hline Apr.-Mar. & $\begin{array}{r}1 \\
7 \\
30\end{array}$ & $\begin{array}{l}11 \\
13 \\
20\end{array}$ & $\begin{array}{l}7.0 \\
9.5 \\
16\end{array}$ & $\begin{array}{l}5.5 \\
8.0 \\
14\end{array}$ & $\begin{array}{l}4.4 \\
6.9 \\
12\end{array}$ & $\begin{array}{l}3.4 \\
5.8 \\
11\end{array}$ & Sept.-Nov. & $\begin{array}{r}1 \\
7 \\
30\end{array}$ & $\begin{array}{l}13 \\
15 \\
24\end{array}$ & $\begin{array}{r}4.7 \\
7.6 \\
14\end{array}$ & $\begin{array}{r}2.0 \\
4.5 \\
11\end{array}$ & $\begin{array}{r}0.8 \\
2.7 \\
10\end{array}$ & $\begin{array}{l}0.2 \\
1.3 \\
9.0\end{array}$ \\
\hline June-Aug. & $\begin{array}{r}1 \\
7 \\
30\end{array}$ & $\begin{array}{l}13 \\
15 \\
35\end{array}$ & $\begin{array}{l}8.3 \\
9.9 \\
21\end{array}$ & $\begin{array}{l}6.3 \\
8.0 \\
17\end{array}$ & $\begin{array}{l}4.9 \\
6.6 \\
14\end{array}$ & $\begin{array}{l}3.5 \\
5.3 \\
11\end{array}$ & Mar.- May & $\begin{array}{r}1 \\
7 \\
30\end{array}$ & $\begin{array}{r}78 \\
93 \\
301\end{array}$ & $\begin{array}{r}50 \\
58 \\
120\end{array}$ & $\begin{array}{l}38 \\
45 \\
71\end{array}$ & $\begin{array}{l}31 \\
36 \\
44\end{array}$ & $\begin{array}{l}24 \\
28 \\
25\end{array}$ \\
\hline
\end{tabular}

Duration of daily flow for indicated periods

\begin{tabular}{l|c|c|c|r|r|r|r|r|r|r|r|r|r}
\hline \multirow{2}{*}{ Period } & \multicolumn{8}{|c|}{ Discharge $\left(\mathrm{ft}^{3} / \mathrm{s}\right)$ which was equaled or exceeded for indicated percent of time } \\
\cline { 2 - 12 } & \multicolumn{1}{|c|}{98} & 95 & 90 & 85 & 80 & 75 & 70 & 60 & 50 & 40 & 30 & 20 & 10 \\
\hline Apr. - Mar. & 12 & 17 & 22 & 27 & 35 & 47 & 63 & 100 & 160 & 250 & 430 & 810 & 2000 \\
May - Nov. & 7.2 & 12 & 16 & 20 & 23 & 26 & 31 & 44 & 67 & 100 & 150 & 250 & 670 \\
June - Aug. & 9.5 & 13 & 17 & 20 & 24 & 29 & 34 & 48 & 65 & 86 & 120 & 180 & 370 \\
Sept. - Nov. & 5.9 & 9.1 & 14 & 17 & 19 & 21 & 23 & 27 & 38 & 61 & 120 & 200 & 520 \\
Dec. - Feb. & 23 & 42 & 73 & 93 & 120 & 150 & 190 & 260 & 350 & 540 & 850 & 1600 & 3000 \\
Mar. - May & 42 & 60 & 85 & 110 & 140 & 180 & 220 & 300 & 430 & 630 & 960 & 1600 & 2800 \\
\hline
\end{tabular}


LOCATION:

Lat $41^{\circ} 14^{\prime} 1^{\prime \prime}$, long $84^{\circ} 23^{\prime} 57^{\prime \prime}$, in NEl/4 sec.9, T.3 N., R.4 E., Defiance County, Hydrologic Unit 04100007, on right bank $125 \mathrm{ft}$ downstream from dam of Toledo Edison Co., $0.2 \mathrm{mi}$ upstream from Jackson ditch, and $3 \mathrm{mi}$ south of Defiance.

DRAINAGE AREA: $\quad 2,318 \mathrm{mi}^{2}$.

TRIBUTARY TO: Maumee River.

DISCHARGE DATA USED: October 1915 to September 1978.

REMARKS:

Flow regulated by dam at former powerplant $125 \mathrm{ft}$ upstream from station; reservoir capacity, 9,800 acre-ft, operation of plant discontinued Jan. 10, 1963; occasional gate operation subsequently. Some diversion by Miami and Erie Canal from Grand Lake into Jennings Creek, tributary to Auglaize River $70 \mathrm{mi}$ upstream from station.

SELECTED DISCHARGE CHARACTERISTICS: Average discharge: $1,705 \mathrm{ft}^{3} / \mathrm{s}$ (63 years). Minimum daily discharge: $0.5 \mathrm{ft}^{3} / \mathrm{s}$ October 1952 during repairs to powerplant dall.

Magnitude and frequency of low flow for indicated periods

\begin{tabular}{|c|c|c|c|c|c|c|}
\hline \multirow{2}{*}{ Period } & \multirow{2}{*}{$\begin{array}{l}\text { Number of } \\
\text { consecu- } \\
\text { tive days }\end{array}$} & \multicolumn{5}{|c|}{$\begin{array}{l}\text { Discharge }\left(\mathrm{ft}^{3} / \mathrm{s}\right) \text { for indicated } \\
\text { recurrence interval (years) }\end{array}$} \\
\hline & & 2 & 5 & 10 & 20 & 50 \\
\hline Apr.-Mar. & $\begin{array}{r}1 \\
7 \\
30\end{array}$ & $\begin{array}{l}26 \\
36 \\
63\end{array}$ & $\begin{array}{l}12 \\
18 \\
31\end{array}$ & $\begin{array}{l}6.4 \\
11 \\
20\end{array}$ & $\begin{array}{r}3.6 \\
6.0 \\
13\end{array}$ & $\begin{array}{l}1.4 \\
2.5 \\
8.0\end{array}$ \\
\hline May- Nov. & $\begin{array}{r}1 \\
7 \\
30\end{array}$ & $\begin{array}{l}28 \\
37 \\
64\end{array}$ & $\begin{array}{l}13 \\
19 \\
36\end{array}$ & $\begin{array}{l}7.1 \\
12 \\
26\end{array}$ & $\begin{array}{r}3.9 \\
7.2 \\
19\end{array}$ & $\begin{array}{l}1.5 \\
3.8 \\
13\end{array}$ \\
\hline June-Aug. & $\begin{array}{r}1 \\
7 \\
30\end{array}$ & $\begin{array}{r}35 \\
56 \\
124\end{array}$ & $\begin{array}{l}22 \\
36 \\
65\end{array}$ & $\begin{array}{l}18 \\
29 \\
48\end{array}$ & $\begin{array}{l}15 \\
24 \\
38\end{array}$ & $\begin{array}{l}12 \\
21 \\
29\end{array}$ \\
\hline
\end{tabular}

\begin{tabular}{c|c|r|r|r|r|r}
\hline \multirow{2}{*}{ Period } & \multirow{2}{*}{$\begin{array}{c}\text { Number of } \\
\text { consecu- } \\
\text { tive days }\end{array}$} & \multicolumn{4}{|c}{$\begin{array}{c}\text { Discharge } \\
\left.\text { recurrence } \mathrm{ft}^{3} / \mathrm{s}\right)\end{array}$ for interval $\begin{array}{c}\text { indicated } \\
\text { (years) }\end{array}$} \\
\cline { 3 - 7 } Sept.-Nov. & 1 & 31 & 14 & 7.6 & 4.1 & 1.8 \\
& 7 & 38 & 18 & 11 & 7.3 & 4.3 \\
& 30 & 66 & 34 & 26 & 21 & 18 \\
Dec.-Feb. & 1 & 44 & 18 & 12 & 8.0 & 5.4 \\
& 7 & 122 & 42 & 22 & 13 & 6.5 \\
& 30 & 378 & 111 & 55 & 30 & 14 \\
& 1 & 69 & 31 & 22 & 17 & 12 \\
& 7 & 269 & 124 & 77 & 50 & 29 \\
& 30 & 876 & 402 & 258 & 175 & 110 \\
\hline
\end{tabular}

Duration of daily flow for indicated periods

\begin{tabular}{|c|c|c|c|c|c|c|c|c|c|c|c|c|c|c|}
\hline \multirow{2}{*}{\multicolumn{2}{|c|}{ Period }} & \multicolumn{13}{|c|}{ Discharge $\left(\mathrm{ft}^{3} / \mathrm{s}\right)$ which was equaled or exceeded for indicated percent of time } \\
\hline & & 98 & 95 & 90 & 85 & 80 & 75 & 70 & 60 & 50 & 40 & 30 & 20 & 10 \\
\hline $\begin{array}{l}\text { Apr. - } \\
\text { May - } \\
\text { June - } \\
\text { Sept. - } \\
\text { Dec. - } \\
\text { Mar. - }\end{array}$ & $\begin{array}{l}\text { Mar. } \\
\text { Nov. } \\
\text { Aug. } \\
\text { Nov. } \\
\text { Feb. } \\
\text { May }\end{array}$ & $\begin{array}{l}20 \\
19 \\
25 \\
13 \\
18 \\
40\end{array}$ & $\begin{array}{r}27 \\
25 \\
29 \\
20 \\
30 \\
130\end{array}$ & $\begin{array}{r}36 \\
31 \\
36 \\
26 \\
45 \\
250\end{array}$ & $\begin{array}{r}44 \\
37 \\
42 \\
30 \\
73 \\
350\end{array}$ & $\begin{array}{r}59 \\
42 \\
51 \\
34 \\
120 \\
450\end{array}$ & $\begin{array}{r}87 \\
50 \\
63 \\
38 \\
180 \\
550\end{array}$ & $\begin{array}{r}130 \\
63 \\
85 \\
42 \\
260 \\
670\end{array}$ & $\begin{array}{r}240 \\
110 \\
140 \\
58 \\
420 \\
960\end{array}$ & $\begin{array}{r}380 \\
190 \\
220 \\
90 \\
640 \\
1400\end{array}$ & $\begin{array}{r}630 \\
300 \\
320 \\
140 \\
1000 \\
2100\end{array}$ & $\begin{array}{r}1100 \\
470 \\
460 \\
230 \\
1800 \\
3100\end{array}$ & $\begin{array}{r}2100 \\
820 \\
750 \\
420 \\
3300 \\
4800\end{array}$ & $\begin{array}{l}4700 \\
1900 \\
1600 \\
1100 \\
6900 \\
8600\end{array}$ \\
\hline
\end{tabular}


MAUMEE RIVER BASIN

04193500 Maumee River at waterville, Ohio

LOCATION: La.t $41^{\circ} 30^{\prime} 00^{\prime \prime}$, long $83^{\circ} 42^{\prime} 46^{\prime \prime}$, Lucas County, Hydrologic Unit 04100009 , on downstream side of second pier from left end of bridge on State Route 64 at Waterville, $3 \mathrm{mi}$ downstream from Tontogany Creek, and $20.7 \mathrm{mi}$ upstream from mouth.

DRAINAGE AREA: $\quad 6,330 \mathrm{mi}^{2}$.

TRIBUTARY TO: Lake Erie.

DISCHARGE DATA USED: October 1939 to September 1978.

REMARKS: Low flow slightly regulated by power plants upstream from station. Small diversion upstream from gage into Portage River basin.

SELECTED DISCHARGE CHARACTERISTICS: Average discharge: $4,922 \mathrm{ft}^{3} / \mathrm{s}_{3}$ (39 years). Minimum daily discharge: $26 \mathrm{ft}^{3} / \mathrm{s}$ October 1964.

Magnitude and frequency of low flow for indicated periods

\begin{tabular}{|c|c|c|c|c|c|c|}
\hline \multirow{2}{*}{ Period } & \multirow{2}{*}{$\begin{array}{l}\text { Number of } \\
\text { consecu- } \\
\text { tive days }\end{array}$} & \multicolumn{5}{|c|}{$\begin{array}{l}\text { Discharge }\left(\mathrm{ft}^{3} / \mathrm{s}\right) \text { for indicated } \\
\text { recurrence interval (years) }\end{array}$} \\
\hline & & 2 & 5 & 10 & 20 & 50 \\
\hline Apr. -Mar. & $\begin{array}{r}1 \\
7 \\
30\end{array}$ & $\begin{array}{l}147 \\
201 \\
264\end{array}$ & $\begin{array}{r}82 \\
124 \\
169\end{array}$ & $\begin{array}{r}57 \\
95 \\
136\end{array}$ & $\begin{array}{r}41 \\
74 \\
114\end{array}$ & $\begin{array}{l}27 \\
56 \\
94\end{array}$ \\
\hline May- Nov. & $\begin{array}{r}1 \\
7 \\
30\end{array}$ & $\begin{array}{l}147 \\
201 \\
268\end{array}$ & $\begin{array}{r}82 \\
124 \\
170\end{array}$ & $\begin{array}{r}57 \\
94 \\
136\end{array}$ & $\begin{array}{r}41 \\
73 \\
113\end{array}$ & $\begin{array}{l}27 \\
55 \\
93\end{array}$ \\
\hline June-Aug. & $\begin{array}{r}1 \\
7 \\
30\end{array}$ & $\begin{array}{l}225 \\
298 \\
492\end{array}$ & $\begin{array}{l}146 \\
197 \\
289\end{array}$ & $\begin{array}{l}117 \\
158 \\
232\end{array}$ & $\begin{array}{r}96 \\
133 \\
198\end{array}$ & $\begin{array}{r}77 \\
109 \\
171\end{array}$ \\
\hline
\end{tabular}

\begin{tabular}{|c|c|c|c|c|c|c|}
\hline \multirow{2}{*}{ Period } & \multirow{2}{*}{$\begin{array}{l}\text { Number of } \\
\text { consecu- } \\
\text { tive days }\end{array}$} & \multicolumn{5}{|c|}{$\begin{array}{l}\text { Discharge }\left(\mathrm{ft}^{3} / \mathrm{s}\right) \text { for indicated } \\
\text { recurrence interval (years) }\end{array}$} \\
\hline & & 2 & 5 & 10 & 20 & 50 \\
\hline Sept.-Nov. & $\begin{array}{r}1 \\
7 \\
30\end{array}$ & $\begin{array}{l}153 \\
207 \\
284\end{array}$ & $\begin{array}{r}85 \\
124 \\
168\end{array}$ & $\begin{array}{r}59 \\
93 \\
137\end{array}$ & $\begin{array}{r}42 \\
73 \\
120\end{array}$ & $\begin{array}{r}28 \\
55 \\
107\end{array}$ \\
\hline Dec. -Feb. & $\begin{array}{r}1 \\
7 \\
30\end{array}$ & $\begin{array}{r}436 \\
527 \\
1220\end{array}$ & $\begin{array}{l}235 \\
282 \\
466\end{array}$ & $\begin{array}{l}169 \\
205 \\
279\end{array}$ & $\begin{array}{l}128 \\
158 \\
183\end{array}$ & $\begin{array}{r}95 \\
119 \\
113\end{array}$ \\
\hline Mar. - May & $\begin{array}{r}1 \\
7 \\
30\end{array}$ & $\begin{array}{r}878 \\
11.30 \\
31.90\end{array}$ & $\begin{array}{r}531 \\
751 \\
1770\end{array}$ & $\begin{array}{r}400 \\
613 \\
1270\end{array}$ & $\begin{array}{l}313 \\
521 \\
910\end{array}$ & $\begin{array}{l}235 \\
435 \\
620\end{array}$ \\
\hline
\end{tabular}

Duration of daily flow for indicated periods

\begin{tabular}{|c|c|c|c|c|c|c|c|c|c|c|c|c|c|}
\hline \multirow{2}{*}{ Period } & \multicolumn{13}{|c|}{ Discharge $\left(\mathrm{ft}^{3} / \mathrm{s}\right)$ which was equaled or exceeded for indicated percent of time } \\
\hline & 98 & 95 & 90 & 85 & 80 & 75 & 70 & 60 & 50 & 40 & 30 & 20 & 10 \\
\hline $\begin{array}{l}\text { Apr. - Mar. } \\
\text { May - Nov. } \\
\text { June - Aug. } \\
\text { Sept. - Nov. } \\
\text { Dec. - Feb. } \\
\text { Mar. - May }\end{array}$ & $\begin{array}{l}140 \\
120 \\
170 \\
100 \\
190 \\
660\end{array}$ & $\begin{array}{l}190 \\
160 \\
210 \\
130 \\
230 \\
890\end{array}$ & $\begin{array}{r}250 \\
210 \\
260 \\
160 \\
300 \\
1200\end{array}$ & $\begin{array}{r}310 \\
250 \\
320 \\
190 \\
380 \\
1600\end{array}$ & $\begin{array}{r}390 \\
300 \\
380 \\
210 \\
510 \\
2000\end{array}$ & $\begin{array}{r}490 \\
360 \\
440 \\
240 \\
650 \\
2300\end{array}$ & $\begin{array}{r}610 \\
420 \\
510 \\
280 \\
850 \\
2700\end{array}$ & $\begin{array}{r}960 \\
580 \\
680 \\
360 \\
1400 \\
3900\end{array}$ & $\begin{array}{r}1500 \\
810 \\
930 \\
460 \\
2000 \\
5400\end{array}$ & $\begin{array}{r}2300 \\
1200 \\
1300 \\
620 \\
3200 \\
7400\end{array}$ & $\begin{array}{r}3900 \\
1800 \\
1800 \\
880 \\
5400 \\
11000\end{array}$ & $\begin{array}{r}7000 \\
3100 \\
2800 \\
1500 \\
9900 \\
15000\end{array}$ & $\begin{array}{r}14000 \\
6300 \\
5500 \\
3500 \\
19000 \\
22000\end{array}$ \\
\hline
\end{tabular}


PORTAGE RIVER BASIN

04195500 Portage River at Woodville, Ohio

LOCATION:

Lat $41^{\circ} 26^{\prime} 58^{\prime \prime}$, long $83^{\circ} 21^{\prime} 41^{\prime \prime}$, in sec. 28, T.6 N., R.13 E., Sandusky County, Hydrologic Unit 04100010, on left bank at upstream side of bridge on U.S. Highway 20 in woodville, $600 \mathrm{ft}$ downstream from unnamed right bank tributary, and $10.3 \mathrm{mi}$ upstream from Sugar Creek.

DRAINAGE AREA: $\quad 428 \mathrm{mi}^{2}$.

TRIBUTARY TO: Lake Erie.

DISCHARGE DATA USED: October 1951 to September 1978.

REMARKS:

Flow supplenented by water imported from Maumee kiver basin for municipal supply for city of Bowling Green $16 \mathrm{mi}$ upstream. The importation of this water began Sept. 1, 1951.

SELECTED DISCHARGE CHARACTERISTICS: Average discharge: $318 \mathrm{ft}^{3} / \mathrm{s}$ (2z years).

Minimum daily discharge: $1.8 \mathrm{ft}^{3} / \mathrm{s}$ September 1955.

Magnitude and frequency of low flow for indicated periods

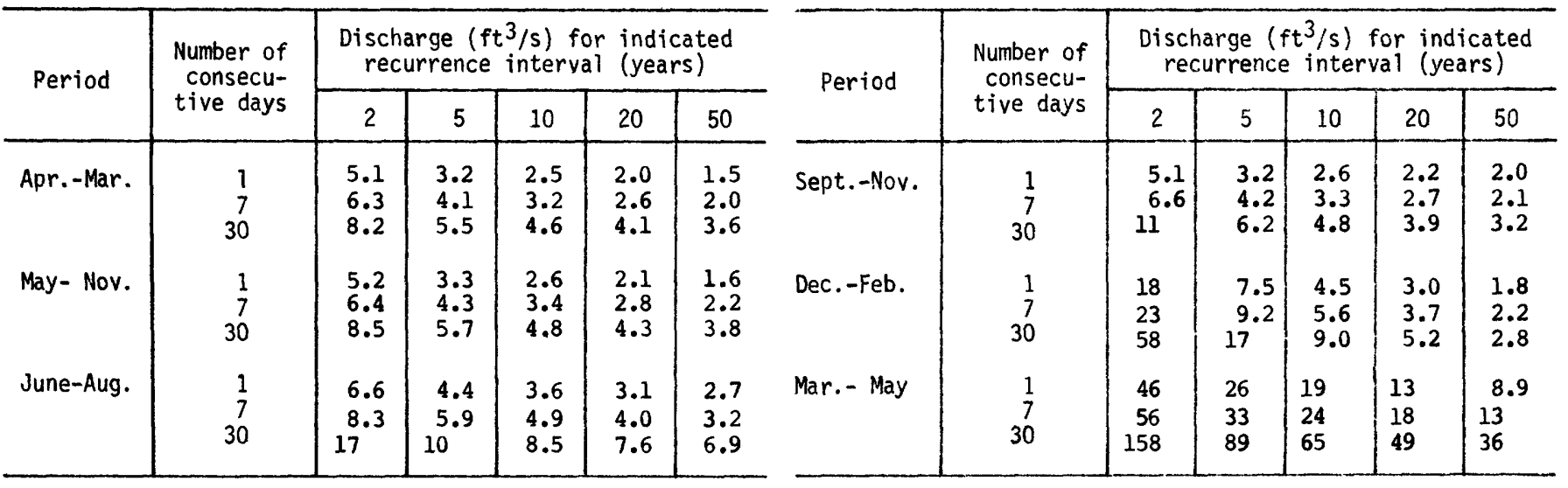

Duration of daily flow for indicated periods

\begin{tabular}{l|r|r|r|r|r|r|r|r|r|r|r|r|r|r}
\hline \multirow{2}{*}{ Period } & \multicolumn{8}{|c|}{ Discharge $\left(\mathrm{ft}^{3} / \mathrm{s}\right)$} & which was equaled or exceeded for indicated percent of time \\
\cline { 2 - 12 } & 98 & 95 & 90 & 85 & 80 & 75 & 70 & 60 & 50 & 40 & 30 & 20 & 10 \\
\hline Apr. - Mar. & 4.4 & 6.2 & 8.2 & 11 & 13 & 17 & 22 & 37 & 63 & 100 & 170 & 330 & 800 \\
May - Nov. & 3.8 & 5.5 & 7.2 & 8.6 & 10 & 12 & 14 & 20 & 29 & 46 & 74 & 130 & 320 \\
June - Aug. & 5.1 & 6.7 & 8.1 & 10 & 12 & 14 & 16 & 23 & 32 & 47 & 69 & 110 & 270 \\
Sept. - Nov. & 3.1 & 4.3 & 5.8 & 6.9 & 7.9 & 9.0 & 10 & 13 & 17 & 32 & 33 & 59 & 170 \\
Dec. - Feb. & 5.0 & 6.7 & 9.7 & 13 & 20 & 30 & 40 & 64 & 100 & 150 & 260 & 510 & 1200 \\
Mar. - May & 28 & 44 & 62 & 77 & 92 & 110 & 130 & 170 & 240 & 330 & 500 & 820 & 1700 \\
\hline
\end{tabular}




\author{
SANDUSKY RIVER BASI!N \\ 04196000 Sandusky River near Bucyrus, Ohio
}

\begin{abstract}
LOCATION :
Lat $40^{\circ} 48^{\prime} 13^{\prime \prime}$, long $83^{\circ} 00^{\prime} 21^{\prime \prime}$, in NEl/4 sec.10, T.3 S., R.16 E. Crawford County, Hydrologic Unit 04100111, on right bank at downstream side of bridge on township road, $1 \mathrm{mi}$ upstream from unnamed left bank tributary, $1.5 \mathrm{mi}$ west of Bucyrus, and $12 \mathrm{mi}$ downstream from Loss Creek.

DRAINAGE AREA: $88.8 \mathrm{mi}^{2}$.

TRIBUTARY TO:

Lake Erie.

DISCHARGE DATA USED:

REMARKS :

September 1925 to November 1935, August 1938 to December 1951, January 1964 to September 1978.

Low flow slightly affected by operation of reservoirs $5.3 \mathrm{mi}$ to

$6.0 \mathrm{mi}$ upstream from station, for municipal supply of Bucyrus.
\end{abstract}

SELECTED DISCHARGE CHARACTERISTICS: Average discharge: $84.9 \mathrm{ft}^{3} / \mathrm{s}^{3}\left(2.4 \mathrm{~m}^{3} / \mathrm{s}\right.$ ) (37 years). Minimum daily discharge: $0.6 \mathrm{ft}^{3} / \mathrm{s}$ September $1941,1946$.

Magnitude and frequency of low flow for indicated periods

\begin{tabular}{|c|c|c|c|c|c|c|c|c|c|c|c|c|c|}
\hline \multirow{2}{*}{ Period } & \multirow{2}{*}{$\begin{array}{l}\text { Number of } \\
\text { consecu- } \\
\text { tive days }\end{array}$} & \multicolumn{5}{|c|}{$\begin{array}{l}\text { Discharge }\left(\mathrm{ft}^{3} / \mathrm{s}\right) \text { for indicated } \\
\text { recurrence interval (years) }\end{array}$} & \multirow{2}{*}{ Period } & \multirow{2}{*}{$\begin{array}{l}\text { Number of } \\
\text { consecu- } \\
\text { tive days }\end{array}$} & \multicolumn{5}{|c|}{$\begin{array}{l}\text { Discharge }\left(\mathrm{ft}^{3} / \mathrm{s}\right) \text { for indicated } \\
\text { recurrence interval (years) }\end{array}$} \\
\hline & & 2 & 5 & 10 & 20 & 50 & & & 2 & 5 & 10 & 20 & 50 \\
\hline Apr. -Mar. & $\begin{array}{r}1 \\
7 \\
30\end{array}$ & $\begin{array}{l}1.7 \\
2.4 \\
3.8\end{array}$ & $\begin{array}{l}1.0 \\
1.4 \\
2.3\end{array}$ & $\begin{array}{l}0.8 \\
1.1 \\
1.7\end{array}$ & $\begin{array}{l}0.7 \\
0.9 \\
1.4\end{array}$ & $\begin{array}{l}0.5 \\
0.7 \\
1.1\end{array}$ & Sept.-Nov. & $\begin{array}{r}1 \\
7 \\
30\end{array}$ & $\begin{array}{l}1.9 \\
2.7 \\
5.0\end{array}$ & $\begin{array}{l}1.1 \\
1.5 \\
2.5\end{array}$ & $\begin{array}{l}0.9 \\
1.2 \\
1.8\end{array}$ & $\begin{array}{l}0.7 \\
1.0 \\
1.5\end{array}$ & $\begin{array}{l}0.6 \\
0.8 \\
1.2\end{array}$ \\
\hline June-Aug. & $\begin{array}{r}1 \\
7 \\
30\end{array}$ & $\begin{array}{l}2.1 \\
3.1 \\
6.1\end{array}$ & $\begin{array}{l}1.3 \\
1.9 \\
3.1\end{array}$ & $\begin{array}{l}1.1 \\
1.5 \\
2.3\end{array}$ & $\begin{array}{l}0.9 \\
1.2 \\
1.8\end{array}$ & $\begin{array}{l}0.7 \\
0.9 \\
1.3\end{array}$ & Mar. - May & $\begin{array}{r}1 \\
7 \\
30\end{array}$ & $\begin{array}{l}11 \\
15 \\
36\end{array}$ & $\begin{array}{r}5.9 \\
8.3 \\
17\end{array}$ & $\begin{array}{r}3.8 \\
5.8 \\
11\end{array}$ & $\begin{array}{l}2.4 \\
4.1 \\
7.7\end{array}$ & $\begin{array}{l}1.3 \\
2.7 \\
5.1\end{array}$ \\
\hline
\end{tabular}

Duration of daily flow for indicated periods

\begin{tabular}{l|c|c|c|c|c|c|c|c|c|c|c|c|c|}
\hline \multirow{2}{*}{ Period } & \multicolumn{8}{|c|}{ Discharge $\left(\mathrm{ft}^{3} / \mathrm{s}\right)$ which was equaled or exceeded for indicated percent of time } \\
\cline { 2 - 13 } & 98 & 95 & 90 & 85 & 80 & 75 & 70 & 60 & 50 & 40 & 30 & 20 & 10 \\
\hline Apr. - Mar. & 1.3 & 2.0 & 3.0 & 3.9 & 5.0 & 6.5 & 8.2 & 14 & 21 & 34 & 54 & 90 & 190 \\
May - Nov. & 1.2 & 1.7 & 2.4 & 3.0 & 3.5 & 4.2 & 4.9 & 6.9 & 9.9 & 14 & 22 & 39 & 86 \\
June - Aug. & 1.2 & 1.8 & 2.5 & 3.1 & 3.7 & 4.3 & 5.0 & 6.6 & 8.7 & 12 & 17 & 29 & 64 \\
Sept.- Nov. & 1.1 & 1.4 & 2.0 & 2.5 & 2.9 & 3.3 & 3.8 & 5.0 & 6.8 & 10 & 16 & 29 & 68 \\
Dec. - Feb. & 1.3 & 2.6 & 5.2 & 9.0 & 12 & 15 & 18 & 27 & 39 & 56 & 82 & 140 & 330 \\
Mar. - May & 6.8 & 10 & 15 & 19 & 23 & 28 & 33 & 45 & 60 & 80 & 110 & 170 & 320 \\
\hline
\end{tabular}


Appendix 3.--Low-flow data--Continued

SANDUSKY RIVER BASIN

04196200 Broken Sword Creek at Nevada, Ohio

LOCATION:

Lat $40^{\circ} 49^{\prime} 34^{\prime \prime}$, long $83^{\circ} 09^{\prime} 11^{\prime \prime}$, Wyandot County, Hydrologic Unit

04100011 , at bridge on State Route $182,1.0 \mathrm{mi}$ northwest of Nevada and $5.0 \mathrm{mi}$ upstream from mouth.

DRAINAGE AREA: $\quad 83.8 \mathrm{mi}^{2}$.

TRIBUTARY TO: Sandusky River.

DISCHARGE DATA USED: Low-flow measurements 1959, 1962-1965, 1967, 1969-1971 water years.

INDEX STATION: $\quad 04196500$ Sandusky River near Upper Sandusky, Ohio.

REMARKS: $\quad$ None.

SELECTED DISCHARGE CHARACTERISTICS: Minimum discharge observed: $0 \mathrm{ft}^{3} / \mathrm{s}$ October 1963 , September. 1964 .

\begin{tabular}{l|c|c|c}
\multicolumn{4}{c}{$\begin{array}{c}\text { Magnitude and frequency of low flow for } \\
\text { indicated periods }\end{array}$} \\
\hline & $\begin{array}{c}\text { Number of } \\
\text { consecu- } \\
\text { tive days }\end{array}$ & $\begin{array}{c}\text { Discharge (ft3/s) for } \\
\text { indicated recurrence } \\
\text { interval (years) }\end{array}$ \\
\cline { 3 - 4 } & 7 & 2 & 10 \\
\hline Apr. - Mar. & 30 & 0.2 & 0 \\
& 7 & 0.2 & 0.1 \\
May - Nov. & 30 & 0.6 & 0 \\
June - Aug. & 7 & 0.5 & 0.1 \\
& 30 & 1.6 & 0.3 \\
Sept. - Nov. & 7 & 0.3 & 0 \\
& 30 & 0.9 & 0.1 \\
\hline
\end{tabular}

Duration of daily flow for indicated periods

\begin{tabular}{l|c|c|c|c|c}
\hline \multirow{2}{*}{ Period } & \multicolumn{5}{|c}{$\begin{array}{c}\text { Discharge ( } \mathrm{ft}^{3} / \mathrm{s} \text { ) which was equaled or } \\
\text { exceeded for indicated percent of time }\end{array}$} \\
\cline { 2 - 6 } & 98 & 95 & 90 & 85 & 80 \\
\hline Apr. - Mar. & 0.1 & 0.2 & 0.5 & 0.9 & 1.4 \\
May - Nov. & 0.1 & 0.1 & 0.3 & 0.5 & 0.7 \\
June - Aug. & 0.1 & 0.2 & 0.4 & 0.6 & 0.9 \\
Sept. - Nov. & 0.0 & 0.1 & 0.2 & 0.2 & 0.4 \\
\hline
\end{tabular}




\section{SANDUSKY RIVER BASIN}

04196500 Sandusky River near Upper Sandusky, Ohio

LOCATION:

DRAINAGE AREA:

TRIBUTARY T0:

DISCHARGE DATA USED:

REMARKS:

SELECTED DISCHARGE CHARACTERISTICS: Average discharge: $242 \mathrm{ft}^{3} / \mathrm{s}$ (54 years).

Minimum daily discharge: $0.6 \mathrm{ft} / \mathrm{s}$ September 1955, October 1963.

Magnitude and frequency of low flow for indicated periods

\begin{tabular}{c|c|c|c|c|c|c}
\hline \multirow{2}{*}{ Period } & \multirow{2}{*}{$\begin{array}{c}\text { Number of } \\
\text { consecu- } \\
\text { tive days }\end{array}$} & \multicolumn{4}{|c}{$\begin{array}{c}\text { Discharge ( } \mathrm{ft}^{3} / \mathrm{s} \text { ) for indicated } \\
\text { recurrence interval (years) }\end{array}$} \\
\cline { 3 - 7 } Apr.-Mar. & 1 & 2 & 5 & 10 & 20 & 50 \\
\hline \multirow{3}{*}{ May- Nov. } & 7 & 5.0 & 2.0 & 1.3 & 0.9 & 0.6 \\
& 30 & 8.4 & 4.1 & 1.7 & 1.2 & 0.8 \\
& 1 & 4.0 & 2.0 & 1.3 & 0.9 & 0.6 \\
& 7 & 5.1 & 2.5 & 1.7 & 1.2 & 0.8 \\
& 30 & 8.4 & 4.1 & 2.8 & 2.0 & 1.3 \\
& 1 & 5.8 & 3.0 & 2.1 & 1.6 & 1.2 \\
& 7 & 7.6 & 4.0 & 2.8 & 2.1 & 1.5 \\
& 30 & 15 & 7.8 & 5.8 & 4.6 & 3.7 \\
\hline
\end{tabular}

\begin{tabular}{|c|c|c|c|c|c|c|}
\hline \multirow{2}{*}{ Period } & \multirow{2}{*}{$\begin{array}{l}\text { Number of } \\
\because \text { secu- } \\
\text { : ive days }\end{array}$} & \multicolumn{5}{|c|}{$\begin{array}{l}\text { Discharge }\left(\mathrm{ft}^{3} / \mathrm{s}\right) \text { for indicated } \\
\text { recurrence interval (years) }\end{array}$} \\
\hline & & 2 & 5 & 10 & 20 & 50 \\
\hline Sept.-Nov. & $\begin{array}{r}1 \\
7 \\
30\end{array}$ & $\begin{array}{l}4.6 \\
5.8 \\
11\end{array}$ & $\begin{array}{l}2.1 \\
2.7 \\
4.4\end{array}$ & $\begin{array}{l}1.4 \\
1.8 \\
2.9\end{array}$ & $\begin{array}{l}1.0 \\
1.3 \\
2.1\end{array}$ & $\begin{array}{l}0.6 \\
0.9 \\
1.5\end{array}$ \\
\hline Dec.-Feb. & $\begin{array}{r}1 \\
7 \\
30\end{array}$ & $\begin{array}{l}22 \\
29 \\
77\end{array}$ & $\begin{array}{l}9.5 \\
12 \\
25\end{array}$ & $\begin{array}{l}6.0 \\
7.5 \\
13\end{array}$ & $\begin{array}{l}4.1 \\
4.9 \\
7.4\end{array}$ & $\begin{array}{l}2.6 \\
3.1 \\
3.9\end{array}$ \\
\hline Mar. - May & $\begin{array}{r}1 \\
7 \\
30\end{array}$ & $\begin{array}{r}41 \\
52 \\
109\end{array}$ & $\begin{array}{l}24 \\
31 \\
56\end{array}$ & $\begin{array}{l}18 \\
23 \\
39\end{array}$ & $\begin{array}{l}13 \\
18 \\
29\end{array}$ & $\begin{array}{l}9.6 \\
13 \\
21\end{array}$ \\
\hline
\end{tabular}

Duration of daily flow for indicated periods

\begin{tabular}{|c|c|c|c|c|c|c|c|c|c|c|c|c|c|}
\hline \multirow{2}{*}{ Period } & \multicolumn{13}{|c|}{ Discharge $\left(\mathrm{ft}^{3} / \mathrm{s}\right)$ which was equaled or exceeded for indicated percent of time } \\
\hline & 98 & 95 & 90 & 85 & 80 & 75 & 70 & 60 & 50 & 40 & 30 & 20 & 10 \\
\hline $\begin{array}{l}\text { Apr. - Mar. } \\
\text { May - Nov. } \\
\text { June - Aug. } \\
\text { Sept. - Nov. } \\
\text { Dec. - Feb. } \\
\text { Mar. - May }\end{array}$ & $\begin{array}{r}2.9 \\
2.3 \\
3.0 \\
1.7 \\
6.4 \\
25\end{array}$ & $\begin{array}{r}4.8 \\
3.6 \\
4.5 \\
2.6 \\
9.4 \\
37\end{array}$ & $\begin{array}{l}7.7 \\
5.5 \\
6.6 \\
4.0 \\
14 \\
50\end{array}$ & $\begin{array}{l}11 \\
7.5 \\
8.7 \\
5.2 \\
20 \\
63\end{array}$ & $\begin{array}{l}14 \\
9.5 \\
11 \\
6.7 \\
29 \\
76\end{array}$ & $\begin{array}{l}18 \\
12 \\
13 \\
8.2 \\
38 \\
90\end{array}$ & $\begin{array}{c}23 \\
14 \\
16 \\
9.7 \\
50 \\
110\end{array}$ & $\begin{array}{r}37 \\
20 \\
21 \\
13 \\
80 \\
140\end{array}$ & $\begin{array}{r}59 \\
28 \\
28 \\
17 \\
120 \\
190\end{array}$ & $\begin{array}{r}98 \\
40 \\
38 \\
23 \\
180 \\
260\end{array}$ & $\begin{array}{r}160 \\
62 \\
55 \\
33 \\
270 \\
370\end{array}$ & $\begin{array}{r}280 \\
110 \\
91 \\
58 \\
470 \\
570\end{array}$ & $\begin{array}{r}610 \\
240 \\
200 \\
160 \\
1000 \\
1000\end{array}$ \\
\hline
\end{tabular}




\section{SANDUSKY RIVER BASIN}

04197000 Sandusky River near Mexico, Ohio

LOCATION:

Lat $41^{\circ} 02^{\prime} 39^{\prime \prime}$, long $83^{\circ} 11^{\prime} 42^{\prime \prime}$, in sec.13, T.1 N., R.14 E., Seneca County, Hydrologic Unit 0410011 , on right bank at downstream side of county road bridge, $4.1 \mathrm{mi}$ upstream from Honey Creek, $4.2 \mathrm{mi}$ north of Mexico, $4 . y$ ul soucn or ' 1 irrin, ana $0.3 \mathrm{mi}$ aownstream ixom mile kun.

DRAINAGE AREA: $\quad 774 \mathrm{mi}^{2}$.

TRIBUTARY TO: Lake Erie.

DISCHARGE DATA USED: March 1923 to December 1935, August 1938 to September 1978.

REMARKS :

None.

SELECTED DISCHARGE CHARACTERISTICS: Average discharge: $577 \mathrm{ft}^{3} / \mathrm{s}$ (52 years).

Minimum daily discharge: $2.0 \mathrm{ft}^{3} / \mathrm{s}$ October. 1943.

Magnitude and frequency of low flow for indicated periods

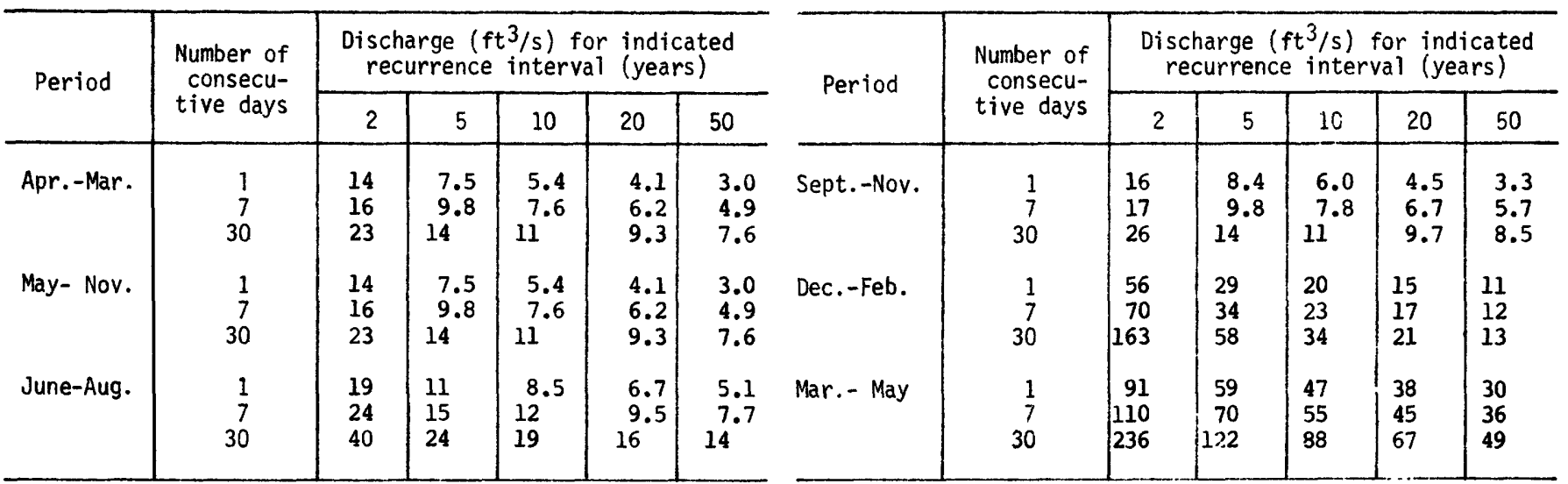

Duration of daily flow for indicated periods

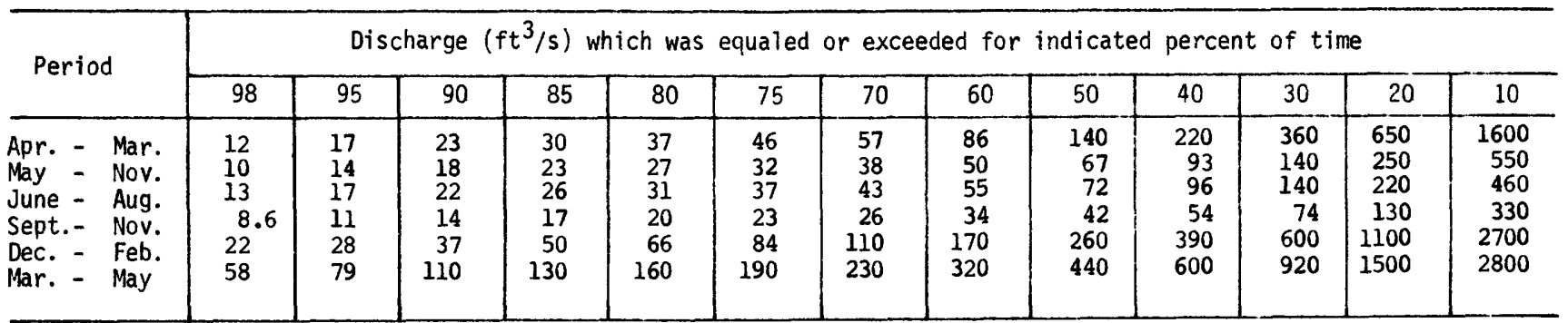




\section{SANDUSKY RIVER BASIN}

04198000 Sandusky River near Fremont, Ohio

LOCATION:

Lat $41^{\circ} 18^{\prime} 28^{\prime \prime}$, long $83^{\circ} 09^{\prime} 32^{\prime \prime}$, in sec.17, T.4 N., R.15 E.,

Sandusky County, Hydrologic unit 04100011 , on left bank at

downstream side of county road bridge, $2.3 \mathrm{mi}$ upstream from

Ballville diversion dam, $2.5 \mathrm{mi}$ downstream from Wolf Creek,

and $3.5 \mathrm{mi}$ southwest of Fremont.

DRAINAGE AREA:

$1,251 \mathrm{mi}^{2}$.

TRIBUTARY TO:

Lake Erie.

DISCHARGE DATA USED: October 1923 to September 1935, August 1938 to September 1978.

REMARKS: $\quad$ None.

SELECTED DISCHARGE CHARACTERISTICS: Average discharge: $956 \mathrm{ft}^{3} / \mathrm{s}$ (32 years).

Minimum daily discharge: $5.0 \mathrm{ft} \mathrm{t}^{3} / \mathrm{s}$ October 1963.

Magnitude and frequency of low flow for indicated periods

\begin{tabular}{c|c|c|c|c|c|c}
\hline \multirow{2}{*}{ Period } & \multirow{2}{*}{$\begin{array}{c}\text { Number of } \\
\text { consecu- } \\
\text { tive days }\end{array}$} & \multicolumn{4}{|c|}{$\begin{array}{c}\text { Discharge ( } \mathrm{ft} 3 / \mathrm{s} \text { ) for indicated } \\
\text { recurrence interval (years) }\end{array}$} \\
\cline { 3 - 7 } Apr.-Mar. & 1 & 21 & 13 & 10 & 8.0 & 6.1 \\
& 7 & 26 & 16 & 12 & 9.6 & 7.3 \\
& 30 & 35 & 22 & 17 & 14 & 11 \\
May- Nov. & 1 & 21 & 13 & 10 & 8.0 & 6.1 \\
& 7 & 26 & 16 & 12 & 9.6 & 7.3 \\
& 30 & 36 & 22 & 17 & 14 & 11 \\
& 1 & 29 & 19 & 16 & 13 & 11 \\
& 7 & 36 & 24 & 20 & 17 & 15 \\
& 30 & 70 & 41 & 32 & 26 & 22 \\
\hline
\end{tabular}

\begin{tabular}{c|c|r|r|r|r|r}
\hline \multirow{2}{*}{ Period } & \multirow{2}{*}{$\begin{array}{c}\text { Number of } \\
\text { consecu- } \\
\text { tive days }\end{array}$} & \multicolumn{4}{|c|}{$\begin{array}{c}\text { Discharge } \\
\text { recurrence }\end{array}$} \\
\cline { 3 - 7 } & 2 & 5 & 10 & 20 & 50 \\
\hline Sept.-Nov. & 1 & 24 & 14 & 10 & 8.0 & 6.0 \\
& 7 & 28 & 16 & 12 & 9.6 & 7.2 \\
& 30 & 39 & 21 & 17 & 15 & 13 \\
Dec.-Feb. & 1 & 92 & 46 & 31 & 23 & 16 \\
& 7 & 109 & 52 & 35 & 25 & 17 \\
& 30 & 267 & 91 & 52 & 32 & 19 \\
& 1 & 164 & 96 & 71 & 54 & 39 \\
& 7 & 200 & 125 & 97 & 79 & 62 \\
& 30 & 441 & 233 & 166 & 126 & 92 \\
\hline
\end{tabular}

Duration of daily flow for indicated periods

\begin{tabular}{l|r|r|r|r|r|r|r|r|r|r|r|r|r}
\hline \multirow{2}{*}{ Period } & \multicolumn{8}{|c|}{ Discharge $\left(\mathrm{ft}^{3} / \mathrm{s}\right.$ ) which was equaled or exceeded for indicated percent of time } \\
\cline { 2 - 12 } & 98 & 95 & 90 & 85 & 80 & 75 & 70 & 60 & 50 & 40 & 30 & 20 & 10 \\
\hline Apr. - Mar. & 19 & 26 & 36 & 45 & 55 & 68 & 86 & 140 & 230 & 370 & 590 & 1100 & 2600 \\
May - Nov. & 15 & 21 & 28 & 35 & 41 & 48 & 56 & 76 & 110 & 160 & 250 & 430 & 920 \\
June - Aug. & 21 & 26 & 34 & 42 & 50 & 58 & 66 & 88 & 120 & 160 & 230 & 370 & 760 \\
Sept.- Nov. & 12 & 16 & 22 & 26 & 31 & 35 & 39 & 49 & 61 & 78 & 110 & 210 & 560 \\
Dec. - Feb. & 33 & 41 & 55 & 74 & 100 & 140 & 180 & 290 & 430 & 640 & 1100 & 1900 & 4400 \\
Mar. - May & 100 & 140 & 200 & 250 & 290 & 350 & 400 & 540 & 740 & 1100 & 1600 & 2500 & 4500 \\
\hline
\end{tabular}


Appendix 3.--Low-flow data--Continued

\section{HURON RIVER BASIN}

04198020 West Branch Huron River near Monroeville, Ohio

LOCATION:

Lat $41^{\circ} 16^{\prime} 40^{\prime \prime}$, long $82^{\circ} 40^{\prime} 30^{\prime \prime}$, Huron County, Hydrologic Unit

04100012 , at bridge on Lamoreaux Road, $2.5 \mathrm{mi}$ northeast of

Monroeville.

DRAINAGE AREA: $\quad 220 \mathrm{mi}^{2}$.

TRIBUTARY TO: Head of Huron River.

DISCHARGE DATA USED: LOW-flow measurements 1960-1967, 1970-1978 water years.

INDEX STATION: $\quad 04199000$ Huron River at Milan, Ohio.

REMARKS: $\quad$ None.

SELECTED DISCHARGE CHARACTERISTICS: Minimum discharge observed: $0.7 \mathrm{ft}^{3} / \mathrm{s}$ October 1973.

\begin{tabular}{c|c|c|c}
\multicolumn{4}{c}{$\begin{array}{c}\text { Magnitude and frequency of low flow for } \\
\text { indicated periods }\end{array}$} \\
\hline \multirow{2}{*}{ Period } & $\begin{array}{c}\text { Number of } \\
\text { consecu- } \\
\text { tive days }\end{array}$ & $\begin{array}{c}\left.\text { Discharge (ft }{ }^{3} / \mathrm{s}\right) \text { for } \\
\text { indicated recurrence } \\
\text { interval (years) }\end{array}$ \\
\cline { 3 - 4 } & 7 & 2 & 10 \\
\hline Apr. - Mar. & 30 & 9.3 & 1.0 \\
May - Nov. & 7 & 5.3 & 2.3 \\
& 30 & 9.0 & 1.0 \\
June - Aug. & 7 & 9.0 & 2.2 \\
& 30 & 16 & 6.3 \\
Sept. - Nov. & 7 & 6.5 & 1.0 \\
& 30 & 11 & 2.4 \\
\hline
\end{tabular}

Duration of daily flow for indicated periods

\begin{tabular}{l|c|c|c|c|c}
\hline \multirow{2}{*}{ Period } & \multicolumn{5}{|c}{$\begin{array}{c}\text { Discharge ( } \mathrm{ft}^{3 / \mathrm{s}} \text { ) which was equaled or } \\
\text { exceeded for indicated percent of time }\end{array}$} \\
\cline { 2 - 6 } & 98 & 95 & 90 & 85 & 80 \\
\hline Apr. - Mar. & 2.4 & 5.3 & 9.0 & 12 & 14 \\
May - Nov. & 1.7 & 3.5 & 6.5 & 9.0 & 10 \\
June - Aug. & 2.6 & 4.4 & 7.9 & 9.5 & 11 \\
Sept. - Nov. & 1.2 & 2.0 & 4.6 & 7.9 & 8.3 \\
\hline
\end{tabular}


HURON RIVER BASIN

04198500 East Branch Huron River near Norwalk, Ohio

LOCATION:

Lat $41^{\circ} 14^{\prime} 58^{\prime \prime}$, long $80^{\circ} 38^{\prime} 52^{\prime \prime}$, Huron County, Hydrologic Unit

04100012 , at highway bridge $1.3 \mathrm{mi}$ northwest of Norwalk and

$1.5 \mathrm{mi}$ below mouth of cole creek.

DRAINAGE AREA: $\quad 85.5 \mathrm{mi}^{2}$.

TRISUTARY TO: West Branch Huron River.

DISCHARGE DATA USED: November 1923 to September 1935.

REMARKS: $\quad$ None.

SELECTED DISCHARGE CHARACTERISTICS: Average discharge: $62.8 \mathrm{ft}^{3} / \mathrm{s}$ (11 years).

Minimum daily discharge: $0.2 \mathrm{ft}^{3} / \mathrm{s}$ July 1934 .

Magnitude and frequency of low flow for indicated periods

\begin{tabular}{|c|c|c|c|c|c|c|c|c|c|c|c|c|c|}
\hline \multirow{2}{*}{ Period } & \multirow{2}{*}{$\begin{array}{l}\text { Number of } \\
\text { consecu- } \\
\text { tive days }\end{array}$} & \multicolumn{5}{|c|}{$\begin{array}{l}\text { Discharge }\left(\mathrm{ft}^{3} / \mathrm{s}\right) \text { for indicated } \\
\text { recurrence interval (years) }\end{array}$} & \multirow{2}{*}{ Period } & \multirow{2}{*}{$\begin{array}{l}\text { Number of } \\
\text { consecu- } \\
\text { tive days }\end{array}$} & \multicolumn{5}{|c|}{$\begin{array}{l}\text { Discharge ( } \mathrm{ft}^{3} / \mathrm{s} \text { ) for indicated } \\
\text { recurrence interval (years) }\end{array}$} \\
\hline & & 2 & 5 & 10 & 20 & 50 & & & 2 & 5 & 10 & 20 & 50 \\
\hline Apr.-Mar. & $\begin{array}{r}1 \\
7 \\
30\end{array}$ & $\begin{array}{l}2.0 \\
2.4 \\
3.6\end{array}$ & $\begin{array}{l}0.8 \\
1.0 \\
1.8\end{array}$ & $\begin{array}{l}0.4 \\
0.6 \\
1.2\end{array}$ & $\begin{array}{l}0.2 \\
0.4 \\
0.9\end{array}$ & $\begin{array}{l}0.1 \\
0.2 \\
0.6\end{array}$ & Sept.-Nov. & $\begin{array}{r}1 \\
7 \\
30\end{array}$ & $\begin{array}{l}2.7 \\
3.4 \\
5.3\end{array}$ & $\begin{array}{l}1.5 \\
1.8 \\
3.0\end{array}$ & $\begin{array}{l}1.0 \\
1.4 \\
2.2\end{array}$ & $\begin{array}{l}0.7 \\
1.1 \\
1.6\end{array}$ & $\begin{array}{l}0.5 \\
0.8 \\
1.1\end{array}$ \\
\hline June-Aug. & $\begin{array}{r}1 \\
7 \\
30\end{array}$ & $\begin{array}{l}2.2 \\
2.7 \\
4.9\end{array}$ & $\begin{array}{l}0.9 \\
1.2 \\
2.1\end{array}$ & $\begin{array}{l}0.5 \\
0.7 \\
1.4\end{array}$ & $\begin{array}{l}0.2 \\
0.4 \\
1.0\end{array}$ & $\begin{array}{l}0.1 \\
0.2 \\
0.7\end{array}$ & Mar. - May & $\begin{array}{r}1 \\
7 \\
30\end{array}$ & $\begin{array}{l}11 \\
12 \\
20\end{array}$ & $\begin{array}{c}6.8 \\
7.4 \\
10\end{array}$ & $\begin{array}{l}5.0 \\
5.7 \\
7.9\end{array}$ & $\begin{array}{l}3.7 \\
4.5 \\
6.6\end{array}$ & $\begin{array}{l}2.6 \\
3.5 \\
5.5\end{array}$ \\
\hline
\end{tabular}

Duration of daily flow for indicated periods

\begin{tabular}{|c|c|c|c|c|c|c|c|c|c|c|c|c|c|}
\hline \multirow{2}{*}{ Period } & \multicolumn{13}{|c|}{ Discharge $\left(\mathrm{ft}^{3} / \mathrm{s}\right)$ which was equaled or exceeded for indicated percent of time } \\
\hline & 98 & 95 & 90 & 85 & 80 & 75 & 70 & 60 & 50 & 40 & 30 & 20 & 10 \\
\hline $\begin{array}{l}\text { Apr. - Mar. } \\
\text { May - Nov. } \\
\text { June - Aug. } \\
\text { Sept. - Nov. } \\
\text { Dec. - Feb. } \\
\text { Mar. - May }\end{array}$ & $\begin{array}{l}1.4 \\
1.0 \\
0.6 \\
1.4 \\
5.2 \\
6.2\end{array}$ & $\begin{array}{l}2.3 \\
1.7 \\
1.3 \\
2.0 \\
7.6 \\
9.0\end{array}$ & $\begin{array}{l}3.5 \\
2.6 \\
1.9 \\
3.0 \\
11 \\
12\end{array}$ & $\begin{array}{l}4.6 \\
3.3 \\
2.6 \\
3.5 \\
13 \\
14\end{array}$ & $\begin{array}{l}5.7 \\
3.9 \\
3.2 \\
4.1 \\
15 \\
16\end{array}$ & $\begin{array}{l}6.9 \\
4.6 \\
3.9 \\
4.7 \\
17 \\
19\end{array}$ & $\begin{array}{l}8.3 \\
5.3 \\
4.5 \\
5.3 \\
19 \\
21\end{array}$ & $\begin{array}{l}12 \\
6.7 \\
5.7 \\
6.4 \\
25 \\
27\end{array}$ & $\begin{array}{l}17 \\
8.6 \\
7.1 \\
7.7 \\
36 \\
35\end{array}$ & $\begin{array}{l}24 \\
11 \\
9.0 \\
9.2 \\
48 \\
49\end{array}$ & $\begin{array}{l}39 \\
16 \\
12 \\
13 \\
69 \\
69\end{array}$ & $\begin{array}{r}64 \\
27 \\
20 \\
23 \\
120 \\
110\end{array}$ & $\begin{array}{r}150 \\
66 \\
58 \\
57 \\
270 \\
220\end{array}$ \\
\hline
\end{tabular}


HURON RIVER BASIN

04199000 Huron River at Milan, Ohio

LOCATION

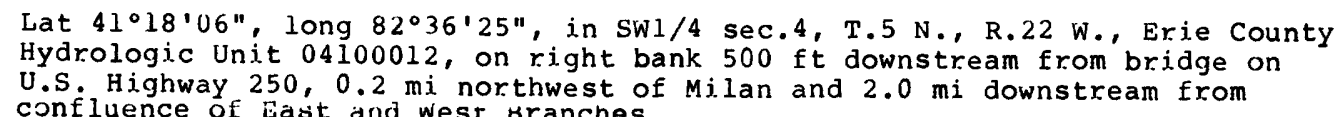
Hydrologic Unit 04100012 , on right bank $500 \mathrm{ft}$ downstream from bridge on confluence of iast and west branches.

DRAINAGE AREA: $\quad 371 \mathrm{mi}^{2}$.

TRIBUTARY TO: Lake Erie.

DISCHARGE DATA USED: April 1950 to September 1978.

REMARKS: None.

SELECTED DISCHARGE CHARACTERISTICS: Average discharge: $300 \mathrm{ft}^{3} / \mathrm{s}$ (28 years). Minimum daily discharge: $3.0 \mathrm{ft}^{3} / \mathrm{s}$ september 1955.

Magnitude and frequency of low flow for indicated periods

\begin{tabular}{|c|c|c|c|c|c|c|c|c|c|c|c|c|c|}
\hline \multirow{2}{*}{ Period } & \multirow{2}{*}{$\begin{array}{l}\text { Number of } \\
\text { consecu- } \\
\text { tive days }\end{array}$} & \multicolumn{5}{|c|}{$\begin{array}{l}\text { Discharge }\left(\mathrm{ft}^{3} / \mathrm{s}\right) \text { for indicated } \\
\text { recurrence interval (years) }\end{array}$} & \multirow{2}{*}{ Period } & \multirow{2}{*}{$\begin{array}{l}\text { Number of } \\
\text { consecu- } \\
\text { tive days }\end{array}$} & \multicolumn{5}{|c|}{$\begin{array}{l}\text { Discharge }\left(\mathrm{ft}^{3} / \mathrm{s}\right) \text { for indicated } \\
\text { recurrence interval (years) }\end{array}$} \\
\hline & & 2 & 5 & 10 & 20 & 50 & & & 2 & 5 & 10 & 20 & 50 \\
\hline Apr.-Mar. & $\begin{array}{r}1 \\
7 \\
30\end{array}$ & $\begin{array}{l}8.4 \\
10 \\
14\end{array}$ & $\begin{array}{l}5.0 \\
6.0 \\
8.3\end{array}$ & $\begin{array}{l}3.8 \\
4.6 \\
6.8\end{array}$ & $\begin{array}{l}3.1 \\
3.7 \\
5.8\end{array}$ & $\begin{array}{l}2.4 \\
2.9 \\
5.1\end{array}$ & Sept.-Nov. & $\begin{array}{r}1 \\
7 \\
30\end{array}$ & $\begin{array}{l}9.1 \\
11 \\
17\end{array}$ & $\begin{array}{l}5.1 \\
6.3 \\
9.1\end{array}$ & $\begin{array}{l}3.8 \\
4.7 \\
7.0\end{array}$ & $\begin{array}{l}3.0 \\
3.7 \\
5.9\end{array}$ & $\begin{array}{l}2.3 \\
2.8 \\
5.0\end{array}$ \\
\hline June-Aug. & $\begin{array}{r}1 \\
7 \\
30\end{array}$ & $\begin{array}{l}11 \\
14 \\
27\end{array}$ & $\begin{array}{r}6.5 \\
8.5 \\
15\end{array}$ & $\begin{array}{r}5.0 \\
6.8 \\
11\end{array}$ & $\begin{array}{l}4.1 \\
5.7 \\
8.9\end{array}$ & $\begin{array}{l}3.2 \\
4.7 \\
7.0\end{array}$ & Mar. - May & $\begin{array}{r}1 \\
7 \\
30\end{array}$ & $\begin{array}{r}50 \\
66 \\
156\end{array}$ & $\begin{array}{l}33 \\
42 \\
91\end{array}$ & $\begin{array}{l}26 \\
33 \\
68\end{array}$ & $\begin{array}{l}22 \\
27 \\
54\end{array}$ & $\begin{array}{l}18 \\
21 \\
41\end{array}$ \\
\hline
\end{tabular}

Duration of daily flow for indicated periods

\begin{tabular}{|c|c|c|c|c|c|c|c|c|c|c|c|c|c|}
\hline \multirow{2}{*}{ Period } & \multicolumn{13}{|c|}{ Discharge $\left(\mathrm{ft}^{3} / \mathrm{s}\right)$ which was equaled or exceeded for indicated percent of time } \\
\hline & 98 & 95 & 90 & 85 & 80 & 75 & 70 & 60 & 50 & 40 & 30 & 20 & 10 \\
\hline $\begin{array}{l}\text { Apr. - Mar. } \\
\text { May - Nov. } \\
\text { June - Aug. } \\
\text { Sept. - Nov. } \\
\text { Dec. - Feb. } \\
\text { Mar. - May }\end{array}$ & $\begin{array}{l}7.0 \\
6.0 \\
7.2 \\
5.0 \\
13 \\
37\end{array}$ & $\begin{array}{l}10 \\
8.3 \\
9.2 \\
6.5 \\
19 \\
54\end{array}$ & $\begin{array}{l}14 \\
11 \\
12 \\
9.4 \\
26 \\
71\end{array}$ & $\begin{array}{l}19 \\
14 \\
13 \\
12 \\
32 \\
88\end{array}$ & $\begin{array}{r}23 \\
16 \\
18 \\
13 \\
41 \\
110\end{array}$ & $\begin{array}{r}28 \\
19 \\
21 \\
15 \\
54 \\
120\end{array}$ & $\begin{array}{r}34 \\
23 \\
24 \\
17 \\
64 \\
140\end{array}$ & $\begin{array}{r}52 \\
30 \\
32 \\
21 \\
93 \\
180\end{array}$ & $\begin{array}{r}79 \\
40 \\
41 \\
27 \\
140 \\
240\end{array}$ & $\begin{array}{r}120 \\
57 \\
54 \\
36 \\
190 \\
320\end{array}$ & $\begin{array}{r}190 \\
83 \\
76 \\
50 \\
280 \\
430\end{array}$ & $\begin{array}{r}320 \\
140 \\
120 \\
75 \\
480 \\
670\end{array}$ & $\begin{array}{r}690 \\
270 \\
260 \\
150 \\
1100 \\
1300\end{array}$ \\
\hline
\end{tabular}


Appendix 3.--Low-flow data--Continued

VERMILION RIVER BASIN

04199300 Vermilion River at Clarksfield, Ohio

LOCATION:

Lat $41^{\circ} 11^{\prime} 45^{\prime \prime}$, long $22^{\circ} 24^{\prime} 5^{\prime \prime}$, Huron County, Hydrologlc Unit 04100012 , at bridge on Zenobia Road at Clarksfield.

DRAINAGE AREA: $\quad 130 \mathrm{mi}^{2}$.

TRIBUTARY TO: Lake Erie.

DISCHARGE DATA USED: Low-flow measurements 1960, 1962-1967, 1970-1971 water years.

INDEX STATION: $\quad 04199500$ Vermilion River near vermilion, Ohio.

REMARKS: $\quad$ None.

SELECTED DISCHARGE CHARACTERISTICS: Minimum discharge observed: No flow October 1963.

\begin{tabular}{l|c|c|c}
\multicolumn{4}{c}{$\begin{array}{c}\text { Magnitude and frequency of low flow for } \\
\text { indicated periods }\end{array}$} \\
\hline & $\begin{array}{c}\text { Number of } \\
\text { consecu- } \\
\text { tive days }\end{array}$ & $\begin{array}{c}\left.\text { Discharge ( } \mathrm{ft}^{3} / \mathrm{s}\right) \text { for } \\
\text { indicated recurrence } \\
\text { interval (years) }\end{array}$ \\
\cline { 3 - 4 } & 7 & 2 & 10 \\
\hline Apr. - Mar. & 30 & 0.2 & 0 \\
May - Nov. & 7 & 0.5 & 0 \\
& 30 & 0.2 & 0 \\
June - Aug. & 7 & 0.5 & 0 \\
& 30 & 1.8 & 0.1 \\
Sept. - Nov. & 7 & 0.3 & 0.4 \\
& 30 & 0.9 & 0.1 \\
\hline
\end{tabular}

Duration of daily flow for indicated periods

\begin{tabular}{l|l|l|l|l|l}
\hline \multirow{2}{*}{ Period } & \multicolumn{5}{|c|}{$\begin{array}{c}\text { Discharge }\left(\mathrm{ft}^{3} / \mathrm{s}\right) \\
\text { exceeded for } \text { wich was equaled or }\end{array}$} \\
\cline { 2 - 6 } & 98 & 95 & 90 & 85 & 80 \\
\hline Apr. - Mar. & 0.1 & 0.3 & 0.5 & 0.9 & 1.5 \\
May - Nov. & 0 & 0.2 & 0.3 & 0.5 & 0.7 \\
June - Aug. & 0.1 & 0.2 & 0.4 & 0.6 & 0.9 \\
Sept. - Nov. & 0 & 0 & 0.2 & 0.3 & 0.4 \\
\hline
\end{tabular}


LOCATION:

Lat $41^{\circ} 22^{\prime} 55^{\prime \prime}$, long $82^{\circ} 19^{\prime} 01^{\prime \prime}$, in T.6 N., R.19 W., Lorain County, Hydrologic Unit 04100012 , on right bank $40 \mathrm{ft}$ downstream from bridge on North Ridge Road, $3.5 \mathrm{mi}$ southeast of Vermilion and $4.5 \mathrm{mi}$ upstream from mouth.

DRAINAGE AREA: $\quad 262 \mathrm{mi}^{2}$.

TRIBUTARY TO: Lake Erie.

DISCHARGE DATA USED: April 1950 to September 1978.

REMARKS:

None

SELECTED DISCHARGE CHARACTERISTICS: Average discharge: $262 \mathrm{ft}^{3} / \mathrm{s}$ (28 years).

Minimum daily discharge: No flow at times in many years.

Magnitude and frequency of low flow for indicated periods

\begin{tabular}{|c|c|c|c|c|c|c|c|c|c|c|c|c|c|}
\hline \multirow{2}{*}{ Period } & \multirow{2}{*}{$\begin{array}{l}\text { Number of } \\
\text { consecu- } \\
\text { tive days }\end{array}$} & \multicolumn{5}{|c|}{$\begin{array}{l}\text { Discharge }\left(\mathrm{ft}^{3} / \mathrm{s}\right) \text { for indicated } \\
\text { recurrence interval (years) }\end{array}$} & \multirow{2}{*}{ Period } & \multirow{2}{*}{$\begin{array}{l}\text { Number of } \\
\text { consecu- } \\
\text { tive days }\end{array}$} & \multicolumn{5}{|c|}{$\begin{array}{l}\text { Discharge }\left(\mathrm{Ft}^{3} / \mathrm{s}\right) \text { for indicated } \\
\text { recurrerice interval (years) }\end{array}$} \\
\hline & & 2 & 5 & 10 & 20 & 50 & & & 2 & 5 & 10 & 20 & 50 \\
\hline Apr.-Mar. & $\begin{array}{r}1 \\
7 \\
30\end{array}$ & $\begin{array}{l}1.1 \\
1.5 \\
3.3\end{array}$ & $\begin{array}{l}0.2 \\
0.3 \\
0.9\end{array}$ & $\begin{array}{l}0 \\
0.1 \\
0.3\end{array}$ & $\begin{array}{l}0 \\
0 \\
0.1\end{array}$ & $\begin{array}{l}0 \\
0 \\
0\end{array}$ & Sept.-Nov. & $\begin{array}{r}1 \\
7 \\
30\end{array}$ & $\begin{array}{l}1.2 \\
1.7 \\
5.3\end{array}$ & $\begin{array}{l}0.2 \\
0.3 \\
1.1\end{array}$ & $\begin{array}{l}0.1 \\
0.1 \\
0.4\end{array}$ & $\begin{array}{l}0 \\
0 \\
0.1\end{array}$ & $\begin{array}{l}0 \\
0 \\
0\end{array}$ \\
\hline June-Aug. & $\begin{array}{r}1 \\
7 \\
30\end{array}$ & $\begin{array}{l}1.9 \\
3.1 \\
11\end{array}$ & $\begin{array}{l}0.5 \\
0.9 \\
3.9\end{array}$ & $\begin{array}{l}0.2 \\
0.4 \\
2.2\end{array}$ & $\begin{array}{l}0.1 \\
0.2 \\
1.4\end{array}$ & $\begin{array}{l}0 \\
0.1 \\
0.8\end{array}$ & Mar. - May & $\begin{array}{r}1 \\
7 \\
30\end{array}$ & $\begin{array}{r}28 \\
38 \\
120\end{array}$ & $\begin{array}{l}17 \\
22 \\
63\end{array}$ & $\begin{array}{l}13 \\
16 \\
44\end{array}$ & $\begin{array}{l}10 \\
13 \\
32\end{array}$ & $\begin{array}{r}8.0 \\
9.9 \\
23\end{array}$ \\
\hline
\end{tabular}

Duration of daily flow for indicated periods

\begin{tabular}{l|c|c|c|c|c|c|c|c|c|c|c|c|c|}
\hline \multirow{2}{*}{ Period } & \multicolumn{8}{|c}{ Discharge $\left(\mathrm{ft}^{3} / \mathrm{s}\right.$ ) which was equaled or exceeded for indicated percent of time } \\
\cline { 2 - 13 } & 98 & 95 & 90 & 85 & 80 & 75 & 70 & 60 & 50 & 40 & 30 & 20 & 10 \\
\hline Apr. - Mar. & 0.6 & 1.6 & 3.2 & 5.6 & 8.7 & 13 & 17 & 31 & 54 & 89 & 150 & 260 & 600 \\
May - Nov. & 0.2 & 1.0 & 1.9 & 2.9 & 4.2 & 5.7 & 7.5 & 13 & 21 & 33 & 52 & 95 & 220 \\
June - Aug. & 0.6 & 1.4 & 2.7 & 3.9 & 5.3 & 6.8 & 8.8 & 13 & 19 & 28 & 43 & 74 & 170 \\
Sept. - Nov. & 0 & 0.3 & 1.2 & 1.8 & 2.5 & 3.1 & 4.1 & 6.7 & 11 & 18 & 32 & 56 & 140 \\
Dec. - Feb. & 5.3 & 9.3 & 14 & 20 & 28 & 39 & 51 & 74 & 110 & 160 & 260 & 470 & 1000 \\
Mar. - May & 19 & 30 & 44 & 57 & 72 & 86 & 100 & 140 & 190 & 260 & 380 & 600 & 1200 \\
\hline
\end{tabular}


Appendix 3.--Low-flow data--Continued

BLACK RIVER BASIN

04200000 East Branch Black River at Elyria, Ohio

LOCATION: Lat $41^{\circ} 20^{\prime} 55^{\prime \prime}$, long $82^{\circ} 05^{\prime} 40^{\prime \prime}$, Lorain County, Hydrologic Unit 04110001 , at Fuller Street Bridge, $1.3 \mathrm{mi}$ southeast of center of Elyria, and $3.0 \mathrm{mi}$ above junction with West Branch.

DRAINAGE AREA: $\quad 217 \mathrm{mi}^{2}$.

TRIBUTARY TO: Head of Black River.

DISCHARGE DATA USED: August 1922 to November 1935.

REMARKS: $\quad$ None.

SELECTED DISCHARGE CHARACTERISTICS: Average discharge: $178 \mathrm{ft}^{3} / \mathrm{s}$ (13 years).

Minimum daily discharge: No flow several days in

August 1930 and in August and September 1933.

Magnitude and frequency of low flow for indicated periods

\begin{tabular}{|c|c|c|c|c|c|c|c|c|c|c|c|c|c|}
\hline \multirow{2}{*}{ Period } & \multirow{2}{*}{$\begin{array}{l}\text { Number of } \\
\text { consecu- } \\
\text { tive days }\end{array}$} & \multicolumn{5}{|c|}{$\begin{array}{l}\text { Discharge }\left(\mathrm{ft}^{3} / \mathrm{s}\right) \text { for indicated } \\
\text { recurrence interval (years) }\end{array}$} & \multirow{2}{*}{ Period } & \multirow{2}{*}{$\begin{array}{l}\text { Number of } \\
\text { consecu- } \\
\text { tive days }\end{array}$} & \multicolumn{5}{|c|}{$\begin{array}{l}\text { Discharge }\left(\mathrm{ft}^{3} / \mathrm{s}\right) \text { for indicated } \\
\text { recurrence interval (years) }\end{array}$} \\
\hline & & 2 & 5 & 10 & 20 & 50 & & & 2 & 5 & 10 & 20 & 50 \\
\hline Apr.-Mar. & $\begin{array}{r}1 \\
7 \\
30\end{array}$ & $\begin{array}{l}0.2 \\
0.4 \\
1.5\end{array}$ & $\begin{array}{l}0.1 \\
0.1 \\
0.3\end{array}$ & $\begin{array}{l}0 \\
0 \\
0.1\end{array}$ & $\begin{array}{l}0 \\
0 \\
0.1\end{array}$ & $\begin{array}{l}0 \\
0 \\
0\end{array}$ & Sept.-Nov. & $\begin{array}{r}1 \\
7 \\
30\end{array}$ & $\begin{array}{l}0.3 \\
0.3 \\
1.8\end{array}$ & $\begin{array}{l}0.1 \\
0.1 \\
0.4\end{array}$ & $\begin{array}{l}0 \\
0.1 \\
0.3\end{array}$ & $\begin{array}{l}0 \\
0 \\
0.2\end{array}$ & $\begin{array}{l}0 \\
0 \\
0.1\end{array}$ \\
\hline June-Aug. & $\begin{array}{r}1 \\
7 \\
30\end{array}$ & $\begin{array}{l}0.4 \\
1.3 \\
3.9\end{array}$ & $\begin{array}{l}0.1 \\
0.2 \\
1.0\end{array}$ & $\begin{array}{l}0 \\
0 \\
0.4\end{array}$ & $\begin{array}{l}0 \\
0 \\
0.2\end{array}$ & $\begin{array}{l}0 \\
0 \\
0.1\end{array}$ & Mar.- May & $\begin{array}{r}1 \\
7 \\
30\end{array}$ & $\begin{array}{l}10 \\
15 \\
45\end{array}$ & $\begin{array}{c}4.1 \\
8.8 \\
20\end{array}$ & $\begin{array}{c}2.3 \\
6.7 \\
14\end{array}$ & $\begin{array}{c}1.3 \\
5.4 \\
11\end{array}$ & $\begin{array}{l}0.7 \\
4.2 \\
8.4\end{array}$ \\
\hline
\end{tabular}

Duration of daily flow for indicated periods

\begin{tabular}{|c|c|c|c|c|c|c|c|c|c|c|c|c|c|c|}
\hline \multirow{2}{*}{\multicolumn{2}{|c|}{ Period }} & \multicolumn{13}{|c|}{ Discharge $\left(\mathrm{ft}^{3} / \mathrm{s}\right)$ which was equaled or exceeded for indicated percent of time } \\
\hline & & 98 & 95 & 90 & 85 & 80 & 75 & 70 & 60 & 50 & 40 & 30 & 20 & 10 \\
\hline $\begin{array}{l}\text { Apr. - } \\
\text { May - } \\
\text { June - } \\
\text { Sept. - } \\
\text { Dec. - } \\
\text { Mar. - }\end{array}$ & $\begin{array}{l}\text { Mar. } \\
\text { Nov. } \\
\text { Aug. } \\
\text { Nov. } \\
\text { Feb. } \\
\text { May }\end{array}$ & $\begin{array}{l}0.2 \\
0.1 \\
0.1 \\
0.1 \\
3.8 \\
8.2\end{array}$ & $\begin{array}{l}0.4 \\
0.3 \\
0.3 \\
0.2 \\
5.4 \\
12\end{array}$ & $\begin{array}{l}1.3 \\
0.6 \\
0.8 \\
0.4 \\
7.4 \\
17\end{array}$ & $\begin{array}{l}2.7 \\
1.1 \\
1.4 \\
0.6 \\
11 \\
22\end{array}$ & $\begin{array}{c}4.1 \\
1.9 \\
2.1 \\
0.9 \\
17 \\
27\end{array}$ & $\begin{array}{l}5.3 \\
2.8 \\
2.9 \\
1.5 \\
22 \\
34\end{array}$ & $\begin{array}{c}6.9 \\
3.7 \\
3.8 \\
2.2 \\
29 \\
42\end{array}$ & $\begin{array}{c}13 \\
5.4 \\
5.1 \\
3.6 \\
41 \\
58\end{array}$ & $\begin{array}{l}26 \\
7.7 \\
6.7 \\
4.9 \\
70 \\
82\end{array}$ & $\begin{array}{c}47 \\
13 \\
9.4 \\
7.4 \\
100 \\
120\end{array}$ & $\begin{array}{r}85 \\
23 \\
15 \\
13 \\
180 \\
190\end{array}$ & $\begin{array}{r}170 \\
51 \\
29 \\
30 \\
340 \\
310\end{array}$ & $\begin{array}{r}460 \\
150 \\
86 \\
120 \\
790 \\
710\end{array}$ \\
\hline
\end{tabular}


BLACK RIVER BASIN

04200500 Black River at Elyria, Ohio

LOCATION:

Lat $41^{\circ} 22^{\prime} 49^{\prime \prime}$, long $82^{\circ} 06^{\prime} 17^{\prime \prime}$, in T.6 N., R.17 W., Lorain County, Hydrologic Unit 04110001 , on left bank in Cascade Park at Elyria, $0.8 \mathrm{mi}$ downstream from confluence of East and West Branches.

DRAINAGE AREA: $\quad 396^{\circ} \mathrm{mi}^{2}$.

TRIBUTARY TO: Lake Erie.

DISCHARGE DATA USED: October 1944 to September 1978.

REMARKS:

Some regulation at low flow for industrial use.

SELECTED DISCHARGE CHARACTERISTICS: Average discharge: $322 \mathrm{ft}^{3} / \mathrm{s}$ (34 years).

Minimum daily discharge: $0.6 \mathrm{ft}^{3} / \mathrm{s}$ october 1945 .

Magnitude and frequency of low flow for indicated periods

\begin{tabular}{|c|c|c|c|c|c|c|c|c|c|c|c|c|c|}
\hline \multirow{2}{*}{ Period } & \multirow{2}{*}{$\begin{array}{l}\text { Number of } \\
\text { consecu- } \\
\text { tive days }\end{array}$} & \multicolumn{5}{|c|}{$\begin{array}{l}\text { Discharge }\left(\mathrm{ft}^{3} / \mathrm{s}\right) \text { for indicated } \\
\text { recurrence interval (years) }\end{array}$} & \multirow{2}{*}{ Period } & \multirow{2}{*}{$\begin{array}{l}\text { Number of } \\
\text { consecu- } \\
\text { tive days }\end{array}$} & \multicolumn{5}{|c|}{$\begin{array}{l}\text { Discharge }\left(\mathrm{ft}^{3} / \mathrm{s}\right) \text { for indicated } \\
\text { recurrence interval (years) }\end{array}$} \\
\hline & & 2 & 5 & 10 & 20 & 50 & & & 2 & 5 & 10 & 20 & 50 \\
\hline Apr.-Mar. & $\begin{array}{r}1 \\
7 \\
30\end{array}$ & $\begin{array}{l}5.0 \\
7.2 \\
9.7\end{array}$ & $\begin{array}{l}3.0 \\
4.7 \\
6.1\end{array}$ & $\begin{array}{l}2.3 \\
3.6 \\
4.5\end{array}$ & $\begin{array}{l}1.6 \\
2.9 \\
4.0\end{array}$ & $\begin{array}{l}1.3 \\
2.2 \\
3.3\end{array}$ & Sept.-Nov. & $\begin{array}{r}1 \\
7 \\
30\end{array}$ & $\begin{array}{l}5.1 \\
7.5 \\
12\end{array}$ & $\begin{array}{l}3.0 \\
4.6 \\
6.4\end{array}$ & $\begin{array}{l}2.2 \\
3.6 \\
5.0\end{array}$ & $\begin{array}{l}1.8 \\
3.0 \\
4.3\end{array}$ & $\begin{array}{l}1.4 \\
2.4 \\
3.7\end{array}$ \\
\hline June-Aug. & $\begin{array}{r}1 \\
7 \\
30\end{array}$ & $\begin{array}{l}7.3 \\
9.6 \\
19\end{array}$ & $\begin{array}{l}4.8 \\
6.4 \\
11\end{array}$ & $\begin{array}{l}3.9 \\
5.3 \\
8.2\end{array}$ & $\begin{array}{l}3.2 \\
4.5 \\
6.6\end{array}$ & $\begin{array}{l}2.7 \\
3.8 \\
5.1\end{array}$ & Mar.- May & $\begin{array}{r}1 \\
7 \\
30\end{array}$ & $\begin{array}{r}35 \\
46 \\
176\end{array}$ & $\begin{array}{l}22 \\
29 \\
90\end{array}$ & $\begin{array}{l}17 \\
23 \\
60\end{array}$ & $\begin{array}{l}14 \\
18 \\
41\end{array}$ & $\begin{array}{l}11 \\
14 \\
26\end{array}$ \\
\hline
\end{tabular}

Duration of daily flow for indicated periods

\begin{tabular}{|c|c|c|c|c|c|c|c|c|c|c|c|c|c|c|}
\hline \multirow{2}{*}{\multicolumn{2}{|c|}{ Period }} & \multicolumn{13}{|c|}{ Discharge $\left(\mathrm{ft}^{3} / \mathrm{s}\right)$ which was equaled or exceeded for indicated percent of time } \\
\hline & & 98 & 95 & 90 & 85 & 80 & 75 & 70 & 60 & 50 & 40 & 30 & 20 & 10 \\
\hline $\begin{array}{l}\text { Apr. - } \\
\text { May - } \\
\text { June - } \\
\text { Sept. - } \\
\text { Dec. - } \\
\text { Mar. - }\end{array}$ & $\begin{array}{l}\text { Mar. } \\
\text { Nov. } \\
\text { Aug. } \\
\text { Nov. } \\
\text { Feb. } \\
\text { May }\end{array}$ & $\begin{array}{l}5.2 \\
4.3 \\
5.3 \\
3.3 \\
6.9 \\
25\end{array}$ & $\begin{array}{l}7.3 \\
5.9 \\
7.3 \\
4.8 \\
11 \\
37\end{array}$ & $\begin{array}{l}9.8 \\
7.9 \\
8.9 \\
6.3 \\
18 \\
55\end{array}$ & $\begin{array}{c}13 \\
9.5 \\
11 \\
7.5 \\
25 \\
73\end{array}$ & $\begin{array}{l}17 \\
11 \\
13 \\
8.7 \\
33 \\
91\end{array}$ & $\begin{array}{c}21 \\
14 \\
15 \\
9.9 \\
43 \\
110\end{array}$ & $\begin{array}{r}25 \\
16 \\
17 \\
11 \\
54 \\
130\end{array}$ & $\begin{array}{r}40 \\
22 \\
23 \\
15 \\
82 \\
180\end{array}$ & $\begin{array}{r}65 \\
30 \\
29 \\
19 \\
120 \\
250\end{array}$ & $\begin{array}{r}110 \\
43 \\
39 \\
26 \\
190 \\
340\end{array}$ & $\begin{array}{r}190 \\
65 \\
55 \\
39 \\
300 \\
500\end{array}$ & $\begin{array}{r}340 \\
120 \\
88 \\
67 \\
540 \\
830\end{array}$ & $\begin{array}{r}800 \\
290 \\
210 \\
180 \\
1200 \\
1600\end{array}$ \\
\hline
\end{tabular}


Appendix 3.--Low-flow data--Continued

ROCKY RIVER BASIN

04201400 West Branch Rocky River at West view, Ohio

LOCATION:

Lat $41^{\circ} 21^{\prime} 00^{\prime \prime}$, long $81^{\circ} 54^{\prime} 15^{\prime \prime}$, Cuyahoga County, Hydrologic

Unit 04110001 , at bridge on State Route 252 at West View.

\author{
DRAINAGE AREA: $\quad 147 \mathrm{mi}^{2}$. \\ TRIBUTARY TO: Head of Rocky River. \\ DISCHARGE DATA USED: Low-flow measurements 1951, 1961, 1962-1967, 1971-1974 \\ water years. \\ INDEX STATION: $\quad 04201500$ Rocky River near Berea, Ohio. \\ REMARKS: $\quad$ None.
}

SELECTED DISCHARGE CHARACTERISTICS: Minimum discharqe observed: $0.3 \mathrm{ft}^{3} / \mathrm{s}$

\begin{tabular}{|c|c|c|c|}
\hline \multirow[t]{2}{*}{ Period } & \multirow{2}{*}{$\begin{array}{l}\text { Number of } \\
\text { consecu- } \\
\text { tive days }\end{array}$} & \multicolumn{2}{|c|}{$\begin{array}{l}\text { Discharge }\left(\mathrm{ft}^{3} / \mathrm{s}\right) \text { for } \\
\text { indicated recurrence } \\
\text { interval (years) }\end{array}$} \\
\hline & & 2 & 10 \\
\hline Apr. - Mar. & $\begin{array}{r}7 \\
30\end{array}$ & $\begin{array}{l}1.7 \\
3.4\end{array}$ & $\begin{array}{l}0.3 \\
0.6\end{array}$ \\
\hline May - Nov. & $\begin{array}{r}7 \\
30\end{array}$ & $\begin{array}{l}1.7 \\
3.4\end{array}$ & $\begin{array}{l}0.3 \\
0.6\end{array}$ \\
\hline June - Aug. & $\begin{array}{r}7 \\
30\end{array}$ & $\begin{array}{l}2.3 \\
6.1\end{array}$ & $\begin{array}{l}0.4 \\
1.1\end{array}$ \\
\hline Sept. - Nov. & $\begin{array}{r}7 \\
30\end{array}$ & $\begin{array}{l}2.3 \\
4.8\end{array}$ & $\begin{array}{l}0.4 \\
0.8\end{array}$ \\
\hline
\end{tabular}

Duration of daily flow for indicated periods

\begin{tabular}{l|c|c|c|c|c}
\hline \multirow{2}{*}{ Period } & \multicolumn{5}{|c}{$\begin{array}{c}\text { Discharge }\left(\mathrm{ft}^{3} / \mathrm{s}\right) \\
\text { exceeded for }\end{array}$ wich was equaled or } \\
\cline { 2 - 6 } & 98 & 95 & 90 & 85 & 80 \\
\hline Apr. - Mar. & 0.7 & 1.5 & 2.6 & 3.9 & 5.7 \\
May - Nov. & 0.5 & 0.9 & 1.7 & 2.4 & 3.1 \\
June - Aug. & 0.4 & 0.9 & 1.7 & 2.3 & 2.9 \\
Sept. - Nov. & 0.4 & 0.8 & 1.4 & 1.9 & 2.5 \\
\hline
\end{tabular}




\section{ROCKY RIVER BASIN \\ 04201500 Rocky River near Berea, Ohio}

LOCATION:

Lat $41^{\circ} 24^{\prime} 24^{\prime \prime}$, long $81^{\circ} 53^{\prime} 14^{\prime \prime}$, in T.6 N., R.15 W., Cuyahoga County Hydrologic Unit 04110001 , on right bank at downstream side of Cedar Point Road Bridge in Rocky River Reservation, just downstream from confluence of East and West Branches, and $3.0 \mathrm{mi}$ northwest of Berea.

DRAINAGE AREA: $\quad 267 \mathrm{mi}^{2}$.

TRIBUTARY TO: Lake Erie.

DISCHARGE DATA USED: November 1923 to September 1935, October 1943 Lo September 1978.

REMARKS: Some regulation at low flow by small reservoirs on East Branch.

SELECTED DISCHARGE CHARACTERISTICS: Average discharge: $257 \mathrm{ft}^{3} / \mathrm{s}$ ( 46 years).

Minimum daily discharge: $0.2 \mathrm{ft}^{3} / \mathrm{s}$ September 1932, August 1933.

Magnitude and frequency of low flow for indicated periods

\begin{tabular}{|c|c|c|c|c|c|c|c|c|c|c|c|c|c|}
\hline \multirow{2}{*}{ Period } & \multirow{2}{*}{$\begin{array}{l}\text { Number of } \\
\text { consecu- } \\
\text { tive days }\end{array}$} & \multicolumn{5}{|c|}{$\begin{array}{l}\text { Discharge }\left(\mathrm{ft}^{3} / \mathrm{s}\right) \text { for indicated } \\
\text { recurrence interval (years) }\end{array}$} & \multirow{2}{*}{ Period } & \multirow{2}{*}{$\begin{array}{l}\text { Number of } \\
\text { consecu- } \\
\text { tive days }\end{array}$} & \multicolumn{5}{|c|}{$\begin{array}{l}\text { Discharge }\left(\mathrm{ft}^{3} / \mathrm{s}\right) \text { for indicated } \\
\text { recurrence interval (years) }\end{array}$} \\
\hline & & 2 & 5 & 10 & 20 & 50 & & & 2 & 5 & 10 & 20 & 50 \\
\hline Apr.-Mar. & $\begin{array}{r}1 \\
7 \\
30\end{array}$ & $\begin{array}{l}4.3 \\
5.5 \\
9.8\end{array}$ & $\begin{array}{l}1.6 \\
2.4 \\
4.1\end{array}$ & $\begin{array}{l}0.9 \\
1.4 \\
2.4\end{array}$ & $\begin{array}{l}0.5 \\
0.8 \\
1.4\end{array}$ & $\begin{array}{l}0.2 \\
0.4 \\
0.7\end{array}$ & Sept.-Nov. & $\begin{array}{r}1 \\
7 \\
30\end{array}$ & $\begin{array}{r}5.6 \\
7.1 \\
13\end{array}$ & $\begin{array}{l}2.1 \\
3.1 \\
4.8\end{array}$ & $\begin{array}{l}1.2 \\
1.8 \\
3.0\end{array}$ & $\begin{array}{l}0.6 \\
1.1 \\
2.1\end{array}$ & $\begin{array}{l}0.3 \\
0.5 \\
1.5\end{array}$ \\
\hline May- Nov. & $\begin{array}{r}1 \\
7 \\
30\end{array}$ & $\begin{array}{l}4.3 \\
5.5 \\
9.8\end{array}$ & $\begin{array}{l}1.6 \\
2.4 \\
4.1\end{array}$ & $\begin{array}{l}0.9 \\
1.4 \\
2.4\end{array}$ & $\begin{array}{l}0.5 \\
0.8 \\
1.4\end{array}$ & $\begin{array}{l}0.2 \\
0.4 \\
0.7\end{array}$ & Dec.-Feb. & $\begin{array}{r}1 \\
7 \\
30\end{array}$ & $\begin{array}{l}31 \\
36 \\
92\end{array}$ & $\begin{array}{l}16 \\
19 \\
38\end{array}$ & $\begin{array}{l}11 \\
14 \\
23\end{array}$ & $\begin{array}{l}8.1 \\
10 \\
16\end{array}$ & $\begin{array}{l}5.7 \\
7.4 \\
9.6\end{array}$ \\
\hline June-Aug. & $\begin{array}{r}1 \\
7 \\
30\end{array}$ & $\begin{array}{l}5.2 \\
7.1 \\
16\end{array}$ & $\begin{array}{l}2.1 \\
2.8 \\
6.6\end{array}$ & $\begin{array}{l}1.2 \\
1.6 \\
3.9\end{array}$ & $\begin{array}{l}0.6 \\
0.9 \\
2.5\end{array}$ & $\begin{array}{l}0.3 \\
0.4 \\
1.4\end{array}$ & Mar. - May & $\begin{array}{r}1 \\
7 \\
30\end{array}$ & $\begin{array}{r}33 \\
44 \\
149\end{array}$ & $\begin{array}{l}18 \\
25 \\
77\end{array}$ & $\begin{array}{l}13 \\
19 \\
52\end{array}$ & $\begin{array}{l}9.5 \\
14 \\
37\end{array}$ & $\begin{array}{l}6.4 \\
11 \\
24\end{array}$ \\
\hline
\end{tabular}

Duration of daily flow for indicated periods

\begin{tabular}{l|c|c|c|c|c|c|c|c|c|r|r|r|r}
\hline \multirow{2}{*}{ Period } & \multicolumn{8}{|c|}{ Discharge $\left(\mathrm{ft}^{3} / \mathrm{s}\right)$ which was equaled or exceeded for indicated percent of time } \\
\cline { 2 - 12 } & 98 & 95 & 90 & 85 & 80 & 75 & 70 & 60 & 50 & 40 & 30 & 20 & 10 \\
\hline Apr. - Mar. & 2.8 & 5.0 & 7.9 & 11 & 15 & 20 & 25 & 42 & 66 & 110 & 170 & 290 & 630 \\
May - Nov. & 2.0 & 3.5 & 5.6 & 7.3 & 9.2 & 11 & 14 & 20 & 29 & 43 & 65 & 110 & 260 \\
June - Aug. & 1.8 & 3.5 & 5.5 & 7.1 & 8.7 & 11 & 13 & 17 & 24 & 33 & 47 & 77 & 170 \\
Sept. - Nov. & 1.6 & 3.0 & 4.7 & 6.2 & 7.6 & 9.3 & 11 & 16 & 22 & 32 & 52 & 91 & 220 \\
Dec. - Feb. & 13 & 18 & 25 & 34 & 44 & 53 & 61 & 92 & 130 & 170 & 250 & 420 & 910 \\
Mar. - May & 23 & 33 & 49 & 62 & 77 & 92 & 110 & 150 & 210 & 280 & 390 & 600 & 1100 \\
\hline
\end{tabular}




\section{CUYAHOGA RIVER BASIN}

04202000 Cuyahoga River at Hiram Rapids, Ohio

LOCATION:

Lat $41^{\circ} 20^{\prime} 26^{\prime \prime}$, long $81^{\circ} 10^{\prime} 01^{\prime \prime}$, in T.5 N., K.7 W., Portage County

Hydrologic Unit 04110002 , on left bank at downstream side of bridge

on Winchell Road at Hiram Rapids, $0.6 \mathrm{mi}$ downstream from Black Brook.

DRAINAGE AREA: $\quad 151 \mathrm{mi}$.

TRIBUTARY TO:

Lake Erie.

DISCHARGE DATA USED:

October 1961 to September 1978.

REMARKS:

Flow regulated by East Branch Reservoir, usat ie capacity 4,140 acre-ft

$14.6 \mathrm{mi}$ upstream since 1939 and by LaDue Reservoir, usable capacity

18,110 acre-ft, $9.8 \mathrm{mi}$ upstream since 1961 .

SELECTED DISCHARGE CHARACTERISTICS: Averaye discharge: $213 \mathrm{ft}^{3} / \mathrm{s}$ (17 years).

Minimum daily discharge: $12 \mathrm{ft} 3 / \mathrm{s}$ september 1967.

Magnitude and frequency of low flow for indicated periods

\begin{tabular}{|c|c|c|c|c|c|c|c|c|c|c|c|c|c|}
\hline \multirow{2}{*}{ Period } & \multirow{2}{*}{$\begin{array}{l}\text { Number of } \\
\text { consecu- } \\
\text { tive days }\end{array}$} & \multicolumn{5}{|c|}{$\begin{array}{l}\text { Discharge }\left(\mathrm{ft}^{3} / \mathrm{s}\right) \text { for indicated } \\
\text { recurrence interval (years) }\end{array}$} & \multirow{2}{*}{ Period } & \multirow{2}{*}{$\begin{array}{l}\text { Number of } \\
\text { consecu- } \\
\text { tive days }\end{array}$} & \multicolumn{5}{|c|}{$\begin{array}{l}\text { Discharge }\left(\mathrm{ft}^{3} / \mathrm{s}\right) \text { for indicated } \\
\text { recurrence interval (years) }\end{array}$} \\
\hline & & 2 & 5 & 10 & 20 & 50 & & & 2 & 5 & 10 & 20 & 50 \\
\hline Apr.-Mar. & $\begin{array}{r}1 \\
7 \\
30\end{array}$ & $\begin{array}{l}20 \\
25 \\
45\end{array}$ & $\begin{array}{l}15 \\
18 \\
35\end{array}$ & $\begin{array}{l}13 \\
16 \\
31\end{array}$ & $\begin{array}{l}12 \\
13 \\
27\end{array}$ & $\begin{array}{l}11 \\
11 \\
23\end{array}$ & Sept.-Nov. & $\begin{array}{r}1 \\
7 \\
30\end{array}$ & $\begin{array}{l}23 \\
27 \\
56\end{array}$ & $\begin{array}{l}15 \\
18 \\
38\end{array}$ & $\begin{array}{l}13 \\
15 \\
31\end{array}$ & $\begin{array}{l}11 \\
13 \\
26\end{array}$ & $\begin{array}{l}9.6 \\
11 \\
22\end{array}$ \\
\hline June-Aug. & $\begin{array}{r}1 \\
7 \\
30\end{array}$ & $\begin{array}{l}24 \\
33 \\
58\end{array}$ & $\begin{array}{l}19 \\
26 \\
50\end{array}$ & $\begin{array}{l}17 \\
23 \\
46\end{array}$ & $\begin{array}{l}15 \\
21 \\
43\end{array}$ & $\begin{array}{l}13 \\
18 \\
39\end{array}$ & Mar. - May & $\begin{array}{r}1 \\
7 \\
30\end{array}$ & $\begin{array}{r}53 \\
73 \\
143\end{array}$ & $\begin{array}{l}40 \\
53 \\
99\end{array}$ & $\begin{array}{l}35 \\
45 \\
82\end{array}$ & $\begin{array}{l}31 \\
40 \\
70\end{array}$ & $\begin{array}{l}28 \\
35 \\
59\end{array}$ \\
\hline
\end{tabular}

Duration of daily flow for indicated periods

\begin{tabular}{l|c|c|c|r|r|r|r|r|r|r|r|r|r}
\hline \multirow{2}{*}{ Period } & \multicolumn{8}{|c|}{ Discharge $\left(\mathrm{ft}^{3} / \mathrm{s}\right)$ which was equaled or exceeded for indicated percent of tine } \\
\cline { 2 - 12 } & 98 & 95 & 90 & 85 & 80 & 75 & 70 & 60 & 50 & 40 & 30 & 20 & 10 \\
\hline Apr. - Mar. & 23 & 32 & 44 & 51 & 60 & 69 & 77 & 95 & 120 & 160 & 210 & 310 & 510 \\
May - Nov. & 20 & 26 & 36 & 43 & 48 & 53 & 59 & 71 & 82 & 97 & 120 & 150 & 240 \\
June - Aug. & 24 & 29 & 36 & 42 & 47 & 51 & 55 & 64 & 72 & 80 & 89 & 110 & 150 \\
Sept.- Nov. & 17 & 21 & 30 & 39 & 45 & 51 & 58 & 73 & 89 & 100 & 130 & 160 & 240 \\
Dec. - Feb. & 42 & 53 & 67 & 82 & 97 & 110 & 120 & 150 & 190 & 230 & 300 & 400 & 610 \\
Mar. - May & 49 & 65 & 87 & 110 & 120 & 140 & 160 & 200 & 250 & 310 & 410 & 530 & 720 \\
\hline
\end{tabular}




\section{CUYAHOGA RIVER BASIN}

04204000 Little Cuyahoga River at Mogadore, Ohio

LOCATION:

Lat $41^{\circ} 03^{\prime} 47^{\prime \prime}$, long $81^{\circ} 23^{\prime} 38^{\prime \prime}$, in T.1 N., R.10 W., Summit County, Hydrologic Unit 04110002 , on left bank at upstream side of bridge on State Route $532,500 \mathrm{ft}$ downstream from Mogadore Reservoir, $0.8 \mathrm{mi}$ upstream from Wingfoot Lake Outlet, and $0.8 \mathrm{mi}$ north of Mogadore.

DRAINAGE AREA: $\quad 17.3 \mathrm{mi}^{2}$.

TRIBUTARY TO: CuYahoga River.

DISCHARGE DATA USED: February 1946 to September 1978.

REMARKS: Records fair. Flow regulated by Mogadore Reservoir, usable capacity, 6,540 acre-ft.

SELECTED DISCHARGE CHARACTERISTICS: Average discharge: $14.6 \mathrm{ft}^{3} / \mathrm{s}$ (32 years). Minimum daily discharge: $0.1 \mathrm{ft}^{3} / \mathrm{s}$ october 1967.

Magnitude and frequency of low flow for indicated periods

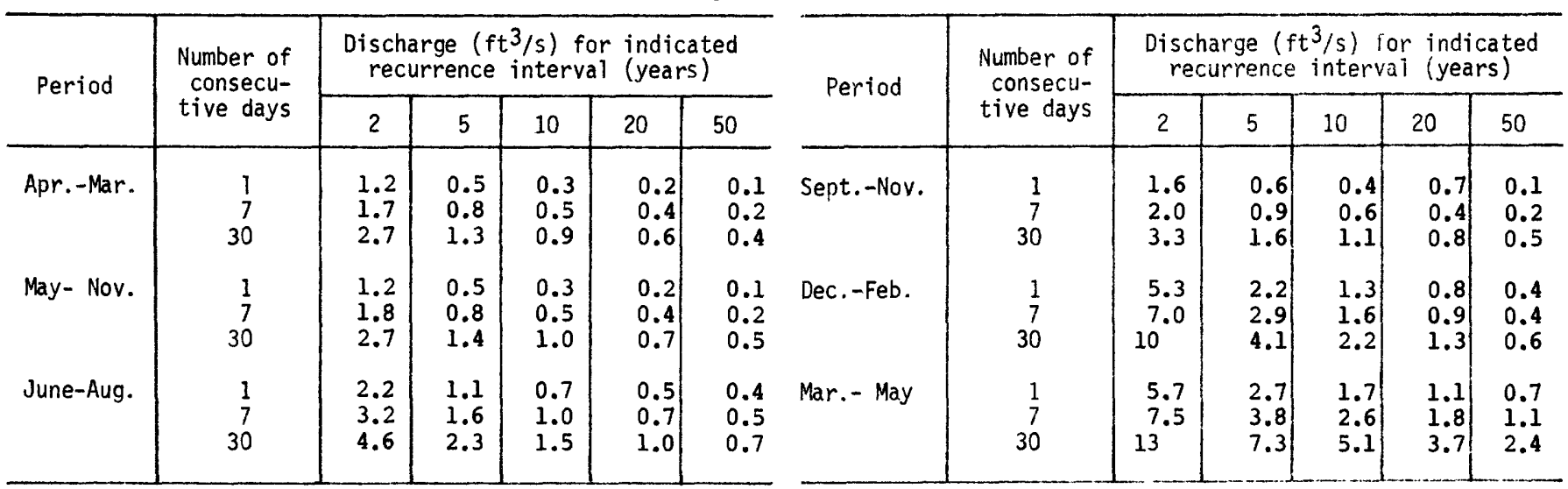

Duration of daily flow for indicated periods

\begin{tabular}{l|c|c|c|c|c|c|c|c|c|c|c|c|c}
\hline \multirow{2}{*}{ Period } & \multicolumn{8}{|c|}{ Discharge $\left(\mathrm{ft}^{3} / \mathrm{s}\right)$ which was equaled or exceeded for indicated percent of time } \\
\cline { 2 - 14 } & 98 & 95 & 90 & 85 & 80 & 75 & 70 & 60 & 50 & 40 & 30 & 20 & 10 \\
\hline Apr. - Mar. & 1.0 & 1.4 & 2.0 & 2.7 & 3.8 & 4.8 & 5.8 & 8.3 & 10 & 13 & 18 & 23 & 33 \\
May - Nov. & 0.9 & 1.2 & 1.6 & 2.1 & 2.5 & 3.2 & 3.9 & 5.4 & 7.1 & 9.1 & 12 & 15 & 23 \\
June - Aug. & 1.1 & 1.4 & 1.9 & 2.4 & 2.9 & 3.6 & 4.2 & 5.5 & 7.0 & 8.9 & 11 & 15 & 22 \\
Sept. - Nov. & 0.7 & 1.0 & 1.3 & 1.6 & 1.9 & 22 & 2.6 & 3.8 & 5.4 & 7.2 & 9.3 & 12 & 16 \\
Dec. - Feb. & 1.2 & 1.7 & 3.9 & 5.4 & 7.4 & 8.6 & 9.2 & 11 & 14 & 17 & 22 & 27 & 38 \\
Mar. - May & 2.8 & 4.8 & 6.9 & 8.5 & 9.6 & 11 & 13 & 16 & 20 & 23 & 20 & 34 & 44 \\
\hline
\end{tabular}


LOCATION:

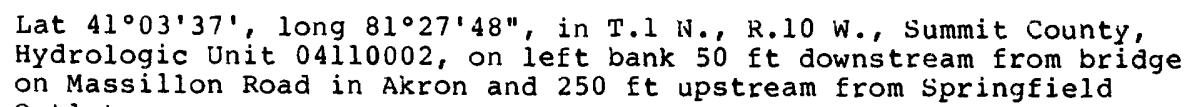

DRAINAGE AREA: $\quad 31.6 \mathrm{mi}^{2}$.

TRIBUTARY TO: CuYahoga River.

DISCHARGE DATA USED: February 1946 to September 1974.

REMARKS :

Flow regulated by Mogadore Reservoir $4.5 \mathrm{mi}$ upstream, usable capacity 6.540 acre-ft and Wingfoot Lake $7.2 \mathrm{mi}$ upstream.

SELECTED DISCHARGE CHARACTERISTICS: Average discharge: $27.6 \mathrm{ft}^{3} / \mathrm{s}$ (28 years).

Minimum daily discharge: $3.1 \mathrm{ft} / \mathrm{s}$ September 1962 , september 1 . 60 .

Magnitude and frequency of low flow for indicated periods

\begin{tabular}{|c|c|c|c|c|c|c|c|c|c|c|c|c|c|}
\hline \multirow{2}{*}{ Period } & \multirow{2}{*}{$\begin{array}{l}\text { Number of } \\
\text { consecu- } \\
\text { tive days }\end{array}$} & \multicolumn{5}{|c|}{$\begin{array}{l}\text { Discharge }\left(\mathrm{ft}^{3} / \mathrm{s}\right) \text { for indicated } \\
\text { recurrence interval (years) }\end{array}$} & \multirow{2}{*}{ Period } & \multirow{2}{*}{$\begin{array}{l}\text { Number of } \\
\text { consecu- } \\
\text { tive days }\end{array}$} & \multicolumn{5}{|c|}{$\begin{array}{l}\text { Discharge }\left(\mathrm{ft}^{3} / \mathrm{s}\right) \text { for indicated } \\
\text { recurrence interval (years) }\end{array}$} \\
\hline & & 2 & 5 & 10 & 20 & 50 & & & 2 & 5 & 10 & 20 & 50 \\
\hline Apr.-Mar. & $\begin{array}{r}1 \\
7 \\
30\end{array}$ & $\begin{array}{l}5.7 \\
6.3 \\
7.9\end{array}$ & $\begin{array}{l}4.1 \\
4.6 \\
5.8\end{array}$ & $\begin{array}{l}3.5 \\
3.9 \\
4.9\end{array}$ & $\begin{array}{l}3.1 \\
3.4 \\
4.3\end{array}$ & $\begin{array}{l}2.7 \\
3.0 \\
3.8\end{array}$ & Sept.-Nov. & $\begin{array}{r}1 \\
7 \\
30\end{array}$ & $\begin{array}{l}6.0 \\
6.8 \\
8.8\end{array}$ & $\begin{array}{l}4.4 \\
5.0 \\
6.4\end{array}$ & $\begin{array}{l}3.8 \\
4.3 \\
5.5\end{array}$ & $\begin{array}{l}3.4 \\
3.7 \\
4.9\end{array}$ & $\begin{array}{l}2.9 \\
3.3 \\
4.4\end{array}$ \\
\hline June-Aug. & $\begin{array}{r}1 \\
7 \\
30\end{array}$ & $\begin{array}{l}7.2 \\
8.4 \\
11\end{array}$ & $\begin{array}{l}4.9 \\
5.6 \\
7.5\end{array}$ & $\begin{array}{l}4.0 \\
4.5 \\
6.0\end{array}$ & $\begin{array}{l}3.4 \\
3.7 \\
5.1\end{array}$ & $\begin{array}{l}2.8 \\
3.0 \\
4.1\end{array}$ & Mar. - May & $\begin{array}{r}1 \\
3 \\
30\end{array}$ & $\begin{array}{l}14 \\
17 \\
25\end{array}$ & $\begin{array}{l}10 \\
12 \\
18\end{array}$ & $\begin{array}{l}8.8 \\
10 \\
15\end{array}$ & $\begin{array}{r}7.9 \\
9.2 \\
13\end{array}$ & $\begin{array}{l}7.0 \\
8.0 \\
11\end{array}$ \\
\hline
\end{tabular}

Duration of daily flow for indicated periods

\begin{tabular}{|c|c|c|c|c|c|c|c|c|c|c|c|c|c|}
\hline \multirow{2}{*}{ Period } & \multicolumn{13}{|c|}{ Discharge $\left(\mathrm{ft}^{3} / \mathrm{s}\right)$ which was equaled or exceeded for indicated percent of time } \\
\hline & 98 & 95 & 90 & 85 & 80 & 75 & 70 & 60 & 50 & 40 & 30 & 20 & 10 \\
\hline $\begin{array}{l}\text { Apr. - Mar. } \\
\text { May - Nov. } \\
\text { June - Aug. } \\
\text { Sept. - Nov. } \\
\text { Dec. - Feb. } \\
\text { Mar. - May }\end{array}$ & $\begin{array}{r}4.6 \\
4.4 \\
4.3 \\
4.2 \\
5.4 \\
12\end{array}$ & $\begin{array}{r}5.8 \\
5.2 \\
5.2 \\
4.9 \\
7.4 \\
14\end{array}$ & $\begin{array}{l}7.0 \\
6.1 \\
6.3 \\
5.8 \\
10 \\
17\end{array}$ & $\begin{array}{l}8.5 \\
6.9 \\
7.2 \\
6.3 \\
12 \\
19\end{array}$ & $\begin{array}{l}10 \\
8.0 \\
8.3 \\
6.8 \\
13 \\
21\end{array}$ & $\begin{array}{l}12 \\
9.0 \\
9.5 \\
7.6 \\
15 \\
24\end{array}$ & $\begin{array}{l}13 \\
10 \\
11 \\
8.4 \\
16 \\
26\end{array}$ & $\begin{array}{l}16 \\
12 \\
13 \\
10 \\
20 \\
32\end{array}$ & $\begin{array}{l}19 \\
15 \\
15 \\
12 \\
23 \\
38\end{array}$ & $\begin{array}{l}24 \\
17 \\
18 \\
14 \\
28 \\
44\end{array}$ & $\begin{array}{l}30 \\
21 \\
22 \\
16 \\
35 \\
51\end{array}$ & $\begin{array}{l}41 \\
28 \\
27 \\
20 \\
45 \\
60\end{array}$ & $\begin{array}{l}57 \\
41 \\
38 \\
28 \\
64 \\
75\end{array}$ \\
\hline
\end{tabular}


CUYAHOGA RIVER BASIN

04205000 Springfield Lake outlet at Akron, Ohio

LOCATION: Lat $41^{\circ} 03^{\prime} 21^{\prime \prime}$, long $81^{\circ} 27^{\prime} 52^{\prime \prime}$, in T.1 N., R.10 W., Summit County Hydrologic Unit 04110002 , on right bank $3.0 \mathrm{mi}$ downstream from springfield Lake in Akron, and $0.3 \mathrm{mi}$ upstream from mouth.

DRAINAGE AREA: $\quad 9.72 \mathrm{mi}^{2}$.

TRIBUTARY TO: Little Cuyahoga River.

DISCHARGE DATA LSED: June 1946 to September 1949, October 1960 to September 1974.

REMARKS:

Flow regulated by Springfield Lake.

SELECTED DISCHARGE CHARACTERISTICS: Average discharge: $4.85 \mathrm{ft}^{3} / \mathrm{s}$ ( 17 years).

Minimum daily discharge: No flow at times in 1961-1967, 1970.

Magnitude and frequency of low flow for indicated periods

\begin{tabular}{c|c|c|c|c|c|c}
\hline \multirow{2}{*}{ Period } & \multirow{2}{*}{$\begin{array}{c}\text { Number of } \\
\text { consecu- } \\
\text { tive days }\end{array}$} & \multicolumn{4}{|c}{$\begin{array}{c}\text { Discharge }\left(\mathrm{ft}^{3} / \mathrm{s} \text { ) for indicated }\right. \\
\text { recurrence interval }\end{array}$} \\
\cline { 3 - 7 } (years)
\end{tabular}

\begin{tabular}{|c|c|c|c|c|c|c|}
\hline \multirow{2}{*}{ Period } & \multirow{2}{*}{$\begin{array}{l}\text { Number of } \\
\text { consecu- } \\
\text { tive days }\end{array}$} & \multicolumn{5}{|c|}{$\begin{array}{l}\left.\text { Discharge ( } \mathrm{ft}^{3} / \mathrm{s}\right) \text { for indicated } \\
\text { recurrence interva } 7 \text { (years) }\end{array}$} \\
\hline & & 2 & 5 & 10 & 20 & 50 \\
\hline Sept.-Nov. & $\begin{array}{r}1 \\
7 \\
30\end{array}$ & $\begin{array}{l}0.3 \\
0.4 \\
0.8\end{array}$ & $\begin{array}{l}0 \\
0 \\
0.2\end{array}$ & $\begin{array}{l}0 \\
0 \\
0.1\end{array}$ & $\begin{array}{l}0 \\
0 \\
0\end{array}$ & $\begin{array}{l}0 \\
0 \\
0\end{array}$ \\
\hline Dec.-Feb. & $\begin{array}{r}1 \\
7 \\
30\end{array}$ & $\begin{array}{l}0.5 \\
0.7 \\
1.2\end{array}$ & $\begin{array}{l}0 \\
0.1 \\
0.3\end{array}$ & $\begin{array}{l}0 \\
0 \\
0.1\end{array}$ & $\begin{array}{l}0 \\
0 \\
0\end{array}$ & $\begin{array}{l}0 \\
0 \\
0\end{array}$ \\
\hline Mar.- May & $\begin{array}{r}1 \\
7 \\
30\end{array}$ & $\begin{array}{l}2.0 \\
2.6 \\
4.8\end{array}$ & $\begin{array}{l}1.0 \\
1.4 \\
2.8\end{array}$ & $\begin{array}{l}0.7 \\
1.1 \\
2.1\end{array}$ & $\begin{array}{l}0.5 \\
0.9 \\
1.6\end{array}$ & $\begin{array}{l}0.3 \\
0.7 \\
1.2\end{array}$ \\
\hline
\end{tabular}

Duration of daily flow for indicated periods

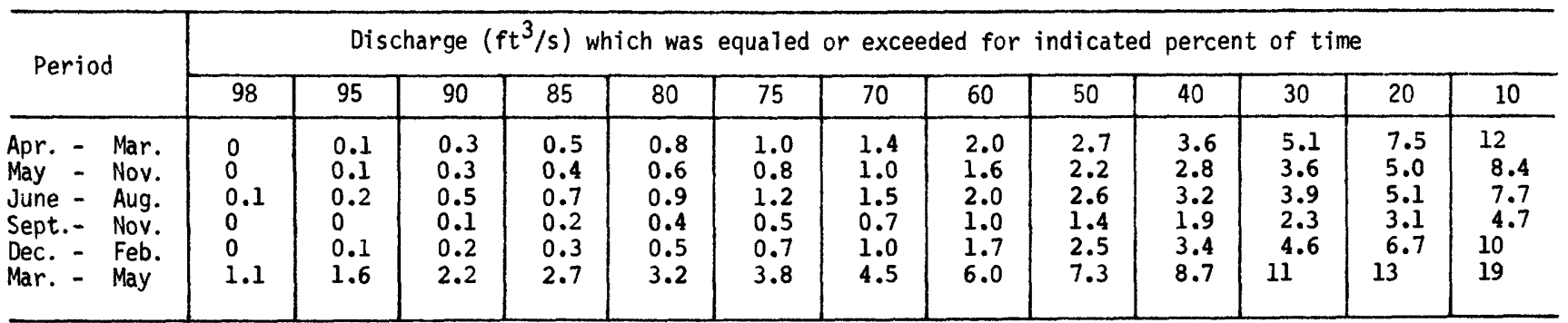


LOCATION:

DRAINAGE AREA:

TRIBITARY TO:

DISCHARGE DATA USED: October 1939 to september 1978.

REMARKS:

Natural flow of stream affected by diversions, storage reservoirs and power pląnts. At Lake Rockwell, $17.7 \mathrm{mi}$ upstream from gage, an average of $78 \mathrm{ft} / \mathrm{s}$ was diverted for municipal supply of city of Akron. Sewage from city enters river $2.9 \mathrm{mi}$ downstream from station. Some diversion from the Tuscarawas drainage into this basin at Portage Lakes.

SELECTED DISCHARGE CHARACTERISTICS: Average discharge: $415 \mathrm{ft}^{3} / \mathrm{s}$ (39 years). Minimum daily discharge: $24 \mathrm{ft} / \mathrm{s}$ September 1964.

Magnitude and frequency of low flow for indicated pericds

\begin{tabular}{|c|c|c|c|c|c|c|}
\hline \multirow{2}{*}{ Period } & \multirow{2}{*}{$\begin{array}{l}\text { Number of } \\
\text { consecu- } \\
\text { tive days }\end{array}$} & \multicolumn{5}{|c|}{$\begin{array}{l}\text { Discharge }\left(\mathrm{ft}^{3} / \mathrm{s}\right) \text { for indicated } \\
\text { recurrence interval (years) }\end{array}$} \\
\hline & & 2 & 5 & 10 & 20 & 50 \\
\hline Apr.-Mar. & $\begin{array}{r}1 \\
7 \\
30\end{array}$ & $\begin{array}{l}52 \\
65 \\
82\end{array}$ & $\begin{array}{l}36 \\
50 \\
61\end{array}$ & $\begin{array}{l}30 \\
44 \\
52\end{array}$ & $\begin{array}{l}26 \\
40 \\
47\end{array}$ & $\begin{array}{l}22 \\
36 \\
41\end{array}$ \\
\hline May- Nov. & $\begin{array}{r}1 \\
7 \\
30\end{array}$ & $\begin{array}{l}53 \\
66 \\
83\end{array}$ & $\begin{array}{l}37 \\
50 \\
61\end{array}$ & $\begin{array}{l}30 \\
44 \\
53\end{array}$ & $\begin{array}{l}26 \\
40 \\
47\end{array}$ & $\begin{array}{l}22 \\
36 \\
42\end{array}$ \\
\hline June-Aug. & $\begin{array}{r}1 \\
7 \\
30\end{array}$ & $\begin{array}{r}65 \\
80 \\
108\end{array}$ & $\begin{array}{l}45 \\
58 \\
75\end{array}$ & $\begin{array}{l}37 \\
50 \\
63\end{array}$ & $\begin{array}{l}31 \\
44 \\
54\end{array}$ & $\begin{array}{l}26 \\
38 \\
46\end{array}$ \\
\hline
\end{tabular}

\begin{tabular}{|c|c|c|c|c|c|c|}
\hline \multirow{2}{*}{ Period } & \multirow{2}{*}{$\begin{array}{l}\text { Number of } \\
\text { consecu- } \\
\text { tive days }\end{array}$} & \multicolumn{5}{|c|}{$\begin{array}{l}\text { Discharge }\left(\mathrm{ft}^{3} / \mathrm{s}\right) \text { for indicated } \\
\text { recurrence interval (years) }\end{array}$} \\
\hline & & 2 & 5 & 10 & 20 & 50 \\
\hline Sept.-Nov. & $\begin{array}{r}1 \\
7 \\
30\end{array}$ & $\begin{array}{l}55 \\
69 \\
95\end{array}$ & $\begin{array}{l}37 \\
51 \\
65\end{array}$ & $\begin{array}{l}31 \\
45 \\
55\end{array}$ & $\begin{array}{l}27 \\
42 \\
49\end{array}$ & $\begin{array}{l}24 \\
38 \\
43\end{array}$ \\
\hline Dec. -Feb. & $\begin{array}{r}1 \\
7 \\
30\end{array}$ & $\begin{array}{l}112 \\
135 \\
218\end{array}$ & $\begin{array}{r}63 \\
81 \\
116\end{array}$ & $\begin{array}{l}47 \\
63 \\
83\end{array}$ & $\begin{array}{l}37 \\
51 \\
63\end{array}$ & $\begin{array}{l}28 \\
41 \\
46\end{array}$ \\
\hline Mar. - May & $\begin{array}{r}1 \\
7 \\
30\end{array}$ & $\begin{array}{l}139 \\
170 \\
350\end{array}$ & $\begin{array}{r}91 \\
119 \\
235\end{array}$ & $\begin{array}{r}72 \\
100 \\
184\end{array}$ & $\begin{array}{r}59 \\
87 \\
149\end{array}$ & $\begin{array}{r}47 \\
76 \\
114\end{array}$ \\
\hline
\end{tabular}

Duration of daily flow for indicated periods

\begin{tabular}{|c|c|c|c|c|c|c|c|c|c|c|c|c|c|c|}
\hline \multirow{2}{*}{\multicolumn{2}{|c|}{ Period }} & \multicolumn{13}{|c|}{ Discharge $\left(\mathrm{ft}^{3} / \mathrm{s}\right)$ which was equaled or exceeded for indicated percent of time } \\
\hline & & 98 & 95 & 90 & 85 & 80 & 75 & 70 & 60 & 50 & 40 & 30 & 20 & 10 \\
\hline $\begin{array}{l}\text { Apr. - } \\
\text { May - } \\
\text { June - } \\
\text { Sept. - } \\
\text { Dec. - } \\
\text { Mar. - }\end{array}$ & $\begin{array}{l}\text { Mar. } \\
\text { Nov. } \\
\text { Aug. } \\
\text { Nov. } \\
\text { Feb. } \\
\text { May }\end{array}$ & $\begin{array}{r}48 \\
46 \\
47 \\
44 \\
53 \\
100\end{array}$ & $\begin{array}{r}57 \\
54 \\
54 \\
52 \\
65 \\
140\end{array}$ & $\begin{array}{r}71 \\
62 \\
62 \\
58 \\
93 \\
190\end{array}$ & $\begin{array}{r}85 \\
72 \\
74 \\
64 \\
120 \\
240\end{array}$ & $\begin{array}{r}99 \\
81 \\
83 \\
71 \\
140 \\
290\end{array}$ & $\begin{array}{r}120 \\
91 \\
92 \\
78 \\
160 \\
340\end{array}$ & $\begin{array}{r}130 \\
100 \\
100 \\
86 \\
190 \\
380\end{array}$ & $\begin{array}{l}180 \\
120 \\
120 \\
100 \\
260 \\
470\end{array}$ & $\begin{array}{l}240 \\
150 \\
150 \\
130 \\
350 \\
570\end{array}$ & $\begin{array}{l}340 \\
200 \\
180 \\
160 \\
450 \\
690\end{array}$ & $\begin{array}{l}460 \\
260 \\
220 \\
210 \\
590 \\
830\end{array}$ & $\begin{array}{r}650 \\
370 \\
310 \\
290 \\
790 \\
1100\end{array}$ & $\begin{array}{r}970 \\
570 \\
470 \\
460 \\
1200 \\
1400\end{array}$ \\
\hline
\end{tabular}


CUYAHOGA RIVER BASIN

04207200 Tinkers Creek at Bedford, Ohio

LOCATION: Lat $41^{\circ} 23^{\prime} 04^{\prime \prime}$, long $81^{\circ} 31^{\prime} 39^{\prime \prime}$, in T.6 N., R.ll W., Cuyahoga County, Hydrologic Unit 04110002 , on left bank at downstream side of bridge on state Route 1.4 in Bedford, $5.5 \mathrm{mi}$ upstream from mouth.

DRAINAGE AREA:

$83.9 \mathrm{mi}^{2}$.

TRIBUTARY TO:

Cuyahoga River.

DISCHARGE DATA USED:

December 1962 to september 1978 .

REMARKS:

None.

SELECTED DISCHARGE CHARACTERISTICS: Average discharge: $125 \mathrm{ft}^{3} / \mathrm{s}(15$ years $)$.

Minimum daily discharge: $5.8 \mathrm{ft} / \mathrm{s}$ August 1964.

Magnitude and frequency of low flow for indicated periods

\begin{tabular}{|c|c|c|c|c|c|c|c|c|c|c|c|c|c|}
\hline \multirow{2}{*}{ Period } & \multirow{2}{*}{$\begin{array}{l}\text { Number of } \\
\text { consecu- } \\
\text { tive days }\end{array}$} & \multicolumn{5}{|c|}{$\begin{array}{l}\text { Discharge }\left(\mathrm{ft}^{3} / \mathrm{s}\right) \text { for indicated } \\
\text { recurrence interval (years) }\end{array}$} & \multirow{2}{*}{ Period } & \multirow{2}{*}{$\begin{array}{l}\text { Number of } \\
\text { consecu- } \\
\text { tive days }\end{array}$} & \multicolumn{5}{|c|}{$\begin{array}{l}\text { Discharge }\left(\mathrm{ft}^{3} / \mathrm{s}\right) \text { for indicated } \\
\text { recurrence interval (years) }\end{array}$} \\
\hline & & 2 & 5 & 10 & 20 & 50 & & & 2 & 5 & 10 & 20 & 50 \\
\hline Apr.-Mar. & $\begin{array}{r}1 \\
7 \\
30\end{array}$ & $\begin{array}{l}12 \\
13 \\
20\end{array}$ & $\begin{array}{r}8.3 \\
9.2 \\
13\end{array}$ & $\begin{array}{l}6.8 \\
7.5 \\
9.9\end{array}$ & $\begin{array}{l}5.7 \\
6.3 \\
7.8\end{array}$ & $\begin{array}{l}4.7 \\
5.2 \\
6.0\end{array}$ & Sept.-Nov. & $\begin{array}{r}1 \\
7 \\
30\end{array}$ & $\begin{array}{l}12 \\
15 \\
29\end{array}$ & $\begin{array}{r}8.3 \\
9.8 \\
15\end{array}$ & $\begin{array}{r}6.8 \\
7.7 \\
11\end{array}$ & $\begin{array}{l}5.8 \\
6.3 \\
7.7\end{array}$ & $\begin{array}{l}4.9 \\
5.0 \\
5.2\end{array}$ \\
\hline June-Aug. & $\begin{array}{r}1 \\
7 \\
30\end{array}$ & $\begin{array}{l}12 \\
14 \\
24\end{array}$ & $\begin{array}{l}8.7 \\
10 \\
16\end{array}$ & $\begin{array}{r}7.1 \\
8.5 \\
12\end{array}$ & $\begin{array}{l}5.9 \\
7.3 \\
9.9\end{array}$ & $\begin{array}{l}4.7 \\
6.2 \\
7.7\end{array}$ & Mar. - May & $\begin{array}{r}1 \\
7 \\
30\end{array}$ & $\begin{array}{l}22 \\
27 \\
69\end{array}$ & $\begin{array}{l}16 \\
21 \\
46\end{array}$ & $\begin{array}{l}13 \\
18 \\
38\end{array}$ & $\begin{array}{l}12 \\
16 \\
32\end{array}$ & $\begin{array}{l}10 \\
14 \\
27\end{array}$ \\
\hline
\end{tabular}

Duration of daily flow for indicated periods

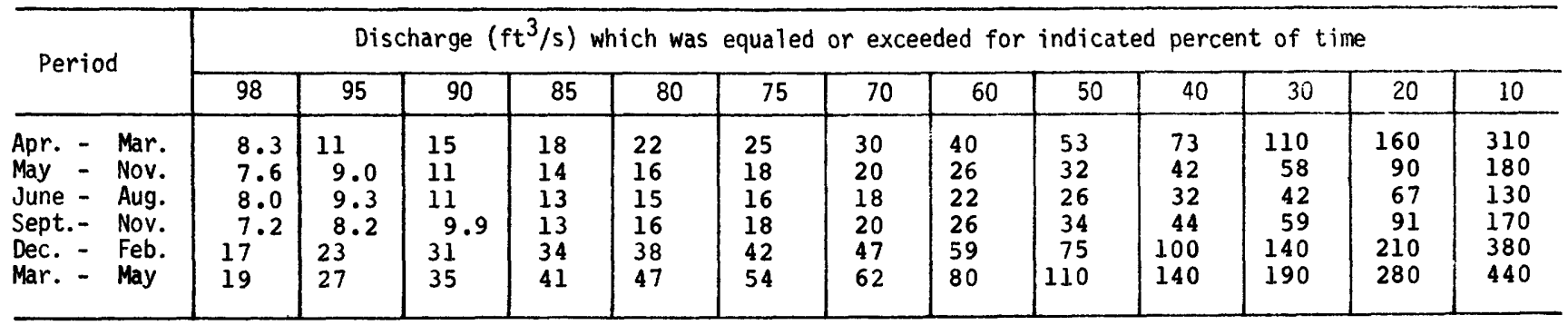


CUYAHOGA RIVER BASIN

04208000 Cuyahoga River at Independence, ohio

LOCATION: Lat $41^{\circ} 23^{\prime} 43^{\prime \prime}$, long $81^{\circ} 37^{\prime} 48^{\prime \prime}$, in T.6 N., R.12 W., Cuyahoga County, Hydrologic Unit 04110002 , on left bank $240 \mathrm{ft}$ downstream from bridge on old Rockside Road, $0.8 \mathrm{mi}$ northeast of Independence, and $3.0 \mathrm{mi}$ downstream from Tinkers Creek.

DRAINAGE AREA: $\quad 707 \mathrm{mi}^{2}$.

TRIBITARY TO: Lake Erie.

DISCHARGE DATA USED: October 1929 to December 1935, April 1940 to September 1978.

REMARKS :

Natural flow of stream affected by diversion, storage reservoirs and power plants. Some diversion from the Tuscarawas drainage into this basin at Portage Lakes. Water diverted into Ohio Canal at Brecksville, $6 \mathrm{mi}$ upstream from station, bypasses station. Theşe records do not include flow in canal except above about $15,000 \mathrm{ft} / \mathrm{s}$ when channels merge.

SELECTED DISCHARGE CHARACTERISTICS: Average discharge: $787 \mathrm{ft}^{3} / \mathrm{s}$ (44 years).

Minimum daily discharge: $21 \mathrm{ft}^{3} / \mathrm{s}$ August 1933.

Magnitude and frequency of low flow for indicated periods

\begin{tabular}{c|c|r|r|r|r|r}
\hline \multirow{2}{*}{ Period } & \multirow{2}{*}{$\begin{array}{c}\text { Number of } \\
\text { consecu- } \\
\text { tive days }\end{array}$} & \multicolumn{3}{|c|}{$\begin{array}{c}\text { Discharge ( } \mathrm{ft}^{3} / \mathrm{s} \text { ) for indicated } \\
\text { recurrence interval (years) }\end{array}$} \\
\cline { 3 - 7 } Apr.-Mar. & 2 & 5 & 10 & 20 & 50 \\
\hline \multirow{3}{*}{ May-Nov. } & 7 & 91 & 57 & 42 & 32 & 23 \\
& 30 & 109 & 77 & 63 & 53 & 44 \\
& 133 & 91 & 75 & 64 & 54 \\
& 7 & 90 & 56 & 42 & 32 & 23 \\
& 30 & 109 & 76 & 62 & 53 & 43 \\
& 132 & 90 & 75 & 64 & 54 \\
& 1 & 102 & 62 & 46 & 35 & 25 \\
& 7 & 126 & 84 & 67 & 55 & 44 \\
& 30 & 174 & 112 & 88 & 73 & 59 \\
\hline
\end{tabular}

\begin{tabular}{|c|c|c|c|c|c|c|}
\hline \multirow{2}{*}{ Period } & \multirow{2}{*}{$\begin{array}{l}\text { Number of } \\
\text { consecu- } \\
\text { tive days }\end{array}$} & \multicolumn{5}{|c|}{$\begin{array}{l}\text { Discharge }\left(\mathrm{ft}^{3} / \mathrm{s}\right) \text { for indicated } \\
\text { recurrence interval (years) }\end{array}$} \\
\hline & & 2 & 5 & 10 & 20 & 50 \\
\hline Sept. -Nov. & $\begin{array}{r}1 \\
7 \\
30\end{array}$ & $\begin{array}{r}91 \\
112 \\
151\end{array}$ & $\begin{array}{l}59 \\
80 \\
99\end{array}$ & $\begin{array}{l}47 \\
68 \\
81\end{array}$ & $\begin{array}{l}36 \\
60 \\
71\end{array}$ & $\begin{array}{l}27 \\
53 \\
61\end{array}$ \\
\hline Dec. -Feb. & $\begin{array}{r}1 \\
7 \\
30\end{array}$ & $\begin{array}{l}197 \\
233 \\
404\end{array}$ & $\begin{array}{l}113 \\
.141 \\
223\end{array}$ & $\begin{array}{r}85 \\
109 \\
165\end{array}$ & $\begin{array}{r}67 \\
89 \\
129\end{array}$ & $\begin{array}{l}51 \\
72 \\
99\end{array}$ \\
\hline Mar. - May & $\begin{array}{r}1 \\
7 \\
30\end{array}$ & $\begin{array}{l}244 \\
304 \\
636\end{array}$ & $\begin{array}{l}153 \\
199 \\
386\end{array}$ & $\begin{array}{l}117 \\
158 \\
282\end{array}$ & $\begin{array}{r}92 \\
131 \\
211\end{array}$ & $\begin{array}{r}70 \\
106 \\
148\end{array}$ \\
\hline
\end{tabular}

Duration of daily flow for indicated periods

\begin{tabular}{l|c|c|c|c|c|c|c|c|c|r|r|r|r}
\hline \multirow{2}{*}{ Period } & \multicolumn{8}{|c|}{ Discharge $\left(\mathrm{ft}^{3} / \mathrm{s}\right)$ which was equaled or exceeded for indicated percent of time } \\
\cline { 2 - 12 } & 98 & 95 & 90 & 85 & 80 & 75 & 70 & 60 & 50 & 40 & 30 & 20 & 10 \\
\hline Apr. - Mar. & 71 & 93 & 110 & 140 & 160 & 180 & 210 & 290 & 400 & 570 & 810 & 1200 & 1900 \\
May - Nov. & 62 & 79 & 100 & 110 & 130 & 140 & 150 & 190 & 240 & 310 & 420 & 620 & 1100 \\
June - Aug. & 59 & 79 & 100 & 120 & 130 & 140 & 160 & 190 & 230 & 290 & 370 & 520 & 850 \\
Sept. - Nov. & 60 & 75 & 90 & 100 & 110 & 120 & 130 & 160 & 190 & 240 & 320 & 460 & 820 \\
Dec. - Feb. & 110 & 140 & 180 & 220 & 260 & 300 & 350 & 460 & 610 & 800 & 1100 & 1500 & 2500 \\
Mar. - May & 170 & 220 & 300 & 390 & 480 & 550 & 640 & 800 & 1000 & 1200 & 1500 & 2000 & 2800 \\
\hline
\end{tabular}


Appendix 3.--Low-flow data--Continued

CUYAHOGA RIVER BASIN

04208900 Aurora Branch near Chagrin Falls, Ohio

LOCATION :

Lat $41^{\circ} 24^{\prime} 40^{\prime \prime}$, long $81^{\circ} 24^{\prime} 44^{\prime \prime}$, Cuyahoga County, Hydrologic

Unit 04l10003, at Solon Road bridge $1.6 \mathrm{mi}$ southwest of

Chagrin Falls and $1.1 \mathrm{mi}$ upstream from mouth.

DRAINAGE AREA: $\quad 57.4 \mathrm{mi}^{2}$.

TRIBUTARY TO: Lake Erie.

DISCHARGE DATA USED: LOW-flow measurements 1954, 1972-1977 water years.

INDEX STATION: $\quad 03093000$ Eagle Creek at Phalanx Station, Ohio.

REMARKS: None. SELECTED DISCHARGE CHARACTERISTICS: $\begin{gathered}\text { Minimum discharge observed: } 7.7 \mathrm{ft}^{3} / \mathrm{s} \\ \text { November } 1953 .\end{gathered}$

Magnitude and frequency of low flow for indicated periods

\begin{tabular}{c|c|c|c}
\hline \multirow{2}{*}{ Period } & $\begin{array}{c}\text { Number of } \\
\text { consecu- } \\
\text { tive days }\end{array}$ & \multicolumn{2}{|c}{$\begin{array}{c}\text { Discharge (ft } 3 / \mathrm{s} \text { ) for } \\
\text { indicated recurrence } \\
\text { interval (years) }\end{array}$} \\
\cline { 3 - 4 } Apr. - Mar. & 7 & 2 & 10 \\
\hline May - Nov. & 30 & 11 & 3.9 \\
& 7 & 7.4 & 5.6 \\
June - Aug. & 30 & 11 & 4.1 \\
& 7 & 9.3 & 4.5 \\
Sept. - Nov. & 30 & 15 & 7.3 \\
& 7 & 7.9 & 4.6 \\
& 30 & 13 & 6.5 \\
\hline
\end{tabular}

Duration of daily flow for indicated periods

\begin{tabular}{l|c|c|c|c|c}
\hline \multirow{2}{*}{ Period } & \multicolumn{5}{|c}{$\begin{array}{c}\text { Discharge ( } \mathrm{ft}^{3} / \mathrm{s} \text { ) which was equaled or } \\
\text { exceeded for indicated percent of time }\end{array}$} \\
\cline { 2 - 6 } & 98 & 95 & 90 & 85 & 80 \\
\hline Apr. - Mar. & 5.1 & 8.0 & 12 & 15 & 18 \\
May - Nov. & 4.4 & 6.2 & 9.3 & 11 & 13 \\
June - Aug. & 4.2 & 6.1 & 8.1 & 11 & 12 \\
Sept. - Nov. & 3.7 & 5.3 & 7.6 & 9.3 & 11 \\
\hline
\end{tabular}


CHAGRIN RIVER BASIN

04209000 Chagrin River at Willoughby, Ohio

LOCATION:

Lat 41.37'51", long $81^{\circ} 24^{\prime} 13^{\prime \prime}$, in T.9 N., R.10 W., Lake County, Hydrologic

Unit 04110003, on left bank, $150 \mathrm{ft}$ downstream from city waterworks dam,

$800 \mathrm{ft}$ downstream from East Branch, $1.0 \mathrm{mi}$ southeast of willoughby, and

$5.0 \mathrm{mi}$ upstream from mouth.

DRAINAGE AREA: $\quad 246 \mathrm{mi}^{2}$.

TRIBUTARY TO: Lake Erie.

DISCHARGE DATA USED: August 1925 to September 1935, October 1939 to September 1978.

REMARKS: Water diverted $200 \mathrm{ft}$ upstream from station for municipal supply of city of Willoughby.

SELECTED OISCHARGE CHARACTERISTICS: Average discharge: $324 \mathrm{ft}^{3} / \mathrm{s}$ (49,years).

Minimum daily discharge: $3.0 \mathrm{ft} / \mathrm{s}$ July 1934.

Magnitude and frequency of low flow for indicated periods

\begin{tabular}{|c|c|c|c|c|c|c|c|c|c|c|c|c|c|}
\hline \multirow{2}{*}{ Period } & \multirow{2}{*}{$\begin{array}{l}\text { Number of } \\
\text { consecu- } \\
\text { tive days }\end{array}$} & \multicolumn{5}{|c|}{$\begin{array}{l}\text { Discharge }\left(\mathrm{ft}^{3} / \mathrm{s}\right) \text { for indicated } \\
\text { recurrence interval (years) }\end{array}$} & \multirow{2}{*}{ Period } & \multirow{2}{*}{$\begin{array}{l}\text { Number of } \\
\text { consecu- } \\
\text { tive days }\end{array}$} & \multicolumn{5}{|c|}{$\begin{array}{l}\text { Discharge }\left(\mathrm{ft}^{3} / \mathrm{s}\right) \text { for indicated } \\
\text { recurrence interval (years) }\end{array}$} \\
\hline & & 2 & 5 & 10 & 20 & 50 & & & 2 & 5 & 30 & 20 & 50 \\
\hline Apr.-Mar. & $\begin{array}{r}7 \\
7 \\
30\end{array}$ & $\begin{array}{l}24 \\
26 \\
36\end{array}$ & $\begin{array}{l}14 \\
17 \\
24\end{array}$ & $\begin{array}{l}10 \\
13 \\
19\end{array}$ & $\begin{array}{l}7.3 \\
10 \\
16\end{array}$ & $\begin{array}{l}5.0 \\
8.2 \\
14\end{array}$ & Sept.-Nov. & $\begin{array}{r}1 \\
7 \\
30\end{array}$ & $\begin{array}{l}25 \\
28 \\
47\end{array}$ & $\begin{array}{l}16 \\
19 \\
27\end{array}$ & $\begin{array}{l}13 \\
16 \\
22\end{array}$ & $\begin{array}{l}11 \\
14 \\
19\end{array}$ & $\begin{array}{l}9.7 \\
13 \\
16\end{array}$ \\
\hline June-Aug. & $\begin{array}{r}1 \\
7 \\
30\end{array}$ & $\begin{array}{l}26 \\
30 \\
46\end{array}$ & $\begin{array}{l}15 \\
19 \\
29\end{array}$ & $\begin{array}{l}11 \\
14 \\
23\end{array}$ & $\begin{array}{l}7.6 \\
11 \\
19\end{array}$ & $\begin{array}{l}5.0 \\
8.3 \\
16\end{array}$ & Mar. - May & $\begin{array}{r}1 \\
7 \\
30\end{array}$ & $\begin{array}{r}82 \\
98 \\
217\end{array}$ & $\begin{array}{r}59 \\
70 \\
139\end{array}$ & $\begin{array}{r}49 \\
58 \\
108\end{array}$ & $\begin{array}{l}41 \\
50 \\
88\end{array}$ & $\begin{array}{l}33 \\
42 \\
68\end{array}$ \\
\hline
\end{tabular}

Duration of daily flow for indicated periods

\begin{tabular}{|c|c|c|c|c|c|c|c|c|c|c|c|c|c|c|}
\hline \multirow{2}{*}{\multicolumn{2}{|c|}{ Period }} & \multicolumn{13}{|c|}{ Discharge $\left(\mathrm{ft}^{3} / \mathrm{s}\right)$ which was equaled or exceeded for indicated percent of time } \\
\hline & & 98 & 95 & 90 & 85 & 80 & 75 & 70 & 60 & 50 & 40 & 30 & 20 & 10 \\
\hline $\begin{array}{l}\text { Apr. - } \\
\text { May - } \\
\text { June - } \\
\text { Sept. - } \\
\text { Dec. - } \\
\text { Mar. - }\end{array}$ & $\begin{array}{l}\text { Mar. } \\
\text { Nov. } \\
\text { Aug. } \\
\text { Nov. } \\
\text { Feb. } \\
\text { May }\end{array}$ & $\begin{array}{l}20 \\
18 \\
15 \\
18 \\
49 \\
65\end{array}$ & $\begin{array}{l}26 \\
22 \\
21 \\
22 \\
61 \\
83\end{array}$ & $\begin{array}{r}33 \\
27 \\
25 \\
26 \\
80 \\
110\end{array}$ & $\begin{array}{r}41 \\
31 \\
29 \\
30 \\
95 \\
130\end{array}$ & $\begin{array}{r}50 \\
36 \\
33 \\
34 \\
110 \\
140\end{array}$ & $\begin{array}{r}60 \\
41 \\
38 \\
37 \\
130 \\
160\end{array}$ & $\begin{array}{r}73 \\
46 \\
42 \\
42 \\
140 \\
180\end{array}$ & $\begin{array}{r}100 \\
60 \\
52 \\
54 \\
180 \\
230\end{array}$ & $\begin{array}{r}140 \\
77 \\
64 \\
70 \\
210 \\
300\end{array}$ & $\begin{array}{r}190 \\
100 \\
79 \\
96 \\
270 \\
390\end{array}$ & $\begin{array}{r}260 \\
140 \\
99 \\
130 \\
370 \\
510\end{array}$ & $\begin{array}{l}410 \\
200 \\
140 \\
200 \\
550 \\
710\end{array}$ & $\begin{array}{r}740 \\
380 \\
250 \\
370 \\
1000 \\
1200\end{array}$ \\
\hline
\end{tabular}




\section{Appendix 3.--Low-flow data--Continued}

GRAND RIVER BASIN

04210000 Phelps Creek near Windsor, Ohio

LOCATION:

Lat $41^{\circ} 30^{\prime} 55^{\prime \prime}$, long $80^{\circ} 56^{\prime} 05^{\prime \prime}$, in T.8 N., R.5 W., Ashtabula County, Hydrologic Unit 04110004 , on left bank at upstream side of bridge on State koute $534,1.4 \mathrm{mi}$ south of Windsor and $1.5 \mathrm{mi}$ upstream from station.

DRAINAGE AREA: $\quad 25.6 \mathrm{mi}^{2}$.

TRIBITARY TO: Grand River.

DISCHARGE DATA USED: May 1942 to June 1959.

REMARKS:

None.

SELECTED DISCHARGE CHARACTERISTICS: Average discharge: $35.6 \mathrm{ft}^{3} / \mathrm{s}$ (16 years). Minimum daily discharge: No flow August 1943.

Magnitude and frequency of low flow for indicated periods

\begin{tabular}{c|c|c|c|c|l|l}
\hline \multirow{2}{*}{ Period } & \multirow{2}{*}{$\begin{array}{c}\text { Number of } \\
\text { consecu- } \\
\text { tive days }\end{array}$} & \multicolumn{4}{|c|}{$\begin{array}{c}\text { Discharge } \\
\text { recurrence } 3 / \mathrm{s} \text { ) for interval } \begin{array}{l}\text { indicated } \\
\text { (years) }\end{array}\end{array}$} \\
\cline { 3 - 7 } Apr.-Mar. & 1 & 0.4 & 0.2 & 0.1 & 0 & 0 \\
& 7 & 0.5 & 0.3 & 0.2 & 0.2 & 0.1 \\
& 30 & 0.7 & 0.4 & 0.4 & 0.3 & 0.3 \\
May-Nov. & 1 & 0.4 & 0.2 & 0.1 & 0 & 0 \\
& 7 & 0.5 & 0.3 & 0.2 & 0.2 & 0.1 \\
& 30 & 0.7 & 0.5 & 0.4 & 0.3 & 0.3 \\
& 1 & 0.5 & 0.3 & 0.3 & 0 & 0 \\
& 7 & 0.6 & 0.3 & 0.2 & 0.2 & 0.1 \\
& 30 & 1.0 & 0.6 & 0.4 & 0.4 & 0.3 \\
\hline
\end{tabular}

\begin{tabular}{|c|c|c|c|c|c|c|}
\hline \multirow{2}{*}{ Period } & \multirow{2}{*}{$\begin{array}{l}\text { Number of } \\
\text { consecu- } \\
\text { tive days }\end{array}$} & \multicolumn{5}{|c|}{$\begin{array}{l}\text { Discharge }\left(\mathrm{ft}^{3} / \mathrm{s}\right) \text { for indicated } \\
\text { recurrence interval (years) }\end{array}$} \\
\hline & & 2 & 5 & 10 & 20 & 50 \\
\hline Sept.-Nov. & $\begin{array}{r}1 \\
7 \\
30\end{array}$ & $\begin{array}{l}0.5 \\
0.5 \\
0.8\end{array}$ & $\begin{array}{l}0.3 \\
0.3 \\
0.5\end{array}$ & $\begin{array}{l}0.2 \\
0.3 \\
0.4\end{array}$ & $\begin{array}{l}0.2 \\
0.3 \\
0.4\end{array}$ & $\begin{array}{l}0.1 \\
0.2 \\
0.3\end{array}$ \\
\hline Dec.-Feb. & $\begin{array}{r}1 \\
7 \\
30\end{array}$ & $\begin{array}{l}3.2 \\
4.2 \\
13\end{array}$ & $\begin{array}{l}1.8 \\
2.2 \\
5.7\end{array}$ & $\begin{array}{l}1.3 \\
1.6 \\
3.6\end{array}$ & $\begin{array}{l}1.0 \\
1.2 \\
2.4\end{array}$ & $\begin{array}{l}0.7 \\
0.8 \\
1.5\end{array}$ \\
\hline Mar.- May & $\begin{array}{r}1 \\
7 \\
30\end{array}$ & $\begin{array}{l}2.7 \\
3.5 \\
17\end{array}$ & $\begin{array}{l}1.7 \\
2.0 \\
9.4\end{array}$ & $\begin{array}{l}1.3 \\
1.5 \\
6.5\end{array}$ & $\begin{array}{l}0.9 \\
1.1 \\
4.7\end{array}$ & $\begin{array}{l}0.6 \\
0.8 \\
3.2\end{array}$ \\
\hline
\end{tabular}

Duration of daily flow for indicated periods

\begin{tabular}{|c|c|c|c|c|c|c|c|c|c|c|c|c|c|}
\hline \multirow{2}{*}{ Period } & \multicolumn{13}{|c|}{ Discharge $\left(\mathrm{ft}^{3} / \mathrm{s}\right)$ which was equaled or exceeded for indicated percent of time } \\
\hline & 98 & 95 & 90 & 85 & 80 & 75 & 70 & 60 & 50 & 40 & 30 & $2 C$ & 10 \\
\hline $\begin{array}{l}\text { Apr. - Mar. } \\
\text { May - Nov. } \\
\text { June - Aug. } \\
\text { Sept. - Nov. } \\
\text { Dec. - Feb. } \\
\text { Mar. - May }\end{array}$ & $\begin{array}{l}0.4 \\
0.4 \\
0.4 \\
0.4 \\
1.8 \\
1.8\end{array}$ & $\begin{array}{l}0.5 \\
0.5 \\
0.5 \\
0.4 \\
2.4 \\
3.2\end{array}$ & $\begin{array}{l}0.7 \\
0.6 \\
0.6 \\
0.5 \\
3.2 \\
4.0\end{array}$ & $\begin{array}{l}0.9 \\
0.7 \\
0.7 \\
0.6 \\
4.2 \\
5.5\end{array}$ & $\begin{array}{l}1.2 \\
0.8 \\
0.8 \\
0.7 \\
5.2 \\
7.5\end{array}$ & $\begin{array}{l}1.6 \\
1.0 \\
0.9 \\
0.8 \\
6.5 \\
10\end{array}$ & $\begin{array}{l}2.1 \\
1.2 \\
1.1 \\
0.9 \\
8.1 \\
13\end{array}$ & $\begin{array}{l}3.5 \\
1.6 \\
1.4 \\
1.3 \\
12 \\
18\end{array}$ & $\begin{array}{l}6.0 \\
2.3 \\
1.9 \\
1.9 \\
16 \\
24\end{array}$ & $\begin{array}{l}11 \\
3.4 \\
2.4 \\
2.9 \\
24 \\
33\end{array}$ & $\begin{array}{l}19 \\
5.4 \\
3.2 \\
4.9 \\
37 \\
49\end{array}$ & $\begin{array}{l}35 \\
11 \\
5.2 \\
8.7 \\
64 \\
78\end{array}$ & $\begin{array}{r}85 \\
28 \\
15 \\
22 \\
150 \\
160\end{array}$ \\
\hline
\end{tabular}


GRAND RIVER BASIN

04212000 Grand River near Madison, Ohio

LOCATION:

Lat $41^{\circ} 44^{\prime \prime 2} 6^{\prime \prime}$, long $81^{\circ} 02^{\prime} 48^{\prime \prime}$, Lake County, Hydrologic Unit

04110004 , on downstream end of center pier of abandoned high-

way bridge $800 \mathrm{ft}$ upstream from State Route $528,0.8 \mathrm{mi}$ upstream

from Griswold Creek and $2.1 \mathrm{mi}$ south of Madison.

DRAINAGE AREA: $\quad 581 \mathrm{mi}^{2}$.

TRIBUTARY TO: Lake Erie.

DISCHARGE DATA USED: October 1922 to September 1955, April 1938 to September 1974.

REMARKS: None.

SELECTED DISCHARGE CHARACTERISTICS: Average discharge: $663 \mathrm{ft}^{3} / \mathrm{s}$ ( 49 years).

Minimum daily discharge: No flow July, August 1934, October 1963.

Magnitude and frequency of low flow for indicated periods

\begin{tabular}{|c|c|c|c|c|c|c|c|c|c|c|c|c|c|}
\hline \multirow{2}{*}{ Period } & \multirow{2}{*}{$\begin{array}{l}\text { Number of } \\
\text { consecu- } \\
\text { tive days }\end{array}$} & \multicolumn{5}{|c|}{$\begin{array}{l}\text { Discharge }\left(\mathrm{ft}^{3} / \mathrm{s}\right) \text { for indicated } \\
\text { recurrence interval (years) }\end{array}$} & \multirow{2}{*}{ Period } & \multirow{2}{*}{$\begin{array}{l}\text { Number of } \\
\text { consecu- } \\
\text { tive days }\end{array}$} & \multicolumn{5}{|c|}{$\begin{array}{l}\text { Discharge ( } \mathrm{ft}^{3} ; \mathrm{s} \text { ) for indicated } \\
\text { recurrerice interval (years) }\end{array}$} \\
\hline & & 2 & 5 & 10 & 20 & 50 & & & 2 & 5 & 10 & 20 & 50 \\
\hline Apr.-Mar. & $\begin{array}{r}1 \\
7 \\
30\end{array}$ & $\begin{array}{l}3.8 \\
5.4 \\
9.5\end{array}$ & $\begin{array}{l}1.1 \\
1.8 \\
4.4\end{array}$ & $\begin{array}{l}0.4 \\
0.9 \\
2.8\end{array}$ & $\begin{array}{l}0.1 \\
0.4 \\
1.9\end{array}$ & $\begin{array}{l}0 \\
0 \\
1.1\end{array}$ & Sept.-Nov. & $\begin{array}{r}1 \\
7 \\
30\end{array}$ & $\begin{array}{l}5.2 \\
6.2 \\
13\end{array}$ & $\begin{array}{l}1.7 \\
2.7 \\
5.0\end{array}$ & $\begin{array}{l}0.7 \\
1.7 \\
3.4\end{array}$ & $\begin{array}{l}0.3 \\
1.1 \\
2.7\end{array}$ & $\begin{array}{l}0 \\
0 \\
2.1\end{array}$ \\
\hline June-Aug. & $\begin{array}{r}1 \\
7 \\
30\end{array}$ & $\begin{array}{r}6.6 \\
9.0 \\
19\end{array}$ & $\begin{array}{l}2.1 \\
3.1 \\
8.4\end{array}$ & $\begin{array}{l}0.9 \\
1.5 \\
5.8\end{array}$ & $\begin{array}{l}0.4 \\
0.8 \\
4.2\end{array}$ & $\begin{array}{l}0 \\
0.3 \\
3.2\end{array}$ & Mar. - May & $\begin{array}{r}1 \\
7 \\
30\end{array}$ & $\begin{array}{r}62 \\
85 \\
322\end{array}$ & $\begin{array}{r}33 \\
45 \\
144\end{array}$ & $\begin{array}{l}23 \\
32 \\
89\end{array}$ & $\begin{array}{l}17 \\
24 \\
59\end{array}$ & $\begin{array}{l}12 \\
17 \\
36\end{array}$ \\
\hline
\end{tabular}

Duration of daily flow for indicated periods

\begin{tabular}{|c|c|c|c|c|c|c|c|c|c|c|c|c|c|}
\hline \multirow{2}{*}{ Period } & \multicolumn{13}{|c|}{ Discharge $\left(\mathrm{ft}^{3} / \mathrm{s}\right)$ which was equaled or exceeded for indicated percent of time } \\
\hline & 98 & 95 & 90 & 85 & 80 & 75 & 70 & 60 & 50 & 40 & 30 & 20 & 10 \\
\hline $\begin{array}{l}\text { Apr. - Mar. } \\
\text { Hay - Nov. } \\
\text { June - Aug. } \\
\text { Sept. - Nov. } \\
\text { Dec. - Feb. } \\
\text { Mar. - May }\end{array}$ & $\begin{array}{l}3.6 \\
2.2 \\
2.2 \\
1.8 \\
32 \\
36\end{array}$ & $\begin{array}{r}6.5 \\
4.6 \\
4.9 \\
3.8 \\
52 \\
60\end{array}$ & $\begin{array}{l}11 \\
7.3 \\
7.8 \\
5.8 \\
84 \\
95\end{array}$ & $\begin{array}{c}15 \\
9.9 \\
11 \\
7.7 \\
130 \\
130\end{array}$ & $\begin{array}{c}21 \\
12 \\
13 \\
9.6 \\
160 \\
170\end{array}$ & $\begin{array}{r}29 \\
15 \\
15 \\
11 \\
200 \\
220\end{array}$ & $\begin{array}{r}41 \\
18 \\
18 \\
14 \\
240 \\
270\end{array}$ & $\begin{array}{r}87 \\
27 \\
25 \\
19 \\
330 \\
390\end{array}$ & $\begin{array}{r}170 \\
42 \\
35 \\
28 \\
460 \\
590\end{array}$ & $\begin{array}{r}300 \\
72 \\
51 \\
47 \\
680 \\
910\end{array}$ & $\begin{array}{r}520 \\
130 \\
79 \\
100 \\
1000 \\
1300\end{array}$ & $\begin{array}{r}1000 \\
270 \\
130 \\
260 \\
1600 \\
1900\end{array}$ & $\begin{array}{r}2000 \\
810 \\
340 \\
830 \\
2900 \\
3000\end{array}$ \\
\hline
\end{tabular}


Appendix 3.--Low-flow data--Continued

ASHTABULA RIVER BASIN

04212500 Ashtabula River near Ashtabula, Ohio

LOCATION:

Lat $41^{\circ} 51^{\prime} 20^{\prime \prime}$, long $80^{\circ} 45^{\prime} 44^{\prime \prime}$, Ashtabula County, Hydrologic unit 04110003 , on left bank at downstream side of State Road bridge, $1.1 \mathrm{mi}$ upstream from Hubbard Run, $1.3 \mathrm{mi}$ southeast of Ashtabula, and $5.5 \mathrm{mi}$ upstream from mouth.

DRAINAGE AREA: $\quad 121 \mathrm{mi}^{2}$.

TRIBUTARY TO: Lake Erie.

DISCHARGE DATA USED: August 1924 to December 1935, March 1939 to October 1947 ,

REMARKS: April 1950 to September 1978 .

None.

SELECTED DISCHARGE CHARACTERISTICS: Average discharge: $154 \mathrm{ft}^{3} / \mathrm{s}$ (47 years).

Minimum daily discharge: No flow at times during most years.

Magnitude and frequency of low flow for iridicated periods

\begin{tabular}{|c|c|c|c|c|c|c|}
\hline \multirow{2}{*}{ Period } & \multirow{2}{*}{$\begin{array}{l}\text { Number of } \\
\text { consecu- } \\
\text { tive days }\end{array}$} & \multicolumn{5}{|c|}{$\begin{array}{l}\text { Discharge }\left(\mathrm{ft}^{3} / \mathrm{s}\right) \text { for indicated } \\
\text { recurrence interval (years) }\end{array}$} \\
\hline & & 2 & 5 & 10 & 20 & 50 \\
\hline Apr.-Mar. & $\begin{array}{r}1 \\
7 \\
30\end{array}$ & $\begin{array}{l}0 \\
0 \\
0.4\end{array}$ & $\begin{array}{l}0 \\
0 \\
0\end{array}$ & $\begin{array}{l}0 \\
0 \\
0\end{array}$ & $\begin{array}{l}0 \\
0 \\
0\end{array}$ & $\begin{array}{l}0 \\
0 \\
0\end{array}$ \\
\hline May- Nov. & $\begin{array}{r}1 \\
7 \\
30\end{array}$ & $\begin{array}{l}0 \\
0 \\
0.4\end{array}$ & $\begin{array}{l}0 \\
0 \\
0\end{array}$ & $\begin{array}{l}0 \\
0 \\
0\end{array}$ & $\begin{array}{l}0 \\
0 \\
0\end{array}$ & $\begin{array}{l}0 \\
0 \\
0\end{array}$ \\
\hline June-Aug. & $\begin{array}{r}1 \\
7 \\
30\end{array}$ & $\begin{array}{l}0 \\
0.1 \\
1.5\end{array}$ & $\begin{array}{l}0 \\
0 \\
0.2\end{array}$ & $\begin{array}{l}0 \\
0 \\
0\end{array}$ & $\begin{array}{l}0 \\
0 \\
0\end{array}$ & $\begin{array}{l}0 \\
0 \\
0\end{array}$ \\
\hline
\end{tabular}

\begin{tabular}{|c|c|c|c|c|c|c|}
\hline \multirow{2}{*}{ Period } & \multirow{2}{*}{$\begin{array}{l}\text { Number of } \\
\text { consecu- } \\
\text { tive days }\end{array}$} & \multicolumn{5}{|c|}{$\begin{array}{l}\text { Discharge }\left(\mathrm{ft}^{3} / \mathrm{s}\right) \text { for indicated } \\
\text { recurrence interval (years) }\end{array}$} \\
\hline & & 2 & 5 & 10 & 20 & 50 \\
\hline Sept.-Nov. & $\begin{array}{r}1 \\
7 \\
30\end{array}$ & $\begin{array}{l}0.2 \\
0.3 \\
1.5\end{array}$ & $\begin{array}{l}0 \\
0 \\
0.2\end{array}$ & $\begin{array}{l}0 \\
0 \\
0\end{array}$ & $\begin{array}{l}0 \\
0 \\
0\end{array}$ & $\begin{array}{l}0 \\
0 \\
0\end{array}$ \\
\hline Dec. -Feb. & $\begin{array}{r}1 \\
7 \\
30\end{array}$ & $\begin{array}{l}24 \\
31 \\
89\end{array}$ & $\begin{array}{l}12 \\
17 \\
48\end{array}$ & $\begin{array}{l}8.2 \\
12 \\
34\end{array}$ & $\begin{array}{c}5.7 \\
9.2 \\
25\end{array}$ & $\begin{array}{l}3.6 \\
6.4 \\
18\end{array}$ \\
\hline Mar. - May & $\begin{array}{r}1 \\
7 \\
30\end{array}$ & $\begin{array}{l}8.8 \\
13 \\
62\end{array}$ & $\begin{array}{c}4.0 \\
6.2 \\
26\end{array}$ & $\begin{array}{c}2.6 \\
4.1 \\
16\end{array}$ & $\begin{array}{c}1.7 \\
2.9 \\
10\end{array}$ & $\begin{array}{l}1.1 \\
2.0 \\
6.0\end{array}$ \\
\hline
\end{tabular}

Duration of daily flow for indicated periods

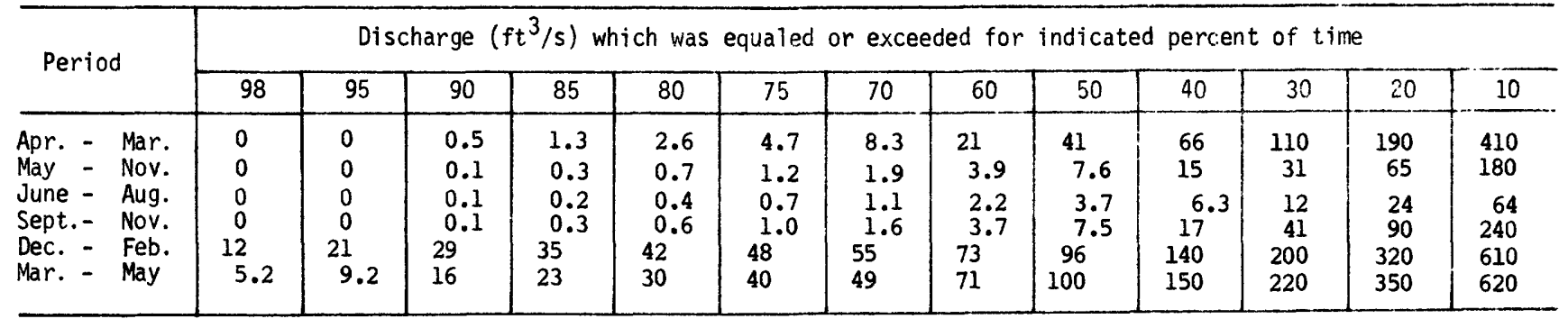


04213000 Conneaut Creek at Conneaut, Ohio

LOCATION: Lat $41^{\circ} 55^{\prime} 37^{\prime \prime}$, long $80^{\circ} 36^{\prime} 15^{\prime \prime}$, Ashtabula County, Hydrologic Unit 04120101 , on right bank at downstream side of Keefus Road bridge at Conneaut, and $6.4 \mathrm{mi}$ upstream from mouth.

DRAINAGE AREA: $\quad 175 \mathrm{mi}^{2}$.

TRIBLTARY TO: Lake Erie.

DISCHARGE DATA USED: July 1922 to December 1935, April 1950 to September 1978.

REMARKS: None.

SELECTED DISCHARGE CHARACTERISTICS: Average discharge: $261 \mathrm{ft}^{3} / \mathrm{s}$.

Minimum daily discharge: $0.3 \mathrm{ft}^{3} / \mathrm{s} \mathrm{July}$, August 1933, August 1934.

Magnitude and frequency of low flow for indicated periods

\begin{tabular}{|c|c|c|c|c|c|c|c|c|c|c|c|c|c|}
\hline \multirow{2}{*}{ Period } & \multirow{2}{*}{$\begin{array}{l}\text { Number of } \\
\text { consecu- } \\
\text { tive days }\end{array}$} & \multicolumn{5}{|c|}{$\begin{array}{l}\text { Discharge }\left(\mathrm{ft}^{3} / \mathrm{s}\right) \text { for indicated } \\
\text { recurrence interval (years) }\end{array}$} & \multirow{2}{*}{ Period } & \multirow{2}{*}{$\begin{array}{l}\text { Number of } \\
\text { consecu- } \\
\text { tive days }\end{array}$} & \multicolumn{5}{|c|}{$\begin{array}{l}\text { Discharge }\left(\mathrm{ft}^{3} / \mathrm{s}\right) \text { for indicated } \\
\text { recurrence interval (years) }\end{array}$} \\
\hline & & 2 & 5 & 10 & 20 & 50 & & & 2 & 5 & 10 & 20 & 50 \\
\hline Apr.-Mar. & $\begin{array}{r}1 \\
7 \\
30\end{array}$ & $\begin{array}{l}5.0 \\
5.9 \\
9.7\end{array}$ & $\begin{array}{l}2.1 \\
2.8 \\
5.1\end{array}$ & $\begin{array}{l}1.2 \\
1.9 \\
3.6\end{array}$ & $\begin{array}{l}0.7 \\
1.3 \\
2.8\end{array}$ & $\begin{array}{l}0.3 \\
0.8 \\
2.0\end{array}$ & Sept.-Nov. & $\begin{array}{r}1 \\
7 \\
30\end{array}$ & $\begin{array}{l}5.9 \\
6.8 \\
14\end{array}$ & $\begin{array}{l}2.6 \\
3.5 \\
6.3\end{array}$ & $\begin{array}{l}1.7 \\
2.6 \\
4.5\end{array}$ & $\begin{array}{l}1.2 \\
2.1 \\
3.6\end{array}$ & $\begin{array}{l}0.8 \\
1.7 \\
2.9\end{array}$ \\
\hline June-Aug. & $\begin{array}{r}1 \\
7 \\
30\end{array}$ & $\begin{array}{r}5.8 \\
7.2 \\
12\end{array}$ & $\begin{array}{l}2.4 \\
3.4 \\
5.9\end{array}$ & $\begin{array}{l}1.3 \\
2.2 \\
4.1\end{array}$ & $\begin{array}{l}0.8 \\
1.5 \\
3.1\end{array}$ & $\begin{array}{l}0.4 \\
0.9 \\
2.3\end{array}$ & Mar. - May & $\begin{array}{r}1 \\
7 \\
30\end{array}$ & $\begin{array}{r}41 \\
48 \\
141\end{array}$ & $\begin{array}{l}23 \\
30 \\
74\end{array}$ & $\begin{array}{l}16 \\
23 \\
51\end{array}$ & $\begin{array}{l}11 \\
18 \\
37\end{array}$ & $\begin{array}{l}7.2 \\
14\end{array}$ \\
\hline
\end{tabular}

Duration of daily flow for indicated periods

\begin{tabular}{|c|c|c|c|c|c|c|c|c|c|c|c|c|c|}
\hline \multirow{2}{*}{ Period } & \multicolumn{13}{|c|}{ Discharge $\left(\mathrm{ft}^{3} / \mathrm{s}\right)$ which was equaled or exceeded for indicated percent of time } \\
\hline & 98 & 95 & 90 & 85 & 80 & 75 & 70 & 60 & 50 & 40 & 30 & 20 & 10 \\
\hline $\begin{array}{l}\text { Apr. - Mar. } \\
\text { May - Nov. } \\
\text { June - Aug. } \\
\text { Sept. - Nov. } \\
\text { Dec. - Feb. } \\
\text { Mar. - May }\end{array}$ & $\begin{array}{l}3.7 \\
2.8 \\
2.3 \\
3.1 \\
36 \\
25\end{array}$ & $\begin{array}{l}5.8 \\
4.4 \\
3.9 \\
4.3 \\
49 \\
38\end{array}$ & $\begin{array}{l}9.2 \\
6.3 \\
5.7 \\
5.9 \\
64 \\
54\end{array}$ & $\begin{array}{c}13 \\
8.3 \\
7.3 \\
7.4 \\
76 \\
68\end{array}$ & $\begin{array}{l}18 \\
10 \\
9.1 \\
9.1 \\
89 \\
81\end{array}$ & $\begin{array}{r}24 \\
13 \\
11 \\
11 \\
100 \\
96\end{array}$ & $\begin{array}{r}33 \\
15 \\
13 \\
14 \\
120 \\
110\end{array}$ & $\begin{array}{r}59 \\
22 \\
17 \\
19 \\
150 \\
150\end{array}$ & $\begin{array}{r}93 \\
31 \\
22 \\
28 \\
190 \\
200\end{array}$ & $\begin{array}{r}140 \\
48 \\
30 \\
46 \\
260 \\
270\end{array}$ & $\begin{array}{r}210 \\
78 \\
41 \\
89 \\
360 \\
370\end{array}$ & $\begin{array}{r}340 \\
130 \\
65 \\
170 \\
550 \\
550\end{array}$ & $\begin{array}{r}690 \\
300 \\
120 \\
400 \\
1100 \\
970\end{array}$ \\
\hline
\end{tabular}



war $+4,4$

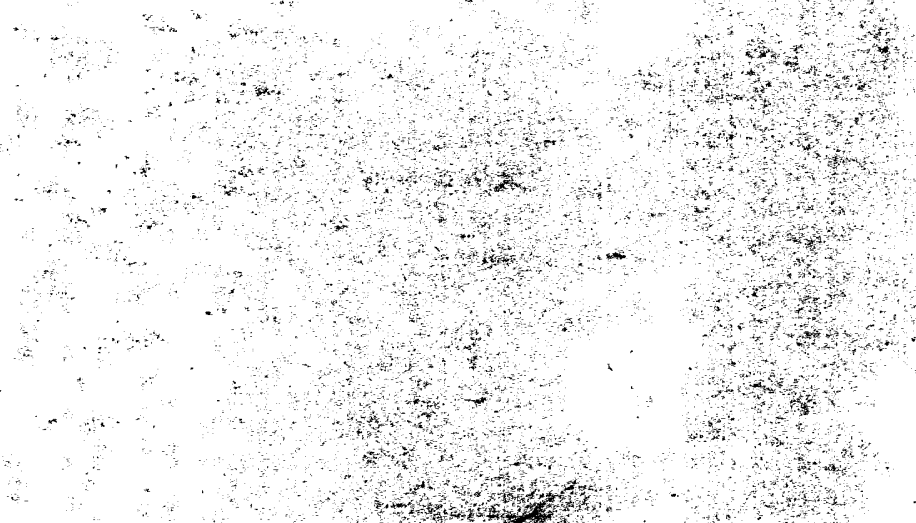

7.

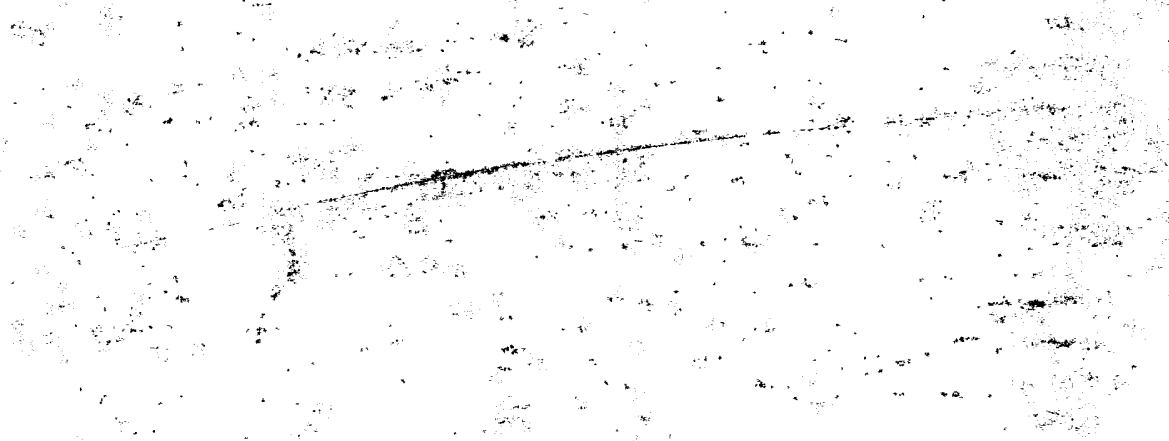

ntwits

$$
\text { - }
$$
thes

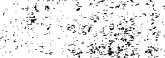

4

7

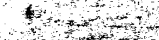

$+4+6$,

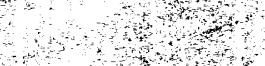

